\title{
MODELING OF DUCTILE FRACTURE \\ IN STEEL STRUCTURES \\ FOR MONOTONIC AND CYCLIC LOADING
}

\author{
A thesis submitted to \\ the Faculty of Graduate and Postdoctoral Affairs \\ in partial fulfillment of the requirements for the degree of \\ Doctor of Philosophy \\ in
}

Civil Engineering

by

Zhiqi Wen

Department of Civil and Environmental Engineering

Carleton University

Ottawa, Ontario

Ottawa-Carleton Institute of Civil and Environmental Engineering

May, 2012

(C) 2012 Zhiqi Wen 
Library and Archives

Canada

Published Heritage

Branch

395 Wellington Street

Ottawa ON K1A ON4

Canada
Bibliothèque et

Archives Canada

Direction du

Patrimoine de l'édition

395 , rue Wellington

Ottawa ON K1A ON4

Canada
Your file Votre référence

ISBN: 978-0-494-89312-8

Our file Notre référence

ISBN: $978-0-494-89312-8$

\section{NOTICE:}

The author has granted a nonexclusive license allowing Library and Archives Canada to reproduce, publish, archive, preserve, conserve, communicate to the public by telecommunication or on the Internet, loan, distrbute and sell theses worldwide, for commercial or noncommercial purposes, in microform, paper, electronic and/or any other formats.

The author retains copyright ownership and moral rights in this thesis. Neither the thesis nor substantial extracts from it may be printed or otherwise reproduced without the author's permission.
AVIS:

L'auteur a accordé une licence non exclusive permettant à la Bibliothèque et Archives Canada de reproduire, publier, archiver, sauvegarder, conserver, transmettre au public par télécommunication ou par l'Internet, prêter, distribuer et vendre des thèses partout dans le monde, à des fins commerciales ou autres, sur support microforme, papier, électronique et/ou autres formats.

L'auteur conserve la propriété du droit d'auteur et des droits moraux qui protege cette thèse. $\mathrm{Ni}$ la thèse ni des extraits substantiels de celle-ci ne doivent être imprimés ou autrement reproduits sans son autorisation.
In compliance with the Canadian Privacy Act some supporting forms may have been removed from this thesis.

While these forms may be included in the document page count, their removal does not represent any loss of content from the thesis.
Conformément à la loi canadienne sur la protection de la vie privée, quelques formulaires secondaires ont été enlevés de cette thèse.

Bien que ces formulaires aient inclus dans la pagination, il n'y aura aucun contenu manquant. 


\begin{abstract}
The performance of structures is greatly dependent on the material behaviour. In order to properly assess the performance, a structure has to be evaluated against a likely range of possible material behaviour. A combined experimental and numerical study has been carried out to develop procedures to generate and calibrate material model parameters to simulate the behaviour and predict fracture of steel structures and structural components under monotonic and cyclic loading. Round specimens of two steel grades with mainly three shape profiles have been tested under a variety of monotonic and cyclic loading conditions in the study. The combined isotropic and nonlinear kinematic hardening model by Chaboche has been found to be suitable for modeling the behaviour of steel based on results of the tests. A systematic procedure has been developed to determine the parameters for the plasticity model to be used in the numerical simulations. A modified Huang and Mahin fracture criterion for predicting fracture of steel subjected to monotonic tension as well as cyclic loading has also been established and validated against the test results of the round specimens.
\end{abstract}

The established procedures to calibrate and generate the material model parameters have been further adapted to study performance of a steel strap tension-only brace. The adapted procedures are able to calibrate and generate the material model parameters for the numerical simulations using only data of monotonic tension material tests when compared to and validated against results of the steel strap tension-only brace 
specimens tested by others. Using the validated procedures and FEA models, a parametric finite element analysis study has been carried out to study the performance of the steel strap tension-only brace for a range of material behaviour using the generated material model parameters and various geometric configurations. Based on results of the study, equations to predict the ductility of the steel strap have been proposed and recommendations on designing steel strap tension-only braces in a Seismic Force Resisting System have been developed. 


\section{ACKNOWLEDGEMENT}

This research project is funded by Steel Structures Education Foundation and the Natural Sciences and Engineering Research Council of Canada.

I would like to thank my supervisor, Dr. Heng-Aik Khoo, for his great guidance, advice and support throughout this research.

I really appreciate the professional help from Stanley Conley and other technicians of the Civil and Environmental Engineering Laboratories at Carleton University.

Thanks should also go to Jianwei Chen, Zhe Tan, Ruogang Zhao and Yu Kang, who have provided valuable suggestions.

Last but not least, I am very grateful to my parents, my sister and my wife for their endless love and encouragement during the period of this research. 
ABSTRACT …...................................................................................................... ii

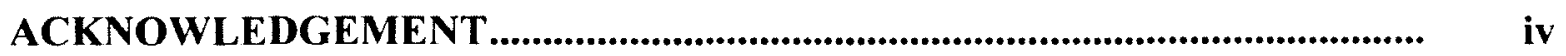

TABLE OF CONTENTS.......................................................................................... v

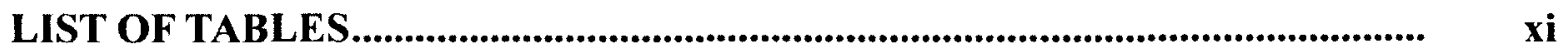

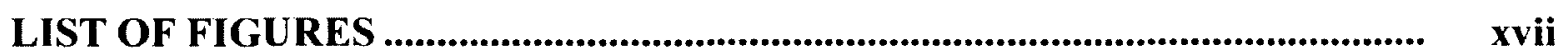

LIST OF SYMBOLS AND ABBREVIATIONS .................................................. x xli

CHAPTER 1 INTRODUCTION ................................................................

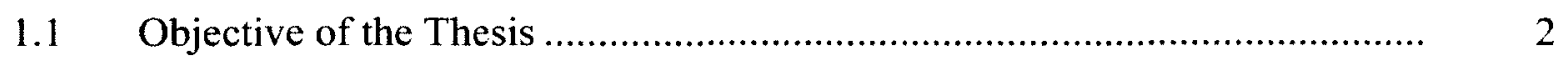

$1.2 \quad$ Methodology Used in the Research ................................................ 3

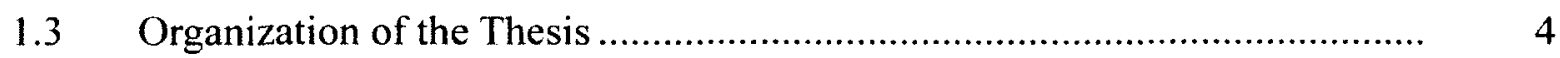

CHAPTER 2 LITERATURE REVIEW ................................................. 7

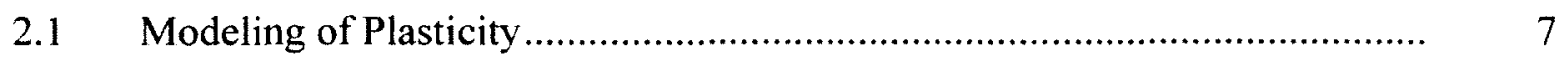

2.1.1 Hardening Models ........................................................... 9

2.1.2 Summary of Combined Isotropic and Kinematic Hardening Model 15

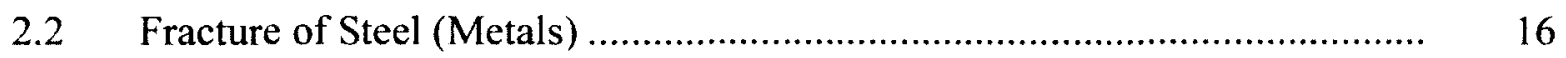

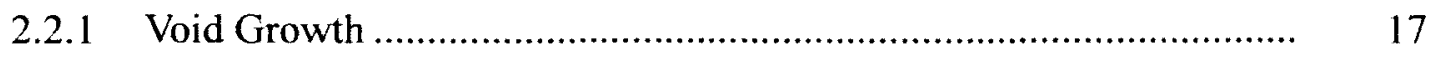

2.2.2 Void Volume Fraction .................................................... 21

2.2.3 Absorbed Strain Energy Density ............................................. 22 
2.2.4 Continuum Damage Mechanics ......................................... 22

2.2.5 Other Damage Criteria ......................................................... 24

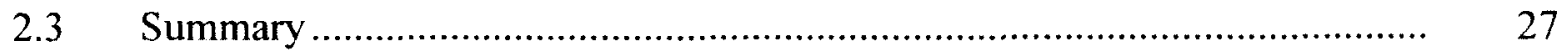

CHAPTER 3 CALIBRATION OF PLASTICITY MODEL PARAMETERS 33

3.1 Testing Program and FEA Modeling .............................................. 34

3.1.1 Specimen Designation ................................................... 35

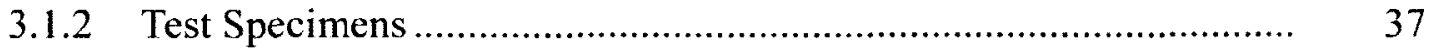

3.1.3 Test Setup and Instrumentation ......................................... 38

3.1.4 FEA Modeling ................................................................ 39

3.2 Stress Components of Cyclic Hardening …...................................... 41

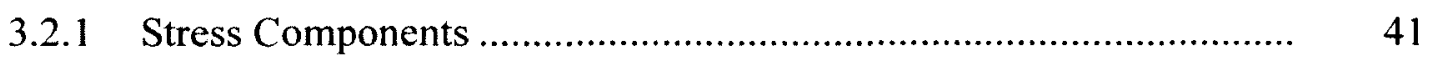

3.2.2 Flow Stress under Monotonic Loading .................................. 44

3.2.3 Equations Describing Flow Stress....................................... 45

3.2.4 Determination of Flow Stress versus Equivalent Plastic Strain Relationship.

3.3 Procedure to Determine Cyclic Hardening Parameters ........................... 49

3.3.1 Curve Fitting of Kinematic Hardening Parameters ....................... 50

3.3.2 Calibration of Kinematic Hardening Parameters ......................... 54

3.3.2.1 Two-Term Kinematic Hardening Parameters .................. 54

3.3.2.1.1 Optimum Saturated Yield Stress ..................... 56 
3.3.2.1.2 Optimum Parameter b ............................... 60

3.3.2.1.3 FEA Simulation.......................................... 62

3.3.2.2 One-Term Kinematic Hardening Parameters.................... 64

3.4 Other Methods to Determine Cyclic Hardening Parameters ....................... 65

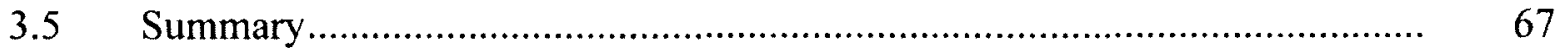

CHAPTER 4 EVALUATION OF FRACTURE CRITERIA .......................... 118

4.1 Evaluated Fracture Criteria ................................................................ 119

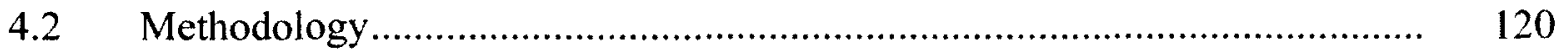

4.2.1 Point of Fracture ............................................................. 121

4.2.2 Calculation of Damage Limit ............................................... 122

4.2.3 Mesh Study of FEA Model................................................ 123

Results of Fracture Prediction .......................................................... 124

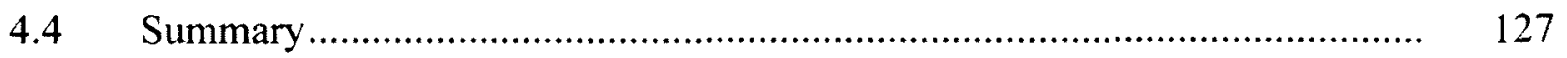

CHAPTER 5 SIMULATIONS OF STRAP TENSION-ONLY BRACE ......... 144

5.1 Experimental Study on Strap Tension-Only Brace ............................... 145

5.2 Material Model Parameters for Tested Steel Straps................................ 146

5.2.1 Bound for Saturated Yield Stress ....................................... 147

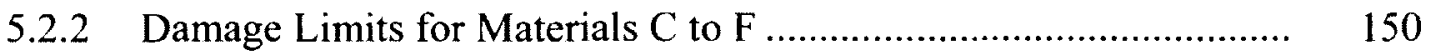

5.2.3 Procedure to Determine Damage Limits for Materials C to F ........ 153

5.3 FEA Simulations of Type A Strap Specimens ................................... 154 
5.3.1 FEA Modeling of Type A Strap Specimens

CHAPTER 6 PARAMETRIC STUDY OF STRAP TENSION-ONLY BRACE

6.1 Generation of Material Properties

6.2 Parameters Used in the Parametric Study.

6.2.1 Rectangular Specimen for Calculating Damage Limit

6.2.2 Flow Stress Parameter $n$

6.2.3 Yield Plateau and Engineering Strain at Ultimate Stress.

6.3 FEA Simulations and Results Analyses.

6.3.1 Materials Used in the Parametric Study

6.3.2 List of Braces.

6.3.3 FEA Simulations and Discussions

6.3.3.1 Effect of Yield Plateau

6.3.3.2 Effect of $A_{n} F_{u} / A_{g} F_{y}$

6.3.3.3 Effect of Slenderness Ratio

6.3.3.4 Effect of $F_{u} / F_{y}$ 
6.4.1 First Regression (RI) for Ductility Prediction Equations ............... 233

6.4.2 Second Regression (RII) for Ductility Prediction Equations ........... 235

6.4.3 Comparisons of Test and Predicted Ductility .............................. 236

6.4.4 Effect of Yield Strength ........................................................... 237

6.4.5 Limitations of Ductility Prediction Equations.............................. 238

6.5 Recommendations for Designing Strap Tension-Only Brace...................... 239

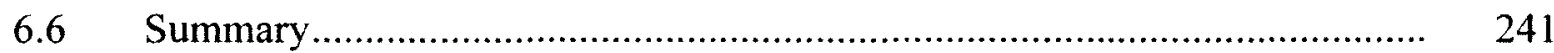

CHAPTER 7 SUMMARY, CONCLUSIONS AND RECOMMENDATIONS 275

7.1 Summary and Conclusions .......................................................... 275

7.1.1 Calibration and Determination of Plasticity Model Parameters....... $\quad 275$

7.1.2 Evaluation of Fracture Criteria....................................................... 277

7.1.3 Validation of Strap Tension-Only Brace Modeling ....................... 277

7.1.4 Parametric Study on Strap Tension-Only Brace............................ 279

7.2 Recommendations for Future Studies.............................................. 280

APPENDIX A COMPARISONS OF TESTS AND FEA SIMULTIONS FOR

MATERIALS A AND B (TWO TERMS) ................................ 283

APPENDIX B COMPARISONS OF TESTS AND FEA SIMULTIONS FOR

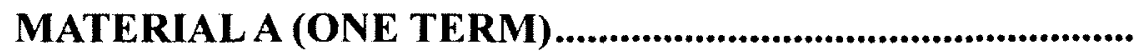


APPENDIX D MESH STUDY OF FEA MODEL FOR PROFILES D10R14N AND D14R19N.

APPENDIX E FEA SIMULATIONS FOR MONOTONIC TENSION AND CYCLIC TESTS OF STRAP SPECIMENS

REFERENCES... 


\section{LIST OF TABLES}

Table

Page

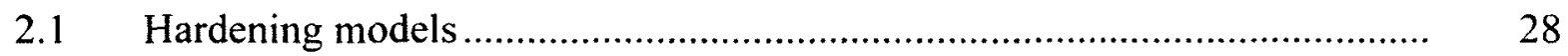

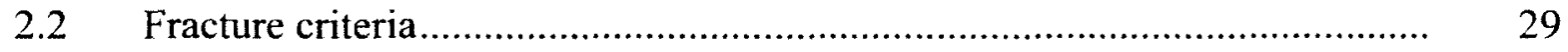

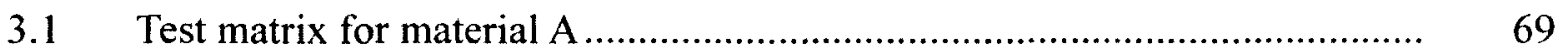

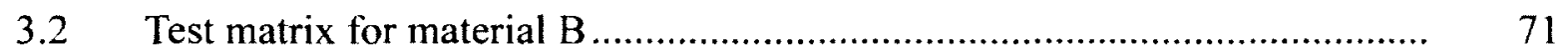

3.3 Material model parameters and properties used in finding optimum kinematic hardening parameters with their associated error Q.................... 73

3.4 Yield stress versus equivalent plastic strain data for material A (two terms) $\quad 74$

3.5 Yield stress versus equivalent plastic strain data for material B (two terms) 75

3.6 Calibrated material model parameters for materials A and B (two terms)... $\quad 76$

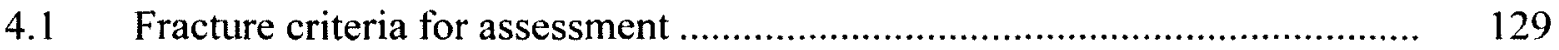

4.2 Test and predicted point of fracture for specimens of material A using the criterion by Kanvinde and Deierlein $(2004,2007)$ with void closing in compression and $\mathrm{c}=1$

4.3 Test and predicted point of fracture for specimens of material A using the modified Huang and Mahin criterion with gross strain energy density, and $\mathrm{c}=1, \mathrm{~m}=3.07$ and $\mathrm{k}=1.44$

4.4 Test and predicted point of fracture for specimens of material B using the criterion by Kanvinde and Deierlein $(2004,2007)$ with void closing in compression and $\mathrm{c}=1$ 
4.5 Test and predicted point of fracture for specimens of material B using the modified Huang and Mahin criterion with gross strain energy density, and $c=1, \mathrm{~m}=2.35$ and $\mathrm{k}=2.13$

5.1 Loading sequence of cyclic test for strap specimen

5.2 Steel material properties

5.3 Calculation of the damage limit for a fictitious material ${ }^{\mathrm{F}} \mathrm{A}$ using the measured cycle number and diameter at fracture for specimens of material $B$ with $\mathrm{c}=1, \mathrm{~m}=3.39$ and $\mathrm{k}=1.12$

5.4 Test and predicted point of fracture for specimens of material A using damage limit $D_{c}=14.92$ calibrated through test results of specimens from material $\mathrm{B}$, and $\mathrm{c}=1, \mathrm{~m}=3.39$ and $\mathrm{k}=1.12$

5.5 Flow stress equation parameters for materials $\mathrm{C}$ to $\mathrm{F}$

5.6 Parameters of two-term kinematic hardening model for materials $\mathrm{C}$ to $\mathrm{F}$.....

5.7 Calculation of the damage limit for a fictitious material ${ }^{\mathrm{F}} \mathrm{C}$ using the measured cycle number and diameter at fracture for specimens of material $\mathrm{B}$ with $\mathrm{c}=1, \mathrm{~m}=2.18$ and $\mathrm{k}=1.48(\eta=0.75)$

5.8 Parameters for the fracture criterion and damage limit for materials $\mathrm{C}$ to $\mathrm{F}$ estimated through test results of specimens from material B

5.9 Type A strap specimens tested by Aghdam (2009)

5.10 Comparison of FEA predictions for strap specimen A8075 with two different boundary constraints 
5.11 Strain rate dependence on the net section fracture to gross section yielding strength ratio (Aghdam 2009).

5.12 FEA predictions for strap specimen A4075 and A2078 using two different mesh schemes

5.13 FEA predictions for tests of strap specimens using the $1 / 4$ model

5.14 FEA predictions for tests of strap specimens using the $1 / 2$ model

5.15 Comparisons of test and FEA predictions for tests of strap specimens of materials $D$ to $F$ using the $1 / 2$ model with the measured maximum deformation reduced by $6 \mathrm{~mm}$ (except A2078e)

6.1 The cross-sectional area change $\ln \left(\mathrm{A}_{0} / \mathrm{A}\right)$ at fracture for different materials

6.2 Flow stress parameter $\mathrm{n}$ for materials $\mathrm{A}$ to $\mathrm{F}$

6.3 FEA predictions for strap specimens A2128 and A4128 with different values of flow stress parameter $n(\eta=0.75)$.

6.4 Material properties to study effect of yield plateau length and engineering strain at ultimate stress.

6.5 Material model parameters for materials MA, MB, NA and NB

6.6 Effect of yield plateau length and engineering strain at ultimate stress on FEA prediction.

6.7 Mechanical properties of materials for the parametric study

6.8 Material model parameters for materials JA, JB, KA and KB

6.9 List of braces for materials JA and JB 
6.10 List of braces for materials $\mathrm{KA}$ and $\mathrm{KB}$

6.11 FEA predictions for braces JA04124 and JB04124 using two different mesh schemes

6.12 FEA predictions for braces of material JA that has no yield plateau

6.13 FEA predictions for braces of material JB that has a yield plateau

6.14 FEA predictions for braces of material KA that has no yield plateau.

6.15 FEA predictions for braces of material $\mathrm{KB}$ that has a yield plateau

6.16 $A_{n} / A_{g}$ ratio at the same thickness but different $F_{u} / F_{y}$ with drilled hole for M20 bolt.

6.17 Constants for the ductility prediction equations of materials without a yield plateau, JA and KA

6.18 Constants for the ductility prediction equations of materials with a yield plateau, JB and KB

6.19 Statistics of the ratio of the predicted ductility from FEA simulation to that by the first regression equation for all four materials

6.20 Statistics of the difference of the predicted ductility between FEA simulation and that by the first regression equation for all four materials ....

6.21 Constants for the ductility prediction equation of materials without a yield plateau, combing JA and KA

6.22 Constants for the ductility prediction equation of materials with a yield plateau, combing JB and KB 
6.23 Statistics of the ratio of the predicted ductility from FEA simulation to that by the second regression equation for the combined materials

6.24 Statistics of the difference of the predicted ductility between FEA simulation to that by the second regression equation for the combined materials

6.25 Comparisons of test and predicted ductility through interpolating and extrapolating from Eqs. (6.18) and (6.19) with the measured maximum deformation reduced by $6 \mathrm{~mm}$ (except specimens of material C)

6.26 Comparisons of test and predicted ductility using Eq. (6.18) for specimens of material $\mathrm{C}$

6.27 Comparisons of the predicted ductility for materials with different yield strengths

B.1 Yield stress versus equivalent plastic strain data for material A (one term).. 305

C.1 Fracture criteria for the preliminary study

C.2 Test and predicted fracture using criterion by Rice and Tracey (1969) with $c=1$

C.3 Test and predicted fracture using criterion by Kanvinde and Deierlein $(2004,2007)$ with void closing in compression and $\mathrm{c}=1$

C.4 Test and predicted fracture using criterion by Huang and Mahin (2008) with $c-0.15$ and $\mathrm{m}=4$

C.5 Test and predicted fracture using criterion by Huang and Mahin (2008) 
with gross strain energy density, and $c=0.44$ and $\mathrm{m}=4$

C.6 Test and predicted fracture using criterion by Freudenthal (1950)..

C.7 Test and predicted fracture using criterion by Cockcroft and Latham (1968)

C.8 Test and predicted fracture using criterion by Brozzo et al. (1972)..............

C.9 Test and predicted fracture using criterion by Norris et al. (1978) with $\mathrm{c}=$ $1.37 \mathrm{GPa}^{-1}$

C.10 Test and predicted fracture using criterion by Oyane et al. (1980) with $\mathrm{c}=$

0.74 


\section{LIST OF FIGURES}

Figure

Page

1.1 Strap tension-only brace with bolted connection (Aghdam 2009) ............... 6

2.1 Bauschinger effect

2.2 Ratchetting.

2.3 Material hardening on principal S1-S2 deviatoric stress plane

3.1 Profile of D10R14N notched specimen

3.2 Profile of D14R19N notched specimen

3.3 Profile of D10R7N notched specimen

3.4 Profile of D14R24U straight specimen.

3.5 Profile of D14R19U straight specimen.

3.6 Test setup shown using $25.4 \mathrm{~mm}$ (one-inch) axial extensometer and diametral extensometer

3.7 Mesh of $25.4 \mathrm{~mm}$ (one-inch) FEA model for D10R14N notched specimen.

3.8 Mesh of $25.4 \mathrm{~mm}$ (one-inch) FEA model for D14R19N notched specimen.

3.9 Mesh of $25.4 \mathrm{~mm}$ (one-inch) FEA model for D10R7N notched specimen ...

3.10 Mesh of $12.7 \mathrm{~mm}$ (half-inch) FEA model for D14R24U and D14R19U straight specimens

3.11 Mesh of half full-length FEA model for straight specimens

3.12 Comparison of simulation results using half full-length and $12.7 \mathrm{~mm}$ (half-inch) FEA models for straight specimens of material A subjected to 
monotonic tension loading

3.13 Relationship between various stress components under monotonic uniaxial tension loading.

3.14 Flow stresses for calculating backstress and yield stress under uniaxial cyclic loading.

3.15 Test and predicted engineering stress versus cross-sectional area change curves for straight specimen ADMa using the same flow stress versus equivalent plastic strain data but different kinematic hardening parameters.

3.16 Curve fitting of the flow stress versus equivalent plastic strain data of monotonic straight specimens with power law Eq. (3.25) and exponential law Eq. (3.28).

3.17 Test and predicted engineering stress versus cross-sectional area change curves for straight specimens of material A using the material parameters determined by least-square fitting for power law Eq. (3.25) and exponential law Eq. (3.28)

3.18 True flow stress versus equivalent plastic strain curves generated by power law Eq. (3.25) and exponential law Eq. (3.28) over a large range of strain with the calibrated parameters

3.19 The true stress versus true plastic strain curves at different cycles for straight specimen ADC 40202

3.20 Assumed saturated true stress versus true plastic strain curve (static values) 
for calibration, tension branch of ADC40202 cycle \#20

3.21 Curve fitting of backstress for small strain with $\sigma_{\mathrm{s}}^{0}=450 \mathrm{MPa}$ using test results from the tension branch of ADC40202 cycle \#20 (two terms)

3.22 Curve fitting of backstress for large strain with $\sigma_{s}^{0}=450 \mathrm{MPa}$ (two terms)

3.23 Curve fitting of backstress for small strain with $\sigma_{\mathrm{s}}^{0}=450 \mathrm{MPa}$ using test results from the tension branch of ADC40202 cycle $\# 20$ (one term)

3.24 Curve fitting of backstress for large strain with $\sigma_{\mathrm{s}}^{0}=450 \mathrm{MPa}$ (one term)

3.25 Curve fitting of backstress for small strain with $\sigma_{s}^{0}=273 \mathrm{MPa}$ at proportional limit using test results from the tension branch of ADC40202 cycle \#20 (two terms)

3.26 Curve fitting of backstress for large strain with $\sigma_{\mathrm{s}}^{0}=273 \mathrm{MPa}$ at proportional limit (two terms)

3.27 Test and predicted engineering stress versus cross-sectional area change curves with different parameter $\mathrm{b}$ for straight specimens of material $\mathrm{A}$ subjected to monotonic tension loading (two terms)

3.28 Test and predicted engineering stress versus engineering strain curves with different parameter $\mathrm{b}$ for straight specimen ADC40202 (two terms)

3.29 Test and predicted engineering stress versus cross-sectional area change curves with different parameter b for straight specimen ADC40202 (two terms) 
3.30 Test and predicted engineering stress versus engineering strain curves with different parameter $\mathrm{b}$ for notched specimen $\mathrm{ABC} 70404$ (two terms).

3.31 Test and predicted engineering stress versus cross-sectional area change curves with different parameter $b$ for notched specimen ABC70404 (two terms)

3.32 Test and predicted engineering stress versus engineering strain curves with different parameter $b$ for notched specimen ABCF 1212 (two terms).

3.33 Test and predicted engineering stress versus cross-sectional area change curves with different parameter $b$ for notched specimen ABCF1212 (two terms)

3.34 Test and predicted engineering stress versus engineering strain curves using $\sigma_{s}^{\prime}=273 \mathrm{MPa}$ and $\mathrm{b}=4.5$ for straight specimen ADC40202 (two terms)

3.35 Test and predicted engineering stress versus cross-sectional area change curves using $\sigma_{\mathrm{s}}^{0}=273 \mathrm{MPa}$ and $\mathrm{b}=4.5$ for straight specimen ADC40202 (two terms)

3.36 Test and predicted engineering stress versus engineering strain curves using $\sigma_{\mathrm{s}}^{0}=273 \mathrm{MPa}$ and $\mathrm{b}=4.5$ for notched specimen $\mathrm{ABC} 20404$ (two terms)

3.37 Curve fitting of backstress for large strain with $\sigma_{\mathrm{s}}^{0}=450 \mathrm{MPa}, \Delta \sigma_{\mathrm{s}}^{0}=$ $10 \mathrm{MPa}$ (two terms) 
3.38 Locations of engineering strains where errors are calculated for notched specimen $\mathrm{ABC} 20404$, shown for cycle $\# 20$.

3.39 Test and predicted engineering stress versus engineering strain curves using $\sigma_{\mathrm{s}}^{0}=450 \mathrm{MPa}$ and $\Delta \sigma_{\mathrm{s}}^{0}=0$ for notched specimen $\mathrm{ABC} 20404$ (two terms)

3.40 Test and predicted engineering stress versus engineering strain curves using $\sigma_{\mathrm{s}}^{0}=450 \mathrm{MPa}$ and $\Delta \sigma_{\mathrm{s}}^{0}=10 \mathrm{MPa}$ for notched specimen ABC20404 (two terms)

3.41 Test and predicted engineering stress versus engineering strain curves using $\sigma_{\mathrm{s}}^{0}=450 \mathrm{MPa}$ and $\Delta \sigma_{\mathrm{s}}^{0}=20 \mathrm{MPa}$ for notched specimen $\mathrm{ABC} 20404$ (two terms)

3.42 Error versus assumed saturated yield stress offset $\left(\Delta \sigma_{\mathrm{s}}^{0}\right)$ for $\sigma_{\mathrm{s}}^{0}=$ $450 \mathrm{MPa}$ (two terms)

3.43 Minimum error versus assumed saturated yield stress $\left(\sigma_{\mathrm{s}}^{0}\right)($ two terms $) \ldots . .$.

3.44 Error versus assumed saturated yield stress offset $\left(\Delta \sigma_{\mathrm{s}}^{0}\right)$ for $\sigma_{\mathrm{s}}^{0}=$ $464 \mathrm{MPa}$ (two terms)

3.45 Test and predicted engineering stress versus cross-sectional area change curves with $b=4.5$ for notched specimen ABC70404 (two terms)

3.46 Test and predicted engineering stress versus cross-sectional area change curves with $b=5.5$ for notched specimen ABC70404 (two terms) 
3.48 Test and predicted engineering stress versus cross-sectional area change curves for straight specimens ADMa, ADMb and AEM (two terms).......... 106

3.49 Test and predicted engineering stress versus engineering strain curves for notched specimen ABM (two terms)

3.50 Test and predicted engineering stress versus cross-sectional area change curves for notched specimen ABM (two terms)

3.51 Test and predicted engineering stress versus engineering strain curves for straight specimen ADC20202 (two terms).

3.52 Test and predicted engineering stress versus cross-sectional area change curves for straight specimen ADC20202 (two terms)

3.53 Test and predicted engineering stress versus cross-sectional area change curves for straight specimen ADC20202 focus of cyclic loading (two terms)

3.54 Test and predicted engineering stress versus cross-sectional area change curves for straight specimen ADC40102 (two terms)

3.55 Test and predicted engineering stress versus cross-sectional area change curves for straight specimen ADCR0201 (two terms)

3.56 Test and predicted engineering stress versus cross-sectional area change curves for straight specimen ADCR0201 focus of cyclic loading (two terms)

3.57 Test and predicted engineering stress versus engineering strain curves for 
notched specimen ABC20404 (two terms)

3.58 Test and predicted engineering stress versus cross-sectional area change curves for notched specimen ABC20404 (two terms)

3.59 Test and predicted engineering stress versus engineering strain curves for notched specimen ABC70404 (two terms)

3.60 Test and predicted engineering stress versus cross-sectional area change curves for notched specimen $\mathrm{ABC} 70404$ (two terms)

3.61 Test and predicted engineering stress versus engineering strain curves for notched specimen ABCF 1212 (two terms)

3.62 Test and predicted engineering stress versus cross-sectional area change curves for notched specimen ABCF1212 (two terms)

3.63 Test and predicted engineering stress versus engineering strain curves for notched specimen ABCF3010 (two terms)

3.64 Test and predicted engineering stress versus cross-sectional area change curves for notched specimen ABCF3010 (two terms)

3.65 Test and predicted engineering stress versus engineering strain curves for notched specimen AACR0804 (two terms)

3.66 Test and predicted engineering stress versus cross-sectional area change curves for notched specimen AACR0804 (two terms)

3.67 Test and predicted engineering stress versus cross-sectional area change curves for straight specimens subjected to monotonic tension loading 
using parameters calibrated by ABAQUS (two terms)......

3.68 Test and predicted engineering stress versus engineering strain curves for straight specimen ADC20202 using parameters calibrated by ABAQUS (two terms)

3.69 Test and predicted engineering stress versus cross-sectional area change curves for straight specimen ADC20202 using parameters calibrated by ABAQUS (two terms)

3.70 Test and predicted engineering stress versus engineering strain curves for notched specimen ABC20404 using parameters calibrated by ABAQUS (two terms).

3.71 FEA simulations of monotonic tension test using material parameters for AW50 steel by Kanvinde and Deierlein (2004)

4.1 Initial slope (modulus) of the average true stress versus cross-sectional area change $\left[\ln \left(\mathrm{A}_{0} / \mathrm{A}\right)\right]$ curve for notched specimen $\mathrm{BBC} 70404$ at each cycle of loading.

4.2 Average true stress versus cross-sectional area change curve for notched specimen BBC70404

4.3 Fracture point for notched specimen ABCF1212

4.4 Softening and pinching behaviours observed in the test of notched specimen BBC70404

4.5 Scheme-2 mesh of $12.7 \mathrm{~mm}$ (half-inch) FEA model for D14R24U and 
4.6 Engineering stress versus engineering strain curves from FEA simulations for straight specimens ADMa and ADMb with two mesh schemes

4.7 Engineering stress versus cross-sectional area change curves from FEA simulations for straight specimens ADMa and ADMb with two mesh schemes

4.8 Calculated damage state D from FEA simulations for straight specimens ADMa and ADMb with two mesh schemes

4.9 Engineering stress versus engineering strain curves from FEA simulations for straight specimen ADC40202 with two mesh schemes

4.10 Engineering stress versus cross section area change curves from FEA simulations for straight specimen ADC40202 with two mesh schemes

4.11 Calculated damage state D from FEA simulations for straight specimen ADC40202 with two mesh schemes

4.12 Engineering stress versus engineering strain curves from FEA simulations for straight specimen ADCR0201 with two mesh schemes.

4.13 Engineering stress versus cross section area change curves from FEA simulations for straight specimen ADCR0201 with two mesh schemes .......

4.14 Calculated damage state D from FEA simulations for straight specimen ADCR0201 with two mesh schemes 
and $\mathrm{ABM}$

4.16 Test and predicted fracture point for notched specimen ABC20404

4.17 Test and predicted fracture point for notched specimens BAM and BBM....

4.18 Test and predicted fracture point for notched specimen BBC20404

$5.1 \quad$ Bracing systems (Aghdam 2009)

5.2 Steel strap specimen with various connection details (Aghdam 2009) .........

5.3 Test setup (Aghdam 2009)

5.4 Curve fitting of backstress for large strain using assumed saturated yield stress of $380 \mathrm{MPa}$ (two terms)

5.5 Test and predicted engineering stress versus engineering strain for notched specimen BBC20404 with bound $\eta$ of $0.75,0.79$ and 1.2

5.6 Test and predicted engineering stress versus engineering strain for notched specimen BBCF1212 with bound $\eta$ of $0.75,0.79$ and 1.2 .

5.7 Test and generated flow stress versus true plastic strain for materials $\mathrm{C}$ to F

5.8 Typical mesh for the FEA model of $1 / 8$ rectangular specimen for materials C to $F($ Chen 2010)

5.9 Type A strap specimen (Aghdam 2009).

5.10 Both ends of strap specimen A4078b of material D at the end of the test (Aghdam 2009)

5.11 Both ends of strap specimen A8075b of material $C$ at the end of the test 
(Aghdam 2009)

5.12 FEA mesh of the $1 / 4$ model, shown for strap specimen A4075 of material C.

5.13 FEA mesh of the $1 / 2$ model, shown for strap specimen A4075 of material C.

5.14 The region around the innermost bolt hole modeled with solid element, shown for strap specimen A4075

5.15 Plan view of the 1/4 FEA model, shown for strap specimen A4075

5.16 Spring elements to induce buckling to the appropriate direction

5.17 Applied transverse displacement ( $Z$ direction) at the spring element support (odd half cycle number corresponds to the start of compression leg)

5.18 Nodes on the bottom face of the solid element around the innermost bolt hole at the upper (loading) end of the specimen constrained in the $\mathrm{Z}$ direction, shown for strap specimen A4075 (same constraint is applied to nodes of the shell element in the similar region at the lower (fixed) end for the $1 / 2$ model)

5.19 Modeling of the bolt and contact surface at the bolt hole

5.20 Results of FEA simulation with the bolt and contact surfaces at the bolt hole

5.21 Nodes on the surface around the innermost bolt hole constrained in the $\mathrm{Z}$ 
5.22 Nodes around the innermost bolt hole at the upper end constrained to have the same displacement in the Y direction, shown for strap specimen A4075

5.23 Nodes around the innermost bolt hole at the lower (fixed) end constrained against deformation in the $\mathrm{Y}$ direction, shown for strap specimen $\mathrm{A} 4075 \ldots$.

5.24 Scheme-2 mesh of the critical region of strap specimen A4075 modeled with solid element

5.25 FEA simulation of load versus deformation curves for strap specimen A4075 using the $1 / 2$ model with two different mesh schemes

5.26 FEA simulation of load versus deformation curves for strap specimen A2078 using the $1 / 4$ model with two different mesh schemes

5.27 Deformation around the region of the innermost bolt hole at close to fracture showing the contour plot of the damage, shown for strap specimen A4075 with $\eta=0.75$

5.28 Test and predicted load versus deformation curves for strap specimen A4075a using the $1 / 4$ model

5.29 Test and predicted load versus deformation curves for strap specimen A $4075 \mathrm{~b}$ using the $1 / 4$ model

5.30 Test and predicted load versus deformation curves for strap specimen A4075a using the $1 / 2$ model

5.31 Test and predicted load versus deformation curves for strap specimen 
A4075b using the $1 / 2$ model.

5.32 Test and predicted load versus deformation curves for strap specimen A4078a using the $1 / 4$ model

5.33 Test and predicted load versus deformation curves for strap specimen A4078b using the $1 / 4$ model.

5.34 Test and predicted load versus deformation curves for strap specimen A4078a using the $1 / 2$ model

5.35 Test and predicted load versus deformation curves for strap specimen A $4078 \mathrm{~b}$ using the $1 / 2$ model.....

5.36 Test and predicted load versus deformation curves for strap specimens A2078d and A2078e using the $1 / 2$ model

5.37 Test and predicted load versus deformation curves for strap specimens A2128d and A2128e using the $1 / 2$ model

5.38 Test and predicted load versus deformation curves for strap specimen A2108b using the $1 / 2$ model.

5.39 Test and predicted load versus deformation curves for strap specimen A4108a using the $1 / 2$ model

5.40 Test and predicted load versus deformation curves for strap specimens A2078d and A2078e using the $1 / 2$ model with the test deformation reduced by $6 \mathrm{~mm}$ for $\mathrm{A} 2078 \mathrm{~d}$

5.41 Test and predicted load versus deformation curves for strap specimens 
A2128d and A2128e using the $1 / 2$ model with the test deformation reduced by $6 \mathrm{~mm}$

5.42 Test and predicted load versus deformation curves for strap specimen A2 $108 \mathrm{~b}$ using the $1 / 2$ model with the test deformation reduced by $6 \mathrm{~mm} \ldots$.

5.43 Test and predicted load versus deformation curves for strap specimen A4108a using the $1 / 2$ model with the test deformation reduced by $6 \mathrm{~mm} \ldots$...

6.1 Test and predicted engineering stress versus cross-sectional area change curves for monotonic tension tests of material $\mathrm{F}$ with different values of flow stress parameter $n(\eta=0.75)$

6.2 FEA simulations of engineering stress versus engineering strain curves for monotonic tension tests of four materials considered in the parametric study, JA, JB, KA and KB

6.3 Predicted ductility versus $A_{n} F_{u} / A_{g} F_{y}$ for $4 m$ braces of material JA

6.4 Predicted ductility versus $A_{n} F_{u} / A_{q} F_{y}$ for $6 \mathrm{~m}$ braces of material JA

6.5 Predicted ductility versus $\mathrm{A}_{n} \mathrm{~F}_{\mathrm{u}} / \mathrm{A}_{\mathrm{g}} \mathrm{F}_{\mathrm{y}}$ for $4 \mathrm{~m}$ braces of material JB

6.6 Predicted ductility versus $A_{n} F_{u} / A_{g} F_{y}$ for $6 \mathrm{~m}$ braces of material JB

6.7 Predicted ductility versus $\mathrm{A}_{n} \mathrm{~F}_{\mathrm{u}} / \mathrm{A}_{\mathrm{g}} \mathrm{F}_{\mathrm{y}}$ for $4 \mathrm{~m}$ braces of material KA...........

6.8 Predicted ductility versus $\mathrm{A}_{n} \mathrm{~F}_{\mathrm{u}} / \mathrm{A}_{\mathrm{g}} \mathrm{F}_{\mathrm{y}}$ for $6 \mathrm{~m}$ braces of material KA ............ 260

6.9 Predicted ductility versus $\mathrm{A}_{n} \mathrm{~F}_{\mathrm{u}} / \mathrm{A}_{\mathrm{g}} \mathrm{F}_{\mathrm{y}}$ for $4 \mathrm{~m}$ braces of material $\mathrm{KB} \ldots \ldots \ldots \ldots . \quad 26 \mathrm{l}$

6.10 Predicted ductility versus $A_{n} F_{u} / A_{2} F_{y}$ for $6 \mathrm{~m}$ braces of material $\mathrm{KB} \ldots \ldots \ldots \ldots . \quad 261$

6.11 Predicted ductility versus slenderness ratio for $4 \mathrm{~m}$ braces of material JA ... $\quad 262$ 
6.12 Predicted ductility versus slenderness ratio for $6 \mathrm{~m}$ braces of material JA ... 262

6.13 Predicted ductility versus slenderness ratio for $4 \mathrm{~m}$ braces of material JB ... $\quad 263$

6.14 Predicted ductility versus slenderness ratio for $6 \mathrm{~m}$ braces of material JB ... 263

6.15 Predicted ductility versus slenderness ratio for $4 \mathrm{~m}$ braces of material KA.. $\quad 264$

6.16 Predicted ductility versus slenderness ratio for $6 \mathrm{~m}$ braces of material KA.. $\quad 264$

6.17 Predicted ductility versus slenderness ratio for $4 \mathrm{~m}$ braces of material KB.. $\quad 265$

6.18 Predicted ductility versus slenderness ratio for $6 \mathrm{~m}$ braces of material $\mathrm{KB}$.. $\quad 265$

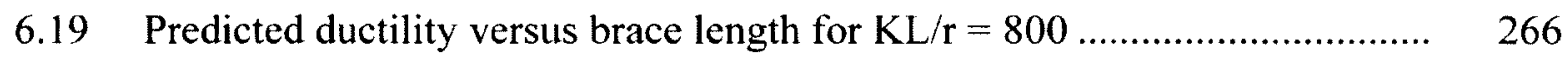

6.20 Predicted ductility versus brace length for $\mathrm{KL} / \mathrm{r}=600$ and $\mathrm{A}_{\mathrm{i}} \mathrm{F}_{\mathrm{u}} / \mathrm{A}_{\mathrm{g}} \mathrm{F}_{\mathrm{y}}=1.1 . \quad 266$

6.21 An/Ag versus $F_{u} / F_{y}$ at the same thickness for different $A_{n} F_{u} / A_{g} F_{y} \ldots \ldots \ldots \ldots . .267$

6.22 Predicted ductility versus $F_{u} / F_{y}$ for braces with $4 \mathrm{~m}$ length and $\mathrm{KL} / \mathrm{r}=400$ of materials without a yield plateau, JA and KA

6.23 Predicted ductility versus $F_{u} / F_{y}$ for braces with $4 \mathrm{~m}$ length and $\mathrm{KL} / \mathrm{r}=800$ of materials without a yield plateau, JA and KA

6.24 Predicted ductility versus $F_{u} / F_{y}$ for braces with $6 \mathrm{~m}$ length and $\mathrm{KL} / \mathrm{r}=800$ of materials without a yield plateau, JA and KA

6.25 Predicted ductility versus $F_{u} / F_{y}$ for braces with $4 \mathrm{~m}$ length and $K L / r=400$ of materials with a yield plateau, JB and $\mathrm{KB}$

6.26 Predicted ductility versus $F_{u} / F_{y}$ for braces with $4 \mathrm{~m}$ length and $\mathrm{KL} / \mathrm{r}=800$ of materials with a yield plateau, JB and $\mathrm{KB}$

6.27 Predicted ductility versus $F_{u} / F_{y}$ for braces with $6 \mathrm{~m}$ length and $\mathrm{KL} / \mathrm{r}=800$ 
6.28 FEA/RI of ductility for materials without a yield plateau, JA and KA ........ $\quad 270$

6.29 FEA/RI of ductility for materials with a yield plateau, JB and KB

6.30 FEA-RI of ductility for materials without a yield plateau, JA and KA .

6.31 FEA-RI of ductility for materials with a yield plateau, JB and KB.

6.32 FEA/RII of ductility for materials without a yield plateau, JA and KA .......

6.33 FEA/RII of ductility for materials with a yield plateau, JB and KB

6.34 FEA-RII of ductility for materials without a yield plateau, JA and KA.......

6.35 FEA-RII of ductility for materials with a yield plateau, JB and KB

A.1 Test and predicted engineering stress versus engineering strain curves for notched specimens AAMa and AAMb (two terms)

A.2 Test and predicted engineering stress versus cross-sectional area change curves for notched specimens AAMa and AAMb (two terms).

A.3 Test and predicted engineering stress versus cross-sectional area change curves for straight specimen ADC40101 (two terms)

A.4 Test and predicted engineering stress versus cross-sectional area change curves for straight specimen ADC40202 (two terms)

A.5 Test and predicted engineering stress versus engineering strain curves for notched specimen AAC20404 (two terms)

A.6 Test and predicted engineering stress versus cross-sectional area change curves for notched specimen AAC20404 (two terms). 
A.7 Test and predicted engineering stress versus engineering strain curves for notched specimen ACC20404 (two terms)

A.8 Test and predicted engineering stress versus cross-sectional area change curves for notched specimen ACC20404 (two terms).

A.9 Test and predicted engineering stress versus engineering strain curves for notched specimen AAC70404 (two terms)

A.10 Test and predicted engineering stress versus cross-sectional area change curves for notched specimen AAC70404 (two terms)

A.11 Test and predicted engineering stress versus engineering strain curves for notched specimen AACF1212 (two terms).

A.12 Test and predicted engineering stress versus cross-sectional area change curves for notched specimen AACF1212 (two terms).

A.13 Test and predicted engineering stress versus engineering strain curves for notched specimen AACF3010 (two terms).

A.14 Test and predicted engineering stress versus cross-sectional area change curves for notched specimen AACF3010 (two terms)

A.15 Test and predicted engineering stress versus cross-sectional area change curves for straight specimens BDMa and BDMb (two terms)

A.16 Test and predicted engineering stress versus engineering strain curves for notched specimen BAM (two terms)

A.17 Test and predicted engineering stress versus cross-sectional area change 
curves for notched specimen BAM (two terms)

A.18 Test and predicted engineering stress versus engineering strain curves for notched specimen BBM (two terms)

A.19 Test and predicted engineering stress versus cross-sectional area change curves for notched specimen BBM (two terms)

A.20 Test and predicted engineering stress versus cross-sectional area change curves for straight specimen BDC20202 (two terms)

A.21 Test and predicted engineering stress versus cross-sectional area change curves for straight specimen BDC40101 (two terms)

A.22 Test and predicted engineering stress versus cross-sectional area change curves for straight specimen BDC40102 (two terms)

A.23 Test and predicted engineering stress versus cross-sectional area change curves for straight specimen BDC40202 (two terms)

A.24 Test and predicted engineering stress versus cross-sectional area change curves for straight specimen BDCR0201 (two terms)

A.25 Test and predicted engineering stress versus engineering strain curves for notched specimen BAC20404 (two terms)

A.26 Test and predicted engineering stress versus cross-sectional area change curves for notched specimen BAC20404 (two terms)

A.27 Test and predicted engineering stress versus engineering strain curves for notched specimen BBC20404 (two terms) 
A.28 Test and predicted engineering stress versus cross-sectional area change curves for notched specimen BBC20404 (two terms).

A.29 Test and predicted engineering stress versus engineering strain curves for notched specimen BAC70404 (two terms)

A.30 Test and predicted engineering stress versus cross-sectional area change curves for notched specimen BAC70404 (two terms)

A.31 Test and predicted engineering stress versus engineering strain curves for notched specimen BBC70404 (two terms)

A.32 Test and predicted engineering stress versus cross-sectional area change curves for notched specimen BBC70404 (two terms)

A.33 Test and predicted engineering stress versus engineering strain curves for notched specimen BACF1212 (two terms).

A.34 Test and predicted engineering stress versus cross-sectional area change curves for notched specimen BACF1212 (two terms).

A.35 Test and predicted engineering stress versus engineering strain curves for notched specimen BBCF1212 (two terms)

A.36 Test and predicted engineering stress versus cross-sectional area change curves for notched specimen BBCF1212 (two terms).

A.37 Test and predicted engineering stress versus engineering strain curves for notched specimen B $A$ CF3010 (two terms)

A.38 Test and predicted engineering stress versus cross-sectional area change 
curves for notched specimen BACF3010 (two terms).

A.39 Test and predicted engineering stress versus engineering strain curves for notched specimen BBCF3010 (two terms).

A.40 Test and predicted engineering stress versus cross-sectional area change curves for notched specimen BBCF3010 (two terms)

A.41 Test and predicted engineering stress versus engineering strain curves for notched specimen BACR0804 (two terms)

A.42 Test and predicted engineering stress versus cross-sectional area change curves for notched specimen BACR0804 (two terms)

B.1 Various components of stresses for material A (one terms)

B.2 Test and predicted engineering stress versus cross-sectional area change curves for straight specimens ADMa, ADMb and AEM (one term) .....

B.3 Test and predicted engineering stress versus engineering strain curves for notched specimens AAMa and AAMb (one term).

B.4 Test and predicted engineering stress versus cross-sectional area change curves for straight specimen ADC40202 (one term)

B.5 Test and predicted engineering stress versus cross-sectional area change curves for straight specimen ADCR0201 (one term).

B.6 Test and predicted engineering stress versus engineering strain curves for notched specimen AAC20404 (one term).

B.7 Test and predicted engineering stress versus cross-sectional area change 
curves for notched specimen AAC20404 (one term).

B.8 Test and predicted engineering stress versus engineering strain curves for notched specimen AAC70404 (one term)

B.9 Test and predicted engineering stress versus cross-sectional area change curves for notched specimen AAC70404 (one term).

B.10 Test and predicted engineering stress versus engineering strain curves for notched specimen AACF 1212 (one term)

B.11 Test and predicted engineering stress versus cross-sectional area change curves for notched specimen AACF1212 (one term)

D.1 Scheme-2 mesh of $25.4 \mathrm{~mm}$ (one-inch) FEA model for D10R14N notched specimen (boundary condition not shown)

D.2 Scheme-2 mesh of $25.4 \mathrm{~mm}$ (one-inch) FEA model for D14R19N notched specimen (boundary condition not shown)

D.3 Engineering stress versus engineering strain curve from FEA simulations for notched specimens AAMa and AAMb with two mesh schemes

D.4 Engineering stress versus cross-sectional area change curves from FEA simulations for notched specimens AAMa and AAMb with two mesh schemes

D.5 Calculated damage state D from FEA simulations for notched specimens AAMa and AAMb with two mesh schemes

D.6 Engineering stress versus engineering strain curve from FEA simulations 
D.7 Engineering stress versus cross-sectional area change curves from FEA simulations for notched specimen AAC70404 with two mesh schemes

D.8 Calculated damage state D from FEA simulations for notched specimen AAC.70404 with two mesh schemes

D.9 Engineering stress versus engineering strain curve from FEA simulations for notched specimen AACF3010 with two mesh schemes

D.10 Engineering stress versus cross-sectional area change curves from FEA simulations for notched specimen AACF3010 with two mesh schemes.......

D.11 Calculated damage state D from FEA simulations for notched specimen AACF3010 with two mesh schemes.

D.12 Engineering stress versus engineering strain curve from FEA simulations for notched specimen AACR0804 with two mesh schemes

D.13 Engineering stress versus cross-sectional area change curves from FEA simulations for notched specimen AACR0804 with two mesh schemes.......

D.14 Calculated damage state D from FEA simulations for notched specimen AACR0804 with two mesh schemes

D.15 Engineering stress versus engineering strain curve from FEA simulations for notched specimen $\mathrm{ABM}$ with two mesh schemes

D.16 Engineering stress versus cross-sectional area change curves from FEA simulations for notched specimen ABM with two mesh schemes 
D.17 Calculated damage state D from FEA simulations for notched specimen ABM with two mesh schemes

D.18 Engineering stress versus engineering strain curve from FEA simulations for notched specimen $\mathrm{ABC} 70404$ with two mesh schemes

D.19 Engineering stress versus cross-sectional area change curves from FEA simulations for notched specimen ABC70404 with two mesh schemes .......

D.20 Calculated damage state D from FEA simulations for notched specimen $\mathrm{ABC} 70404$ with two mesh schemes

D.21 Engineering stress versus engineering strain curve from FEA simulations for notched specimen ABCF3010 with two mesh schemes.

D.22 Engineering stress versus cross-sectional area change curves from FEA simulations for notched specimen ABCF3010 with two mesh schemes

D.23 Calculated damage state D from FEA simulations for notched specimen ABCF3010 with two mesh schemes

E.1 Test and predicted load versus deformation curves for strap specimen A6075a using the $1 / 2$ model

E.2 Test and predicted load versus deformation curves for strap specimen A6075b using the $1 / 2$ model

E.3 Test and predicted load versus deformation curves for strap specimen A8075a using the $1 / 2$ model

E.4 Test and predicted load versus deformation curves for strap specimen 
A $8075 b$ using the $1 / 2$ model

E.5 Test and predicted load versus deformation curves for strap specimen A2078a using the $1 / 2$ model

E.6 Test and predicted load versus deformation curves for strap specimen A2078b using the $1 / 2$ model

E.7 Test and predicted load versus deformation curves for strap specimen A2078c using the $1 / 2$ model

E.8 Test and predicted load versus deformation curves for strap specimen A2108a using the $1 / 2$ model

E.9 Test and predicted load versus deformation curves for strap specimen A4108b using the $1 / 2$ model.

E.10 Test and predicted load versus deformation curves for strap specimen A2128a using the $1 / 2$ model

E.11 Test and predicted load versus deformation curves for strap specimen A2128b using the $1 / 2$ model

E.12 Test and predicted load versus deformation curves for strap specimen A2128c using the $1 / 2$ model

E.13 Test and predicted load versus deformation curves for strap specimen A4128a using the $1 / 2$ model

E.14 Test and predicted load versus deformation curves for strap specimen A4128b using the $1 / 2$ model 


\section{LIST OF SYMBOLS AND ABBREVIATIONS}

A

$\mathrm{A}_{0}$

$A_{f}$

$A_{\mathrm{g}}$

$A_{n}$

$A_{\text {puah }}$

$a_{0}, a_{1}, a_{2}$

b

C

$C_{k}$

c

D

$\mathrm{D}_{\mathrm{c}}$

$\overline{\mathrm{D}_{\mathrm{c}}}$

$d_{1}$
- current cross-sectional area

- undeformed cross-sectional area

- cross-sectional area at fracture

- gross cross-sectional area

- net cross-sectional area

- cross-sectional area at peak stress

- constants

- material parameter for isotropic hardening, or for the exponential law equation by Voce

- material parameter for kinematic hardening

- material parameter for kinematic hardening term $\mathrm{k}$

- material parameter for the damage evolution, or for the models by Prager, Manson and Coffin, McClintock, Rice and Tracey, Kanvinde and Deierlein, Norris et al., Oyane et al., Atkins

- state of damage, or damage parameter for the model by Manson and Coffin

- damage limit

- arithmetical average of the damage at fracture

- initial diameter 


$$
\begin{aligned}
& d_{\mathrm{f}} \\
& \text { - measured diameter at fracture } \\
& d_{p} \\
& \text { - predicted diameter at fracture } \\
& (\mathrm{dW} / \mathrm{dV})_{\mathrm{c}} \quad-\text { critical strain energy density } \\
& \mathrm{d} \lambda \quad-\text { constant of proportionality } \\
& \mathrm{d} \mu \quad-\quad \text { multiplier or constant of proportionality } \\
& \text { E - elastic modulus } \\
& \mathrm{E}^{\mathrm{p}} \quad-\text { plastic modulus in the model by Dafalias and Popov } \\
& E_{1}^{p}, E_{2}^{p}, \ldots, E_{n}^{p}-\text { plastic moduli in the model by Mroz } \\
& \text { ER - error in the predicted damage at fracture } \\
& \text { F } \quad-\text { load } \\
& \text { FB - flat bar } \\
& \mathrm{F}_{\mathrm{b}}^{\mathrm{r}} \quad-\text { relative growth factor for the model by McClintock } \\
& \text { FEA - finite element analysis } \\
& F_{n} \quad-\quad \text { ultimate strength } \\
& F_{y} \quad-\text { yield strength } \\
& \text { f } \quad-\text { void volume fraction } \\
& f_{0}, f_{1}, \ldots, f_{n} \quad-\text { hypersurfaces in the model by Mroz } \\
& f_{c} \quad-\text { critical void volume fraction } \\
& \mathrm{f}_{\mathrm{F}} \quad-\text { void volume fraction at fracture } \\
& \mathrm{f}^{*} \quad \text { - function of void volume fraction }
\end{aligned}
$$


K

L

$\mathrm{L}_{0}$

m

$\mathrm{NBCC}$

$\mathrm{N}_{\mathrm{f}}$

n

\section{Q}

$\mathrm{p}_{1}, \mathrm{p}_{2}, \ldots, \mathrm{p}_{7}$

$\mathrm{q}_{1}, \mathrm{q}_{2}, \mathrm{q}_{3}$

$\mathbf{R}$

$\mathrm{R}_{0}$
- material parameter for the power law equations by Hollomon, Naqvi and Khoo, or for the model by Tvergaard, and Tvergaard and Needleman, or effective length factor

- material parameter for the damage evolution

- ratio of the hoop to the axial strain increment of a cylindrical solid, or the nominal length of specimen measured between the middle bolt holes

- specimen length

- material parameter for the damage evolution, or for the power law equation by Hollomon

- National Building Code of Canada

- number of cycle to fracture

- material parameter for the power law equation by Naqvi and Khoo, or for the exponential law equation by Chinh et al., or for the model by Oyane et al.

- constants for the ductility prediction equations

- error measurement

- adjustable parameters for the model by Tvergaard, and Tvergaard and Needleman

- void radius

- initial void radius 


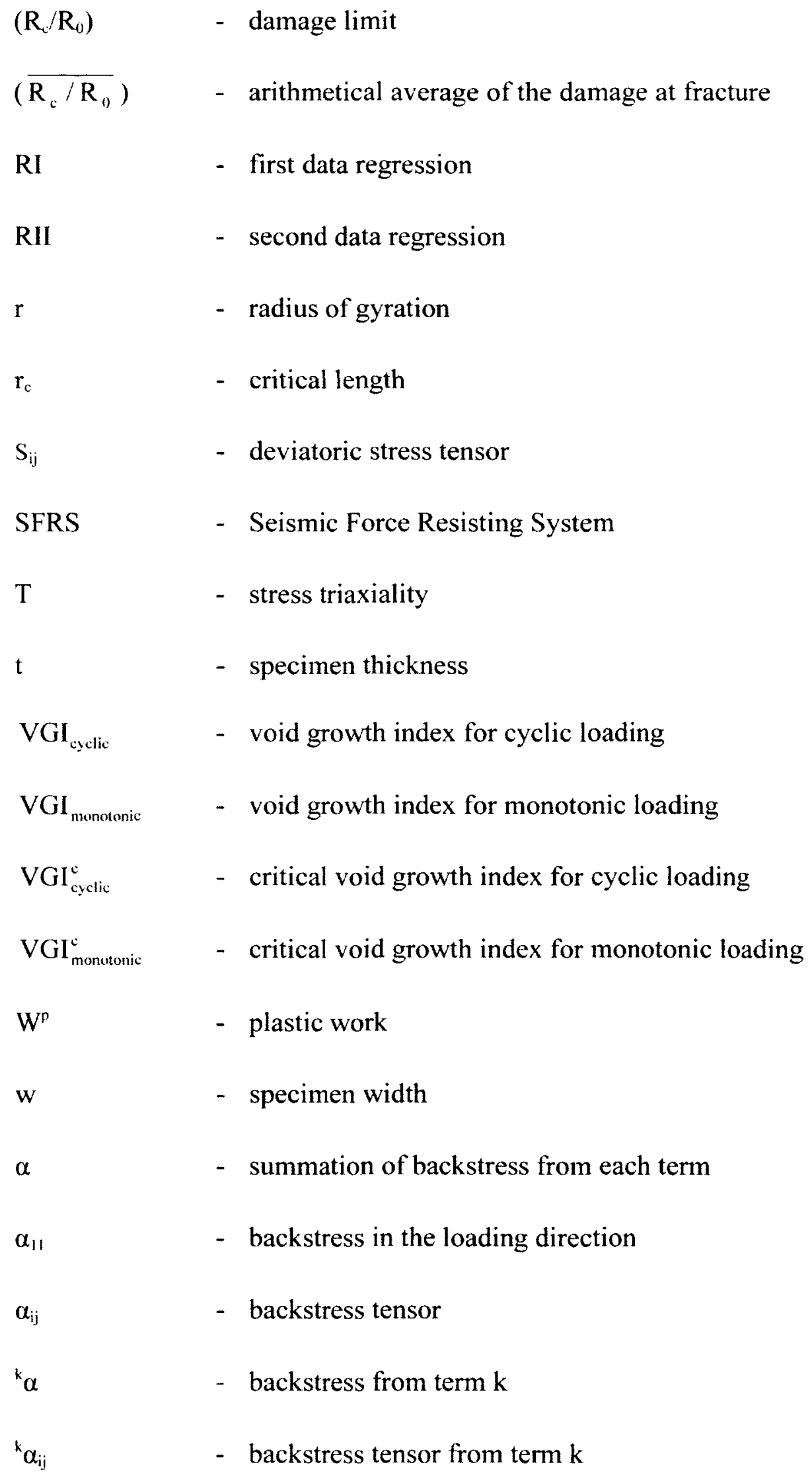




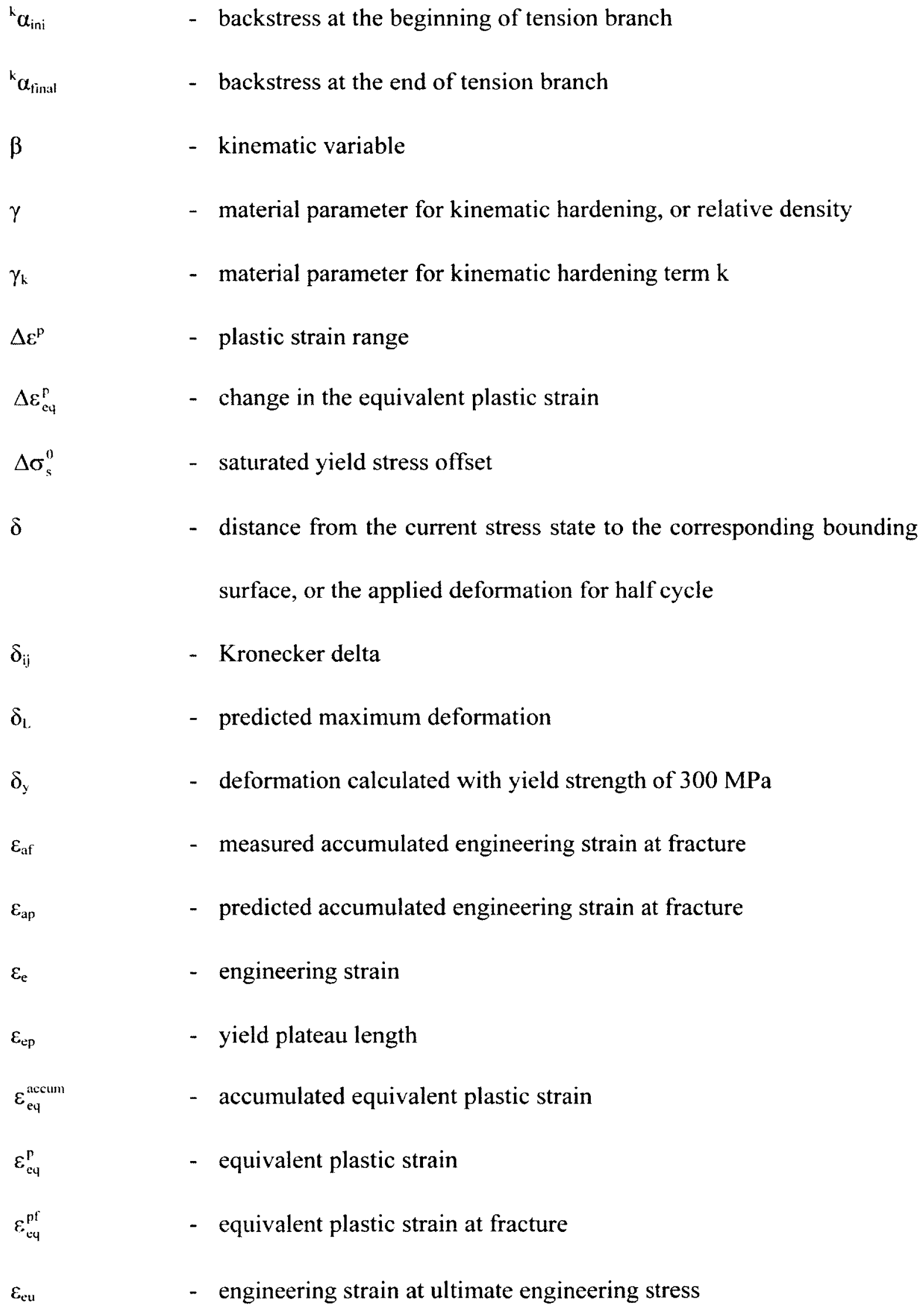

${ }^{k} \alpha_{\text {ini }}$

${ }^{k} \alpha_{\text {final }}$

$\beta$

$\gamma$

$\gamma_{k}$

$\Delta \varepsilon^{p}$

$\Delta \varepsilon_{\mathrm{eq}}^{\mathrm{p}}$

$\Delta \sigma_{\mathrm{s}}^{0}$

$\delta$

$\delta_{i j}$

$\delta_{\mathrm{l}}$

$\delta_{y}$

$\varepsilon_{\mathrm{af}}$

$\varepsilon_{\mathrm{ap}}$

$\varepsilon_{\mathrm{e}}$

$\varepsilon_{\text {ep }}$

$\varepsilon_{\text {eq }}^{\text {accum }}$

$\varepsilon_{\mathrm{eq}}^{\mathrm{p}}$

$\varepsilon_{\text {eq }}^{\mathrm{pf}}$

$\varepsilon_{\mathrm{cu}}$

- backstress at the beginning of tension branch

- backstress at the end of tension branch

- kinematic variable

- material parameter for kinematic hardening, or relative density

- material parameter for kinematic hardening term $\mathrm{k}$

- plastic strain range

- change in the equivalent plastic strain

- saturated yield stress offset

- distance from the current stress state to the corresponding bounding surface, or the applied deformation for half cycle

- Kronecker delta

- predicted maximum deformation

- deformation calculated with yield strength of $300 \mathrm{MPa}$

- measured accumulated engineering strain at fracture

- predicted accumulated engineering strain at fracture

- engineering strain

- yield plateau length

- accumulated equivalent plastic strain

- equivalent plastic strain

- equivalent plastic strain at fracture

- engineering strain at ultimate engineering stress 


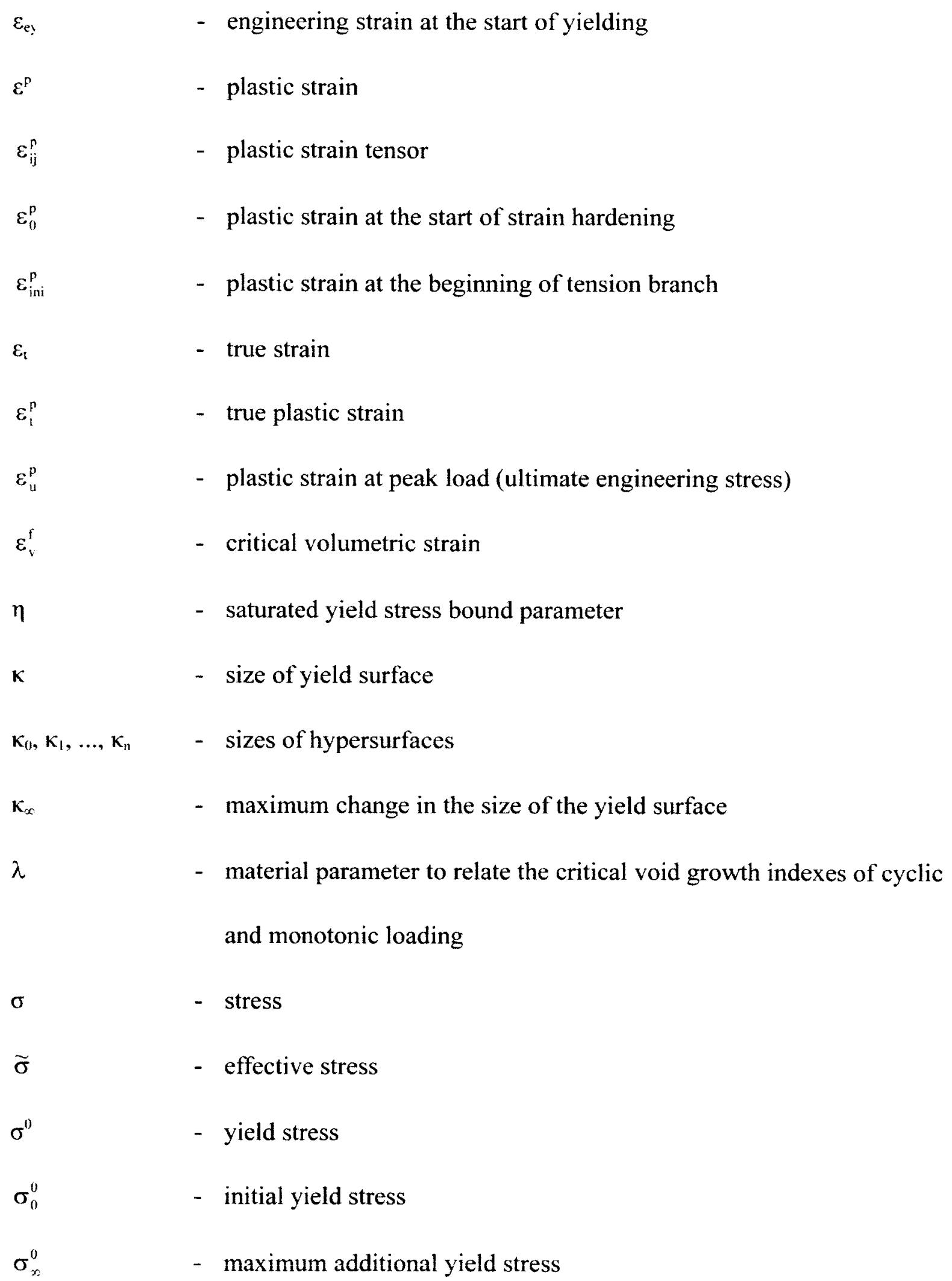




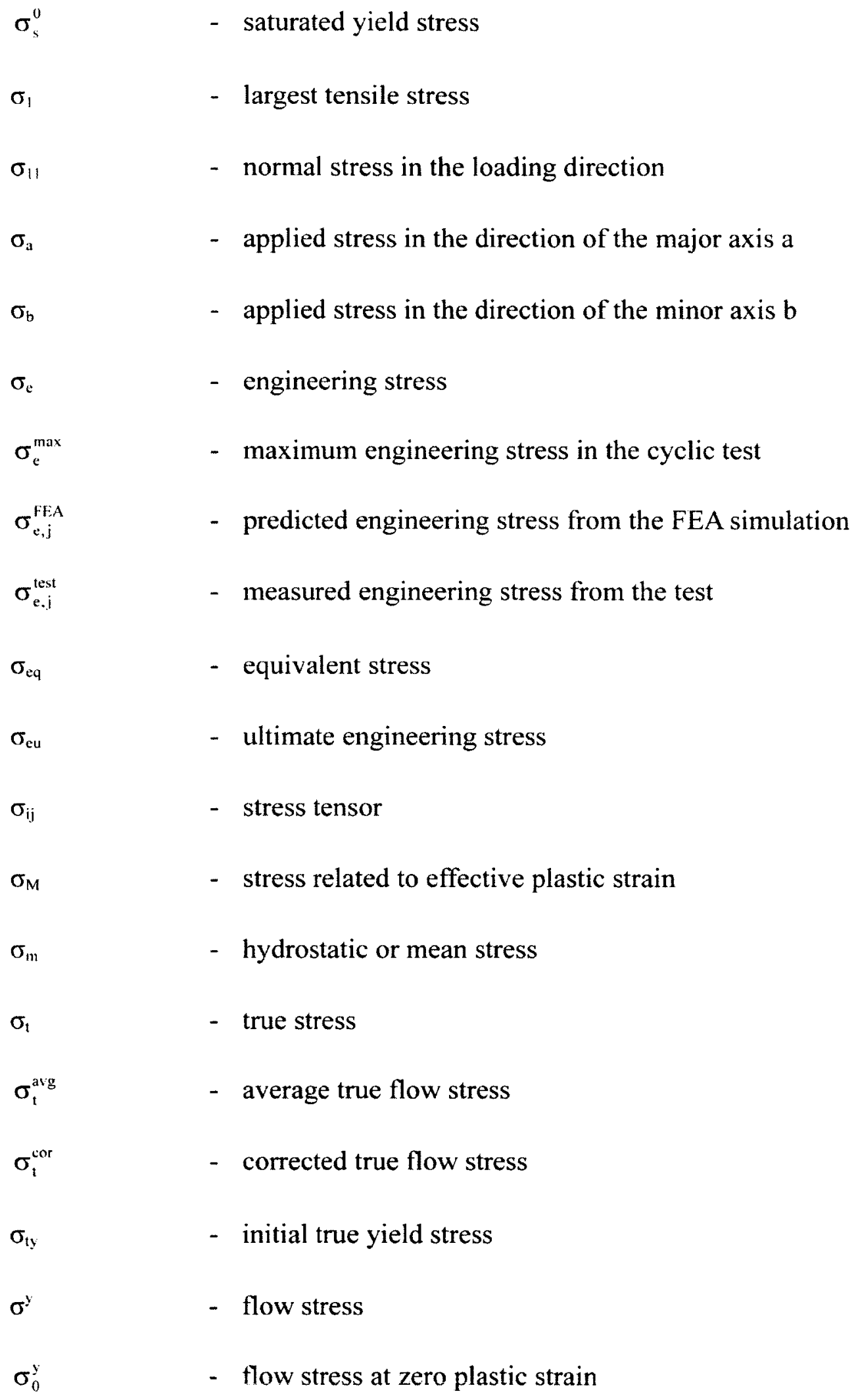




$\begin{array}{ll}\sigma_{x}^{y} & - \text { maximum flow stress } \\ \sigma_{u}^{y} & - \text { flow stress at peak load (ultimate engineering stress) } \\ \sigma_{11}^{c} & - \text { flow stress for the reversed branch in uniaxial cyclic loading } \\ \sigma_{11}^{\mathrm{l}} & - \text { flow stress for the forward branch in uniaxial cyclic loading } \\ v & - \text { Poisson's ratio } \\ \phi & - \text { material parameter for the model by Armstrong and Frederick }\end{array}$




\section{CHAPTER 1 INTRODUCTION}

The performance of structures under any loading condition is greatly dependent on the material behaviour. Knowing the actual material behaviour is especially important in designing a structure to withstand seismic load. The philosophy adopted in the seismic design is based on the capacity-design approach where ductile components in the Seismic Force Resisting System (SFRS) of the structure are expected to be able to undergo considerable cyclic plastic deformation to dissipate energy during a severe seismic event. Consequently the rest of the structure has to be designed to withstand forces generated by the SFRS undergoing the cyclic plastic deformation. The material stress versus strain curve and fracture limit have a significant influence on the ductility of the SFRS and the forces generated by the system. However, the steel used in the structure does not have a unique stress versus strain curve. Thus, it is essential to assess the performance of the SFRS with different material properties (stress-strain curves).

There are many types of Seismic Force Resisting Systems. One of them is a concentrically braced frame system. A brace is required to have the ductility to dissipate energy during an earthquake. In the National Building Code of Canada (NBCC) 2010 (NRCC 2010), a normal building is permitted to deflect up to $2.5 \%$ in storey drift due to seismic load. Ideally, a brace in a SFRS should be able to sustain this deformation under cyclic loading condition without any significant loss of strength. Other than the slenderness ratio, Al-Kharat and Rogers (2007) and Aghdam (2009) have found that the ductility of a steel strap tension-only brace is significantly affected by the net section fracture to gross section yielding strength ratio of the bolted (screwed) connection. An 
example of an isolated strap tension-only brace with the bolted connection in a test is shown in Fig. 1.1. Although testing is the most accurate option to study the performance of the brace, it is very costly to carry out the tests for all different combinations of various factors. Therefore, an economical alternative is to carry out the numerical testing for these combinations.

Many plasticity models and ductile fracture criteria have been proposed for steel (metals) from a large number of studies. However, most of these studies seldom evaluate both the material behaviour modeling together with the fracture prediction under many different loading conditions. Furthermore, parametric finite element analysis studies of the structural system under cyclic loading were carried out mainly using either the material stress-strain curve from the specimen tested (Berman and Bruneau 2006) or various stress-strain curves that are generated by simple functions (Tremblay et al. 1997), such as a bi-linear stress-strain curve for the linear kinematic hardening. Thus, it will be useful to develop a systematic approach to generate the material property input data required in the finite element analysis for a cyclic plasticity model that can reasonably represent the material response over a large range of loading conditions with only a few parameters. This will enable the input data for a wide range of material behaviour response to be generated easily and used in the parametric finite element analysis study to assess the performance of the structural system.

\subsection{Objective of the Thesis}

The objective of the research is to develop a systematic procedure to generate the material property input data of steel for a wide range of material behaviour in the finite 
element analysis of a structural system or component, and to establish the fracture criterion for steel under a variety of loading scenarios including monotonic and cyclic loading. Using the procedure developed and the fracture criterion established, a parametric finite element analysis study is carried out for various brace geometric configuration and material behaviour combinations to assess the performance of a steel strap tension-only brace, and to provide recommendations in its design as a brace in a SFRS.

\subsection{Methodology Used in the Research}

A total of thirty nine round specimens fabricated from round rods from ASTM A572 (ASTM 2007) Grade 50 and CSA G40.20/21 (CSA 2004) Grade 300W steels were tested to failure. These specimens consist mainly of three profiles of one straight and two notched shapes. The specimens were tested under (1) monotonic tension, (2) cyclic loading followed by tension and (3) cyclic only loading conditions. Specimens of different profiles were tested under various loading conditions in order to allow for a more comprehensive study to be conducted in the development of the fracture criterion and the procedure in generating input data for different material behaviours (properties) to be used in the finite element analysis. A number of material property equations and fracture criteria were assessed.

Based on the material property equations selected, a systematic procedure has been devised to allow the material property input data for a wide range of material response to be generated from a few parameters and used in the finite element analysis. Values of some of these parameters are determined in relation to the material property 
characteristics that can be expected in the structural steel such as the range of the ultimate to yield strength ratio and the strain at ultimate stress, while other parameters are varied at some predetermined intervals.

The parametric finite element analysis study of the numerical testing were carried out with ABAQUS (Simulia 2009) for the bolted strap tension-only brace loaded according to ATC-24 Guidelines for Cyclic Seismic Testing of Components of Steel Structures (ATC 1992) for single specimen testing program with the selected fracture criterion and plasticity model implemented in the user-defined material subroutine. Braces with various lengths, slenderness ratios and net section fracture to gross section yielding strength ratios were simulated for a range of material behaviour response generated with the material property equations for the selected range of the equation parameters' values. The fracture limit used in the finite element analysis for each generated material property set has been calibrated with results of round specimen tests by taking the ductility obtained in the tests to be representative of CSA G40.20/21 (CSA 2004) Grade 300W and Grade 350W hot-rolled steels. Results of the finite element analyses were validated against that from the strap tension-only brace tests conducted by Aghdam (2009). Using results of the parametric study, recommendations on using a strap tension-only brace in a SFRS were developed.

\subsection{Organization of the Thesis}

Chapter 2 presents a brief literature review on various metal plasticity models and ductile fracture criteria. 
In Chapter 3, details on round specimen tests are presented. The calibration procedure for the material model parameters is developed. Results of numerical simulations with the calibrated material model parameters are compared to those of the tests to assess the suitability of the adopted material model and equations used in generating material property input data.

Chapter 4 presents the evaluation of fracture criteria based on the experimental results of round specimen tests.

In Chapter 5, the experimental study of the strap tension-only brace by Aghdam (2009) is briefly introduced. Procedures to generate material property input data for the numerical simulations of the steel strap specimens based on the tension material test are described. The FEA models of the strap specimen are also developed. Proposed procedures for generating the material property input data and the adopted fracture criterion are validated against results of the tests by Aghdam (2009) through numerical simulations.

Chapter 6 presents the parametric finite element analysis study on strap tensiononly brace. Effects of material properties and brace geometric configurations are discussed. Equations for predicting the ductility of the brace are proposed and recommendations on designing steel strap tension-only braces in a SFRS are developed.

Chapter 7 consists of the summary and conclusions of this research as well as the recommendations for future work.

Additional results of the research are listed in the appendices. 


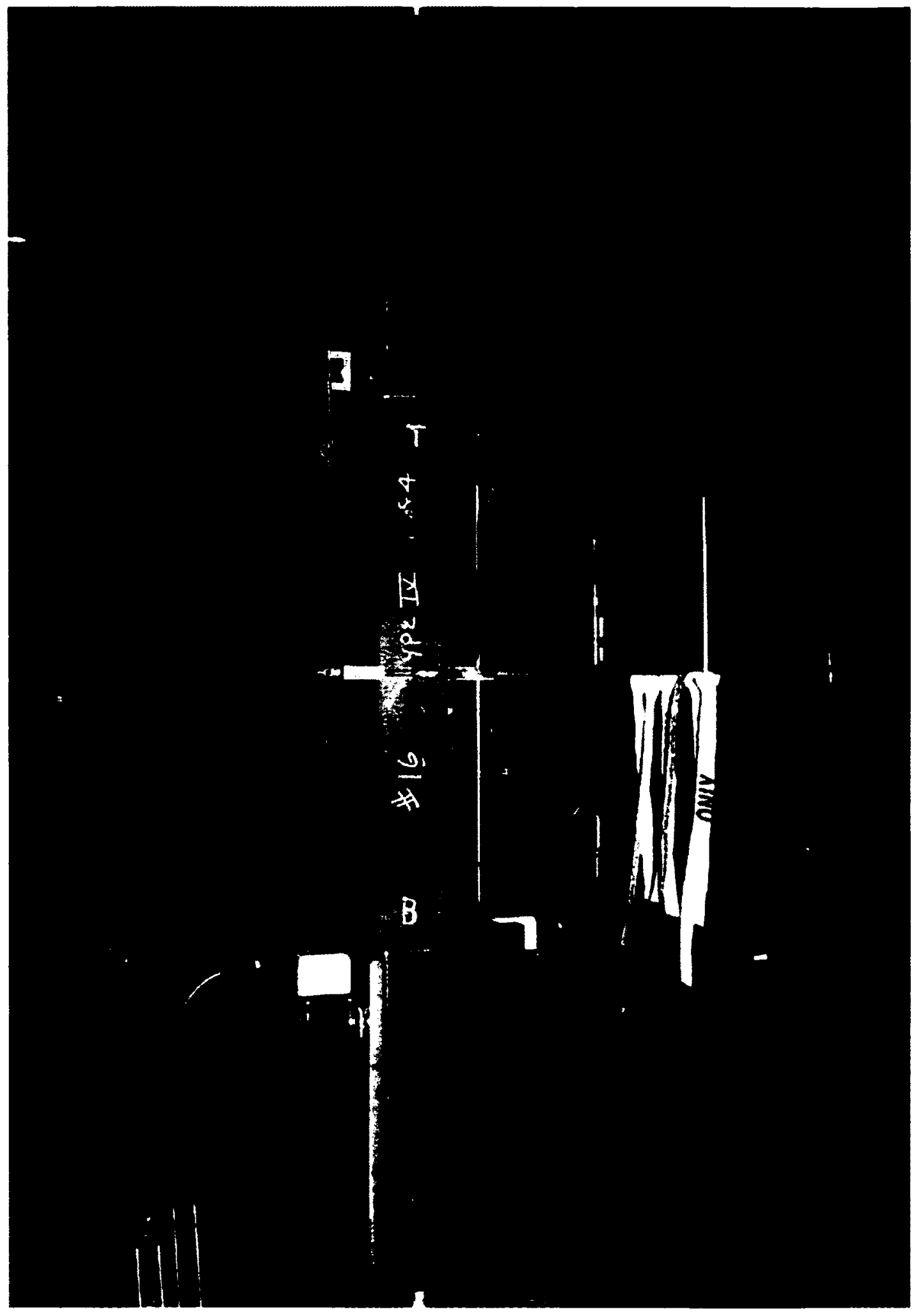

Figure 1.1 Strap tension-only brace with bolted connection (Aghdam 2009) 


\section{CHAPTER 2 LITERATURE REVIEW}

The behaviour of structural steel can be considered to be ductile under quasi-static monotonic loading condition. When subjected to cycles of large stress reversal, such as in a seismic event, components of a steel structure may experience low-cycle fatigue failures without undergoing a large overall deformation. Nevertheless, an accurate simulation of the behaviour and prediction of low-cycle fatigue failure of steel depends greatly on the hardening model and fracture criterion used. When steel is subjected to cyclic loading beyond elastic limit, behaviours such as Bauschinger effect and ratchetting are exhibited. Figure 2.1 shows the Bauschinger effect, which is characterized by a reduced yield stress on load reversal after the initial yielding has occurred. Ratchetting in the material response when subjected to a constant range of unsymmetric cycles of stress (non-zero average stress) is shown in Fig.2.2. Some of the hardening models and fracture criteria proposed for steel are discussed in the following sections.

\subsection{Modeling of Plasticity}

There are two basic work hardening rules that are widely used in describing the plastic behaviour of steel (metals): isotropic hardening and kinematic hardening. As shown in Fig. 2.3, isotropic hardening occurs when the yield surface expands uniformly in the stress space during plastic deformation. In kinematic hardening, the yield surface does not change its form and orientation but translates in the stress space similar to a rigid body translation, which manifests as Bauschinger effect. When combining isotropic and 
kinematic hardening rules to describe the plastic behaviour, the yield surface translates in the stress space as well as expanding or contracting in its size.

Strain hardening is a result of an increase in barriers to slip deformation or dislocation entanglements in the material as the plastic strain increases. Consequently, a larger force (stress) is required to increase the deformation. It was also described by Lemaitre and Chaboche (1990) that there is a deformation incompatibility at grain boundaries with permanent deformations. This incompatibility is accommodated by elastic microdeformations. These deformations remain partially locked in when the load is released, which induce microscopic residual stresses. As a result, the effect of this is exhibited as kinematic hardening as the stresses required to initiate the additional plastic deformation vary with the direction. A more detailed treatment of the material behaviour at the microscopic level can be found in McClintock and Argon (1966), and Lemaitre and Chaboche (1990).

Some basic concepts of plasticity are introduced below to help facilitate the discussion of various hardening models. The yield function can be defined as

$$
f\left(\sigma_{i j}-\alpha_{i j}, \kappa\right)=0
$$

where $\sigma_{i \mathrm{ij}}$ is the stress tensor, $\alpha_{\mathrm{ij}}$ is the backstress tensor and $\kappa$ is a scalar that defines the size of the yield surface. For metals, von Mises yield criterion for isotropic material that combines isotropic and kinematic hardenings can be expressed as

$$
\left(S_{i j}-\alpha_{i j}\right)\left(S_{i j}-\alpha_{i j}\right)-\kappa^{2}=0,
$$

where $\mathrm{S}_{\mathrm{ij}}$ is the deviatoric stress tensor defined as

$$
S_{i j}=\sigma_{i j}-\frac{1}{3} \sigma_{i k} \delta_{i j}=\sigma_{i j}-\sigma_{i n} \delta_{i j},
$$


where $\sigma_{m}$ is the hydrostatic or mean stress and $\delta_{i j}$ is the Kronecker delta. The material experiences no isotropic hardening when the change in $\kappa$ is zero $(d \kappa=0)$ and no kinematic hardening when the change in $\alpha_{\mathrm{ij}}$ is zero $\left(\mathrm{d} \alpha_{\mathrm{ij}}=0\right)$.

The plastic deformation of metals is normally assumed to follow the associated flow rule, which is defined as

$$
\mathrm{d} \varepsilon_{\mathrm{ij}}^{\mathrm{p}}=\frac{\partial \mathrm{f}}{\partial \sigma_{\mathrm{ij}}} \mathrm{d} \lambda,
$$

where $d \varepsilon_{i j}^{p}$ is the change in the plastic strain tensor and $d \lambda$ is a constant of proportionality with the equivalent plastic strain increment defined as

$$
\mathrm{d} \varepsilon_{\mathrm{e} 4}^{\mathrm{p}}=\sqrt{\frac{2}{3} \mathrm{~d} \varepsilon_{\mathrm{ij}}^{\mathrm{p}} \mathrm{d} \varepsilon_{\mathrm{ij}}^{\mathrm{p}}} .
$$

Similarly, the equivalent stress associated with plastic deformation can be defined as

$$
\sigma_{e q}=\sqrt{\frac{3}{2} S_{i j} S_{i j}} .
$$

\subsubsection{Hardening Models}

There have been many hardening models proposed to describe the cyclic behaviour of metals. Only some of these models are briefly presented below.

Prager (1956) first proposed a linear kinematic hardening model to describe the cyclic behaviour of metals. The evolution rule of kinematic hardening in the model is given by

$$
\mathrm{d} \alpha_{\mathrm{ij}}=\mathrm{cd} \varepsilon_{\mathrm{ij}}^{\mathrm{p}}
$$


where $\alpha_{\mathrm{ij}}$ is the backstress tensor, $\mathrm{d} \varepsilon_{\mathrm{ij}}^{\mathrm{p}}$ is the increment of the plastic strain tensor and $\mathrm{c}$ is a material constant. Ziegler (1959) modified the kinematic hardening model proposed by Prager to

$$
\mathrm{d} \alpha_{\mathrm{ij}}=\left(\sigma_{\mathrm{ij}}-\alpha_{\mathrm{ij}}\right) \mathrm{d} \mu
$$

where $\sigma_{\mathrm{ij}}$ is the stress tensor and $\mathrm{d} \mu$ is a multiplier. The use of tensor $\left(\sigma_{\mathrm{ij}}-\alpha_{\mathrm{ij}}\right)$ allows the hardening rule to account for the influence of mean stress on the evolution of the kinematic internal variable. In the case of incompressible plasticity where the von Mises yield criterion of Eq. (2.2) is used together with the associated flow rule, both models by Prager and Ziegler are identical (Lemaitre and Chaboche 1990). Although a linear kinematic hardening model can represent the Bauschinger effect under cyclic loading, it cannot describe ratchetting.

Mroz (1967) introduced the concept of representing the work hardening modulus by a number of hypersurfaces in the stress space. In this model, the stress versus strain curve is approximated by $n$ linear segments of constant plastic moduli $E_{1}^{p}, E_{2}^{p}, \ldots, E_{n}^{p}$. In the stress space, this approximation can be represented by $n$ hypersurfaces $f_{0}, f_{1}, \ldots, f_{n}$ with the size $\kappa_{n}, \kappa_{1}, \ldots, \kappa_{n}$, where $f_{n}$ is the initial yield surface. The work hardening modulus is assumed to be constant in the region between the hypersurfaces. The change in the backstress tensor is given by

$$
d \alpha_{i j}^{m}=\frac{d \mu}{\kappa^{m}}\left[\left(\kappa^{m+1}-\kappa^{m}\right) \sigma_{i j}^{m}-\left(\alpha_{i j}^{m} \kappa^{m+1}-\alpha_{i j}^{m+1} \kappa^{m}\right)\right]
$$

where $d \mu$ is a constant of proportionality, superscript $m$ is the current yield hypersurface, and the stress points at the initial and final yield surfaces have the same outward normal. One obvious shortcoming of this model is that a large number of variables are needed, 
which leads to a heavier computing demand for the numerical solution. It also lacks the ability to model the ratchetting behaviour under an unsymmetrical loading condition (non-zero average stress).

Similar to that proposed by Mroz (1967), Dafalias and Popov (1975, 1976) proposed a simplified bounding surface plasticity model that can capture the nonlinear kinematic hardening effect. All the hypersurfaces in the model by Mroz (1967) are replaced by an inner yield surface and an outer bounding surface. Two-surface model has also been proposed by Krieg (1975) independently of Dafalias and Popov (1975, 1976). In the model proposed by Dafalias and Popov $(1975,1976)$, a function $\hat{E}^{p}\left(\delta, \delta_{\text {in }}\right)$ is introduced to define the continuous variation of the plastic modulus instead of discrete moduli, which simplifies the computational effort. The plastic modulus $E^{p}$ is defined by

$$
E^{p}=\hat{E}^{p}\left(\delta, W^{p}\right)
$$

where $\delta$ is the distance from the current stress state to the corresponding bounding surface, and $W^{p}$ is the plastic work defined as

$$
W^{p}=\int \sigma_{i j} d \varepsilon_{i j}^{p}
$$

Even though this model allows the yield and bounding surfaces to change in size, only kinematic hardening has been considered in the examples presented. Petersson and Popov (1977) proposed improvements to this model by introducing a weighting function and intermediate surfaces between the yield and the bounding surfaces. Nevertheless, Dafalias and Popov (1976), and Petersson and Popov (1977) showed that there was a good agreement between their respective experimental and predicted stress-strain curve of the uniaxial cyclic test at a moderate strain. Adopting the idea similar to that by Mroz (1967), the distance from the current stress state to the bounding surface is given by the 
contact point on the yield surface to a corresponding point on the bounding surface with the stress state that has the same outward normal.

Tseng and Lee (1983) proposed another hardening model based on the twosurface concept by Dafalias and Popov $(1975,1976)$. The yield surface was assumed to follow a combined isotropic and kinematic hardening rule while the bounding surface only experiences isotropic hardening. There is no coupled movement of yield and bounding surfaces until both surfaces are in contact. There was a good correlation between the prediction given by the model and the experimental data of the nonproportional cyclic strain path test at a moderate strain for a small number of cycles. There were also other modifications to the bounding surface model proposed by Minagawa et al. (1987), Mizuno et al. (1992), Montáns (2000), Geng and Wagoner (2002) and Lee et al. (2007).

In models by Mroz (1967), Dafalias and Popov (1975, 1976), and Tseng and Lee (1983), the nonlinearity of kinematic hardening was based on the concept of multiple bounding surfaces. Unlike these models, Armstrong and Frederick (1966) introduced nonlinear kinematic hardening by defining the evolution of backstress directly as

$$
\mathrm{d} \alpha_{\mathrm{ij}}=\phi\left[\mathrm{d} \varepsilon_{\mathrm{ij}}^{\mathrm{p}}-\frac{\alpha_{\mathrm{ij}}}{\mathrm{h}\left(\varepsilon_{\mathrm{eq}}^{\mathrm{p}}\right)} \mathrm{d} \varepsilon_{\mathrm{eq}}^{\mathrm{p}}\right],
$$

where $\phi$ is a material constant, $\varepsilon_{\mathrm{eq}}^{\mathrm{p}}$ is the equivalent plastic strain and $\mathrm{h}\left(\varepsilon_{\mathrm{eq}}^{\mathrm{p}}\right)$ is a scalar function of $\varepsilon_{\mathrm{eq}}^{\mathrm{p}}$. The yield surface can also change its size while its centre translates.

A version of the combined isotropic and nonlinear kinematic hardening model by Armstrong and Frederick (1966) was presented by Chaboche (1986), and Lemaitre and 
Chaboche (1990) by taking the scalar function $h\left(\varepsilon_{\mathrm{eq}}^{\mathrm{p}}\right)$ to be a constant. Thus, the evolution of backstress becomes

$$
\mathrm{d} \alpha_{\mathrm{ij}}=\mathrm{Cd} \varepsilon_{\mathrm{ij}}^{\mathrm{p}}-\gamma \alpha_{\mathrm{ij}} \mathrm{d} \varepsilon_{\mathrm{eq}}^{\mathrm{p}}
$$

where $\mathrm{C}$ and $\gamma$ are the material constants. The evolution of nonlinear kinematic hardening was further combined with the yield surface evolution function for isotropic hardening modified from that proposed by Voce (1955), which is given as

$$
\kappa=\kappa_{0}+\kappa_{\infty}\left(1-\mathrm{e}^{-b \varepsilon_{c y}^{\mathrm{p}}}\right)
$$

where $\kappa_{0}$ is the size of the initial yield surface, $\kappa_{\infty}$ is the maximum change in the size of the yield surface and $b$ is a material constant. Although this model can represent many characteristics of cyclic behaviour, it cannot accurately simulate out-of-phase cyclic loading and effects of strain range. Various improvements on the mixed-mode hardening model with the combined Eqs. (2.13) and (2.14) have also been suggested by Chaboche (1986). Some of these modifications include using multiple terms of Eq. (2.13) to improve the simulation of ratchetting. Effects of strain range can be modeled by allowing the saturated stress given by Eq. (2.14) to be a function of the strain range.

Bower (1989) modified the Armstrong and Frederick (1966) model by introducing a second kinematic variable $\beta_{\mathrm{ij}}$ in the recall term as

$$
\mathrm{d} \alpha_{\mathrm{ij}}=\mathrm{Cd} \varepsilon_{\mathrm{ij}}^{\mathrm{p}}-\gamma_{1}\left(\alpha_{\mathrm{ij}}-\beta_{\mathrm{ij}}\right) \mathrm{d} \varepsilon_{\mathrm{eq}}^{\mathrm{p}}
$$

where $\beta_{\mathrm{ij}}$ is defined as

$$
\mathrm{d} \beta_{\mathrm{ij}}=\gamma_{2}\left(\alpha_{\mathrm{ij}}-\beta_{\mathrm{ij}}\right) \mathrm{d} \varepsilon_{\mathrm{eq}}^{\mathrm{p}}
$$

and $\gamma_{1}$ and $\gamma_{2}$ are material constants. Setting $\gamma_{2}=0$, Eq. (2.15) is similar to Eq. (2.13). This modification improves the modeling of the rail steel behaviour in which the 
ratchetting rate was observed to decrease with continued cycling. In order to improve the simulation of ratchetting behaviour, Ohno and Wang (1993a, b) decomposed the kinematic hardening variable in a multi-term kinematic hardening Armstrong and Frederick (1966) model into components based on the assumption that each component has a critical state for its recovery to be activated fully. Basuroychowdhury and Voyiadjis (1998) also proposed modifications to the kinematic hardening term by Armstrong and Frederick (1966) to improve the modeling of the behaviour due to nonproportional loading. Other modifications to the kinematic hardening term by Armstrong and Frederick (1966) have also been proposed by Chun et al. (2002) and Chung et al. (2005) to model sheet forming process. For modeling of non-proportional loading response, Francois $(2001)$, Feigenbaum and Dafalias $(2007,2008)$ proposed using a yield surface that distorts with loading.

There have been many numerical simulations carried out for steel structures under cyclic loading. Most of the analyses have been carried out with the plasticity model based on a variation of Dafalias and Popov $(1975,1976)$ model or Armstrong and Frederick (1966) model. Shen et al. (1995), Goto et al. (1998), Jiang et al. (2002), Chiou and Hsiao (2005) and Mamaghani (2006) performed the numerical analyses using Dafalias and Popov (1975, 1976) based hardening models, while Chou and Wu (2006), and Berman and Bruneau (2006) used Armstrong and Frederick (1966) based hardening models. 


\subsubsection{Summary of Combined Isotropic and Kinematic Hardening Model}

Numerous studies from Lemaitre and Chaboche (1990), and Kanvinde and Deierlein $(2004,2007)$ have shown that the combined isotropic and nonlinear kinematic hardening model by Chaboche (1986) based on Eqs. (2.2) and (2.13) was able to reasonably predict the behaviour of a steel where the loading is primarily close to proportional. Beside the elastic modulus and Poisson's ratio, a few model parameters have to be defined to carry out the numerical simulation. These parameters determine the predicted material response when undergoing monotonic and cyclic plastic deformations. The predicted response is described by the relationship between the parameter $\kappa$ in Eq. (2.2) versus the equivalent plastic strain $\varepsilon_{\mathrm{e} 4}^{\mathrm{p}}$ for isotropic hardening to define the size of the yield surface and the parameters $\mathrm{C}$ and $\gamma$ (multiple terms if required) for kinematic hardening in Eq. (2.13) to define the evolution of backstress. The challenge is to determine a single set of parameters that can reasonably model the behaviour for both monotonic and cyclic loading until fracture.

Even though using isotropic hardening does not allow the model to accurately simulate out-of-phase cyclic loading, this is not expected to significantly affect results of most analyses. When a steel structure is subjected to seismic loading, the critical point in the structural component mainly experiences close to proportional and not significantly out-of-phase loading condition. For example, the stress at the flange tip of the I-shape beam is primarily uniaxial. The model is also expected to be less capable of modeling the effect of strain range in cyclic loading if only one strain range independent hardening relationship $\kappa$ versus $\varepsilon_{\mathrm{eq}}^{\mathrm{p}}$ is considered. 


\subsection{Fracture of Steel (Metals)}

In steel structures, ductile fracture may occur due to low-cycle fatigue and overload. There are different approaches used in predicting fracture of a structural component depending on the loading and the type of analysis employed. Parameters that relate to the local condition at the tip of a crack or flaw due to the applied load, such as stress intensity factor, J-integral and crack-tip opening displacement have been widely used to predict fracture in steel structures. For low-cycle fatigue failure prediction, the relationship proposed by Manson (1953) and Coffin (1954) has been widely used to relate the cyclic strain range to the number of cycle to fracture. It can be expressed as

$$
\Delta \varepsilon^{\mathrm{p}}=\mathrm{D}\left(\mathrm{N}_{\mathrm{f}}\right)^{\mathrm{c}},
$$

where $\Delta \varepsilon^{p}$ is the range of plastic strain in cyclic loading, $N_{f}$ is the number of cycle to fracture, $\mathrm{D}$ and $\mathrm{c}$ are material constants. This relationship has subsequently been modified to account for multiaxial non-proportional loading. Some of the recent studies in this area were carried out by Yang and Itoh (2008), Itoh and Ozaki (2008), and Susmel et al. (2008). However, local fracture criteria that are integrated over the stress and strain history are better suitable in a finite element analysis for predicting ductile fracture of a structural steel component due to low-cycle fatigue and monotonic loading since in most situations the local stress and strain paths are more complex and not known in advance.

The mechanism for ductile fracture in metals is microvoid coalescence. Microvoids formed in ductile metals as the deformation increases due to either particle cracking, or the separation of inclusions and precipitate particles from the surrounding matrix. These microvoids grow and coalesce, and eventually lead to fracture. Numerous criteria have been proposed to predict ductile fracture of metals. Many fracture criteria 
have been developed for monotonic loading based on the observed physical process. Some of these criteria are critical void growth rate, critical void volume fraction, absorbed strain energy till fracture, critical damage state and other limits integrated over the deformation history. A few of these criteria have also been modified and extended to low-cycle fatigue.

\subsubsection{Void Growth}

McClintock et al. (1966) used a physical model to describe the phenomenon of the growth and eventual merging of holes from microscopic inclusions within the material that result in fracture. In this physical model, large numbers of cylindrical holes are assumed to be scattered uniformly throughout a material. The solid can be considered to be divided into a number of elements, with each element containing a single centrallylocated hole. Fracture occurs when the holes touch the boundaries of their elements. Based on the assumed model, McClintock (1968) analyzed the expansion of a long cylindrical void subjected to the transverse tensile stresses. From the study, the fracture limit is given by $F_{b}^{f}$ as

$$
\ln F_{b}^{f}=\int_{0}^{\varepsilon_{\mathrm{cq}}^{\mathrm{nd}}}\left[\frac{\sqrt{3}}{2(1-\mathrm{c})} \sinh \left(\frac{\sqrt{3}(1-\mathrm{c})}{2} \frac{\left(\sigma_{\mathrm{a}}+\sigma_{\mathrm{b}}\right)}{\sigma_{\mathrm{eq}}}\right)+\frac{3}{4} \frac{\left(\sigma_{\mathrm{a}}-\sigma_{\mathrm{b}}\right)}{\sigma_{\mathrm{eq}}}\right] \mathrm{d} \varepsilon_{\mathrm{eq}}^{\mathrm{p}},
$$

where

$F_{b}^{f}=$ relative growth factor, which is the ratio of the width and the spacing of the ellipse in the minor axis b,

$\sigma_{\mathrm{eq}} \quad=$ equivalent stress,

$\sigma_{\mathrm{a}} \quad=$ applied stress in the direction of the major axis a, 
$\sigma_{\mathrm{b}} \quad=$ applied stress in the direction of the minor axis $b$,

$\varepsilon_{\text {eq }}^{\mathrm{n}} \quad=$ equivalent plastic strain,

$\varepsilon_{\mathrm{eq}}^{\mathrm{ri}} \quad=$ equivalent plastic strain at fracture,

c $\quad=$ hardening coefficient.

In order to predict the fracture strain, information regarding inclusion shape, size and spacing and void growth rates are required.

Instead of the long cylindrical void considered by McClintock (1966), Rice and Tracey (1969) used a more realistic shape of an isolated spherical void in a remotely uniform stress and strain rate field to determine the relationship between void growth and stress triaxiality. The void growth rate can be expressed as

$$
\frac{\mathrm{dR}}{\mathrm{R}}=\mathrm{c} \exp (1.5 \mathrm{~T}) \mathrm{d} \varepsilon_{\mathrm{eq}}^{\mathrm{p}},
$$

where $R$ is the instantaneous void radius, $c$ is a material constant and $T$ is the stress triaxiality defined as the ratio of the mean stress to the equivalent stress defined as

$$
\mathrm{T}=\frac{\sigma_{\mathrm{m}}}{\sigma_{\mathrm{cq}}}
$$

Integrating Eq. (2.19), the void radius $\mathrm{R}$ can be expressed as

$$
\ln \left(\frac{\mathrm{R}}{\mathrm{R}_{0}}\right)=\int_{0}^{\varepsilon_{\mathrm{eq}}^{\mathrm{pr}}} c \exp (1.5 \mathrm{~T}) \mathrm{d} \varepsilon_{\mathrm{eq}}^{\mathrm{p}},
$$

where $R_{0}$ is the initial void radius. The expression in Eq. (2.19) was developed neglecting the interaction of multiple voids. The left side of Eq. (2.21) can be interpreted as the critical void ratio assuming that void growth to be the controlling aspect of the fracture process. However, the coefficient of 1.5 in the exponential term has been found to vary for different materials. The coefficient in the range of 1.1 to 2.3 was found to 
best fit the fracture test data by Hancock and Brown (1983) for 50D and 50D N.N. British steels, and 2.12 by Johnson and Cook (1985) for AISI 4340 steel.

Banks-Sills and Budiansky (1982) studied the collapse of voids in compression. Studies on void growth and collapse in cyclic loading have also been carried out by Hori and Nemat-Nasser (1988) and Ristinmaa (1997). Kanvinde and Deierlein $(2004,2007)$ proposed a fracture criterion under cyclic loading based on the void growth fracture criterion by Rice and Tracey (1969). In order to account for the reversed load under cyclic loading condition, the absolute value of the stress triaxiality term $\mathrm{T}$ is used in Eq. (2.19). The sign of stress triaxiality was also added to differentiate between expanding and shrinking of the void due to tension versus compression loading, and Eq. (2.19) was correspondingly modified to

$$
\frac{\mathrm{dR}}{\mathrm{R}}=\operatorname{sign}(\mathrm{T}) \exp (|1.5 \mathrm{~T}|) \mathrm{d} \varepsilon_{\mathrm{eq}}^{\mathrm{p}}
$$

Equation (2.22) can be integrated over its loading history by subdividing the loading cycles into tensile and compressive components as

$$
\ln \left(\frac{\mathrm{R}}{\mathrm{R}_{0}}\right)_{\text {cyctic }}=\sum_{\substack{\text { tensile } \\ \text { cycles }}} \mathrm{c}_{1} \int_{\varepsilon_{\mathrm{equ} 1}^{\mathrm{p}}}^{\varepsilon_{\mathrm{eq} 2}^{\mathrm{p}}} \exp (|1.5 \mathrm{~T}|) \mathrm{d} \varepsilon_{\mathrm{eq}}^{\mathrm{p}}-\sum_{\substack{\text { compressive } \\ \text { cycles }}} \mathrm{c}_{2} \int_{\varepsilon_{\mathrm{eq}}^{\mathrm{p}}}^{\varepsilon_{\mathrm{q} q 2}^{\mathrm{p}}} \exp (|1.5 \mathrm{~T}|) \mathrm{d} \varepsilon_{\mathrm{eq}}^{\mathrm{p}},
$$

where $\varepsilon_{\mathrm{eq} 1}^{\mathrm{p}}$ and $\varepsilon_{\mathrm{eq} 2}^{\mathrm{p}}$ are the equivalent plastic strains at the beginning and the end of each cycle, $c_{1}$ and $c_{2}$ are the material constants. The negative sign on the second term reflects that plastic straining will cause void shrinkage when the triaxial stress is negative.

Assuming that $c_{1}$ and $c_{2}$ are equal, and represented with c, Eq. (2.23) can be simplified to 


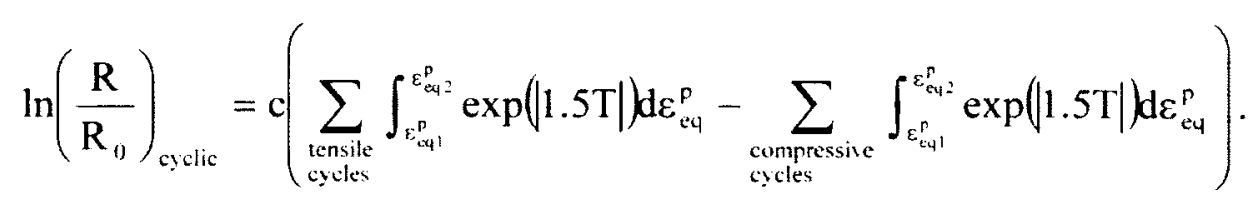

In addition, a new term defined as void growth index was introduced in order to improve the prediction of fracture. The void growth index (VGI) for cyclic loading is calculated as

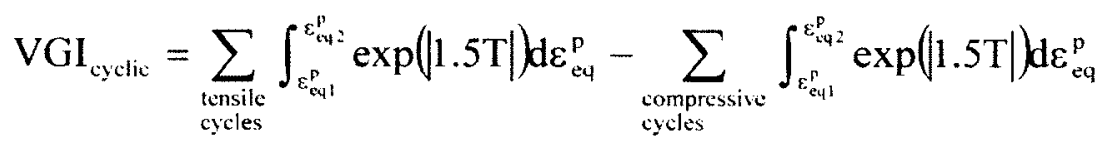

$$
\begin{aligned}
& =\frac{1}{\mathrm{c}} \ln \left(\frac{\mathrm{R}}{\mathrm{R}_{0}}\right)_{\mathrm{cyclic}} .
\end{aligned}
$$

Fracture is assumed to occur when

$$
\mathrm{VGI}_{\text {cyclic }}>\mathrm{VGI}_{\text {cyclic }}^{\mathrm{c}}=\frac{1}{\mathrm{c}} \ln \left(\frac{\mathrm{R}_{\mathrm{c}}}{\mathrm{R}_{0}}\right)_{\text {cyclic }} .
$$

Similarly, for monotonic loading, fracture is assumed to occur when

$$
\mathrm{VGI}_{\text {monotonic }}>\mathrm{VGI}_{\text {munntonic }}^{\mathrm{c}}=\frac{1}{\mathrm{c}} \ln \left(\frac{\mathrm{R}_{\mathrm{c}}}{\mathrm{R}_{0}}\right)_{\text {monotonic }} .
$$

A relationship is assumed to exist between the critical void growth index for cyclic and monotonic loading through

$$
\mathrm{VGI}_{\mathrm{cyclic}}^{\mathrm{c}}=\mathrm{VGI}_{\text {monotonic }}^{\mathrm{c}} \exp \left(\lambda \varepsilon_{\mathrm{cu}}^{\text {accum }}\right),
$$

where $\lambda$ is a material constant and $\varepsilon_{\mathrm{eq}}^{\text {accum }}$ is the accumulated equivalent plastic strain from all the preceding tensile legs of the loading cycle. Comparisons with the test results from various straight and notched specimens were considered to be encouraging although there was significant scatter. 


\subsubsection{Void Volume Fraction}

Gurson $(1977 \mathrm{a}, \mathrm{b})$ proposed a constitutive model that incorporates nucleation and growth of voids in ductile fracture. The plastic potential of the model is a function of the void volume fraction and mean stress in addition to the deviatoric stress. It was further modified by Tvergaard (1982), and Tvergaard and Needleman (1984). The potential function is defined as

$$
\Phi=\frac{3 S_{i j} S_{i j}}{2 \sigma_{M}^{2}}+2 f^{*} q_{1} \cosh \left(\frac{q_{2} \sigma_{k k}}{2 \sigma_{M}}\right)-\left[1+\left(q_{3} f^{*}\right)^{2}\right]=0
$$

where $q_{1}, q_{2}$ and $q_{3}$ are adjustable parameters, $f^{*}$ is a function of void volume fraction $f$ and $\sigma_{M}$ depends on the effective plastic strain through a uniaxial true stress versus natural $\log$ strain curve. The growth rate of the void volume fraction consists of void growth and void nucleation. Fracture is assumed to occur when the void volume fraction reaches a value $f_{F}$. For $q_{2}=0$ and $q_{1}=q_{3}$, local fracture is assumed to occur when $f^{*}=1 / q_{1}$. $f^{*}$ is defined as

$$
f^{*}=\left\{\begin{array}{cc}
f & \text { for } f \leq f_{c}, \text { and } \\
f_{c}+K\left(f-f_{c}\right) & \text { for } f>f_{c}
\end{array}\right.
$$

where $f_{c}$ is the critical void volume fraction and $K$ is a parameter which is related to $q_{1}, f_{c}$ and the void volume fraction at fracture $f_{F}$.

Sun et al. (1989) found that there was a good agreement between the numerical solutions and test results on compact tension specimens and notched round rods. However, Shi et al. (1991) found from tests on cracked specimens that the void volume fraction at the onset of crack growth depends on the geometric constraints and stress triaxiality. Pardoen and Hutchinson (2000) and Benzerga et al. (2004) studied effects of various void porosity, void spacing and initial void shape on the fracture limit. Other 
studies based on Gurson or modified Gurson models have been carried out by Mear and Hutchinson (1985), Leblond et al. (1995), Besson and Guillemer-Neel (2003), and Cedergren et al. (2004) where the original Gurson model has been modified to include kinematic hardening.

\subsubsection{Absorbed Strain Energy Density}

Gillemot (1976) and Matic et al. (1987) used the absorbed critical strain energy density $(\mathrm{dW} / \mathrm{dV})_{\mathrm{c}}$ till fracture as a limit to trigger fracture initiation. The strain energy density can be written as

$$
\frac{d W}{d V}=\int \sigma_{i j} d \varepsilon_{i j} .
$$

In the test, Gillemot (1976) found that $(\mathrm{dW} / \mathrm{dV})_{\mathrm{c}}$ is almost constant for tests with different loading conditions of tension only, compression to tension and cyclic to tension. However, Matic et al. (1987) found that $(\mathrm{dW} / \mathrm{dV})_{\mathrm{c}}$ to be dependent on the loading type whether it is proportional or non-proportional loading. Nevertheless, in large plastic deformation, the absorbed strain energy density is almost independent of the mean stress because the yield function used is normally in terms of only deviatoric stress tensor as in Eq. (2.2). However, McClintock (1968), and Rice and Tracey (1969) found that hydrostatic tension has an inverse effect on the strain at fracture.

\subsubsection{Continuum Damage Mechanics}

Lemaitre $(1984,1985)$ applied continuum damage mechanics to model the effect of void and crack formation, which is reflected in the parameter D. The damage 
parameter $\mathrm{D}$ is incorporated into the material model to relate the effective stress $\widetilde{\sigma}$ and nominal stress $\sigma$ through

$$
\tilde{\sigma}=\frac{\sigma}{1-D}
$$

The nominal stress is the average stress over the gross area including voids and cracks, while the effective stress is the actual stress experienced by the material matrix. Lemaitre (1984) proposed one of the damage rate expressions for isotropic damage as

$$
\begin{aligned}
& \mathrm{dD}=(\mathrm{cy})^{\mathrm{m}} \mathrm{d} \varepsilon_{\mathrm{eq}}^{\mathrm{p}}, \text { and } \\
& \mathrm{y}=\frac{\sigma_{\mathrm{eq}}{ }^{2}}{2 \mathrm{E}(1-\mathrm{D})^{2}}\left[\frac{2}{3}(1+v)+3(1-2 v)\left(\frac{\sigma_{\mathrm{m}}}{\sigma_{\mathrm{eq}}}\right)^{2}\right],
\end{aligned}
$$

where $\mathrm{c}$ and $\mathrm{m}$ are material constants with $\mathrm{m}$ taken as $1, \mathrm{E}$ is the elastic modulus and $v$ is the Poisson's ratio. Fracture is assumed to occur when the critical damage limit $D_{c}$ is reached.

For cyclic loading, Huang and Mahin (2008) used a slightly varied form of damage evolution by Lemaitre (1992) as

$$
\mathrm{dD}=\left\{\begin{array}{cc}
(\mathrm{cy})^{\mathrm{m}} \mathrm{d} \varepsilon_{\mathrm{eq}}^{\mathrm{p}} & \text { for } \frac{\sigma_{\mathrm{m}}}{\sigma_{\mathrm{eq}}}>-\frac{1}{3}, \text { and } \\
0 & \text { otherwise }
\end{array}\right.
$$

Huang and Mahin found that the continuum damage mechanics fracture criterion was able to predict the results that were consistent with the low-cycle fatigue fracture calculated according to the Manson-Coffin relationship. However, no comparison has been made to any actual test. Although the multi-term nonlinear kinematic hardening rule by Chaboche (1986) has been adopted in the study, the actual number of terms used 
was not stated. It was also not clear if a continuum damage mechanics based constitutive model has been employed.

Other studies on ductile fracture using continuum damage mechanics have also been carried by Dufailly and Lemaitre (1995) on very low-cycle fatigue, Bonora (1997) on an alternate form of fracture limit for monotonic loading, Lemaitre et al. (2000) on anisotropic damage evolutions and Barbier et al. (2008) on effects of mean stress on lowcycle fatigue. Pirondi and Bonora (2003) extended the proposed fracture limit by Bonora (1997) to include cyclic loading. Steglich et al. (2005) carried out a finite element analysis study to compare cyclic plasticity between porous metal plasticity model by Leblond et al. (1995) and continuum damage mechanics model by Pirondi and Bonora (2003).

\subsubsection{Other Damage Criteria}

Most of the studies on local damage have been focused on criteria based on void growth, void volume fraction and continuum damage mechanics as discussed in the preceding sections. Nevertheless, there are other criteria. Some of the other proposed criteria are briefly listed below.

Freudenthal (1950) proposed a fracture criterion based on the energy or the generalized plastic work. The damage limit $D_{c}$ can be expressed as

$$
\mathrm{D}_{\mathrm{c}}=\int_{0}^{\varepsilon_{\mathrm{cq}}^{\mathrm{pr}}} \sigma_{\mathrm{eq}} \mathrm{d} \varepsilon_{\mathrm{eq}}^{\mathrm{p}}
$$

However, this criterion assumes that mean stress has no effect on the fracture strain, which is contrary to the observation in the tests. 
Cockcroft and Latham (1968) modified the criterion by Freudenthal (1950) by using the largest principal stress instead of the equivalent stress. The expression for damage limit was modified to

$$
\mathrm{D}_{\mathrm{c}}=\int_{0}^{\varepsilon_{\mathrm{eq}}^{\mathrm{pr}}} \sigma_{\mathrm{eq}}\left(\frac{\sigma_{1}}{\sigma_{\mathrm{eq}}}\right) \mathrm{d} \varepsilon_{\mathrm{eq}}^{\mathrm{p}}=\int_{0}^{\varepsilon_{\mathrm{eq}}^{\mathrm{pq}}} \sigma_{1} \mathrm{~d} \varepsilon_{\mathrm{eq}}^{\mathrm{p}},
$$

where the ratio $\sigma_{1} / \sigma_{\mathrm{eq}}$ is a non-dimensional stress concentration factor representing the effect of the largest tensile stress $\sigma_{1}$. This criterion was developed for the metal forming operation. Research has shown that this criterion agreed with upsetting tests but failed to predict fracture in other tests including pure shear.

By introducing the effect of mean stress, Brozzo et al. (1972) modified the criterion by Cockcroft and Latham (1968) to

$$
\mathrm{D}_{\mathrm{c}}=\int_{0}^{\varepsilon_{\mathrm{eq}}^{\mathrm{pq}}} \frac{2 \sigma_{1}}{3\left(\sigma_{1}-\sigma_{\mathrm{m}}\right)} \mathrm{d} \varepsilon_{\mathrm{eq}}^{\mathrm{p}}
$$

On the other hand, Norris et al. (1978) proposed a criterion based on the integral of a function of the mean stress over the plastic strain path to fracture. The damage accumulation is given by

$$
\mathrm{D}_{\mathrm{c}}=\int_{0}^{\mathrm{e}_{\mathrm{eq}}^{\mathrm{pe}}}\left(\frac{1}{1-\mathrm{c} \sigma_{\mathrm{m}}}\right) \mathrm{d} \varepsilon_{\mathrm{eq}}^{\mathrm{p}} \text {, }
$$

where $\mathrm{c}$ is a material constant. Fracture is assumed to occur when D reaches the critical value $D_{c}$ over a critical length $r_{c}$. Validation of the criterion was carried out against the test results from a number of simple tension, circumferentially notched tension, Charpy V-notch and compact tension specimens. 
Oyane et al. (1980) proposed a ductile fracture criterion for metal forming based on the plasticity theory for porous materials. Fracture is assumed to occur when the volumetric strain reaches a critical value $\varepsilon_{\vee}^{\mathrm{f}}$. The criterion can be expressed as

$$
\begin{aligned}
& \int_{0}^{\varepsilon_{\mathrm{c}}^{\mathrm{p}}(}\left(1+\frac{\sigma_{\mathrm{m}}}{\mathrm{c} \sigma_{\mathrm{eq}}}\right) \mathrm{d} \varepsilon_{\mathrm{eq}}^{\mathrm{p}}=\mathrm{A}, \text { and } \\
& \mathrm{A}=\int_{0}^{\varepsilon_{v}^{\mathrm{r}}}\left(\frac{\mathrm{f}^{2} \gamma^{2 n-1}}{\mathrm{c}}\right) \mathrm{d} \varepsilon_{\mathrm{v}},
\end{aligned}
$$

where $\mathrm{c}$ and $\mathrm{n}$ are material constants, $\mathrm{f}$ is a function of the relative density $\gamma$ which is the ratio of the apparent density of the porous material to the density of its pore-free matrix. The calculated fracture strains were in good agreement with those measured experimentally in tension and compression tests.

Based on the test results of cylindrical specimens, Atkins (1981) modified the criterion by Norris et al. (1978) to include effects of the principal strain ratio path as

$$
D_{c}=\int_{0}^{e_{c 4}^{p_{e}^{p}}}\left(\frac{1+\frac{1}{2 L}}{1-c \sigma_{n y}}\right) d \varepsilon_{e q}^{p},
$$

where $\mathrm{L}$ is the ratio of the hoop to the axial strain increment of a cylindrical solid. There is no damage accumulation when $\mathrm{L}=-0.5$. A better agreement with the test results from sheet metal forming experiments was achieved with this modification. Subsequently, additional criteria have been proposed by other researchers including Tai and Yang (1987), Goijaerts et al. (2000) and Han and Kim (2003). 


\subsection{Summary}

A number of plastic hardening models have been reviewed. These models are listed in Table 2.1. Linear kinematic hardening models have been proposed by Prager (1956) and Ziegler (1959). Mroz (1967), Dafalias and Popov (1975, 1976), and Tseng and Lee (1983) proposed nonlinear kinematic hardening models based on the concept of multiple bounding surfaces. Another approach to treating nonlinear kinematic hardening is by defining the backstress evolution directly, as proposed by Armstrong and Frederick (1966). Variations of the Armstrong and Frederick (1966) model haven been proposed by Chaboche (1986) and Bower (1989). Numerous studies have shown that the proposed model by Chaboche (1986) was able to reasonably predict the behaviour of steel under close to a proportional loading.

A number of ductile fracture criteria for metals have also been reviewed. These criteria are listed in Table 2.2. Gurson (1977a, b), Tvergaard (1982), and Tvergaard and Needleman (1984) based the fracture criteria on the void volume fraction, while Gillemot (1976) and Matic et al. (1987), and a number of other researches based the criteria on the cumulative work or the integration of the stress function over the plastic strain. Another two criteria discussed are based on void growth by McClintock (1968), Rice and Tracey (1969), and on continuum damage mechanics by Lemaitre $(1984,1985)$. Kanvinde and Deierlein $(2004,2007)$ extended the criterion by Rice and Tracey (1969) to low-cycle fatigue. Similarly, Huang and Mahin (2008) introduced a slight variation to the damage evolution by Lemaitre ((1992) for low-cycle fatigue. 
Table 2.1 Hardening models

\begin{tabular}{|c|c|}
\hline Model & Equation \\
\hline Prager (1956) & $\mathrm{d} \alpha_{\mathrm{ij}}=\mathrm{cd} \varepsilon_{\mathrm{ij}}^{\mathrm{p}}$ \\
\hline Ziegler (1959) & $\mathrm{d} \alpha_{\mathrm{ij}}=\left(\sigma_{\mathrm{ij}}-\alpha_{\mathrm{ij}}\right) \mathrm{d} \mu$ \\
\hline $\operatorname{Mroz}(1967)$ & $d \alpha_{i j}^{m}=\frac{d \mu}{\kappa^{m}}\left[\left(\kappa^{m+1}-\kappa^{m}\right) \sigma_{i j}^{m}-\left(\alpha_{i j}^{m} \kappa^{m+1}-\alpha_{i j}^{m+1} \kappa^{m}\right)\right]$ \\
\hline Dafalias and Popov $(1975,1976)$ & $\begin{array}{r}E^{p}=\hat{E}^{p}\left(\delta, W^{p}\right) \\
W^{p}=\int \sigma_{i j} d \varepsilon_{i j}^{p}\end{array}$ \\
\hline Armstrong and Frederick (1966) & $\mathrm{d} \alpha_{\mathrm{ij}}=\phi\left[\mathrm{d} \varepsilon_{\mathrm{ij}}^{\mathrm{p}}-\frac{\alpha_{\mathrm{ij}}}{\mathrm{h}\left(\varepsilon_{\mathrm{eq}}^{\mathrm{p}}\right)} \mathrm{d} \varepsilon_{\mathrm{eq}}^{\mathrm{p}}\right]$ \\
\hline Chaboche (1986) & $\begin{array}{l}\mathrm{d} \alpha_{\mathrm{ij}}=\mathrm{Cd} \varepsilon_{\mathrm{ij}}^{\mathrm{p}}-\gamma \alpha_{\mathrm{ij}} \mathrm{d} \varepsilon_{\mathrm{eq}}^{\mathrm{p}} \\
\kappa=\kappa_{0}+\kappa_{x}\left(1-\mathrm{e}^{-\mathrm{b \textrm {e } _ { \mathrm { eq } } ^ { \mathrm { p } }}}\right)\end{array}$ \\
\hline Bower (1989) & $\begin{array}{c}\mathrm{d} \alpha_{\mathrm{ij}}=\mathrm{Cd} \varepsilon_{\mathrm{ij}}^{\mathrm{p}}-\gamma_{1}\left(\alpha_{\mathrm{ij}}-\beta_{\mathrm{ij}}\right) \mathrm{d} \varepsilon_{\mathrm{eq}}^{\mathrm{p}} \\
\mathrm{d} \beta_{\mathrm{ij}}=\gamma_{2}\left(\alpha_{\mathrm{ij}}-\beta_{\mathrm{ij}}\right) \mathrm{d} \varepsilon_{\mathrm{eq}}^{\mathrm{p}}\end{array}$ \\
\hline
\end{tabular}


Table $2.2 \quad$ Fracture criteria

\begin{tabular}{|c|c|}
\hline Model & Equation \\
\hline \multirow{2}{*}{$\begin{array}{l}\text { McClintock } \\
(1966)\end{array}$} & \multirow{2}{*}{$\ln F_{\mathrm{b}}^{\mathrm{f}}=\int_{0}^{\varepsilon_{\mathrm{eq}}^{\mathrm{p}}}\left[\frac{\sqrt{3}}{2(1-\mathrm{c})} \sinh \left(\frac{\sqrt{3}(1-\mathrm{c})}{2} \frac{\left(\sigma_{\mathrm{a}}+\sigma_{\mathrm{b}}\right)}{\sigma_{\mathrm{eq}}}\right)+\frac{3}{4} \frac{\left(\sigma_{\mathrm{a}}-\sigma_{\mathrm{b}}\right)}{\sigma_{\mathrm{eq}}}\right] \mathrm{d} \varepsilon_{\mathrm{eq}}^{\mathrm{p}}$} \\
\hline & \\
\hline $\begin{array}{c}\text { Rice and } \\
\text { Tracey }(1969)\end{array}$ & $\ln \left(\frac{\mathrm{R}_{\mathrm{c}}}{\mathrm{R}_{0}}\right)=\int_{0}^{\varepsilon_{\mathrm{cu}}^{\mathrm{pt}}} c \exp (1.5 \mathrm{~T}) \mathrm{d} \varepsilon_{\mathrm{eq}}^{\mathrm{p}}$ \\
\hline $\begin{array}{l}\text { Kanvinde and } \\
\text { Deierlein } \\
(2004,2007)\end{array}$ & 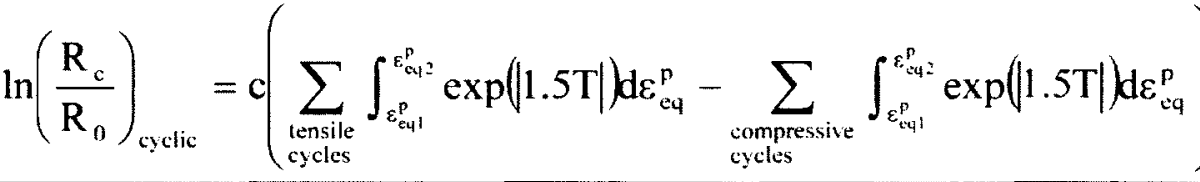 \\
\hline $\begin{array}{c}\text { Tvergaard } \\
\text { (1982), and } \\
\text { Tvergaard and } \\
\text { Needleman } \\
(1984)\end{array}$ & $\begin{array}{c}\Phi=\frac{3 S_{i j} S_{i j}}{2 \sigma_{M}{ }^{2}}+2 f^{*} q_{1} \cosh \left(\frac{q_{2} \sigma_{k k}}{2 \sigma_{M}}\right)-\left[1+\left(q_{3} f^{*}\right)^{2}\right]=0 \\
f^{*}=\left\{\begin{array}{cc}f & \text { for } f \leq f_{c}, \text { and } \\
f_{c}+K\left(f-f_{c}\right) & \text { for } f>f_{c}\end{array}\right.\end{array}$ \\
\hline $\begin{array}{l}\text { Lemaitre } \\
\text { (1984) }\end{array}$ & $\mathrm{dD}=(\mathrm{cy})^{\mathrm{m}} \mathrm{d} \varepsilon_{\mathrm{eq}}^{\mathrm{p}}$ \\
\hline $\begin{array}{l}\text { Huang and } \\
\text { Mahin (2008) }\end{array}$ & $\begin{array}{c}\mathrm{dD}=\left\{\begin{array}{cc}(\mathrm{cy})^{\mathrm{m}} \mathrm{d} \varepsilon_{\mathrm{eq}}^{\mathrm{p}} & \text { for } \frac{\sigma_{\mathrm{m}}}{\sigma_{\mathrm{eq}}}>-\frac{1}{3}, \text { and } \\
0 & \text { otherwise }\end{array}\right. \\
\mathrm{y}=\frac{\sigma_{\mathrm{eq}}{ }^{2}}{2 \mathrm{E}(1-\mathrm{D})^{2}}\left[\frac{2}{3}(1+v)+3(1-2 v)\left(\frac{\sigma_{\mathrm{m}}}{\sigma_{\mathrm{eq}}}\right)^{2}\right. \\
\mathrm{D}_{\mathrm{c}}=\int_{0}^{\varepsilon_{\mathrm{eq}}^{\mathrm{pr}} \mathrm{dD}}\end{array}$ \\
\hline $\begin{array}{c}\text { Freudenthal } \\
\text { (1950) }\end{array}$ & $\mathrm{D}_{\mathrm{c}}=\int_{0}^{\mathrm{s}_{\mathrm{ce}}^{\mathrm{r}}} \sigma_{\mathrm{eq}} \mathrm{d} \varepsilon_{\mathrm{eq}}^{\mathrm{p}}$ \\
\hline $\begin{array}{l}\text { Cockcroft and } \\
\text { Latham (1968) }\end{array}$ & $\mathrm{D}_{\mathrm{c}}=\int_{0}^{\mathrm{s}_{\mathrm{eq}}^{\mathrm{pq}}} \sigma_{1} \mathrm{~d} \varepsilon_{\mathrm{eq}}^{\mathrm{p}}$ \\
\hline $\begin{array}{c}\text { Brozzo et al. } \\
(1972)\end{array}$ & $\mathrm{D}_{\mathrm{c}}=\int_{0}^{\varepsilon_{\mathrm{eq}}^{\mathrm{ri}}} \frac{2 \sigma_{1}}{3\left(\sigma_{1}-\sigma_{\mathrm{m}}\right)} \mathrm{d} \varepsilon_{\mathrm{eq}}^{\mathrm{p}}$ \\
\hline $\begin{array}{l}\text { Norris et al. } \\
\text { (1978) }\end{array}$ & $\mathrm{D}_{\mathrm{c}}=\int_{0}^{\mathrm{E}_{\mathrm{cu}}^{\mathrm{p}}}\left(\frac{1}{1-\mathrm{c} \sigma_{\mathrm{m}}}\right) \mathrm{d} \varepsilon_{\mathrm{cu}}^{\mathrm{p}}$ \\
\hline
\end{tabular}


Table 2.2 Continue

\begin{tabular}{|c|c|}
\hline Model & Equation \\
\hline $\begin{array}{c}\text { Oyane et al. } \\
(1980)\end{array}$ & $\mathrm{D}_{\mathrm{c}}=\int_{0}^{\varepsilon_{\mathrm{cu}}^{\mathrm{p}}}\left(1+\frac{\sigma_{\mathrm{m}}}{\mathrm{c} \sigma_{\mathrm{eq}}}\right) \mathrm{d} \varepsilon_{\mathrm{eq}}^{\mathrm{p}}$ \\
\hline Atkins (1981) & $\mathrm{D}_{\mathrm{c}}=\int_{0}^{\varepsilon_{\mathrm{eq}}^{\mathrm{pr}}}\left(\frac{1+\frac{1}{2 \mathrm{~L}}}{1-\mathrm{c} \sigma_{\mathrm{m}}}\right) \mathrm{d} \varepsilon_{\mathrm{eq}}^{\mathrm{p}}$ \\
\hline $\begin{array}{c}\text { Gillemot } \\
(1976) \text { and } \\
\text { Matic et al. } \\
(1987)\end{array}$ & $\left(\frac{\mathrm{dW}}{\mathrm{dV}}\right)_{\mathrm{c}}=\int_{0}^{\varepsilon_{\mathrm{cq}}^{\mathrm{pr}}} \sigma_{\mathrm{ij}} \mathrm{d} \varepsilon_{\mathrm{ij}}$ \\
\hline
\end{tabular}




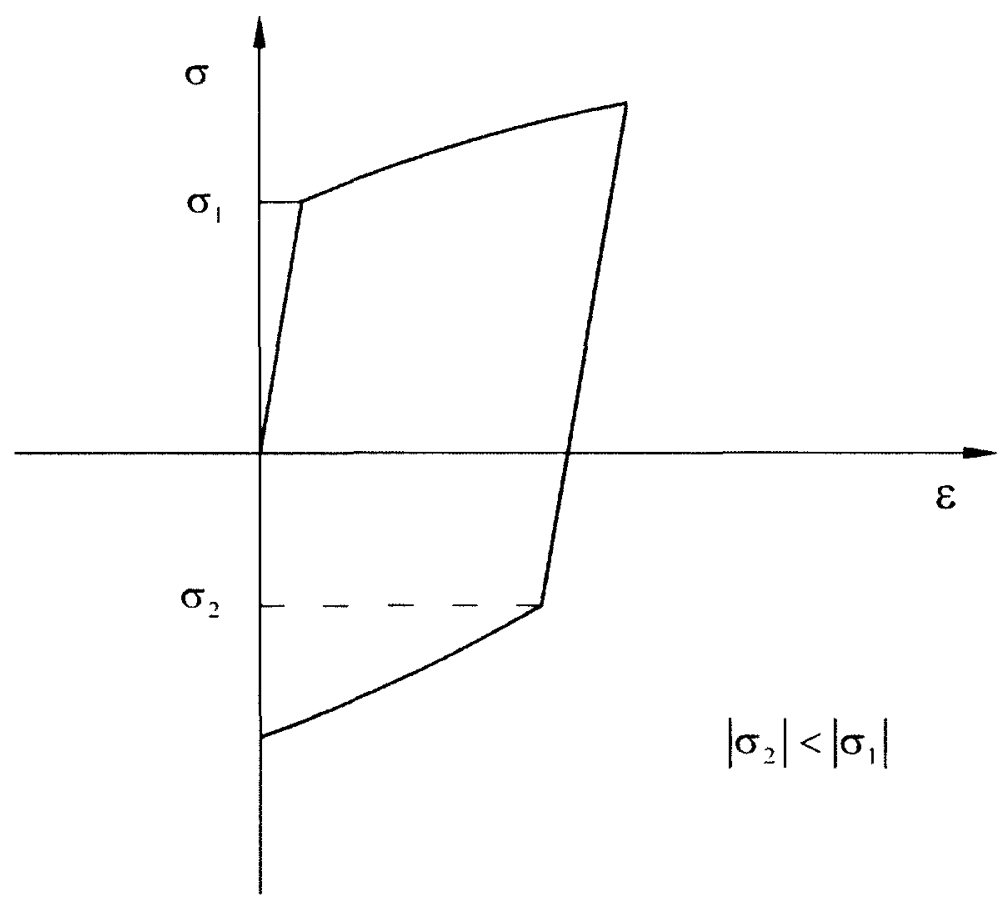

Figure 2.1 Bauschinger effect

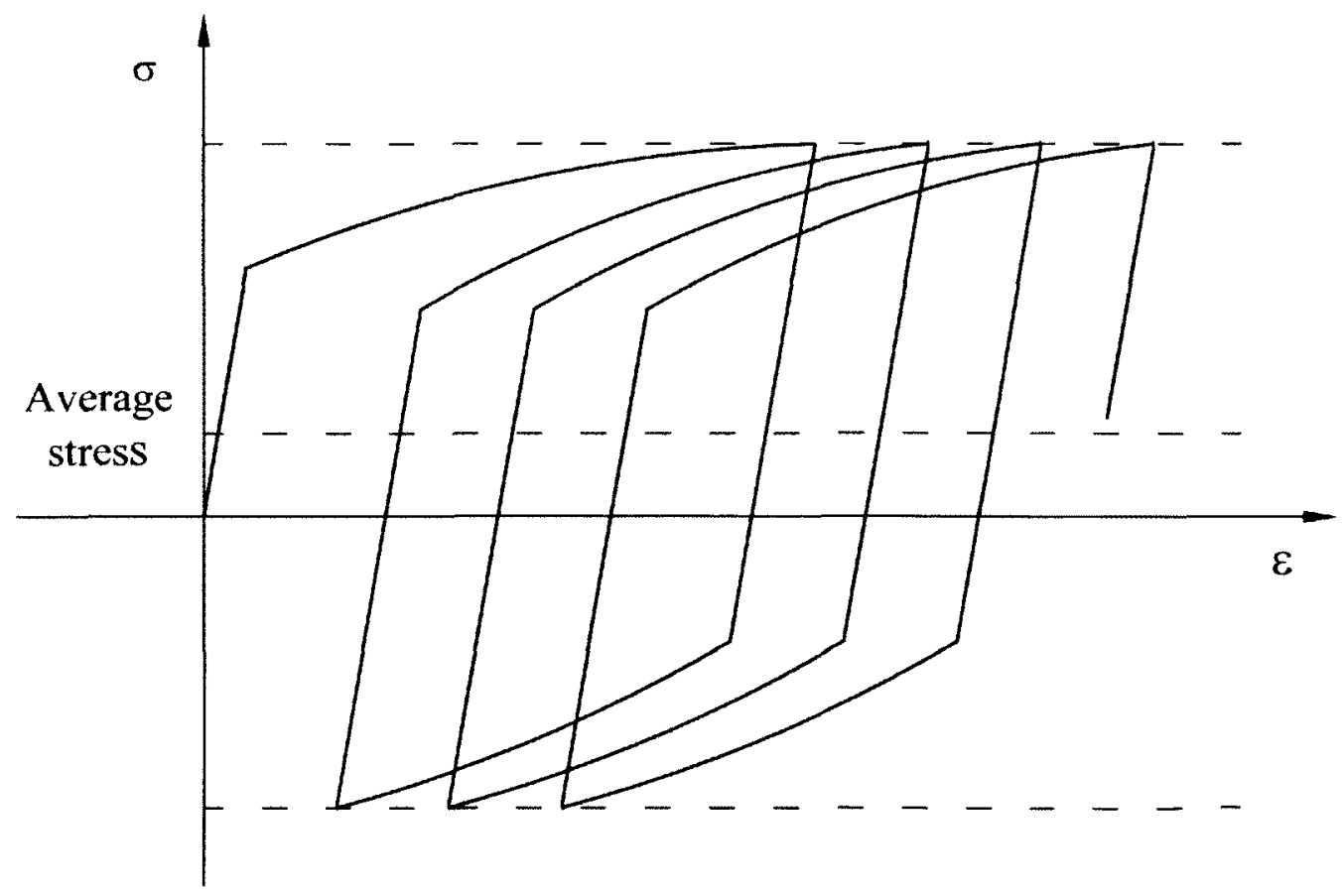

Figure 2.2 Ratchetting 


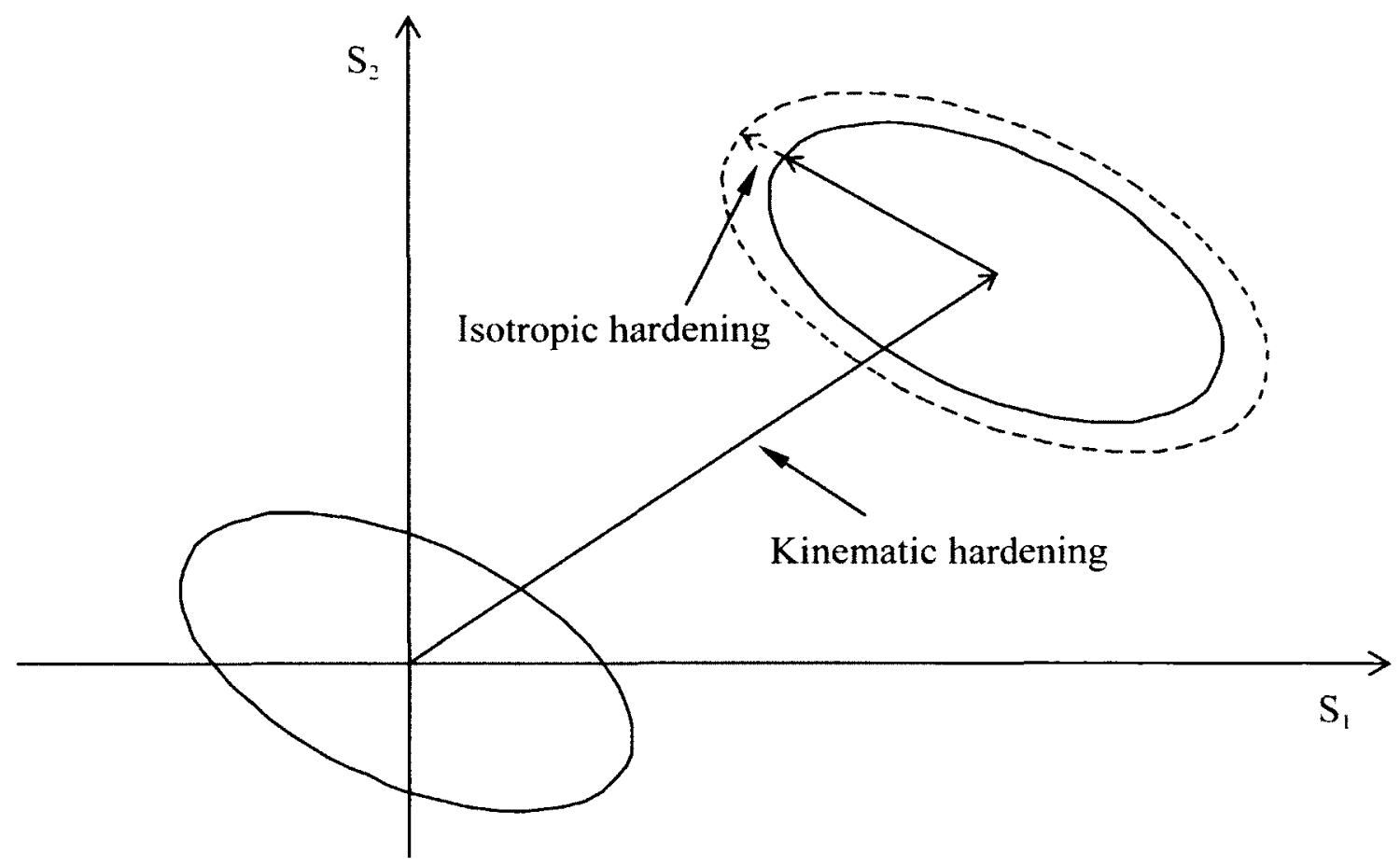

Figure 2.3 Material hardening on principal S1-S2 deviatoric stress plane 


\section{CHAPTER 3 CALIBRATION OF PLASTICITY MODEL PARAMETERS}

From the literature review, it is clear that the behaviour of steel under cyclic loading can be modeled with different levels of complexity. However, as the complexity of the material model used increases, the number of parameters that need to be calibrated and the effort required in implementing the material model into a finite element analysis software also increases. With these in mind, the combined isotropic and nonlinear kinematic hardening model by Chaboche (1986) with one or two nonlinear kinematic hardening terms of Eq. (2.13) appears to be a reasonable model based on the ease of implementing the model into a finite element analysis software and the small number of kinematic hardening parameters that have to be calibrated ( $\mathrm{C}$ and $\gamma$ ). However, as stated in Chapter 2, there are limitations to the model. The small number of parameters allows the parametric study of structural components and systems with different material properties to be carried out with reasonable effort. This model has also been implemented in the finite element analysis software package ABAQUS (Simulia 2009). The suitability of the model will be assessed in the following study.

One of the challenges in the calibration for the material model parameters is to determine values of $\mathrm{C}$ and $\gamma$ that are appropriate for a wide range of loading since the ductile fracture criteria established have to be applicable to fracture prediction for monotonic tension, low and ultra-low cycle fatigue. The procedure to calibrate the kinematic hardening parameters $\mathrm{C}$ and $\gamma$ as well as the isotropic hardening relationship is thus developed and discussed in detail in the following sections. 


\subsection{Testing Program and FEA Modeling}

The objective of the testing program is to determine the essential mechanical properties (parameters) and fracture limit of steel to be used in the numerical simulations. Monotonic tests have been carried out to determine the flow stress versus true strain relationship up to fracture, while cyclic tests have been conducted to determine the kinematic hardening parameters $\mathrm{C}$ and $\gamma$, and the isotropic hardening relationship. All the test results are also used in the evaluation of the fracture criteria in Chapter 4.

Kanvinde and Deierlein $(2004,2007)$ have conducted many ultra low-cycle fatigue tests on Grade $50\left(F_{y}=345 \mathrm{MPa}\right)$ and higher strength steels. Although these tests yield useful information, there were not enough test data published to allow for a thorough study on the fracture criteria. For this reason, new tests were carried out in the current study. Nevertheless, the test program by Kanvinde and Deierlein $(2004,2007)$ has been used as a reference in designing the test matrix for the current research.

Effects of mean stress, strain range and loading sequence are investigated in the current test program. Tests were carried out with different mean to effective stress ratio $\left(\sigma_{\mathrm{m}} / \sigma_{\mathrm{eq}}\right)$ by using specimens with straight and notched profiles. The ductility of steel has been found in many studies (Bridgman 1947, Hancock and Brown 1983, and Johnson and Cook 1985) to be significantly affected by the mean stress. Three loading sequences up to fracture consist of monotonic tension, cyclic loading followed by tension, and cyclic loading only were considered. Tests by Kanvinde and Deierlein $(2004,2007)$ consisted of specimens subjected to a large strain range but a small number of up to 20 cycles, but mainly less than 10 cycles. In addition to tests at the similar loading cycle number considered by Kanvinde and Deierlein $(2004,2007)$, the current test program also 
considered tests at a larger number of loading cycle but at a smaller strain range. The cross-section dimension of the specimen was measured throughout the test in the current test program. This will allow the true stress and true strain to be approximated from the load and deformed cross-sectional area instead of relying entirely on numerical simulations.

\subsubsection{Specimen Designation}

The following designation is adopted to describe the material, profile, test type and strain range applied to the test specimen.

$\alpha \beta \eta \xi \chi \chi \omega \omega \rho$

(1) $\alpha$

The first letter represents the material.
$\mathrm{A}=\quad$ ASTM A572 (ASTM 2007) Grade 50 steel.

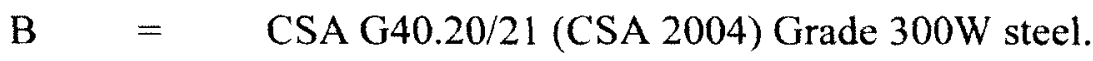

(2) $\beta$

The second letter represents the specimen profile.
$\mathrm{A}=\quad$ Notched profile D10R 14N shown in Fig. 3.1.
$\mathrm{B}=\quad$ Notched profile D14R19N shown in Fig. 3.2.
$\mathrm{C}=\quad$ Notched profile D10R7N shown in Fig. 3.3.
D = Straight profile D14R24U shown in Fig. 3.4.
$\mathrm{E}=\quad$ Straight profile D14R19U shown in Fig. 3.5. 
The profile description Dxx denotes the diameter is $\mathrm{xx} \mathrm{mm}$, Rxx denotes the transition radius is $\mathrm{xx} \mathrm{mm}, \mathrm{U}$ indicates it is a straight specimen and $\mathrm{N}$ indicates it is a notched specimen.

(3) $\eta$

The third letter denotes the test type.

$\mathrm{M}=$ Monotonic tension test.

C $=$ Cyclic test.

(4) $\xi \chi \chi \omega \omega$

These letters are only used in the cyclic test and are omitted in the monotonic tension test. $\xi$ denotes the loading sequence applied until fracture occurs. It is represented by $F$ when the specimen is loaded cyclically at a single strain range until it reaches failure, or by $R$ when the strain range changes during the cyclic loading, or by a numerical value which represents 10 times of the loading cycle number before the specimen is being pulled to fracture. For example, a numerical value 2 indicates that the specimen is subjected to 20 cycles of loading and then pulled in tension to failure, while 7 indicates the specimen is subjected to 70 cycles of loading and then pulled in tension to failure. $\chi \chi \omega \omega$ denotes the cyclic strain range applied in the tests. In the case where the cyclic strain range changes during the test, the first two digits $(\chi \chi)$ are the first strain range in percentage and the last two digits $(\omega \omega)$ are the second strain range in percentage with the number of loading cycle for each strain range noted in Tables 3.1 and 3.2. Both strain ranges have a zero mean strain. For other tests, the first two digits $(\chi \chi)$ are the 
upper limit of strain in percentage and the last two digits $(\omega \omega))$ are the absolute value of the negative strain in percentage at the lower limit.

(5)

$\rho$

This final lowercase letter of either ' $a$ ' or ' $b$ ' represents specimens with the same material and profile tested at two different loading rates. It is omitted if there is only one specimen tested for the loading sequence, material and specimen profile combination.

For example, ABM is a specimen of material A with D14R19N profile subjected to monotonic tension loading up to failure and ADC20404 is a specimen of material A with D14R24U profile subjected to 20 cycles of loading at the strain range of $-4 \%$ to $+4 \%$ before being pulled to failure in tension.

\subsubsection{Test Specimens}

All specimens are round cross-sectional shape machined from $25.4 \mathrm{~mm}$ (one-inch) diameter round rods. Twenty one specimens of ASTM A572 (ASTM 2007) Grade 50 steel and eighteen specimens of CSA G40.20/21 (CSA 2004) Grade 300W steel were tested in the study. The test matrices are listed in Tables 3.1 and 3.2. The specimens consist of mainly notched profile of $A$ and $B$, and straight profile D. These different profiles were considered in order to quantify the effect of mean stress on fracture. There is only one specimen each of profile $\mathrm{C}$ and $\mathrm{E}$ for material $\mathrm{A}$. These are specimens from the preliminary study included to provide additional data for the evaluation.

Five straight and five notched specimens with two notched profiles were tested in monotonic tension. Ten straight and nineteen notched specimens with three notched 
profiles were tested in cyclic loading. The engineering strain in Tables 3.1 and 3.2 is that calculated using the gauge length of the axial extensometer while the true strain is an approximation based on

$$
\varepsilon_{\mathrm{t}}=\ln \left(\frac{\mathrm{A}_{0}}{\mathrm{~A}}\right)
$$

where $\mathrm{A}_{0}$ and $\mathrm{A}$ are the undeformed and current cross-sectional areas, respectively. The true strain is estimated from the numerical simulation using the approximated flow stress versus true plastic strain relationship from the preliminary tests.

It is particularly important to have an accurate profile of the notched specimen in the numerical modeling. All specimens were fabricated by a computer controlled lathe that can machine the specimen accurately to the exact profile, thus eliminates the need to measure the profile of each specimen using a shadowgraph instrument. Nevertheless, a Mitutoyo PJ-300 shadowgraph instrument has been used to verify a few profiles of the specimens. The instrument measures the projected outline of the specimen in a twodimensional plane with the accuracy of two ten-thousandth of $25.4 \mathrm{~mm}$ (an inch).

\subsubsection{Test Setup and Instrumentation}

The test setup is shown in Fig. 3.6. Tests were conducted on a MTS 810 Material Testing System which has the capacity of $500 \mathrm{kN}$. For straight specimens with the D14R24U profile, tests were controlled through a MTS $634.12 \mathrm{E}-2425.4 \mathrm{~mm}$ (one-inch) axial extensometer. All other tests were controlled through a MTS 634.25E-24 50.8 mm (two-inch) axial extensometer. A MTS 632.18E-20 diametral extensometer was used to measure the diameter of the specimen. For notched specimens, a digital caliper was used to measure the diameter after the diametral extensometer was removed when its range has 
been exhausted. For straight specimens, both the digital caliper and diametral extensometer were used to measure the diameter. However, the diametral extensometer was removed once necking has initiated. During the test, static reading of the load was also measured. The testing machine was put on hold for about thirty seconds before the static load was recorded. Chen (2010) has shown that the loading rate has some effect on the dynamic stress, but has very little effect on the static stress. Thus in the following discussions, any reference to the stress is considered as static stress unless noted otherwise.

\subsubsection{FEA Modeling}

Numerical analyses were carried out with ABAQUS (Simulia 2009) using nonlinear geometry option to account for the effect of geometric nonlinearity in large deformation and large strain analyses. All the specimens were modeled using the CAX8R element, an eight-node biquadratic axisymmetric element with reduced integration. The minimum number of increment specified for half cycle of loading is 100 .

Since all the tests were carried out under axial extensometer control, only the segment of a specimen between the gauge length of an axial extensometer was modeled in order to accurately apply the same displacement in the numerical simulation as that of a cyclic test. While it is necessary to model the exact applied displacement of a cyclic test, it is not required for a monotonic test. From the study by Naqvi and Khoo (2007) for a straight specimen under monotonic tension, it was found that the stress versus change in the minimum cross-sectional area at the necking region does not depend on the exact end displacement as long as the displacement is increasing in tension. Therefore in the 
numerical modeling for a straight specimen under monotonic tension, the FEA mesh for profile D14R24U with a shorter gauge length was also used for profile D14R19U.

Figures 3.7 to 3.9 show the FEA meshes used for the three notched specimen profiles. Considering symmetry of the specimen, only half the gauge length or $25.4 \mathrm{~mm}$ (one-inch) segment was modeled. In a straight specimen, it is assumed that the end point of the axial extensometer gauge is sufficiently far away from the necking region so that the displacement (strain) at that point can be considered as uniform. Thus for straight specimens, the $12.7 \mathrm{~mm}$ (half-inch) segment model between the $25.4 \mathrm{~mm}$ (one-inch) gauge length of the MTS 634.12E-24 axial extensometer has been used in the numerical simulation. Figure 3.10 shows the FEA mesh used for the two straight specimen profiles. In order to enable necking to initiate at the mid-length of the specimen, the mesh has a slight taper with a $0.14 \%$ slope along the outer edge, which yields a $0.25 \%$ smaller radius at the mid-length of the specimen. All the FEA meshes used can be divided into different mesh zones in the longitudinal direction. The refined zone is located at the middle of the gauge length where necking is expected to be triggered, while the transition zone is located between the refined and general zones in order to provide a more accurate modeling close to the location of necking. The rest of the model is considered as the general zone which has a larger element size than the transition zone. All the nodes along the mid-length of the specimen have been constrained with YSYMM boundary condition, and the loading was applied by imposing a uniform displacement in the $\mathrm{Y}$ direction on the nodes at the end point of the axial extensometer gauge while allowing the nodes to slide in the $\mathrm{X}$ direction. A mesh study has been carried out and showed doubling the element number in both directions produced no noticeable change in the 
simulation results. The mesh study is discussed and illustrated in Chapter 4 and Appendix C.

Figure 3.11 shows the half model of a straight specimen which shares the same mesh configuration at the mid-length as that for the $12.7 \mathrm{~mm}$ (half-inch) segment model in Fig. 3.10 but without the taper. Numerical simulations were carried out for the straight specimen of material A under monotonic tension loading using the half full-length and $12.7 \mathrm{~mm}$ (half-inch) segment models with the parameters for the exponential law flow stress equation established in Section 3.2.4. Figure 3.12 shows the predicted stress versus cross-section change curves by both models are almost identical, which validates that the straight specimen can be modeled with the $12.7 \mathrm{~mm}$ (half-inch) segment model.

\subsection{Stress Components of Cyclic Hardening}

In order to facilitate the discussion on the determination of the cyclic hardening parameters, it is necessary to first introduce equations describing flow stress, yield stress (isotropic hardening) and backstress (kinematic hardening).

\subsubsection{Stress Components}

For the mixed-mode hardening model proposed by Chaboche (1986), the equivalent flow stress can be defined as

$$
\sigma^{y}=\sqrt{\frac{3}{2} S_{i j} S_{i j}},
$$

with the size of the yield surface based on von Mises yield criterion given by

$$
\sigma^{0}=\sqrt{\frac{3}{2}\left(S_{i j}-\alpha_{i j}\right)\left(S_{i j}-\alpha_{i j}\right)},
$$


where $S_{i j}$ is the deviatoric stress tensor and $\alpha_{i j}=\sum{ }^{k} \alpha_{i j}$ is the summation of backstress tensor from each kinematic hardening term $\mathrm{k}$. Henceforth, $\sigma^{0}$ will be referred to as the yield stress. Under uniaxial tension loading, the flow stress reduces to

$$
\begin{aligned}
\sigma^{y} & =\left|\sigma_{11}\right|=\sigma^{0}+\alpha, \text { and } \\
\alpha & =\operatorname{sgn}\left(\alpha_{11}\right) \sqrt{\frac{3}{2} \alpha_{i j} \alpha_{i j}} \\
& =\sum \operatorname{sgn}\left({ }^{k} \alpha_{11}\right) \sqrt{\frac{3^{k}}{2} \alpha_{i j}{ }^{k} \alpha_{i j}}=\sum{ }^{k} \alpha
\end{aligned}
$$

where $\sigma_{11}$ and $\alpha_{11}$ are the normal stress and backstress in the loading direction, $\alpha$ is the summation of ${ }^{k} \alpha$ from each kinematic hardening term $k$, and $\operatorname{sgn}\left(\alpha_{11}\right)$ is 1 when $\alpha_{11}$ is positive and -1 when $\alpha_{11}$ is negative. Any backstress symbol without pre-superscript denotes the summation of backstress from all of the kinematic hardening terms. From Eq. (2.13), the evolution of the backstress for each kinematic hardening term $\mathrm{k}$ under cyclic loading for uniaxial condition is reduced to

$$
\mathrm{d}^{\mathrm{k}} \alpha=\mathrm{C}_{\mathrm{k}} \cdot \operatorname{sgn}\left(\sigma_{11}-\alpha\right) \mathrm{d} \varepsilon^{\mathrm{p}}-^{\mathrm{k}} \alpha \gamma_{\mathrm{k}} \mathrm{d} \varepsilon^{\mathrm{p}},
$$

where $\varepsilon^{\mathfrak{p}}$ is the accumulated plastic strain in the loading direction given by

$$
\begin{aligned}
& \mathrm{d} \varepsilon^{\mathrm{p}}=\left|\mathrm{d} \varepsilon_{11}^{\mathrm{p}}\right|=\left|\mathrm{d} \varepsilon_{11}-\frac{\mathrm{d} \sigma_{11}}{\mathrm{E}}\right|, \text { and } \\
& \mathrm{d} \varepsilon^{\mathrm{p}}=\mathrm{d} \varepsilon_{\mathrm{eq}}^{\mathrm{p}}=\sqrt{\frac{2}{3} \mathrm{~d} \varepsilon_{\mathrm{ij}}^{\mathrm{p}} \mathrm{d} \varepsilon_{\mathrm{ij}}^{\mathrm{p}}} .
\end{aligned}
$$

where $\mathrm{d} \varepsilon_{\mathrm{ij}}^{\mathrm{p}}$ is the change in plastic strain tensor. Rearranging Eq. (3.6) gives

$$
\frac{\mathrm{d}^{\mathrm{k}} \alpha}{{ }^{\mathrm{k}} \alpha-\frac{\mathrm{C}_{\mathrm{k}} \cdot \operatorname{sgn}\left(\sigma_{11}-\alpha\right)}{\gamma_{\mathrm{k}}}}=-\gamma_{\mathrm{k}} \mathrm{d} \varepsilon^{\mathrm{p}} .
$$


Integrating Eq. (3.9) gives

$$
\begin{gathered}
\left.\ln \left|{ }^{k} \alpha-\frac{C_{k} \cdot \operatorname{sgn}\left(\sigma_{11}-\alpha\right)}{\gamma_{k}}\right|\right|_{\alpha_{1}} ^{k} \alpha_{2}=-\left.\gamma_{k} \varepsilon^{p}\right|_{\varepsilon_{1}^{p}} ^{\varepsilon_{2}^{p}}, \\
{ }^{k} \alpha_{2}=\left({ }^{k} \alpha_{1}-\frac{C_{k} \cdot \operatorname{sgn}\left(\sigma_{11}-\alpha\right)}{\gamma_{k}}\right) e^{-\gamma_{k}\left(\varepsilon_{2}^{p}-\varepsilon_{1}^{p}\right)}+\frac{C_{k} \cdot \operatorname{sgn}\left(\sigma_{11}-\alpha\right)}{\gamma_{k}} \\
={ }^{k} \alpha_{1} e^{-\gamma_{k}\left(\varepsilon_{2}^{p}-\varepsilon_{1}^{p}\right)}+\frac{C_{k} \cdot \operatorname{sgn}\left(\sigma_{11}-\alpha\right)}{\gamma_{k}}\left[1-\mathrm{e}^{-\gamma_{k}\left(\varepsilon_{2}^{p}-\varepsilon_{1}^{p}\right)}\right],
\end{gathered}
$$

where ${ }^{k} \alpha_{1}$ and $\varepsilon_{1}^{p}$ are the backstress and accumulated plastic strain at the beginning of the loading step, and ${ }^{k} \alpha_{2}$ and $\varepsilon_{2}^{p}$ are the backstress and accumulated plastic strain at the end of the loading step. Equation (3.11) assumes there is no change in the loading direction within the loading step. The relationship between the various stress components under monotonic uniaxial tension loading is shown in Fig. 3.13. The equivalent stress for the yield surface can be rewritten from Eq. (3.4) as

$$
\begin{aligned}
\sigma^{0} & =\left|\sigma^{y}-\alpha\right| \text { or } \\
& =\sigma_{11}^{t}-\alpha=\alpha-\sigma_{11}^{c},
\end{aligned}
$$

where $\sigma_{11}^{1}$ and $\sigma_{11}^{c}$ are the stresses for the forward and reversed branches in uniaxial cyclic loading. At the point of loading direction change $i$, the backstress and the equivalent yield stress can be calculated from Eq. (3.12) as

$$
\begin{aligned}
& \alpha_{i}=\frac{{ }^{i} \sigma_{11}^{\mathrm{t}}+{ }^{\mathrm{i}} \sigma_{11}^{\mathrm{c}}}{2}, \text { and } \\
& \sigma_{i}^{0}=\frac{{ }^{i} \sigma_{11}^{\mathrm{t}}-{ }^{i} \sigma_{11}^{\mathrm{c}}}{2}
\end{aligned}
$$


The flow stresses used in Eqs. (3.13) and (3.14) are shown in Fig. 3.14. Using Eqs. (3.7) and (3.14), the relationship between the yield stress and the equivalent plastic strain can be established for each unloading or reloading point from the uniaxial cyclic loading test as $\sigma_{i}^{0}$ and $\varepsilon_{i}^{p}$. For uniaxial monotonic loading, Eq. (3.11) for the backstress reduces to

$$
{ }^{k} \alpha=\frac{C_{k}}{\gamma_{k}}\left(1-e^{-\gamma_{k} \varepsilon^{p}}\right) .
$$

Combining $\sigma_{i}^{0}$ from the cyclic test calculated with Eq. (3.14) and the flow stress ${ }^{i} \sigma_{11}$ from the uniaxial monotonic tension test at the same accumulated plastic strain $\varepsilon_{i}^{p}$, the backstress for the uniaxial monotonic tension test at the plastic strain $\varepsilon_{\mathrm{i}}^{p}$ can be calculated as

$$
\alpha_{i}=\sigma_{11}-\sigma_{i}^{0}
$$

Thus, a multi-variable least-square error procedure can be applied to fit Eq. (3.15) through the data from Eq. (3.16) to find the kinematic hardening parameters $C_{k}$ and $\gamma_{k}$.

\subsubsection{Flow Stress under Monotonic Loading}

In the mixed-mode hardening model proposed by Chaboche (1986), the true flow stress $\left(\sigma^{y}\right)$ under uniaxial monotonic loading consists of yield stress $\left(\sigma^{0}\right)$ and backstress ( $\alpha$. Under monotonic loading, the predicted load versus deformation curve of the round specimen from the FEA analysis can be considered to be independent of the kinematic hardening parameters as long as the flow stress versus equivalent plastic strain relationship remains unchanged. The comparisons are shown in Fig. 3.15 for the test and predicted engineering stress versus cross-sectional area change curves for specimen ADMa using the same flow stress versus equivalent plastic strain data but different 
kinematic hardening parameters. The analyses were carried out using the flow stress versus equivalent plastic strain data determined in Appendix B. Thus, the flow stress determined from the monotonic tests can be used to calculate the relationship of the yield stress (isotropic hardening) versus equivalent plastic strain through Eq. (3.16) based on the selected kinematic hardening parameters $\mathrm{C}$ and $\gamma$.

\subsubsection{Equations Describing Flow Stress}

For a standard straight specimen, the stress and strain are uniform across the reduced section of the specimen before necking occurs. The true stress $\sigma_{t}$ for a straight specimen can be taken as

$$
\begin{aligned}
\sigma_{\mathrm{t}} & =\frac{\mathrm{F}}{\mathrm{A}}=\frac{\mathrm{F}}{\mathrm{A}_{0}}\left(\frac{\mathrm{A}_{0}}{\mathrm{~A}}\right)=\sigma_{\mathrm{e}}\left(\frac{\mathrm{A}_{0}}{\mathrm{~A}}\right), \text { and approximated as } \\
& \approx \sigma_{\mathrm{e}}\left(1+\varepsilon_{\mathrm{e}}\right),
\end{aligned}
$$

where $F$ is the load, $A$ is the current cross-sectional area, $A_{0}$ is the undeformed crosssectional area, $\sigma_{\mathrm{e}}$ is the engineering stress and $\varepsilon_{\mathrm{e}}$ is the engineering strain. The true strain is taken as

$$
\begin{aligned}
\varepsilon_{1} & =\ln \left(1+\varepsilon_{\mathrm{e}}\right), \text { and approximated as } \\
& \approx \ln \left(\frac{A_{0}}{A}\right),
\end{aligned}
$$

with the true plastic strain given by

$$
\varepsilon_{\mathrm{t}}^{\mathrm{p}}=\varepsilon_{\mathrm{t}}-\frac{\sigma_{\mathrm{t}}}{\mathrm{E}}
$$

where $\mathrm{E}$ is the elastic modulus. After yielding, the true stress can be taken as the flow stress in Eq. (3.4) and the change in plastic strain in Eq. (3.7) can be taken as 


$$
\begin{aligned}
\mathrm{d} \varepsilon^{\mathrm{p}} & =\mathrm{d} \varepsilon_{\mathrm{t}}-\frac{\mathrm{d} \sigma_{\mathrm{t}}}{\mathrm{E}}=\mathrm{d} \varepsilon_{\mathrm{t}}^{\mathrm{p}} \\
& \approx \mathrm{d}\left[\ln \left(\frac{\mathrm{A}_{0}}{\mathrm{~A}}\right)\right]-\frac{\mathrm{d} \sigma_{\mathrm{t}}}{E} .
\end{aligned}
$$

Equations (3.17) to (3.21) give relationships of various stresses and strains for a straight specimen under uniaxial monotonic loading. These equations are valid up to the peak load point before necking occurs. Due to the non-uniform stress and strain distribution at the necking region, only Eqs. (3.17), (3.20) and (3.21) can be used as the approximate calculations after necking has started.

Hollomon (1945) proposed an empirical power law equation to describe the flow stress with plastic strain that can be defined as

$$
\sigma^{y}=\sigma_{0}^{y}+K\left(\varepsilon^{p}\right)^{m}
$$

where $\sigma_{0}^{y}$ is the flow stress at zero plastic strain, and $\mathrm{K}$ and $\mathrm{m}$ are the material constants. Naqvi and Khoo (2004) proposed a variation of power law equation which can be written as

$$
\varepsilon^{p}=K\left[\left(\frac{\sigma^{y}}{\sigma_{0}^{y}}\right)^{n}-1\right]+\varepsilon_{0}^{p},
$$

where $\varepsilon_{0}^{\mathfrak{p}}$ is the plastic strain at the start of strain hardening, and $\mathrm{K}$ and $\mathrm{n}$ are the material constants.

Besides the power law equation, Voce (1955) proposed an alternative relationship using an exponential law equation to describe flow stress versus plastic strain with

$$
\sigma^{y}=\sigma_{\infty}^{y}-\left(\sigma_{\infty}^{y}-\sigma_{0}^{y}\right) e^{-b \varepsilon^{p}}
$$


where $\sigma_{x}^{y}$ is the maximum flow stress and $\mathrm{b}$ is a material constant. Lemaitre and Chaboche (1990) used an exponential law equation to describe the yield stress for isotropic hardening as

$$
\sigma^{0}=\sigma_{0}^{0}+\sigma_{x_{i}}^{0}\left(1-\mathrm{e}^{-b \varepsilon^{\mathrm{p}}}\right)
$$

where $\sigma_{0}^{0}$ is the initial yield stress and $\sigma_{\infty}^{0}$ is the maximum additional yield stress. Chinh et al. (2004) found that a slightly modified Eq. (3.27) can be used to describe the flow stress based on the results of tests at a very large strain. Introducing the plastic strain at the start of strain hardening $\left(\varepsilon_{0}^{p}\right)$ into the equation, Eq. (3.27) can be rewritten for the flow stress equation proposed by Chinh et al. (2004) as

$$
\sigma^{y}=\sigma_{0}^{y}+\sigma_{x}^{y}\left[1-\mathrm{e}^{-b\left(\varepsilon^{n}-\varepsilon_{i}^{p}\right)^{y}}\right]
$$

where $\mathrm{n}$ is a material constant. Forcing Eq. (3.28) to pass through the test point at peak stress, the flow stress $\sigma_{u}^{y}$ at peak load can be expressed as

$$
\sigma_{u}^{y}=\sigma_{0}^{y}+\sigma_{s}^{y}\left[1-\mathrm{e}^{-b\left(\varepsilon_{u}^{p}-\varepsilon_{i n}^{n}\right)^{n}}\right]
$$

where $\varepsilon_{u}^{p}$ is the plastic strain at peak load. Rearranging Eq. (3.29), the maximum additional flow stress after the start of strain hardening can be expressed as

$$
\sigma_{x}^{y}=\frac{\sigma_{u}^{y}-\sigma_{0}^{y}}{1-\mathrm{e}^{-b\left(\varepsilon_{u}^{p}-\varepsilon_{0}^{p}\right)^{p}}} .
$$

Therefore combining Eqs. (3.28) and (3.30), different flow stress versus equivalent plastic strain relationships can be generated by varying the parameters $b$ and $n$. Since the flow stress can be calculated directly up to peak load from the test data, it only has to be generated after the peak load with either Eq. (3.25) or (3.28). 


\subsubsection{Determination of Flow Stress versus Equivalent Plastic Strain Relationship}

Chen (2010) has proposed using an empirical equation to estimate the flow stress versus equivalent plastic strain curve after the peak load directly from the test data. After necking has occurred, the true flow stress can be corrected to be

$$
\sigma_{t}^{\mathrm{cor}}=0.5 \sigma_{\mathrm{t}}^{\mathrm{avg}}\left(1+\sqrt{\frac{\mathrm{A}}{\mathrm{A}_{\text {peak }}}}\right),
$$

where $\mathrm{A}$ is the current cross-sectional area, $\mathrm{A}_{\text {peak }}$ is the cross-sectional area at peak stress, $\sigma_{1}^{a v g}$ is the average true flow stress given by

$$
\sigma_{1}^{\text {avg }}=\frac{F}{A}
$$

Equations (3.31) and (3.32) together with (3.17) to (3.21) can be used to estimate the flow stress versus equivalent plastic strain curve from the monotonic tension test. Figure 3.16 shows the results of using power law Eq. (3.25) and exponential law Eq. (3.28) to fit the full flow stress versus equivalent plastic strain data from the three monotonic tension tests of straight specimens for material A with $\sigma_{0}^{y}=362 \mathrm{MPa}$ and $\varepsilon_{0}^{\mathrm{p}}=0.0053$ averaged from the three tests with errors $\pm 2 \mathrm{MPa}$ for $\sigma_{0}^{y}$ and \pm 0.0003 for $\varepsilon_{0}^{\mathrm{p}}$. Parameters calibrated through least-square fitting for Eq. (3.25) are $\mathrm{K}=0.00434, \mathrm{n}=6.63$, and for Eq. (3.28) are $\sigma_{\infty}^{y}=623 \mathrm{MPa}, \mathrm{b}=1.33$ and $\mathrm{n}=0.48$. The simulations were carried out with the measured elastic modulus $\mathrm{E}=205600 \mathrm{MPa}$ averaged from the three tests with an error $\pm 600 \mathrm{MPa}$ and an assumed Poission's ratio $v=0.3$. As seen in Fig. 3.17, there is a good agreement between the test and predicted curves using the flow stress versus true plastic strain curve generated by the parameters determined from least-square fitting. Figure 3.18 shows the true flow stress versus equivalent plastic strain curves generated by 
the power law Eq. (3.25) and exponential law Eq. (3.28) over a large strain with the calibrated parameters. Obviously, the flow stress generated by the exponential law Eq. (3.28) saturates much sooner than by the power law Eq. (3.25), with the flow stress for the latter continues to increase even at the plastic strain greater than 20 . In a preliminary study not discussed here, it was found that a better simulation result of cyclic test can be obtained using the exponential law Eq. (3.28) with one kinematic hardening term. Chinh et al. (2004) also found that the exponential law Eq. (3.28) is more suitable for describing the stress-strain relationship over a large range of strain. Therefore, the exponential law Eq. (3.28) has been adopted for the current study even though both equations are able to generate the true flow stress versus equivalent plastic strain required to simulate the monotonic tension test. Only procedures on finding the material model parameters associated with the flow stress generated using Eq. (3.28) will be discussed in the following sections.

\subsection{Procedure to Determine Cyclic Hardening Parameters}

Equations to describe the flow stress, yield stress (isotropic hardening) and backstress (kinematic hardening) are discussed in Section 3.2. Based on these equations, a new procedure to calculate the kinematic hardening parameters from the test results as well as the yield stress versus plastic strain relationship for isotropic hardening is developed and discussed in detail in the following sections. 


\subsubsection{Curve Fitting of Kinematic Hardening Parameters}

The strain range applied in the test program varies from $2 \%$ to $40 \%$. From results of the preliminary tests, it can be expected that structural steel can have a large accumulated plastic strain under cyclic loading before fracture occurs. For this reason, the calibration of the parameters for the kinematic hardening considers the behaviour at both small and large strains.

For the calibration at small strain, data from a stabilized cycle under uniaxial cyclic loading can be used to approximate the backstress with Eqs. (3.13) and (3.14) as the yield stress can be considered as constant within the loading cycle. For the straight specimen, which was subjected to cyclic loading between strain range of $\pm 2 \%$, there was very little change in the stress versus strain curve after 20 cycles, and the yield stress is assumed to have reached a steady state or saturated value. Therefore, straight specimen ADC40202 is chosen for the calculation of the hardening model parameters for material A. Similarly, BDC40202 is chosen for material B, but is not described here. The true stress versus true plastic strain curves at different loading cycles of ADC40202 are shown in Fig. 3.19. Although the yield stress can be approximated with Eq. (3.14), the hardening model parameters calibrated with a slightly larger assumed saturated yield stress has been found able to produce the stress-strain curves that fit better the test data. Figure 3.19 shows the assumed saturated yield stress $\left(\sigma_{\mathrm{s}}^{0}\right)$ relative to the calculated value based on Eq. (3.14). Only one branch of the stabilized stress-strain curve due to either tension or compression is needed for the calibration. Using the tension branch, the assumed static true stress versus true plastic strain curve used in the calibration is shown in Fig. 3.20. For calibration purposes, the start of the assumed curve is taken as twice the 
assumed saturated yield stress $\left(2 \sigma_{s}^{0}\right)$ above the point at the beginning of the tension branch. It is taken to be a constant until it is exceeded by the test data. As discussed previously, flow stress is a summation of yield stress and backstress. Thus, the backstress can be obtained by subtracting the assumed saturated yield stress from the static true stress versus true plastic strain curve shown in Fig. 3.20. Plotting relative to the start of the tension branch, the calculated backstress is shown in Fig. 3.21 where 15 evenly spaced data points along the strain range have been selected in the calibration for $\sigma_{\mathrm{s}}^{0}=450 \mathrm{MPa}$. From Eq. (3.11), backstress can be expressed as

$$
{ }^{k} \alpha={ }^{k} \alpha_{i n i} e^{\left.1-\gamma_{h}\left(\varepsilon^{p}-\varepsilon_{m}^{p}\right)\right]}+\frac{C_{k}}{\gamma_{k}}\left\{1-e^{\left[-\gamma_{k}\left(\varepsilon^{p}-\varepsilon \varepsilon_{i n}^{p}\right)\right]}\right\},
$$

where ${ }^{k} \alpha_{\mathrm{ini}}$ and $\varepsilon_{\mathrm{ini}}^{\mathrm{p}}$ are the backstress and plastic strain at the beginning of tension branch. If defining $\varepsilon_{\mathrm{ini}}^{\mathrm{p}}=0$ and $\varepsilon^{\mathrm{p}}$ as the incremental plastic strain from the beginning of tension branch as shown in Fig. 3.21, Eq. (3.33) can be rewritten as

$$
{ }^{k} \alpha={ }^{k} \alpha_{\text {ini }} e^{\left(-\gamma_{k} \varepsilon^{p}\right)}+\frac{C_{k}}{\gamma_{k}}\left[1-e^{\left(-\gamma_{k} \varepsilon^{p}\right)}\right] .
$$

At the end of tension cycle, the backstress is given by

$$
{ }^{k} \alpha_{\text {tinal }}={ }^{k} \alpha_{\text {ini }} e^{\left(1-\gamma_{k} \Delta \varepsilon^{p}\right)}+\frac{C_{k}}{\gamma_{k}}\left[1-e^{\left(-\gamma_{h} \Delta \varepsilon^{p}\right)}\right],
$$

where $\Delta \varepsilon^{p}$ is the plastic strain range in the tension branch. Using the stabilized cycle for symmetric loading as shown in Fig. 3.19, the relationship between backstress at the start and end of the tension branch can be established as

$$
{ }^{k} \alpha_{\text {final }}=-{ }^{k} \alpha_{\text {ini }} .
$$

Combining Eqs. (3.35) and (3.36), the initial backstress can be rewritten as 


$$
{ }^{k} \alpha_{\text {ini }}=-\frac{C_{k}}{\gamma_{k}} \frac{\left[1-\mathrm{e}^{\left(-\gamma_{k} \Delta \varepsilon^{p}\right)}\right]}{\left[1+\mathrm{e}^{\left(-\gamma_{k} \Delta \varepsilon^{p}\right)}\right]}
$$

Thus, backstress can be expressed as

$$
{ }^{k} \alpha=-\frac{C_{k}}{\gamma_{k}} \frac{\left[1-\mathrm{e}^{\left(-\gamma_{k} \Delta \varepsilon^{p}\right)}\right]}{\left[1+e^{\left(-\gamma_{k} \Delta \varepsilon^{p}\right)}\right]} e^{\left(-\gamma_{h} \varepsilon^{p}\right)}+\frac{C_{k}}{\gamma_{k}}\left[1-e^{\left(-\gamma_{k} \varepsilon^{p}\right)}\right] .
$$

Summing on all the kinematic hardening terms gives the total backstress $\alpha$ as

$$
\alpha=\sum_{k=1}^{n}{ }^{k} \alpha
$$

Equations (3.38) and (3.39) can be used to calibrate the kinematic hardening parameters to fit the backstress versus plastic strain curve in Fig. 3.21.

The data used in calibrating the kinematic hardening parameters at large strain range curve can be generated from the equation describing the flow stress and the assumed saturated yield stress. As discussed in the previous section, the modified Chinh et al. (2004) exponential law Eq. (3.28) has been adopted to describe the flow stress versus plastic strain relationship. Based on the parameters solved for Eq. (3.28) and the assumed saturated yield stress, the backstress versus plastic strain data can be calculated using Eq. (3.4). From results of the cyclic test of a straight specimen at $\pm 2 \%$ strain, the stress-strain curve is close to be stabilized only after a few cycles of loading, as shown in Fig. 3.19. Thus, for the purpose of calibrating kinematic hardening parameters at large strain, the yield stress can be assumed to be saturated at a strain of 0.2 when generating the data points for the calibration. It has been found that using of data points up to the strain of 10 in the calibration is sufficient to ensure that the stress generated with the calibrated hardening parameters to be constant after the strain of 10 . Figure 3.22 shows the backstress versus plastic strain curve used in calibrating the kinematic hardening 
parameters based on Eq. (3.15) for $\sigma_{\mathrm{s}}^{0}=450 \mathrm{MPa}$, where 30 evenly spaced data points have been selected from the strain range of 0.2 to 10 as compared to the 15 data points for small strain. The 2 to 1 ratio in the number of data points for large strain versus small strain has been found to give a good result in the calibration.

Kinematic hardening parameters $C_{k}$ and $\gamma_{k}$ can be calibrated by least-square fitting the backstress data points for both small and large strain in Figs. 3.21 and 3.22 simultaneously. Figures 3.21 and 3.22 show results of the calibration using two kinematic hardening terms where backstress $1\left(C_{1}, \gamma_{1}\right)$ and backstress $2\left(C_{2}, \gamma_{2}\right)$ represent backstresses from the two kinematic hardening terms. Results of the calibration using only one kinematic hardening term are shown in Figs 3.23 and 3.24. Obviously, the fit using only one kinematic hardening term is not as good as that using two terms. Figures 3.25 and 3.26 show results of the calibration using two kinematic hardening terms with the saturated yield stress $\left(\sigma_{\mathrm{s}}^{0}=273 \mathrm{MPa}\right)$ based on the value at the proportional limit determined from the test results of $\mathrm{ADC} 40202$ at the $20^{\text {th }}$ cycle. Based on the comparisons in Figs. $3.21,3.22,3.25$ and 3.26 , it appears that using two kinematic hardening terms are sufficient to provide a good fit to the backstress versus plastic strain data. In order to minimize the complexity of generating the material stress-strain curve for the parametric study of strap tension-only braces, it is desirable to keep the number of kinematic hardening term low. Therefore, the discussions in the following sections focus only on the calibration procedures of the parameters for the hardening model with one and two kinematic hardening terms. 


\subsubsection{Calibration of Kinematic Hardening Parameters}

Although the kinematic hardening parameters calculated in the previous section are suitable to simulate some tests for specimens with straight profiles, they may not be appropriated to simulate other tests with different loading sequences, strain ranges and specimen profiles. For this reason, iterative procedures have been established to calibrate the kinematic hardening parameters that can better simulate a wider range of tests in the numerical analyses. Overall, the procedures are similar for calibrating parameters of the hardening model with both one and two kinematic hardening terms except for some small differences. The detailed procedures are discussed in the following sections.

\subsubsection{Two-Term Kinematic Hardening Parameters}

When using two kinematic hardening terms in the mixed-mode plasticity model to simulate cyclic tests, the value of parameter $b$ in the exponential law flow stress Eq. (3.28) has been found to have little effect on the predicted engineering stress versus engineering strain curves. However, it has a greater effect on the engineering stress versus crosssectional area change curves for notched specimens. Nevertheless, it is important to first investigate the influence of parameter $b$ on the predicted stress-strain (deformation) curve for the straight specimens tested monotonically. It can be seen in Fig. 3.27 that there is very little difference in the predicted engineering stress versus cross-sectional area change curve for the value of $b$ from 1.5 to 4.5 . Using parameters for the kinematic hardening calibrated with the saturated yield stress $\sigma_{\mathrm{s}}^{0}=464 \mathrm{MPa}$, results of numerical simulations for tests under cyclic loading for straight specimen ADC40202, and notched specimens $\mathrm{ABC} 70404$ and $\mathrm{ABCF} 1212$ are shown in Figs. 3.28 to 3.33. It can be seen in 
Figs. 3.28, 3.30 and 3.32 that the value of parameter $b$ has little effect on the predicted engineering stress versus engineering strain curves. Similarly, there is also little difference in the predicted engineering stress versus cross-sectional area change curve with different parameter $b$ for the straight specimen, as shown in Fig. 3.29. However, Figs. 3.31 and 3.33 show that changing parameter $b$ has a significant effect on the predicted engineering stress versus cross-sectional area change curve for notched specimens. Furthermore, using a value of 2.5 for $b$, the predicted curve for specimen ABC70404 in Fig. 3.31 shifts to the left with increasing loading cycle while the curve for the actual test shifts to the right. Thus, a starting value of 4.5 for $b$ was used in the calibration of the parameters for the hardening model. This reduces the curve fitting of the flow stress versus equivalent plastic strain data outlined in Section 3.2.4 to find values of parameters $\sigma_{x}^{y}$ and $\mathrm{n}$ for Eq. (3.28).

It is expected that the numerical simulation will be able to reasonably predict the engineering stress versus engineering strain responses of most tests if the appropriate parameters for the hardening model and the flow stress Eq. (3.28) are used. Since the predicted engineering stress versus cross-sectional area change curve for a straight specimen is not particularly sensitive to the parameter b of the flow stress Eq. (3.28), the calibration of the material model parameters has been carried out using test data from notched specimens $\mathrm{ABC} 20404$ and $\mathrm{ABC} 70404$. Other notched specimens can also be used in the calibration. Nevertheless, parameters for kinematic hardening have been determined through curve fitting of the test data of ADC40202 as described in Section 3.3.1, and the parameter $\mathrm{b}$ in the flow stress Eq. (3.28) has been calculated through curve fitting of the test data of straight specimens under monotonic tension (ADMa, ADMb and 
AEM) as described in Section 3.2.4. Test results of notched specimens are used in the calibration process to find the optimum $C, \gamma$ and $b$.

\subsection{Optimum Saturated Yield Stress}

As discussed in Section 3.3.1, parameters $C_{k}$ and $\gamma_{k}$ for the hardening using two kinematic hardening terms can be approximated based on the assumed saturated yield stress. An error minimizing approach on the test and predicted stress has been adopted to find the optimum assumed saturated yield stress to be used in the calibration. The error measurement is taken as

$$
Q_{i}=\sum_{j=1}^{m}\left[\sigma_{e, j}^{t e s t}-\sigma_{e . j}^{F E \Lambda}\left({ }^{i} \sigma_{s}^{0}\right)\right]^{2},
$$

where ${ }^{i} \sigma_{s}^{0}$ is the assumed saturated yield stress of trial number $i, \sigma_{e, j}^{\text {test }}$ is the measured engineering stress from the test and $\sigma_{e, j}^{\mathrm{FEA}}$ is the predicted engineering stress from the FEA simulation at the corresponding engineering strain and cycle number, $j$ is the data point number among the total $\mathrm{m}$ data points used in the calibration. To assist in minimizing $Q_{i}$, the error term is assumed to be represented by a second order polynomial function of $\sigma_{s}^{0}$ as

$$
\mathrm{Q}=\mathrm{a}_{0}+\mathrm{a}_{1} \sigma_{\mathrm{s}}^{0}+\mathrm{a}_{2}\left(\sigma_{\mathrm{s}}^{0}\right)^{2}
$$

where $a_{0}$ to $a_{2}$ are constants to be determined from various trial values of $\sigma_{s}^{0}$ as

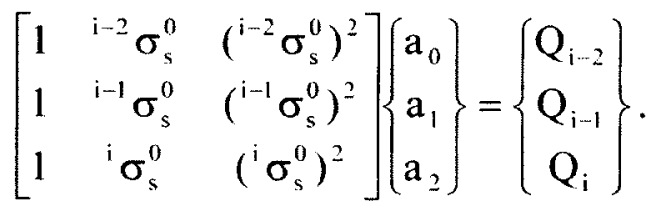


The next trial value of ${ }^{i-1} \sigma_{s}^{0}$ can be obtained by solving the derivatives of Eq. (3.41) with respect to $\sigma_{\mathrm{s}}^{0}$ as

$$
\frac{d Q}{d \sigma_{s}^{0}}=a_{1}+2 a_{2}{ }^{i+1} \sigma_{s}^{n}=0
$$

Generally, the procedure outlined in Section 3.3.1 can approximate the kinematic hardening parameters that are able to reasonably predict the load-deformation curve of the test without going through any iteration. Using a saturated yield stress $\sigma_{\mathrm{s}}^{0}=273 \mathrm{MPa}$ based on the proportional limit of the $20^{\text {th }}$ cycle of ADC40202 and the flow stress parameter $b=4.5$, the kinematic hardening parameters are found to be $C_{1}=99491 \mathrm{MPa}$, $\gamma_{1}=469.58, \mathrm{C}_{2}=1571 \mathrm{MPa}$ and $\gamma_{2}=5.33$ through least-square fitting. Results of the numerical simulations are compared to the stress versus deformation curves of ADC40202 and ABC20404 in Figs. 3.34 to 3.36. It can be seen that the predicted curves are not too different from that of the test.

However, a better result can be obtained by using a different assumed saturated yield stress. An improvement to the calibrated parameters is sought in order to reduce any uncertainty associated with the accuracy of modeling the material behaviour in the study to follow on the evaluation of fracture criteria. Although the stress versus strain curve of ADC40202 may appear to be stabilized at the $20^{\text {th }}$ cycle, the yield stress at the $20^{\text {th }}$ cycle may actually be different from the final saturated yield stress at a very large strain. For this reason, two assumed yield stresses are used to calculate the backstress data in least-square fitting to obtain the kinematic hardening parameters. This also allows a slightly different weight to be applied to the data for small strain and large strain in the curve fitting. Thus, the assumed saturated yield stress used at large strain becomes 
$\sigma_{\mathrm{s}}^{0}+\Delta \sigma_{\mathrm{s}}^{0}$, as shown in Fig. 3.37 for $\sigma_{\mathrm{s}}^{0}=450 \mathrm{MPa}$ and assumed saturated yield stress offset $\Delta \sigma_{\mathrm{s}}^{0}=10 \mathrm{MPa}$. The backstress data for small strain in the curve fitting are still calculated based on $\sigma_{\mathrm{s}}^{0}$.

Test data of $\mathrm{ABC} 20404$ are used in determining the optimum kinematic hardening parameters based on the error minimization through Eqs. (3.40) to (3.43) instead of $\mathrm{ABC70404}$ since the numerical simulation of $\mathrm{ABC} 20404$ requires less time and the engineering stress versus engineering strain curve does not change much after the first few cycles in both tests. Four points per alternate cycle at the engineering strain in the order of $0.002,-0.0088,-0.002$ and 0.0088 have been selected to calculate the difference between the measured and predicted stress. The locations of these strains are shown in Fig. 3.38 for the $20^{\text {th }}$ cycle of $\mathrm{ABC} 20404$. The strains at \pm 0.002 represent points close to the end of the elbow of the stress-strain curve in each of the tension and compression leg of the loading, and \pm 0.0088 are the points close to the start of load reversal.

There are three steps in finding the optimum kinematic hardening parameters.

(1) Step one involved finding the assumed saturated yield stress that gives the least error Q according to Eq. (3.40) by trial and error at an increment of $25 \mathrm{MPa}$ on $\sigma_{\mathrm{s}}^{0}$ with $\Delta \sigma_{\mathrm{s}}^{0}=0$. Parameters for the kinematic hardening are calculated through curve fitting of the test data of ADC40202 as described in Section 3.3.1 and parameters for the flow stress Eq. (3.28) are calculated through curve fitting of the test data of straight specimens (ADMa, ADMb and AEM) under monotonic tension as described in Section 3.2.4 with $b=$ 4.5. Material model parameters and properties used in the numerical simulations for various trial values are listed in Table 3.3 with the error Q. 
The yield stress versus equivalent plastic strain data for isotropic hardening is the net between flow stress from Eq. (3.28) and backstress from Eq. (3.15). As can be seen in Table 3.3, the assumed saturated yield stress that gives the least error lies between 450 to $500 \mathrm{MPa}$, based on trial values of $\sigma_{\mathrm{s}}^{0}=450$, 475 and $500 \mathrm{MPa}$.

(2) The second step involved finding the optimum kinematic hardening parameters at $\sigma_{\mathrm{s}}^{0}=450,475$ and $500 \mathrm{MPa}$ by varying $\Delta \sigma_{\mathrm{s}}^{0}$. Table 3.3 shows the errors from the numerical simulations for $\sigma_{\mathrm{s}}^{0}=450, \Delta \sigma_{\mathrm{s}}^{0}=0,10$ and $20 \mathrm{MPa}$ and parameters used in the simulations. Figures 3.39 to 3.41 show the test and predicted engineering stress versus engineering strain curves for $\sigma_{\mathrm{s}}^{0}=450, \Delta \sigma_{\mathrm{s}}^{0}=0,10$ and $20 \mathrm{MPa}$. Actually, the difference between the three predicted curves is not very big. Results of the error are plotted in Fig. 3.42. Using Eqs. (3.40) to (3.43) and substituting $\sigma_{\mathrm{s}}^{0}+\Delta \sigma_{\mathrm{s}}^{0}$ for $\sigma_{\mathrm{s}}^{0}$ in the equations, the minimum error occurrs at $\sigma_{\mathrm{s}}^{0}+\Delta \sigma_{\mathrm{s}}^{0}=467 \mathrm{MPa}$ or $\Delta \sigma_{\mathrm{s}}^{0}=17 \mathrm{MPa}$. For $\sigma_{\mathrm{s}}^{0}=475$ and $500 \mathrm{MPa}$, the optimum $\Delta \sigma_{\mathrm{s}}^{0}$ are 12 and $8 \mathrm{MPa}$ respectively. By adding an appropriate $\Delta \sigma_{\mathrm{s}}^{0}$, the error reduces from 18930,11058 and $29783 \mathrm{MPa}^{2}$ to 4825,3690 and $26375 \mathrm{MPa}^{2}$ respectively for $\sigma_{\mathrm{s}}^{0}=450,475$ and $500 \mathrm{MPa}$.

(3) The third step involved finding the optimum $\sigma_{\mathrm{s}}^{0}$ and $\Delta \sigma_{\mathrm{s}}^{0}$. Using $\sigma_{\mathrm{s}}^{0}=450$, 475 and $500 \mathrm{MPa}$ with their associated minimum errors, the optimum $\sigma_{\mathrm{s}}^{0}$ has been determined to be $464 \mathrm{MPa}$ by Eqs. (3.41) to (3.43). The assumed 
saturated yield stress versus minimum error curve is shown in Fig. 3.43. Further optimization is carried out by varying $\Delta \sigma_{\mathrm{s}}^{0}$ at $\sigma_{\mathrm{s}}^{0}=464 \mathrm{MPa}$. Errors from numerical simulations with $\Delta \sigma_{\mathrm{s}}^{0}=0,10$ and $20 \mathrm{MPa}$ are shown in Fig. 3.44 , and their associated parameters calculated and used in the numerical simulations are listed in Table 3.3. The optimum assumed saturated yield stress offset is found to be $14 \mathrm{MPa}$ with error $\mathrm{Q}=3225 \mathrm{MPa}^{2}$. The calculated kinematic hardening parameters for $\sigma_{\mathrm{s}}^{0}=464 \mathrm{MPa}$ and $\Delta \sigma_{\mathrm{s}}^{0}=$ $14 \mathrm{MPa}$ are $\mathrm{C}_{1}=3628 \mathrm{MPa}, \gamma_{1}=39.46, \mathrm{C}_{2}=708 \mathrm{MPa}$ and $\gamma_{2}=3.37$

\subsection{Optimum Parameter b}

It can be seen in Figs. 3.28 to 3.33 that the predicted stress versus cross-sectional area change relationship by the numerical simulations for straight specimens are not very sensitive to the value of parameter $b$ in the flow stress Eq. (3.28) as compared to notched specimens. For notched specimens, there is a significant difference between test and predicted engineering stress versus cross-sectional area change curve with different $b$ even though there is no significant difference between test and predicted engineering stress versus engineering strain curve. For uniaxial loading before necking, the crosssectional area change is closely related to the axial strain (engineering strain) as given by Eqs. (3.19) and (3.20). However, this does not apply to a notched specimen as the stress and strain are not uniform over the gauge length of the extensometer. Since the calibration of the kinematic hardening parameters are carried out using the engineering stress versus engineering strain data, the match in the test and predicted engineering stress versus cross-sectional area change curve for a notched specimen is not expected to 
be as good. The value of $b$ affects the generated flow stress versus equivalent plastic strain curve, which affects the kinematic hardening parameters. This in turn affects the stress and strain distribution at the notch. The difference between the predicted engineering stress versus cross-sectional area curve for different $b$ is expected to increase with the accumulated strain since the parameters for the flow stress Eq. (3.28) have been calibrated against the flow stress versus equivalent plastic strain test data of a straight specimen up to a strain of 1.0 .

Both AAC70404 and ABC70404 underwent the most loading cycle and cyclic deformation. Thus, test data from $\mathrm{ABC} 70404$ are used to find an optimum b to improve the simulations of all notched specimens. Numerical simulations have been carried out for $\mathrm{ABC} 70404$ using kinematic hardening parameters calculated for $\sigma_{\mathrm{s}}^{0}=464 \mathrm{MPa}$ and $\Delta \sigma_{s}^{0}=14 \mathrm{MPa}$ with $\mathrm{b}=4.5$ and 5.5. Parameters for Eq. (3.28) are determined to be $\sigma_{x_{i}}^{y}$ $=406 \mathrm{MPa}, \mathrm{n}=0.84$, and kinematic hardening parameters $\mathrm{C}_{1}=3570 \mathrm{MPa}, \gamma_{1}=39.00, \mathrm{C}_{2}$ $=770 \mathrm{MPa}$ and $\gamma_{2}=3.87$ for $\mathrm{b}=5.5$. Results of the simulations are shown in Figs. 3.45 and 3.46 for $b=4.5$ and 5.5 respectively. In order to improve the simulations of all the tests of notched specimens, a linear interpolation is used to obtain the optimum by making the average of the maximum and minimum predicted $\ln \left(A_{0} / A\right)$ at zero stress during unloading to be the same as that of the test. As this approach is adopted, there is no significant over-prediction in the reduction of the minimum diameter and the predicted cross-sectional area change is closer to within the bound of the measured deformation of the test. Using interpolation, an improved $b$ is determined to be 4.54 . After another numerical simulation with $b=4.54$, interpolating the results with $b=4.5$ and 4.54 gives the optimum $b$ to be 4.53 . Results of the simulation for $\mathrm{ABC} 70404$ with $b=4.53$ will be 
shown in the following section. They are not expected to be much different from Fig. 3.45 for $b=4.5$.

\subsection{FEA Simulation}

For material $\mathrm{A}$, the optimum hardening parameters can be determined with $\sigma_{\mathrm{s}}^{0}=$ $464 \mathrm{MPa}$ and $\Delta \sigma_{\mathrm{s}}^{0}=14 \mathrm{MPa}$. With an assumed Poisson's ratio $v=0.3$, the measured elastic modulus $E=205600 \mathrm{MPa}, \sigma_{0}^{y}=362 \mathrm{MPa}$ and $\varepsilon_{0}^{\mathrm{p}}=0.0053$, the rest of the parameters for the hardening model have been determined to be $\sigma_{\infty}^{y}=417 \mathrm{MPa}, \mathrm{n}=0.77$, $\mathrm{b}=4.53, \mathrm{C}_{1}=3625 \mathrm{MPa}, \gamma_{1}=39.46, \mathrm{C}_{2}=711 \mathrm{MPa}$ and $\gamma_{2}=3.39$. The yield stress versus equivalent plastic strain data are calculated by subtracting the backstress from the flow stress generated with Eq. (3.28) according to the relationship in Eq. (3.4). Various components of the stress for material A are shown in Fig. 3.47 and the yield stress versus equivalent plastic strain data are listed in Table 3.4 .

Using the above parameters, numerical simulations have been carried out for all test specimens of material A. Comparisons of test results and numerical simulations are shown in Figs. 3.48 to 3.66. Some of the comparisons for notched specimens for material A with similar loading sequence as that shown in Figs. 3.48 to 3.66 but different profiles are presented in Appendix A. As stated previously for the monotonic tension test of a straight specimen, the engineering stress versus engineering strain curve is also not a good choice to be used in the comparison to the cyclic test results of a straight specimen when there is necking before fracture. Thus, only engineering stress versus crosssectional area change curves are shown in the comparison to the test results for straight specimens in Appendix A. As can be seen in all the comparisons, there is an agreement 
between the test and predicted engineering stress versus engineering strain (crosssectional area change for straight specimens) even the tests with multiple strain ranges in Figs. 3.55 and 3.65, and non-zero mean strain in Figs. 3.54 and 3.63. As expected, there are some differences between the test and predicted engineering stress versus crosssectional area change curves for notched specimens under cyclic loading, with the specimens that have been subjected to more loading cycles, ABC70404 in Fig. 3.60 and AECR0804 in Fig. 3.66, showing the larger differences. Also note that the numerical simulations slightly overestimate the stress at the elbow of the engineering stress versus engineering strain curves under cyclic loading, as shown in Figs. 3.51, 3.57 and 3.59. This occurs over a small strain range where the deformation transitions from elastic to plastic in each loading cycle. The region is small compared to the overall or accumulated plastic strain of the specimen. However, there is an agreement between the test and predicted stress beyond this transition region.

It is not known if there is any study that has compared the numerical simulation results to both the measured engineering stress versus engineering strain and engineering stress versus cross-sectional area change of a notched specimen over many cycles of plastic deformation. Nevertheless, the focus of this research is on calibrating and generating material model parameters to assess the performance of steel structures and structural components, and is not on developing a new plasticity model. Thus in view of the good agreement of the simulation results for the straight specimens and the engineering stress versus engineering strain curves for the notched specimens, the mixedmode hardening model with two kinematic hardening terms is considered to be suitable for modeling structural components subjected to close to uniaxial or proportional loading. 
Thus, no further investigation has been conducted to explore if a better predicted engineering stress versus cross-sectional area change can be achieved.

The same calibration procedure for material A has been applied to determine the optimum hardening parameters of material $\mathrm{B}$ with $\sigma_{\mathrm{s}}^{0}=384 \mathrm{MPa}$ and $\Delta \sigma_{\mathrm{s}}^{0}=15 \mathrm{MPa}$. With an assumed Poisson's ratio $v=0.3$, the measured elastic modulus $E=207100 \mathrm{MPa}$, $\sigma_{0}^{y}=332 \mathrm{MPa}$ and $\varepsilon_{0}^{\mathrm{r}}=0.0161$ averaged from two tests with errors $\pm 400 \mathrm{MPa}$ for $\mathrm{E}$, $\pm 5 \mathrm{MPa}$ for $\sigma_{0}^{\mathrm{y}}$ and \pm 0.0003 for $\varepsilon_{0}^{\mathrm{p}}$, the rest of the parameters for the hardening model have been determined to be $\sigma_{\infty}^{y}=448 \mathrm{MPa}, \mathrm{n}=0.65, \mathrm{~b}=3.03, \mathrm{C}_{1}=3965 \mathrm{MPa}, \gamma_{1}=$ 29.30, $\mathrm{C}_{2}=583 \mathrm{MPa}$ and $\gamma_{2}=2.37$. Table 3.5 shows the yield stress versus equivalent plastic strain data for material B. Comparisons of test results and numerical simulations for specimens of material B using two kinematic hardening terms are shown in Appendix A. In general, comments on the comparisons of the test results and numerical simulations for specimens of material A can also be applied to specimens of material B.

\subsubsection{One-Term Kinematic Hardening Parameters}

The suitability of modeling cyclic loading with one kinematic hardening term has also been evaluated. Unlike numerical simulations with two kinematic hardening terms, both the predicted engineering stress versus engineering strain and engineering stress versus cross-sectional area change curves for cyclic tests of straight and notched specimens are affected by the parameter $b$ in the flow stress Eq. (3.28). As a result, the optimization of the kinematic hardening parameters has to be carried out together with the parameter $b$ for the engineering stress and engineering strain curve of $\triangle B C 20404$. Comparisons of some of the test results and numerical simulations for material $A$ are 
shown in Appendix B. Overall, there are larger differences between the test and predicted engineering stress and engineering strain curve, and especially the engineering stress versus cross-sectional area change curve of the cyclic tests. For these reasons, the mixed-mode hardening model with one kinematic hardening term is not considered as a suitable option to model structural components subjected to cyclic loading. Therefore, the procedure to calibrate the parameters using one kinematic hardening term is not described here.

\subsection{Other Methods to Determine Cyclic Hardening Parameters}

ABAQUS (Simulia 2009) includes a built-in calibration procedure for kinematic hardening parameters. Comparisons of test results and numerical simulations using parameters determined by the built-in calibration procedure for two kinematic hardening terms are shown in Figs. 3.67 to 3.70. The kinematic hardening parameters have been determined using the flow stress and plastic strain data from the tension branch of the $20^{\text {th }}$ cycle of straight specimen ADC40202 taken as the stabilized curve similar to the flow stress shown in Fig. 3.25, and the yield stress versus equivalent plastic strain data have been calculated using Eq. (3.14). For the monotonic tension test results shown in Fig. 3.67, the FEA simulation with the ABAQUS calibrated parameters cannot reproduce the engineering stress versus cross-sectional area change curve of the tests. The stress is predicted to peak at a much lower deformation compared to the tests, and exhibits a very sharp drop with the deformation thereafter. Figures 3.68 and 3.69 show the test and predicted engineering stress versus engineering strain and engineering stress versus crosssectional area change curves of the straight specimen ADC40202 that was subjected to 
cyclic loading then pulled to fracture. While there is a good agreement between the test and predicted engineering stress versus engineering strain curves shown in Fig. 3.68 for cyclic loading, the FEA simulation with the ABAQUS calibrated parameters cannot predict the engineering stress versus cross-sectional area change curve, as shown in Fig. 3.69. For the comparisons with the cyclic test of the notched specimen ABC20404 shown in Fig. 3.69, the numerical simulation significantly under-predicts the stress of the specimen. This clearly shows that the built-in ABAQUS calibration procedure for kinematic hardening parameters cannot be applied to a general loading condition.

Kanvinde and Deierlein (2004) have carried out a study on fracture of steels using mixed-mode hardening model with one kinematic hardening term to simulate monotonic tension and cyclic tests. However, separate material model parameters were used depending on whether the loading is monotonic or cyclic. The calibration procedure has also not been provided in the report. Figure 3.71 shows results of the monotonic tension test simulations of a straight specimen using parameters of AW50 steel for monotonic and cyclic tests provided by Kanvinde and Deierlein (2004). Obviously, results of the simulations are significantly different with either set of the parameters. Since the loading type that leads to fracture in a real situation is not known a priori, only a single set of material model parameters have to be used in a general numerical simulation to model both monotonic and cyclic loading. Therefore, the approach by Kanvinde and Deierlein (2004) using two separate material model parameter sets is not considered as a suitable option for the current study. 


\subsection{Summary}

In this chapter, a testing program to furnish test data for determination of the material model parameters and evaluation of fracture criteria in Chapter 4 was discussed. The testing program consists of thirty nine round cross-sectional shape specimens from two steel grades with twenty one specimens from ASTM A572 (ASTM 2007) Grade 50 steel and eighteen specimens from CSA G40.20/21 (CSA 2004) Grade 300W steel, together with mainly two notched profiles and one straight profile. Three loading sequences consist of monotonic tension, cyclic loading followed by tension, and cyclic loading only were considered in the testing program. Corresponding FEA meshes used in the numerical simulations were created for these specimens.

A systematic procedure to calibrate the parameters for the plasticity model by Chaboche (1986) has been developed based on results of the tests and numerical simulations. Backstress curves calculated with an assumed saturated yield stress for small strain using results from the stabilized tension branch of the stress-strain curve from a straight cyclic test and for large strain using the flow stress generated with the exponential law equation by Chinh et al. (2004), were used in the determination of parameters $\mathrm{C}$ and $\gamma$ for kinematic hardening. Values of the kinematic hardening parameters were further improved through an iterative error minimizing approach as well as adjusting the parameter $b$ for the flow stress equation. It was found that a sufficiently good fit to the backstress versus plastic strain data can be achieved using only two kinematic hardening terms. The calibrated material model parameters using two kinematic hardening terms for materials $\mathrm{A}$ and $\mathrm{B}$ are listed in Table 3.6. Comparisons of results from the tests and numerical simulations for all specimens of both materials have 
been presented. There is a good agreement between test and predicted load versus crosssectional deformation of straight specimens and load versus axial deformation of notched specimens. However, there are larger differences between test and predicted load versus cross-sectional deformation of notched specimens. Modeling cyclic loading with one kinematic hardening term has also been evaluated. Comparisons of some of the test results for material $A$ with that from numerical simulations using one kinematic hardening term have shown that it is not suitable to use just one kinematic hardening term. ABAQUS (Simulia 2009) built-in calibration procedure and the approach by Kanvinde and Deierlein (2004) using separate material model parameters for monotonic or cyclic loading have also been found to be unsuitable for modeling the behaviour of steel under a general loading condition. 
Table 3.1 Test matrix for material A

\begin{tabular}{|c|c|c|c|c|c|c|}
\hline Specimen & Profile & Test type & $\begin{array}{l}\text { Nominal engineering } \\
\text { cyclic strain range }\end{array}$ & $\begin{array}{c}\text { Nominal true } \\
\text { cyclic strain range }\end{array}$ & $\begin{array}{c}\text { Nominal engineering } \\
\text { strain rate }\end{array}$ & $\begin{array}{c}\text { Nominal true } \\
\text { strain rate }\end{array}$ \\
\hline ADMa & D14R24U & Monotonic test & - & - & $8 \% / \mathrm{min}$ & $8 \% / \min ^{1}$ \\
\hline $\mathrm{ADMb}$ & D14R24U & Monotonic test & - & - & $1 \% / \mathrm{min}$ & $1 \% / \min ^{1}$ \\
\hline AEM & D14R19U & Monotonic test & - & - & $1 \% / \mathrm{min}$ & $1 \% / \min ^{1}$ \\
\hline AAMa & D10R14N & Monotonic test & - & - & $1.2 \% / \mathrm{min}$ & $8 \% / \min { }^{1}$ \\
\hline $\mathrm{AAMb}$ & D10R14N & Monotonic test & - & - & $0.184 \% / \mathrm{min}$ & $1 \% / \min ^{\prime}$ \\
\hline $\mathrm{ABM}$ & D14R19N & Monotonic test & - & - & $0.9 \% / \mathrm{min}$ & $4 \% / \min ^{1}$ \\
\hline $\mathrm{ADC} 20202$ & D14R24U & 20 cycles, pull to failure & $\pm 2 \%$ & $\pm 2 \%$ & $8 \% / \mathrm{min}$ & $8 \% / \min$ \\
\hline ADC40101 & D14R24U & 40 cycles, pull to faiture & $\pm 1 \%$ & $\pm 1 \%$ & $8 \% / \mathrm{min}$ & $8 \% / \min$ \\
\hline ADC40102 & $\mathrm{D} 14 \mathrm{R} 24 \mathrm{U}$ & 40 cycles, pull to failure & $-2 \%$ to $1 \%$ & $-2 \%$ to $1 \%^{2}$ & $8 \% / \mathrm{min}$ & $8 \% / \mathrm{min}$ \\
\hline $\mathrm{ADC} 40202$ & D14R24U & 40 cycles, pull to failure & $\pm 2 \%$ & $\pm 2 \%$ & $8 \% / \mathrm{min}$ & $8 \% / \min$ \\
\hline ADCR0201 & D14R24U & 30 cycles, pull to failure & $\pm 2 \%, \pm 1 \%$ & $\pm 2 \%, \pm 1 \%^{3}$ & $8 \% / \mathrm{min}$ & $8 \% / \min$ \\
\hline AAC20404 & D10R14N & 20 cycles, pull to failure & $\pm 0.6 \%$ & $\pm 4 \%$ & $1.2 \% / \mathrm{min}$ & $8 \% / \mathrm{min}$ \\
\hline $\mathrm{ABC} 20404$ & D14R19N & 20 cycles, pull to faiture & $\pm 0.9 \%$ & $\pm 4 \%$ & $0.9 \% / \mathrm{min}$ & $4 \% / \min$ \\
\hline $\mathrm{ACC} 20404$ & D10R7N & 20 cycles, pull to failure & $\pm 0.5 \%$ & $\pm 4 \%$ & $0.5 \% / \mathrm{min}$ & $4 \% / \mathrm{min}$ \\
\hline $\mathrm{AAC} 70404$ & D10R14N & 70 cycles, pull to failure & $\pm 0.6 \%$ & $\pm 4 \%$ & $1.2 \% / \mathrm{min}$ & $8 \% / \mathrm{min}$ \\
\hline $\mathrm{ABC} 70404$ & D14R19N & 70 cycles, pull to failure & $\pm 0.9 \%$ & $\pm 4 \%$ & $1.8 \% / \mathrm{min}$ & $8 \% / \min$ \\
\hline AACF1212 & D10R14N & Cycle to faihure & $-1.8 \%$ to $1.65 \%$ & $\pm 12 \%$ & $1.2 \% / \mathrm{min}$ & $8 \% / \mathrm{min}$ \\
\hline $\mathrm{ABCF} 1212$ & D14R19N & Cycle to failure & $-2.8 \%$ to $2.3 \%$ & $\pm 12 \%$ & $1.8 \% / \mathrm{min}$ & $8 \% / \min$ \\
\hline AACF3010 & D10R14N & Cycle to failure & $-1.5 \%$ to $3.67 \%$ & $-10 \%$ to $30 \%$ & $1.2 \% / \mathrm{min}$ & $8 \% / \min$ \\
\hline $\mathrm{ABCF} 3010$ & D14R19N & Cycle to failure & $-2.3 \%$ to $5 \%$ & $-10 \%$ to $30 \%$ & $1.8 \% / \mathrm{min}$ & $8 \% / \mathrm{min}$ \\
\hline AACR0804 & D10R14N & Cycle to failure & $\pm 1.2 \%, \pm 0.6 \%$ & $\pm 8 \%, \pm 4 \%{ }^{4}$ & $1.2 \% / \min$ & $8 \% / \mathrm{min}$ \\
\hline
\end{tabular}


Notes:

1 The true strain rate approximation is only valid up to peak load.

2 Specimen ADC40102 was loaded first in compression.

3 Specimen ADCR0201 was first subjected to 10 cycles of loading between strain range of $-2 \%$ to $+2 \%$, then it was subjected to 20 cycles of loading between strain range of $-1 \%$ to $+1 \%$ before being pulled to failure in tension.

4 Specimen AACR0804 was first subjected to 8 cycles of loading between nominal true strain range of $-8 \%$ to $+8 \%$, then it was subjected to cyclic loading between nominal true strain range of $-4 \%$ to $+4 \%$ until failure. 
Table 3.2 Test matrix for material B

\begin{tabular}{|c|c|c|c|c|c|c|}
\hline Specimen & Profile & Test type & $\begin{array}{l}\text { Nominal engineering } \\
\text { cyclic strain range }\end{array}$ & $\begin{array}{c}\text { Nominal true } \\
\text { cyclic strain range }\end{array}$ & $\begin{array}{c}\text { Nominal engineering } \\
\text { strain rate }\end{array}$ & $\begin{array}{c}\text { Nominal true } \\
\text { strain rate }\end{array}$ \\
\hline $\mathrm{BDMa}$ & D14R24U & Monotonic test & - & - & $8 \% / \mathrm{min}$ & $8 \% / \min ^{1}$ \\
\hline BDMb & D14R24U & Monotonic test & - & - & $1 \% / \mathrm{min}$ & $1 \% / \min ^{\prime}$ \\
\hline BAM & D10R14N & Monotonic test & - & - & $1.2 \% / \mathrm{min}$ & $8 \% / \min ^{1}$ \\
\hline BBM & D14R19N & Monotonic test & - & - & $1.8 \% / \mathrm{min}$ & $8 \% / \min 1$ \\
\hline $\mathrm{BDC} 20202$ & D14R24U & 20 cycles, pull to faihure & $\pm 2 \%$ & $\pm 2 \%$ & $8 \% / \mathrm{min}$ & $8 \% / \min$ \\
\hline BDC 40101 & D14R24U & 40 cycles, pull to faihure & $\pm 1 \%$ & $\pm 1 \%$ & $8 \% / \min$ & $8 \% / \mathrm{min}$ \\
\hline BDC40102 & D14R24U & 40 cycles, pull to failure & $-2 \%$ to $1 \%$ & $-2 \%$ to $1 \%^{2}$ & $8 \% / \mathrm{min}$ & $8 \% / \mathrm{min}$ \\
\hline BDC40202 & D14R24U & 40 cycles, pull to failure & $\pm 2 \%$ & $\pm 2 \%$ & $8 \% / \mathrm{min}$ & $8 \% / \min$ \\
\hline BDCR0201 & D14R24U & 30 cycles, pull to failure & $\pm 2 \%, \pm 1 \%$ & $\pm 2 \%, \pm 1 \%{ }^{3}$ & $8 \% / \mathrm{min}$ & $8 \% / \mathrm{min}$ \\
\hline BAC20404 & D10R14N & 20 cycles, pull to failure & $\pm 0.6 \%$ & $\pm 4 \%$ & $1.2 \% / \mathrm{min}$ & $8 \% / \mathrm{min}$ \\
\hline BBC20404 & D14R19N & 20 cycles, pull to failure & $\pm 0.9 \%$ & $\pm 4 \%$ & $1.8 \% / \mathrm{min}$ & $8 \% / \mathrm{min}$ \\
\hline BAC 70404 & D10R14N & 70 cycles, pull to failure & $\pm 0.6 \%$ & $\pm 4 \%$ & $1.2 \% / \mathrm{min}$ & $8 \% / \mathrm{min}$ \\
\hline $\mathrm{BBC} 70404$ & D14R19N & 70 cycles, pull to faihure & $\pm 0.9 \%$ & $\pm 4 \%$ & $1.8 \% / \mathrm{min}$ & $8 \% / \mathrm{min}$ \\
\hline $\mathrm{BACF} 1212$ & DI0R14N & Cycle to failure & $-1.8 \%$ to $1.65 \%$ & $\pm 12 \%$ & $1.2 \% / \mathrm{min}$ & $8 \% / \mathrm{min}$ \\
\hline BBCF 1212 & D14R19N & Cycle to failure & $-2.8 \%$ to $2.3 \%$ & $\pm 12 \%$ & $1.8 \% / \mathrm{min}$ & $8 \% / \mathrm{min}$ \\
\hline BACF3010 & D10R14N & Cycle to failure & $-1.5 \%$ to $3.67 \%$ & $-10 \%$ to $30 \%$ & $1.2 \% / \min$ & $8 \% / \mathrm{min}$ \\
\hline BBCF3010 & $\mathrm{D} 14 \mathrm{R} 19 \mathrm{~N}$ & Cycle to failure & $-2.3 \%$ to $5 \%$ & $-10 \%$ to $30 \%$ & $1.8 \% / \mathrm{min}$ & $8 \% / \mathrm{min}$ \\
\hline BACR0804 & D10R14N & Cycle to failure & $\pm 1.2 \%, \pm 0.6 \%$ & $\pm 8 \%, \pm 4 \% \%^{4}$ & $1.2 \% / \mathrm{min}$ & $8 \% / \min$ \\
\hline
\end{tabular}


Notes:

1 The true strain rate approximation is only valid up to peak load.

2 Specimen BDC40102 was loaded first in compression.

3 Specimen BDCR0201 was first subjected to 10 cycles of loading between strain range of $-2 \%$ to $+2 \%$, then it was subjected to 20 cycles of loading between strain range of $-1 \%$ to $+1 \%$ before being pulled to failure in tension.

4 Specimen BACR0804 was first subjected to 8 cycles of loading between nominal true strain range of $-8 \%$ to $+8 \%$, then it was subjected to cyclic loading between nominal true strain range of $-4 \%$ to $+4 \%$ until failure. 
Table 3.3 Material model parameters and properties used in finding optimum kinematic hardening parameters with their associated error $\mathrm{Q}$

\begin{tabular}{|c|c|c|c|c|c|c|}
\hline \multicolumn{2}{|c|}{$\begin{array}{l}E=205600 \mathrm{MPa} \\
\sigma_{\infty}^{y}=417 \mathrm{MPa}\end{array}$} & \multicolumn{2}{|l|}{$\begin{array}{l}v=0.3 \\
\mathrm{n}=0.77\end{array}$} & \multicolumn{2}{|c|}{$\begin{array}{l}\sigma_{0}^{y}=362 \mathrm{MPa} \\
\mathrm{b}=4.5\end{array}$} & $\varepsilon_{0}^{p}=0.005$ \\
\hline$\sigma_{\mathrm{s}}^{0}(\mathrm{MPa})$ & $\Delta \sigma_{\mathrm{s}}^{0}(\mathrm{MPa})$ & $\mathrm{C}_{1}(\mathrm{MPa})$ & $\gamma_{1}$ & $\mathrm{C}_{2}(\mathrm{MPa})$ & $\gamma_{2}$ & $\mathrm{Q}\left(\mathrm{MPa}^{2}\right)$ \\
\hline 273 & 0 & 99491 & 469.58 & 1571 & 5.33 & 68780 \\
\hline \multirow{4}{*}{450} & 0 & 5055 & 63.37 & 982 & 3.93 & 18930 \\
\hline & 10 & 5116 & 62.42 & 892 & 3.75 & 7299 \\
\hline & 20 & 5174 & 61.69 & 809 & 3.58 & 5170 \\
\hline & 17 & 5157 & 61.89 & 834 & 3.63 & 4825 \\
\hline \multirow{4}{*}{475} & 0 & 2613 & 27.44 & 714 & 3.41 & 11058 \\
\hline & 10 & 2652 & 28.55 & 666 & 3.30 & 3958 \\
\hline & 20 & 2688 & 29.66 & 619 & 3.18 & 6407 \\
\hline & 12 & 2659 & 28.77 & 656 & 3.27 & 3690 \\
\hline \multirow{4}{*}{500} & 0 & 473 & 8.75 & 881 & 3.91 & 29783 \\
\hline & 10 & 412 & 11.68 & 910 & 3.88 & 26530 \\
\hline & 20 & 425 & 14.08 & 867 & 3.78 & 33260 \\
\hline & 8 & 415 & 11.14 & 914 & 3.90 & 26375 \\
\hline \multirow{4}{*}{464} & 0 & 3544 & 38.63 & 801 & 3.57 & 12501 \\
\hline & 10 & 3605 & 39.21 & 734 & 3.43 & 3998 \\
\hline & 20 & 3661 & 39.86 & 672 & 3.29 & 4845 \\
\hline & 14 & 3628 & 39.46 & 708 & 3.37 & 3225 \\
\hline
\end{tabular}


Table 3.4 Yield stress versus equivalent plastic strain data for material A (two terms)

\begin{tabular}{|c|c|}
\hline Equivalent plastic strain $(\mathrm{mm} / \mathrm{mm})$ & Yield stress (MPa) \\
\hline 0.000 & 360.3 \\
\hline 0.005 & 341.2 \\
\hline 0.010 & 353.3 \\
\hline 0.019 & 363.1 \\
\hline 0.028 & 371.3 \\
\hline 0.038 & 379.4 \\
\hline 0.047 & 387.7 \\
\hline 0.056 & 395.6 \\
\hline 0.066 & 403.4 \\
\hline 0.075 & 410.6 \\
\hline 0.084 & 417.3 \\
\hline 0.093 & 423.2 \\
\hline 0.106 & 431.3 \\
\hline 0.115 & 436.5 \\
\hline 0.124 & 441.2 \\
\hline 0.135 & 446.2 \\
\hline 0.145 & 450.8 \\
\hline 0.156 & 454.9 \\
\hline 0.168 & 459.1 \\
\hline 0.176 & 461.5 \\
\hline 0.189 & 464.9 \\
\hline 0.203 & 468.3 \\
\hline 0.244 & 475.8 \\
\hline 0.295 & 481.3 \\
\hline 0.313 & 482.6 \\
\hline 0.354 & 484.6 \\
\hline 0.413 & 485.9 \\
\hline 0.501 & 486.0 \\
\hline 0.597 & 485.0 \\
\hline 0.701 & 483.6 \\
\hline 0.818 & 482.0 \\
\hline 0.915 & 481.0 \\
\hline 0.991 & 480.3 \\
\hline 1.200 & 479.0 \\
\hline 2.000 & 477.8 \\
\hline 5.000 & 477.7 \\
\hline 10.000 & 477.7 \\
\hline 20.000 & 477.7 \\
\hline
\end{tabular}


Table 3.5 Yield stress versus equivalent plastic strain data for material B (two terms)

\begin{tabular}{|c|c|}
\hline Equivalent plastic strain $(\mathrm{mm} / \mathrm{mm})$ & Yield stress (MPa) \\
\hline 0.000 & 326.5 \\
\hline 0.016 & 271.6 \\
\hline 0.028 & 308.9 \\
\hline 0.037 & 318.0 \\
\hline 0.047 & 324.7 \\
\hline 0.056 & 330.5 \\
\hline 0.065 & 335.9 \\
\hline 0.075 & 341.0 \\
\hline 0.084 & 345.9 \\
\hline 0.093 & 350.5 \\
\hline 0.099 & 353.5 \\
\hline 0.108 & 357.7 \\
\hline 0.117 & 361.6 \\
\hline 0.126 & 365.3 \\
\hline 0.135 & 368.8 \\
\hline 0.144 & 372.0 \\
\hline 0.153 & 375.1 \\
\hline 0.162 & 378.0 \\
\hline 0.172 & 380.8 \\
\hline 0.186 & 384.4 \\
\hline 0.195 & 386.6 \\
\hline 0.219 & 391.3 \\
\hline 0.259 & 397.2 \\
\hline 0.290 & 400.3 \\
\hline 0.325 & 402.8 \\
\hline 0.368 & 404.6 \\
\hline 0.413 & 405.7 \\
\hline 0.492 & 406.0 \\
\hline 0.583 & 405.2 \\
\hline 0.689 & 403.7 \\
\hline 0.774 & 402.5 \\
\hline 0.868 & 401.1 \\
\hline 1.000 & 399.7 \\
\hline 1.400 & 397.5 \\
\hline 2.000 & 397.4 \\
\hline 5.000 & 399.2 \\
\hline 10.000 & 399.3 \\
\hline 20.000 & 399.3 \\
\hline
\end{tabular}


Table 3.6 Calibrated material model parameters for materials A and B (two terms)

\begin{tabular}{|c|c|c|c|c|c|c|c|c|c|}
\hline \multirow{2}{*}{ Material } & \multicolumn{4}{|c|}{ Flow stress parameters } & \multicolumn{3}{c|}{ Kinematic hardening parameters } \\
\cline { 2 - 10 } & $\sigma_{0}^{y}(\mathrm{MPa})$ & $\varepsilon_{0}^{\mathrm{p}}$ & $\sigma_{\%}^{\mathrm{y}}(\mathrm{MPa})$ & $\mathrm{b}$ & $\mathrm{n}$ & $\mathrm{C}_{1}(\mathrm{MPa})$ & $\gamma_{1}$ & $\mathrm{C}_{2}(\mathrm{MPa})$ & $\gamma_{2}$ \\
\hline $\mathrm{A}$ & 362 & 0.0053 & 417 & 4.53 & 0.77 & 3625 & 39.46 & 711 & 3.39 \\
\hline $\mathrm{B}$ & 332 & 0.0161 & 448 & 3.03 & 0.65 & 3965 & 29.30 & 583 & 2.37 \\
\hline
\end{tabular}




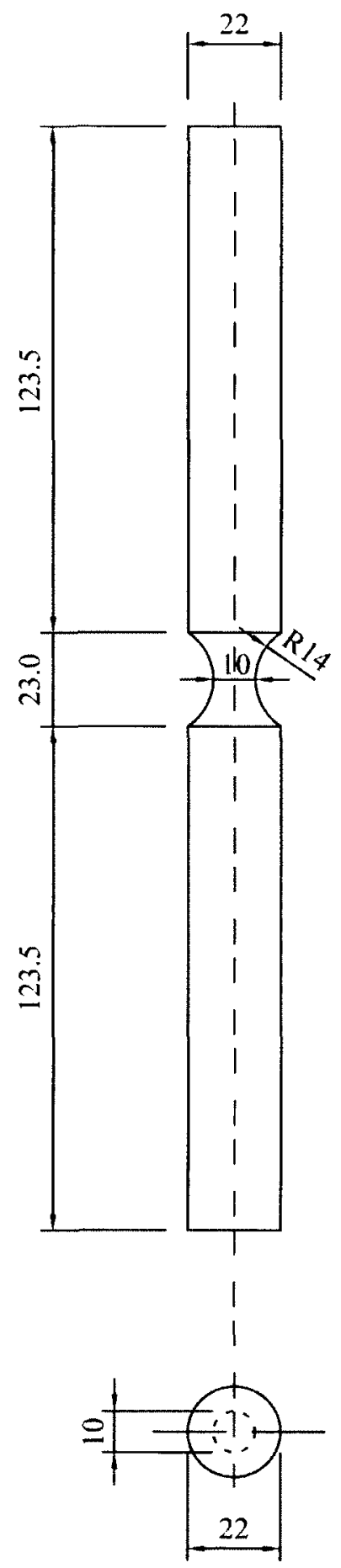

All dimensions in $\mathrm{mm}$

Figure 3.1 Profile of D10R14N notched specimen 


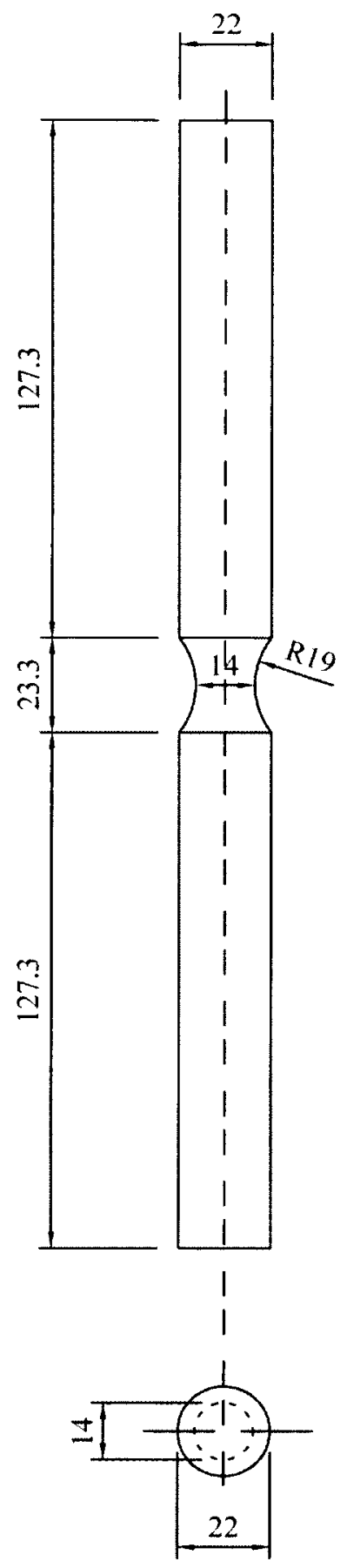

All dimensions in mm

Figure 3.2 Profile of D14R19N notched specimen 


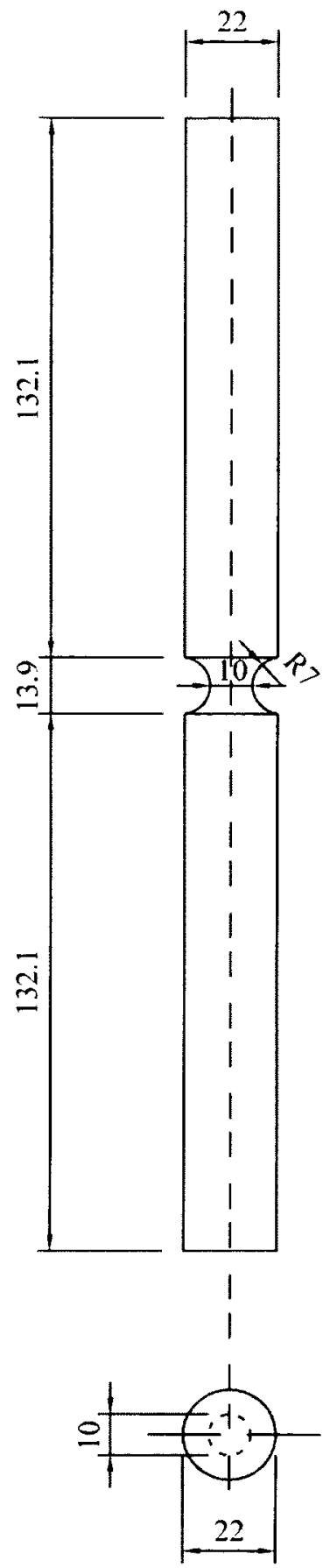

All dimensions in $\mathrm{mm}$

Figure 3.3 Profile of D10R7N notched specimen 


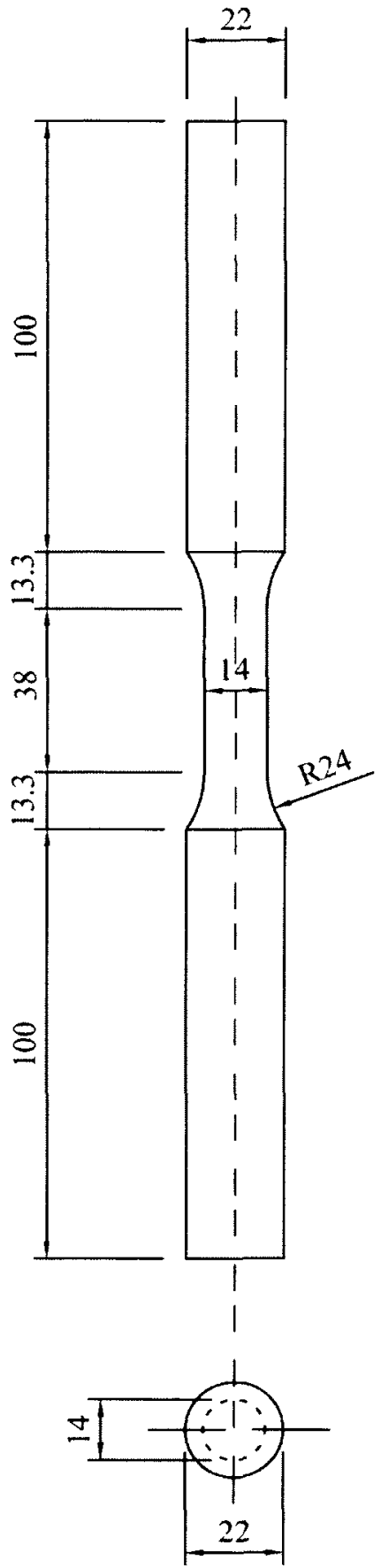

All dimensions in $\mathrm{mm}$

Figure 3.4 Profile of DI4R24U straight specimen 


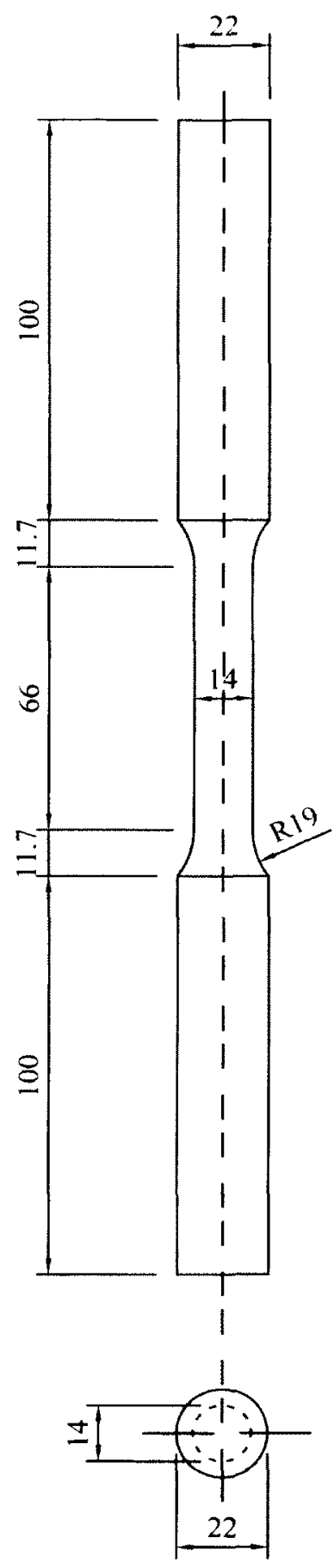

All dimensions in mm

Figure 3.5 Profile of D14R19U straight specimen 


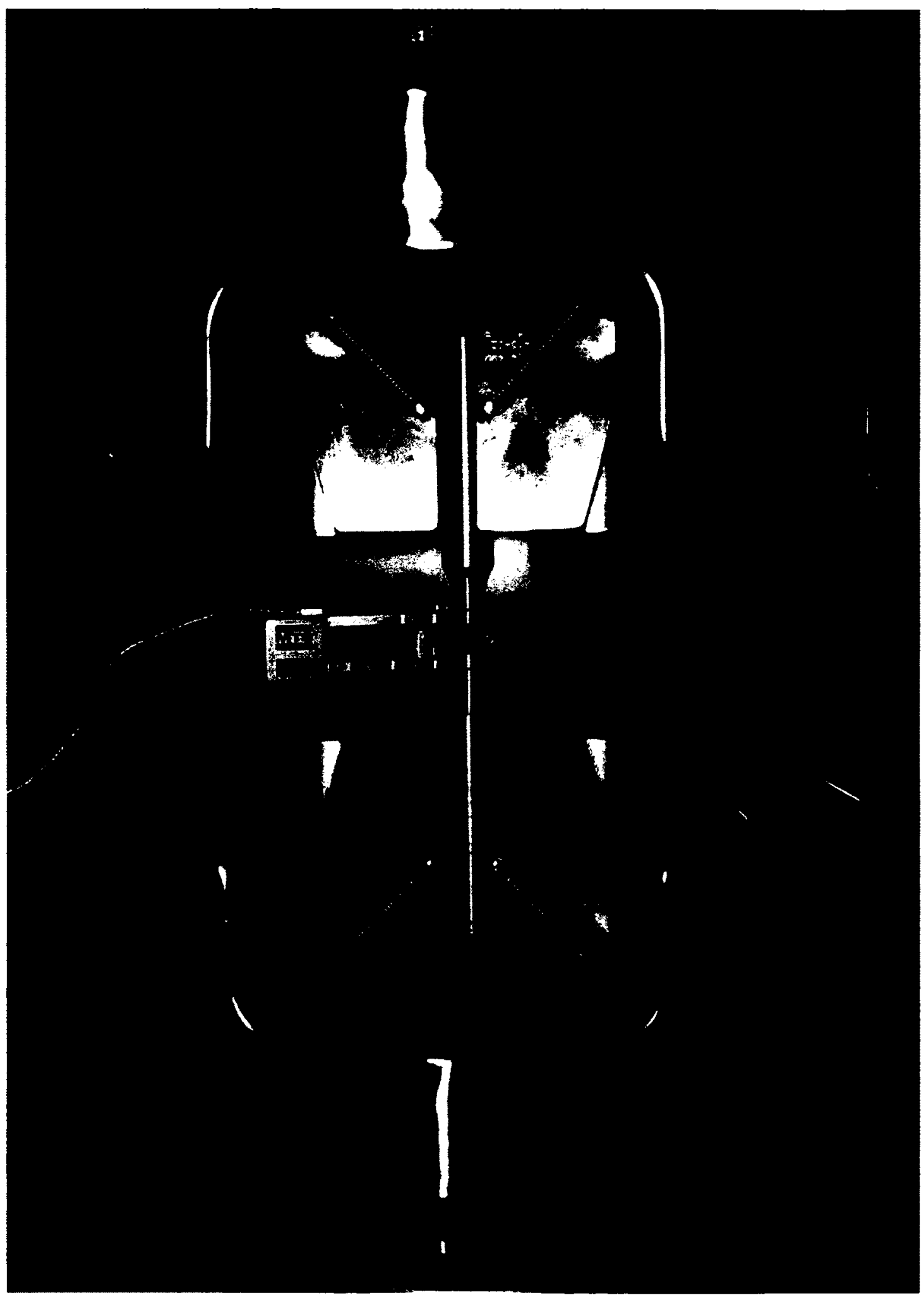

Figure 3.6 Test setup shown using $25.4 \mathrm{~mm}$ (one-inch) axial extensometer and diametral extensometer 


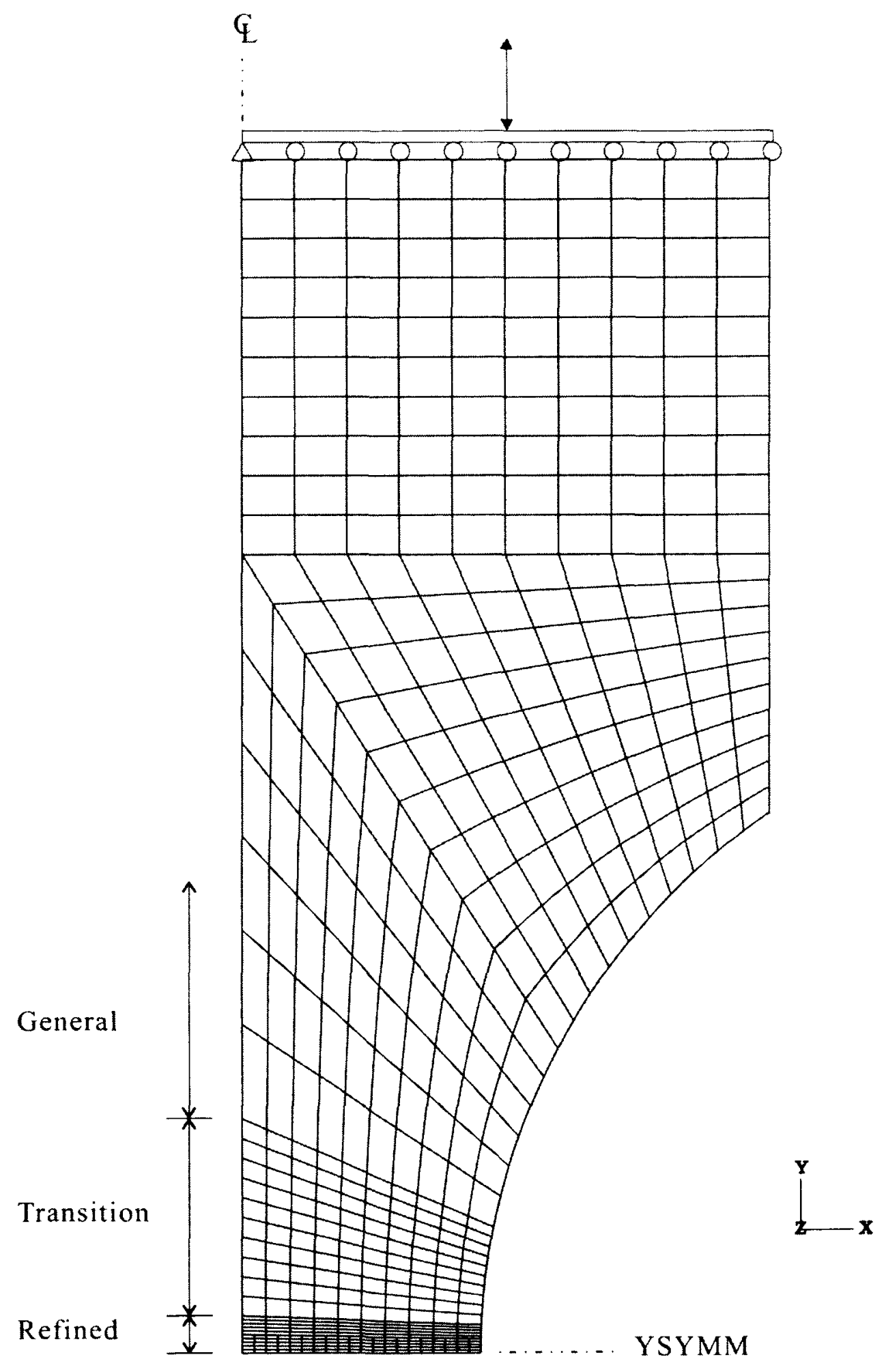

Figure 3.7 Mesh of $25.4 \mathrm{~mm}$ (one-inch) FEA model for D10R14N notched specimen 


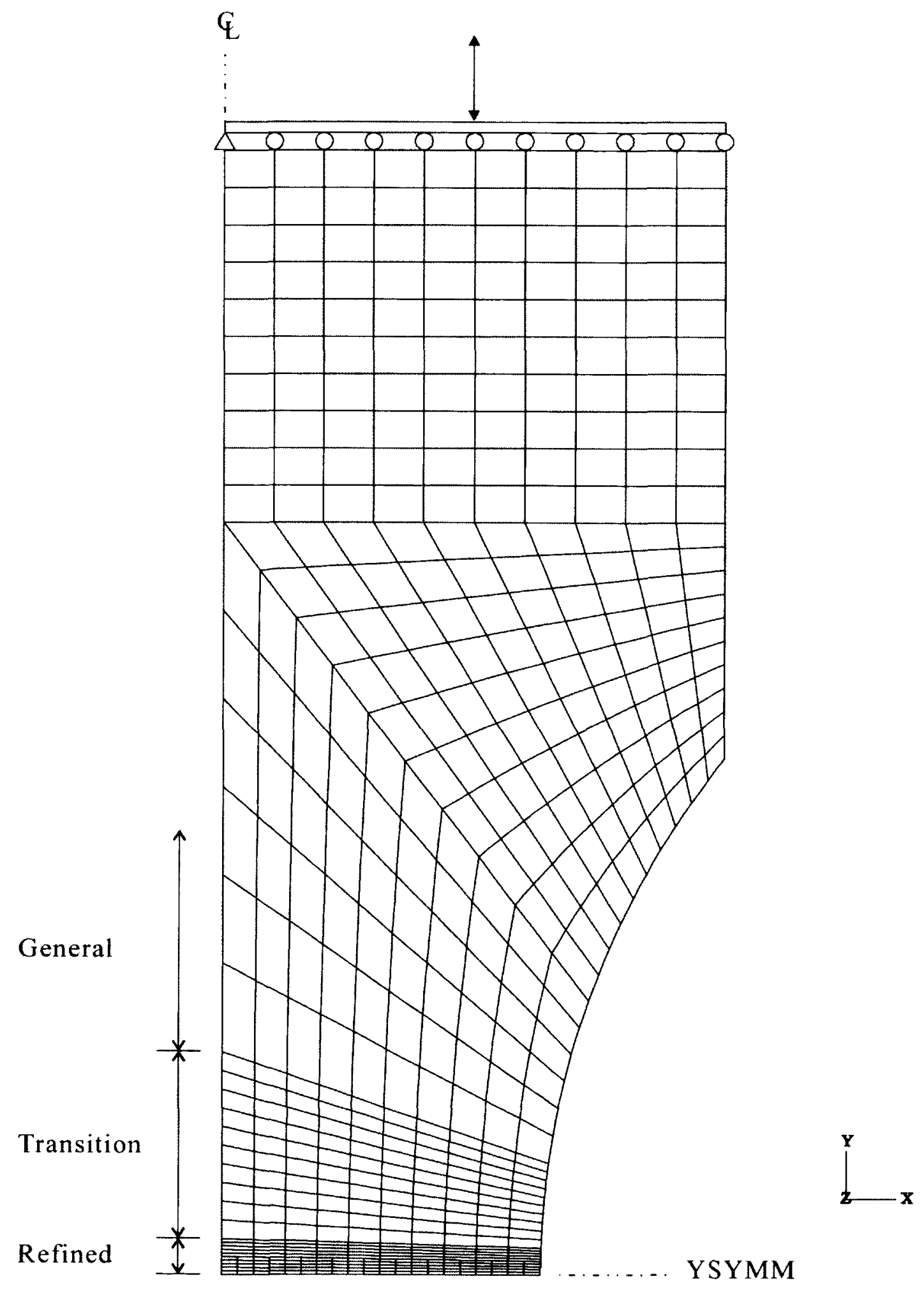

Figure 3.8 Mesh of $25.4 \mathrm{~mm}$ (one-inch) FEA model for D14R19N notched specimen 


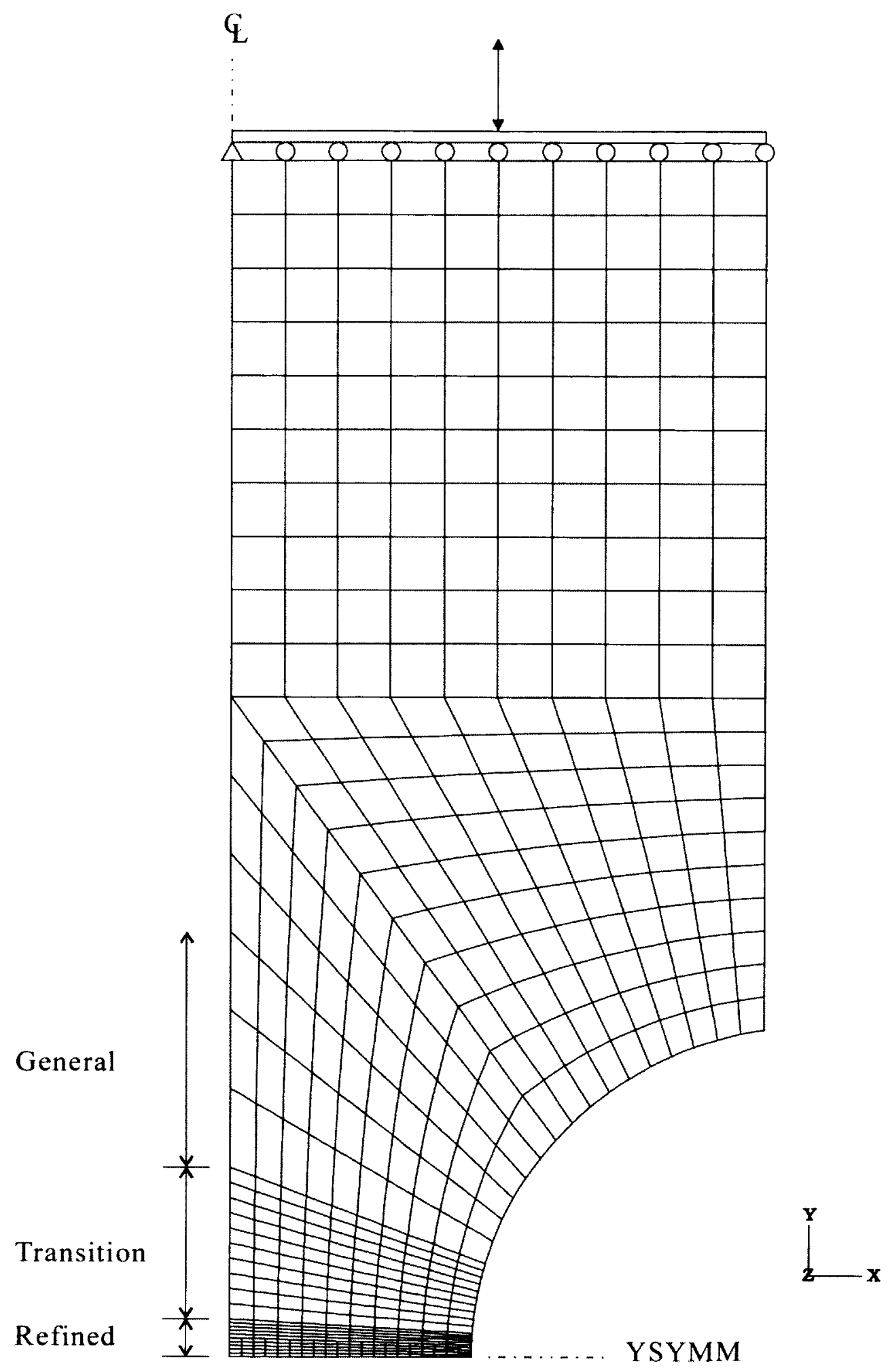

Figure 3.9 Mesh of $25.4 \mathrm{~mm}$ (one-inch) FEA model for DI0R7N notched specimen 


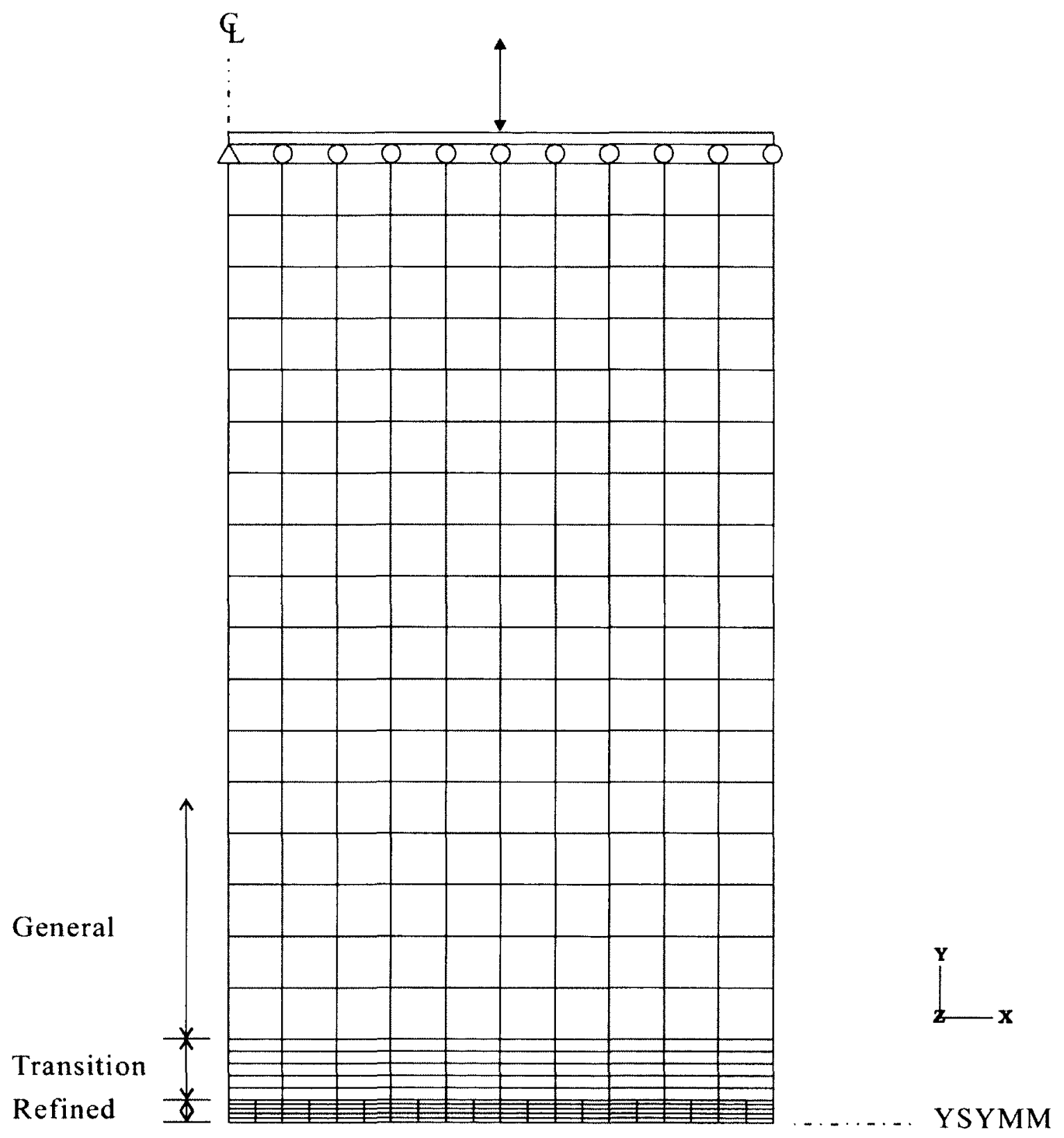

Figure 3.10 Mesh of $12.7 \mathrm{~mm}$ (half-inch) FEA model for D14R24U and D14R19U straight specimens 


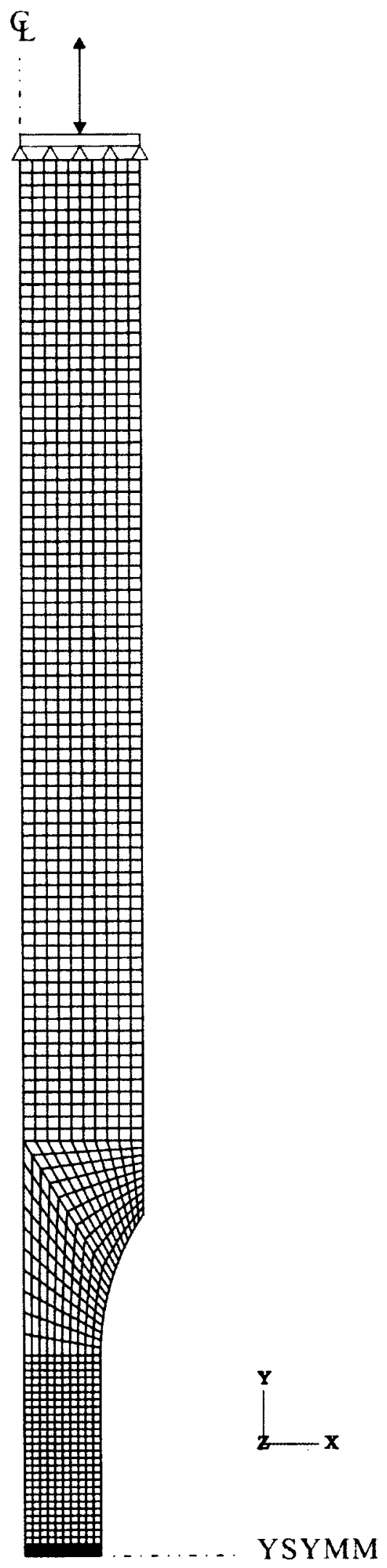

Figure 3.11 Mesh of half full-length FEA model for straight specimens 


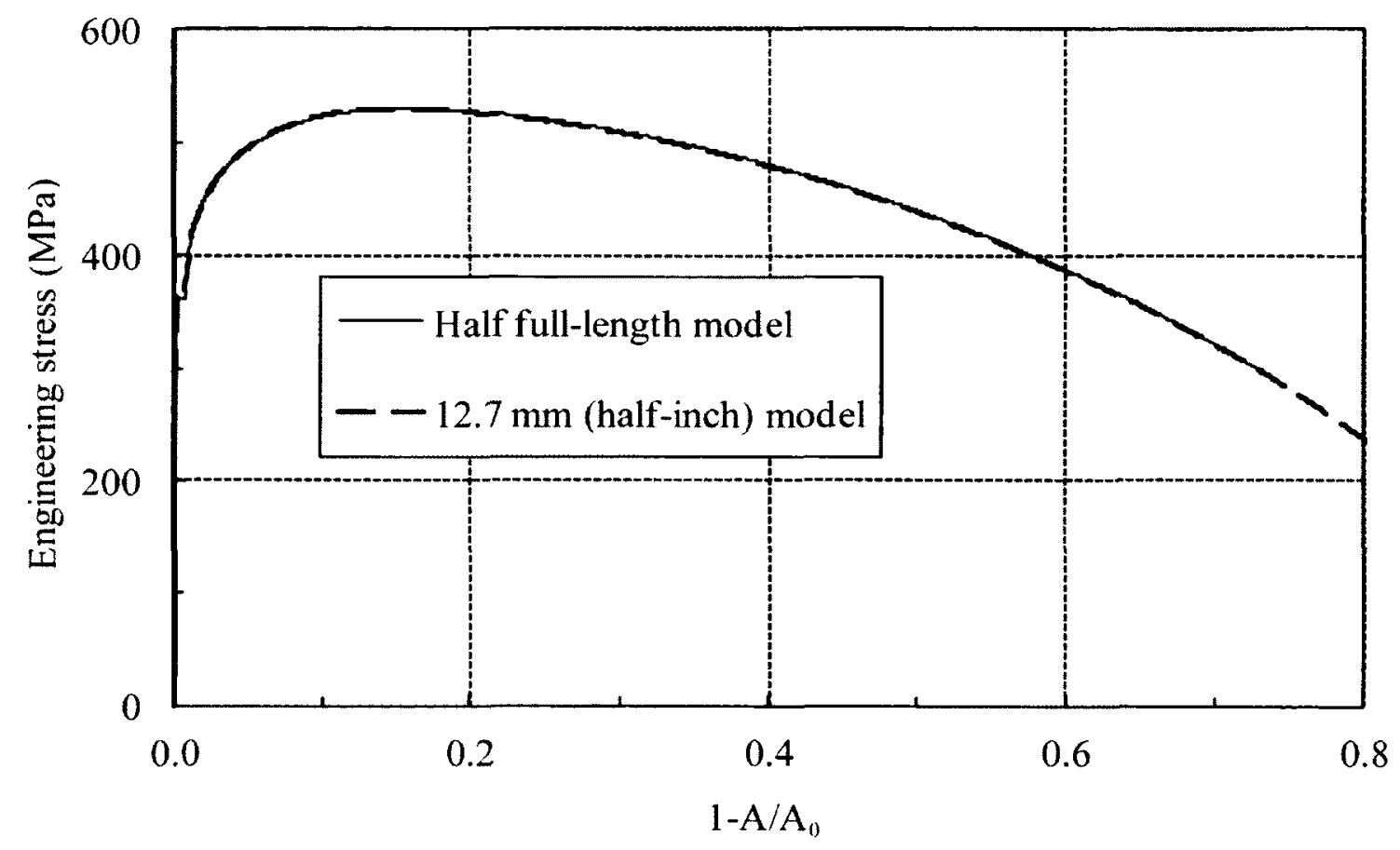

Figure 3.12 Comparison of simulation results using half full-length and $12.7 \mathrm{~mm}$ (halfinch) FEA models for straight specimens of material A subjected to monotonic tension loading

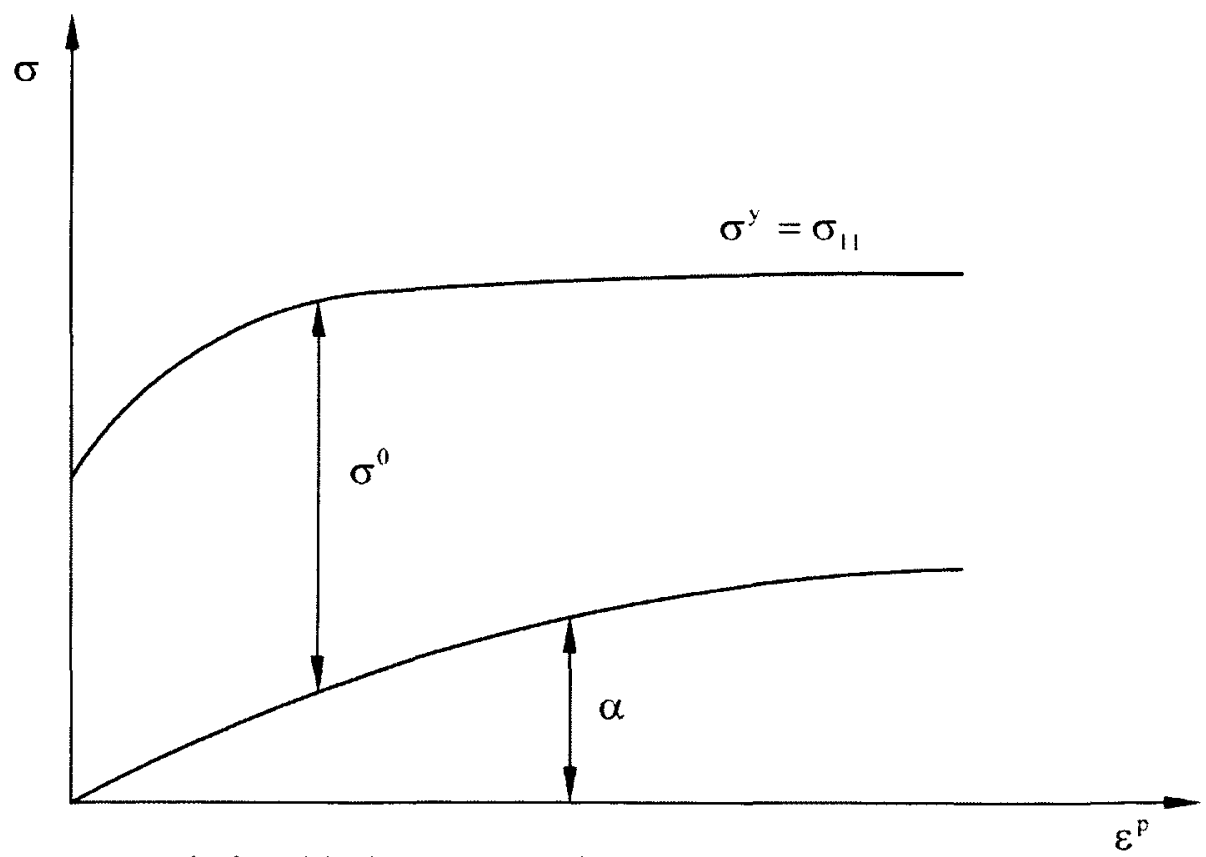

Figure 3.13 Relationship between various stress components under monotonic uniaxial tension loading 


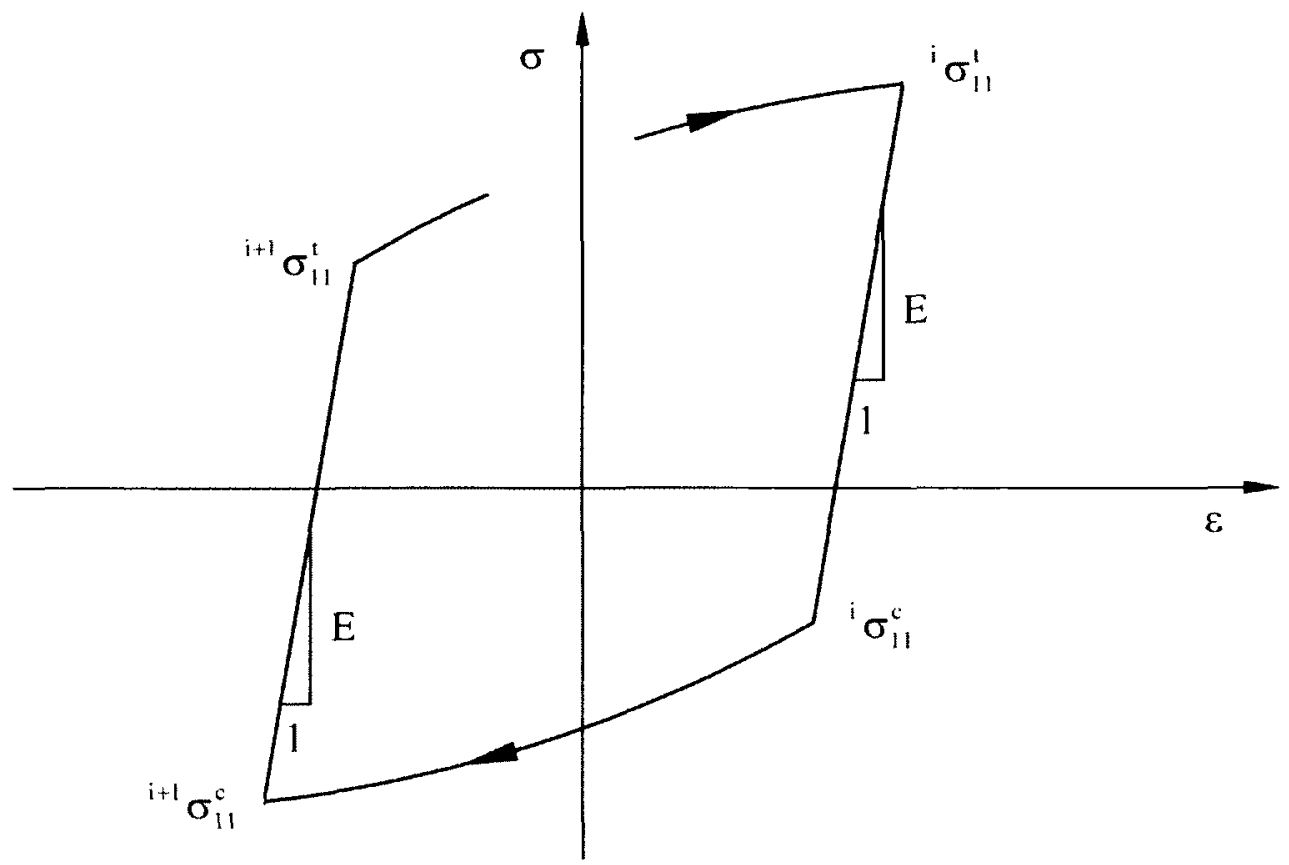

Figure 3.14 Flow stresses for calculating backstress and yield stress under uniaxial cyclic loading

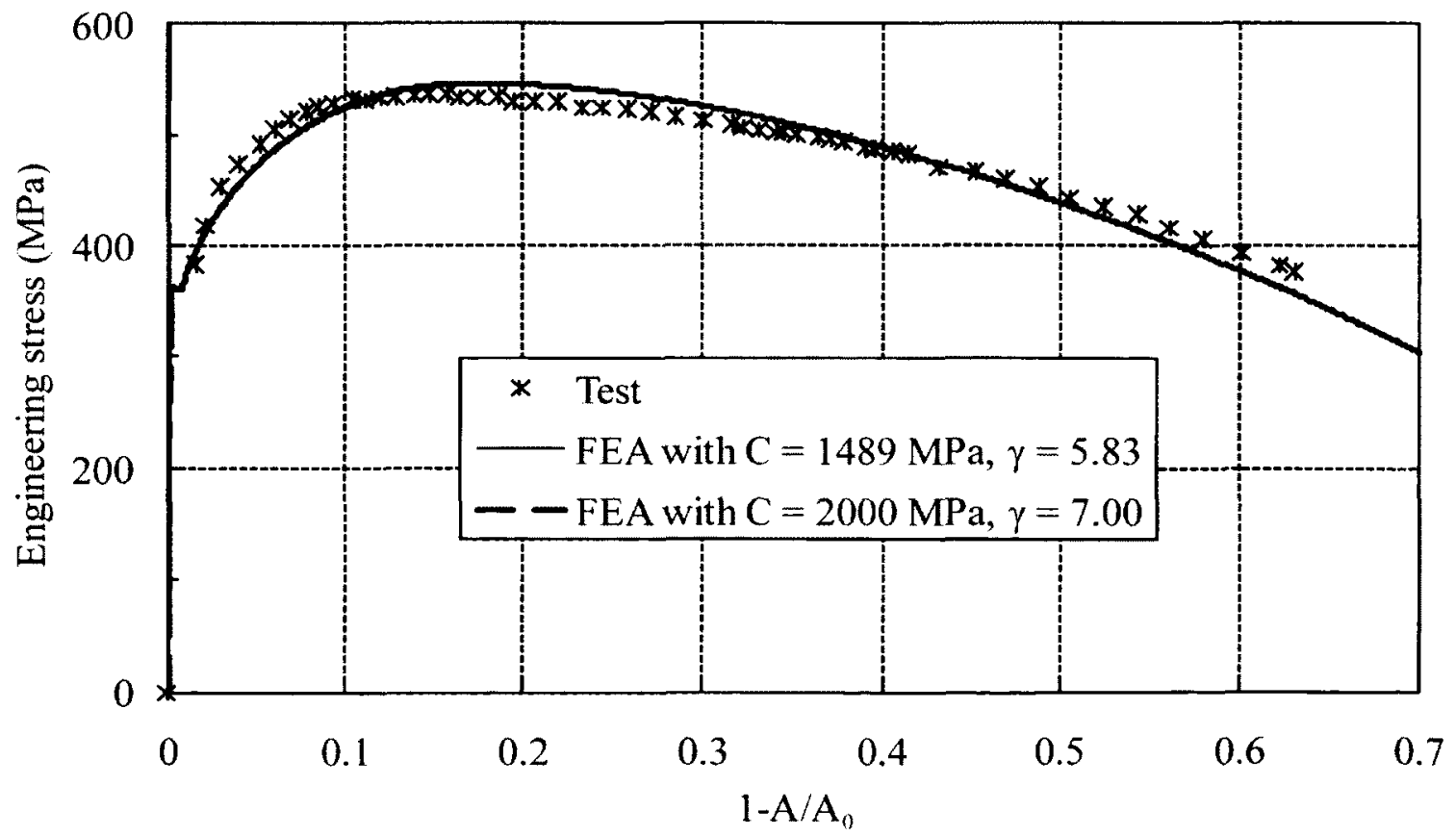

Figure 3.15 Test and predicted engineering stress versus cross-sectional area change curves for straight specimen ADMa using the same flow stress versus equivalent plastic strain data but different kinematic hardening parameters 


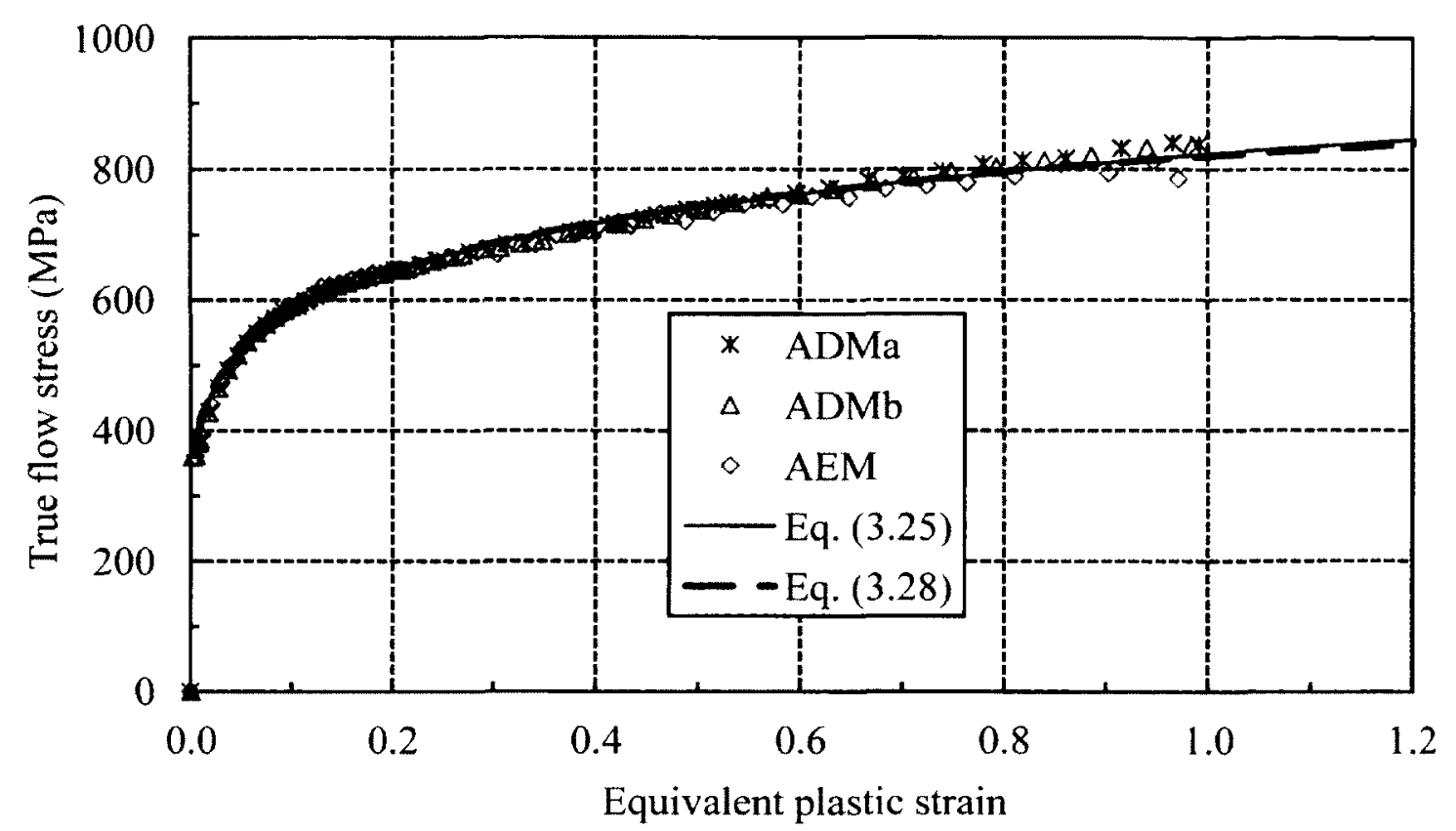

Figure 3.16 Curve fitting of the flow stress versus equivalent plastic strain data of monotonic straight specimens with power law Eq. (3.25) and exponential law Eq. (3.28)

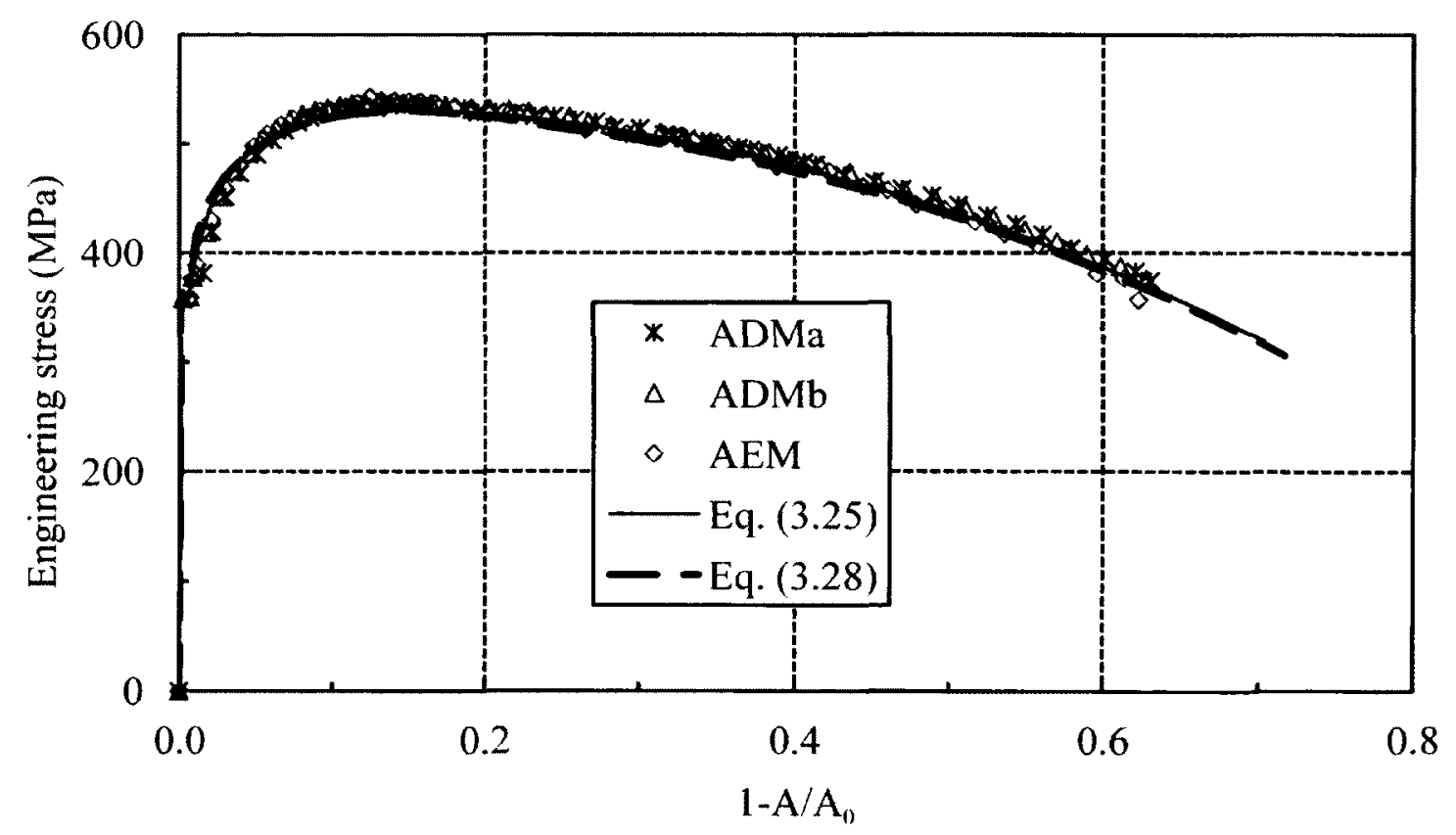

Figure 3.17 Test and predicted engineering stress versus cross-sectional area change curves for straight specimens of material A using the material parameters determined by least-square fitting for power law Eq. (3.25) and exponential law Eq. (3.28) 


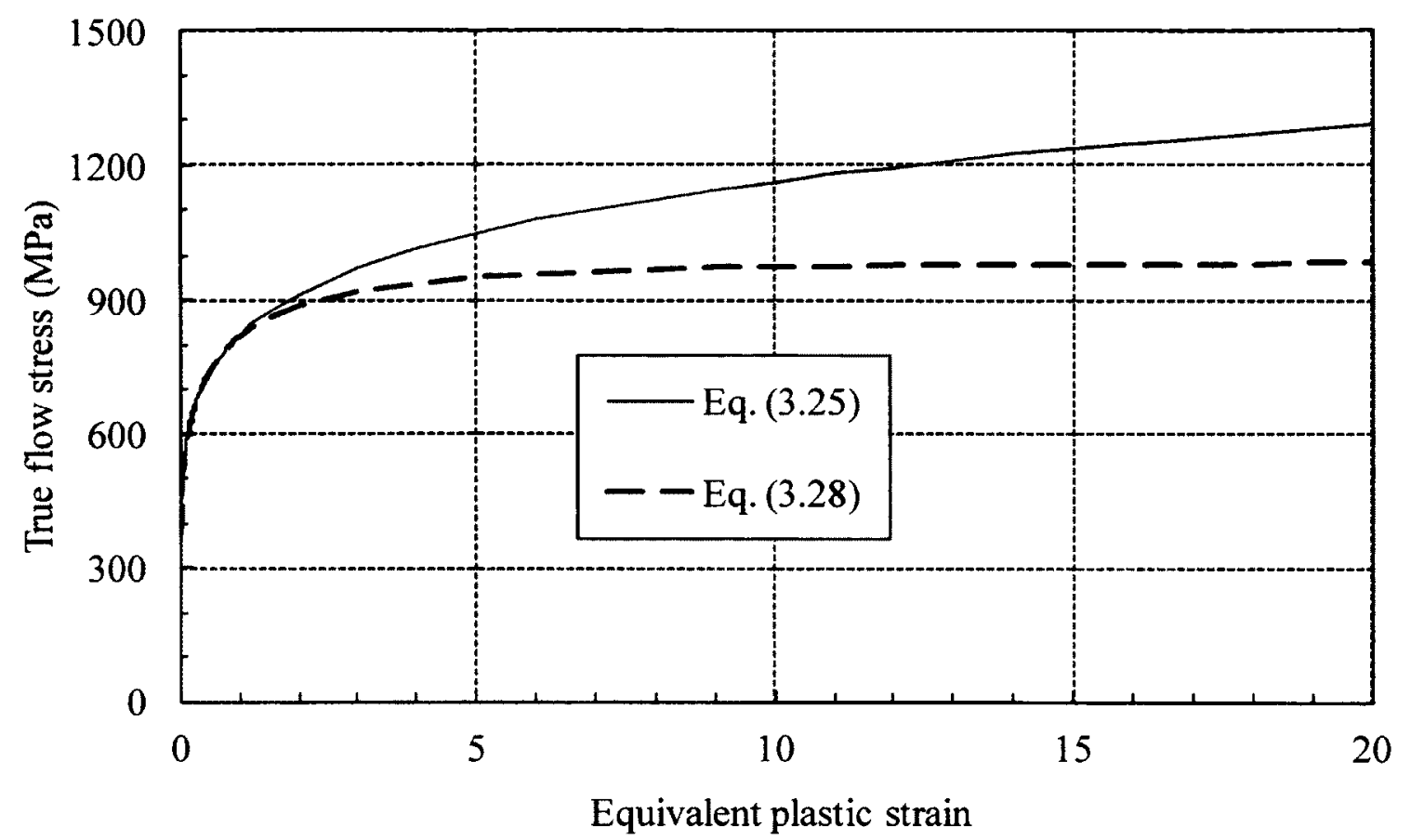

Figure 3.18 True flow stress versus equivalent plastic strain curves generated by power law Eq. (3.25) and exponential law Eq. (3.28) over a large range of strain with the calibrated parameters

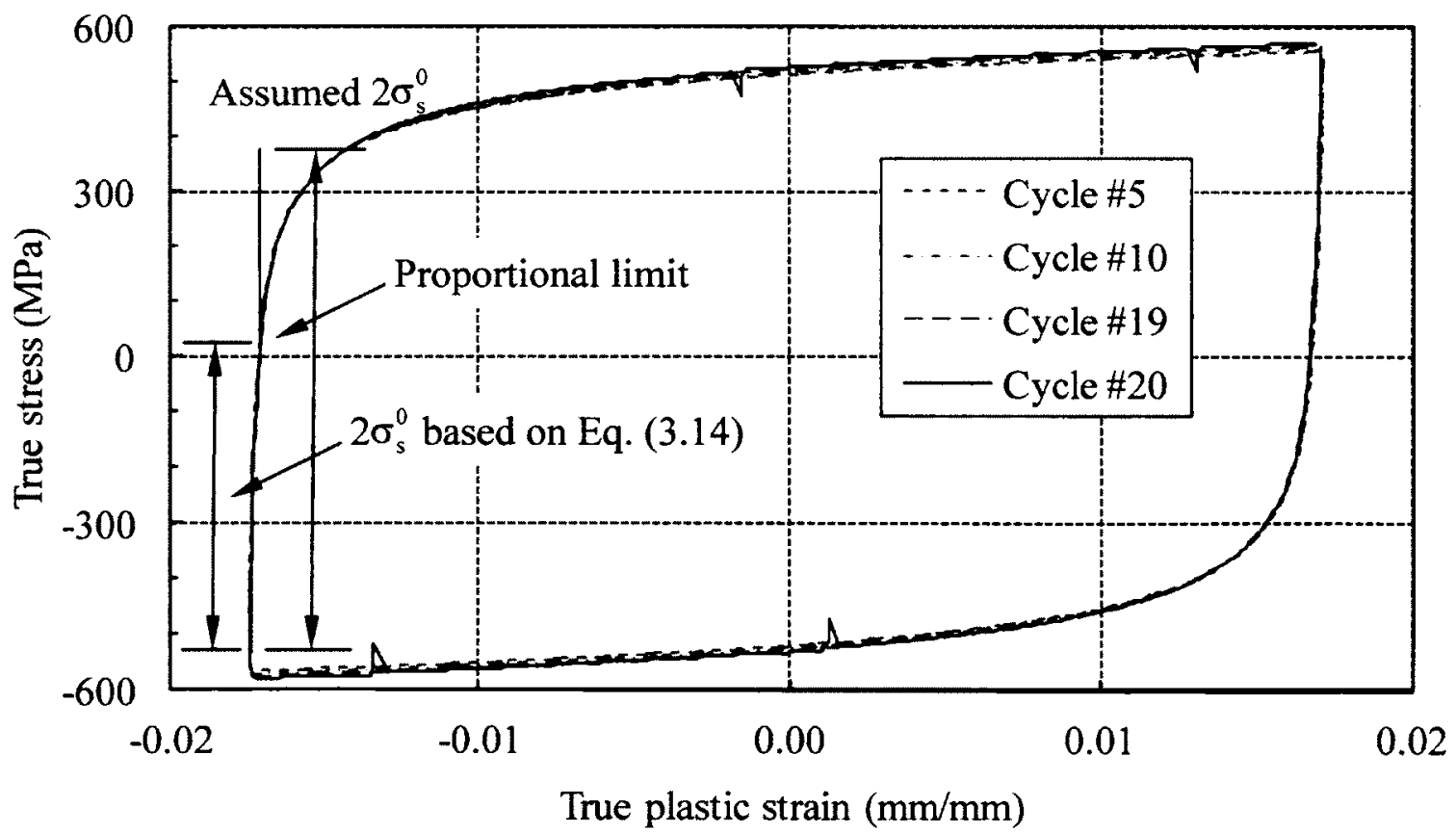

Figure 3.19 The true stress versus true plastic strain curves at different cycles for straight specimen ADC40202 


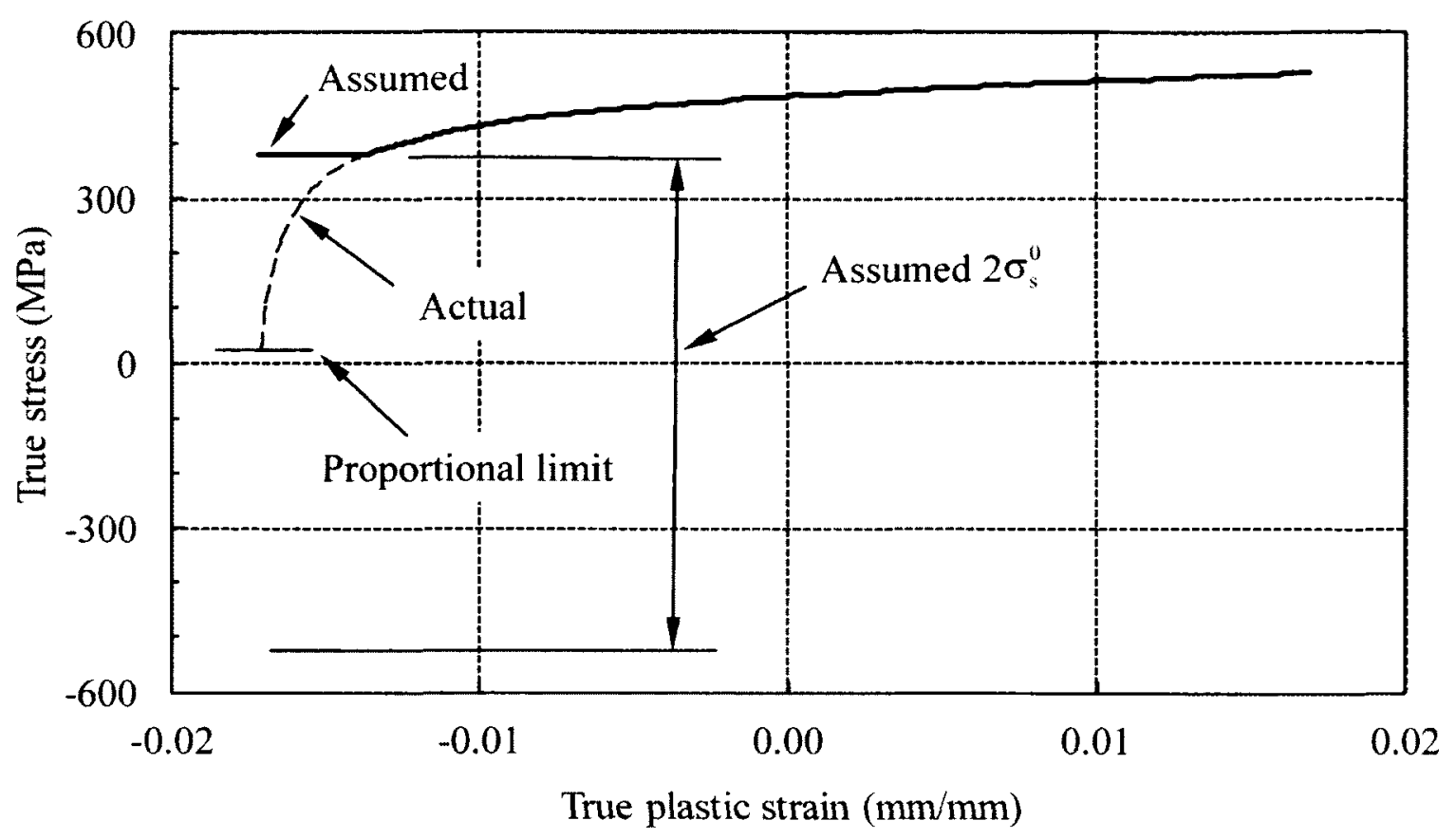

Figure 3.20 Assumed saturated true stress versus true plastic strain curve (static values) for calibration, tension branch of ADC40202 cycle \#20

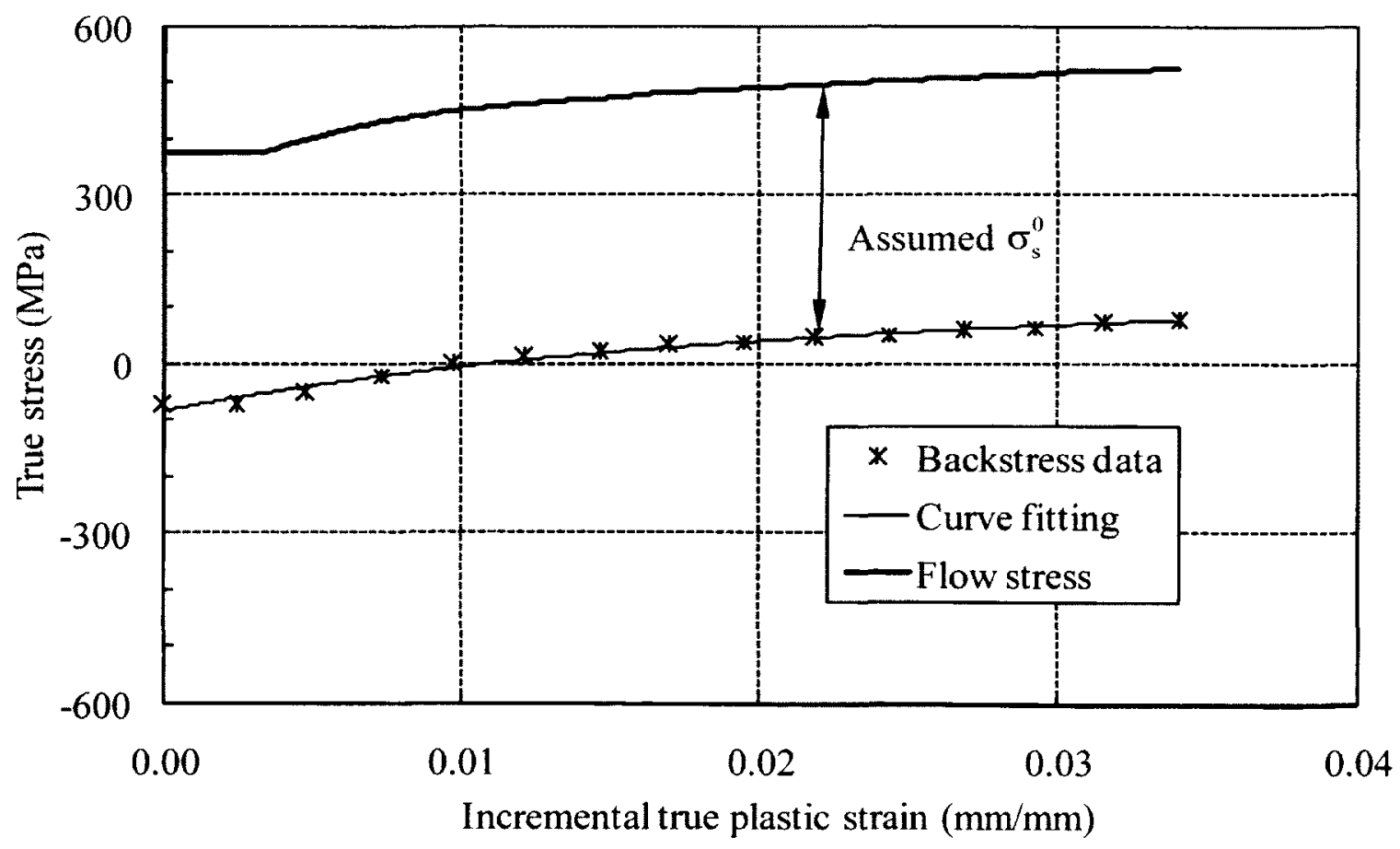

Figure 3.21 Curve fitting of backstress for small strain with $\sigma_{\mathrm{s}}^{0}=450 \mathrm{MPa}$ using test results from the tension branch of ADC40202 cycle \#20 (two terms) 


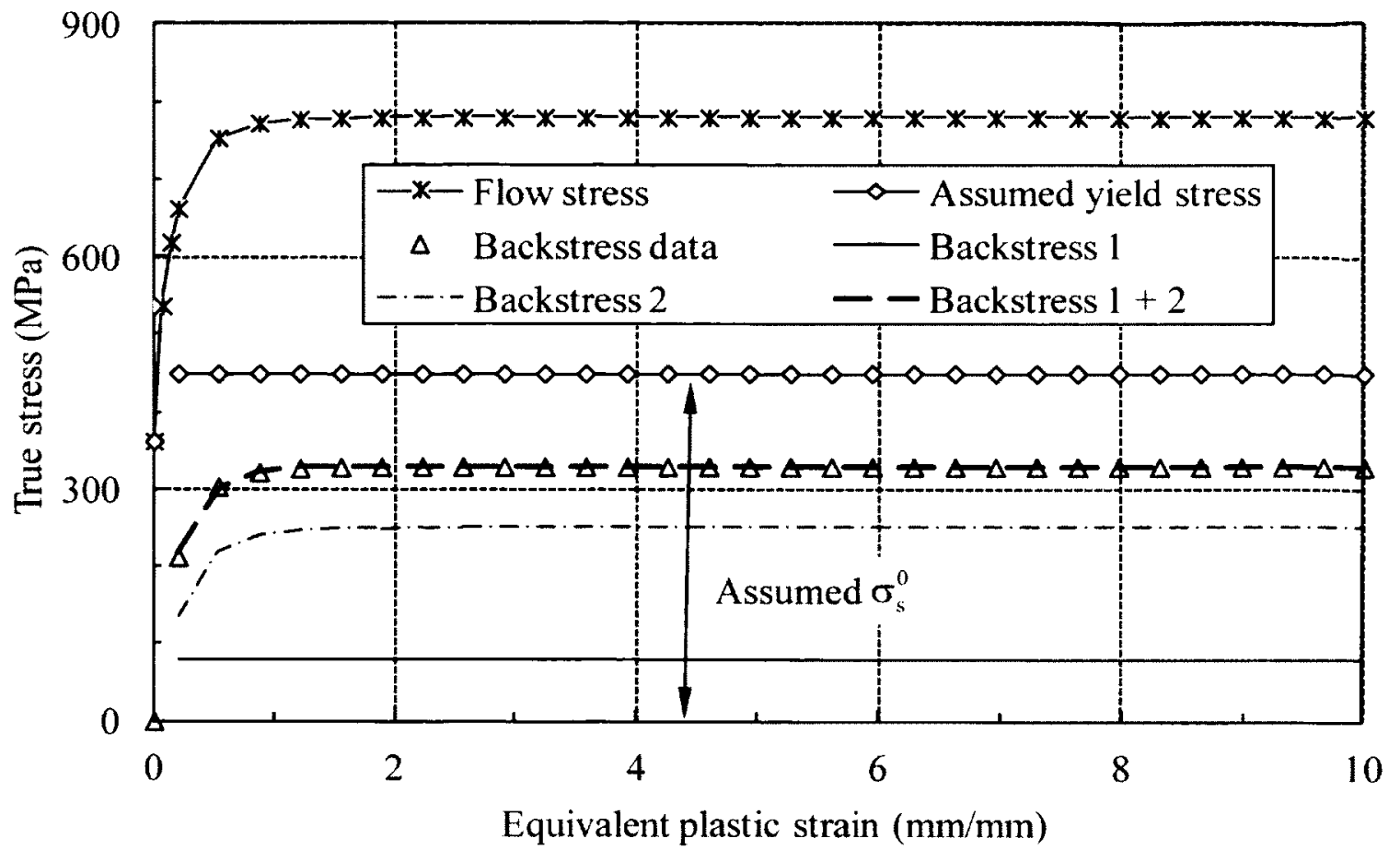

Figure 3.22 Curve fitting of backstress for large strain with $\sigma_{\mathrm{s}}^{0}=450 \mathrm{MPa}$ (two terms)

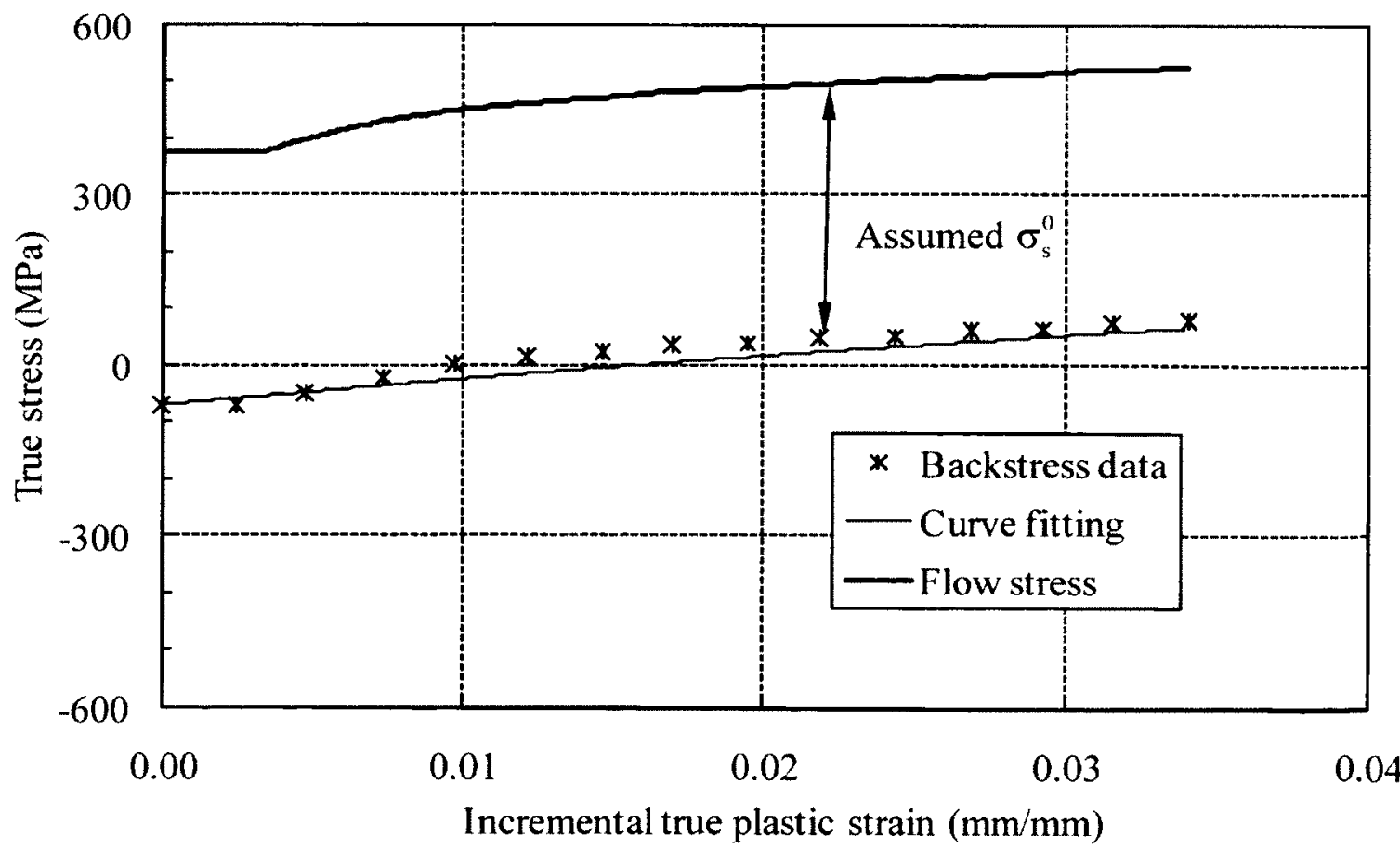

Figure 3.23 Curve fitting of backstress for small strain with $\sigma_{\mathrm{s}}^{0}=450 \mathrm{MPa}$ using test results from the tension branch of ADC 40202 cycle \#20 (one term) 


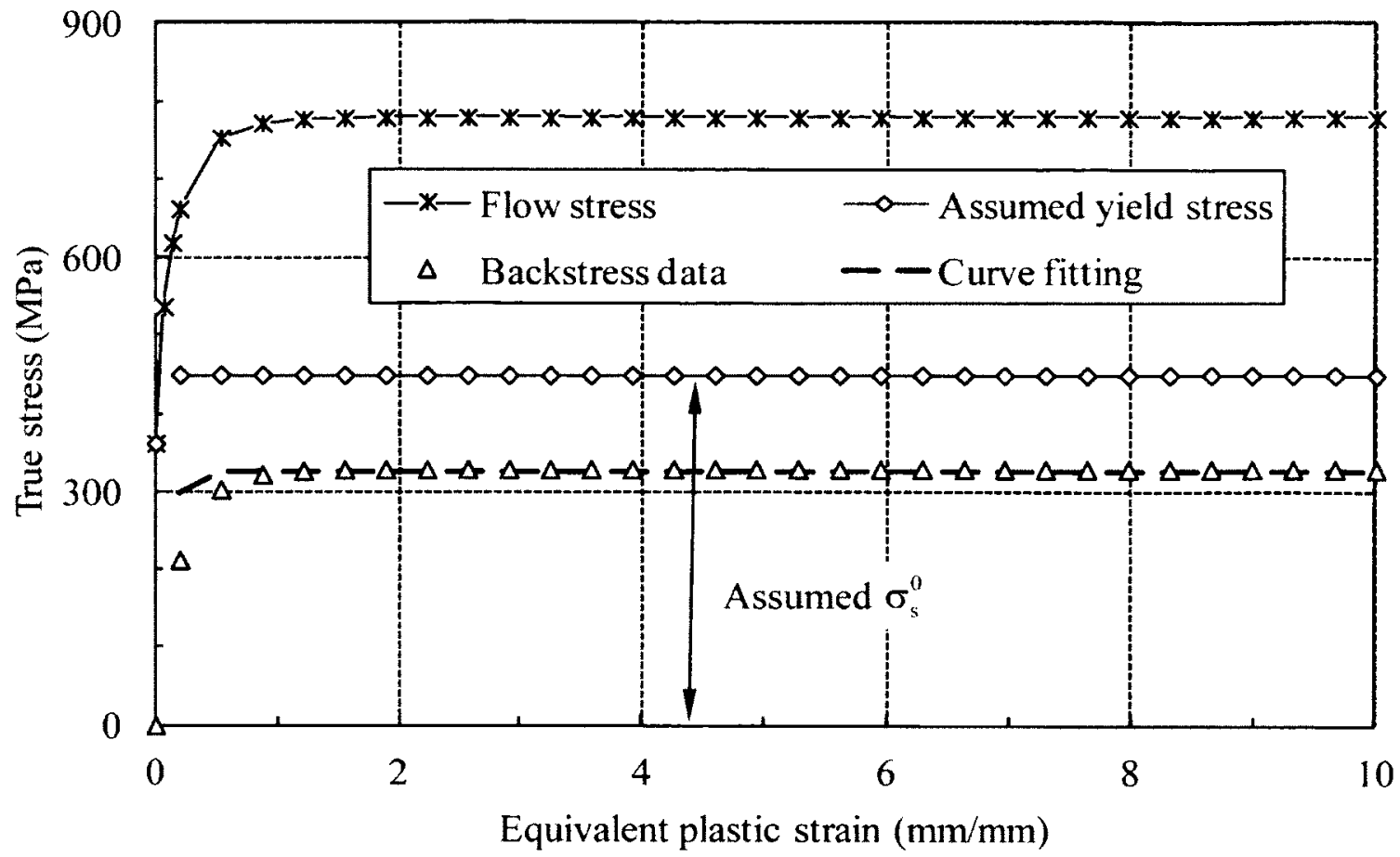

Figure 3.24 Curve fitting of backstress for large strain with $\sigma_{\mathrm{s}}^{0}=450 \mathrm{MPa}$ (one term)

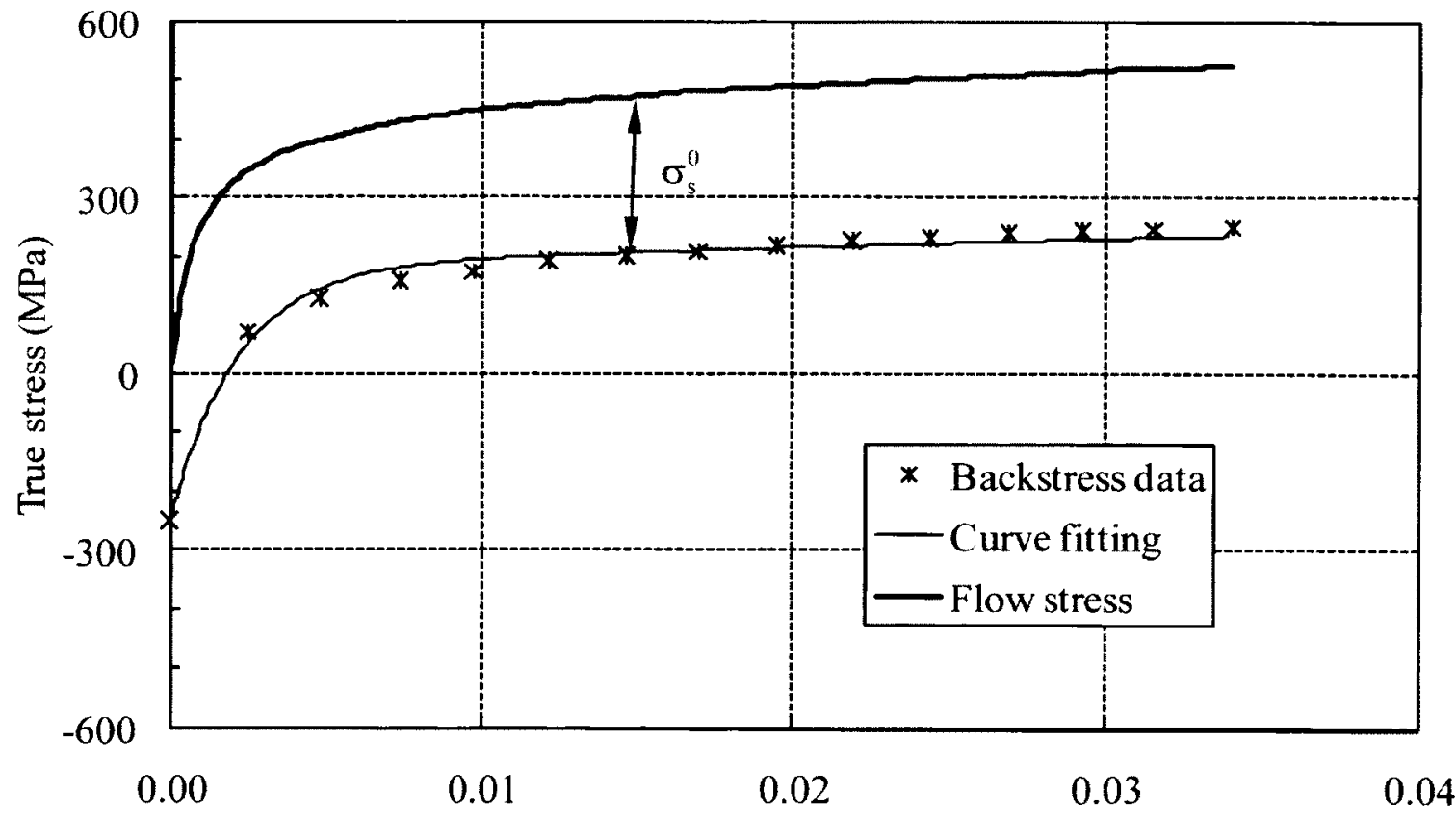

Incremental true plastic strain $(\mathrm{mm} / \mathrm{mm})$

Figure 3.25 Curve fitting of backstress for small strain with $\sigma_{\mathrm{s}}^{0}=273 \mathrm{MPa}$ at proportional limit using test results from the tension branch of ADC40202 cycle \#20 (two terms) 


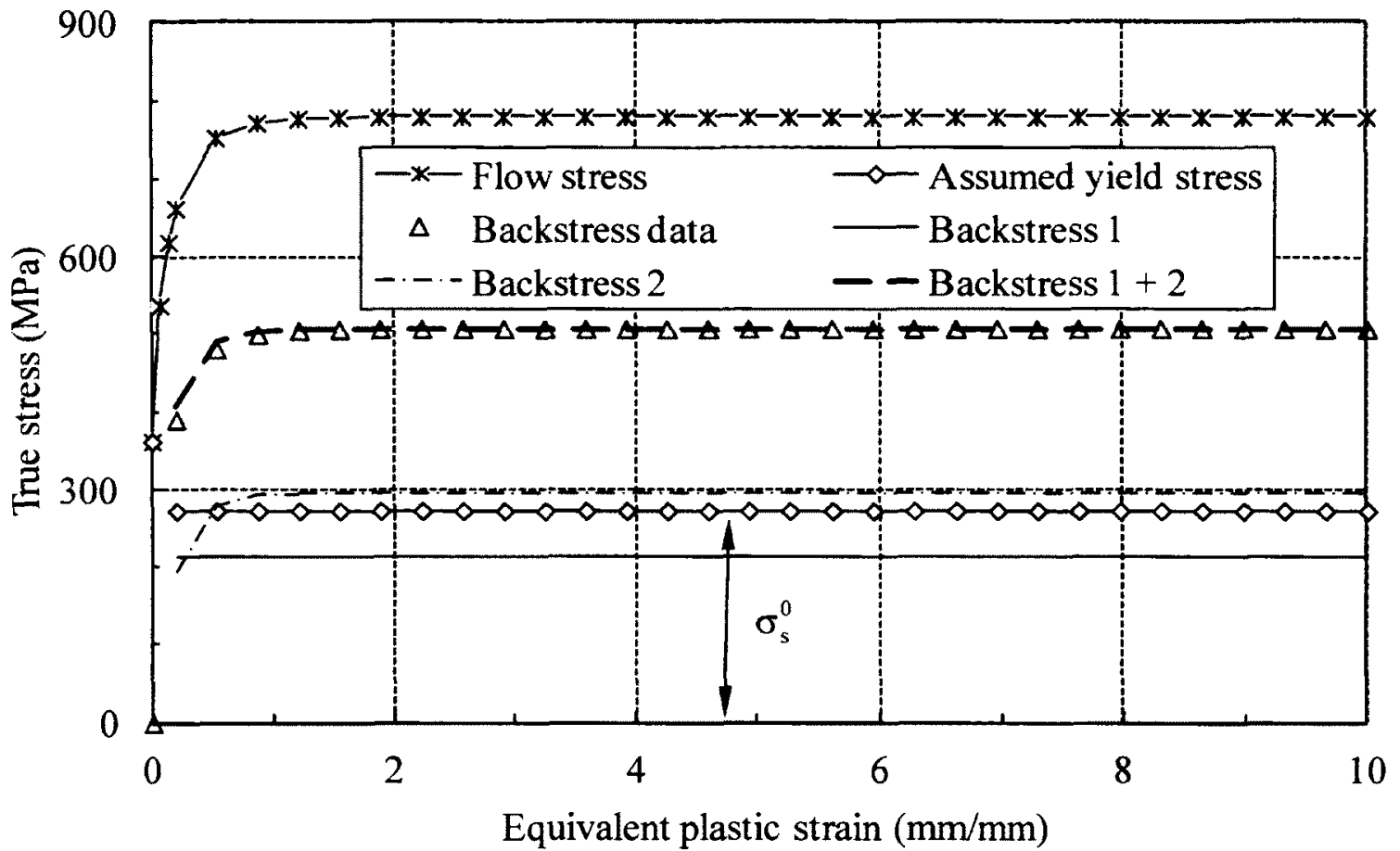

Figure 3.26 Curve fitting of backstress for large strain with $\sigma_{\mathrm{s}}^{0}=273 \mathrm{MPa}$ at proportional limit (two terms)

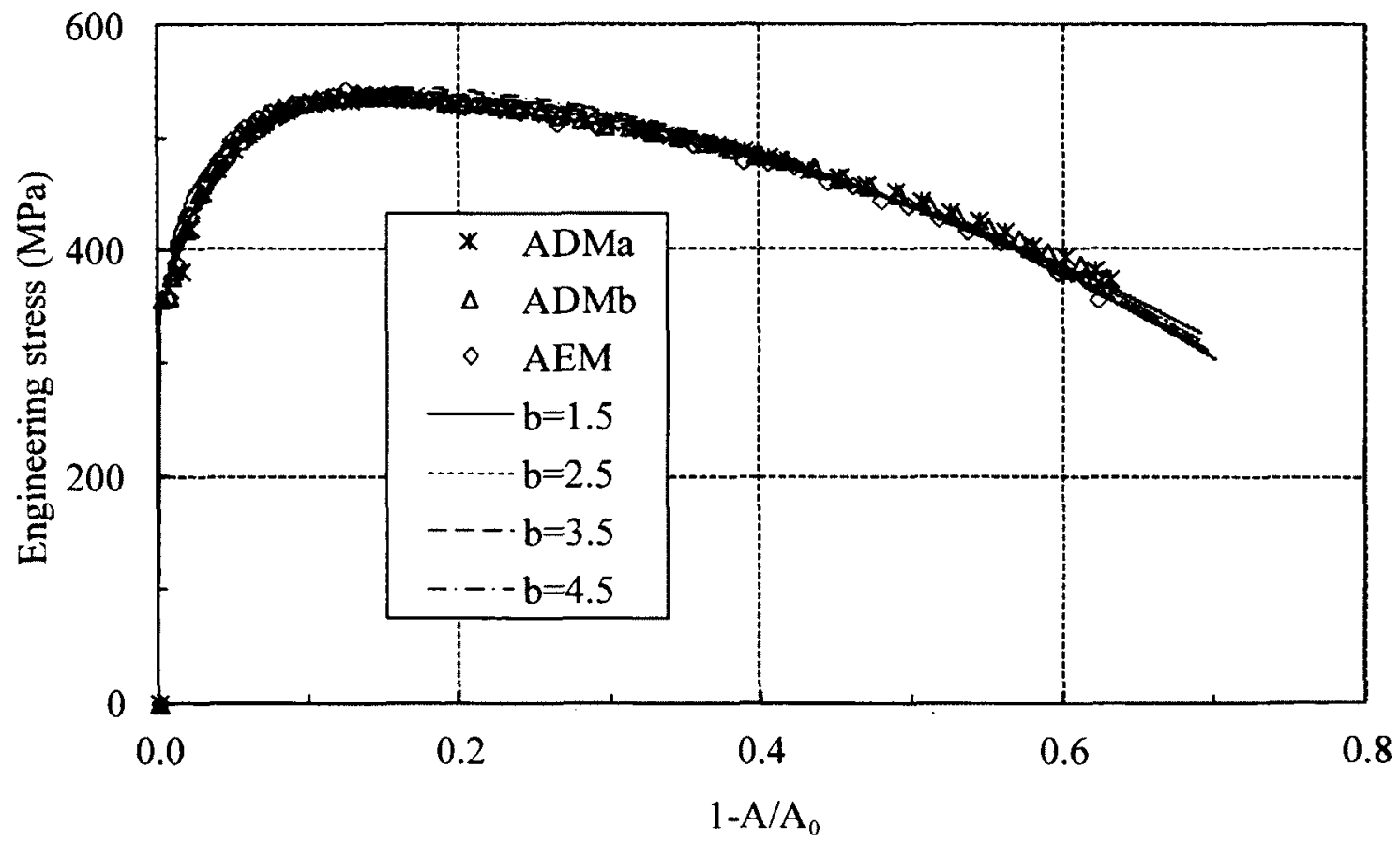

Figure 3.27 Test and predicted engineering stress versus cross-sectional area change curves with different parameter $\mathrm{b}$ for straight specimens of material $\mathrm{A}$ subjected to monotonic tension loading (two terms) 


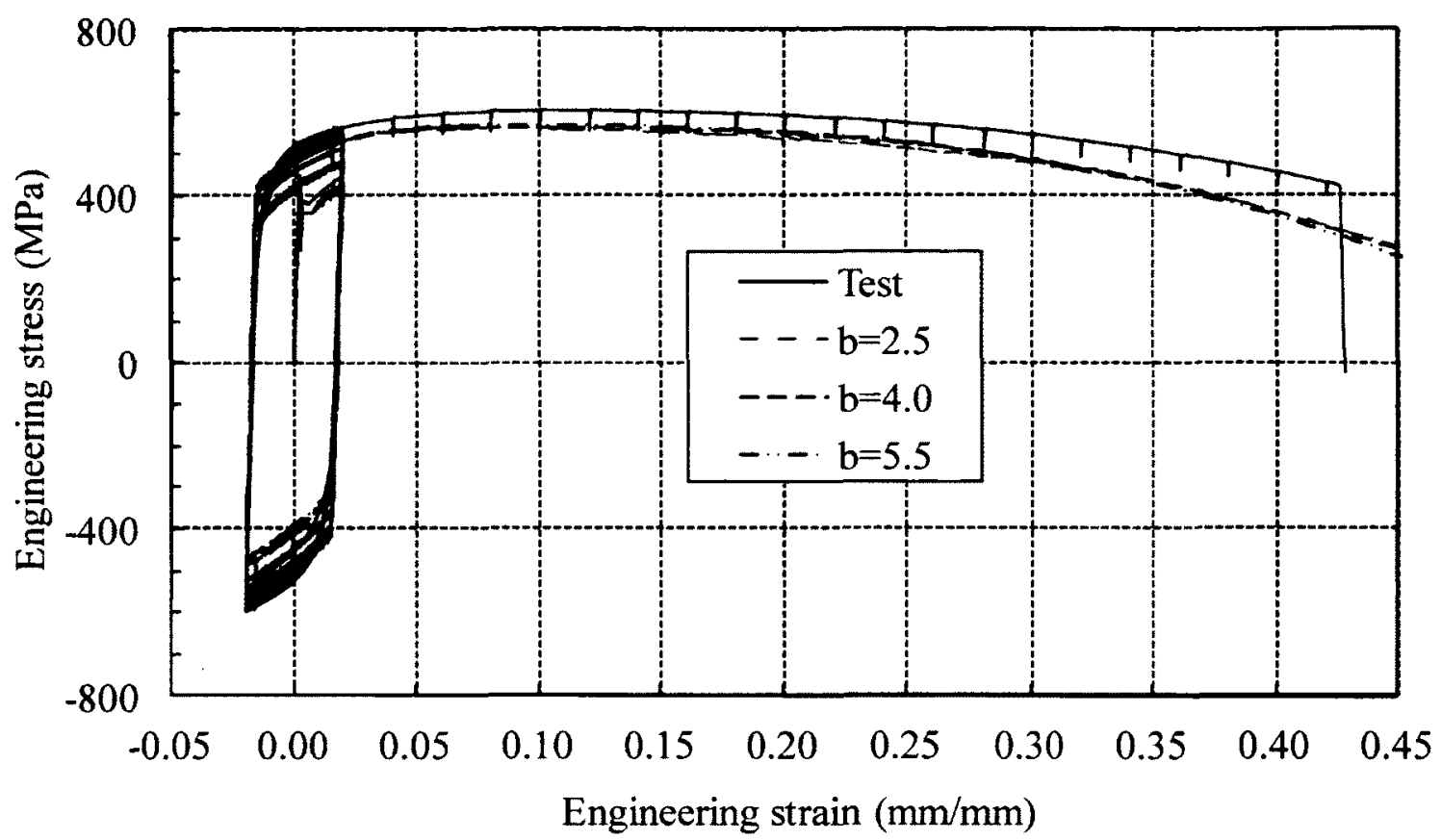

Figure 3.28 Test and predicted engineering stress versus engineering strain curves with different parameter $b$ for straight specimen ADC40202 (two terms)

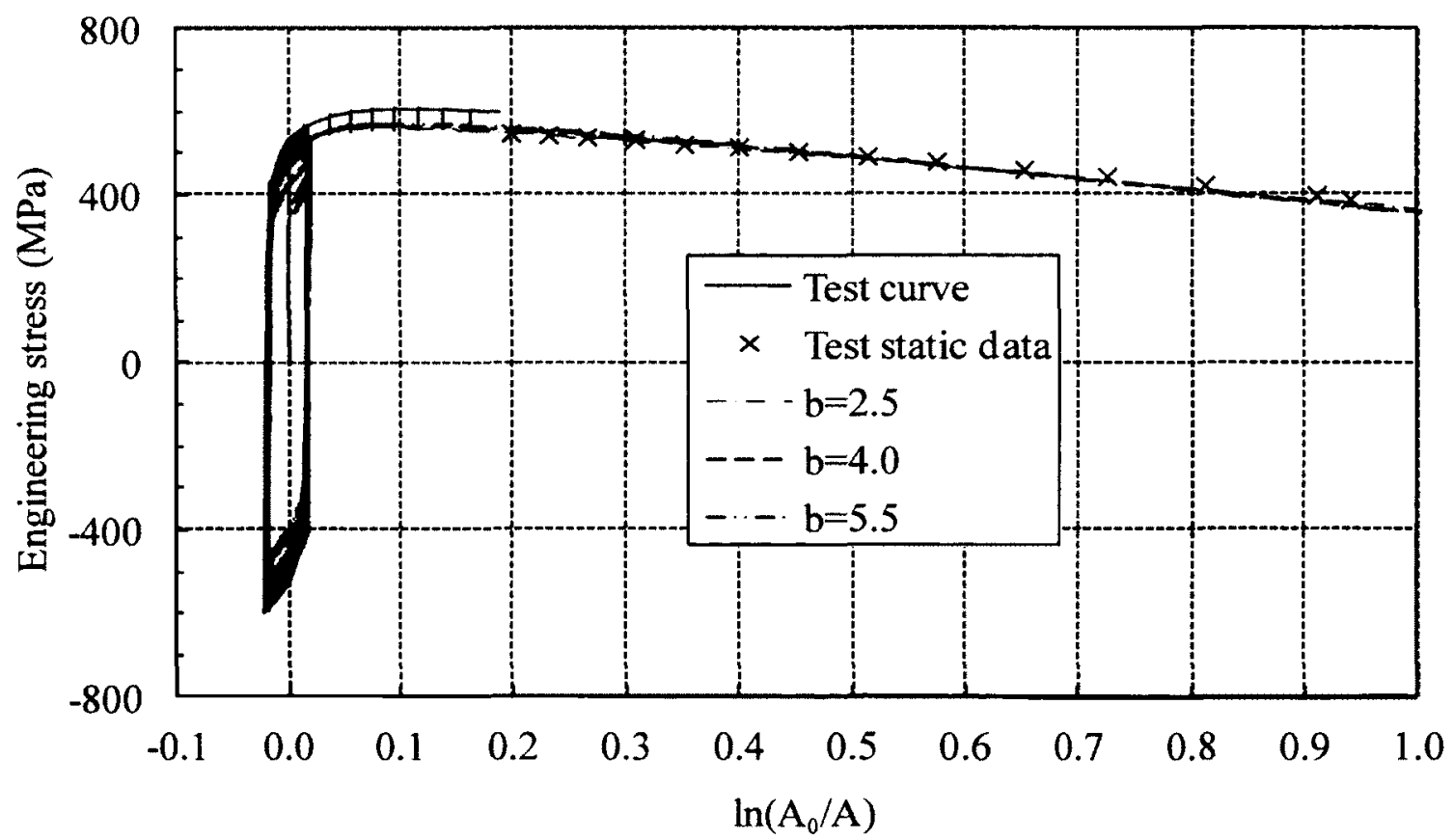

Figure 3.29 Test and predicted engineering stress versus cross-sectional area change curves with different parameter $b$ for straight specimen ADC40202 (two terms) 


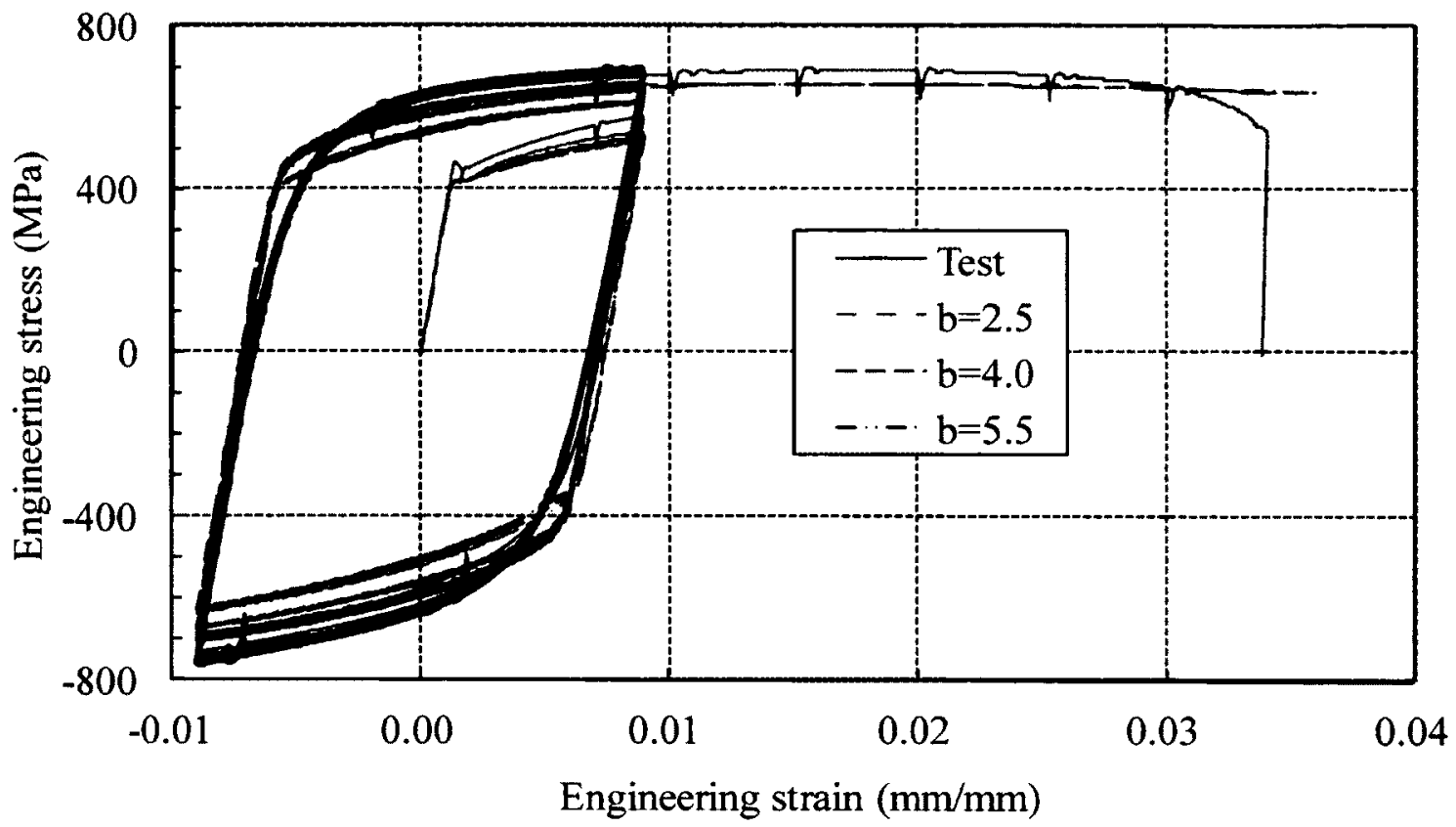

Figure 3.30 Test and predicted engineering stress versus engineering strain curves with different parameter $b$ for notched specimen ABC70404 (two terms)

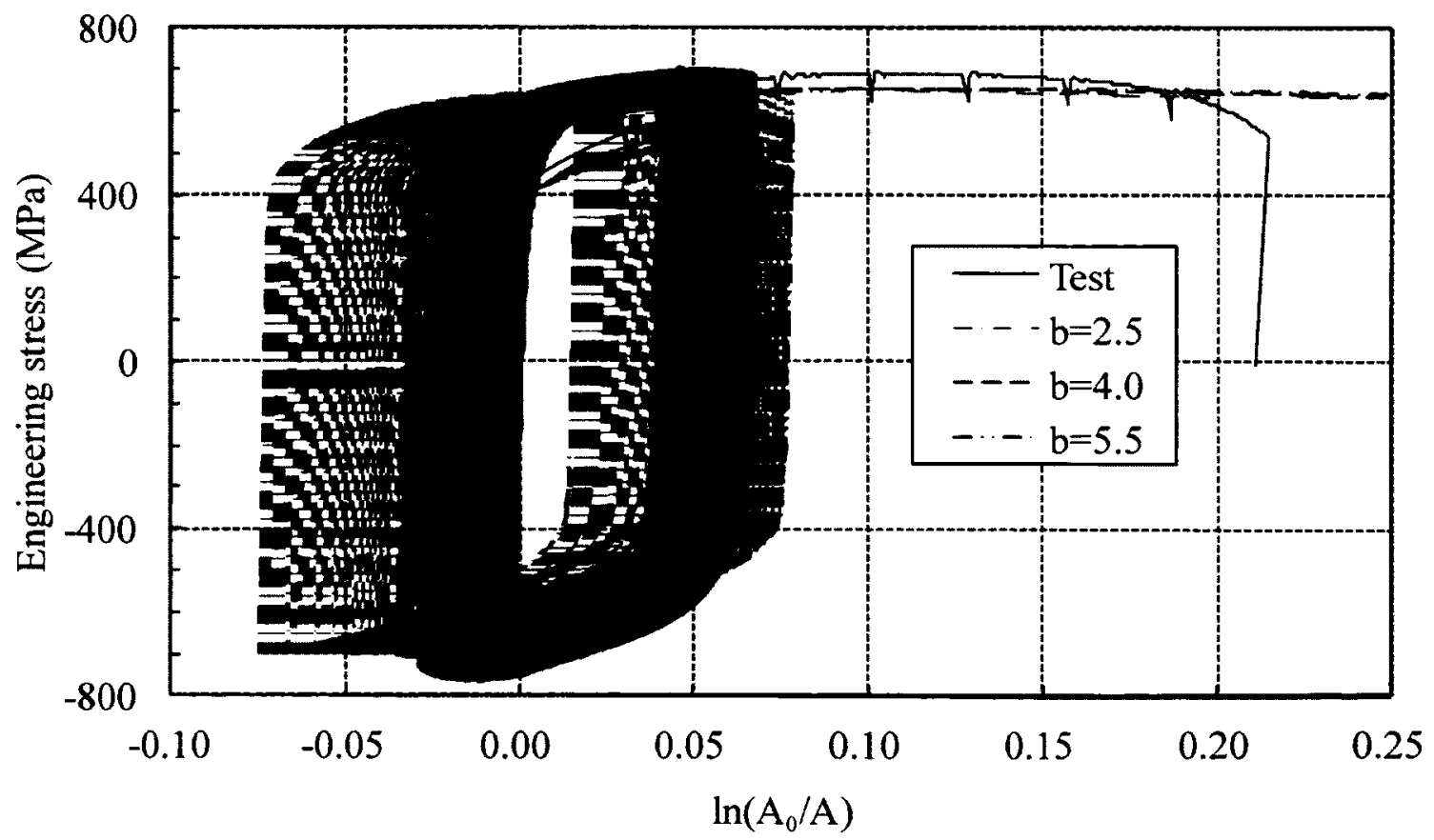

Figure 3.31 Test and predicted engineering stress versus cross-sectional area change curves with different parameter $b$ for notched specimen ABC70404 (two terms) 


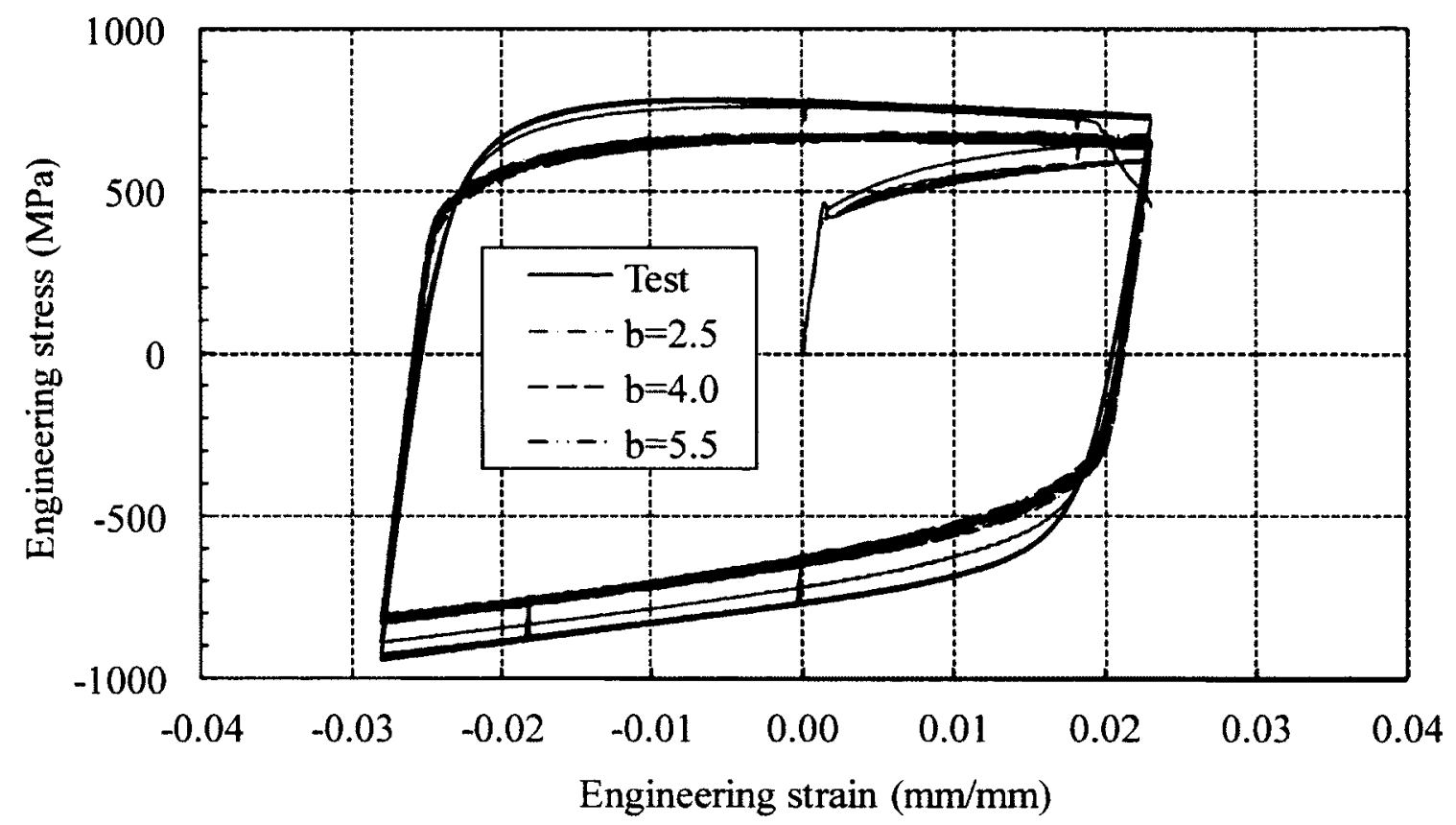

Figure 3.32 Test and predicted engineering stress versus engineering strain curves with different parameter $b$ for notched specimen ABCF1212 (two terms)

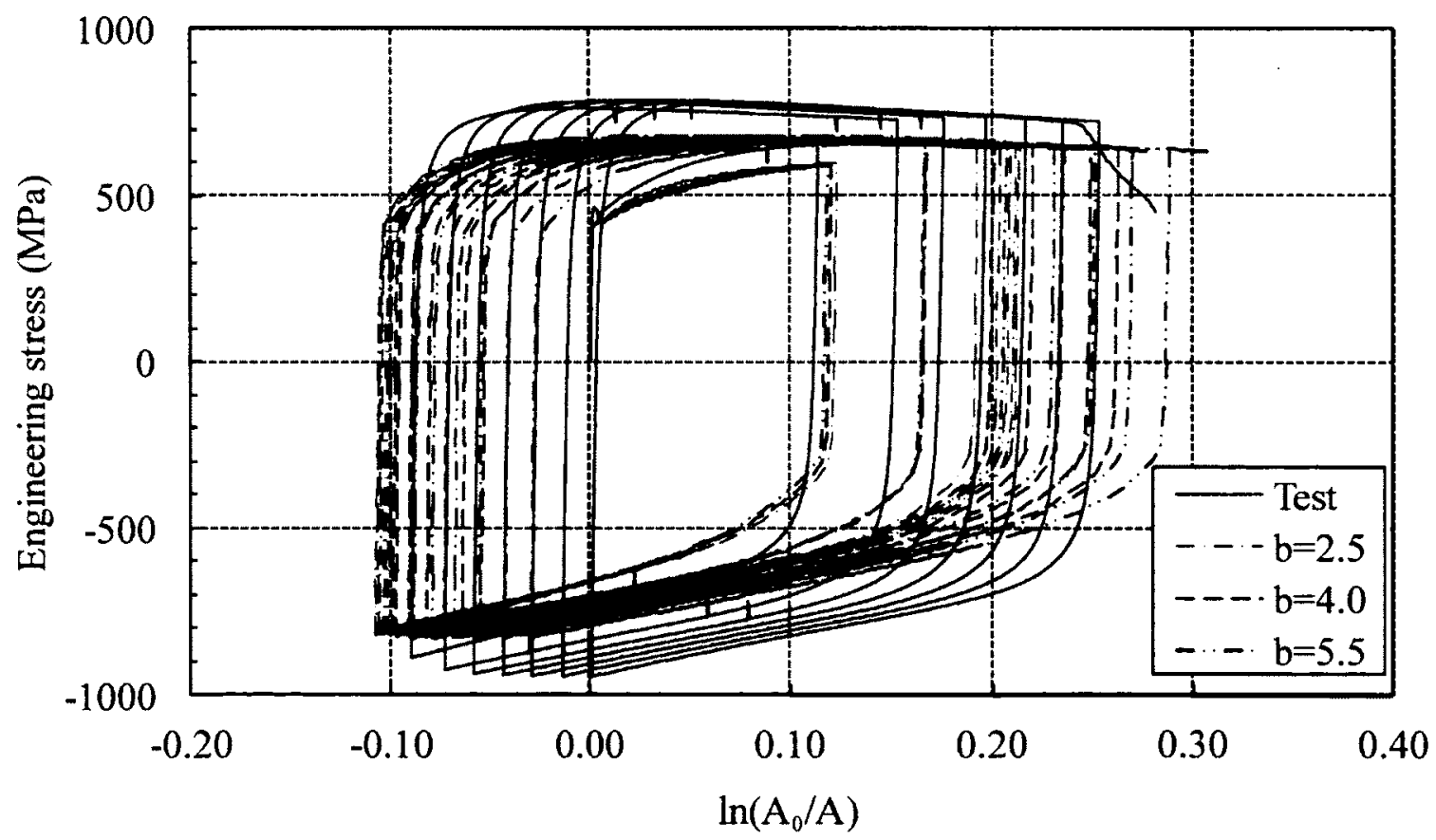

Figure 3.33 Test and predicted engineering stress versus cross-sectional area change curves with different parameter $b$ for notched specimen ABCF1212 (two terms) 


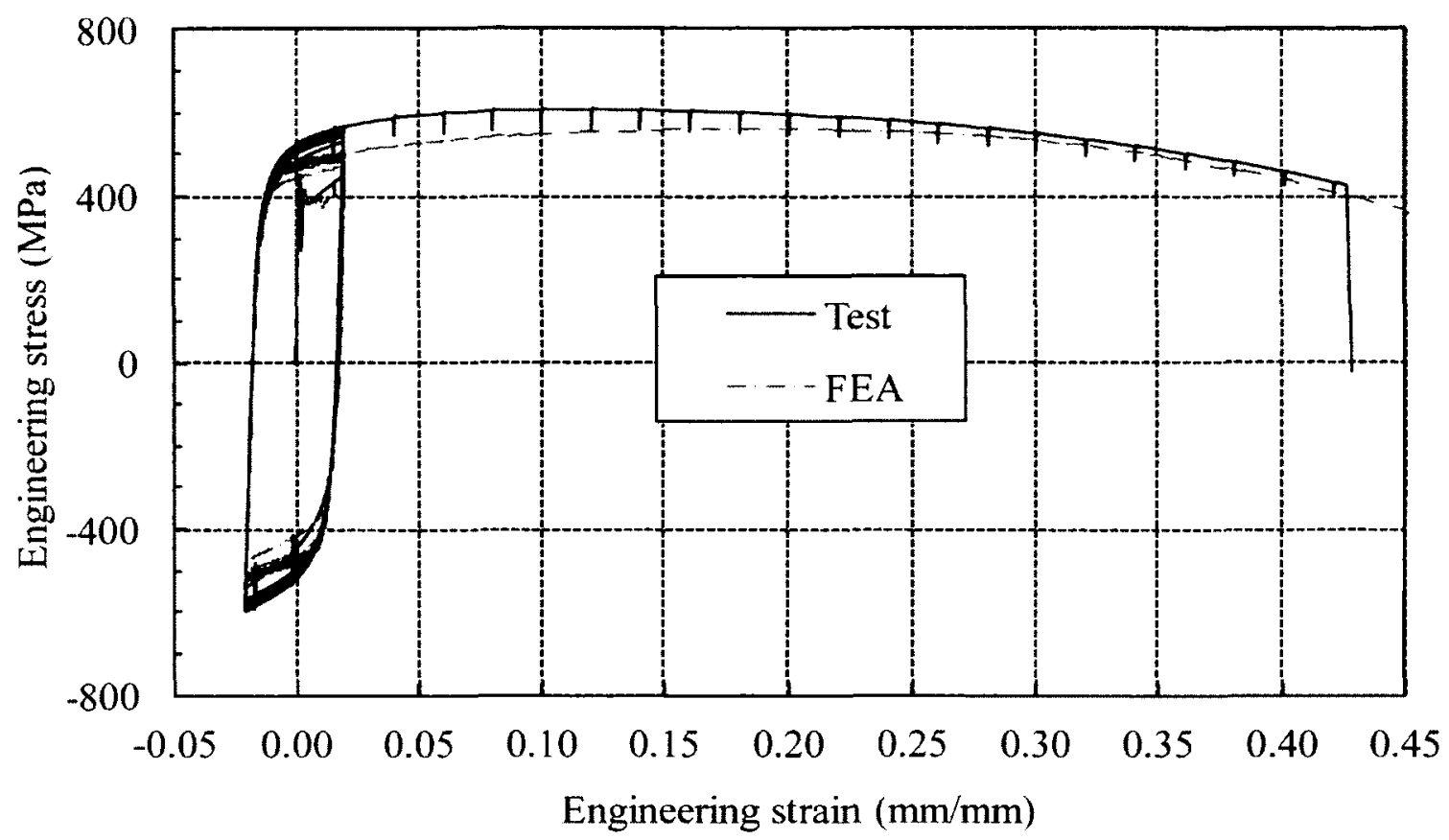

Figure 3.34 Test and predicted engineering stress versus engineering strain curves using $\sigma_{\mathrm{s}}^{0}=273 \mathrm{MPa}$ and $\mathrm{b}=4.5$ for straight specimen ADC40202 (two terms)

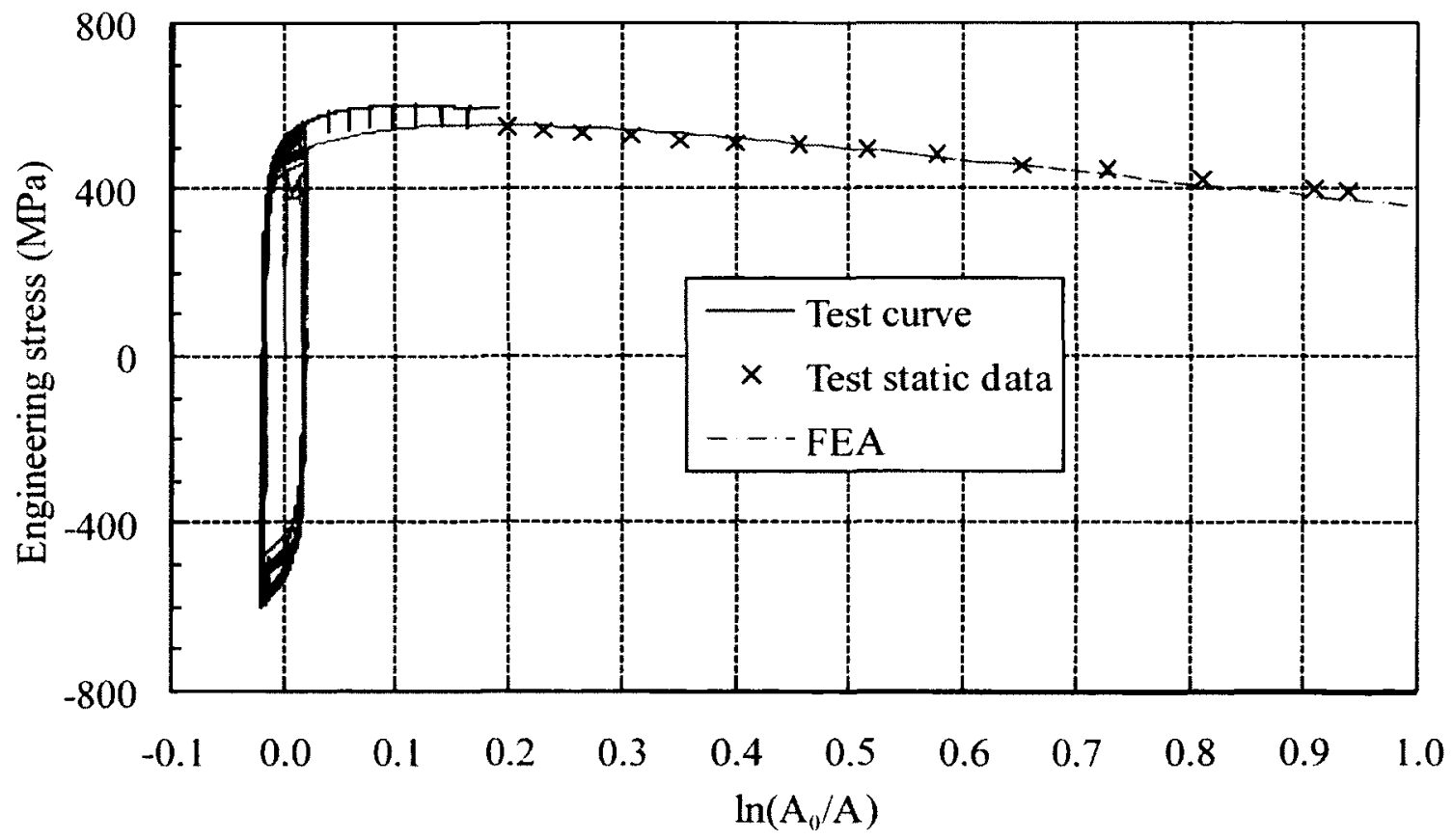

Figure 3.35 Test and predicted engineering stress versus cross-sectional area change curves using $\sigma_{\mathrm{s}}^{0}=273 \mathrm{MPa}$ and $\mathrm{b}=4.5$ for straight specimen ADC40202 (two terms) 


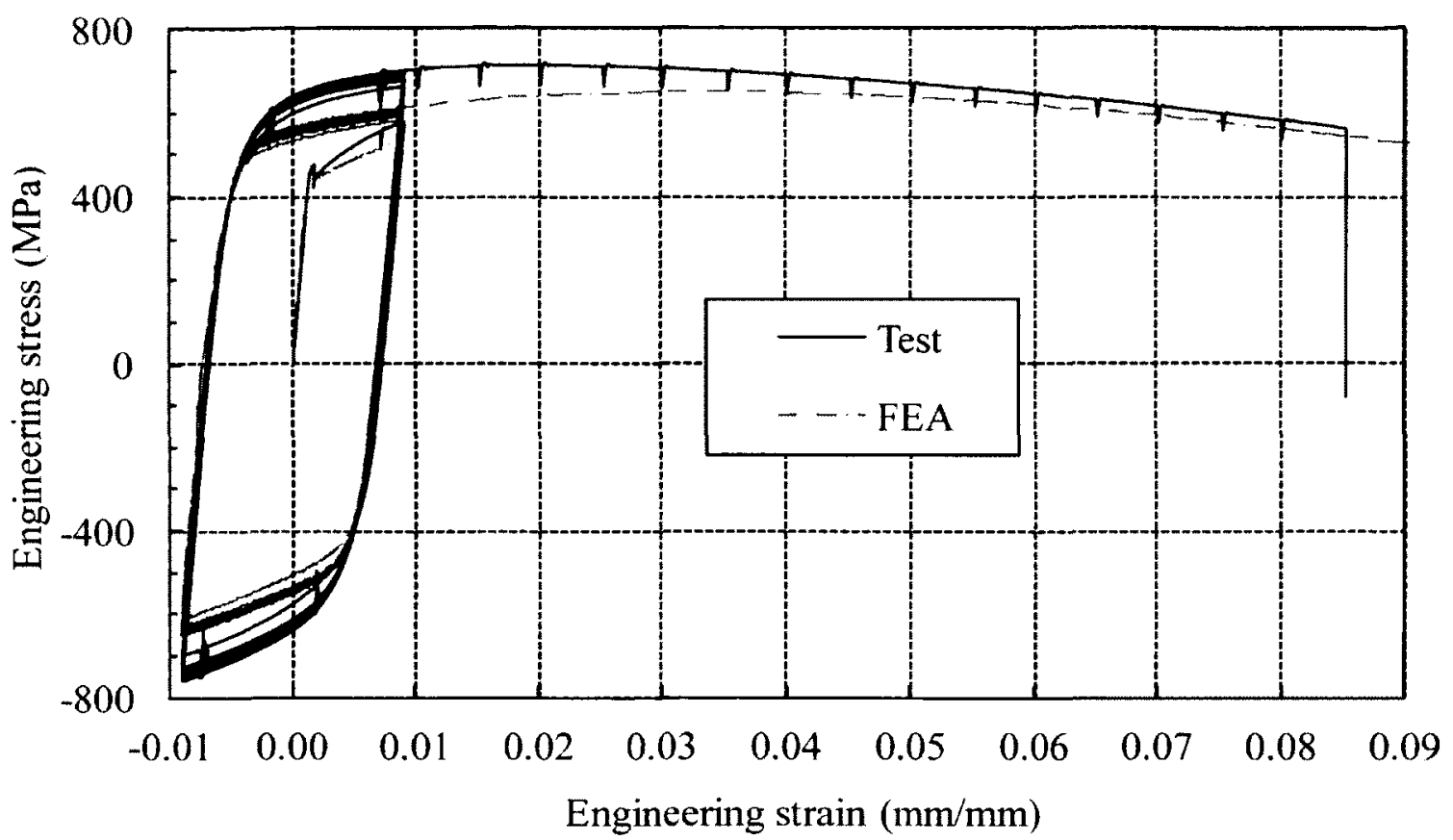

Figure 3.36 Test and predicted engineering stress versus engineering strain curves using $\sigma_{\mathrm{s}}^{0}=273 \mathrm{MPa}$ and $\mathrm{b}=4.5$ for notched specimen ABC20404 (two terms)

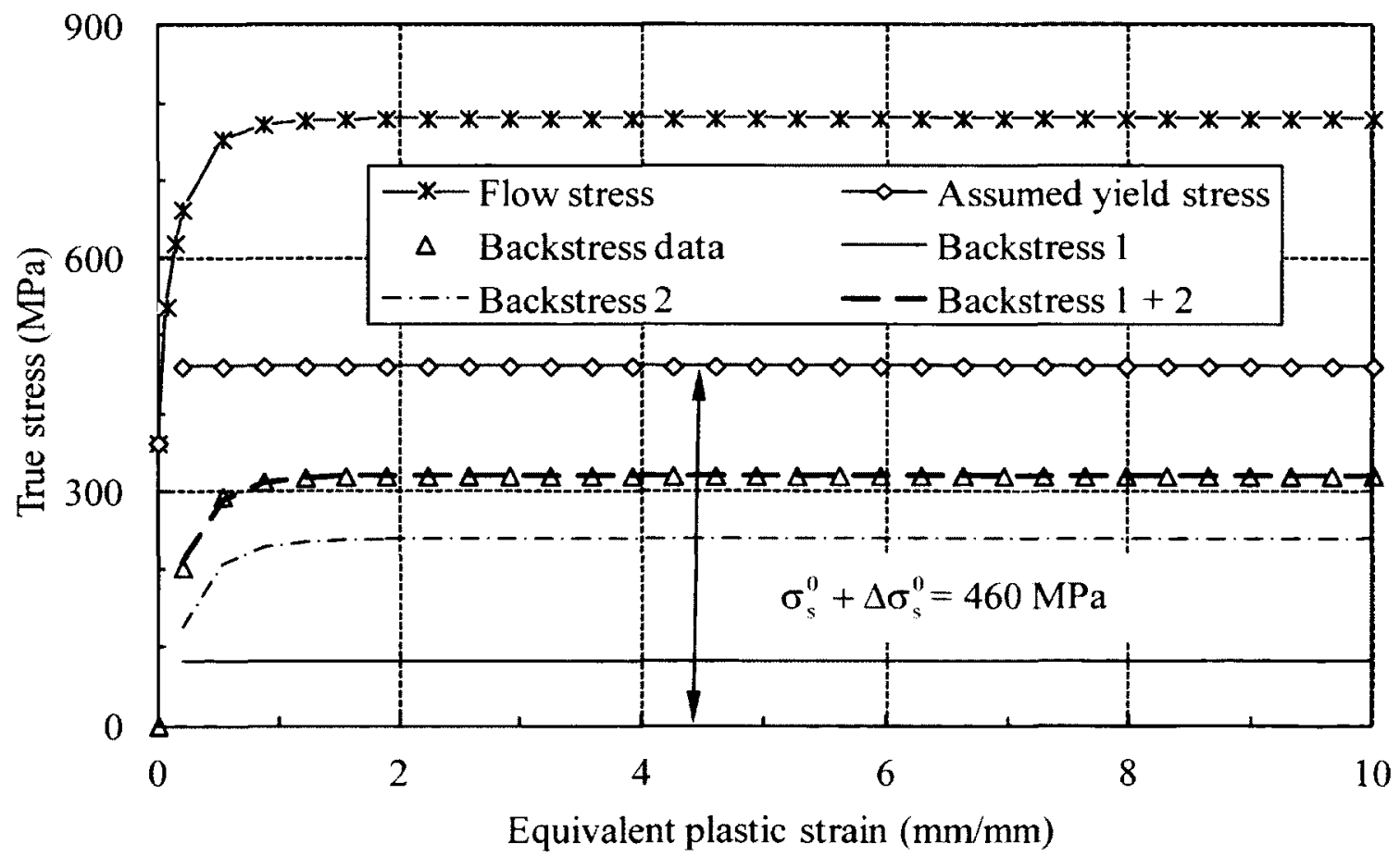

Figure 3.37 Curve fitting of backstress for large strain with $\sigma_{s}^{0}=450 \mathrm{MPa}, \Delta \sigma_{\mathrm{s}}^{0}=10$ MPa (two terms) 


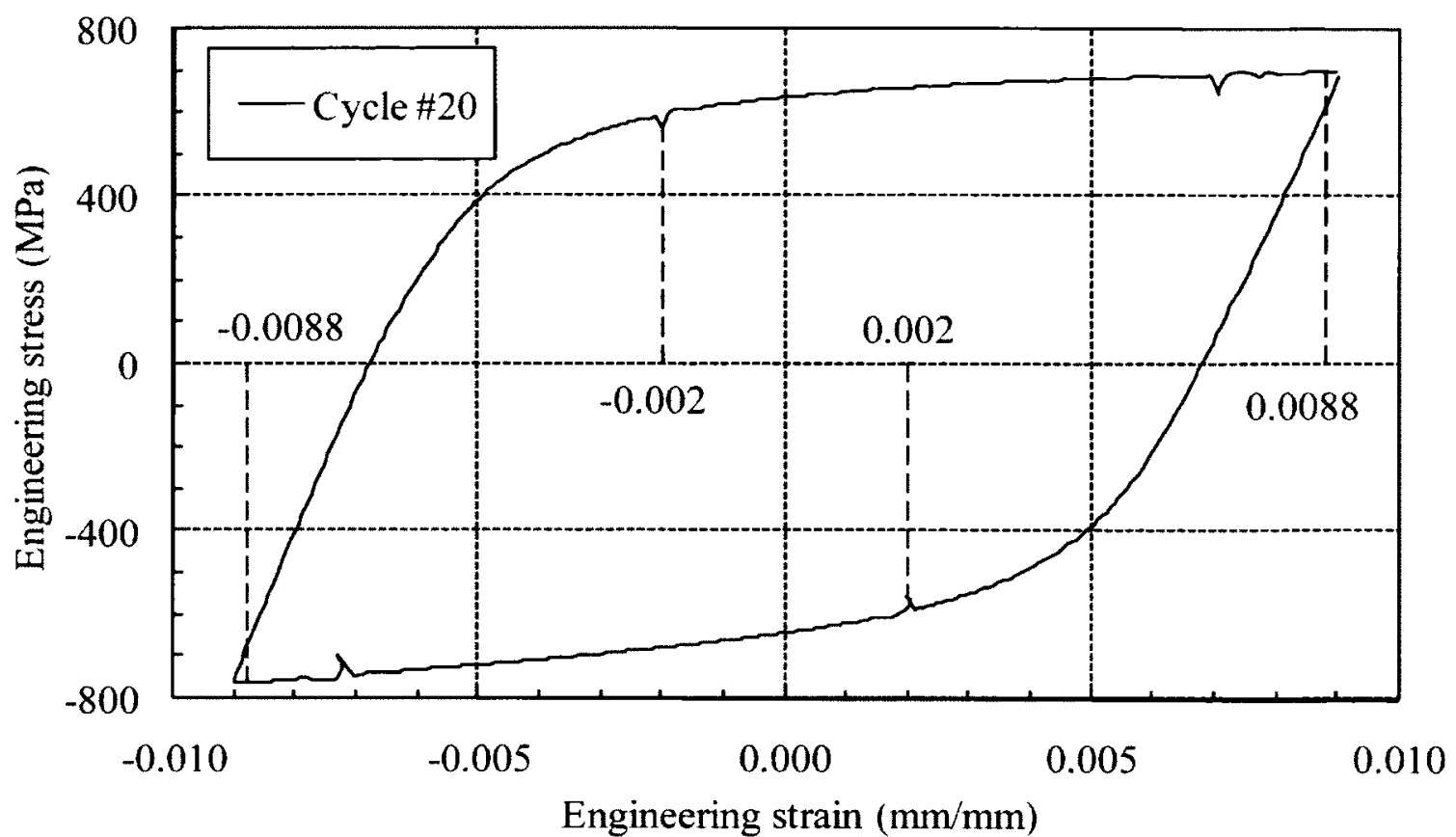

Figure 3.38 Locations of engineering strains where errors are calculated for notched specimen ABC20404, shown for cycle \#20

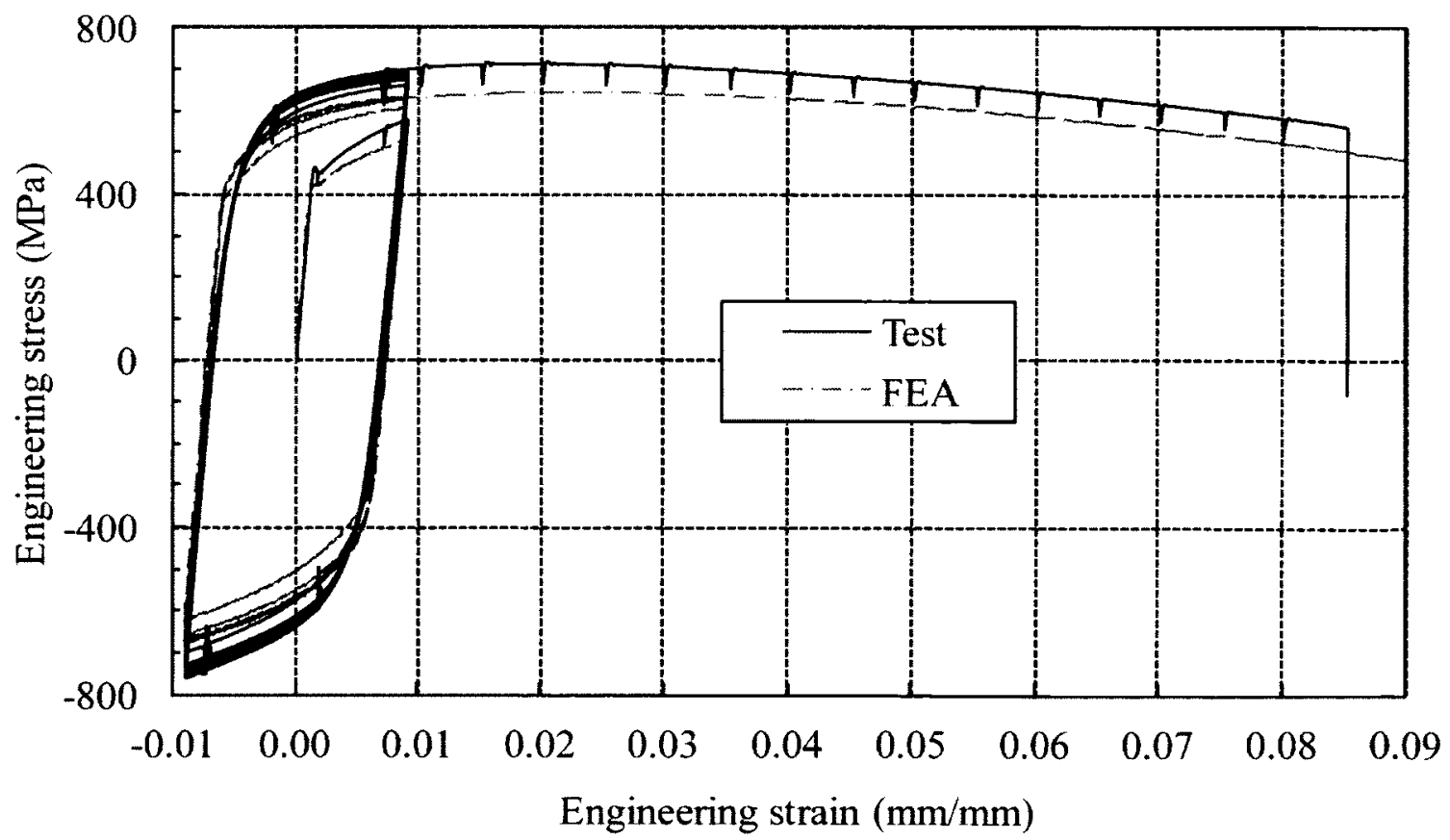

Figure 3.39 Test and predicted engineering stress versus engineering strain curves using $\sigma_{\mathrm{s}}^{0}=450 \mathrm{MPa}$ and $\Delta \sigma_{\mathrm{s}}^{0}=0$ for notched specimen $\mathrm{ABC} 20404$ (two terms) 


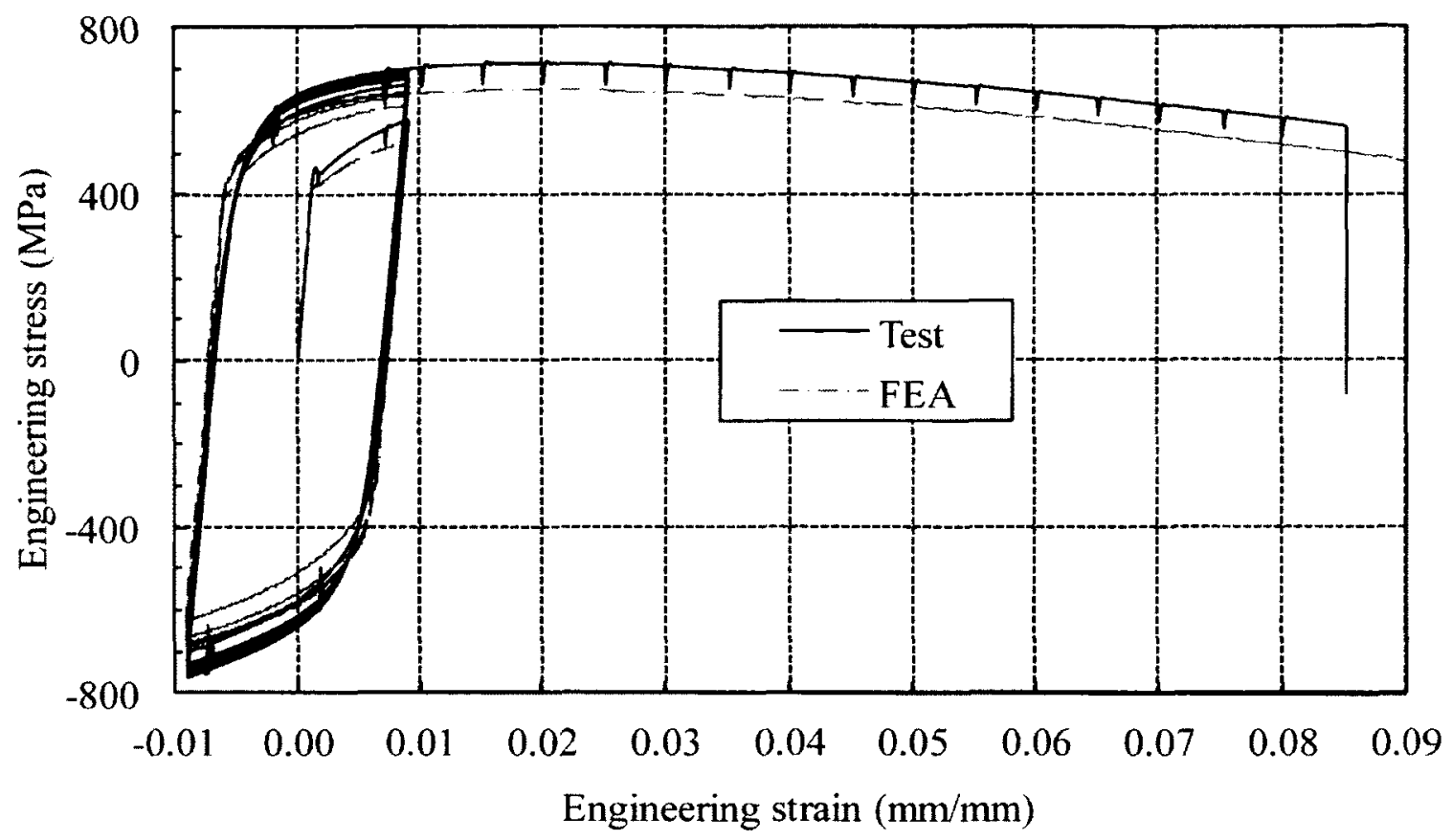

Figure 3.40 Test and predicted engineering stress versus engineering strain curves using $\sigma_{\mathrm{s}}^{0}=450 \mathrm{MPa}$ and $\Delta \sigma_{\mathrm{s}}^{0}=10 \mathrm{MPa}$ for notched specimen ABC20404 (two terms)

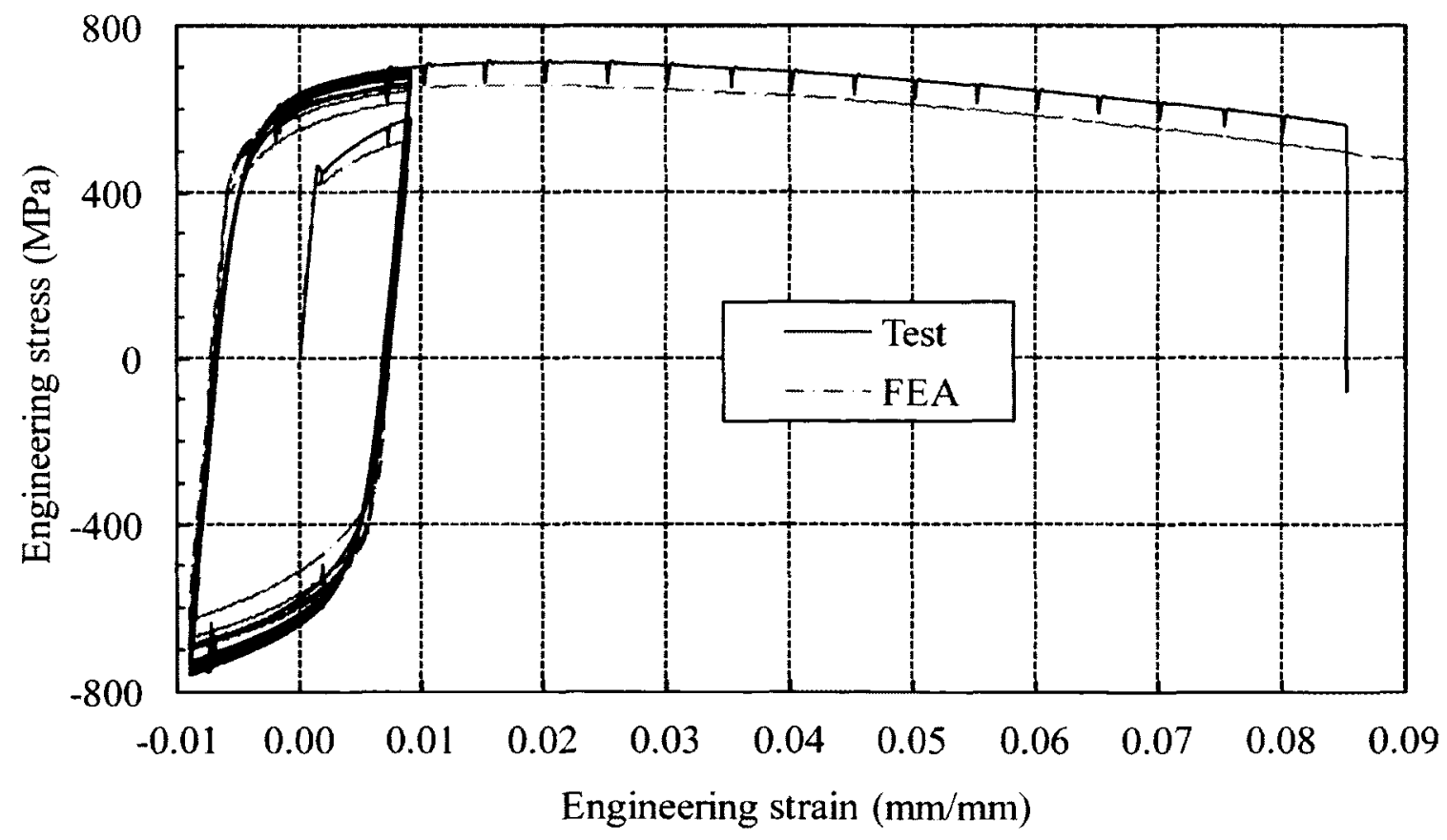

Figure 3.41 Test and predicted engineering stress versus engineering strain curves using $\sigma_{\mathrm{s}}^{0}=450 \mathrm{MPa}$ and $\Delta \sigma_{\mathrm{s}}^{0}=20 \mathrm{MPa}$ for notched specimen $\mathrm{ABC} 20404$ (two terms) 


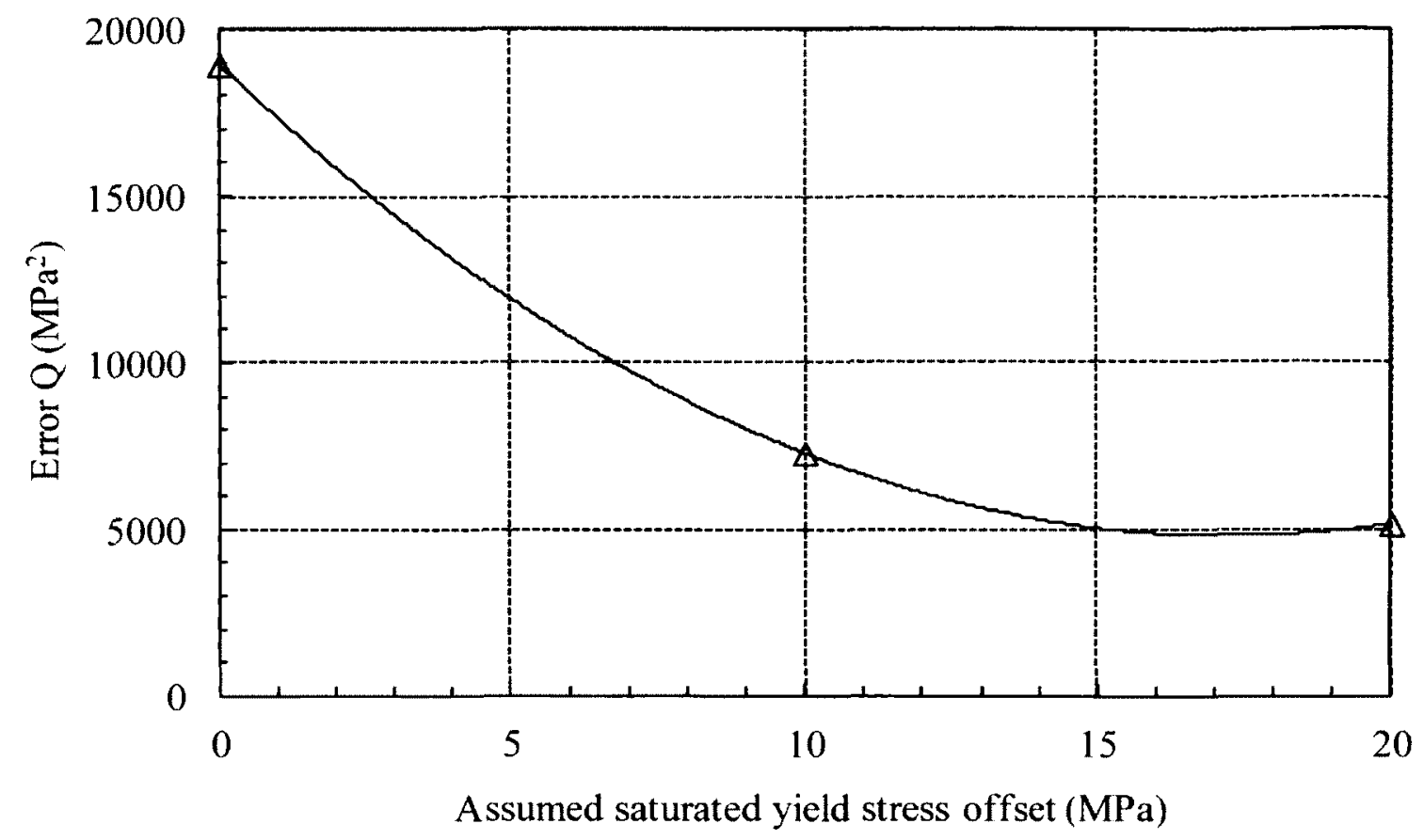

Figure 3.42 Error versus assumed saturated yield stress offset $\left(\Delta \sigma_{\mathrm{s}}^{0}\right)$ for $\sigma_{\mathrm{s}}^{0}=$ $450 \mathrm{MPa}$ (two terms)

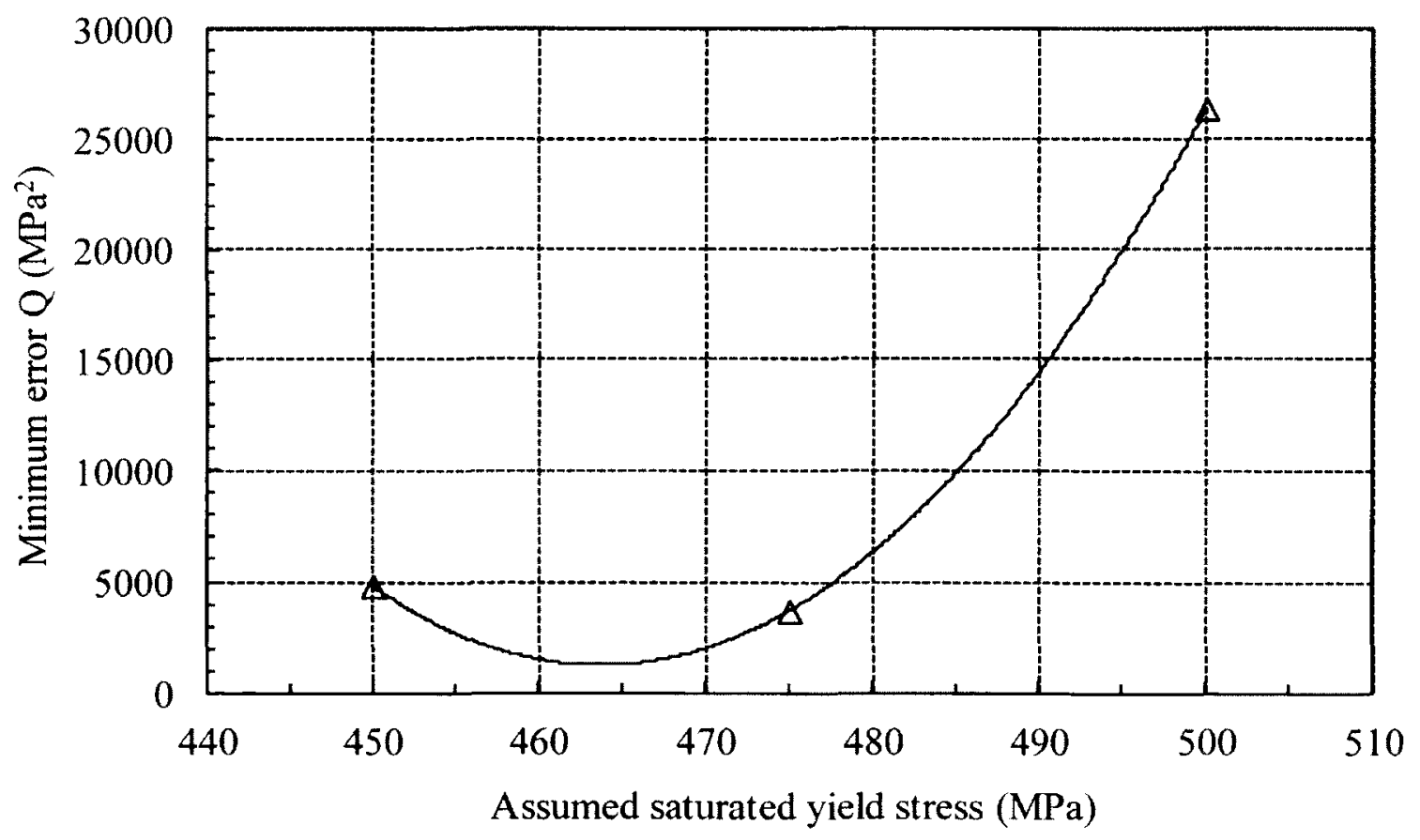

Figure 3.43 Minimum error versus assumed saturated yield stress ( $\left.\sigma_{\mathrm{s}}^{0}\right)$ (two terms) 


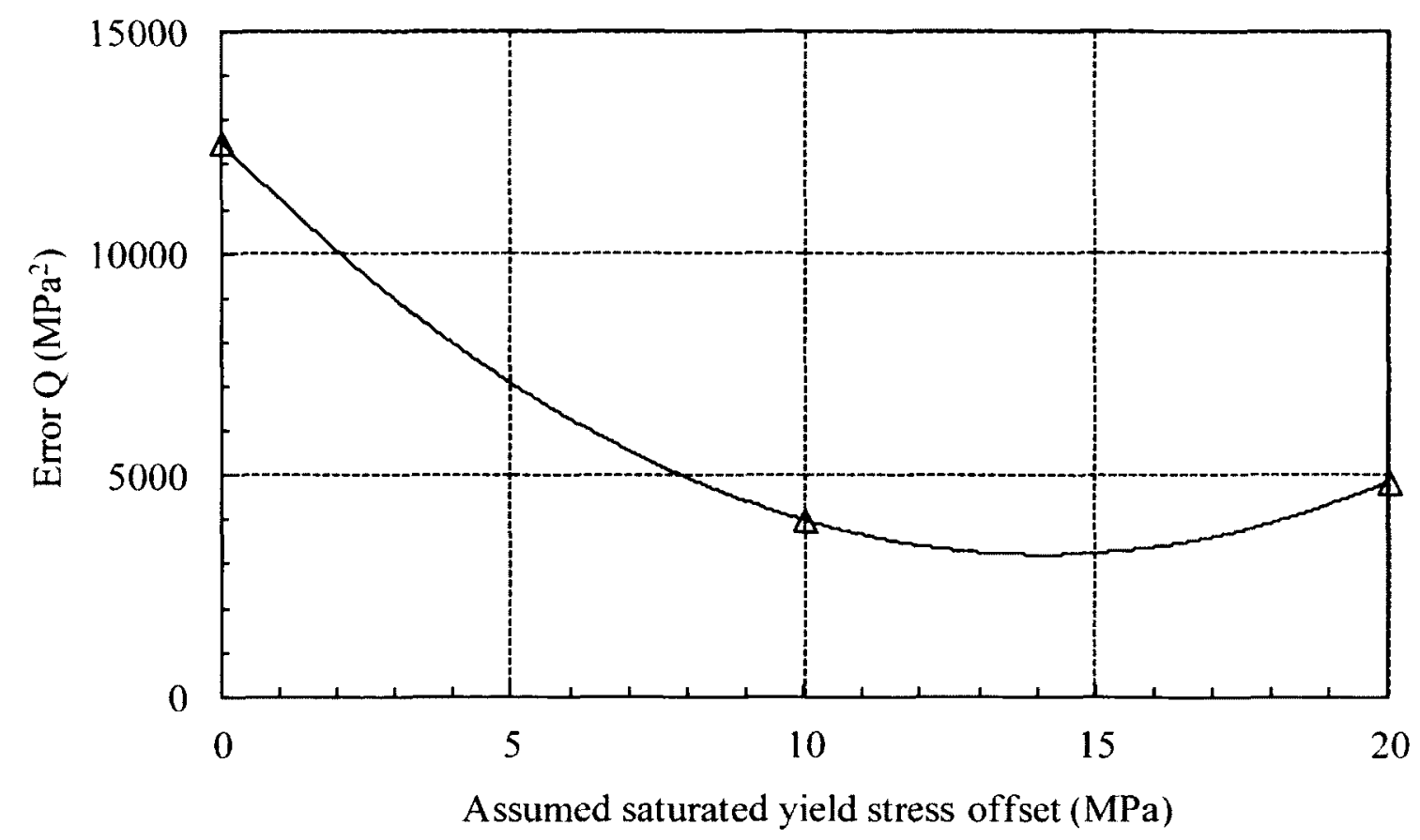

Figure 3.44 Error versus assumed saturated yield stress offset $\left(\Delta \sigma_{\mathrm{s}}^{0}\right)$ for $\sigma_{\mathrm{s}}^{0}=$ $464 \mathrm{MPa}$ (two terms)

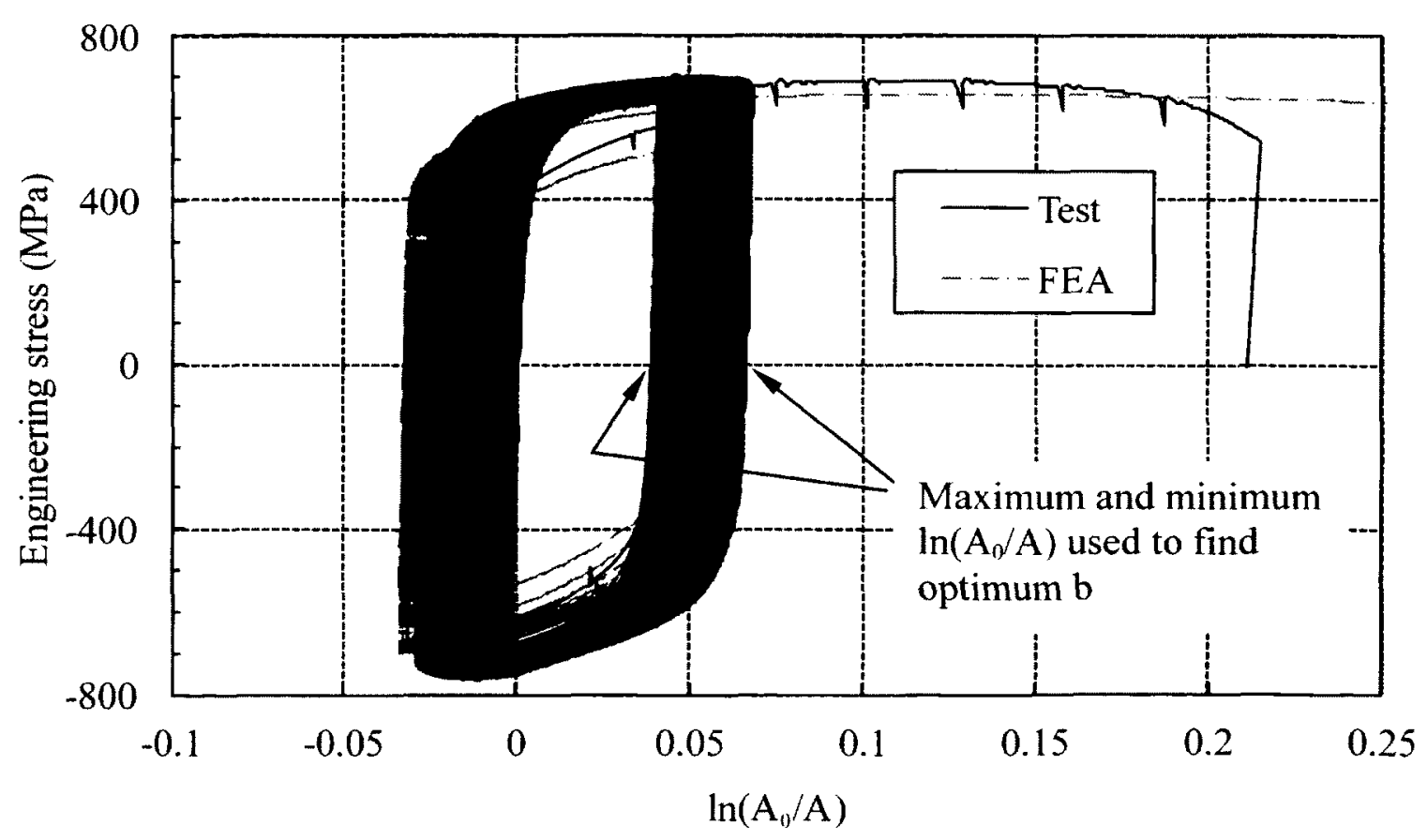

Figure 3.45 Test and predicted engineering stress versus cross-sectional area change curves with $b=4.5$ for notched specimen $\mathrm{ABC} 70404$ (two terms) 


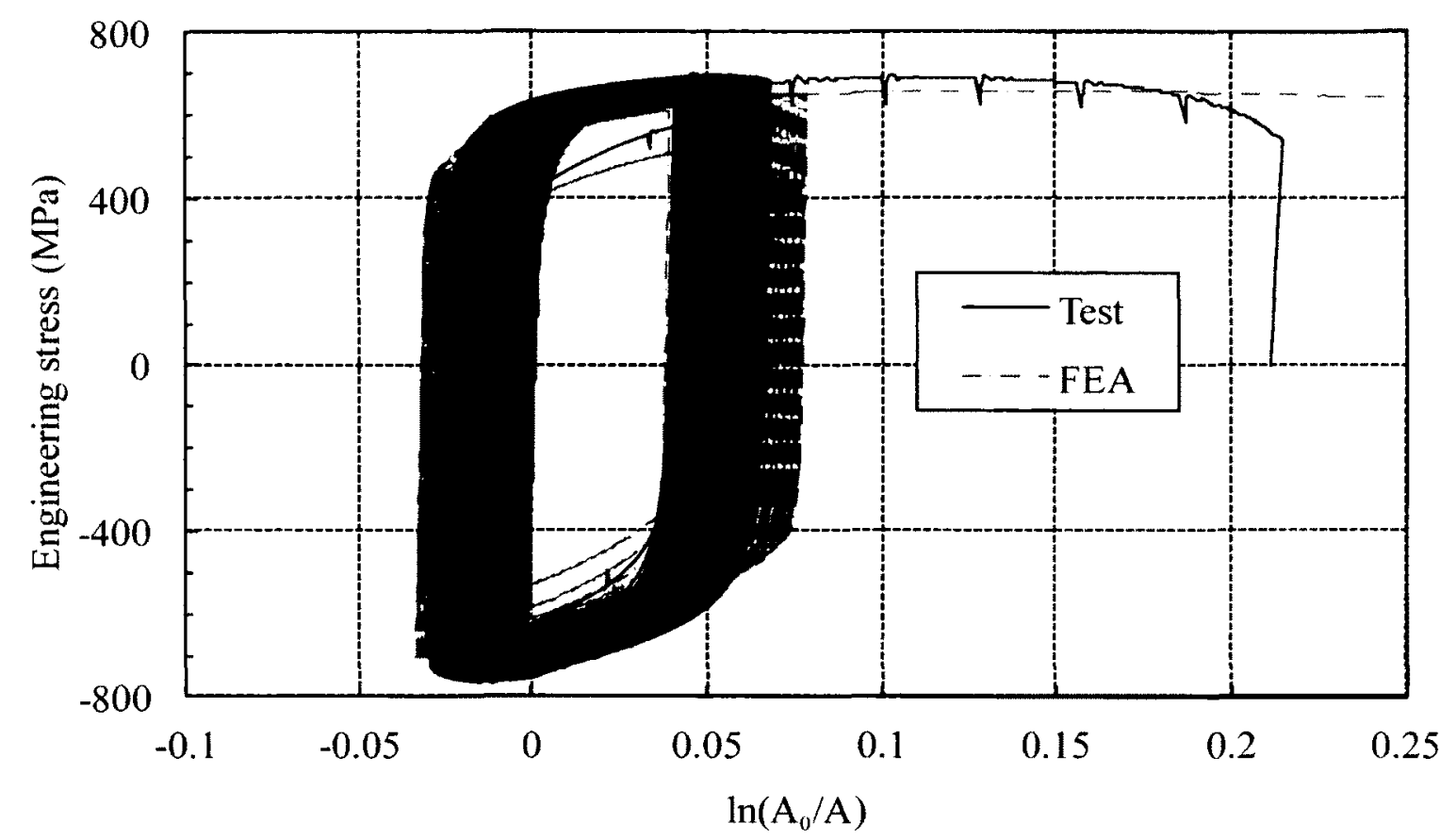

Figure 3.46 Test and predicted engineering stress versus cross-sectional area change curves with $b=5.5$ for notched specimen ABC70404 (two terms)

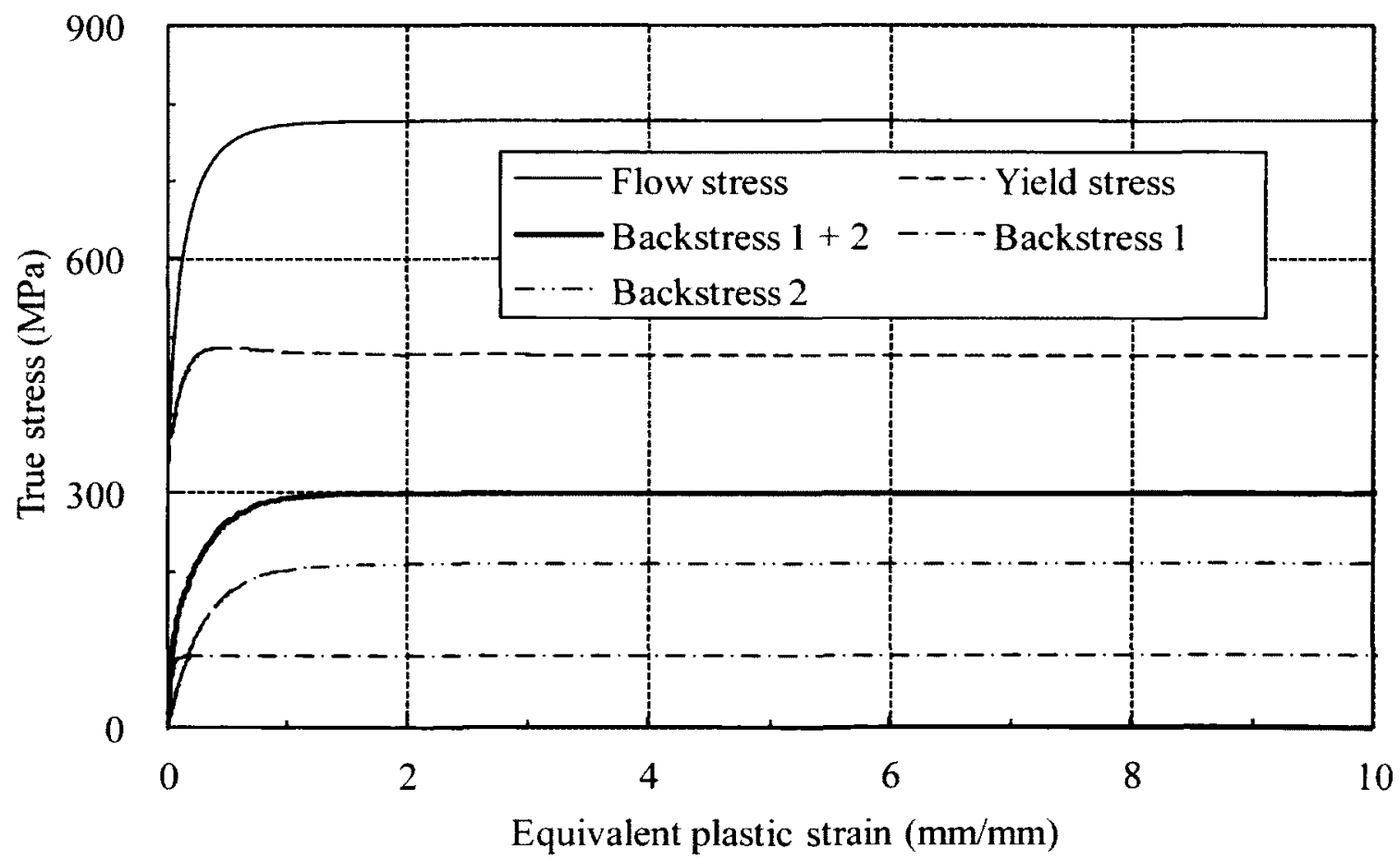

Figure 3.47 Various components of stresses for material A (two terms) 


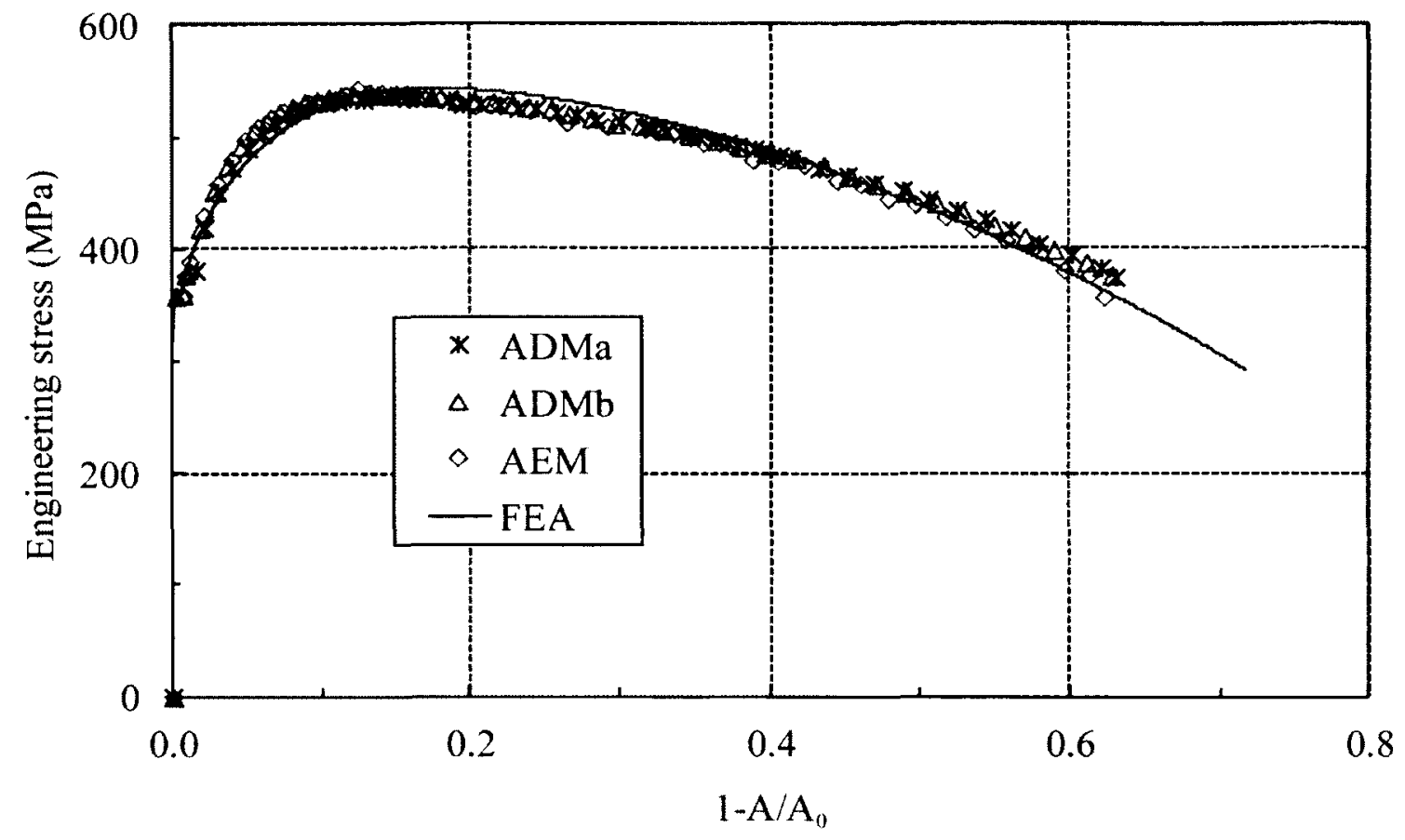

Figure 3.48 Test and predicted engineering stress versus cross-sectional area change curves for straight specimens ADMa, ADMb and AEM (two terms)

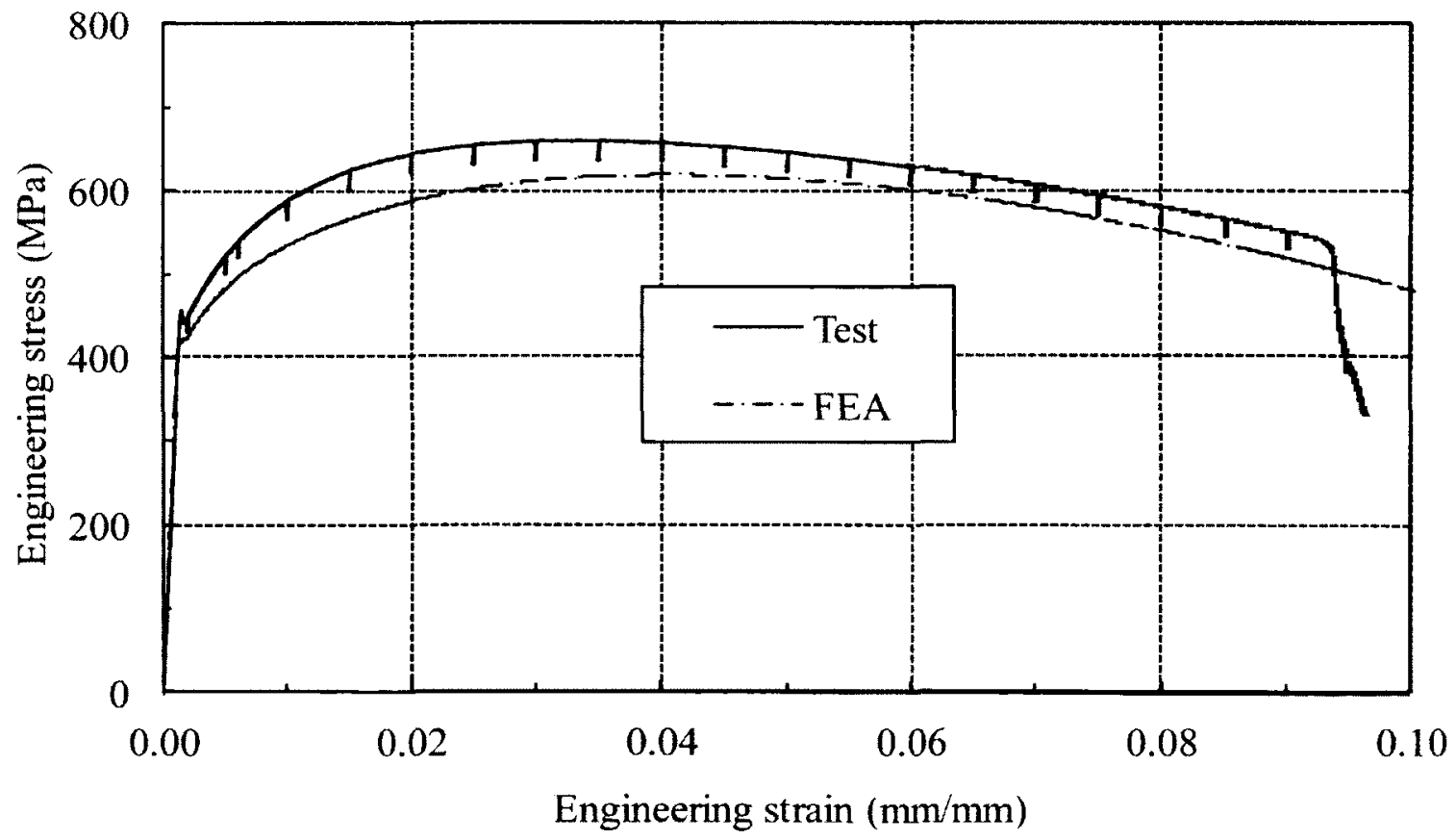

Figure 3.49 Test and predicted engineering stress versus engineering strain curves for notched specimen ABM (two terms) 


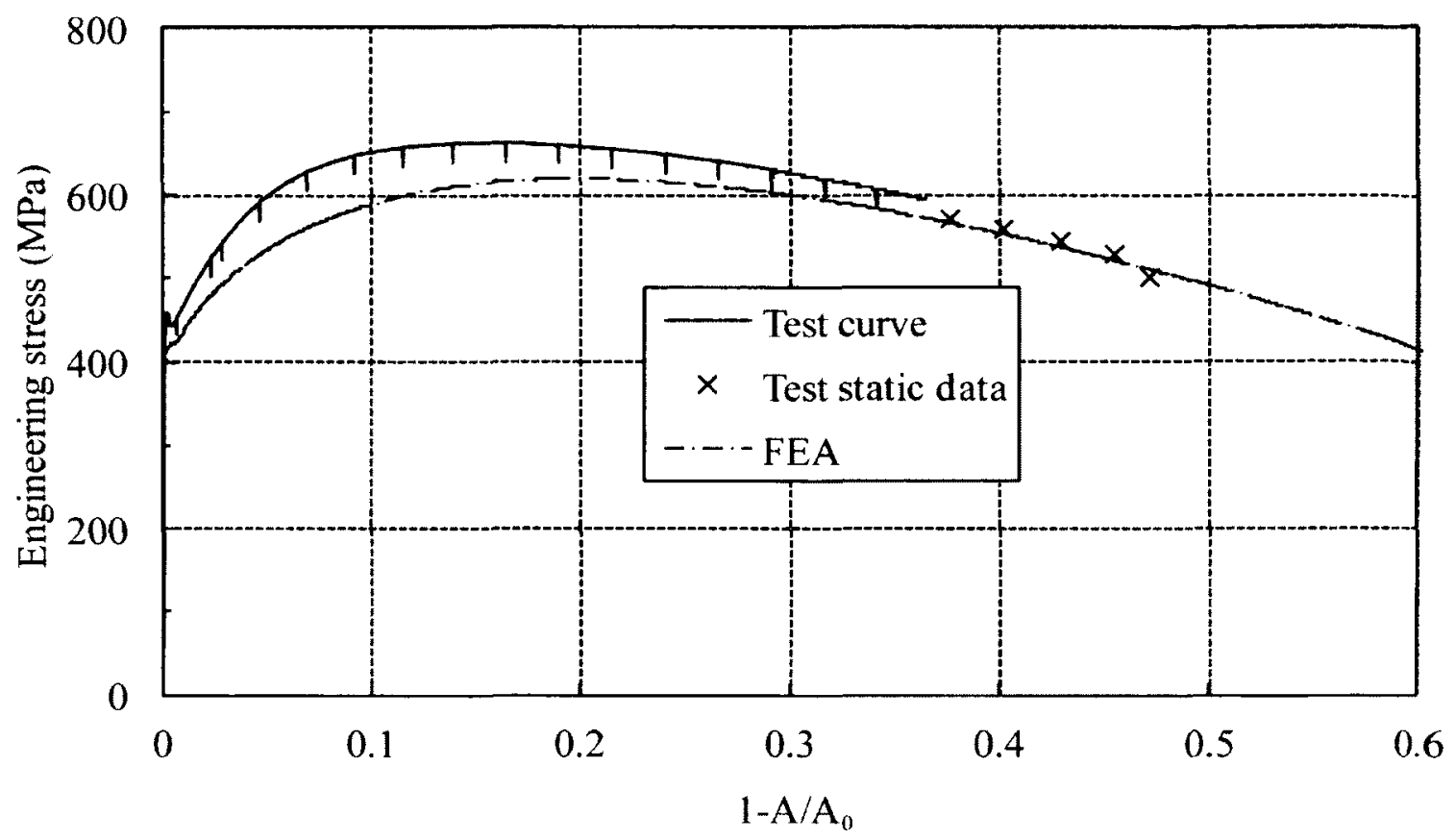

Figure 3.50 Test and predicted engineering stress versus cross-sectional area change curves for notched specimen ABM (two terms)

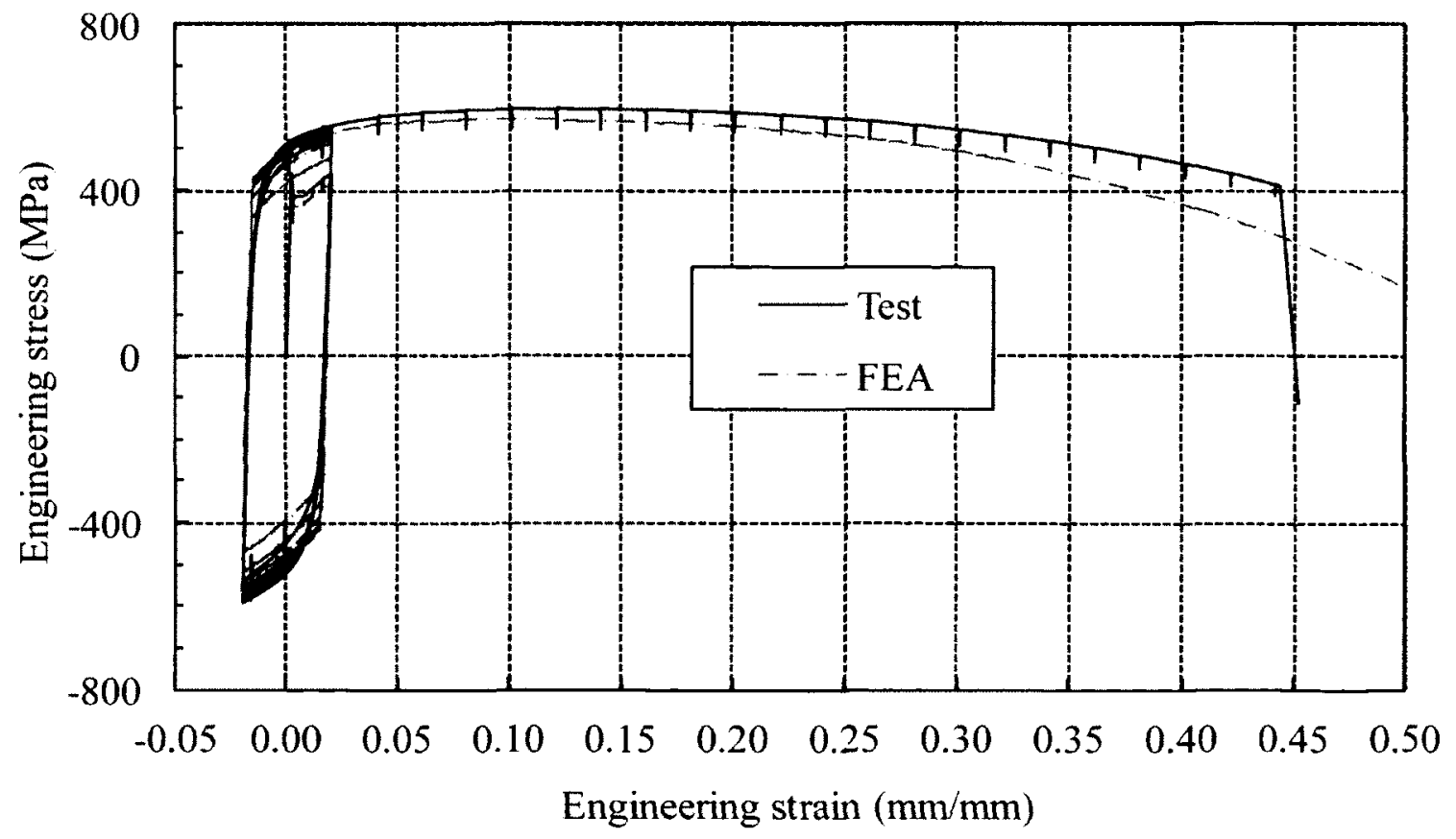

Figure 3.51 Test and predicted engineering stress versus engineering strain curves for straight specimen ADC20202 (two terms) 


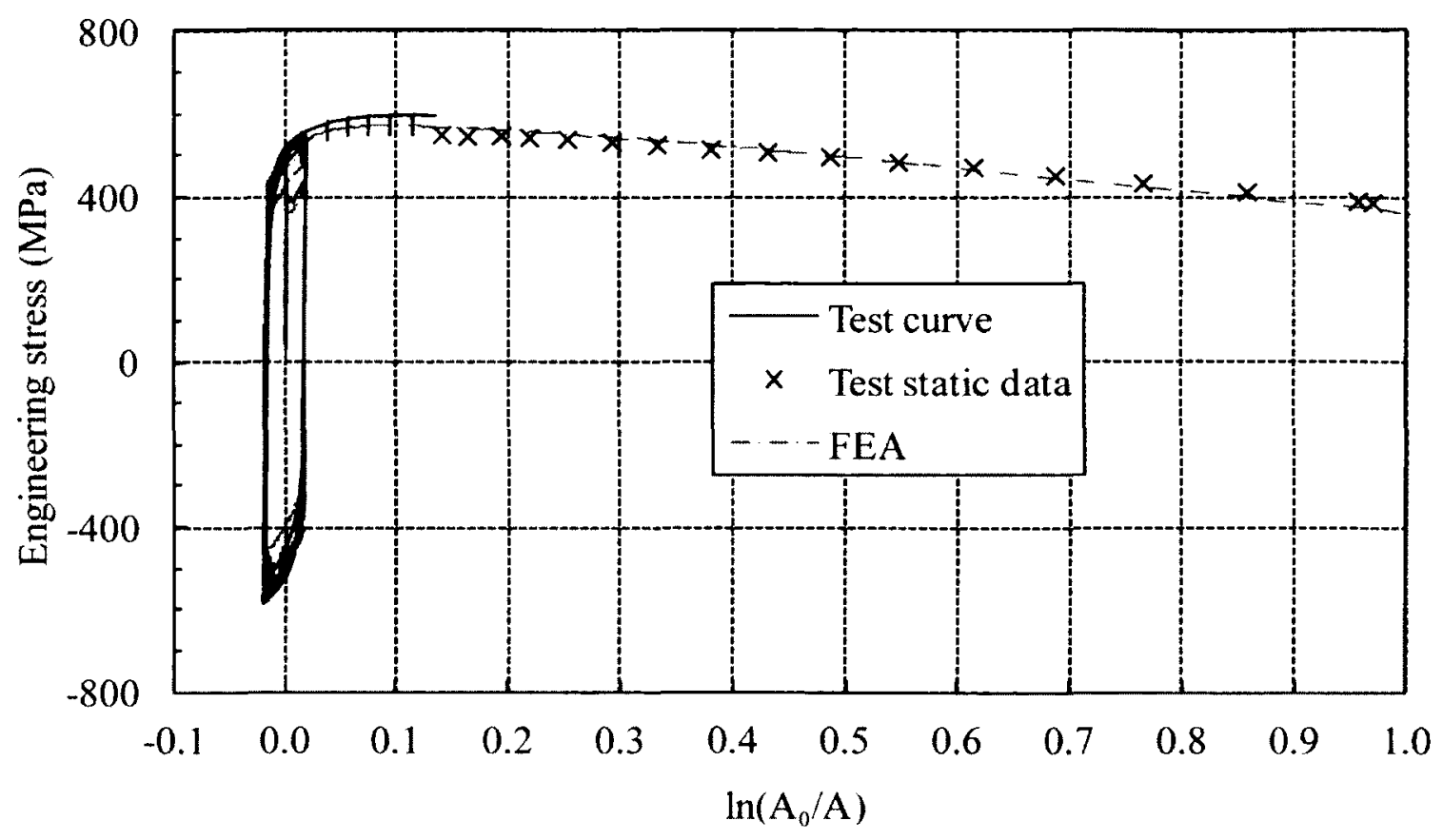

Figure 3.52 Test and predicted engineering stress versus cross-sectional area change curves for straight specimen ADC20202 (two terms)

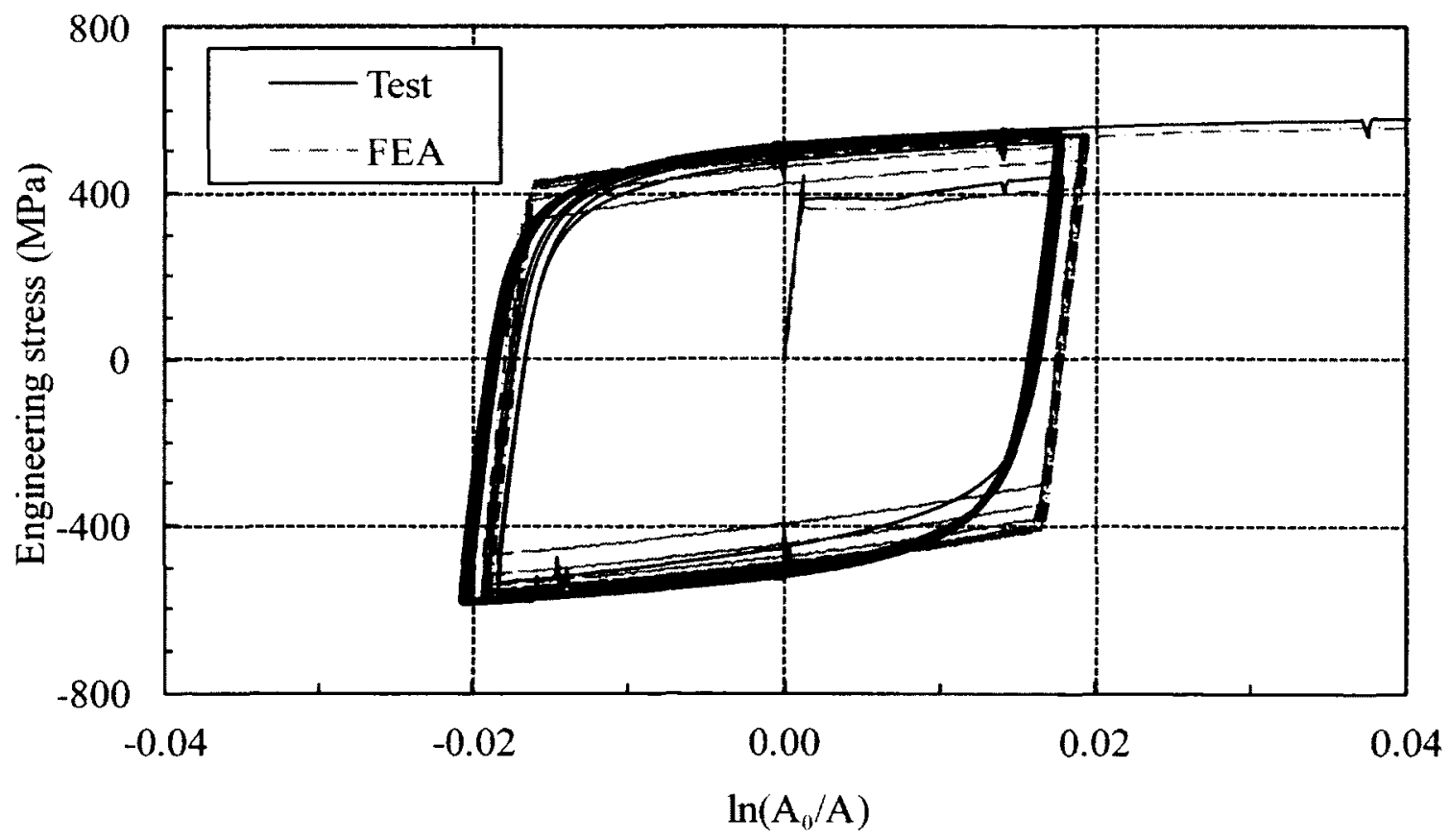

Figure 3.53 Test and predicted engineering stress versus cross-sectional area change curves for straight specimen ADC20202 focus of cyclic loading (two terms) 


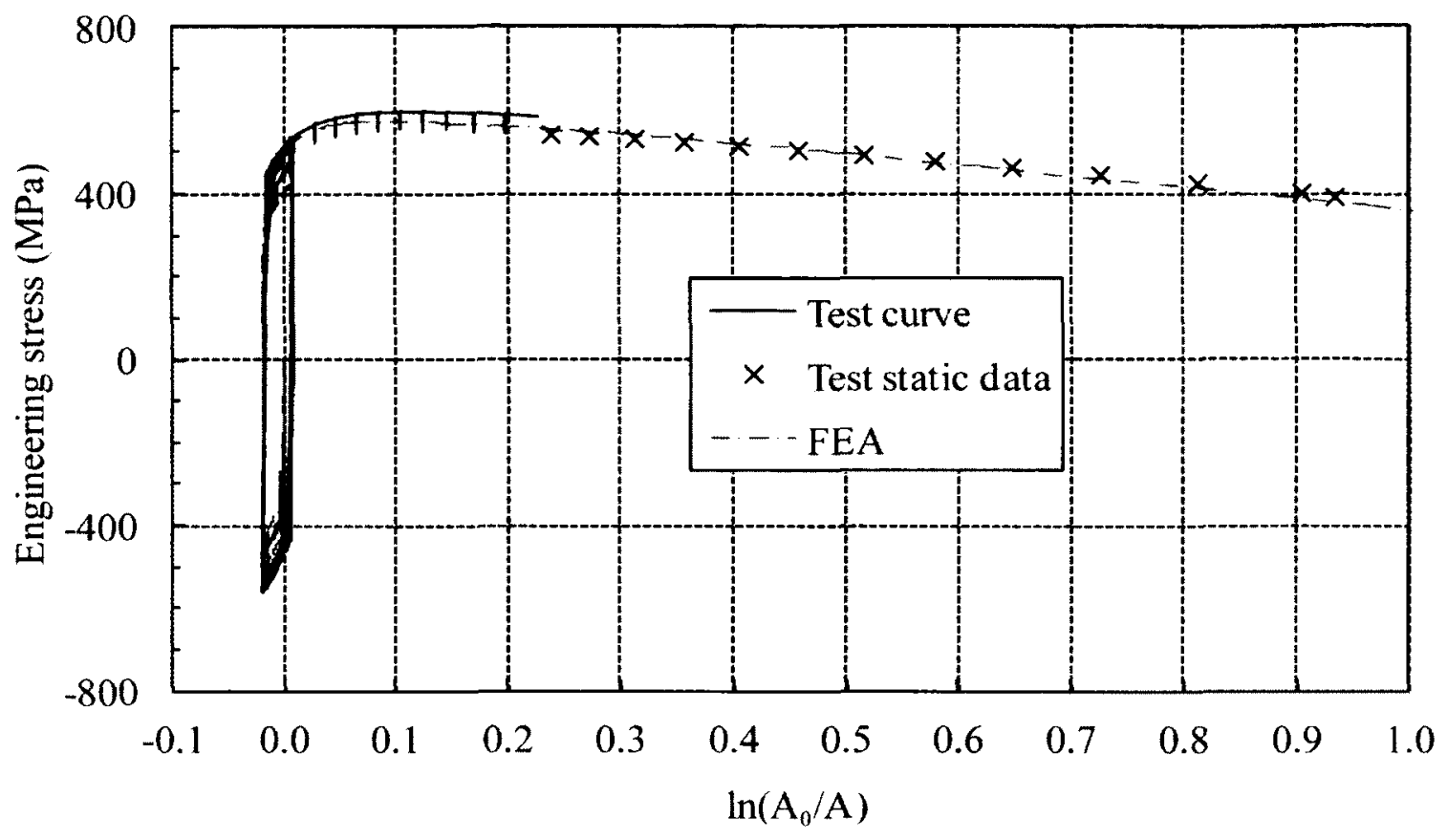

Figure 3.54 Test and predicted engineering stress versus cross-sectional area change curves for straight specimen ADC40102 (two terms)

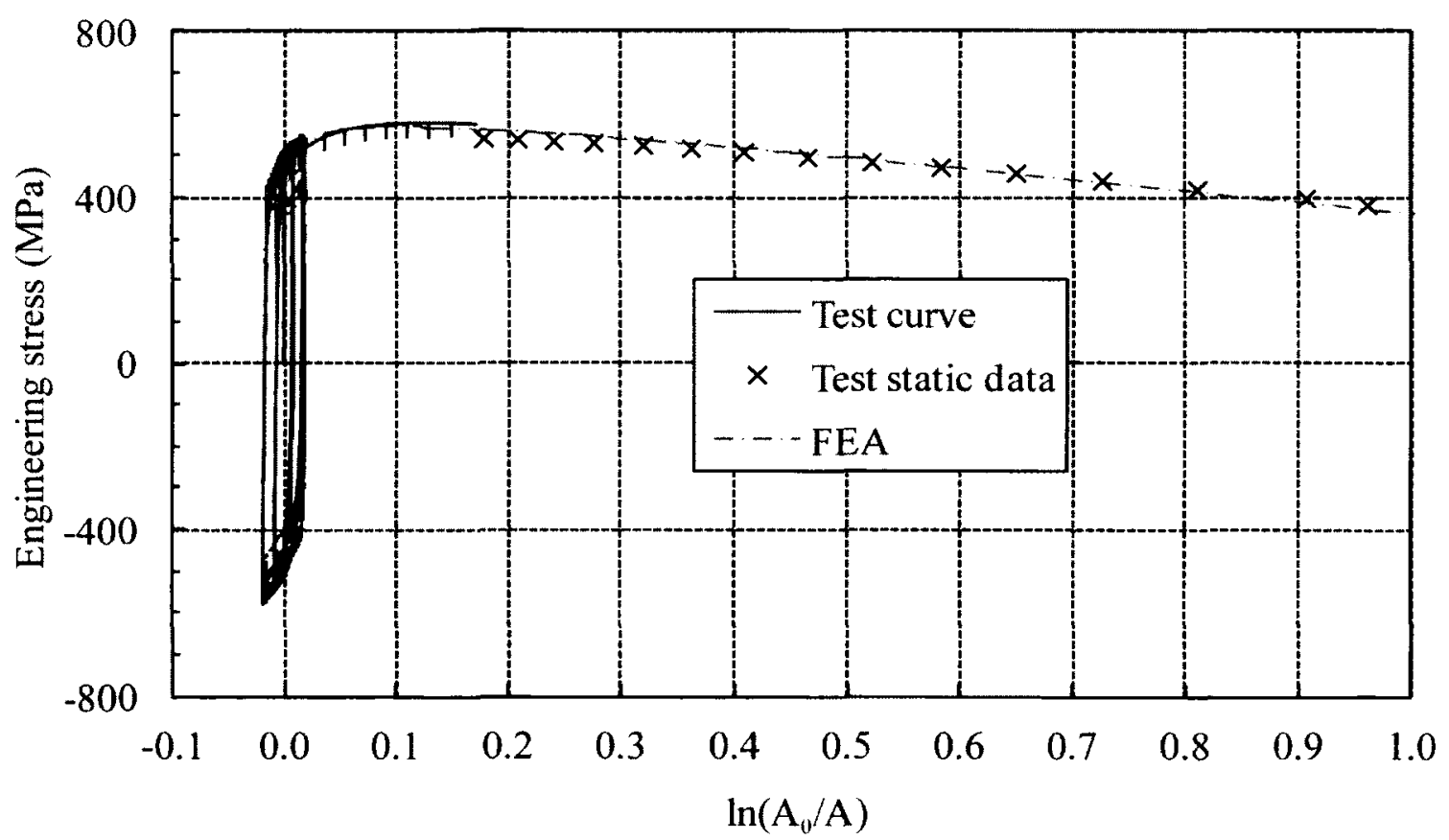

Figure 3.55 Test and predicted engineering stress versus cross-sectional area change curves for straight specimen ADCR0201 (two terms) 


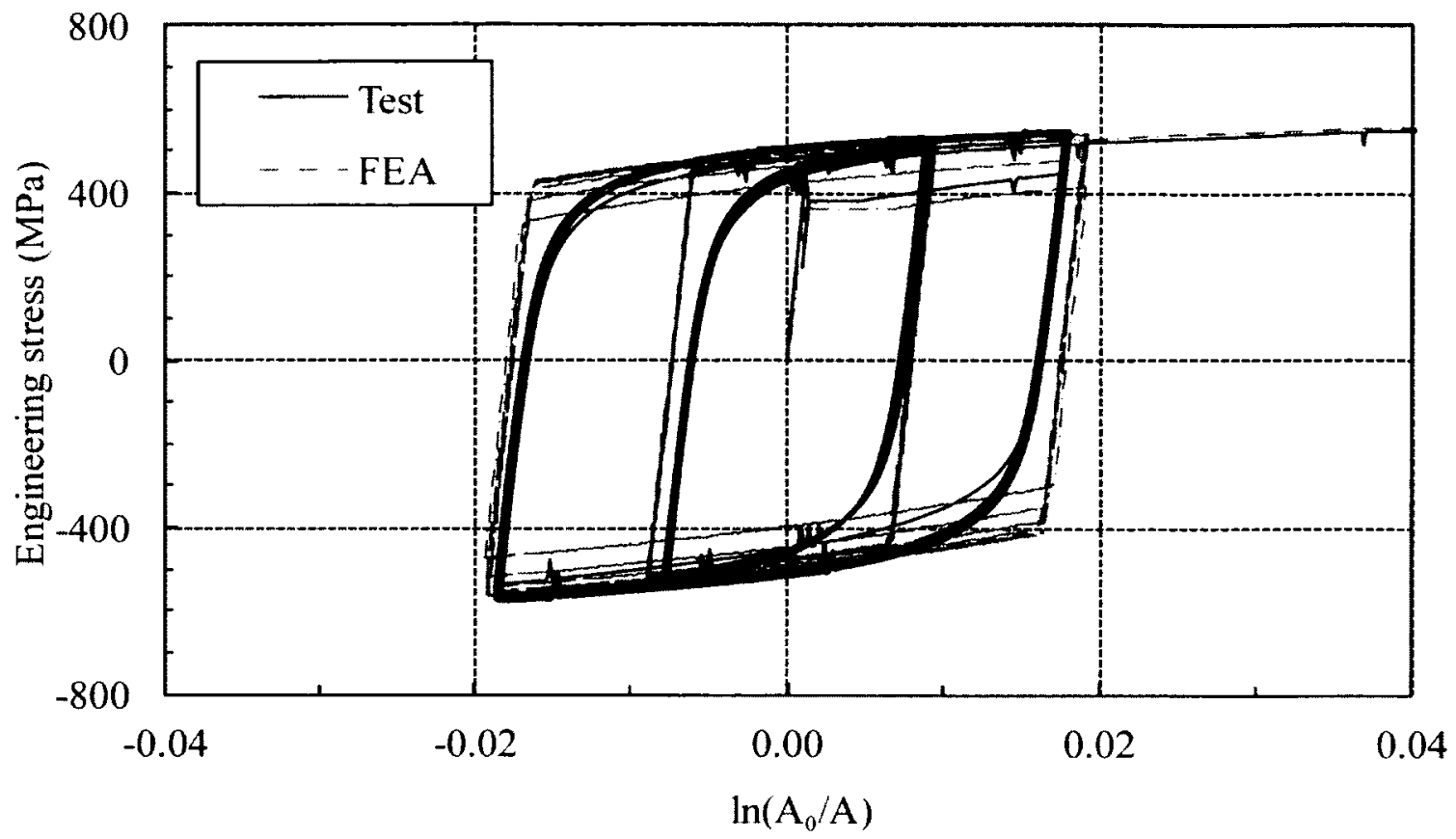

Figure 3.56 Test and predicted engineering stress versus cross-sectional area change curves for straight specimen ADCR0201 focus of cyclic loading (two terms)

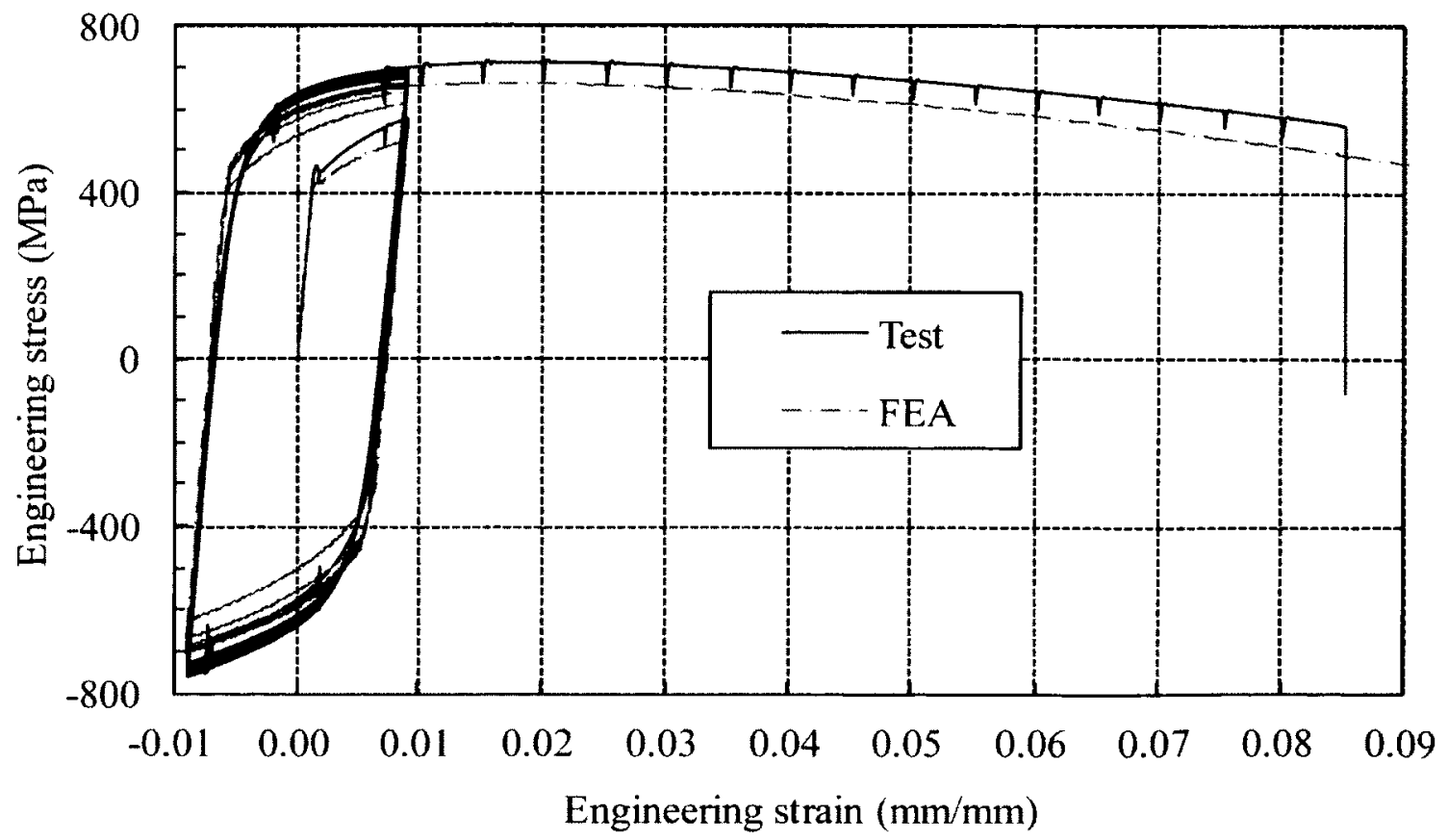

Figure 3.57 Test and predicted engineering stress versus engineering strain curves for notched specimen $\mathrm{ABC} 20404$ (two terms) 


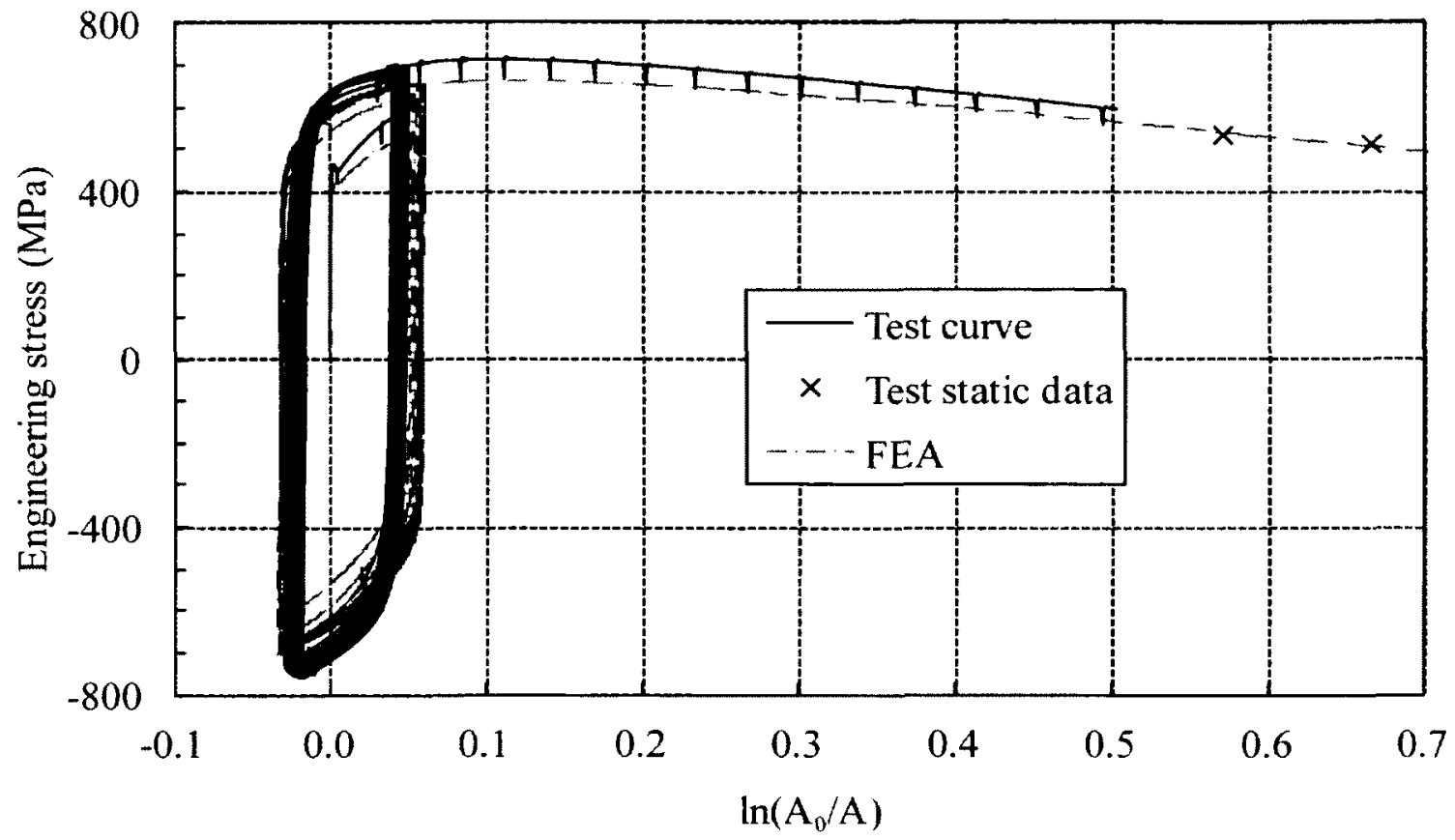

Figure 3.58 Test and predicted engineering stress versus cross-sectional area change curves for notched specimen ABC20404 (two terms)

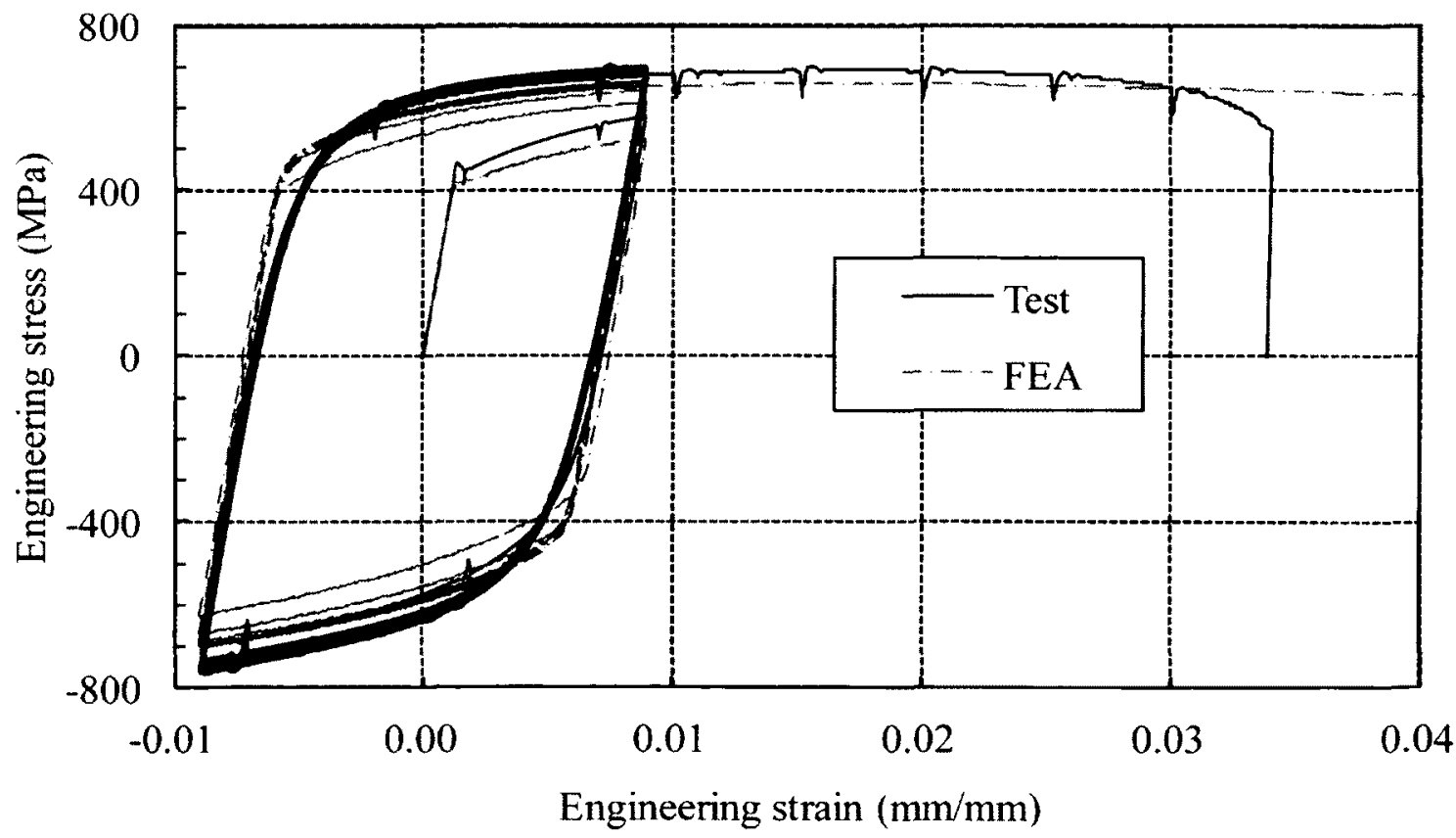

Figure 3.59 Test and predicted engineering stress versus engineering strain curves for notched specimen ABC70404 (two terms) 


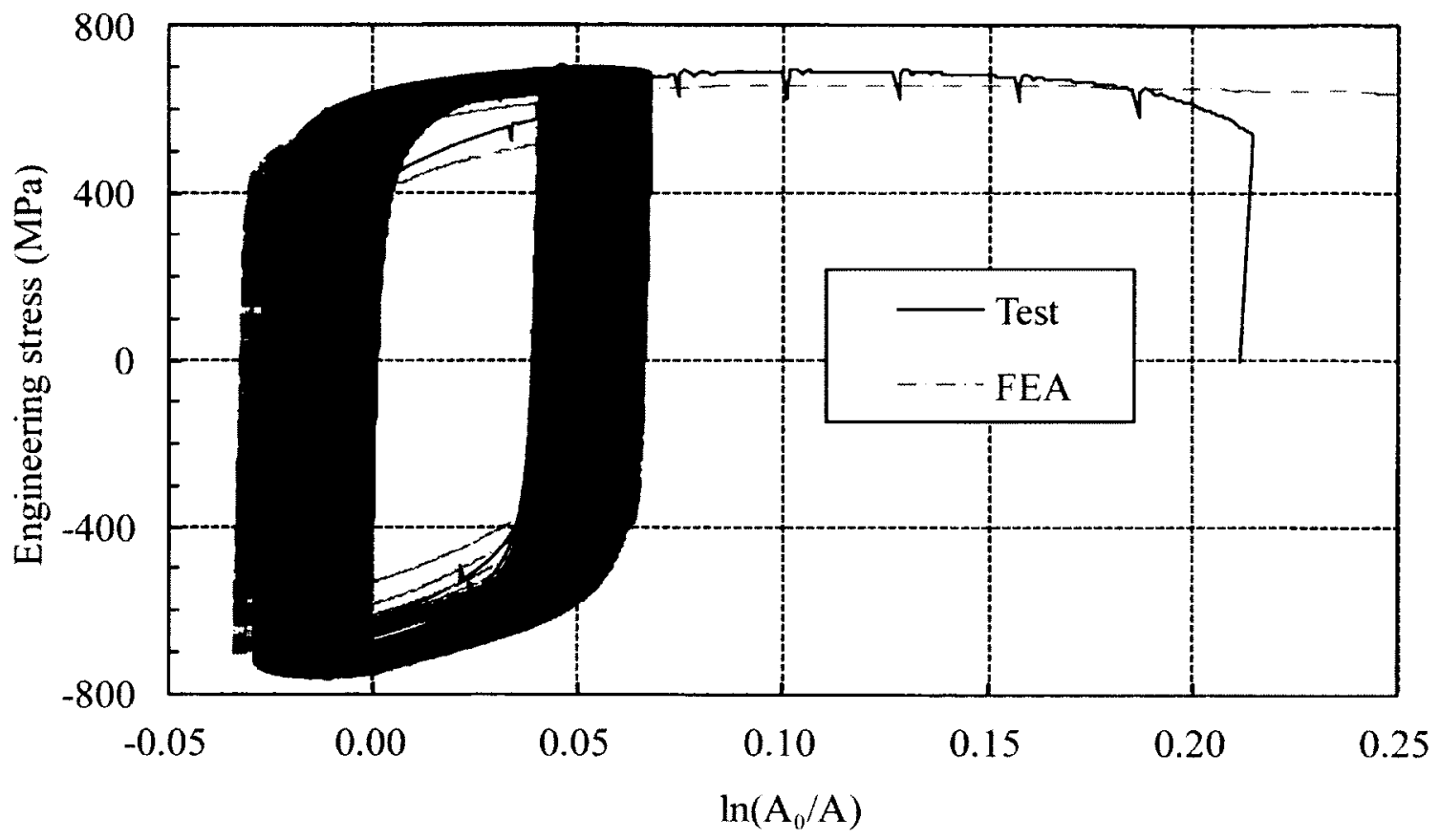

Figure 3.60 Test and predicted engineering stress versus cross-sectional area change curves for notched specimen ABC70404 (two terms)

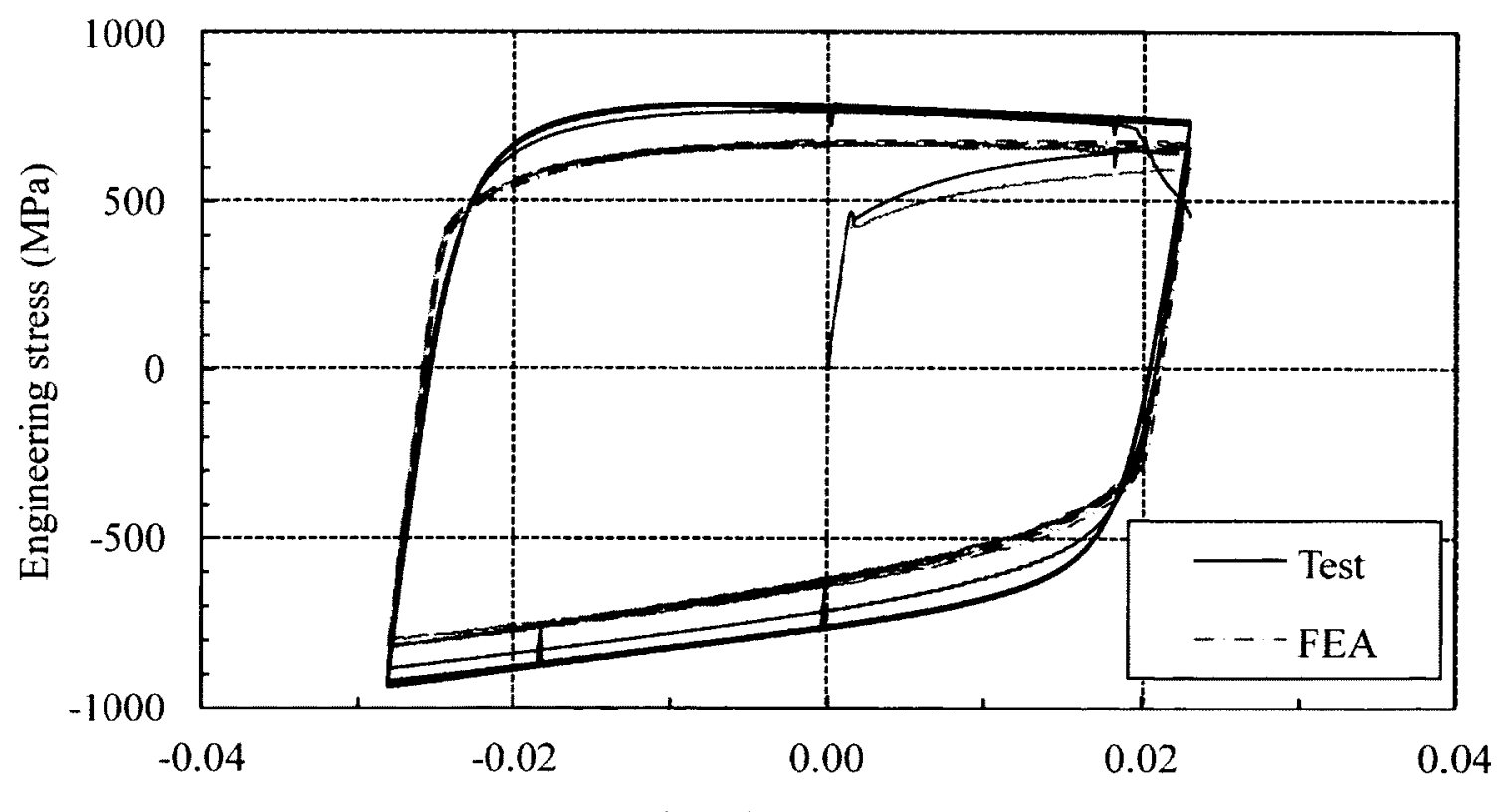

Engineering strain $(\mathrm{mm} / \mathrm{mm})$

Figure 3.61 Test and predicted engineering stress versus engineering strain curves for notched specimen ABCF 1212 (two terms) 


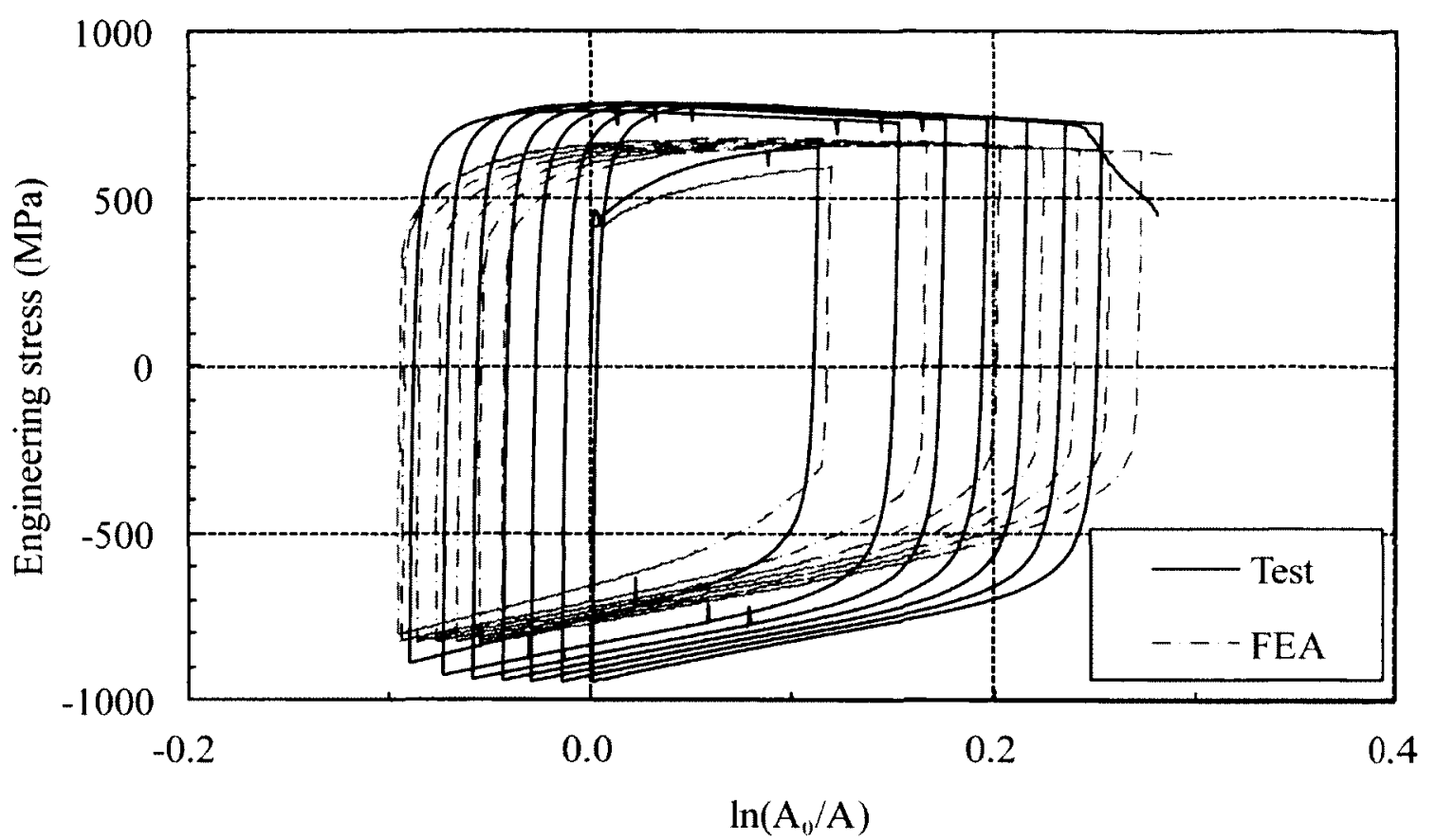

Figure 3.62 Test and predicted engineering stress versus cross-sectional area change curves for notched specimen ABCF 1212 (two terms)

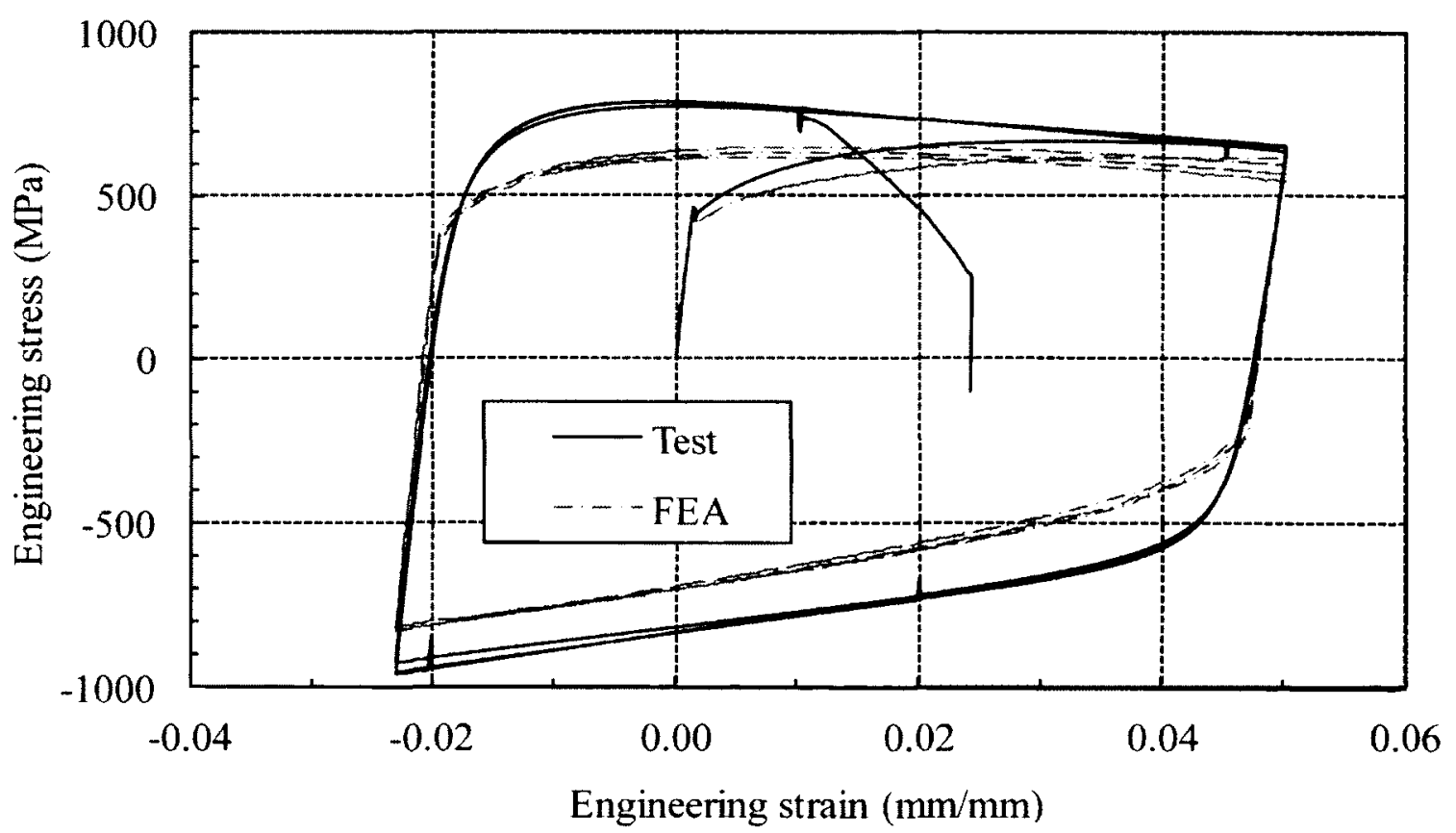

Figure 3.63 Test and predicted engineering stress versus engineering strain curves for notched specimen ABCF3010 (two terms) 


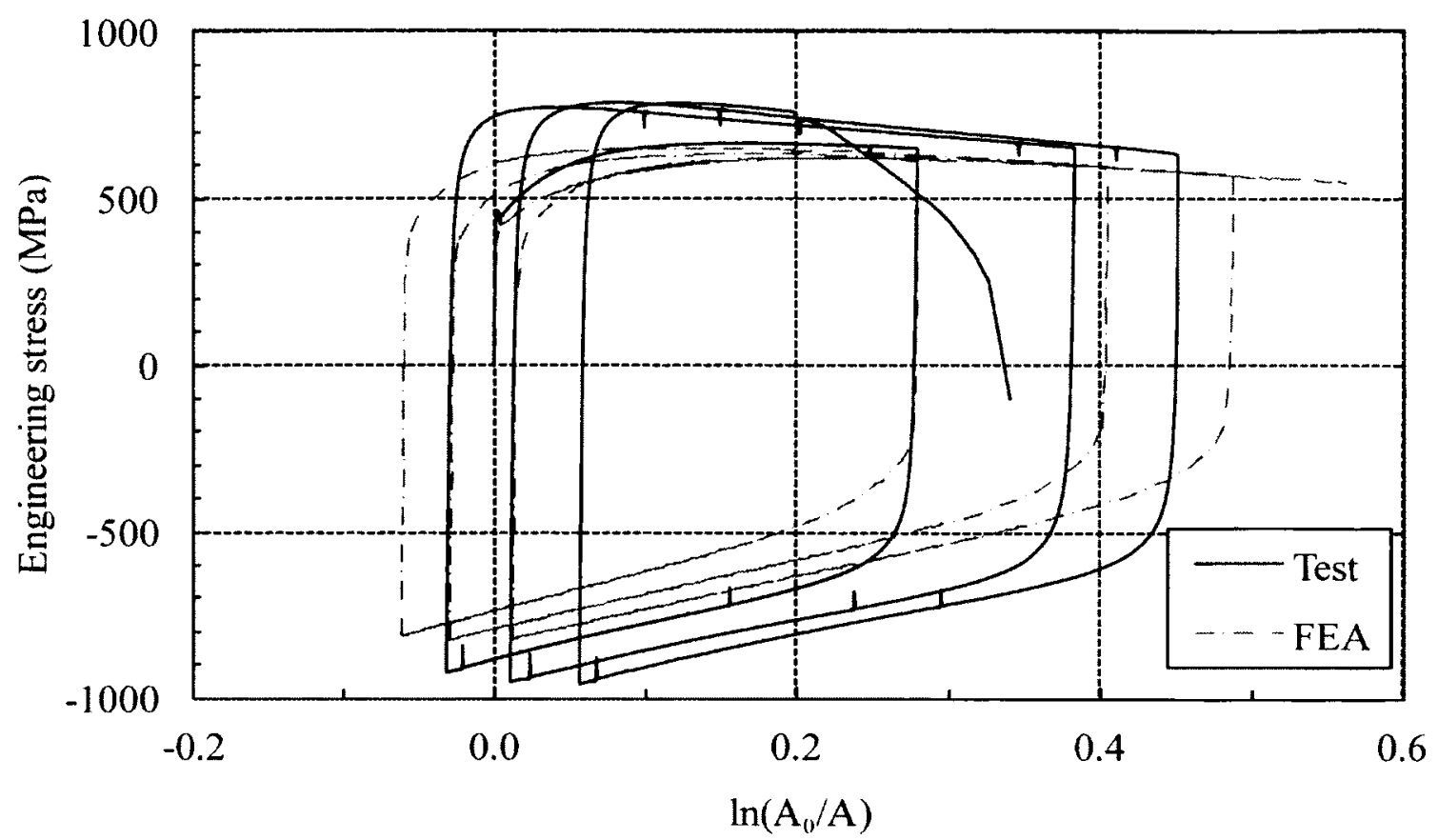

Figure 3.64 Test and predicted engineering stress versus cross-sectional area change curves for notched specimen ABCF3010 (two terms)

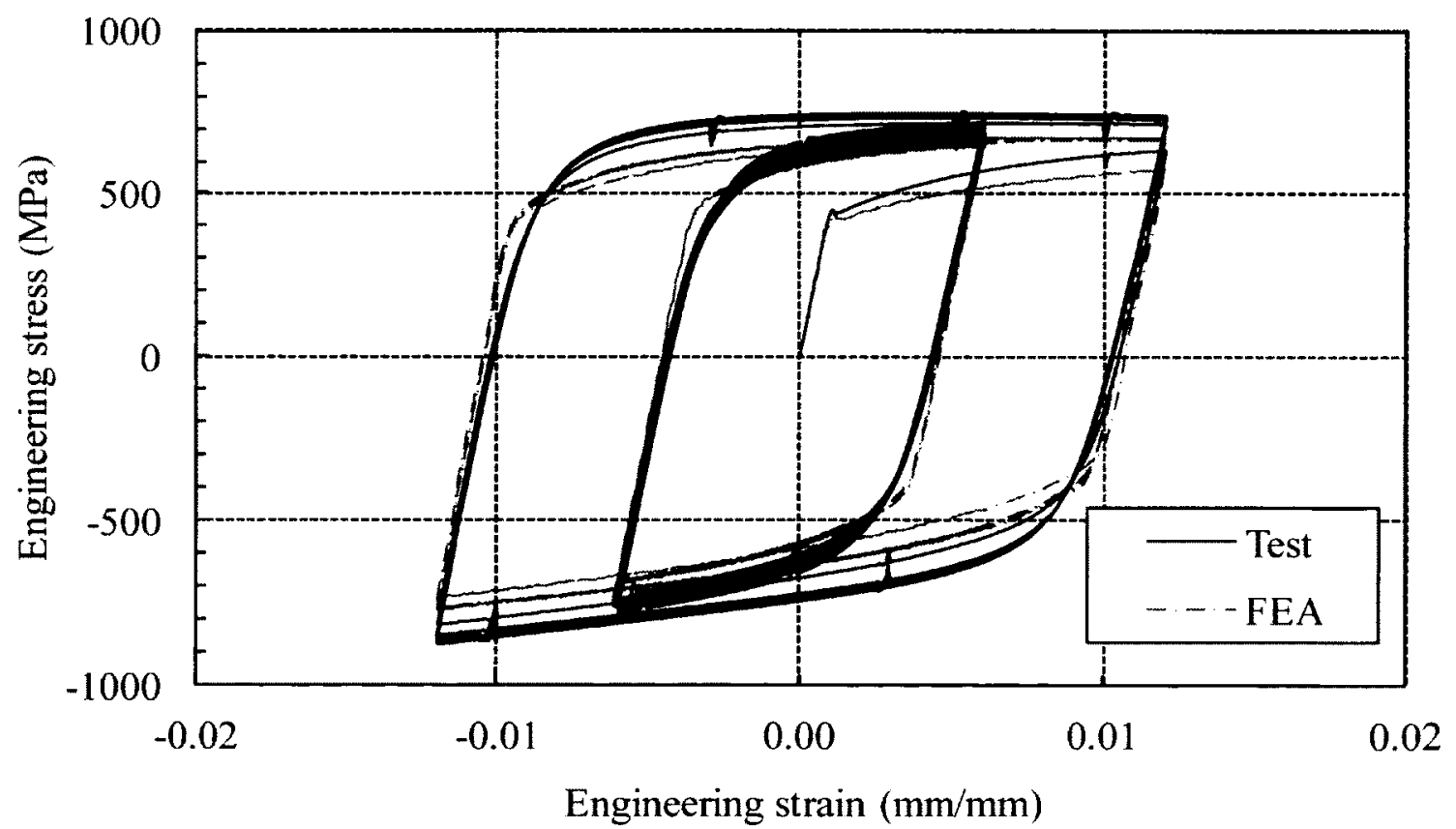

Figure 3.65 Test and predicted engineering stress versus engineering strain curves for notched specimen AACR0804 (two terms) 


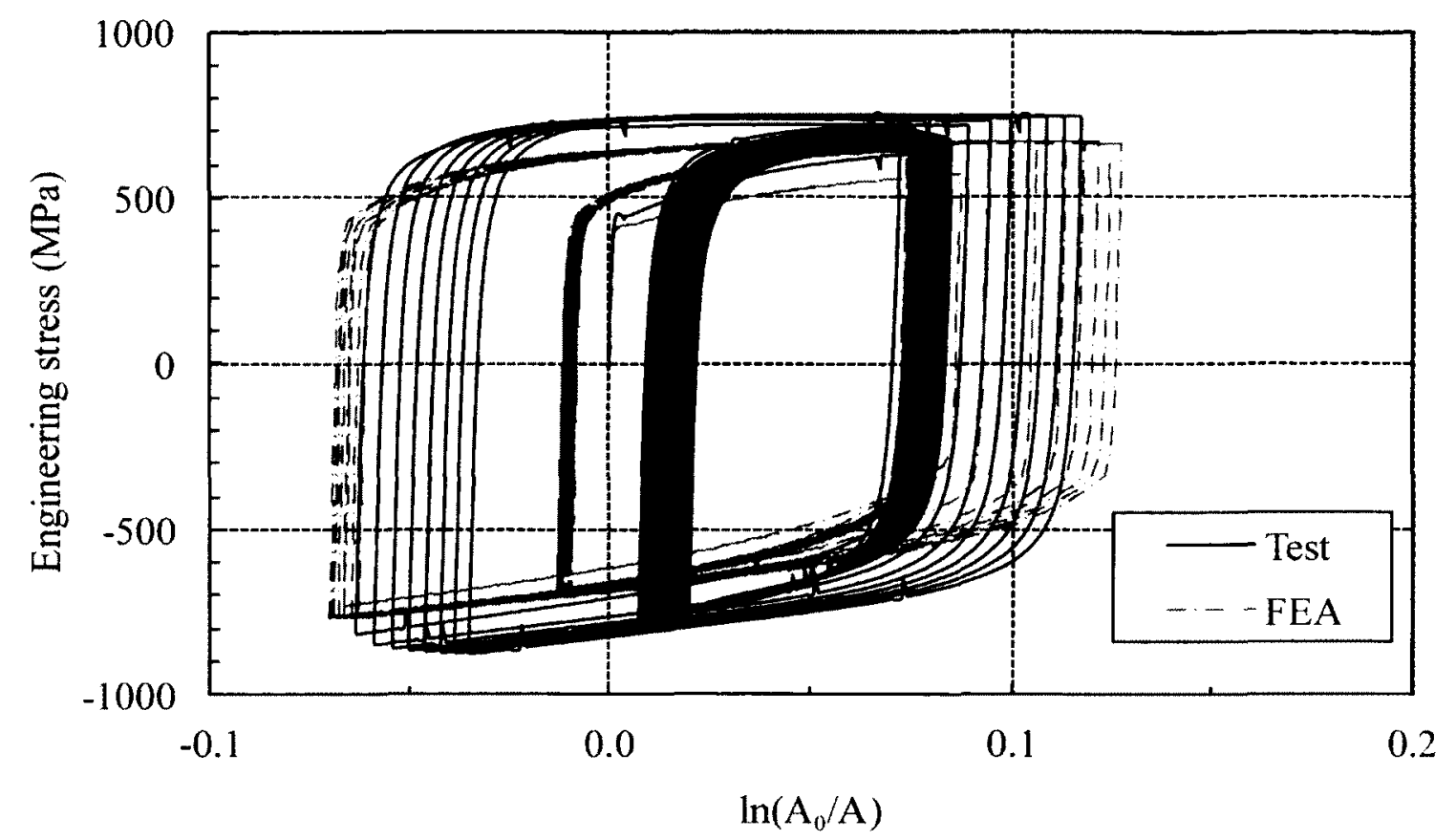

Figure 3.66 Test and predicted engineering stress versus cross-sectional area change curves for notched specimen AACR0804 (two terms)

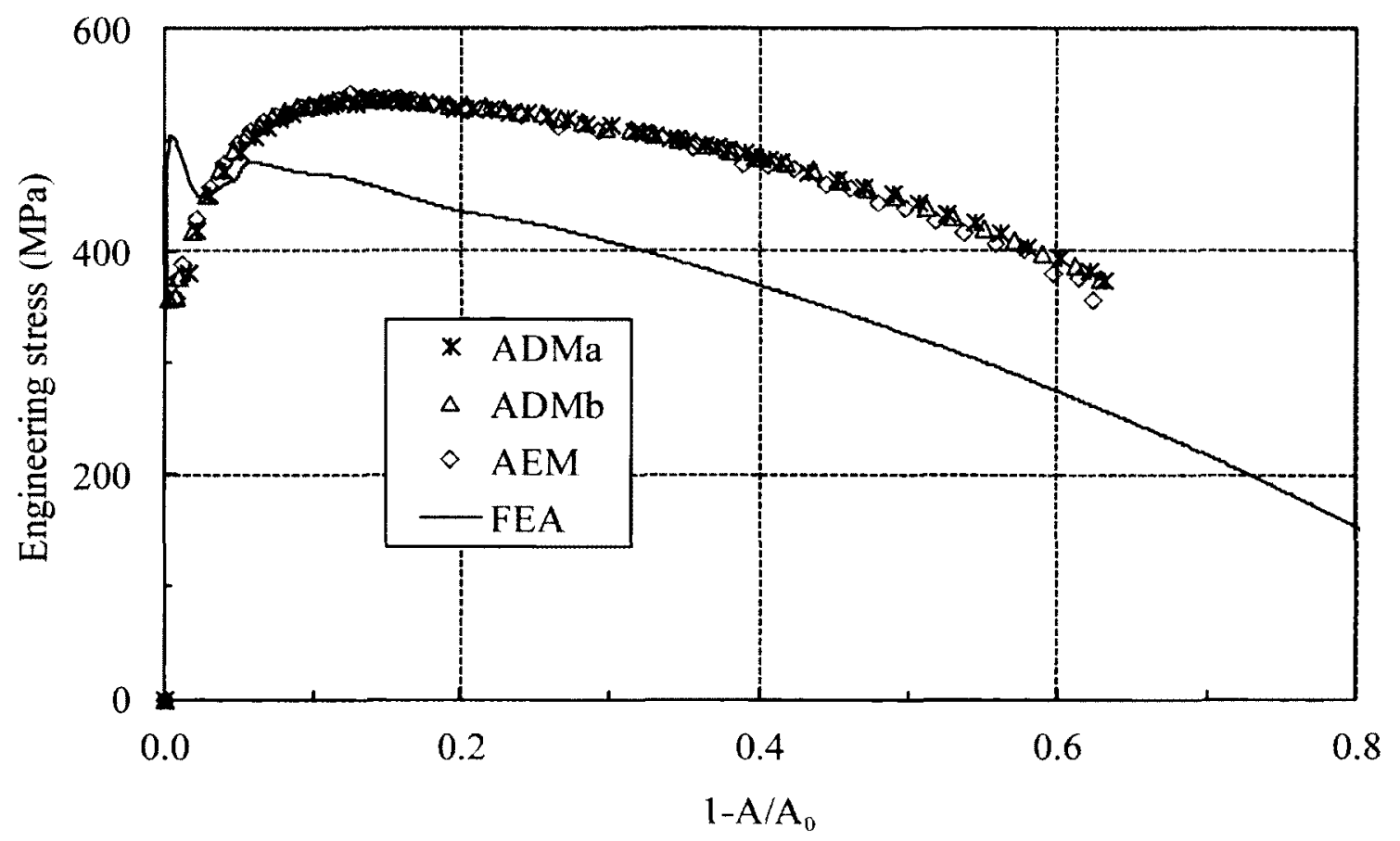

Figure 3.67 Test and predicted engineering stress versus cross-sectional area change curves for straight specimens subjected to monotonic tension loading using parameters calibrated by ABAQUS (two terms) 


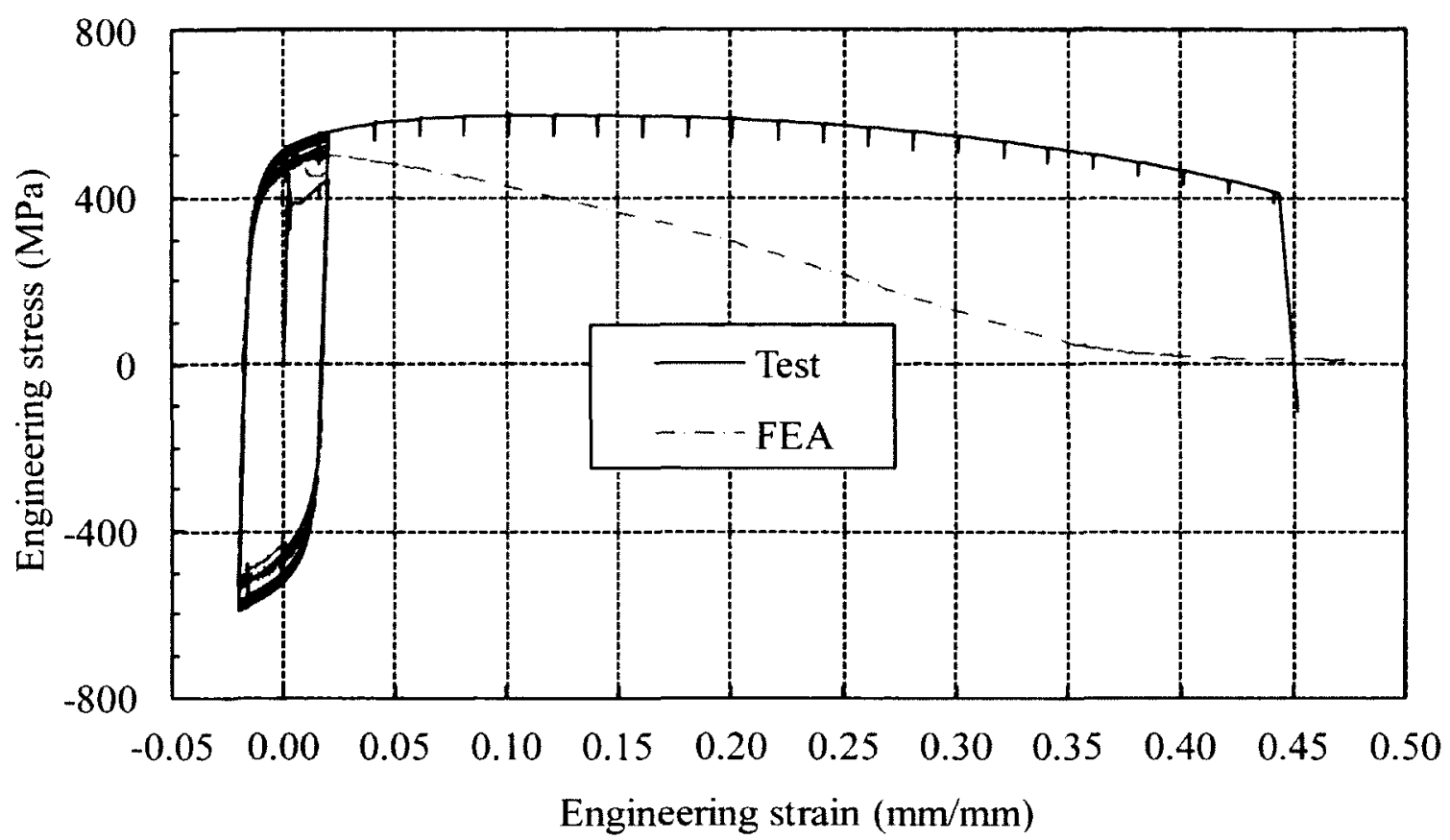

Figure 3.68 Test and predicted engineering stress versus engineering strain curves for straight specimen ADC20202 using parameters calibrated by ABAQUS (two terms)

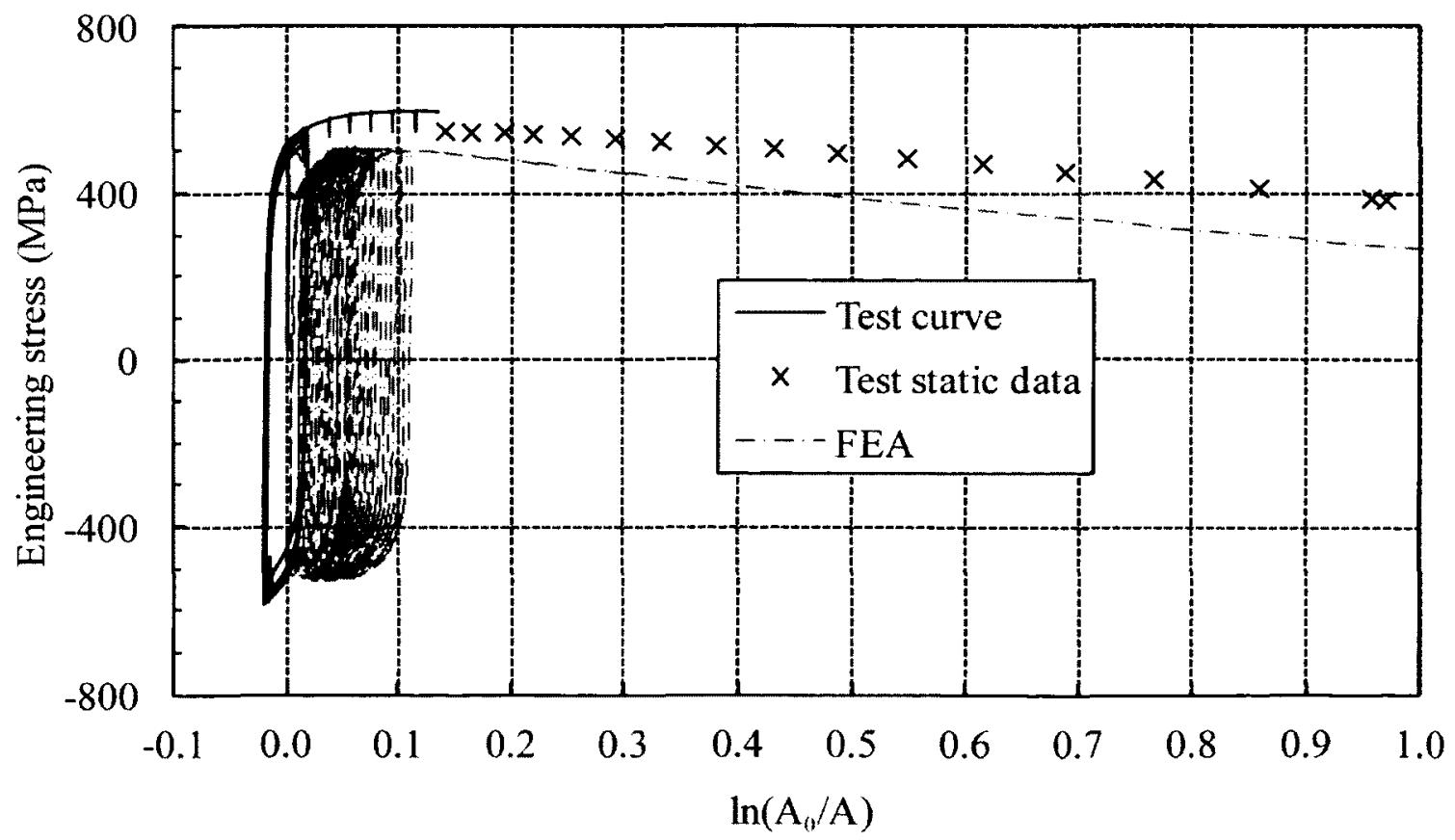

Figure 3.69 Test and predicted engineering stress versus cross-sectional area change curves for straight specimen ADC20202 using parameters calibrated by ABAQUS (two terms) 


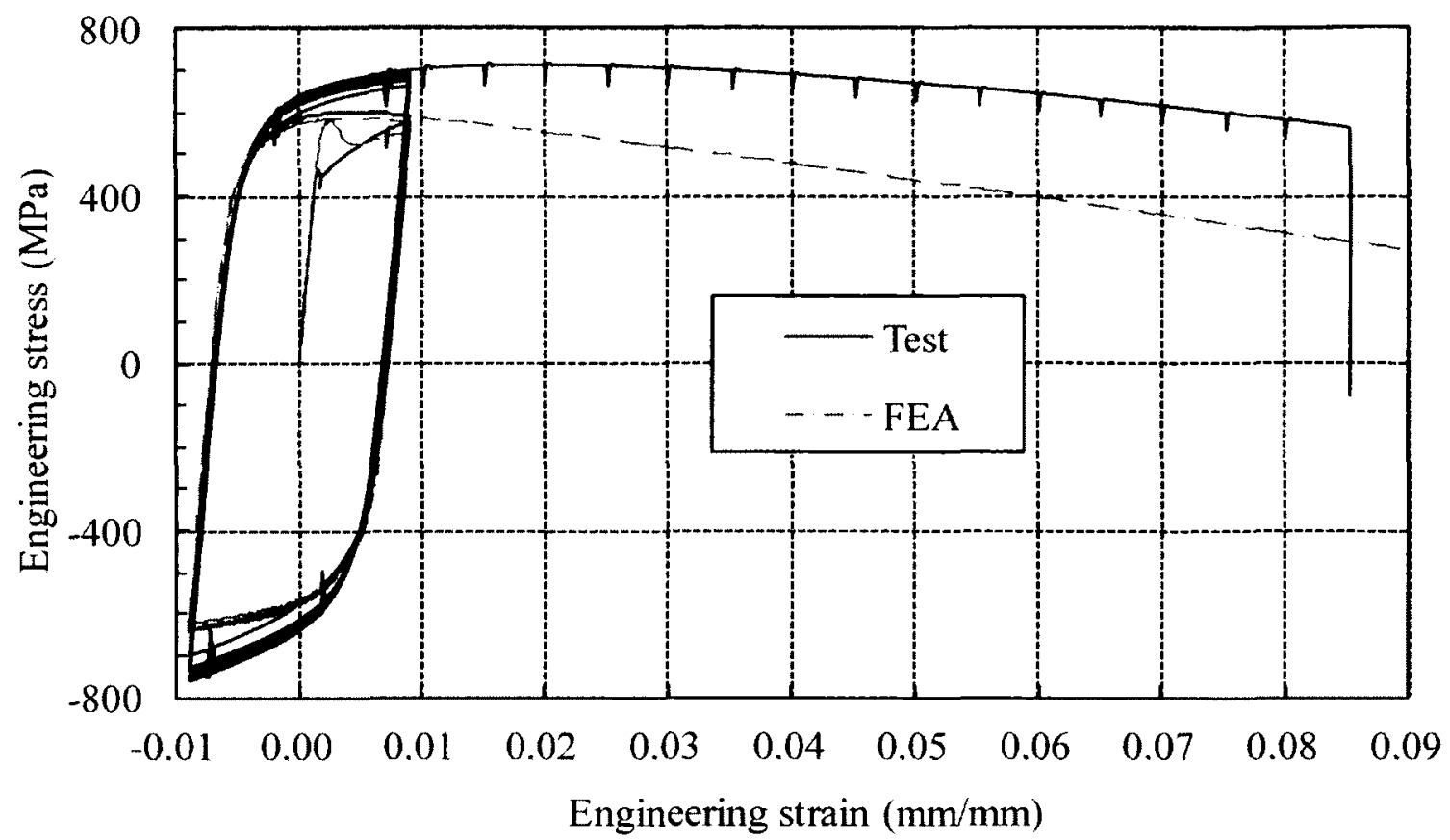

Figure 3.70 Test and predicted engineering stress versus engineering strain curves for notched specimen ABC20404 using parameters calibrated by ABAQUS (two terms)

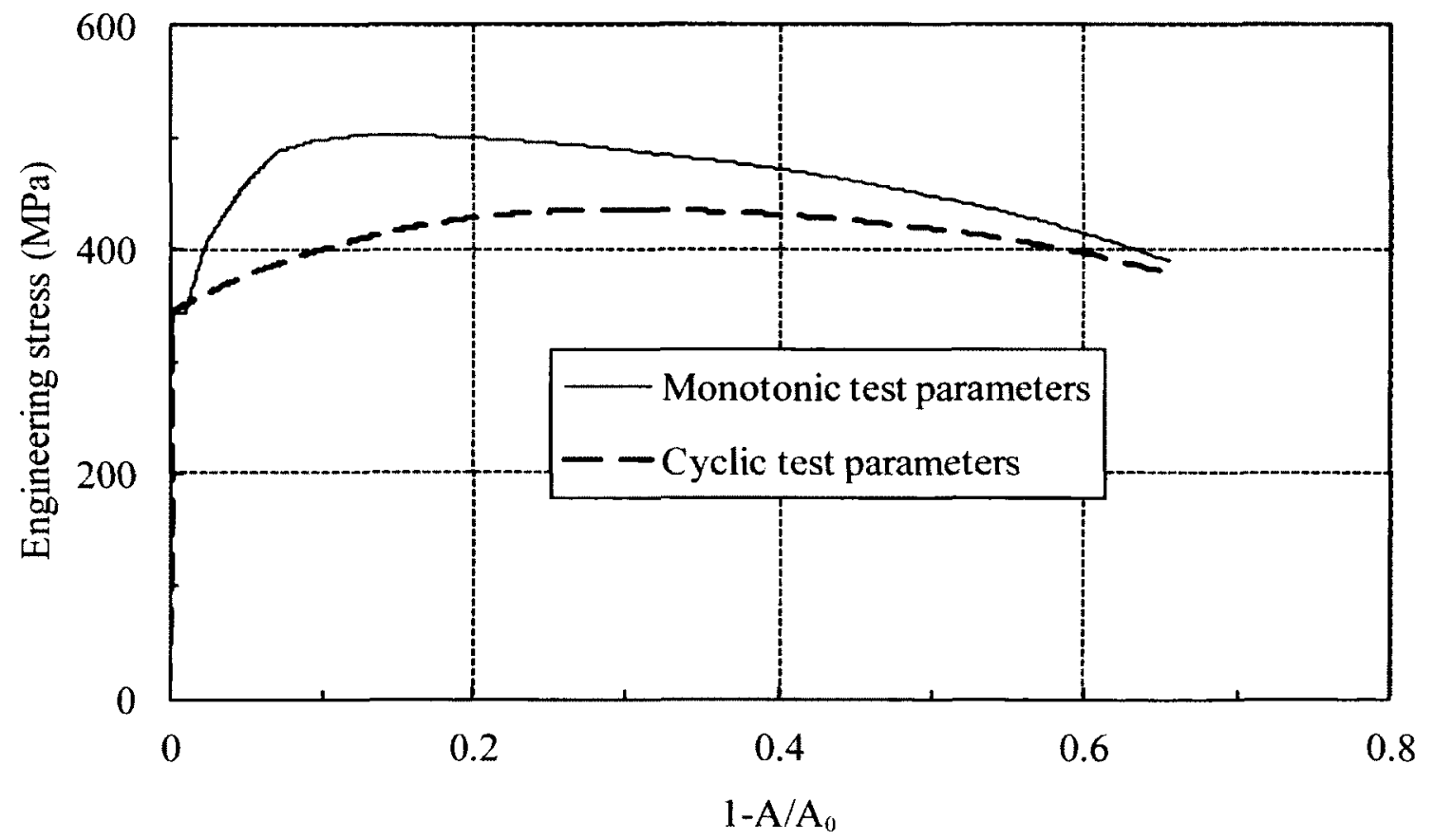

Figure 3.71 FEA simulations of monotonic tension test using material parameters for AW50 steel by Kanvinde and Deierlein (2004) 


\section{CHAPTER 4 EVALUATION OF FRACTURE CRITERIA}

There have been many ductile fracture criteria proposed for metals. A few of those have been discussed in Chapter 2. Some of the criteria couple the state of damage to the material constitutive model, and thus increase the complexity in developing a procedure to generate different material stress-strain curves to be used in any parametric finite element analysis study. However in most situations, the damage is localized. Consequently, the localized strength degradation may not significantly affect the overall strength of a structural component. Furthermore, the level of damage may not be high before fracture occurs. For example, Fig. 4.1 shows the calculated initial slope (modulus) of the average true stress versus cross-sectional area change $\left[\ln \left(A_{0} / A\right)\right]$ curve from Fig. 4.2 during tension and compression loading at each cycle for notched specimen BBC70404. Specimen BBC70404 is considered to have fractured at cycle 64, however, no significant degradation of the modulus is seen in Fig. 4.1 until after the $64^{\text {th }}$ cycle during compression loading. This shows that the level of damage may not be large enough to affect the material behaviour before fracture. Thus, damage may be considered to uncouple from the constitutive model without significantly affecting the simulation of the material behaviour. For this reason, current study will focus only on fracture criteria that do not couple the state of damage to the material constitutive model.

Numerical analyses using material model parameters determined in Chapter 3 have been carried out to simulate the cylindrical specimen tests in order to assess the suitability of various fracture criteria in predicting fracture of steel subjected to monotonic tension loading as well as cyclic loading. 


\subsection{Evaluated Fracture Criteria}

In the preliminary study, nine fracture criteria have been assessed. These criteria cover the widely used void growth and continuum damage mechanics based criteria as well as other damage limits including those by Freudenthal (1950), Cockcroft and Latham (1968), Brozzo et al. (1972), Norris et al. (1978) and Oyane et al. (1980). Two criteria based on void growth proposed by Rice and Tracey (1969) have also been considered. One criterion neglects void closing in compression, and the other considers void closing when the mean stress is negative, as proposed by Kanvinde and Deierlein (2004, 2007). Two fracture criteria based on the damage evolution proposed by Lemaitre (1984) have also been considered. One of the criteria considered is adopted from the damage evolution proposed by Huang and Mahin (2008), and the other is a slight modification of Eq. (2.34) where the gross strain energy density is used in calculating the damage evolution as

$$
\begin{aligned}
& \mathrm{dD}=(\mathrm{cy})^{\mathrm{m}} \mathrm{d} \varepsilon_{\mathrm{eq}}^{\mathrm{p}}, \text { and } \\
& \mathrm{y}=\frac{\sigma_{\mathrm{eq}}^{2}}{2 \mathrm{E}}\left[\frac{2}{3}(1+v)+3(1-2 v)\left(\frac{\sigma_{\mathrm{m}}}{\sigma_{\mathrm{eq}}}\right)^{2}\right],
\end{aligned}
$$

where $c$ and $m$ are the material constants. The term (1-D) has been removed from Eq. (2.34) in Eq. (4.2). For cyclic loading, the damage evolution remains the same as

$$
\mathrm{dD}=\left\{\begin{array}{cc}
(\mathrm{cy})^{\mathrm{m}} \mathrm{d} \varepsilon_{\mathrm{eq}}^{\mathrm{p}} & \text { for } \frac{\sigma_{\mathrm{m}}}{\sigma_{\mathrm{cq}}}>-\frac{1}{3}, \text { and } \\
0 & \text { otherwise }
\end{array}\right.
$$

Unlike the traditional continuum damage mechanics, the state of damage is assumed to be uncoupled from the material constitutive relationship in the current adopted approach. 
Thus, c can be simply taken as 1 . Preliminary study and comparisons of the fracture prediction with these fracture criteria are described in Appendix C.

Based on the results of the preliminary study, it is decided to carry out the further assessment of the criteria by Kanvinde and Deierlein $(2004,2007)$ with void closing in compression and Huang and Mahin (2008) with gross strain energy density. Since both versions of the Huang and Mahin based criteria have been found to give almost the same fracture prediction, only the version that considers gross strain energy density is assessed further. However, in order to include the effect of $\sigma_{\mathrm{m}} / \sigma_{\mathrm{ey}}$ more generally, Eq. (4.2) is modified to a more general form as

$$
y=\frac{\sigma_{\mathrm{eq}}^{2}}{2 \mathrm{E}}\left[\frac{2}{3}(1+v)+3(1-2 v)\left(\frac{\sigma_{\mathrm{nt}}}{\sigma_{\mathrm{eq}}}\right)^{\mathrm{k}}\right] \text {, }
$$

where $\mathrm{k}$ is a material constant. Equation (4.4) is no longer the exact strain energy density. This modified Huang and Mahin criterion with gross strain energy density and the criterion by Kanvinde and Deierlein $(2004,2007)$ with void closing in compression are listed in Table 4.1.

\subsection{Methodology}

Twenty one specimens of ASTM A572 (ASTM 2007) Grade 50 steel listed in Table 3.1 and eighteen specimens of CSA G40.20/21 (CSA 2004) Grade 300W steel listed in Table 3.2 were tested in the study. All these specimens consist of monotonic and cyclic tests are used in the assessment of the fracture criteria. Numerical simulations of the specimens have been carried out with ABAQUS (Simulia 2009) using material model 
parameters with two kinematic hardening terms listed in Section 3.3.2.1.3. Details of the study are discussed in the following sections.

\subsubsection{Point of Fracture}

Bridgman (1947) found that the steel specimen was able to neck almost to a point when sufficiently large hydrostatic pressure was applied. Thus, it can be assumed that damage accumulation only occurs in the tension leg of the loading cycle. Except for the Kanvinde and Deierlein $(2004,2007)$ criterion with void closing in compression, all other criteria evaluated do not allow for damage recovery in compression.

As seen in Figs. 3.49 and 3.51, for monotonic tension tests and most cyclic tests, it is not difficult to identify the fracture point. Similarly, for some ultra-low cycle fatigue tests, as can be seen in Fig. 4.3, the load-displacement curve shows a sudden drop in the slope. The point at which the drop occurs can be considered as the point of fracture. The sharp drop of the curve beyond this point indicates that tearing occurred at the center of the specimen where crack is initiated. If the specimen does not experience any sharp load dropping but exhibit softening and pinching behaviour, as seen in Fig. 4.4, a criterion has been developed to help in determining the cycle number at fracture. The criterion considered fracture has occurred in the $i^{\text {th }}$ cycle if

$$
\left|\sigma_{\mathrm{e}}^{i+1}-\sigma_{\mathrm{e}}^{i}\right| \geq 0.01 \sigma_{\mathrm{e}}^{\max }
$$

where $\sigma_{e}^{i+1}$ and $\sigma_{e}^{i}$ are the engineering stresses at the end of $i+1^{\text {th }}$ and $i^{\text {th }}$ cycles respectively, $\sigma_{c}^{\max }$ is the maximum engineering stress in the cyclic test. Nevertheless, the specimen is also considered to have fractured if pinching appears on the compression leg of the stress-strain curve. The cycle number at fracture determined by Eq. (4.5) cannot be 
larger than that when pinching is first noticed on the stress-strain curve. For specimen BBC 70404, the cycle number determined by Eq. (4.5) happens to coincide with the cycle number when the slope (modulus) exhibits a sharp drop with the loading cycle, as seen in Fig. 4.1. The criterion based on Eq. (4.5) is used because it may be difficult to identify the loading cycle when pinching is first noticed on the stress-strain curve.

\subsubsection{Calculation of Damage Limit}

Since fracture of the specimen has been observed to initiate from the center at the mid-length, the stresses and strains of the middle node at the mid-length of the specimen (middle of the notch and necking) are used to assess the fracture criteria. A simple trapezoidal integration rule is employed to calculate the damage evolution and void growth as

$$
\int_{0}^{\varepsilon_{\mathrm{it}}^{\mathrm{p}}} \mathrm{f}(\sigma) \mathrm{d} \varepsilon_{\mathrm{eq}}^{\mathrm{p}} \approx \sum_{\mathrm{i}=1}^{\mathrm{m}}\left[\frac{\mathrm{f}(\sigma)_{\mathrm{i}-1}+\mathrm{f}(\sigma)_{\mathrm{i}}}{2}\right] \Delta \varepsilon_{\mathrm{eq}}^{\mathrm{p}},
$$

where $i$ is the current time increment step of the total $m$ increment steps, $f(\sigma)$ is the damage evolution function of damage criterion listed in Table $4.1, \Delta \varepsilon_{\mathrm{eq}}^{\mathrm{p}}$ is the change in the equivalent plastic strain at the increment step $i, \varepsilon_{\mathrm{eq}}^{\mathrm{nf}}$ is the equivalent plastic strain at fracture.

For each fracture criterion, the damage measurement at fracture $D_{c}$ or $\left(R_{c} / R_{0}\right)$ for each specimen is calculated with Eq. (4.6) up to the cross-sectional reduction equal to that of the test at fracture. The arithmetical average of the damage at fracture $\bar{D}_{c}$ or $\left(\overline{R_{c} / R_{0}}\right)$ of twenty one specimens of material $A$ and eighteen specimens of material $B$ is regarded as the material constant or damage limit of that fracture criterion for each 
material respectively. For criteria with parameters that can be varied, these parameters are adjusted until the error ER in the predicted damage at fracture is minimized according to

$$
E R=\sum_{j=1}^{p}\left|\frac{\left(D_{c}\right)_{j}-\bar{D}_{c}}{\bar{D}_{c}}\right|,
$$

where $\mathrm{j}$ is the specimen number for a total of $\mathrm{p}$ specimens. The predicted diameter and/or loading cycle at fracture for each specimen is assumed to occur when the accumulated damage reaches $\bar{D}_{c}$. For criterion by Kanvinde and Deierlein $(2004,2007)$, the parameter $\mathrm{c}$ can be factored out in the error minimization of Eq. (4.7) and thus is taken as 1. Similarly for the modified Huang and Mahin criterion with gross strain energy density, the parameter c can also be factored out and is taken as 1 in the calculation.

\subsubsection{Mesh Study of FEA Model}

A mesh study has also been carried out to ensure that the calculated damage is not significantly affected by a further mesh refinement or increased mesh density. Finite element analyses have been carried out using three profiles of straight and notched specimens covering monotonic tension and different cyclic loading conditions. Since materials $\mathrm{A}$ and $\mathrm{B}$ have quite a similar behaviour, the mesh study has been carried out with only properties and material model parameters for material A. Since there is only one specimen of profile D10R7N tested, no mesh study has been carried out for this profile. For this reason, the mesh density of profile DIOR14N is used for profile D10R7N shown in Fig. 3.9. 
Figure 4.5 shows the scheme-2 mesh for D14R24U and D14R19U straight specimens. Although not shown, its boundary condition is the same as the scheme-1 mesh in Fig. 3.10. The only difference between both meshes is that the element size of scheme-2 is half that of scheme-1. Figures 4.6 to 4.14 are results of FEA simulations on the engineering stress versus engineering strain, engineering stress versus cross-sectional area change and the calculated damage state D based on Eqs. (4.3) and (4.4) with $c=1, m$ $=3.07$ and $\mathrm{k}=1.44$ using both mesh schemes for various loading conditions. Similar comparisons for profiles D10R14N and D14R19N of notched specimens are shown in Appendix D. The comparisons show that the simulation results using scheme-1 and scheme- 2 meshes are almost identical. Thus, the mesh for scheme- 1 is considered to be sufficiently fine that no further refinement is required.

\subsection{Results of Fracture Prediction}

Detailed results of fracture prediction based on FEA simulations with two kinematic hardening terms using material model parameters listed in Section 3.3.2.1.3 are listed in Tables 4.2 to 4.5 , where $d_{0}$ is the initial diameter of the specimen, $d_{f}$ and $d_{p}$ are the measured and predicted diameters at fracture, $\varepsilon_{\mathrm{af}}$ and $\varepsilon_{\mathrm{ap}}$ are the measured and predicted accumulated engineering strain at fracture. Calculations of $d_{p} / d_{f},\left(d_{0}-d_{p}\right) /\left(d_{0}-d_{f}\right)$ and $\varepsilon_{\mathrm{ap}} / \varepsilon_{\mathrm{af}}$ are also listed in the tables. The cycle number followed by ' + ' indicates that fracture occurs when pulling the specimen after undergoing the indicated number of loading cycle.

For monotonic tension tests, the fracture criteria are evaluated based on the test results and predicted diameter at fracture, $d_{p} / d_{f}$ and $\left(d_{0}-d_{p}\right) /\left(d_{0}-d_{f}\right)$. However for cyclic 
tests, the cycle number at fracture can be a more important measurement than the diameter at fracture since the change in the diameter is small within the loading cycle. For the notched specimen, the ratio of predicted and measured accumulated engineering strain at fracture is also a good measurement for both monotonic tension and cyclic tests. For the criterion by Kanvinde and Deierlein $(2004,2007)$, the larger of the damage (void growth) value at fracture or at the end of the tension leg of the preceding loading cycle before fracture is listed in the tables because the damage value at fracture can become unreasonably small if the specimen fails immediately after the compression leg of the loading cycle.

For the criterion by Kanvinde and Deierlein $(2004,2007)$ with void closing in compression, the authors also proposed an additional modification to the fracture limit with Eq. (2.28) using the equivalent plastic strain accumulated in each tension leg of the loading cycle. However with this modification, the critical void growth index of the monotonic tension test $\mathrm{VGI}_{\text {monotonic }}^{\mathrm{c}}$ is assumed to be no less than that of the cyclic test $\mathrm{VGI}_{\text {cyclic }}^{\mathrm{c}}$. This contradicts the results for specimens of both materials $\mathrm{A}$ and $\mathrm{B}$ listed in Tables 4.2 and 4.4 where the damage (void growth) values at fracture for some cyclic tests are greater than that of the monotonic tension test. It can be seen in Tables 4.2 and 4.4 that the damage value for $\mathrm{ABC} 20404$ is much higher than $\mathrm{ABM}$ and $\mathrm{BBC} 20404$ is much higher than BBM. This shows that this modification is not suitable for general application. Thus, the modification with Eq. (2.28) is not explored further in this research.

As can be seen in the listed prediction results, both criteria are able to provide a reasonably good fracture prediction for monotonic tension tests of both straight and 
notched specimens. Although the criterion by Kanvinde and Deierlein $(2004,2007)$ with void closing in compression can predict the point of fracture for most of the cyclic tests reasonably well, it cannot predict the cycle number that specimens (A/B)ACR0804 fractured at with any reasonable accuracy. It can be seen that the predicted cycle number is more than twice that of the test. On the other hand, the modified Huang and Mahin criterion based on Eqs. (4.3) and (4.4) is able to predict the point of fracture for specimens (A/B)ACR0804 very well, and also provide a reasonable good prediction for other cyclic tests. The best value of the parameter $k$ in Eq. (4.4) for the modified criterion has been found to be 1.44 and 2.13 for materials $\mathrm{A}$ and $\mathrm{B}$ respectively. These $\mathrm{k}$ values provide a better fracture prediction compared to that if the value is fixed at 2 . Comparisons of test and predicted point of fracture on the stress-strain curves for a few notched specimens under monotonic tension and cyclic loading are shown in Figs. 4.15 to 4.18.

Using the damage limit determined from the proposed procedure, the modified Huang and Mahin criterion tends to overestimate the ductility of notched specimens under cyclic loading at very large strain ranges for specimens xxCF1212 and xxCF3010 and monotonic tension, but underestimate those cyclic tests of notched specimens at a moderate nominal true strain range of $\pm 4 \%$. Nevertheless, for notched specimens under monotonic tension and straight specimens, the predicted change in the diameter at fracture is within $10 \%$ of the measured value for most specimens except for $A B M$, which is within $15 \%$. Similarly for notched specimens under cyclic loading, the predicted accumulated engineering strain at fracture is within $10 \%$ of the measure value for most specimens except for ABC70404 and ABCF 1212, which are within 15\%, and BACF3010, 
BBCF3010 and BBC70404 at a ratio of $1.35,1.33$ and 0.82 for $\varepsilon_{\mathrm{ap}} / \varepsilon_{\mathrm{aff}}$. Specimens BACF3010 and BBCF3010 fractured in only 2 cycles while they are predicted to fracture in 3 cycles. If the specimen fractured at close to the end of the tension leg of the loading cycle, a small difference in the prediction can easily result in the specimen being predicted to fracture in the next cycle. Thus, this can significantly increase the predicted accumulated engineering strain at fracture relative to the measured value for a specimen which fractured at a very low number of cycle because normally failure is not predicted to occur immediately after the compression loading leg due to the damage evolution equation used. For BACF3010 and BBCF3010, most of the damage accumulation occurs in the second half of the tension loading leg of each loading cycle due to the increase in stress triaxiality as the cross-sectional area at the root of the notch decreases in size. Therefore, fracture is likely to be predicted to occur in the second half of the tension loading leg. This may partly explain for the much larger error in the ratio of $\varepsilon_{\mathrm{ap}} / \varepsilon_{\mathrm{af}}$ for specimens BACF3010 and BBCF3010 once fracture is predicted to occur at the loading cycle. Overall, the modified Huang and Mahin criterion based on Eqs. (4.3) and (4.4) provides a reasonably good prediction of the point of fracture for all specimens, and thus is considered as a suitable criterion to be used to predict fracture in the strap tension-only brace in the numerical simulations.

\subsection{Summary}

In the preliminary study described in Appendix $\mathrm{C}$, nine fracture criteria including void growth and continuum damage mechanics based criteria have been assessed to predict fracture of steel subjected to monotonic and cyclic loading. The preliminary 
study showed that the fracture criteria based on void growth with void closing in compression by Kanvinde and Deierlein $(2004,2007)$, and continuum damage mechanics by Huang and Mahin (2008) and its modification using gross strain energy density, are capable of providing a reasonably good fracture prediction for a limited number of tests. Nevertheless, both versions of the Huang and Mahin based criteria have been found to give almost the same fracture prediction. From results of the test, it has also been found that the level of damage may not be large enough to affect the material behaviour before fracture. This implies that damage may be uncoupled from the constitutive model without significantly affecting the simulation of material behaviour. Thus, only a further modified Huang and Mahin based criterion with gross strain energy density that allows for a more general consideration of the effect of stress triaxiality $\left(\sigma_{\mathrm{m}} / \sigma_{\mathrm{eq}}\right)$ and the criterion by Kanvinde and Deierlein $(2004,2007)$ with void closing in compression have been evaluated in this chapter.

Results of numerical simulations, which have been carried out with ABAQUS (Simulia 2009) using calibrated material model parameters listed in Table 3.6 for all the thirty nine round cross-sectional shape specimens were used in assessing both fracture criteria. The assessment showed that only the modified Huang and Mahin criterion was able to reasonably predict the point of fracture for all the tests. 
Table 4.1 Fracture criteria for assessment

\begin{tabular}{|c|c|}
\hline Criterion & Equation \\
\hline $\begin{array}{l}\text { Kanvinde and } \\
\text { Deierlein }(2004, \\
2007)\end{array}$ & 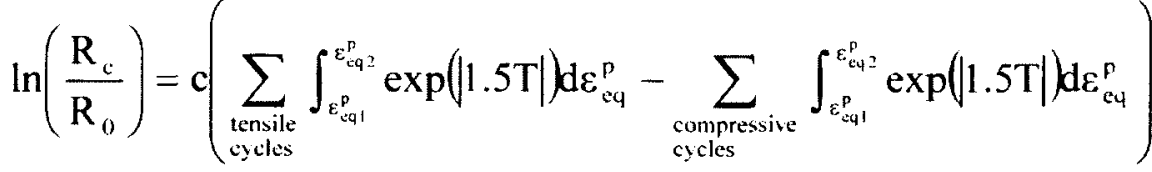 \\
\hline $\begin{array}{l}\text { Modified Huang } \\
\text { and Mahin with } \\
\text { gross strain } \\
\text { energy density }\end{array}$ & 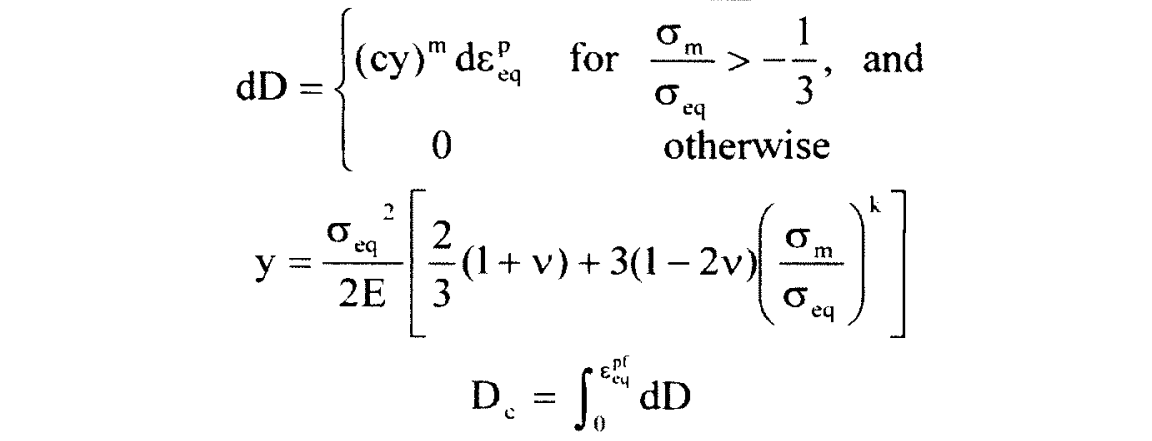 \\
\hline
\end{tabular}


Table 4.2 Test and predicted point of fracture for specimens of material A using the criterion by Kanvinde and Deierlein $(2004,2007)$ with void closing in compression and $\mathrm{c}=1$

\begin{tabular}{|c|c|c|c|c|c|c|c|c|c|c|c|}
\hline Specimen & $\begin{array}{l}\text { Test type } \\
\text { fracture }\end{array}$ & $\begin{array}{l}\text { Damage at } \\
\text { fracture }\end{array}$ & $\mathrm{d}_{0}(\mathrm{~mm})$ & $d_{f}(m m)$ & $d_{p}(m m)$ & $\mathrm{d}_{\mathrm{p}} / \mathrm{d}_{\mathrm{f}}$ & $\left(d_{0}-d_{p}\right) /\left(d_{0}-d_{i}\right)$ & $\begin{array}{c}\text { Predicted } \\
\text { fracture cycle }\end{array}$ & $\varepsilon_{\mathrm{af}}$ & $\varepsilon_{\text {ap }}$ & $\varepsilon_{\mathrm{at}} / \varepsilon_{\mathrm{ap}}$ \\
\hline ADMa & Monotonic & 2.84 & 14.00 & 8.51 & 8.84 & 1.04 & 0.94 & - & - & - & - \\
\hline $\mathrm{ADMb}$ & Monotonic & 2.81 & 14.00 & 8.54 & 8.84 & 1.04 & 0.94 & - & - & - & - \\
\hline $\mathrm{AEM}$ & Monotonic & 2.74 & 13.96 & 8.57 & 8.82 & 1.03 & 0.95 & - & - & - & - \\
\hline AAMa & Monotonic & 2.26 & 10.00 & 7.14 & 6.98 & 0.98 & 1.05 & - & 0.070 & 0.071 & 1.01 \\
\hline $\mathrm{AAMb}$ & Monotonic & 2.36 & 10.00 & 7.07 & 6.98 & 0.99 & 1.03 & - & 0.070 & 0.071 & 1.01 \\
\hline ABM & Monotonic & 2.10 & 14.00 & 10.18 & 9.79 & 0.96 & 1.10 & - & 0.094 & 0.099 & 1.06 \\
\hline ADC 20202 & 20 cycles + & 2.89 & 14.00 & 8.63 & 9.01 & 1.04 & 0.93 & $20+$ & - & - & - \\
\hline ADC 40101 & 40 cyclest & 2.51 & 14.00 & 8.97 & 8.99 & 1.00 & 1.00 & $40+$ & - & - & - \\
\hline ADC40102 & 40 cyclest & 2.73 & 14.00 & 8.78 & 9.02 & 1.03 & 0.95 & $40+$ & - & - & - \\
\hline ADC40202 & 40 cyclest & 2.76 & 14.00 & 8.75 & 9.02 & 1.03 & 0.95 & $40+$ & - & - & - \\
\hline ADCR0201 & 30 cycles + & 2.85 & 14.00 & 8.66 & 9.01 & 1.04 & 0.93 & $30+$ & - & - & - \\
\hline AAC20404 & 20 cycles + & 2.80 & 10.00 & 7.27 & 7.51 & 1.03 & 0.91 & $20+$ & 0.544 & 0.533 & 0.98 \\
\hline ABC 20404 & 20 cycles + & 3.00 & 14.00 & 10.04 & 10.57 & 1.05 & 0.87 & $20+$ & 0.805 & 0.792 & 0.98 \\
\hline ACC20404 & 20 cyclest & 2.59 & 10.00 & 7.92 & 8.00 & 1.01 & 0.96 & $20+$ & 0.441 & 0.437 & 0.99 \\
\hline $\mathrm{AAC} 70404$ & 70 cycles + & 2.71 & 10.00 & 7.94 & 8.16 & 1.03 & 0.90 & $70+$ & 1.729 & 1.720 & 0.99 \\
\hline ABC 70404 & 70 cyclest & 1.90 & 14.00 & 12.57 & 11.67 & 0.93 & 1.63 & $70+$ & 2.554 & 2.569 & 1.01 \\
\hline $\mathrm{AACF} 1212$ & 9 cycles & 2.12 & 10.00 & 9.57 & 8.83 & 0.92 & 2.75 & 10 & 0.621 & 0.706 & 1.14 \\
\hline $\mathrm{ABCF} 1212$ & 7 cycles & 2.13 & 14.00 & 12.38 & 12.08 & 0.98 & 1.18 & 8 & 0.734 & 0.838 & 1.14 \\
\hline AACF3010 & 3 cycles & 2.33 & 10.00 & 8.23 & 8.06 & 0.98 & 1.10 & 3 & 0.339 & 0.341 & 1.01 \\
\hline ABCF3010 & 3 cycles & 2.35 & 13.98 & 12.65 & 11.36 & 0.90 & 1.96 & 3 & 0.448 & 0.473 & 1.06 \\
\hline AACR0804 & 48 cycles & 1.45 & 10.00 & 9.59 & - & - & - & $>120$ & 1.350 & - & - \\
\hline \multicolumn{2}{|c|}{ Mean } & 2.49 & \multicolumn{9}{|c|}{-} \\
\hline \multicolumn{2}{|c|}{ Standard deviation $=$} & 0.39 & \multicolumn{4}{|c|}{ Coefficient of variation $=16 \%$} & \multicolumn{3}{|c|}{-} & & \\
\hline
\end{tabular}


Table 4.3 Test and predicted point of fracture for specimens of material A using the modified Huang and Mahin criterion with gross strain energy density, and $\mathrm{c}=1, \mathrm{~m}=3.07$ and $\mathrm{k}=1.44$

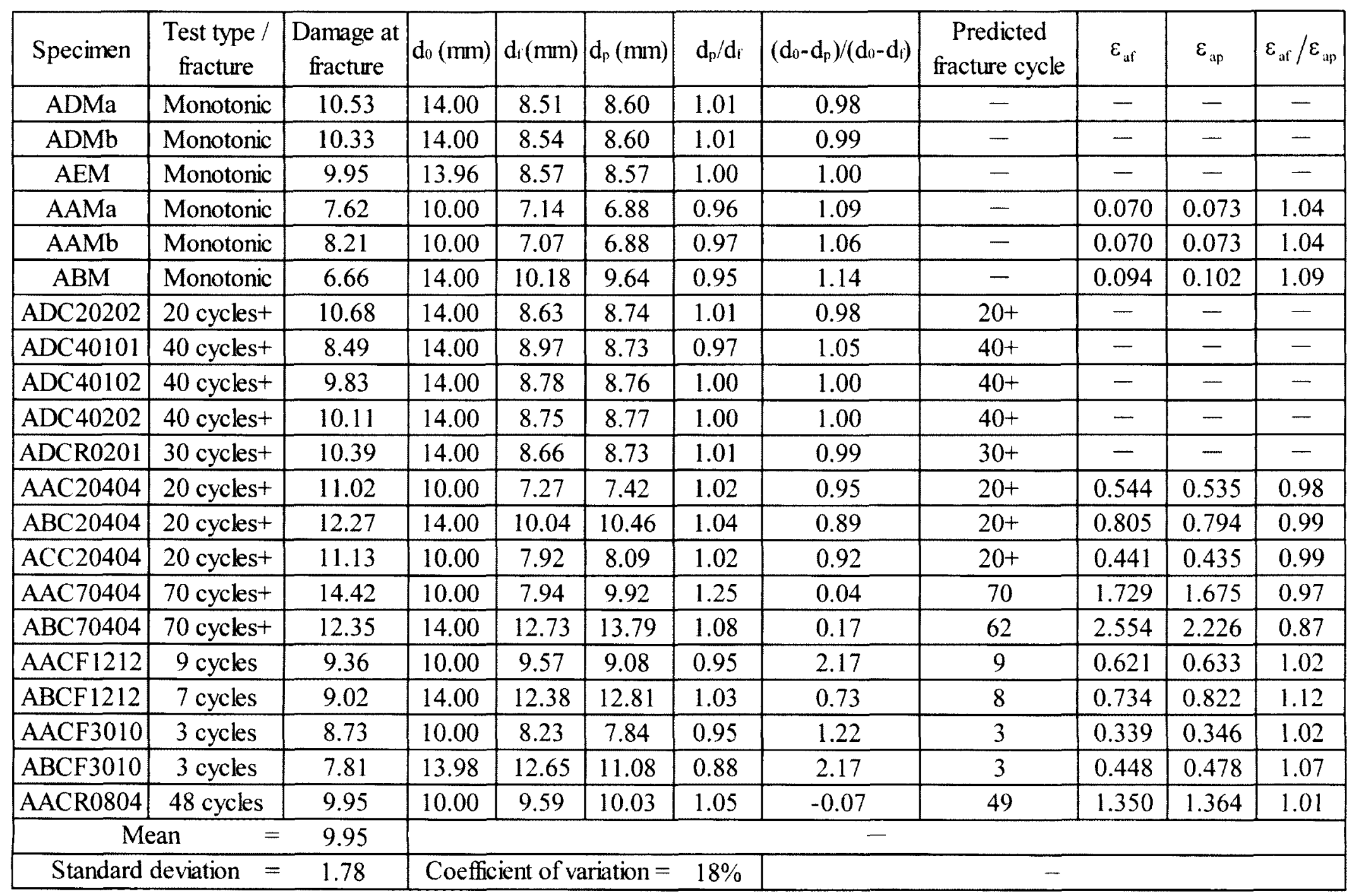


Table 4.4 Test and predicted point of fracture for specimens of material B using the criterion by Kanvinde and Deierlein $(2004,2007)$ with void closing in compression and $c=1$

\begin{tabular}{|c|c|c|c|c|c|c|c|c|c|c|c|}
\hline Specimen & $\begin{array}{l}\text { Test type / } \\
\text { fracture }\end{array}$ & $\begin{array}{c}\text { Damage at } \\
\text { fracture }\end{array}$ & $\mathrm{d}_{0}(\mathrm{~mm})$ & $d_{f}(m m)$ & $d_{p}(m m)$ & $\mathrm{d}_{\mathrm{p}} / \mathrm{d}_{\mathrm{i}}$ & $\left(\mathrm{d}_{0}-\mathrm{d}_{\mathrm{p}}\right) /\left(\mathrm{d}_{0}-\mathrm{d}_{\mathrm{f}}\right)$ & $\begin{array}{c}\text { Predicted } \\
\text { fracture cycle }\end{array}$ & $\varepsilon_{\mathrm{aft}}$ & $\varepsilon_{\text {ap }}$ & $\varepsilon_{\mathrm{ati}} / \varepsilon_{\mathrm{ap}}$ \\
\hline $\mathrm{BDMa}$ & Monotonic & 2.08 & 14.00 & 9.19 & 9.44 & 1.03 & 0.95 & - & - & - & - \\
\hline $\mathrm{BDMb}$ & Monotonic & 2.19 & 14.00 & 9.05 & 9.44 & 1.04 & 0.92 & - & - & - & - \\
\hline BAM & Monotonic & 1.85 & 10.00 & 7.40 & 7.38 & 1.00 & 1.01 & - & 0.069 & 0.066 & 0.95 \\
\hline $\mathrm{BBM}$ & Monotonic & 1.82 & 14.00 & 10.41 & 10.34 & 0.99 & 1.02 & - & 0.097 & 0.092 & 0.95 \\
\hline $\mathrm{BDC} 20202$ & 20 cyclest & 2.12 & 14.00 & 9.30 & 9.61 & 1.03 & 0.93 & $20+$ & - & - & - \\
\hline BDC40101 & 40 cyclest & 1.93 & 14.00 & 9.52 & 9.60 & 1.01 & 0.98 & $40+$ & - & - & - \\
\hline BDC 40102 & 40 cycles + & 2.12 & 14.00 & 9.30 & 9.62 & 1.03 & 0.93 & $40+$ & - & - & - \\
\hline BDC 40202 & 40 cyclest & 2.10 & 14.00 & 9.33 & 9.61 & 1.03 & 0.94 & $40+$ & - & - & - \\
\hline BDCR0201 & 30 cyclest & 2.06 & 14.00 & 9.37 & 9.61 & 1.03 & 0.95 & $30+$ & - & - & - \\
\hline BAC 20404 & 20 cycles + & 2.27 & 10.00 & 7.55 & 7.92 & 1.05 & 0.85 & $20+$ & 0.542 & 0.527 & 0.97 \\
\hline BBC 20404 & 20 cycles + & 2.38 & 14.00 & 10.49 & 11.13 & 1.06 & 0.82 & $20+$ & 0.809 & 0.784 & 0.97 \\
\hline BAC 70404 & 57 cycles & 0.99 & 10.00 & 9.73 & 8.79 & 0.90 & 4.51 & $70+$ & 1.374 & 1.708 & 1.24 \\
\hline BBC 70404 & 64 cycles & 1.26 & 14.00 & 13.57 & 12.57 & 0.93 & 3.30 & $70+$ & 2.313 & 2.553 & 1.10 \\
\hline BACF 1212 & 7 cycles & 1.80 & 10.00 & 9.03 & 9.22 & 1.02 & 0.81 & 8 & 0.496 & 0.565 & 1.14 \\
\hline $\mathrm{BBCF} 1212$ & 7 cycles & 1.81 & 14.00 & 12.83 & 12.62 & 0.98 & 1.18 & 7 & 0.720 & 0.730 & 1.01 \\
\hline BACF3010 & 2 cycles & 1.83 & 10.00 & 8.27 & 8.22 & 0.99 & 1.03 & 2 & 0.240 & 0.242 & 1.01 \\
\hline BBCF3010 & 2 cycles & 1.89 & 14.00 & 11.43 & 11.45 & 1.00 & 0.99 & 2 & 0.338 & 0.337 & 1.00 \\
\hline BACR0804 & 36 cycles & 1.26 & 10.00 & 9.63 & 9.69 & 1.01 & 0.84 & 75 & 1.062 & 1.998 & 1.88 \\
\hline $\mathrm{Me}$ & $\tan$ & 1.88 & \multirow{3}{*}{\multicolumn{9}{|c|}{ - }} \\
\hline Standard & deviation $=$ & 0.37 & & & & & & & & & \\
\hline Coefficient 0 & f variation $=$ & $20 \%$ & & & & & & & & & \\
\hline
\end{tabular}


Table 4.5 Test and predicted point of fracture for specimens of material B using the modified Huang and Mahin criterion with gross strain energy density, and $\mathrm{c}=1, \mathrm{~m}=2.35$ and $\mathrm{k}=2.13$

\begin{tabular}{|c|c|c|c|c|c|c|c|c|c|c|c|}
\hline Specimen & $\begin{array}{l}\text { Test type / } \\
\text { fracture }\end{array}$ & \begin{tabular}{|c} 
Damage at \\
fracture
\end{tabular} & $\mathrm{d}_{0}(\mathrm{~mm})$ & $d_{r}(m m)$ & $\mathrm{d}_{\mathrm{p}}(\mathrm{mm})$ & $\mathrm{d}_{\mathrm{p}} / \mathrm{d}_{\mathrm{i}}$ & $\left(\mathrm{d}_{0}-\mathrm{d}_{\mathrm{p}}\right) /\left(\mathrm{d}_{0}-\mathrm{d}_{\mathrm{i}}\right)$ & $\begin{array}{c}\text { Predicted } \\
\text { fracture cycle }\end{array}$ & $\varepsilon_{\mathrm{at}}$ & $\varepsilon_{\text {up }}$ & $\varepsilon_{\text {at }} / \varepsilon_{\text {ap }}$ \\
\hline BDMa & Monotonic & 2.29 & 14.00 & 9.19 & 9.15 & 1.00 & 1.01 & - & - & - & - \\
\hline $\mathrm{BDMb}$ & Monotonic & 2.50 & 14.00 & 9.05 & 9.15 & 1.01 & 0.98 & - & - & - & - \\
\hline BAM & Monotonic & 1.88 & 10.00 & 7.40 & 7.20 & 0.97 & 1.08 & - & 0.069 & 0.070 & 1.01 \\
\hline $\mathrm{BBM}$ & Monotonic & 1.82 & 14.00 & 10.41 & 10.09 & 0.97 & 1.09 & - & 0.097 & 0.097 & 1.00 \\
\hline BDC20202 & 20 cycles + & 2.35 & 14.00 & 9.30 & 9.30 & 1.00 & 1.00 & $20+$ & - & - & - \\
\hline BDC 40101 & 40 cycles + & 2.02 & 14.00 & 9.52 & 9.29 & 0.98 & 1.05 & $40+$ & - & - & - \\
\hline BDC40102 & 40 cyclest & 2.41 & 14.00 & 9.30 & 9.34 & 1.00 & 0.99 & $40+$ & - & - & - \\
\hline BDC 40202 & 40 cyclest & 2.42 & 14.00 & 9.33 & 9.37 & 1.00 & 0.99 & $40+$ & - & - & - \\
\hline BDCR0201 & 30 cycles + & 2.23 & 14.00 & 9.37 & 9.29 & 0.99 & 1.02 & $30+$ & - & - & - \\
\hline BAC20404 & 20 cycles + & 2.81 & 10.00 & 7.55 & 7.80 & 1.03 & 0.90 & $20+$ & 0.542 & 0.530 & 0.98 \\
\hline BBC 20404 & 20 cyclest & 3.03 & 14.00 & 10.49 & 10.98 & 1.05 & 0.86 & $20+$ & 0.809 & 0.787 & 0.97 \\
\hline $\mathrm{BAC} 70404$ & 57 cycles & 2.35 & 10.00 & 9.73 & 10.15 & 1.04 & -0.55 & 58 & 1.374 & 1.388 & 1.01 \\
\hline BBC 70404 & 64 cycles & 2.93 & 14.00 & 13.57 & 14.04 & 1.03 & -0.09 & 53 & 2.313 & 1.905 & 0.82 \\
\hline BACF 1212 & 7 cycles & 2.35 & 10.00 & 9.03 & 10.39 & 1.15 & -0.41 & 8 & 0.496 & 0.536 & 1.08 \\
\hline BBCF 1212 & 7 cycles & 2.51 & 14.00 & 12.83 & 13.44 & 1.05 & 0.48 & 7 & 0.720 & 0.711 & 0.99 \\
\hline BACF3010 & 2 cycles & 2.02 & 10.00 & 8.27 & 9.03 & 1.09 & 0.56 & 3 & 0.240 & 0.325 & 1.35 \\
\hline $\mathrm{BBCF} 3010$ & 2 cycles & 2.04 & 14.00 & 11.43 & 12.74 & 1.11 & 0.49 & 3 & 0.338 & 0.449 & 1.33 \\
\hline BACR0804 & 36 cycles & 2.31 & 10.00 & 9.63 & 9.73 & 1.01 & 0.73 & 37 & 1.062 & 1.085 & 1.02 \\
\hline $\mathrm{Me}$ & ean & 2.35 & \multirow{3}{*}{\multicolumn{9}{|c|}{ - }} \\
\hline Standard & deviation & 0.33 & & & & & & & & & \\
\hline Coefficient o & f variation $=$ & $14 \%$ & & & & & & & & & \\
\hline
\end{tabular}




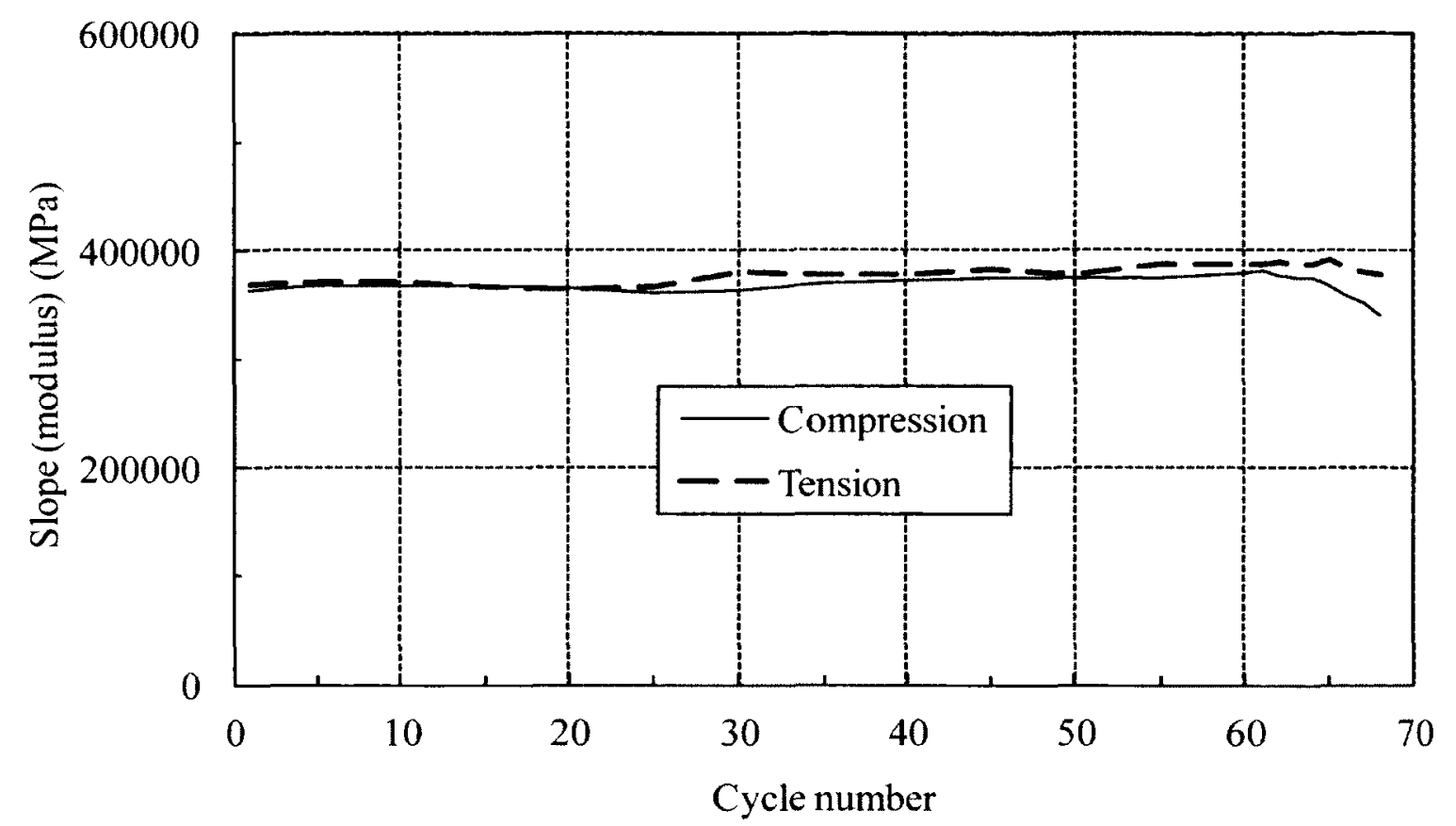

Figure 4.1 Initial slope (modulus) of the average true stress versus cross-sectional area change $\left[\ln \left(\mathrm{A}_{0} / \mathrm{A}\right)\right]$ curve for notched specimen $\mathrm{BBC} 70404$ at each cycle of loading

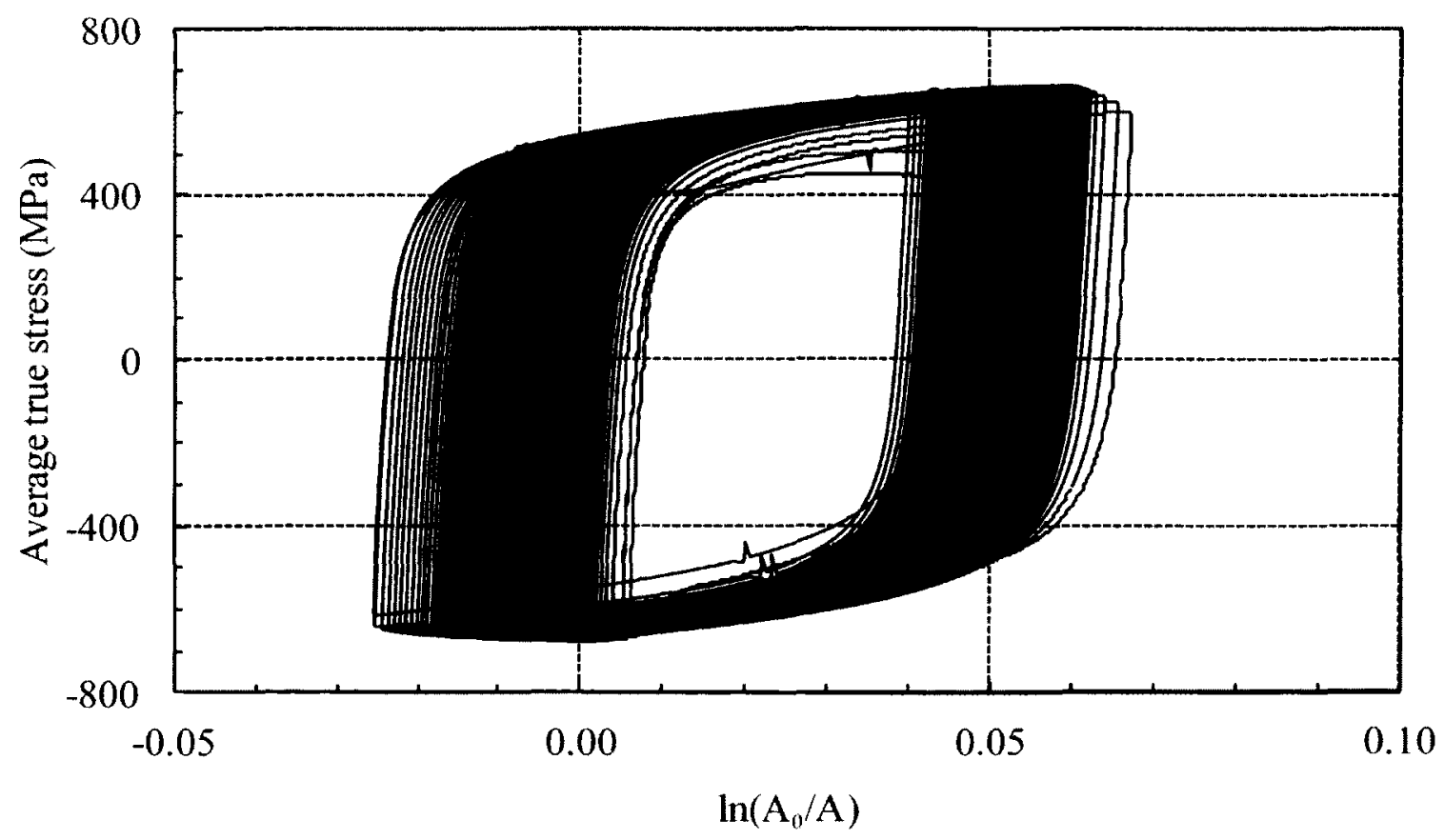

Figure 4.2 Average true stress versus cross-sectional area change curve for notched specimen BBC70404 


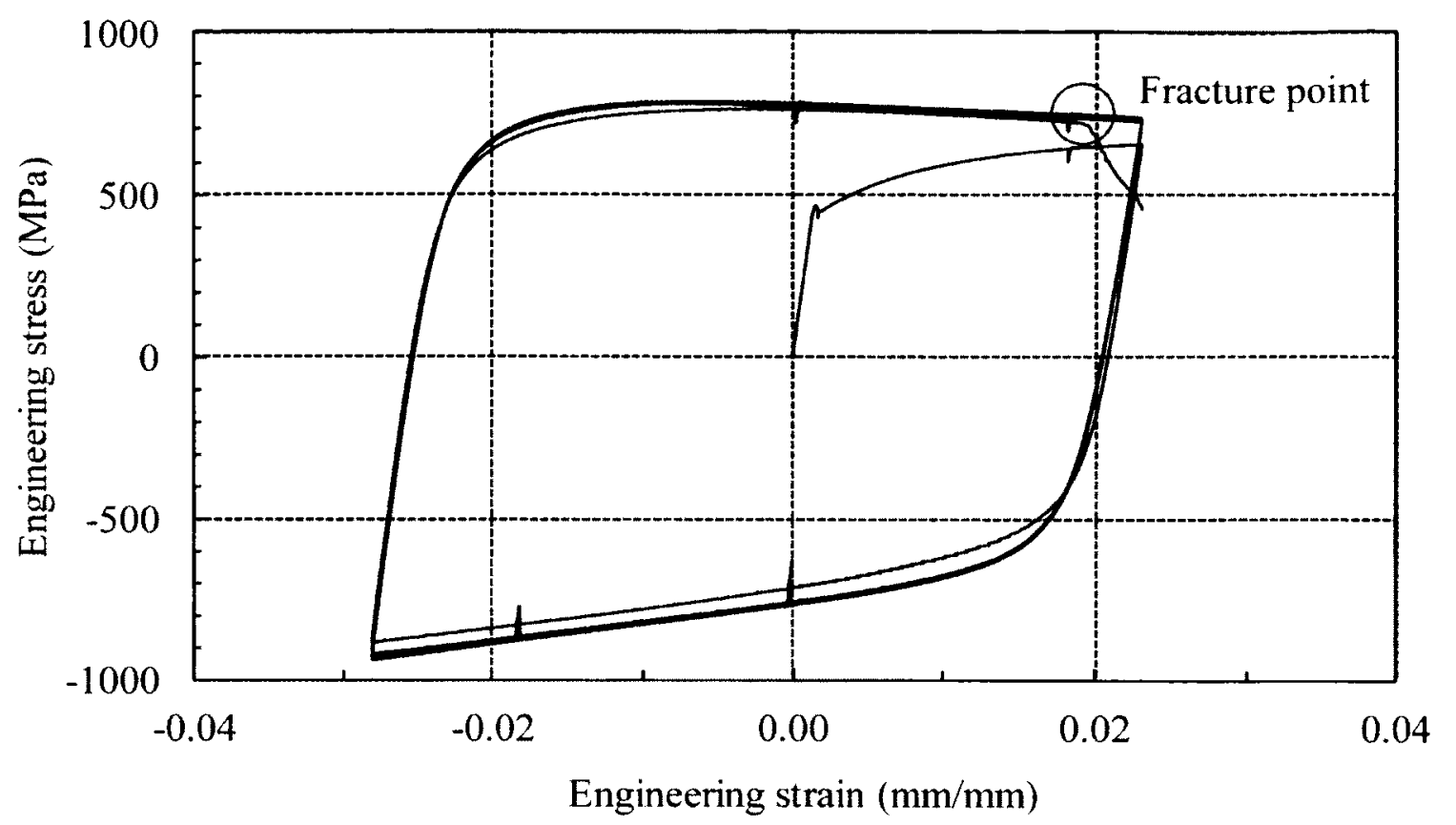

Figure 4.3 Fracture point for notched specimen ABCF 1212

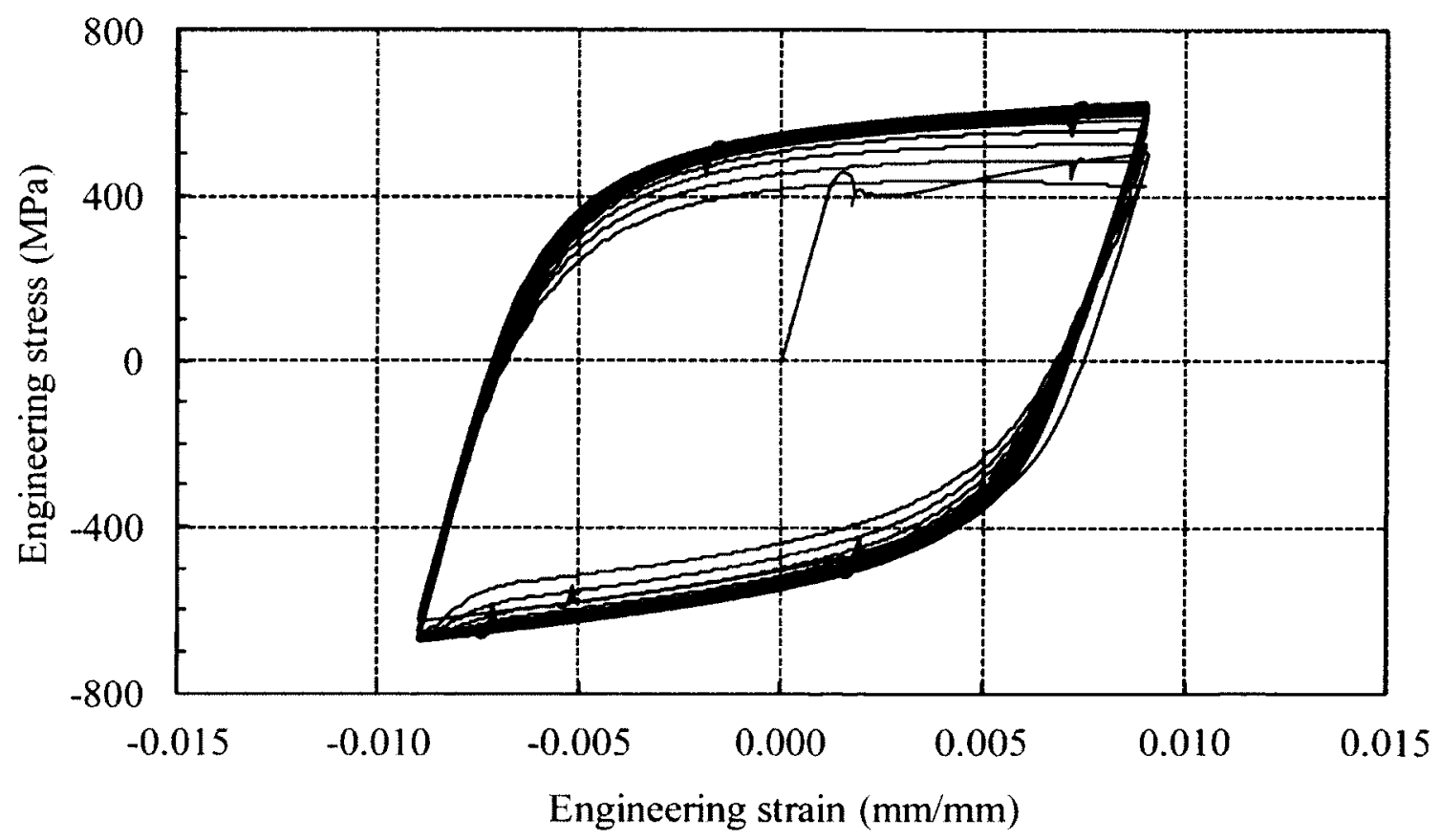

Figure 4.4 Softening and pinching behaviours observed in the test of notched specimen BBC70404 

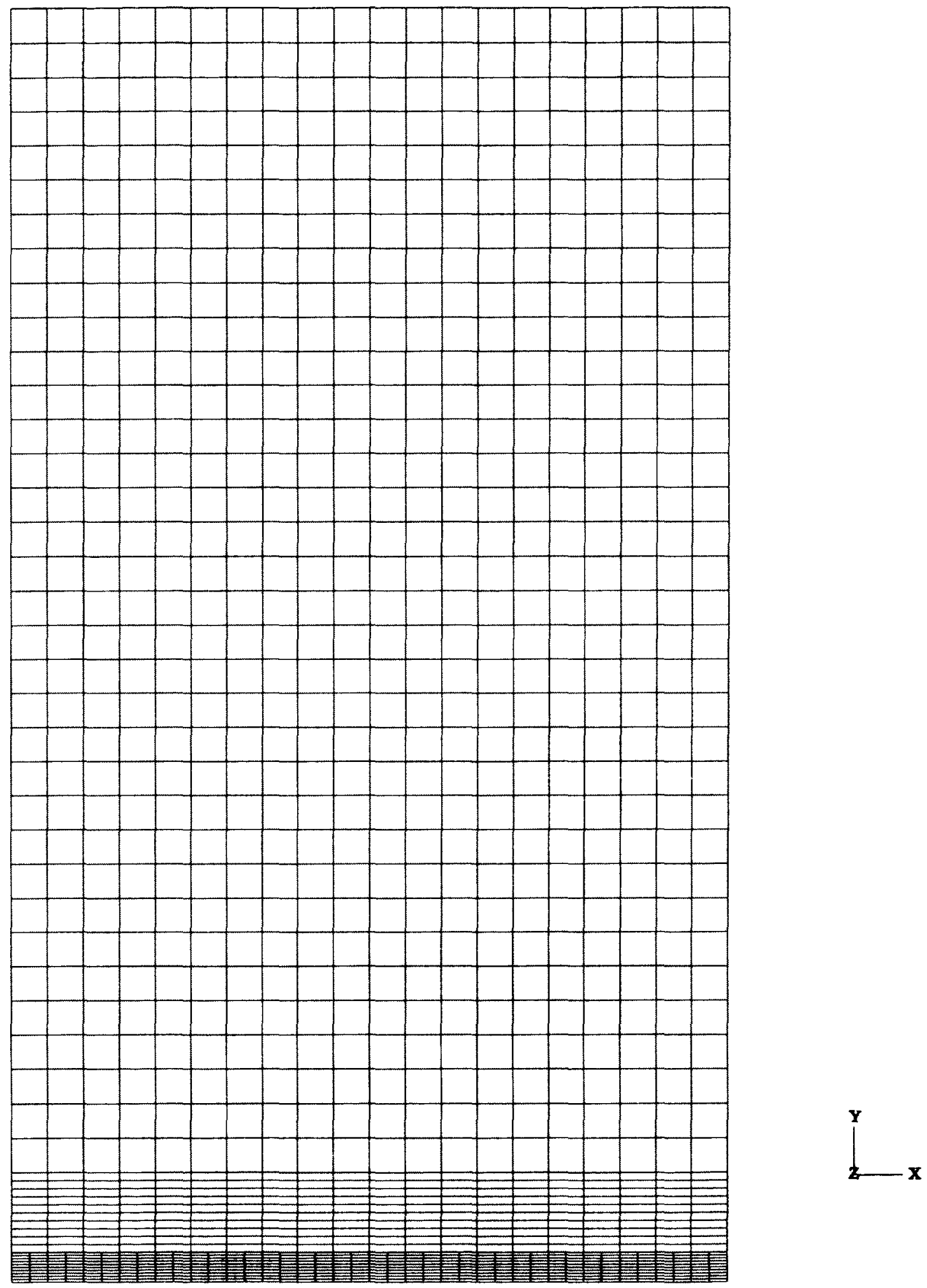

Figure 4.5 Scheme-2 mesh of $12.7 \mathrm{~mm}$ (half-inch) FEA model for D14R24U and D14R19U straight specimens (boundary condition not shown) 


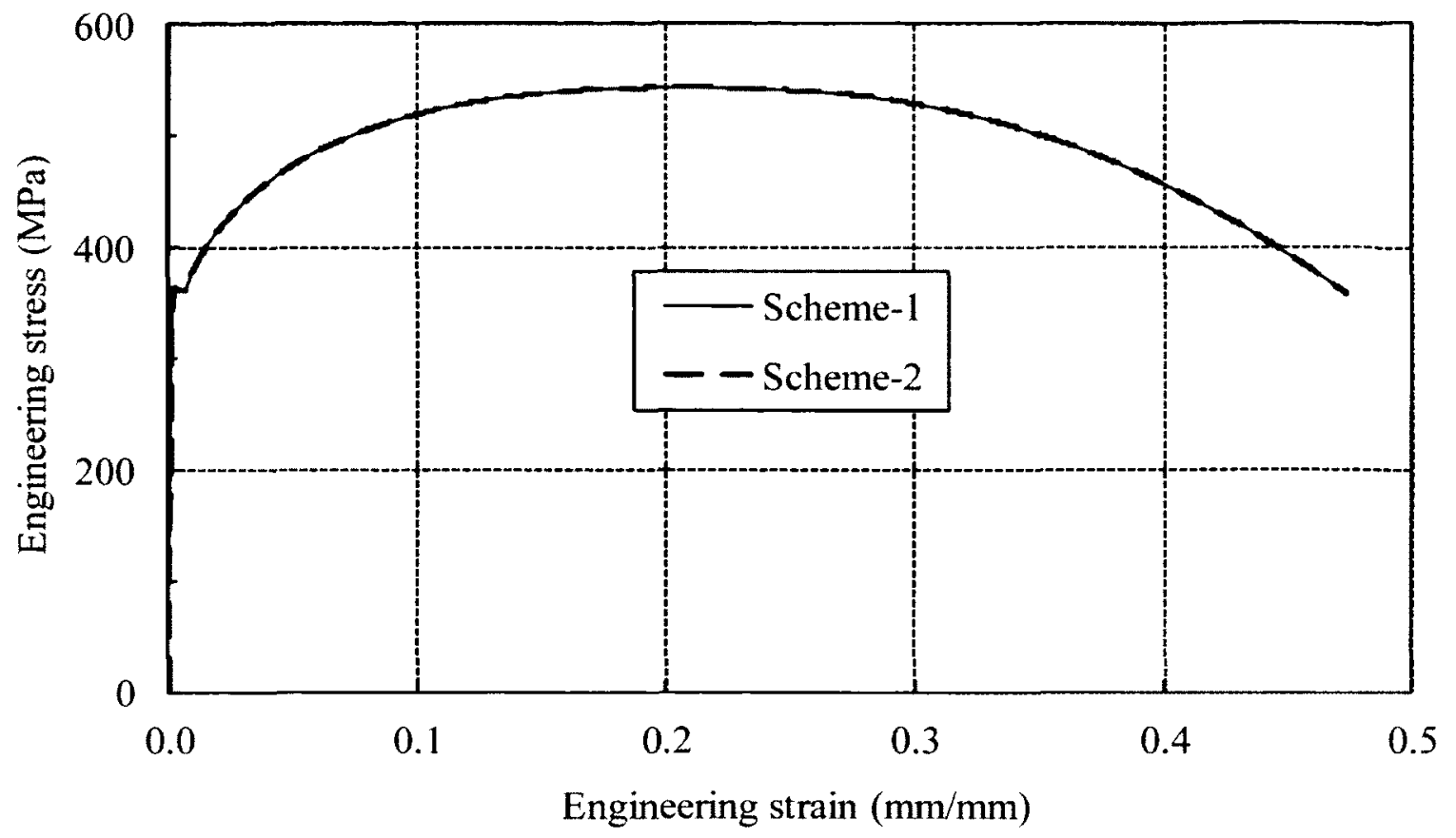

Figure 4.6 Engineering stress versus engineering strain curves from FEA simulations for straight specimens ADMa and ADMb with two mesh schemes

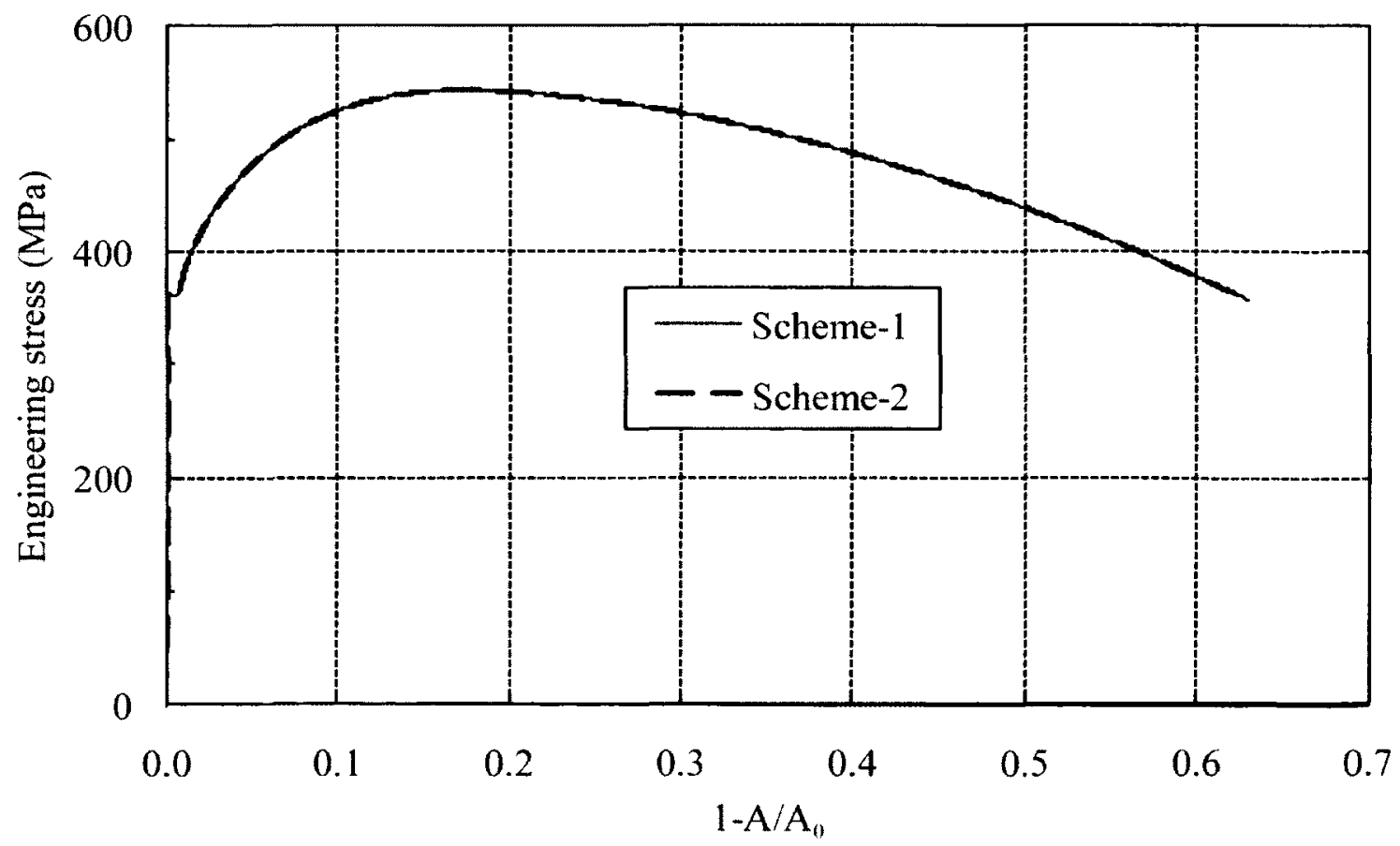

Figure 4.7 Engineering stress versus cross-sectional area change curves from FEA simulations for straight specimens ADMa and ADMb with two mesh schemes 


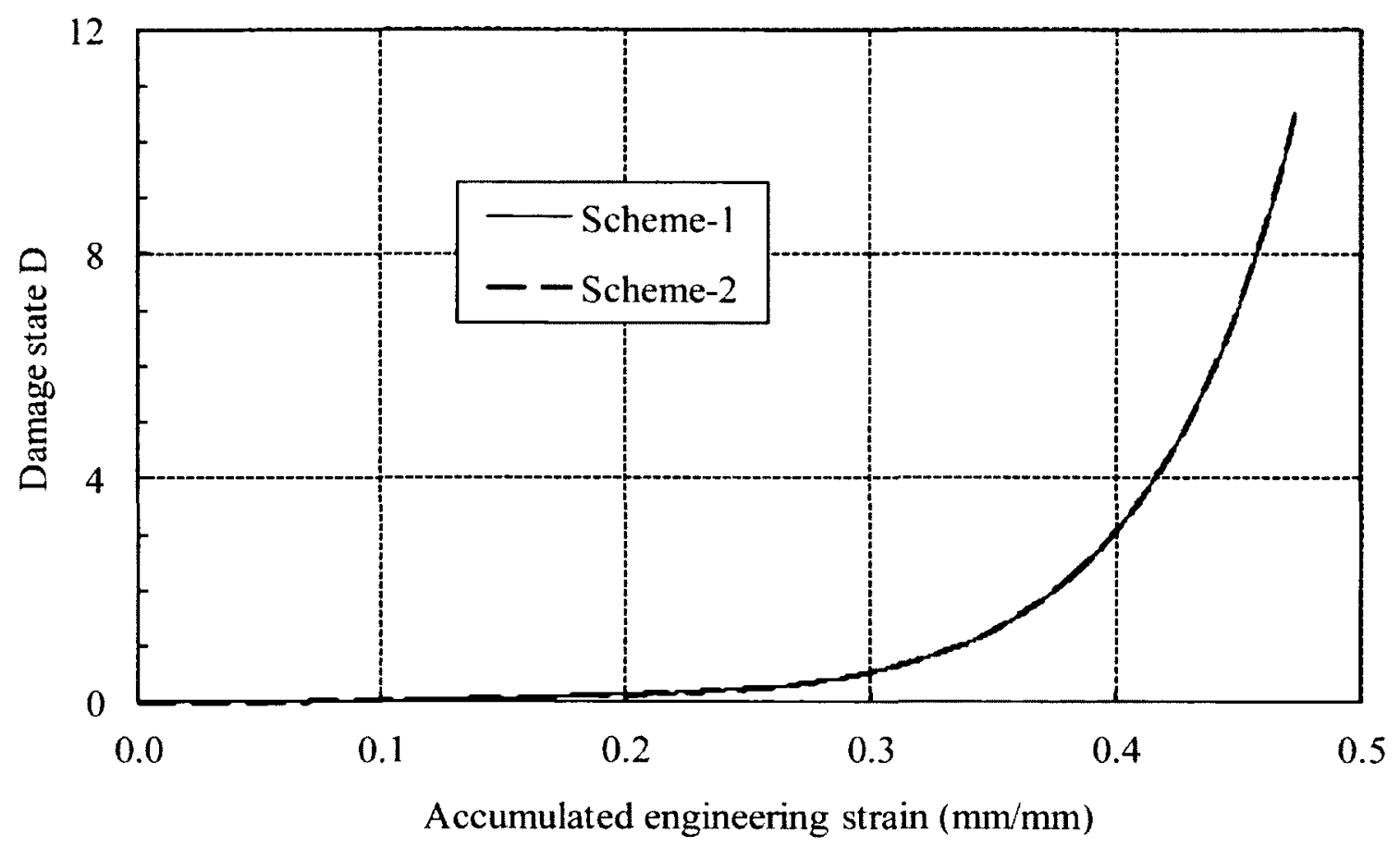

Figure 4.8 Calculated damage state D from FEA simulations for straight specimens $\mathrm{ADMa}$ and $\mathrm{ADMb}$ with two mesh schemes

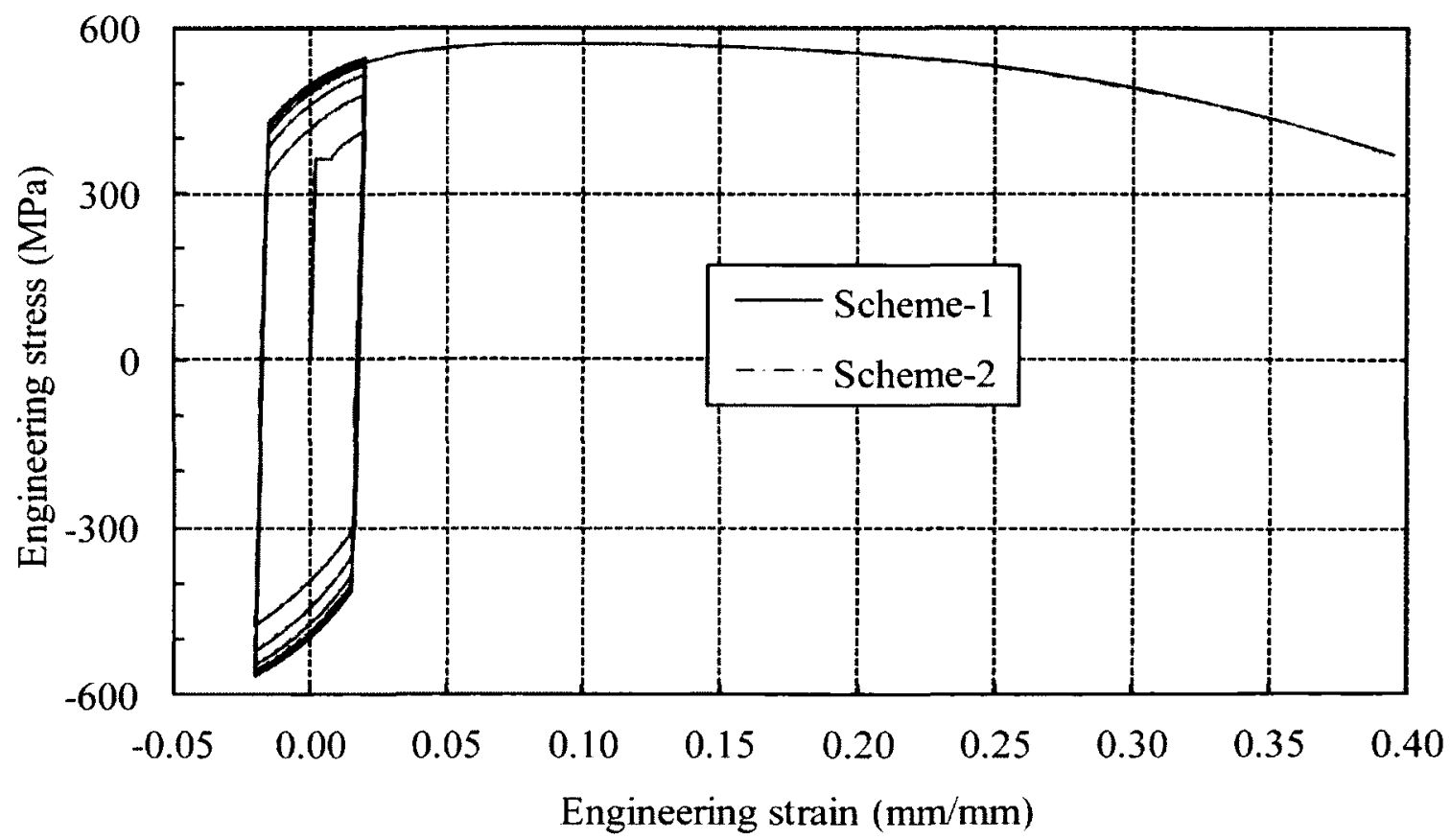

Figure 4.9 Engineering stress versus engineering strain curves from FEA simulations for straight specimen ADC40202 with two mesh schemes 


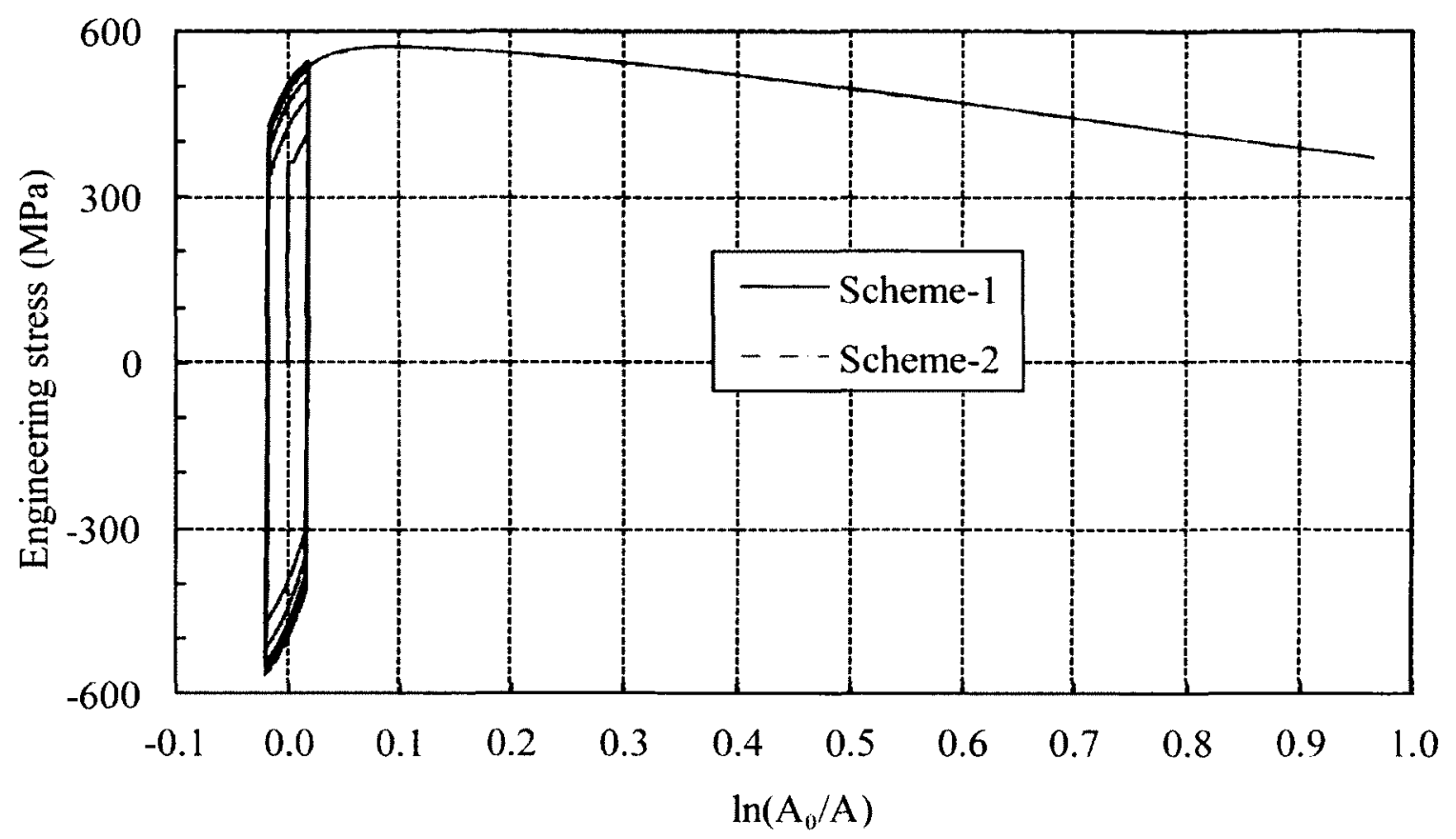

Figure 4.10 Engineering stress versus cross section area change curves from FEA simulations for straight specimen ADC40202 with two mesh schemes

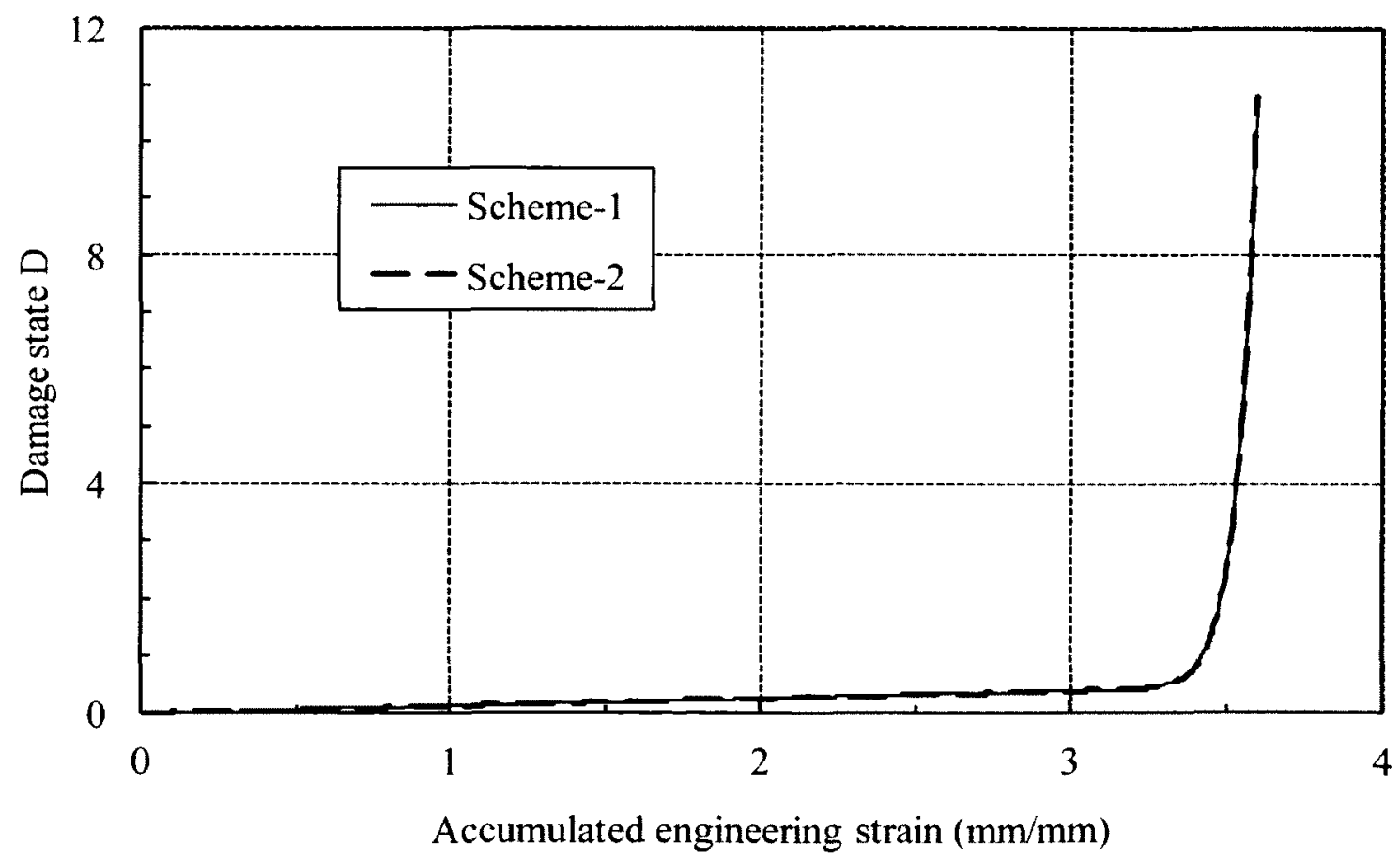

Figure 4.11 Calculated damage state D from FEA simulations for straight specimen ADC40202 with two mesh schemes 


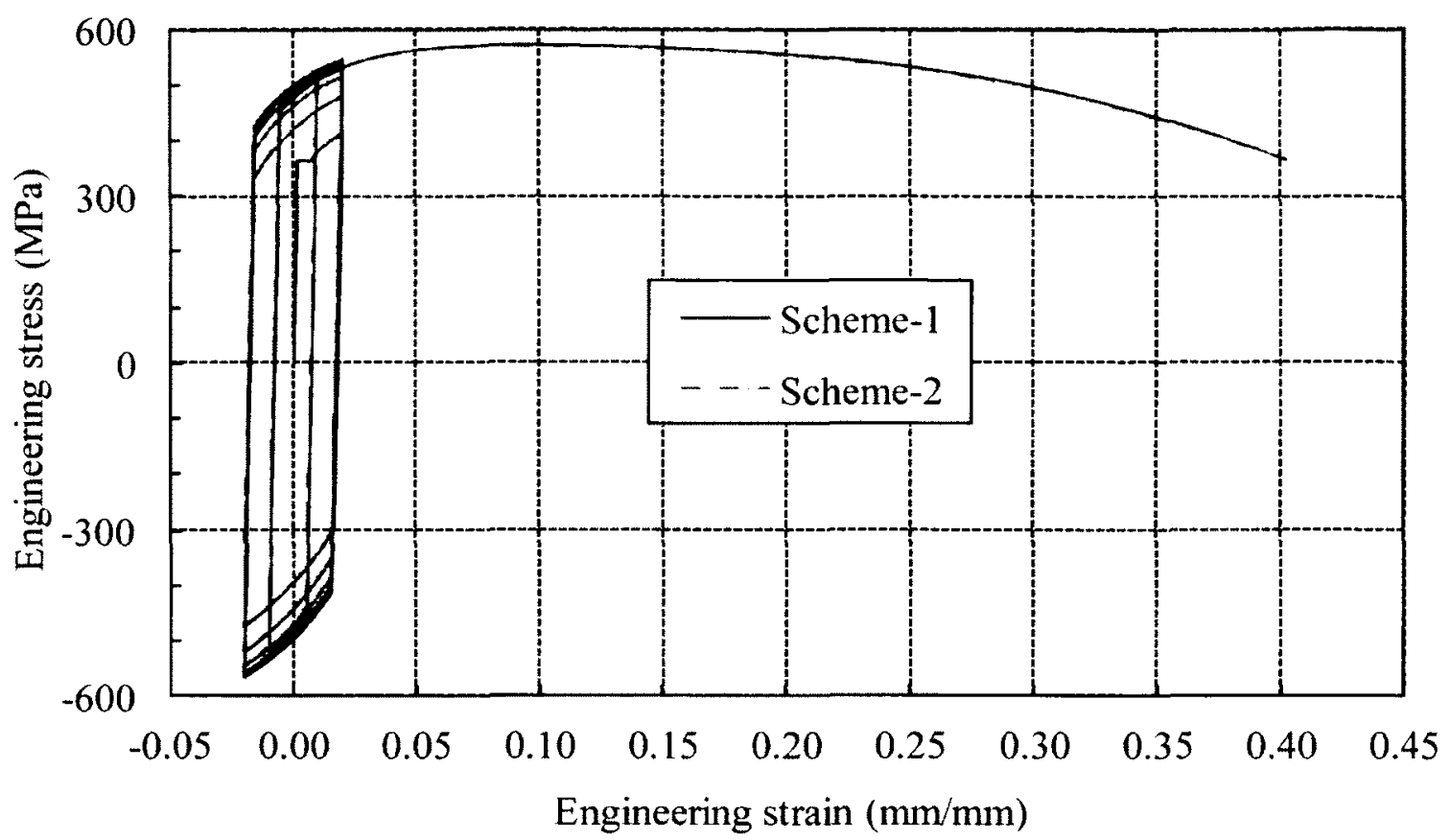

Figure 4.12 Engineering stress versus engineering strain curves from FEA simulations for straight specimen ADCR0201 with two mesh schemes

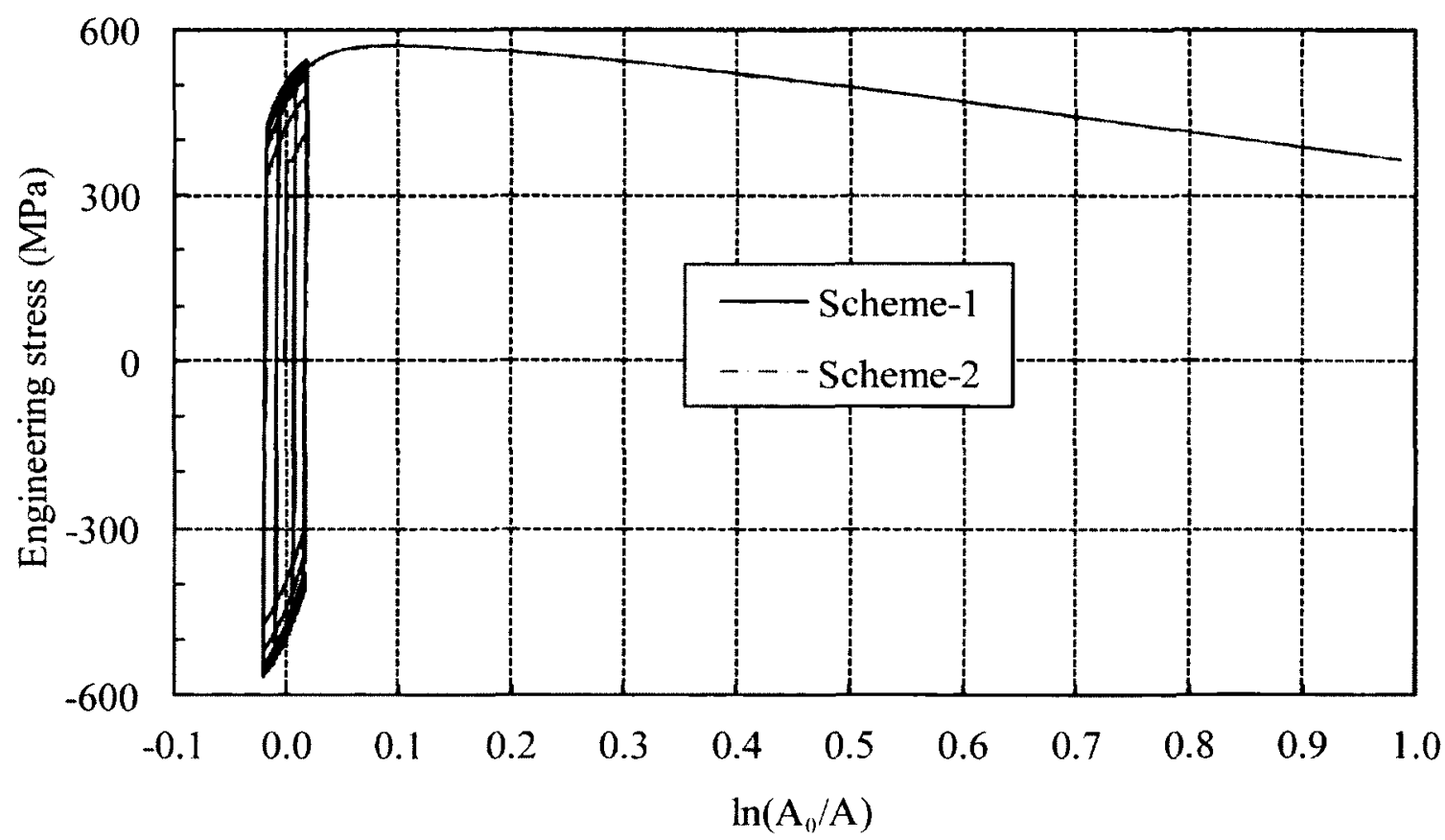

Figure 4.13 Engineering stress versus cross section area change curves from FEA simulations for straight specimen ADCR0201 with two mesh schemes 


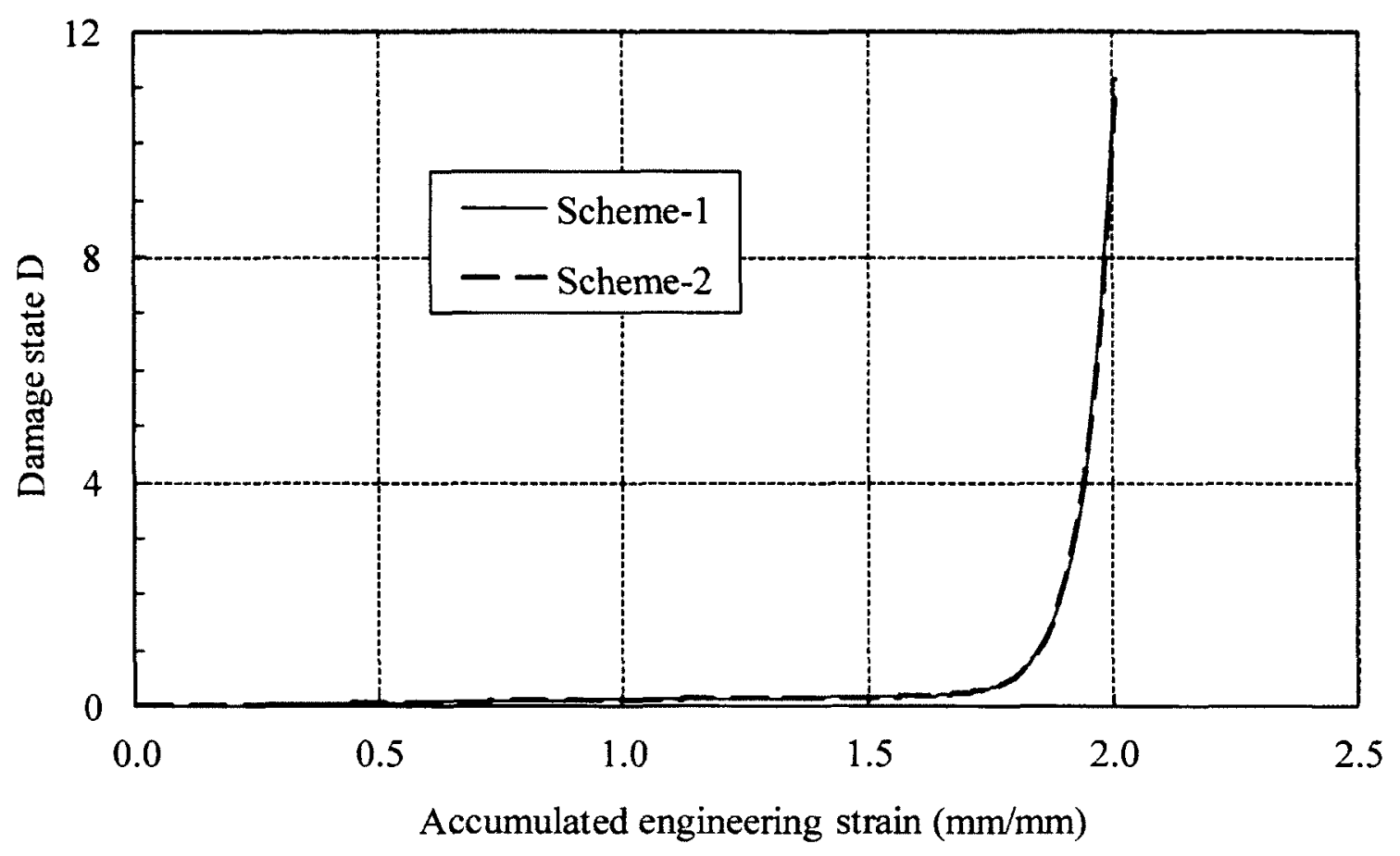

Figure 4.14 Calculated damage state D from FEA simulations for straight specimen ADCR0201 with two mesh schemes

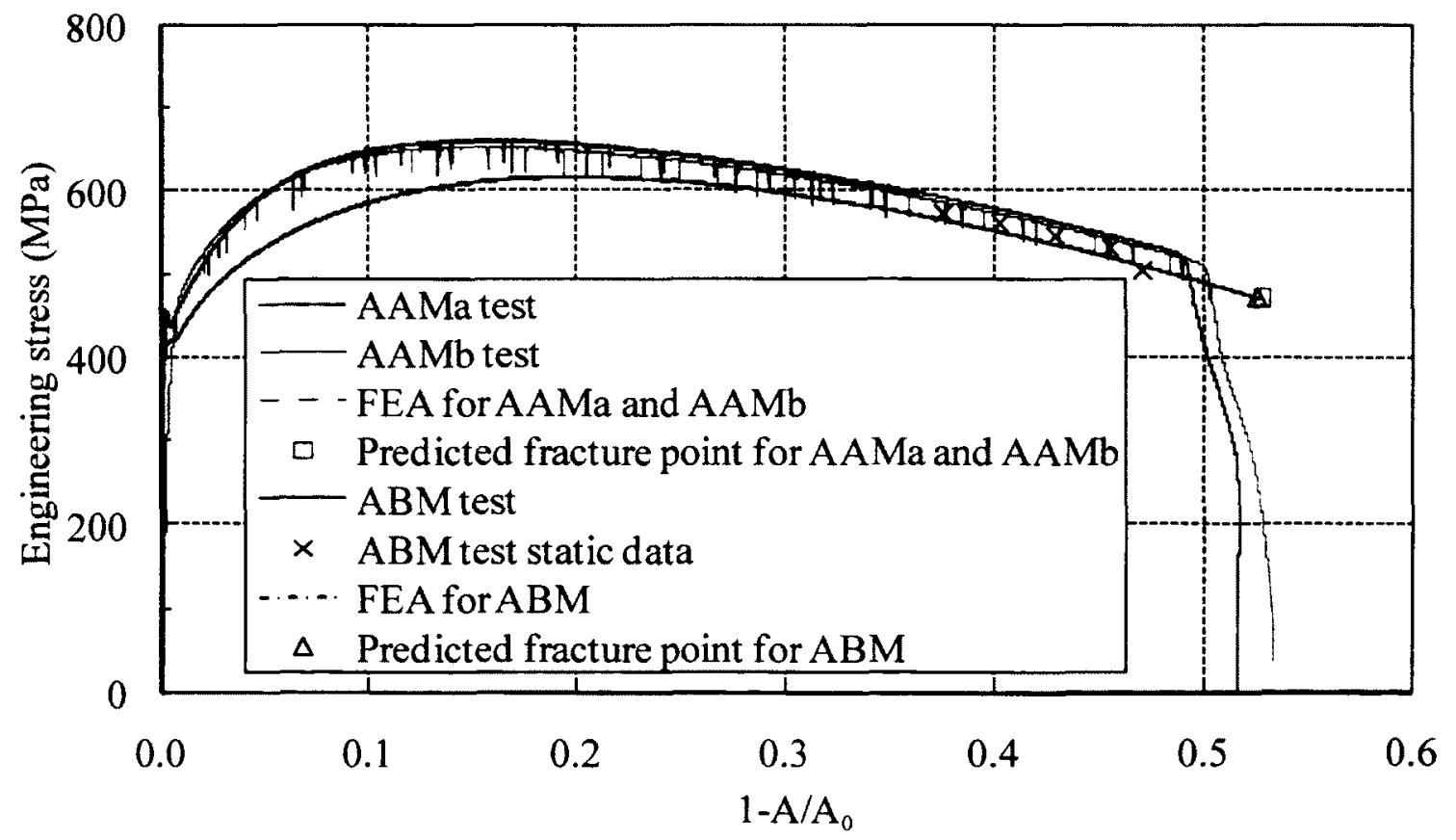

Figure 4.15 Test and predicted fracture point for notched specimens AAMa, AAMb and $\mathrm{ABM}$ 


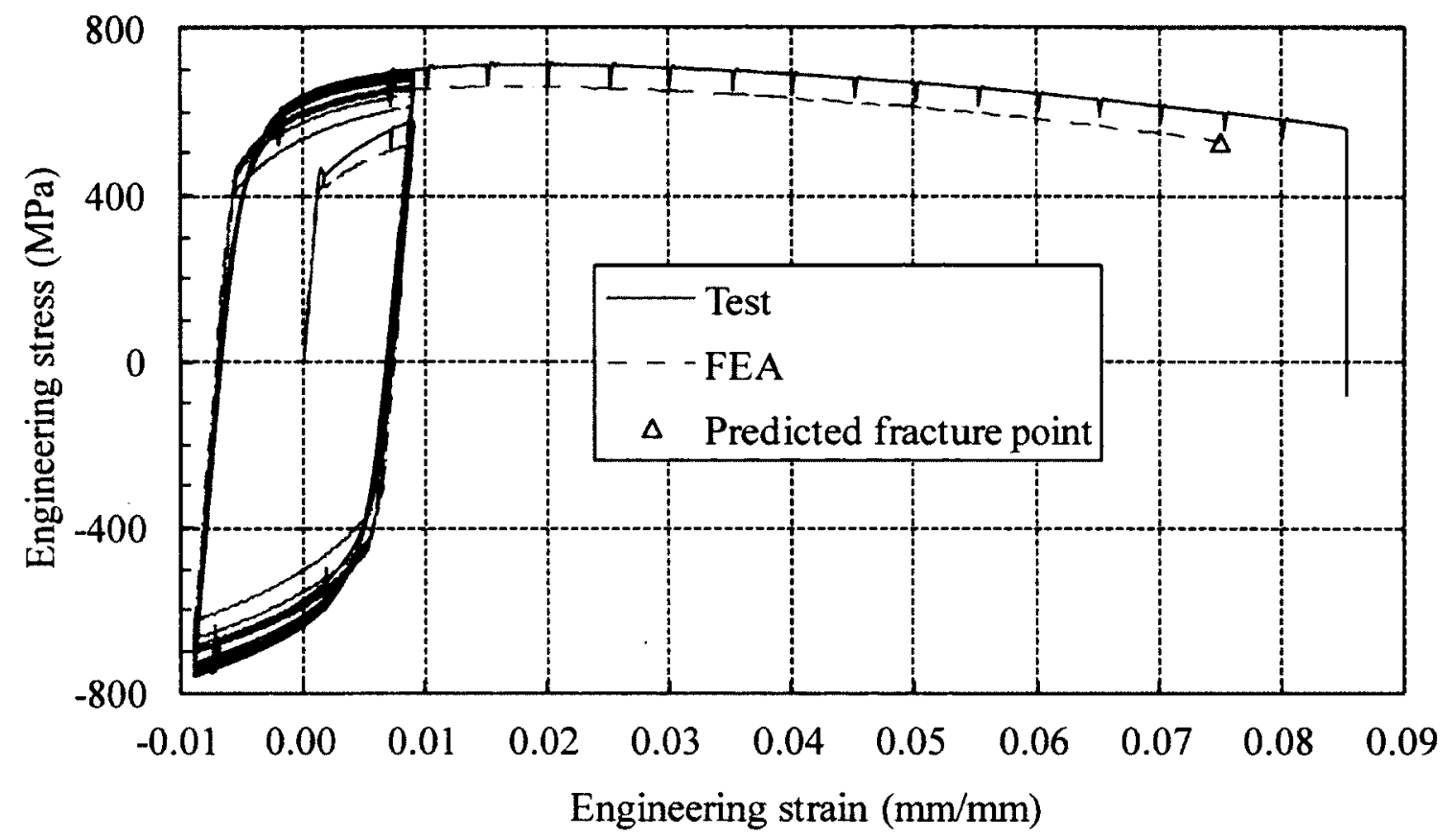

Figure 4.16 Test and predicted fracture point for notched specimen ABC20404

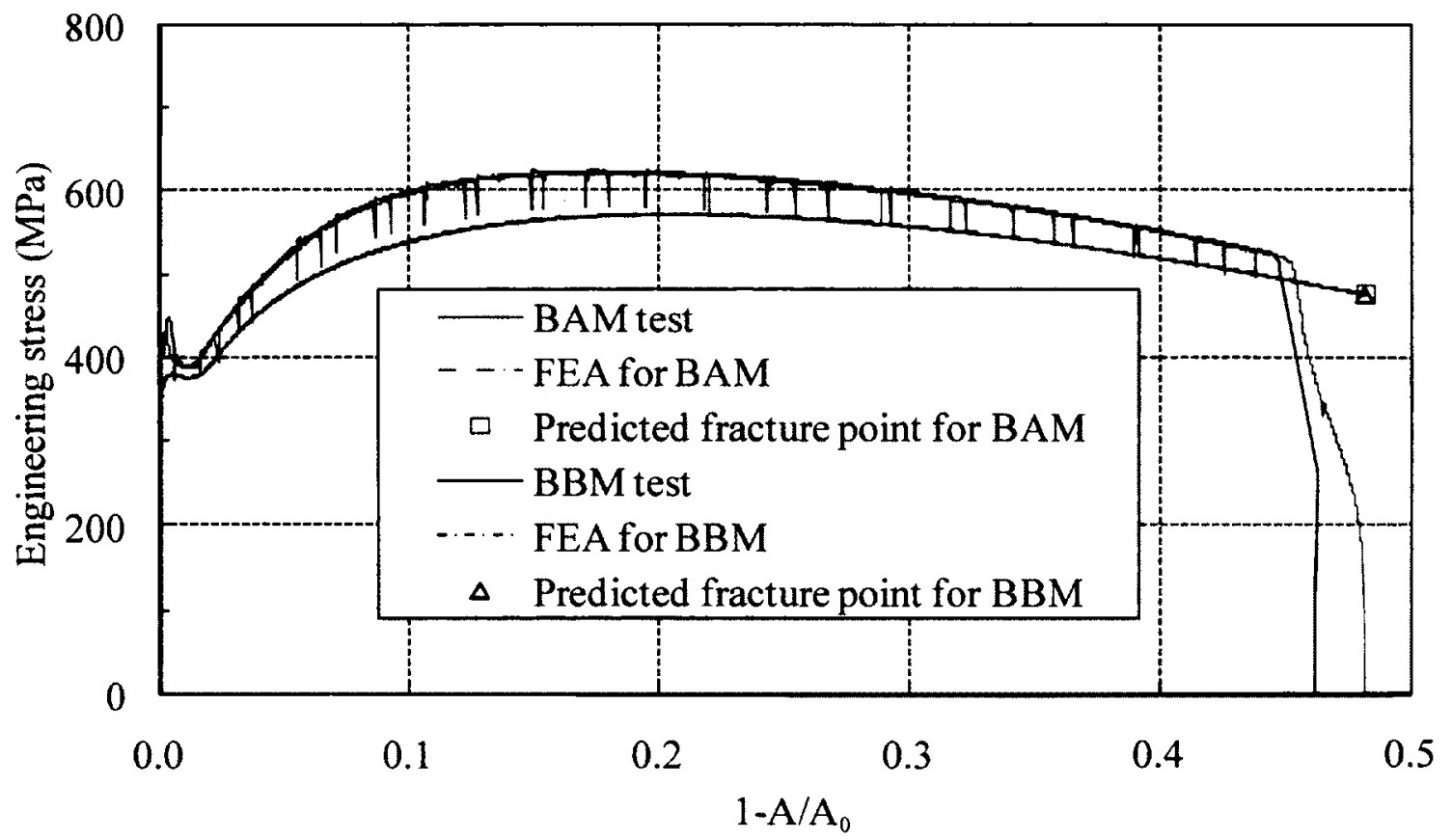

Figure 4.17 Test and predicted fracture point for notched specimens BAM and BBM 


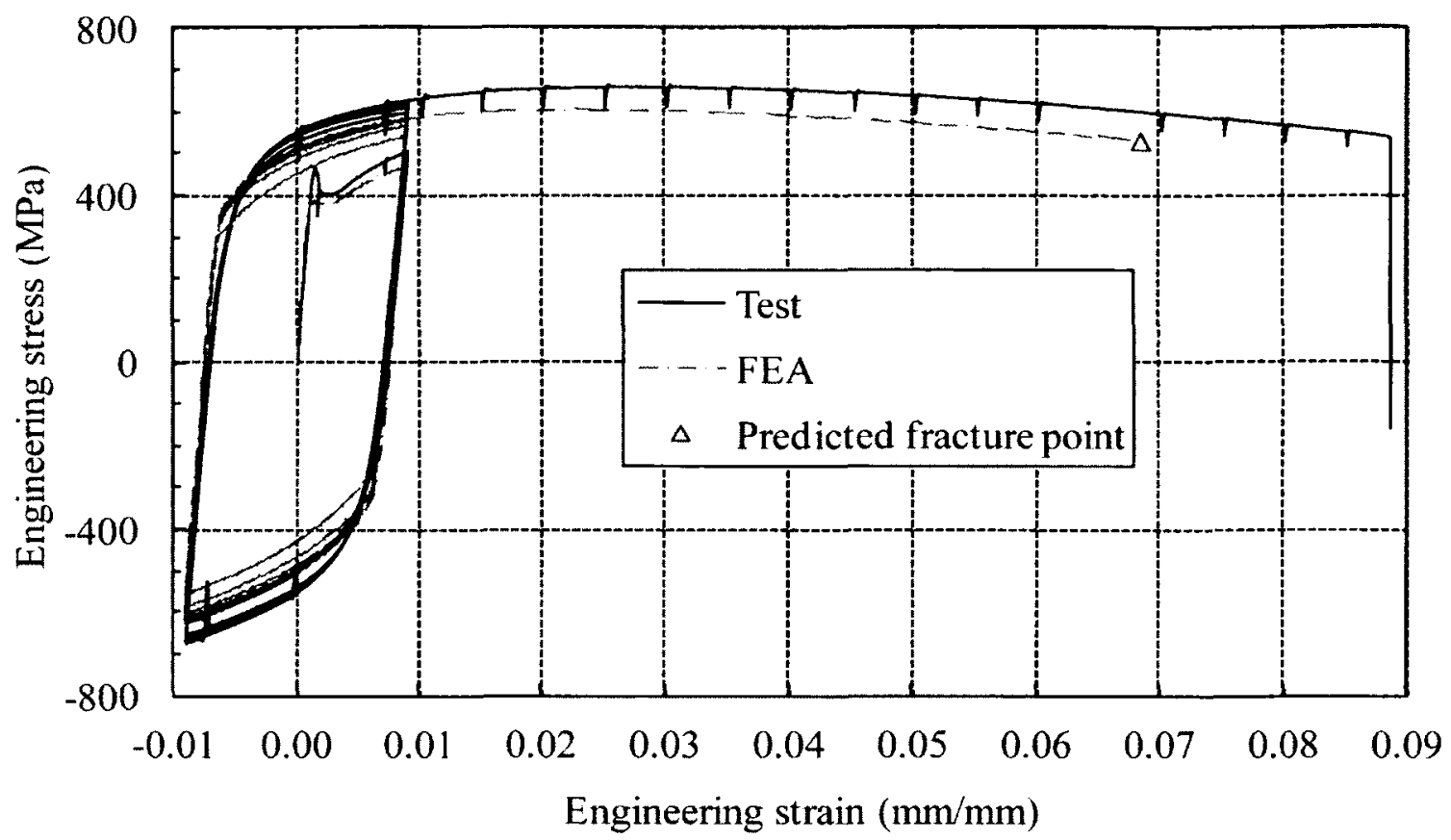

Figure 4.18 Test and predicted fracture point for notched specimen BBC20404 


\section{CHAPTER 5 SIMULATIONS OF STRAP TENSION-ONLY BRACE}

Vertical bracing configurations shown in Fig. 5.1 are commonly used in steel frames to provide lateral support. When designed as a member of Seismic Force Resisting System, energy is dissipated through inelastic deformation of a bracing member during an earthquake. The brace is required to have the ductility to dissipate energy during an earthquake. A stocky brace will resist the loading in both compression and tension that eventually leads to the formation of plastic hinges. The localization of the deformation to the plastic hinges will accelerate the failure of the bracing system. However, using a tension-only brace can delay the formation of plastic hinges and improve the ductility of the bracing system as the brace can buckle elastically up to a larger compressive deformation.

In the National Building Code of Canada (NBCC) 2010 (NRCC 2010), a normal building is permitted to deflect up to $2.5 \%$ in storey drift due to seismic load. Ideally, a brace in a Seismic Force Resisting System should be able to sustain this deformation under cyclic loading without any significant loss of strength.

Aghdam (2009) has conducted an experimental study on slender tension-only braces fabricated with steel straps. However, the experimental study can only investigate limited parametric combinations of the brace member. Therefore, the study on tensiononly brace for wider parametric combinations is being conducted through numerical testing. In this chapter, modeling of the strap specimen and the approximation of the material properties to be used in the numerical simulations are discussed. The modified Huang and Mahin fracture criterion based on Eqs. (4.3) and (4.4), and the mixed-mode 
hardening model by Chaboche (1986) with two kinematic hardening terms are used together in the numerical simulations of the tests by Aghdam (2009).

\subsection{Experimental Study on Strap Tension-Only Brace}

Aghdam (2009) has conducted an experimental study to evaluate the feasibility of using steel straps in a tension-only bracing system for seismic design. A total of forty nine flexible hot-rolled steel strap specimens with the slenderness ratio between 200 and 800 , and three connection details were tested under monotonic tension and cyclic loading to study effects of slenderness ratio, connection detail and ultimate to yield strength ratio on the performance of the brace. The bolted connection details investigated are shown in Fig. 5.2 consisting of no reinforcement (Detail A), clamped with an extra plate (Detail B) and welded with an extra plate (Detail C). The cyclic tests were conducted according to the loading protocol of ATC-24 Guidelines for Cyclic Seismic Testing of Components of Steel Structures (ATC 1992) for single specimen testing program. Figure 5.3 shows the test setup for the strap tension-only brace experiment. The loading sequence used in the cyclic test is listed in Table 5.1. $\delta$ is the applied deformation for half cycle and $\delta_{y}$ is the deformation defined as

$$
\delta_{y}=\frac{F_{y} L}{E}
$$

where $F_{y}$ is the nominal yield strength taken as $300 \mathrm{MPa}, \mathrm{L}$ is the nominal length of the specimen measured between the middle bolt holes and $\mathrm{E}$ is the elastic modulus taken as $200000 \mathrm{MPa}$.

Type A specimens without reinforcement at the connection was observed to exhibit the least ductility in the tests. This was followed by Type B specimens clamped 
with an extra plate. The Type $\mathrm{C}$ specimens welded with an extra plate at the connection exhibited the highest ductility since the net section $\left(A_{n}\right)$ at the bolt hole is larger than the gross section $\left(A_{\underline{g}}\right)$ for the rest of the specimen.

All Type $C$ specimens tested achieved and exceeded the ductility requirement for the 2.5\% storey drift limit in NBCC 2010 (NRCC 2010). Unlike Type C specimens, some Type A specimens fractured at a much lower ductility that is significantly lower than the requirement for the $2.5 \%$ storey drift limit. On the other hand, the behaviour of a Type B specimen falls between that of Type A and C, but is closer to Type A. Therefore, this research only focuses on Type A specimens. The ductility of Type A specimen has been found to be greatly influenced by the net section fracture to the gross section yielding strength ratio $\left(A_{n} F_{u} / A_{g} F_{y}\right)$, and to a lesser extent the ultimate to yield strength ratio $\left(F_{u} / F_{y}\right)$, net to gross section area ratio $\left(A_{n} / A_{g}\right)$, slenderness ratio and specimen length.

\subsection{Material Model Parameters for Tested Steel Straps}

Four steel straps of materials $\mathrm{C}$ to $\mathrm{F}$ listed in Table 5.2 were used in the tests by Aghdam (2009). In order to carry out the numerical simulations of these tests, parameters of the material model and damage criterion for these materials have to be determined. As discussed in Chapter 3, material tests including monotonic tension and cyclic tests should be conducted to determine these parameters. However, only a few monotonic tension tests have been conducted using the standard material testing specimen (straight profile). Procedures are required to be established in order to find the appropriate material model parameters to be used in the numerical simulations of the strap specimens. These procedures are discussed in the following sections. 


\subsubsection{Bound for Saturated Yield Stress}

In Chapter 3, it has been demonstrated that the parameters for kinematic hardening can be approximated using the flow stress versus equivalent plastic strain curve and an assumed saturated yield stress. While the flow stress versus equivalent plastic strain curve can be established from a standard tension material test through the procedure outlined in Chapter 3, the optimum assumed yield stress is determined through an iterative process that uses data from cyclic tests. However in most situations, only standard tension material tests are conducted for a material to obtain the material properties. Instead of using a specific value for the saturated yield stress, it may be speculated that the saturated yield stress to be within a bound established based on values calibrated for other tests. Thus, the numerical simulations may be carried out using the kinematic hardening parameters determined for both the lower and upper bound saturated yield stresses.

In addition to materials $\mathrm{A}$ and $\mathrm{B}$ from the current study, Kanvinde and Deierlein $(2004,2007)$ have also conducted some ultra low-cycle fatigue tests on steels of Grade 50 (50 ksi yield strength) and higher (labeled as AW50, AP50, AP110, AP70HP, JP50. JP50HP and JW50 in Table 5.2). Kanvinde and Deierlein $(2004,2007)$ have provided the input data (the saturated yield stress $\sigma_{s}^{0}$ can be calculated accordingly) used in the numerical simulations of their specimens with ABAQUS. Material properties from the current study together with those from Aghdam (2009) and Kanvinde and Deierlein $(2004,2007)$ are listed in Table 5.2. A relationship (a bound for the saturated yield stress) is thus established between the saturated yield stress to initial true yield stress ratio 
$\left(\sigma_{s}^{01} / \sigma_{t y}\right)$ and the ultimate to yield strength ratio $\left(\mathrm{F}_{\mathrm{u}} / \mathrm{F}_{\mathrm{y}}\right)$ as the saturated yield stress bound parameter to be

$$
\eta=\frac{\sigma_{\mathrm{s}}^{0} / \sigma_{\mathrm{ty}}}{\mathrm{F}_{\mathrm{u}} / \mathrm{F}_{\mathrm{z}}},
$$

where $\sigma_{\mathrm{ty}}=\mathrm{F}_{\mathrm{y}}\left(1+\varepsilon_{\mathrm{ey}}\right), \varepsilon_{\mathrm{ey}}$ is the engineering strain at the start of yielding. Values of $\eta$ based on calibrated material model parameters for materials A and B and materials by Kanvinde and Deierlein $(2004,2007)$ are listed in Table 5.2. It can be seen that it varies from 0.79 to 1.15 . Based on these limited data, $\eta$ for $300 \mathrm{~W}$ to $350 \mathrm{~W}$ grade structural steels is assumed to vary from 0.75 to 1.2 . Therefore, the saturated yield stress of materials C to F for steel straps tested by Aghdam (2009) is estimated to fall between the values given by Eq. (5.2) as no cyclic material tests have been conducted. The average of the measured cross-sectional area change at fracture for straight specimens tested under monotonic tension loading, $\ln \left(\mathrm{A}_{0} / \mathrm{A}_{\mathrm{f}}\right)$, are also listed in Table 5.2. The cross-sectional area at fracture $\left(\mathrm{A}_{\mathrm{f}}\right)$ for materials $\mathrm{C}$ to $\mathrm{F}$ is calculated based on the product of the averages of the maximum and minimum thickness, and the maximum and minimum width of the cross-section at the location of necking.

In Section 3.3, the established procedure of estimating the kinematic hardening parameters uses results of the cyclic test of straight specimen together with an assumed saturated yield stress. Since there were no cyclic material test conducted for materials C to $\mathrm{F}$, the established procedure has to be modified in order to estimate the kinematic hardening parameters for materials $\mathrm{C}$ to $\mathrm{F}$. In the modified procedure, the kinematic hardening parameters are determined by least-square fitting through the backstress data 
points for large strain only since there are no backstress data points for small strain similar to those shown in Fig. 3.21.

In order to verify the feasibility of using the modified procedure and the assumed saturated yield stress bound parameter $\eta$, the numerical simulations have been carried out for a number of specimens for material $\mathrm{B}$ using the kinematic hardening parameters determined by the modified procedure with the saturated yield stress calculated for the assumed lower and upper bound of $\eta$. Since no cyclic material test has been conducted for materials $\mathrm{C}$ to $\mathrm{F}$ and the parameter $\mathrm{b}$ for the flow stress Eq. (3.28) is not sensitive to the predicted engineering stress versus engineering strain curve as discussed in Chapter 3 , only the flow stress parameters determined with the monotonic tension test results are used in the determination of the kinematic hardening parameters. For material B, values of the parameters for the flow stress Eq. (3.28) have been determined to be $\sigma_{0}^{y}=$ $332 \mathrm{MPa}, \varepsilon_{0}^{\mathrm{p}}=0.0161, \sigma_{x}^{y}=564 \mathrm{MPa}, \mathrm{n}=0.52$ and $\mathrm{b}=1.63$. For $\eta=0.75$, the corresponding saturated yield stress $\sigma_{\mathrm{s}}^{0}$ is $380 \mathrm{MPa}$ base on Eq. (5.2) and the measured $\mathrm{F}_{\mathrm{u}} / \mathrm{F}_{\mathrm{y}}$ in Table 5.2. The backstress data used in estimating the kinematic hardening parameters can be calculated by subtracting the assumed saturated yield stress from the flow stress, as shown in Fig. 5.4. The kinematic hardening parameters estimated are $C_{1}=$ $1641 \mathrm{MPa}, \gamma_{1}=5.73, \mathrm{C}_{2}=164 \mathrm{MPa}$ and $\gamma_{2}=0.73$. For the optimum assumed saturated yield stress of $384 \mathrm{MPa}$ and offset saturated yield stress of $15 \mathrm{MPa}$ determined in Chapter 3 , the corresponding values are $\eta=0.79, \mathrm{C}_{1}=1432 \mathrm{MPa}, \gamma_{1}=5.25, \mathrm{C}_{2}=157 \mathrm{MPa}$ and $\gamma_{2}$ $=0.72$. Similarly, for $\eta=1.2$, the kinematic hardening parameters estimated are $C_{1}=$ $255 \mathrm{MPa}, \gamma_{1}=0.97, \mathrm{C}_{2}=3.43 \mathrm{MPa}$ and $\gamma_{2}=0.10$. Comparisons of test results and numerical simulations with ABAQUS (Simulia 2009) for specimens BBC20404 and 
BBCF1212 are shown in Figs. 5.5 to 5.6 for $\eta$ of $0.75,0.79$ and 1.2. It can be seen that the test results are bounded by the results of the simulations for the assumed bound parameter $\eta=0.75$ and 1.2. Obviously, there is a better agreement with results of the simulations using the parameters from the optimum assumed saturated yield stress although the agreement is not good compared to that in Chapter 3 using the parameters calibrated with the original procedure. Thus, it is established that the modified procedure can be used to estimate the kinematic hardening parameters, and is employed to estimate the kinematic hardening parameters for materials $\mathrm{C}$ to $\mathrm{F}$ and materials used in the parametric finite element analysis study of the strap tension-only brace.

\subsubsection{Damage Limits for Materials $\mathrm{C}$ to $\mathrm{F}$}

The procedure adopted to determine the damage limit for materials A and B using results of numerical simulations of the test specimens has been discussed in details in Section 4.2. However, this procedure is not applicable to materials $\mathrm{C}$ to $\mathrm{F}$ since only monotonic tension tests of straight specimens have been conducted for these materials. Therefore, some modifications are made to the procedure in Section 4.2 in order to estimate the damage evolution and limit in the numerical simulations for materials $\mathrm{C}$ to $\mathrm{F}$. Since most Grade 300W and 350W structural steels are expected to have comparable ductility, the modifications have been made based on the following assumptions:

(1) Specimens from two different materials (heats) are expected to fracture at close to the same cycle number and reduction in the cross-sectional dimension (diameter) if the straight specimens of these two materials (heats) 
have the same cross-sectional area change at fracture $\left[\ln \left(A_{1} / A_{f}\right)\right]$ under monotonic tension loading.

(2) The relative ductility between test specimens of different profiles and loading sequences from the same material (heat) does not vary significantly from that for a different material (heat).

It follows from assumption (1) that the damage limit of a material which has the same $\ln \left(A_{v} / A_{f}\right)$ at fracture for a straight specimen under monotonic tension loading as that for either material $\mathrm{A}$ or $\mathrm{B}$ can be determined with the procedure in Section 4.2 using the test results of either material A or B. The numerical simulations of the specimens used in the determination of the damage limit are carried out using the material properties (hardening model parameters) for that material, but using the cycle number and reduction in the cross-sectional dimension (diameter) at fracture for test specimens of either material A or B. Since it is rare that straight specimens of two materials under monotonic tension loading will have the same cross-sectional area change $\left[\ln \left(\mathrm{A}_{0} / \mathrm{A}_{\mathrm{f}}\right)\right]$ at fracture, assumption (2) is used to relate the calculated damage limit through assumption (1) to that of a material with a different $\ln \left(A_{0} / A_{f}\right)$.

Based on assumption (2), the relationship between the predicted and measured cross-sectional area at fracture for two materials 1 and 2 that have the same material properties (hardening model parameters) but different ductility can be established as

$$
\frac{\ln \left(A_{0} / A_{f}\right)_{1}}{\ln \left(A_{0} / A_{p}\right)_{1}}=\frac{\ln \left(A_{0} / A_{f}\right)_{2}}{\ln \left(A_{0} / A_{p}\right)_{2}}
$$

where $A_{p}$ is the predicted cross-sectional area at fracture by the modified Huang and Mahin fracture criterion based on the damage evolution Eqs. (4.3) and (4.4) and the same $\mathrm{c}, \mathrm{m}$ and $\mathrm{k}$. Test results of a straight specimen under monotonic tension loading are used 
to calculate the damage limit with Eq. (5.3). Material 1 is the material that the damage limit is to be determined while material 2 is a fictitious material that has the same ductility as either material A or B.

In order to demonstrate the above procedure more clearly and also to verify its feasibility, test results of material $\mathrm{B}$ are used to calculate the damage limit and to predict the fracture of specimens for material A. Thus, material A is taken as material 1 in the illustration. Numerical simulations are carried out for specimens of material B using the calculated hardening model parameters for material A in Chapter 3. The corresponding damage at the point of fracture for each specimen of material B is calculated from the numerical simulation with hardening model parameters for material A. Table 5.3 shows the calculated damage for each specimen of material B and the parameters for the damage evolution Eqs. (4.3) and (4.4) determined for the fictitious material ${ }^{\mathrm{F}} \mathrm{A}$. Based on the determined damage limit and parameters, the diameter at fracture for the straight specimens ( ${ }^{\mathrm{F}} \mathrm{ADMa}$ and ${ }^{\mathrm{F}} \mathrm{ADMb}$ ) under monotonic tension loading is predicted to be 9.17 $\mathrm{mm}$ and $\ln \left(\mathrm{A}_{\mathrm{v}} / \mathrm{A}_{\mathrm{p}}\right)=0.845$. The average measured $\ln \left(\mathrm{A}_{\mathrm{v}} / \mathrm{A}_{\mathrm{f}}\right)$ of straight specimens under monotonic tension loading for materials A and B are 0.986 and 0.857 , as listed in Table 5.2. According to Eq. (5.3), the predicted cross-sectional area change at fracture for material A can be rewritten as

$$
\begin{aligned}
\ln \left(A_{0} / A_{p}\right)_{1} & =\frac{\ln \left(A_{0} / A_{p}\right)_{2}}{\ln \left(A_{0} / A_{r}\right)_{2}} \cdot \ln \left(A_{0} / A_{f}\right)_{1} \\
& =\frac{0.845}{0.857} \cdot(0.986) \\
& =0.972
\end{aligned}
$$


The damage limit corresponds to the predicted $\ln \left(\mathrm{A}_{0} / \mathrm{A}_{\mathrm{p}}\right)_{1}=0.972$ at fracture is calculated to be $\mathrm{D}_{\mathrm{c}}=14.92$ from the numerical simulation of the straight specimen ADMa or ADMb with $\mathrm{c}=1, \mathrm{~m}=3.39$ and $\mathrm{k}=1.12$ for Eqs. (4.3) and (4.4). Comparisons of test and predicted point of fracture for specimens of material A using these damage limit and damage evolution parameters are listed in Table 5.4. The predicted change in diameter at fracture for the straight specimens is within $5 \%$ of the measured value. Even for the notched specimens, the error in the predicted change in diameter for monotonic tests and the accumulated engineering strain at fracture for cyclic tests are less than $15 \%$ for all but three specimens, which are still within $30 \%$. Overall, the prediction can be considered to be reasonably good even though not as precise as that in Chapter 4 where the damage limit has been calibrated directly from the test results.

\subsubsection{Procedure to Determine Damage Limits for Materials C to F}

Chen (2010) has conducted a number of monotonic tension material tests using rectangular specimens for materials C to F. The empirical Eq. (3.31) by Chen (2010) has been used to calculate the true stress versus true plastic strain data to calibrate the parameters $\sigma_{\llcorner}^{y}, \mathrm{~b}$ and $\mathrm{n}$ for the flow stress Eq. (3.28). Values of these parameters for materials $\mathrm{C}$ to $\mathrm{F}$ are listed in Table 5.5. The test and generated flow stress versus true plastic strain for the materials are shown in Fig. 5.7.

Since only tests of straight specimens under monotonic tension have been conducted for materials $\mathrm{C}$ to $\mathrm{F}$, there are insufficient data to determine the parameters for the kinematic hardening model. As discussed in Section 5.2.1, the stress-strain relationship of a material can be considered to be bounded by the assumed $\eta$ limits. For 
each material, the hardening model parameters are determined for the lower and upper bound of $\eta$ at 0.75 and 1.2, and are listed in Table 5.6. Parameters for the damage limit for materials $\mathrm{C}$ to $\mathrm{F}$ are estimated through test results of specimens for material $\mathrm{B}$ using the procedure described in Section 5.2.2. Results of the numerical simulation of a rectangular specimen are used to determine the damage limit with Eq. (5.4). The typical mesh of the FEA model of the rectangular specimen tested by Chen (2010) is shown in Fig. 5.8. The mesh for $1 / 8$ rectangular specimen is modeled with the $\mathrm{C} 3 \mathrm{D} 20 \mathrm{R}$ element, which is a tri-quadratic isoparametric solid element with reduced integration. It is adopted directly from that created by Chen (2010). An example of the calculation for the damage limit of material $\mathrm{C}$ is shown in Table 5.7. Values of the parameters for the fracture criterion and the damage limits for materials $\mathrm{C}$ to $\mathrm{F}$ determined through test results of specimens for material B are listed in Table 5.8.

\subsection{FEA Simulations of Type A Strap Specimens}

In this research, only Type A strap specimens are investigated because they are the least ductile and Type $\mathrm{C}$ strap specimens have no problem meeting the ductility requirement for seismic design. Since Type B strap specimens are only slightly more ductile than Type A specimens, they are not investigated in this research. Two different types of FEA model are discussed in the following sections. Applying the material model parameters established in Section 5.2.3 in the FEA simulations, using two kinematic hardening terms and the damage evolution Eqs. (4.3) and (4.4), the predicted ductility of the strap specimen is compared to the test results by Aghdam (2009). The fracture criterion is implemented into a user-defined material subroutine for the mixed- 
mode hardening model by Chaboche (1986) which has been developed by Khoo (2010). The strap specimen is considered to have fractured if any point within the specimen exceeds the damage limit.

\subsubsection{FEA Modeling of Type A Strap Specimens}

The profile of Type A strap specimen is shown in Fig. 5.9 and the detailed information of these specimen are listed in Table 5.9. The two strap specimens forced to buckle in the direction towards the gusset plate are not listed and included in the FEA simulations as they exhibited greater ductility because bending occurred away from the bolt hole.

\subsubsection{FEA Models}

If everything is in a perfect symmetry, only $1 / 4$ of strap specimen has to be modeled. FEA simulations using a $1 / 4$ model assume that the upper and lower bolt hole regions of the strap have the same deformation. However in the actual test, the deformations at both ends of the strap are not identical. Figures 5.10 and 5.11 show both ends of specimen A4078b of material D and A8075b of material C at the end of the test. For specimen A4078b, the deformations at both ends appear to be close. This indicates that it may be possible to carry out the numerical simulation using a $1 / 4$ model for this situation. However for specimen A8075b, there appears to be a bigger difference in the deformation at both ends. For this situation, the numerical analysis using a 1/4 model may not be appropriate. Therefore, a 1/2 model has also been created for the FEA simulation. Comparisons of the predictions using $1 / 4$ and 1/2 FEA models are briefly 
discussed in the following section. While not as accurate, it is easier to construct a $1 / 4$ model than a $1 / 2$ model.

To facilitate the discussions, both $1 / 4$ and 1/2 models of specimen A4075 are shown in Figs. 5.12 and 5.13 respectively for illustration. As can be seen in Figs. 5.2 and 5.3, a Type A strap specimen is connected to the gusset plates by M20 ASTM A490 high strength bolts. In the cyclic test, the section at the innermost bolt hole experienced considerable bending and necking, but no bending and necking have been observed at the section of the other two bolt holes. Thus, the region around the innermost bolt hole at the upper (loading) end is modeled with the 8-node tri-linear C3D8R solid element with reduced integration in order to better model the increase in stress triaxiality due to necking. After carrying out a few trial simulations, it was found that the simulation was not significantly affected by modeling the region around the remaining two bolt holes with either solid or shell element. Therefore, the region around the remaining two bolt holes is modeled with the 4-node S4R bi-linear shell element with reduced integration. The transition from shell to solid element and vice versa, is defined using Shell to Solid Coupling option. This coupling constrained the displacement and rotation of each shell node on the contact edge to the displacement and rotation of the solid surface in contact. For the $1 / 2$ model shown in Fig. 5.13, the mesh is designed to induce fracture and necking to occur at the end modeled with the solid element. Thus, only the end where fracture is designed to occur has to be modeled with the solid element. For this reason, the region around the innermost bolt hole at the lower (fixed) end is also modeled with the S4R shell element but with a finer mesh compared to the region of the other two bolt holes in order to enable a more accurate modeling of bending and deformation around the 
hole. The size of the shell element around the innermost bolt hole at the lower (fixed) end is double that of the solid element (planar dimension) at the upper (loading) end. Larger element sizes are used there because no significant necking is expected to occur around the innermost bolt hole at the lower end. The maximum width and length of the shell element at both fixed and loading ends are both $6 \mathrm{~mm}$, and the maximum width of the solid element is $3 \mathrm{~mm}$.

A finer mesh is provided closer to the bolt hole to model the higher stress and strain gradients. Figure 5.14 shows mesh around the innermost bolt hole modeled with solid elements and the typical mesh refinement towards the center of the hole. Around the innermost bolt hole, a finer mesh is also employed towards the line along the minimum cross-sectional area section where bending and necking are expected to occur. Shown in Fig. 5.15 for a 1/4 model, remaining part of the specimen outside the bolt hole region is modeled with the S4R shell element. Similarly, the shell elements are coupled to the solid elements on their contact edges. That part is divided into refined, transition and general regions. Similar four regions are employed to both halves of a 1/2 model. The refined region has a length of $50 \mathrm{~mm}$, each of the two transition regions has a length of $150 \mathrm{~mm}$ and the rest is the general region (for specimens with a short length of $1120 \mathrm{~mm}$, only $50 \mathrm{~mm}$ refined region and two $145 \mathrm{~mm}$ transition regions are used). The shell element length in the $\mathrm{Y}$ direction (along the length of the strap) is $2.5 \mathrm{~mm}$ in the refined region and $10 \mathrm{~mm}$ in the general region. It varies from $2.5 \mathrm{~mm}$ to $9.7 \mathrm{~mm}$ over 28 rows of elements at the transition region close to the mid-length, and from $9.8 \mathrm{~mm}$ to 5 $\mathrm{mm}$ over 21 rows of elements at the transition region close to the bolt hole. The maximum width of the shell element in these four regions is $5.4 \mathrm{~mm}$. 
Except for the two excluded from the current study, all strap specimens buckled away from the gusset plate (positive direction in the $\mathrm{Z}$ coordinate according to Fig. 5.16). Therefore, 2-node SPRING2 spring elements are utilized in the FEA models to induce buckling away from the gusset plate, as shown in Figs. 5.16. These spring elements have a nonlinear stiffness which is close to zero in tension but is very high $\left(10^{10} \mathrm{kN} / \mathrm{m}\right)$ in compression. A very small spring displacement is applied at the start of every compression leg of the loading in order to initiate buckling in the desired direction. Figure 5.17 shows the applied transverse displacement at the spring element support with the odd half cycle number corresponds to the start of the compression loading. The applied displacement is smaller than the actual displacement of the test at the mid-length after a few cycles of loading. Also due to the slenderness of the specimen, this displacement only induces a small strain in the member compared to the overall strain. Thus, the large spring stiffness in compression prevents the specimen from buckling towards the gusset plate, while the small spring stiffness in tension implies that there is minimal force imposed by the spring when the specimen buckles. Since the spring displacement is imposed during the unloading from tension, the stress and strain induced by the spring is not expected to generate significant additional damage. Hence, the spring elements have very little overall effect on the FEA prediction of the specimen failure. These spring elements are removed for the FEA simulations of monotonic tension tests since no buckling occurs. 


\subsubsection{Boundary Conditions of FEA Models}

Three M20 ASTM A490 high-strength bolts were used at each end to fasten the steel strap to the gusset plate. At each end, bending was observed to occur only at the section across the innermost bolt hole. For simplicity, regions around the other two bolt holes can be considered to be almost fixed to the gusset plate and thus are constrained from moving out-of-plane ( $Z$ direction) in the modeling. These represent regions around the two bolt holes that are modeled with shell element at the loading end. These regions are similarly constrained at the lower (fixed) end of the specimen for the $1 / 2$ model. At the region around the innermost bolt hole, only nodes at the bottom surface of the solid element contacting with the gusset plate shown in Fig. 5.18 are constrained in the $\mathrm{Z}$ direction while leaving the rest of the region free to bend. For the $1 / 2$ model, the corresponding nodes of the shell elements at the lower (fixed) end of the specimen that covers over the same area as those in Fig. 5.18 are constrained in the $\mathrm{Z}$ direction since only the loading end is modeled with solid element.

It is observed in the test that the edge of bolt hole gradually stretched out of the bolt head (nut) due to the bending and deformation at the innermost bolt hole. Together with necking at the section across the innermost bolt hole, the effective contact area has been reduced to less than half of the bolt head (nut). This phenomenon can be seen in Fig. 5.10. In the trial FEA simulation shown in Fig. 5.19, an ANALYTICAL rigid surface and a rigid body together with a spring element have been used to model the bolt, and contact surfaces are defined around the bolt hole to simulate the contact between the bolt and the specimen. The minimum bolt pre-tension force according to CSA S16-09 (CSA 2009) taken from Handbook of Steel Construction (CISC 2010) was applied through the 
spring to simulate the pre-tension. Results of the FEA simulation are shown in Fig. 5.20. It shows that the simulation was able to capture the stretching of the bolt hole and the loss of some contact between the bolt and the specimen. Although a modeling that considers the interaction of the bolt and contact surfaces would be more realistic, the simulation had difficulty reaching convergence as the number of loading cycle increases. Therefore, a simpler modeling is used in the current study to replace the more complex modeling using contact elements. The simplified model constrains $3 / 8$ of the nodes of the solid element that initially are in contact with the bolt head from moving in the $\mathrm{Z}$ direction (out-of-plane), thus leaving other nodes around the perimeter of the bolt hole to move freely such that necking can occur at the section of the minimum cross-sectional area across the innermost bolt hole. Figure 5.21 shows nodes around the innermost bolt hole constrained in the $\mathrm{Z}$ direction due to clamping of the bolt. The measured dimension (28.9 $\mathrm{mm}$ diameter) of the bolt head is used to determine the nodes to be constrained. No additional constraint in the $\mathrm{Z}$ direction is applied to the region around the innermost bolt hole at the lower (fixed) end of the 1/2 model other than to the nodes that cover the same area as those shown in Fig. 5.18.

For both $1 / 4$ and $1 / 2$ models, all the nodes along the centre line of the specimen are constrained with the XSYMM boundary condition. For the $1 / 4$ model, nodes at the mid-length of the specimen are constrained with YSYMM boundary condition. In the FEA simulations, the loading (displacement) is applied at the end of the specimen through the boundary nodes around a quarter of the innermost bolt hole perimeter and the complete perimeter of the other two bolt holes. The nodes around a quarter of the innermost bolt hole perimeter where the loading is applied are shown in Fig. 5.22. These 
nodes are assumed to be effectively in contact with the innermost bolt when the specimen is in tension. This neglects any slippage that may occur at the bolted connection. For the $1 / 2$ model, the modeling is designed to have fracture occurs at the upper (loading) end. Therefore, in order to prevent necking and failure at the lower (fixed) end, all the nodes around the innermost bolt hole perimeter as shown in Fig. 5.23 in addition to the nodes around the perimeter of the other two bolt holes are also constrained from deformation in the $\mathrm{Y}$ direction. It assumes that the load is transferred by the innermost bolt at the fixed end through shear friction, and not by bearing. The load may be transferred through shear friction for a thin strap when the friction resistance due to pretension is greater than the failure load. It produces a conservative ductility prediction of the strap specimen. While being more conservative in the ductility prediction, this will only slightly affect the predicted deformation at fracture since the deformation around the bolt hole is small when there is no considerable necking. Numerical simulations have been carried out for strap specimen A8075 with two different boundary conditions at the fixed end. One boundary condition constrains all the nodes along the perimeter of the innermost bolt hole against movement in the $\mathrm{Y}$ direction similar to Fig. 5.23, and the other allows the same nodes to move. Results of the simulations are listed in Table 5.10. The maximum deformation for $\mathrm{A} 8075 \mathrm{a}$ is between the predicted deformations given by the two boundary conditions. For $\mathrm{A} 8075 \mathrm{~b}$, the predicted maximum deformations are higher than the measured deformation. The difference between the predicted maximum deformations using these two boundary conditions is around $3.5 \mathrm{~mm}$ with the model that constrained the nodes along the perimeter of the innermost bolt hole providing a closer prediction for this instance. Nevertheless, it is expected that the maximum difference in the predicted 
deformation at fracture to be around $2 \mathrm{~mm}$ between models that considering the force is transferred through bearing by the innermost bolt at the fixed end and nodes along the perimeter of the innermost bolt hole constrained.

\subsubsection{FEA Simulations of the Strap Specimen Tests}

All the numerical analyses of the Type A strap specimen tests by Aghdam (2009) have been carried out with ABAQUS (Simulia 2009) through a user-defined material subroutine incorporated the modified Huang and Mahin fracture criterion based on Eqs. (4.3) and (4.4) using material model parameters from Tables 5.2, 5.5, 5.6 and 5.8 and Poisson's ratio taken as 0.3 . Nonlinear geometry option is used to account for the effect of geometric nonlinearity in large deformation and large strain analyses. Failure of the strap specimen is assumed to occur and the analysis terminated when the accumulated damage in any part of the model reached the damage limit. The minimum number of increment specified for half cycle of loading is 10 , but normally the simulation required around 20 increments or more to complete a half cycle of loading.

The static net section fracture to the gross section yielding strength ratios $\left(A_{n} F_{u} / A_{g} F_{y}\right)$ of the strap specimens are listed in Table 5.9 with specimens of material $C$ having a ratio slightly larger than 1.0. However, the yield and ultimate strengths of steel change with the loading rate. Table 5.11 lists the net section fracture to the gross section yielding strength ratios of materials $\mathrm{C}$ to $\mathrm{F}$ at various strain rates. It can be seen in the table that at a higher loading rate of $0.01 \mathrm{~s}^{-1}$, a strap specimen of material $\mathrm{C}$ will fail in net section fracture before gross section yielding occurs. Since the static $A_{n} F_{u} / A_{y} F_{y}$ of a strap specimen of material $\mathrm{C}$ is just marginally larger than 1.0 , it is conceivable that it will fail 
in net section fracture before gross section yielding occurs if the loading rate is sufficiently high. From the load versus deformation curve observed for the specimen of material C shown in Fig. 5.25 for A4075, it is clear that the net section fractured before the gross section has yielded since the length (strain) of the yield plateau of material $\mathrm{C}$ is 0.024. In the test, the loading rate for Type A strap specimens varied from $0.0002 \mathrm{~s}^{-1}$ to $0.001 \mathrm{~s}^{-1}$. The average drop in the $A_{n} F_{u} / A_{k} F_{y}$ ratio from the strain rate of $0.0001 \mathrm{~s}^{-1}$ to $0.01 \mathrm{~s}^{-1}$ of materials $\mathrm{C}$ to $\mathrm{F}$ is $5 \%$. However, the numerical simulations have been carried out without considering the strain rate effect on the material properties. Thus, the initial yield stress of the flow stress versus true plastic strain curve is conservatively increased by $5 \%$, based on the average drop of the $A_{n} F_{u} / A_{g} F_{y}$ ratio from the strain rate of $0.0001 \mathrm{~s}^{-1}$ to $0.01 \mathrm{~s}^{-1}$ to reflect the effect of strain rate on $\mathrm{A}_{11} \mathrm{~F}_{\mathrm{u}} / \mathrm{A}_{\mathrm{g}} \mathrm{F}_{y}$ ratio and the mode of failure. This change only applies to material $\mathrm{C}$ as a small change in its yield and ultimate strengths could significantly affect the mode of failure and the specimen ductility because of the yield plateau in the material stress versus strain relationship. For other materials, the value of static $A_{n} F_{u} / A_{g} F_{y}$ is sufficiently greater than 1.0 that the effect of loading rate is unlikely to change the mode of failure.

A mesh study has been carried out to determine the optimum mesh density used for the numerical simulation of the strap model. The finite element analyses have been carried out for the 1/2 model of specimen A4075 of material $C$ and the 1/4 model of specimen A2078 of material D with the material model parameters for $\eta=0.75$. Since the model is designed to fracture and have necking occurring in the solid element at the loading end, the mesh configuration of the shell element shown in Figs. 5.12, 5.13 and 5.23 is considered to be fine enough to model the deformation of the specimen with 
sufficient accuracy. Thus, the study only focuses on the mesh of solid element. The typical mesh for the solid element around the innermost bolt hole of scheme-2 is shown in Fig. 5.24 where the size of the solid element is half of that of scheme-1 shown in Fig. 5.14. The FEA simulations of load versus deformation curves for specimens A4075 and A2078 using scheme-1 and scheme-2 are shown in Figs. 5.25 and 5.26, and the predicted cycle number and maximum deformation at fracture are listed in Table 5.12. The comparisons show that there is only a small difference between results from both mesh schemes. Even though the difference in the predicted maximum deformation is about $6 \%$ for specimen A2078, the difference in the loading cycle number at fracture is only 1 . Therefore, considering the significant increase in computing cost going from scheme-1 to scheme-2 (about 10 times) while providing only a small improvement in the prediction, the mesh for scheme-1 has been adopted in the numerical simulations of strap specimens. The total number of degree of freedom is about 47000 for the $1 / 4$ model and 57800 for the $1 / 2$ model of specimen A4075.

Numerical simulations have been carried out using the $1 / 2$ model for all the strap specimen tests, and the $1 / 4$ model for most of the tests. Figure 5.27 shows the typical failure at the innermost bolt hole predicted by the FEA simulations, which is similar to that observed in the test where fracture has initiated from the edge of the bolt hole at the section of the minimum cross-sectional area after extensive necking. It is expected that the $1 / 2$ model will predict a lower ductility compared to that by the $1 / 4$ model. The computation time for the $1 / 2$ model on average was about 1.1 to 1.2 times of that required for the $1 / 4$ model. The benefit of the $1 / 4$ model is the ease of modeling compared to the $1 / 2$ model. While the $1 / 2$ model is applicable to most materials, configurations and 
loading sequences, the $1 / 4$ model is expected to give a reasonable prediction for a material with a reasonable length of yield plateau and the gross section yielding occurs before net section fractures. Results of all the simulations are compared to the test results in Table 5.13 for the $1 / 4$ model and Table 5.14 for the $1 / 2$ model. In proportion to the test deformation, it can be seen in the tables that the $1 / 4$ model has significantly overpredicted the maximum deformation of specimens of material $\mathrm{C}$, but has only predicted a slightly higher maximum deformation compared to that by the $1 / 2$ model for materials $D$ to $\mathrm{F}$. The maximum difference in the predicted maximum deformation is $10 \mathrm{~mm}$. Since net section fracture occurred before gross section yielding for specimens of material $\mathrm{C}$, the overall deformation at fracture is small and the additional deformation at the bolt hole of the fixed end due to the necking of the section because of the symmetry assumed in the $1 / 4$ model is significant in proportion. However for specimens of materials D to F, the contribution of this additional deformation is small in proportion because the overall deformation at fracture is considerably larger as a result of net section fracture being preceded by gross section yielding. Figures 5.28 to 5.31 show the test and predicted load versus deformation curves from both 1/4 and 1/2 models for specimens A4075a and A4075b of material C, and Figs. 5.32 to 5.35 for specimens A4078a and A4078b of material D. It is clear that the $1 / 4$ model has significantly over-predicted the ductility for specimens of material $\mathrm{C}$ compared to the $1 / 2$ model, but is able to provide comparable results to that of the $1 / 2$ model for material D. As stated previously, the material behaviour of materials $\mathrm{C}$ to $\mathrm{F}$ are assumed to be bound by $\eta$ of 0.75 to 1.2 . Shown in Figs. 5.28 to 5.35 , the FEA simulations with $\eta=1.2$ have predicted a higher strength and 
ductility compared to $\eta=0.75$. This is also reflected in Tables 5.13 and 5.14 on the predicted ductility of the strap specimen.

Figure 5.36 shows the test and predicted load versus deformation curves of specimens A2078d and A2078e under monotonic tension using the $1 / 2$ model. Comparing the test results of A2078d and A2078e, there is a clear indication of slippage at the bolt hole based on the load versus deformation curve of A2078d. Compared to A2078e, the slippage in A2078d appears to be 7 to $9 \mathrm{~mm}$ larger. The bolt hole size for the test setup was specified to have a clearance of $2 \mathrm{~mm}$. Since the cable transducer used in measuring the displacement was attached to the gusset plate, the maximum possible measured slippage is thus $4 \mathrm{~mm}$ at one end, and $8 \mathrm{~mm}$ for the whole specimen. There is little difference between the predicted load versus deformation curves for $\eta$ of 0.75 and 1.2 under monotonic tension except for the ductility. Based on the comparisons of test and predicted load versus deformation curves, slippage appears to occur in specimens A4078a and A4078b (Figs. 5.34 and 5.35), but does not appear to occur in specimens A4075a and A4075b (Figs. 5.30 and 5.31) because the failure load at $115 \mathrm{kN}$ is small compared to the friction force generated by the pretension applied to the bolt (friction force of $137 \mathrm{kN}$ for Class A contact surface with a minimum tension of $178 \mathrm{kN} / \mathrm{bolt}$ ). As can be seen in Fig. 5.36, the majority of slippage occurred at a load larger than $113 \mathrm{kN}$ for specimen A2078d and around $180 \mathrm{kN}$ for specimen A2078e. For specimens A2128d and A2128e shown in Fig. 5.37, slippage only occurred at a load larger than around 120 $\mathrm{kN}$. Figures 5.38 and 5.39 show the test and predicted load versus deformation curves for specimens A2108b and A4108a. It is clear that the specimen appears to be stiffer in the simulation. In addition, the constraint in the $\mathrm{Y}$ direction applied to the nodes around the 
innermost bolt hole at the fixed end, shown in Fig. 5.23, also reduces the predicted deformation by close to $2 \mathrm{~mm}$ when the load transfer is by bearing instead of shear friction. By considering a slippage of $6 \mathrm{~mm}$ including $2 \mathrm{~mm}$ due to the constraint in Fig. 5.23, Figs. 5.40 to 5.43 show the test and predicted load versus deformation curves for specimens A2078d, A2128d, A2128e, A2108b and A4108a with the test deformation reduced by $6 \mathrm{~mm}$. With the shift, there is a better agreement between the test and predicted load versus deformation curves. Since the numerical simulations have been based on material model parameters for the static behaviour, and after taking into consideration of a possible $6 \mathrm{~mm}$ shift in the load versus deformation curve, it can be considered that test load versus deformation curve is within the bound of predicted curves with $\eta$ between 0.75 and 1.2. As can be seen in Table 5.14 , analyses with $\eta=0.75$ gives a better prediction of the maximum deformation for specimens of material $\mathrm{C}$, and $\eta=1.2$ for specimens of materials $D$ to $F$. The predicted maximum deformation on average is larger than that of the test for specimens of material $\mathrm{C}$, and smaller for specimens of materials D to F. However, if a shift (slippage) of $6 \mathrm{~mm}$ is assumed to occur for specimens of materials D to F (except A2078e), the simulations with $\eta=0.75$ appear to give an improved maximum deformation prediction for all tests. No shifting is considered for specimens of material $\mathrm{C}$ since the failure load appears to be smaller than the friction force generated by the pretension. Table 5.15 shows the comparisons of test and predicted maximum deformations with the measured maximum deformation for specimens of materials D to F reduced by $6 \mathrm{~mm}$ except for A2078e. The maximum test deformation in general is bounded by predicted deformations with $\eta$ of 0.75 and 1.2 . This validates the procedures adopted to generate material model parameters as outlined 
in Section 5.2 and the $1 / 2$ model of the strap specimen in Section 5.3.1. An estimation of the material model parameters for each material may be made by adjusting the parameter $\eta$ until the simulations agree with the tests. This process has not been undertaken in this study. Comparisons of test and predicted load versus deformation curves using the $1 / 2$ model for rest of the specimens without reducing the test deformation by $6 \mathrm{~mm}$ are shown in Appendix E.

Based on the comparisons of test and FEA simulation results, the $1 / 2$ model with $\eta=0.75$ will be used in the parametric finite element analysis study of the strap tensiononly brace as it gives a lower bound prediction of the maximum deformation compared to $\eta=1.2$. It is also more conservative to neglect the slippage due to the bolt hole clearance and to consider the load transferred by shear friction at the innermost bolt hole of the fixed end when predicting the maximum deformation of the brace. Although the $1 / 4$ model is more efficient and easier to construct, it is not applicable for a general condition.

\subsection{Summary}

In this chapter, the experimental study on strap tension-only brace by Aghdam (2009) was briefly discussed. Current study only focuses on Type A (no reinforcement) specimens because they were the least ductile among specimens tested by Aghdam (2009) with some having fractured at a significantly lower ductility than the requirement of $2.5 \%$ storey drift limit in NBCC 2010. The ductility of Type A specimen has been found to be greatly influenced by the net section fracture to the gross section yielding strength ratio $\left(A_{n} F_{u} / A_{g} F_{y}\right)$, and to a lesser extent the ultimate to yield strength ratio $\left(F_{u} / F_{y}\right)$, net to gross section area ratio $\left(A_{n} / A_{g}\right)$, slenderness ratio and specimen length. 
Since only monotonic tension material tests have been conducted for the steel straps, the procedure to determine the material model parameters using both monotonic and cyclic test results developed in Chapter 3 was modified with the assumption that the saturated yield stress is bounded within a certain range based on results from the current and other studies on various grades of steel. A new procedure has also been developed to establish the damage limit and the damage evolution parameters for the modified Huang and Mahin fracture criterion using results of round specimen tests from the current study and monotonic tension material tests of steel straps by Aghdam (2009).

Two FEA models, $1 / 4$ and 1/2 models, for Type A strap specimens have been constructed and analyzed. Using the material model parameters determined by the established procedures, numerical simulations of steel strap specimens have been carried out with ABAQUS (Simulia 2009) through a user-defined material subroutine incorporated the modified Huang and Mahin fracture criterion. It was found that only the $1 / 2$ model, not the $1 / 4$ model, is suitable for most materials, configurations and loading sequences. Comparisons of test and FEA simulation results using the $1 / 2$ model shows that the results of cyclic tests in general are bounded by the FEA simulations with the saturated yield stress bound parameter $\eta$ of 0.75 and 1.2. Since the FEA model considers there is no slippage at the bolted connection, some of the test results are adjusted by 6 $\mathrm{mm}$ to account for slippage and the effect of model idealization in the comparisons. The simulation with $\eta=0.75$ predicted a lower maximum deformation compared to $\eta=1.2$. Therefore, the $1 / 2$ model with $\eta=0.75$ has been selected to be used in the parametric finite element analysis study of the strap tension-only brace. 
Table 5.1 Loading sequence of cyclic test for strap specimen

\begin{tabular}{|c|c|}
\hline Number of cycle & Applied deformation for half cycle, $\delta$ \\
\hline 3 & $0.25 \delta_{y}$ \\
\hline 3 & $0.5 \delta_{y}$ \\
\hline 3 & $0.6 \delta_{y}$ \\
\hline 3 & $0.8 \delta_{y}$ \\
\hline 3 & $\delta_{y}$ \\
\hline 3 & $2 \delta_{y}$ \\
\hline 3 & $3 \delta_{y}$ \\
\hline 2 & $4 \delta_{y}$ \\
\hline 2 & $>4 \delta_{y}$ \\
\hline & Increment of $\delta_{y}$ every 2 cycles \\
\hline
\end{tabular}


Table 5.2 Steel material properties

\begin{tabular}{|c|c|c|c|c|c|c|c|}
\hline Material & Grade & $\begin{array}{l}\text { Cross section } \\
(\mathrm{mm})\end{array}$ & $\begin{array}{c}\text { Elastic } \\
\text { modulus } \\
(\mathrm{MPa})\end{array}$ & $\begin{array}{c}\text { Yield } \\
\text { plateau } \\
\varepsilon_{\text {ep }}\end{array}$ & $\begin{array}{c}\text { Yield } \\
\text { strength } \\
\mathrm{F}_{\mathrm{y}}(\mathrm{MPa})\end{array}$ & $\begin{array}{c}\text { Engineering } \\
\text { strain at ultimate } \\
\text { stress, } \varepsilon_{\mathrm{cu}}\end{array}$ & $\begin{array}{l}\text { Ultimate } \\
\text { strength } \\
\mathrm{Fu}_{\mathrm{u}}(\mathrm{MPa})\end{array}$ \\
\hline $\mathrm{A}^{1}$ & ASTM A572 Grade 50 & Round $\phi 25.4$ & 205600 & 0.0053 & 359.7 & 0.153 & 537.9 \\
\hline $\mathrm{B}^{1}$ & CSA $300 W(44 W)$ & Round $\phi 25.4$ & 207100 & 0.0163 & 326.0 & 0.187 & 505.8 \\
\hline $\mathrm{C}^{2}$ & CSA $300 W(44 W)$ & FB $76 \times 4.8$ & 210700 & 0.0236 & 305.0 & 0.216 & 440.9 \\
\hline $\mathrm{D}^{2}$ & CSA $300 W(44 W)$ and $345 W(50 W)$ & FB $76 \times 7.9$ & 205100 & 0.0035 & 319.2 & 0.162 & 553.8 \\
\hline$E^{2}$ & CSA $300 W(44 W)$ & FB $102 \times 7.9$ & 204200 & 0.0065 & 348.1 & 0.151 & 562.1 \\
\hline$F^{2}$ & CSA $300 \mathrm{~W}(44 \mathrm{~W})$ and $345 \mathrm{~W}(50 \mathrm{~W})$ & FB $127 \times 7.9$ & 208300 & 0.0134 & 328.5 & 0.186 & 494.2 \\
\hline $\mathrm{AW} 50^{3}$ & ASTM A572 Grade 50 & - & 200000 & - & 344.1 & 0.159 & 503.0 \\
\hline $\mathrm{AP}^{2} 0^{3}$ & ASTM A572 Grade 50 & - & 200000 & - & 361.1 & 0.113 & 584.8 \\
\hline $\mathrm{AP} 110^{3}$ & ASTM A514 Grade 110 & - & 200000 & - & 766.7 & 0.015 & 816.6 \\
\hline $\mathrm{AP} 70 \mathrm{HP}^{3}$ & HPS70W & - & 200000 & - & 558.3 & 0.113 & 683.8 \\
\hline $\mathrm{JP} 50^{3}$ & JIS-SN490B Grade 50 & - & 200000 & - & 350.6 & 0.189 & 524.3 \\
\hline $\mathrm{JP}^{2} 0 \mathrm{HP}^{3}$ & JIS-SM490YBTMC-5L Grade 50 & - & 200000 & - & 416.7 & 0.128 & 518.6 \\
\hline $\mathrm{JW} 50^{3}$ & JIS-SN490B Grade 50 & - & 200000 & - & 349.5 & 0.097 & 476.8 \\
\hline
\end{tabular}


Table 5.2 Continue

\begin{tabular}{|c|c|c|c|c|c|c|}
\hline Material & $\mathrm{F}_{\mathrm{u}} / \mathrm{F}_{\mathrm{y}}$ & $\ln \left(\mathrm{A}_{0} / \mathrm{A}_{\mathrm{f}}\right)$ & $\begin{array}{c}\text { True initial yield } \\
\text { stress } \sigma_{\mathrm{ty}}(\mathrm{MPa})\end{array}$ & $\begin{array}{c}\text { Saturated yield stress } \\
\sigma_{\mathrm{s}}^{0}(\mathrm{MPa})\end{array}$ & $\sigma_{\mathrm{s}}^{0} / \sigma_{\mathrm{ty}}$ & $\eta$ \\
\hline $\mathrm{A}^{1}$ & 1.50 & 0.986 & 360.3 & 478.0 & 1.33 & 0.89 \\
\hline $\mathrm{B}^{1}$ & 1.55 & 0.857 & 326.5 & 399.0 & 1.22 & 0.79 \\
\hline $\mathrm{C}^{2}$ & 1.45 & 0.867 & 305.4 & - & - & - \\
\hline $\mathrm{D}^{2}$ & 1.73 & 0.791 & 319.7 & - & - & - \\
\hline $\mathrm{E}^{2}$ & 1.61 & 0.870 & 348.7 & - & - & - \\
\hline $\mathrm{F}^{2}$ & 1.50 & 0.896 & 329.0 & - & - & - \\
\hline $\mathrm{AW}^{3}$ & 1.46 & - & 344.7 & 461.9 & 1.34 & 0.92 \\
\hline $\mathrm{AP50}^{3}$ & 1.62 & - & 361.8 & 461.9 & 1.28 & 0.79 \\
\hline $\mathrm{AP}^{3} 10^{3}$ & 1.07 & - & 769.6 & 723.9 & 0.94 & 0.88 \\
\hline $\mathrm{AP70HP}^{3}$ & 1.22 & - & 559.9 & 703.3 & 1.26 & 1.03 \\
\hline $\mathrm{JP50}^{3}$ & 1.50 & - & 351.2 & 482.6 & 1.37 & 0.92 \\
\hline $\mathrm{JP}^{3} 5 \mathrm{HP}^{3}$ & 1.24 & - & 417.6 & 586.1 & 1.40 & 1.13 \\
\hline $\mathrm{JW}^{3} 0^{3}$ & 1.36 & - & 350.1 & 551.6 & 1.58 & 1.15 \\
\hline
\end{tabular}

Notes:

1 Average values from the current study with maximum errors $\pm 600 \mathrm{MPa}$ for the elastic modulus, $\pm 5.2 \mathrm{MPa}$ for the measured stresses, \pm 0.0003 for $\varepsilon_{\mathrm{ep}}, \pm 0.009$ for $\varepsilon_{\mathrm{eu}}$ and \pm 0.014 for $\ln \left(\mathrm{A}_{\mathrm{y}} / \mathrm{A}_{\mathrm{f}}\right)$.

2 Values from Aghdam (2009) averaged over a number of tests with maximum errors $\pm 12000 \mathrm{MPa}$ for the elastic modulus, $\pm 5.8 \mathrm{MPa}$ for the measured stresses, \pm 0.0014 for $\varepsilon_{\mathrm{ep}}, \pm 0.010$ for $\varepsilon_{\mathrm{eu}}$ and \pm 0.031 for $\ln \left(\mathrm{A}_{0} / \mathrm{A}_{\mathrm{i}}\right)$.

${ }^{3}$ Values from Kanvinde and Deierlein $(2004,2007)$ based on input data given for the numerical simulations. 
Table 5.3 Calculation of the damage limit for a fictitious material ${ }^{\mathrm{H}} \mathrm{A}$ using the measured cycle number and diameter at fracture for specimens of material $\mathrm{B}$ with $\mathrm{c}=1, \mathrm{~m}=3.39$ and $\mathrm{k}=1.12$

\begin{tabular}{|c|c|c|c|c|c|}
\hline $\begin{array}{l}\text { Fictitious specimen } \\
\text { for material A }\end{array}$ & $\begin{array}{c}\text { Test type / } \\
\text { fracture }\end{array}$ & $\begin{array}{l}\text { Damage at } \\
\text { fracture }\end{array}$ & $\mathrm{d}_{0}(\mathrm{~mm})$ & $\begin{array}{l}\mathrm{d}_{\mathrm{f}}(\mathrm{mm}) \text { from } \\
\text { material B }\end{array}$ & $\mathrm{d}_{\mathrm{p}}(\mathrm{mm})$ \\
\hline${ }^{\mathrm{F}} \mathrm{ADMa}$ & Monotonic & 10.34 & 14.00 & 9.19 & 9.17 \\
\hline${ }^{F} \mathrm{ADMb}$ & Monotonic & 11.30 & 14.00 & 9.05 & 9.17 \\
\hline FAM & Monotonic & 8.41 & 10.00 & 7.40 & - \\
\hline${ }^{\mathrm{F}} \mathrm{ABM}$ & Monotonic & 8.16 & 14.00 & 10.41 & - \\
\hline${ }^{\mathrm{F}} \mathrm{ADC} 20202$ & 20 cyclest & 10.53 & 14.00 & 9.30 & - \\
\hline${ }^{F} \mathrm{ADC} 40101$ & 40 cycles + & 9.00 & 14.00 & 9.52 & - \\
\hline${ }^{\mathrm{F}} \mathrm{ADC} 40102$ & 40 cycles + & 10.69 & 14.00 & 9.30 & - \\
\hline${ }^{\mathrm{F}} \mathrm{ADC} 40202$ & 40 cycles + & 10.61 & 14.00 & 9.33 & - \\
\hline${ }^{\mathrm{F}} \mathrm{ADCR} 0201$ & 30 cyclest & 10.02 & 14.00 & 9.37 & - \\
\hline${ }^{\mathrm{F} A A C 20404}$ & 20 cycles + & 12.49 & 10.00 & 7.55 & - \\
\hline${ }^{\mathrm{F}} \mathrm{ABC} 20404$ & 20 cyclest & 13.55 & 14.00 & 10.49 & - \\
\hline${ }^{\mathrm{F}} \mathrm{AAC} 70404$ & 57 cycles & 10.23 & 10.00 & 9.73 & - \\
\hline $\mathrm{ABC} 70404$ & 64 cycles & 13.16 & 14.00 & 13.57 & - \\
\hline${ }^{\mathrm{F}} \mathrm{AACF} 1212$ & 7 cycles & 10.30 & 10.00 & 9.03 & - \\
\hline${ }^{\mathrm{F}} \mathrm{ABCF} 1212$ & 7 cycles & 11.34 & 14.00 & 12.83 & - \\
\hline${ }^{\mathrm{F}} \mathrm{AACF} 3010$ & 2 cycles & 8.30 & 10.00 & 8.27 & - \\
\hline${ }^{\mathrm{F}} \mathrm{ABCF} 3010$ & 2 cycles & 8.61 & 13.98 & 11.43 & - \\
\hline FACR0804 & 36 cycles & 10.41 & 10.00 & 9.63 & - \\
\hline \multicolumn{2}{|l|}{ Mean } & 10.41 & \multirow{3}{*}{-} & \multirow{3}{*}{\multicolumn{2}{|c|}{-}} \\
\hline \multicolumn{2}{|c|}{ Standard deviation } & 1.58 & & & \\
\hline \multicolumn{2}{|c|}{ Coefficient of variation } & $15 \%$ & & & \\
\hline
\end{tabular}


Table 5.4 Test and predicted point of fracture for specimens of material A using damage limit $\mathrm{D}_{c}=14.92$ calibrated through test results of specimens from material $B$, and $c=1, m=3.39$ and $k=1.12$

\begin{tabular}{|c|c|c|c|c|c|c|c|c|c|c|}
\hline Specimen & $\begin{array}{c}\text { Test type / } \\
\text { fracture }\end{array}$ & $\mathrm{d}_{0}(\mathrm{~mm})$ & $\mathrm{d}_{\mathrm{f}}(\mathrm{mm})$ & $\mathrm{d}_{\mathrm{p}}(\mathrm{mm})$ & $\mathrm{d}_{\mathrm{p}} / \mathrm{d}_{\mathrm{f}}$ & $\left(\mathrm{d}_{0}-\mathrm{d}_{\mathrm{p}}\right) /\left(\mathrm{d}_{0}-\mathrm{d}_{\mathrm{f}}\right)$ & $\begin{array}{c}\text { Predicted } \\
\text { fracture cycle }\end{array}$ & $\varepsilon_{\text {af }}$ & $\varepsilon_{\text {ap }}$ & $\varepsilon_{\text {af }} / \varepsilon_{\text {ap }}$ \\
\hline ADMa & Monotonic & 14.00 & 8.51 & 8.63 & 1.01 & 0.98 & - & - & - & - \\
\hline ADMb & Monotonic & 14.00 & 8.54 & 8.63 & 1.01 & 0.98 & - & - & - & - \\
\hline AEM & Monotonic & 13.96 & 8.57 & 8.61 & 1.00 & 0.99 & - & - & - & - \\
\hline AAMa & Monotonic & 10.00 & 7.14 & 6.85 & 0.96 & 1.10 & - & 0.070 & 0.073 & 1.05 \\
\hline AAMb & Monotonic & 10.00 & 7.07 & 6.85 & 0.97 & 1.07 & - & 0.070 & 0.073 & 1.05 \\
\hline ABM & Monotonic & 14.00 & 10.18 & 9.60 & 0.94 & 1.15 & - & 0.094 & 0.103 & 1.09 \\
\hline ADC20202 & 20 cycles+ & 14.00 & 8.63 & 8.75 & 1.01 & 0.98 & $20+$ & - & - & - \\
\hline ADC40101 & 40 cycles+ & 14.00 & 8.97 & 8.74 & 0.97 & 1.05 & $40+$ & - & - & - \\
\hline ADC40102 & 40 cycles+ & 14.00 & 8.78 & 8.77 & 1.00 & 1.00 & $40+$ & - & - & - \\
\hline ADC40202 & 40 cycles+ & 14.00 & 8.75 & 8.78 & 1.00 & 0.99 & $40+$ & - & - & - \\
\hline ADCR0201 & 30 cycles+ & 14.00 & 8.66 & 8.75 & 1.01 & 0.98 & $30+$ & - & - & - \\
\hline AAC20404 & 20 cycles+ & 10.00 & 7.27 & 7.31 & 1.01 & 0.99 & $20+$ & 0.544 & 0.537 & 0.99 \\
\hline ABC20404 & 20 cycles+ & 14.00 & 10.04 & 10.30 & 1.03 & 0.93 & $20+$ & 0.805 & 0.797 & 0.99 \\
\hline ACC20404 & 20 cycles+ & 10.00 & 7.92 & 7.86 & 0.99 & 1.03 & $20+$ & 0.441 & 0.439 & 1.00 \\
\hline AAC70404 & 70 cycles+ & 10.00 & 7.94 & 8.71 & 1.10 & 0.63 & $70+$ & 1.729 & 1.709 & 0.99 \\
\hline ABC70404 & 70 cycles+ & 14.00 & 12.73 & 13.07 & 1.03 & 0.73 & $70+$ & 2.554 & 2.539 & 0.99 \\
\hline AACF1212 & 9 cycles & 10.00 & 9.57 & 9.02 & 0.94 & 2.31 & 10 & 0.621 & 0.702 & 1.13 \\
\hline ABCF1212 & 7 cycles & 14.00 & 12.38 & 13.01 & 1.05 & 0.61 & 9 & 0.734 & 0.918 & 1.25 \\
\hline AACF3010 & 3 cycles & 10.00 & 8.23 & 8.63 & 1.05 & 0.77 & 4 & 0.339 & 0.429 & 1.26 \\
\hline ABCF3010 & 3 cycles & 13.98 & 12.65 & 10.72 & 0.85 & 2.44 & 3 & 0.448 & 0.485 & 1.08 \\
\hline AACR0804 & 48 cycles & 10.00 & 9.59 & 9.74 & 1.01 & 0.65 & 60 & 1.350 & 1.635 & 1.21 \\
\hline
\end{tabular}


Table 5.5 Flow stress equation parameters for materials $\mathrm{C}$ to $\mathrm{F}$

\begin{tabular}{|c|c|c|c|c|c|}
\hline Material & $\sigma_{0}^{\mathrm{y}}(\mathrm{MPa})$ & $\varepsilon_{0}^{\mathrm{p}}$ & $\sigma_{x}^{\mathrm{y}}(\mathrm{MPa})$ & $\mathrm{b}$ & $\mathrm{n}$ \\
\hline $\mathrm{C}$ & 312.6 & 0.0232 & 436.8 & 1.82 & 0.64 \\
\hline $\mathrm{D}$ & 320.8 & 0.0035 & 535.2 & 2.82 & 0.57 \\
\hline $\mathrm{E}$ & 351.0 & 0.0065 & 529.8 & 2.00 & 0.52 \\
\hline $\mathrm{F}$ & 333.4 & 0.0133 & 514.8 & 1.80 & 0.57 \\
\hline
\end{tabular}

Table 5.6 Parameters of two-term kinematic hardening model for materials $\mathrm{C}$ to $\mathrm{F}$

\begin{tabular}{|c|c|c|c|c|c|}
\hline Material & $\eta$ & $\mathrm{C}_{1}(\mathrm{MPa})$ & $\gamma_{1}$ & $\mathrm{C}_{2}(\mathrm{MPa})$ & $\gamma_{2}$ \\
\hline \multirow{2}{*}{$\mathrm{C}$} & 0.75 & 1376 & 5.99 & 228 & 1.07 \\
\cline { 2 - 6 } & 1.2 & 269 & 1.10 & 0 & 1.00 \\
\hline \multirow{2}{*}{$\mathrm{D}$} & 0.75 & 2134 & 7.69 & 252 & 1.58 \\
\cline { 2 - 6 } & 1.2 & 287 & 1.53 & 0 & 1.00 \\
\hline \multirow{2}{*}{$\mathrm{E}$} & 0.75 & 2089 & 7.13 & 174 & 0.94 \\
\cline { 2 - 6 } & 1.2 & 235 & 1.05 & 0 & 1.00 \\
\hline \multirow{2}{*}{$\mathrm{F}$} & 0.75 & 1649 & 6.16 & 201 & 0.93 \\
\cline { 2 - 6 } & 1.2 & 269 & 1.03 & 0 & 1.00 \\
\hline
\end{tabular}


Table 5.7 Calculation of the damage limit for a fictitious material ${ }^{\mathrm{r}} \mathrm{C}$ using the measured cycle number and diameter at fracture for specimens of material B with $\mathrm{c}=1, \mathrm{~m}=2.18$ and $\mathrm{k}=1.48(\eta=0.75)$

\begin{tabular}{|c|c|c|c|c|c|}
\hline $\begin{array}{l}\text { Fictitious specimen } \\
\text { for material } C\end{array}$ & $\begin{array}{c}\text { Test type / } \\
\text { fracture }\end{array}$ & $\begin{array}{l}\text { Damage at } \\
\text { fracture }\end{array}$ & $\mathrm{d}_{0}(\mathrm{~mm})$ & $\begin{array}{c}\mathrm{d}_{\mathrm{i}}(\mathrm{mm}) \text { from } \\
\text { material } \mathrm{B}\end{array}$ & $\mathrm{d}_{\mathrm{p}}(\mathrm{mm})$ \\
\hline${ }^{\mathrm{F}} \mathrm{CDMa}$ & Monotonic & 1.32 & 14.00 & 9.19 & 9.42 \\
\hline${ }^{\mathrm{F}} \mathrm{CDMb}$ & Monotonic & 1.43 & 14.00 & 9.05 & 9.42 \\
\hline${ }^{\mathrm{F}} \mathrm{CAM}$ & Monotonic & 0.97 & 10.00 & 7.40 & - \\
\hline${ }^{\mathrm{F}} \mathrm{CBM}$ & Monotonic & 0.94 & 14.00 & 10.41 & - \\
\hline${ }^{\mathrm{F}} \mathrm{CDC} 20202$ & 20 cycles + & 1.31 & 14.00 & 9.30 & - \\
\hline${ }^{\mathrm{F}} \mathrm{CDC} 40101$ & 40 cycles + & 1.15 & 14.00 & 9.52 & - \\
\hline${ }^{\mathrm{F}} \mathrm{CDC} 40102$ & 40 cyclest & 1.33 & 14.00 & 9.30 & - \\
\hline${ }^{\mathrm{F}} \mathrm{CDC} 40202$ & 40 cyclest & 1.32 & 14.00 & 9.33 & - \\
\hline${ }^{\mathrm{F}} \mathrm{CDCR} 0201$ & 30 cycles + & 1.26 & 14.00 & 9.37 & - \\
\hline${ }^{\mathrm{F}} \mathrm{CAC} 20404$ & 20 cyclest & 1.28 & 10.00 & 7.55 & - \\
\hline${ }^{\mathrm{F}} \mathrm{CBC} 20404$ & 20 cyclest & 1.37 & 14.00 & 10.49 & - \\
\hline${ }^{\mathrm{I} C A C 70404}$ & 57 cycles & 1.39 & 10.00 & 9.73 & - \\
\hline${ }^{\mathrm{F}} \mathrm{CBC} 70404$ & 64 cycles & 1.69 & 14.00 & 13.57 & - \\
\hline${ }^{\mathrm{F}} \mathrm{CACF} 1212$ & 7 cycles & 0.67 & 10.00 & 9.03 & - \\
\hline${ }^{\mathrm{F}} \mathrm{CBCF} 1212$ & 7 cycles & 0.71 & 14.00 & 12.83 & - \\
\hline${ }^{\mathrm{F}} \mathrm{CACF} 3010$ & 2 cycles & 0.66 & 10.00 & 8.27 & - \\
\hline${ }^{\mathrm{F}} \mathrm{CBCF} 3010$ & 2 cycles & 0.67 & 14.00 & 11.43 & - \\
\hline${ }^{\mathrm{F} C A C R} 0804$ & 36 cycles & 1.15 & 10.00 & 9.63 & - \\
\hline \multicolumn{2}{|l|}{ Mean } & 1.15 & \multirow{3}{*}{\multicolumn{3}{|c|}{-}} \\
\hline \multicolumn{2}{|c|}{ Standard deviation } & 0.31 & & & \\
\hline \multicolumn{2}{|c|}{ Coefficient of variation } & $27 \%$ & & & \\
\hline
\end{tabular}


Table 5.8 Parameters for the fracture criterion and damage limit for materials $\mathrm{C}$ to $\mathrm{F}$ estimated through test results of specimens from material B

\begin{tabular}{|c|c|c|c|c|c|c|}
\hline \multirow{2}{*}{ Material } & \multicolumn{3}{|c|}{$\eta=0.75$} & \multicolumn{3}{c|}{$\eta=1.2$} \\
\cline { 2 - 7 } & $\mathrm{m}$ & $\mathrm{k}$ & Damage limit, $\mathrm{D}_{\mathrm{c}}$ & $\mathrm{m}$ & $\mathrm{k}$ & Damage limit, $\mathrm{D}_{\mathrm{c}}$ \\
\hline $\mathrm{C}$ & 2.18 & 1.48 & 1.64 & 7.59 & 0.65 & 112.61 \\
\hline $\mathrm{D}$ & 2.09 & 1.72 & 2.67 & 8.68 & 0.61 & 3840.49 \\
\hline $\mathrm{E}$ & 2.29 & 2.09 & 3.43 & 9.16 & 0.54 & 12171.18 \\
\hline $\mathrm{F}$ & 2.17 & 1.71 & 2.22 & 7.96 & 0.71 & 579.66 \\
\hline
\end{tabular}

Table 5.9 Type A strap specimens tested by Aghdam (2009)

\begin{tabular}{|c|c|c|c|c|}
\hline Material & Specimen & $\begin{array}{c}\text { Dimension }(\mathrm{mm}) \\
\text { Length } \mathrm{x} \text { width } \mathrm{x} \text { thickness }\end{array}$ & $\mathrm{KL} / \mathrm{r}$ & $A_{n} F_{v} / A_{g} F_{y}$ \\
\hline \multirow{3}{*}{$\mathrm{C}$} & A4075a-b & $1360 \times 76 \times 4.8$ & 400 & \multirow{3}{*}{1.03} \\
\hline & A6075a-b & $1930 \times 76 \times 4.8$ & 600 & \\
\hline & A8075a-b & $2510 \times 76 \times 4.8$ & 800 & \\
\hline \multirow{2}{*}{$\mathrm{D}$} & A2078a-e & $1120 \times 76 \times 7.9$ & 200 & \multirow{2}{*}{1.23} \\
\hline & A4078a-b & $2050 \times 76 \times 7.9$ & 400 & \\
\hline \multirow{2}{*}{$\mathrm{E}$} & $A 2108 a-b$ & $1120 \times 102 \times 7.9$ & 200 & \multirow{2}{*}{1.27} \\
\hline & A4108a-b & $2050 \times 102 \times 7.9$ & 400 & \\
\hline \multirow{2}{*}{$\mathrm{F}$} & A2128a-e & $1120 \times 127 \times 7.9$ & 200 & \multirow{2}{*}{1.24} \\
\hline & A4128a-b & $2050 \times 127 \times 7.9$ & 400 & \\
\hline
\end{tabular}


Table 5.10 Comparison of FEA predictions for strap specimen A8075 with two different boundary constraints

\begin{tabular}{|c|c|c|c|c|c|c|c|c|c|}
\hline \multirow[b]{2}{*}{ Specimen } & \multicolumn{2}{|c|}{ Test } & \multirow[b]{2}{*}{$\begin{array}{l}\text { Constraint } \\
\text { in the } Y \\
\text { direction }\end{array}$} & \multicolumn{3}{|c|}{$1 / 2 \operatorname{model}(\eta=0.75)$} & \multicolumn{3}{|c|}{$1 / 2 \operatorname{model}(\eta=1.2)$} \\
\hline & $\begin{array}{c}\text { Half cycle } \\
\text { no. at } \\
\text { fracture }\end{array}$ & $\begin{array}{c}\text { Max. } \\
\text { deformation } \\
(\mathrm{mm})(\mathrm{A})\end{array}$ & & $\begin{array}{c}\text { Half cycle no. } \\
\text { at fracture }\end{array}$ & $\begin{array}{l}\text { Max. deformation } \\
(\mathrm{mm})(\mathrm{B})\end{array}$ & $(\mathrm{A}) /(\mathrm{B})$ & $\begin{array}{c}\text { Half cycle no. } \\
\text { at fracture }\end{array}$ & $\begin{array}{l}\text { Max. deformation } \\
(\mathrm{mm})(\mathrm{C})\end{array}$ & $(\mathrm{A}) /(\mathrm{C})$ \\
\hline $\mathrm{A} 8075 \mathrm{a}$ & 47 & 11.75 & \multirow{2}{*}{ Yes } & \multirow{2}{*}{43} & \multirow{2}{*}{10.39} & 1.13 & \multirow{2}{*}{43} & \multirow{2}{*}{10.46} & 1.12 \\
\hline $\mathrm{A} 8075 \mathrm{~b}$ & 43 & 9.92 & & & & 0.95 & & & 0.95 \\
\hline $\mathrm{A} 8075 \mathrm{a}$ & 47 & 11.75 & \multirow{2}{*}{ No } & \multirow{2}{*}{47} & \multirow{2}{*}{13.83} & 0.85 & \multirow{2}{*}{47} & \multirow{2}{*}{13.92} & 0.84 \\
\hline $\mathrm{A} 8075 \mathrm{~b}$ & 43 & 9.92 & & & & 0.72 & & & 0.71 \\
\hline
\end{tabular}

Table 5.11 Strain rate dependent on the net section fracture to gross section yielding strength ratio (Aghdam 2009)

\begin{tabular}{|c|c|c|c|c|}
\hline \multirow{3}{*}{ Material } & \multicolumn{4}{|c|}{$\mathrm{A}_{\mathrm{n}} \mathrm{F}_{\mathrm{u}} / \mathrm{A}_{\mathrm{g}} \mathrm{F}_{\mathrm{v}}$} \\
\cline { 2 - 5 } & Static strain rate & \multicolumn{3}{|c|}{ Dynamic strain rate } \\
\cline { 2 - 5 } & $0.0001 \mathrm{~s}^{-1}$ & $0.0001 \mathrm{~s}^{-1}$ & $0.01 \mathrm{~s}^{-1}$ & $0.5 \mathrm{~s}^{-1}$ \\
\hline $\mathrm{C}$ & 1.03 & 1.02 & 0.94 & 0.89 \\
\hline $\mathrm{D}$ & 1.23 & 1.20 & 1.14 & 1.12 \\
\hline $\mathrm{E}$ & 1.27 & 1.22 & 1.18 & 1.16 \\
\hline $\mathrm{F}$ & 1.24 & 1.25 & 1.12 & 1.12 \\
\hline
\end{tabular}


Table 5.12 FEA predictions for strap specimen A4075 and A2078 using two different mesh schemes

\begin{tabular}{|c|c|c|c|c|c|}
\hline \multirow{2}{*}{ Specimen } & Mesh & $\begin{array}{c}\text { Half cycle } \\
\text { no. at } \\
\text { fracture }\end{array}$ & $\begin{array}{c}1 / 4 \text { model }(\eta=0.75) \\
\text { deformation } \\
(\mathrm{mm})\end{array}$ & $\begin{array}{c}\text { Half cycle } \\
\text { no. at } \\
\text { fracture }\end{array}$ & $\begin{array}{c}\text { Max. } \\
\text { deformation } \\
(\mathrm{mm})\end{array}$ \\
\hline \multirow{2}{*}{ A4075 } & Scheme-1 & - & - & 51 & 8.70 \\
\cline { 2 - 6 } & Scheme-2 & - & - & 49 & 8.70 \\
\hline \multirow{2}{*}{ A2078 } & Scheme-1 & 93 & 21.98 & - & - \\
\cline { 2 - 6 } & Scheme-2 & 91 & 20.70 & - & - \\
\hline
\end{tabular}


Table 5.13 FEA predictions for tests of strap specimens using the $1 / 4$ model

\begin{tabular}{|c|c|c|c|c|c|c|c|c|c|c|c|}
\hline \multirow[b]{3}{*}{ Material } & \multirow[b]{3}{*}{ Specimen } & \multirow{2}{*}{\multicolumn{2}{|c|}{ Test }} & \multicolumn{8}{|c|}{ FEA simulation } \\
\hline & & & & \multicolumn{4}{|c|}{$\eta=0.75$} & \multicolumn{4}{|c|}{$\eta=1.2$} \\
\hline & & $\begin{array}{c}\text { Half cycle } \\
\text { no. at } \\
\text { fracture }(A)\end{array}$ & $\begin{array}{c}\text { Max. } \\
\text { deformation } \\
(\mathrm{mm})(\mathrm{B})\end{array}$ & $\begin{array}{c}\text { Half cycle } \\
\text { no. at } \\
\text { fracture }(C)\end{array}$ & $(\mathrm{A}) /(\mathrm{C})$ & \begin{tabular}{|c|} 
Max. \\
deformation \\
$(\mathrm{mm})(\mathrm{D})$ \\
\end{tabular} & $(\mathrm{B}) /(\mathrm{D})$ & $\begin{array}{l}\text { Half cycle } \\
\text { no. at } \\
\text { fracture }(E)\end{array}$ & $(\mathrm{A}) /(\mathrm{E})$ & \begin{tabular}{|c|} 
Max. \\
deformation \\
$(\mathrm{nmm})(\mathrm{F})$ \\
\end{tabular} & $(\mathrm{B}) /(\mathrm{F})$ \\
\hline \multirow{6}{*}{$\mathrm{C}$} & A4075a & 55 & 9.14 & \multirow{2}{*}{71} & 0.77 & \multirow{2}{*}{17.40} & 0.53 & \multirow{2}{*}{83} & 0.66 & \multirow{2}{*}{22.62} & 0.40 \\
\hline & $\mathrm{A} 4075 \mathrm{~b}$ & 51 & 6.74 & & 0.72 & & 0.39 & & 0.61 & & 0.30 \\
\hline & $\mathrm{A} 6075 \mathrm{a}$ & 43 & 7.52 & \multirow{2}{*}{ - } & - & \multirow{2}{*}{-} & - & \multirow{2}{*}{-} & - & \multirow{2}{*}{ - } & - \\
\hline & $\mathrm{A} 6075 \mathrm{~b}$ & 47 & 8.53 & & - & & & & - & & \\
\hline & $\mathrm{A} 8075 \mathrm{a}$ & 47 & 11.75 & \multirow{2}{*}{-} & - & \multirow{2}{*}{-} & - & \multirow{2}{*}{-} & - & \multirow{2}{*}{-} & - \\
\hline & $\mathrm{A} 8075 \mathrm{~b}$ & 43 & 9.92 & & - & & - & & - & & - \\
\hline \multirow{7}{*}{$\mathrm{D}$} & $\mathrm{A} 2078 \mathrm{a}$ & 107 & 23.95 & \multirow{3}{*}{93} & 1.15 & \multirow{3}{*}{21.98} & 1.09 & \multirow{3}{*}{97} & 1.10 & \multirow{3}{*}{23.41} & 1.02 \\
\hline & A2078b & 115 & 26.48 & & 1.24 & & 1.20 & & 1.19 & & 1.13 \\
\hline & $\mathrm{A} 2078 \mathrm{c}$ & 123 & 28.96 & & 1.32 & & 1.32 & & 1.27 & & 1.24 \\
\hline & A2078d & - & 29.47 & \multirow{2}{*}{ - } & - & \multirow{2}{*}{25.02} & 1.18 & \multirow{2}{*}{-} & - & \multirow{2}{*}{26.99} & 1.09 \\
\hline & $\mathrm{A} 2078 \mathrm{e}$ & - & 20.32 & & - & & 0.81 & & - & & 0.75 \\
\hline & $\mathrm{A} 4078 \mathrm{a}$ & 91 & 37.83 & \multirow{2}{*}{75} & 1.21 & \multirow{2}{*}{30.52} & 1.24 & \multirow{2}{*}{87} & 1.05 & \multirow{2}{*}{38.80} & 0.97 \\
\hline & $\mathrm{A} 4078 \mathrm{~b}$ & 87 & 34.36 & & 1.16 & & 1.13 & & 1.00 & & 0.89 \\
\hline
\end{tabular}


Table 5.13 Continue

\begin{tabular}{|c|c|c|c|c|c|c|c|c|c|c|c|}
\hline \multirow[b]{3}{*}{ Material } & \multirow[b]{3}{*}{ Specimen } & \multirow{2}{*}{\multicolumn{2}{|c|}{ Test }} & \multicolumn{8}{|c|}{ FEA simulation } \\
\hline & & & & \multicolumn{4}{|c|}{$\eta=0.75$} & \multicolumn{4}{|c|}{$\eta=1.2$} \\
\hline & & $\begin{array}{l}\text { Half cycle } \\
\text { no. at } \\
\text { fracture (A) }\end{array}$ & $\begin{array}{c}\text { Max. } \\
\text { deformation } \\
(\mathrm{mm})(\mathrm{B})\end{array}$ & $\begin{array}{c}\text { Half cycle } \\
\text { no. at } \\
\text { fracture (C) }\end{array}$ & $(\mathrm{A}) /(\mathrm{C})$ & $\begin{array}{c}\text { Max. } \\
\text { deformation } \\
(\mathrm{mm})(\mathrm{D})\end{array}$ & $(\mathrm{B}) /(\mathrm{D})$ & $\begin{array}{c}\text { Half cycle } \\
\text { no. at } \\
\text { fracture }(E)\end{array}$ & $(\mathrm{A}) /(\mathrm{E})$ & \begin{tabular}{|c|} 
Max. \\
deformation \\
$(\mathrm{mmm})(\mathrm{F})$
\end{tabular} & $(\mathrm{B}) /(\mathrm{F})$ \\
\hline \multirow{4}{*}{$E$} & $\mathrm{~A} 2108 \mathrm{a}$ & 125 & 28.32 & \multirow{2}{*}{101} & 1.24 & \multirow{2}{*}{24.81} & 1.14 & \multirow{2}{*}{107} & 1.17 & \multirow{2}{*}{26.14} & 1.08 \\
\hline & $\mathrm{A} 2108 \mathrm{~b}$ & 127 & 29.45 & & 1.26 & & 1.19 & & 1.19 & & 1.13 \\
\hline & A4108a & 99 & 42.48 & \multirow{2}{*}{81} & 1.22 & \multirow{2}{*}{36.04} & 1.18 & \multirow{2}{*}{95} & 1.04 & \multirow{2}{*}{44.30} & 0.96 \\
\hline & $A 4108 b$ & 107 & 47.35 & & 1.32 & & 1.31 & & 1.13 & & 1.07 \\
\hline \multirow{7}{*}{$\mathrm{F}$} & A2128a & 139 & 32.82 & \multirow{3}{*}{107} & 1.30 & \multirow{3}{*}{26.12} & 1.26 & \multirow{3}{*}{127} & 1.09 & \multirow{3}{*}{33.11} & 0.99 \\
\hline & $\mathrm{A} 2128 \mathrm{~b}$ & 143 & 35.11 & & 1.34 & & 1.34 & & 1.13 & & 1.06 \\
\hline & $\mathrm{A} 2128 \mathrm{c}$ & 137 & 32.17 & & 1.28 & & 1.23 & & 1.08 & & 0.97 \\
\hline & $\mathrm{A} 2128 \mathrm{~d}$ & - & 42.26 & \multirow{2}{*}{-} & - & \multirow{2}{*}{41.42} & 1.02 & \multirow{2}{*}{-} & - & \multirow{2}{*}{42.42} & 1.00 \\
\hline & $\mathrm{A} 2128 \mathrm{e}$ & - & 43.26 & & - & & 1.04 & & - & & 1.02 \\
\hline & A4128a & 119 & 55.61 & \multirow{2}{*}{99} & 1.20 & \multirow{2}{*}{47.15} & 1.18 & \multirow{2}{*}{121} & 0.98 & \multirow{2}{*}{63.81} & 0.87 \\
\hline & $\mathrm{A} 4128 \mathrm{~b}$ & 119 & 54.23 & & 1.20 & & 1.15 & & 0.98 & & 0.85 \\
\hline
\end{tabular}


Table 5.14 FEA predictions for tests of strap specimens using the $1 / 2$ model

\begin{tabular}{|c|c|c|c|c|c|c|c|c|c|c|c|}
\hline \multirow[b]{3}{*}{ Material } & \multirow[b]{3}{*}{ Specimen } & \multirow{2}{*}{\multicolumn{2}{|c|}{ Test }} & \multicolumn{8}{|c|}{ FEA simulation } \\
\hline & & & & \multicolumn{4}{|c|}{$\eta=0.75$} & \multicolumn{4}{|c|}{$\eta=1.2$} \\
\hline & & $\begin{array}{c}\text { Half cycle } \\
\text { no. at } \\
\text { fracture }(A)\end{array}$ & $\begin{array}{c}\text { Max. } \\
\text { deformation } \\
(\mathrm{mm})(\mathrm{B})\end{array}$ & $\begin{array}{c}\text { Half cycle } \\
\text { no. at } \\
\text { fracture (C) }\end{array}$ & $(\mathrm{A}) /(\mathrm{C})$ & $\begin{array}{c}\text { Max. } \\
\text { deformation } \\
(\mathrm{mm})(\mathrm{D})\end{array}$ & (B)/(D) & $\begin{array}{c}\text { Half cycle } \\
\text { no. at } \\
\text { fracture }(E)\end{array}$ & $(\mathrm{A}) /(\mathrm{E})$ & \begin{tabular}{|c|} 
Max. \\
deformation \\
$(\mathrm{mm})(\mathrm{F})$
\end{tabular} & $(\mathrm{B}) /(\mathrm{F})$ \\
\hline \multirow{6}{*}{$\mathrm{C}$} & A4075a & 55 & 9.14 & \multirow{2}{*}{51} & 1.08 & \multirow{2}{*}{8.70} & 1.05 & \multirow{2}{*}{57} & 0.96 & \multirow{2}{*}{12.16} & 0.75 \\
\hline & $\mathrm{A} 4075 \mathrm{~b}$ & 51 & 6.74 & & 1.00 & & 0.78 & & 0.89 & & 0.55 \\
\hline & $\mathrm{A} 6075 \mathrm{a}$ & 43 & 7.52 & \multirow{2}{*}{43} & 1.00 & \multirow{2}{*}{9.87} & 0.76 & \multirow{2}{*}{47} & 0.91 & \multirow{2}{*}{10.36} & 0.73 \\
\hline & $\mathrm{A} 6075 \mathrm{~b}$ & 47 & 8.53 & & 1.09 & & 0.86 & & 1.00 & & 0.82 \\
\hline & $\mathrm{A} 8075 \mathrm{a}$ & 47 & 11.75 & \multirow{2}{*}{43} & 1.09 & \multirow{2}{*}{10.39} & 1.13 & \multirow{2}{*}{43} & 1.09 & \multirow{2}{*}{10.46} & 1.12 \\
\hline & $A 8075 b$ & 43 & 9.92 & & 1.00 & & 0.95 & & 1.00 & & 0.95 \\
\hline \multirow{7}{*}{$\mathrm{D}$} & A2078a & 107 & 23.95 & \multirow{3}{*}{81} & 1.32 & \multirow{3}{*}{17.83} & 1.34 & \multirow{3}{*}{89} & 1.20 & \multirow{3}{*}{20.67} & 1.16 \\
\hline & A2078b & 115 & 26.48 & & 1.42 & & 1.49 & & 1.29 & & 1.28 \\
\hline & A2078c & 123 & 28.96 & & 1.52 & & 1.62 & & 1.38 & & 1.40 \\
\hline & $\mathrm{A} 2078 \mathrm{~d}$ & - & 29.47 & \multirow{2}{*}{ - } & - & \multirow{2}{*}{17.84} & 1.65 & \multirow{2}{*}{ - } & - & \multirow{2}{*}{18.82} & 1.57 \\
\hline & $\mathrm{A} 2078 \mathrm{e}$ & - & 20.32 & & - & & 1.14 & & - & & 1.08 \\
\hline & $\mathrm{A} 4078 \mathrm{a}$ & 91 & 37.83 & \multirow{2}{*}{63} & 1.44 & \multirow{2}{*}{24.35} & 1.55 & \multirow{2}{*}{79} & 1.15 & \multirow{2}{*}{33.30} & 1.14 \\
\hline & $\mathrm{A} 4078 \mathrm{~b}$ & 87 & 34.36 & & 1.38 & & 1.41 & & 1.10 & & 1.03 \\
\hline
\end{tabular}


Table 5.14 Continue

\begin{tabular}{|c|c|c|c|c|c|c|c|c|c|c|c|}
\hline \multirow[b]{3}{*}{ Material } & \multirow[b]{3}{*}{ Specimen } & \multirow{2}{*}{\multicolumn{2}{|c|}{ Test }} & \multicolumn{8}{|c|}{ FEA simulation } \\
\hline & & & & \multicolumn{4}{|c|}{$\eta=0.75$} & \multicolumn{4}{|c|}{$\eta=1.2$} \\
\hline & & $\begin{array}{c}\text { Half cycle } \\
\text { no. at } \\
\text { fracture }(A)\end{array}$ & $\begin{array}{c}\text { Max. } \\
\text { deformation } \\
(\mathrm{mm})(\mathrm{B})\end{array}$ & $\begin{array}{c}\text { Half cycle } \\
\text { no. at } \\
\text { fracture (C) }\end{array}$ & $(\mathrm{A}) /(\mathrm{C})$ & \begin{tabular}{|c|} 
Max. \\
deformation \\
$(\mathrm{mm})(\mathrm{D})$
\end{tabular} & (B) $/(\mathrm{D})$ & $\begin{array}{c}\text { Half cycle } \\
\text { no. at } \\
\text { fracture }(E)\end{array}$ & $(\mathrm{A}) /(\mathrm{E})$ & $\begin{array}{c}\text { Max. } \\
\text { deformation } \\
(\mathrm{mm})(\mathrm{F}) \\
\end{array}$ & $(\mathrm{B}) /(\mathrm{F})$ \\
\hline \multirow{4}{*}{$\mathrm{E}$} & A2108a & 125 & 28.32 & \multirow{2}{*}{87} & 1.44 & \multirow{2}{*}{19.31} & 1.47 & \multirow{2}{*}{99} & 1.26 & \multirow{2}{*}{23.45} & 1.21 \\
\hline & $\mathrm{A} 2108 \mathrm{~b}$ & 127 & 29.45 & & 1.46 & & 1.53 & & 1.28 & & 1.26 \\
\hline & A4108a & 99 & 42.48 & \multirow{2}{*}{71} & 1.39 & \multirow{2}{*}{27.70} & 1.53 & \multirow{2}{*}{85} & 1.16 & \multirow{2}{*}{38.84} & 1.09 \\
\hline & $\mathrm{A} 4108 \mathrm{~b}$ & 107 & 47.35 & & 1.51 & & 1.71 & & 1.26 & & 1.22 \\
\hline \multirow{7}{*}{ F } & $\mathrm{A} 2128 \mathrm{a}$ & 139 & 32.82 & \multirow{3}{*}{103} & 1.35 & \multirow{3}{*}{24.82} & 1.32 & \multirow{3}{*}{121} & 1.15 & \multirow{3}{*}{31.69} & 1.04 \\
\hline & $\mathrm{A} 2128 \mathrm{~b}$ & 143 & 35.11 & & 1.39 & & 1.41 & & 1.18 & & 1.11 \\
\hline & A2128c & 137 & 32.17 & & 1.33 & & 1.30 & & 1.13 & & 1.02 \\
\hline & $\mathrm{A} 2128 \mathrm{~d}$ & - & 42.26 & \multirow{2}{*}{-} & - & \multirow{2}{*}{33.16} & 1.27 & \multirow{2}{*}{-} & - & \multirow{2}{*}{34.42} & 1.23 \\
\hline & $\mathrm{A} 2128 \mathrm{e}$ & - & 43.26 & & - & & 1.30 & & - & & 1.26 \\
\hline & A4128a & 119 & 55.61 & \multirow{2}{*}{91} & 1.31 & \multirow{2}{*}{41.60} & 1.34 & \multirow{2}{*}{115} & 1.03 & \multirow{2}{*}{59.55} & 0.93 \\
\hline & $\mathrm{A} 4128 \mathrm{~b}$ & 119 & 54.23 & & 1.31 & & 1.30 & & 1.03 & & 0.91 \\
\hline
\end{tabular}


Table 5.15 Comparisons of test and FEA predictions for tests of strap specimens of materials D to $F$ using the $1 / 2$ model with the measured maximum deformation reduced by $6 \mathrm{~mm}$ (except A2078e)

\begin{tabular}{|c|c|c|c|c|c|c|}
\hline \multirow{3}{*}{ Material } & \multirow{3}{*}{ Specimen } & \multirow{3}{*}{$\begin{array}{c}\text { Test } \\
\text { Adjusted max. } \\
\text { deformation }(\mathrm{mm})(\mathrm{A})\end{array}$} & \multicolumn{4}{|c|}{ FEA simulation } \\
\hline & & & \multicolumn{2}{|l|}{$\eta=0.75$} & \multicolumn{2}{|l|}{$\eta=1.2$} \\
\hline & & & $\begin{array}{c}\text { Max. deformation } \\
(\mathrm{mm})(\mathrm{B})\end{array}$ & $(\mathrm{A}) /(\mathrm{B})$ & $\begin{array}{c}\text { Max. deformation } \\
(\mathrm{mm})(\mathrm{C})\end{array}$ & $(\mathrm{A}) /(\mathrm{C})$ \\
\hline \multirow{7}{*}{$\mathrm{D}$} & A2078a & 17.95 & \multirow{3}{*}{17.83} & 1.01 & \multirow{3}{*}{20.67} & 0.87 \\
\hline & $\mathrm{A} 2078 \mathrm{~b}$ & 20.48 & & 1.15 & & 0.99 \\
\hline & $\mathrm{A} 2078 \mathrm{c}$ & 20.96 & & 1.18 & & 1.01 \\
\hline & $\mathrm{A} 2078 \mathrm{~d}$ & 23.47 & \multirow{2}{*}{17.84} & 1.32 & \multirow{2}{*}{18.82} & 1.25 \\
\hline & $\mathrm{A} 2078 \mathrm{e}$ & 20.32 & & 1.14 & & 1.08 \\
\hline & A4078a & 31.83 & \multirow{2}{*}{24.35} & 1.31 & \multirow{2}{*}{33.30} & 0.96 \\
\hline & $\mathrm{A} 4078 \mathrm{~b}$ & 28.36 & & 1.16 & & 0.85 \\
\hline \multirow{4}{*}{$\mathrm{E}$} & A2108a & 22.32 & \multirow{2}{*}{19.31} & 1.16 & \multirow{2}{*}{23.45} & 0.95 \\
\hline & $\mathrm{A} 2108 \mathrm{~b}$ & 23.45 & & 1.21 & & 1.00 \\
\hline & A4108a & 36.48 & \multirow{2}{*}{27.70} & 1.32 & \multirow{2}{*}{38.84} & 0.94 \\
\hline & $\mathrm{A} 4108 \mathrm{~b}$ & 41.35 & & 1.49 & & 1.06 \\
\hline \multirow{7}{*}{$\mathrm{F}$} & $\mathrm{A} 2128 \mathrm{a}$ & 26.82 & \multirow{3}{*}{24.82} & 1.08 & \multirow{3}{*}{31.69} & 0.85 \\
\hline & $\mathrm{A} 2128 \mathrm{~b}$ & 29.11 & & 1.17 & & 0.92 \\
\hline & $\mathrm{A} 2128 \mathrm{c}$ & 26.17 & & 1.05 & & 0.83 \\
\hline & A2128d & 36.26 & \multirow{2}{*}{33.16} & 1.09 & \multirow{2}{*}{34.42} & 1.05 \\
\hline & $\mathrm{A} 2128 \mathrm{e}$ & 37.26 & & 1.12 & & 1.08 \\
\hline & $\mathrm{A} 4128 \mathrm{a}$ & 49.61 & \multirow{2}{*}{41.60} & 1.19 & \multirow{2}{*}{59.55} & 0.83 \\
\hline & $\mathrm{A} 4128 \mathrm{~b}$ & 48.23 & & 1.16 & & 0.81 \\
\hline
\end{tabular}




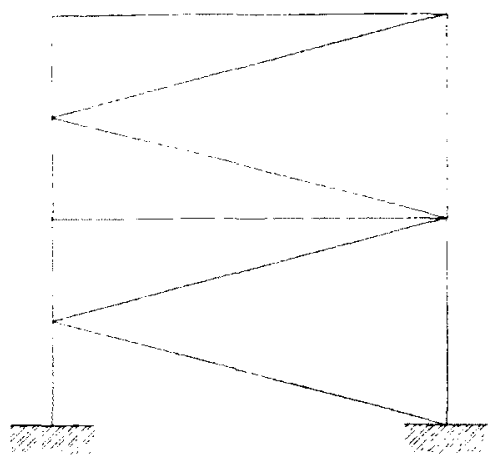

$\mathrm{K}$ bracing

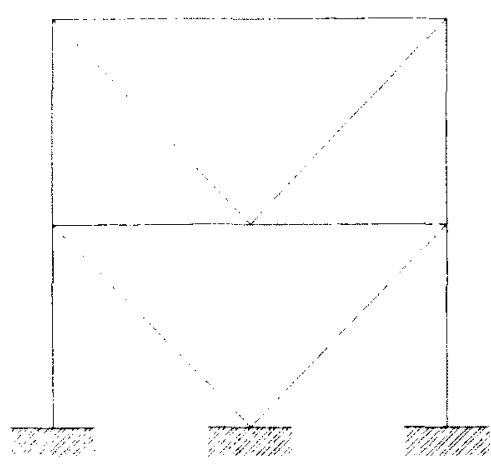

$\mathrm{V}$ bracing

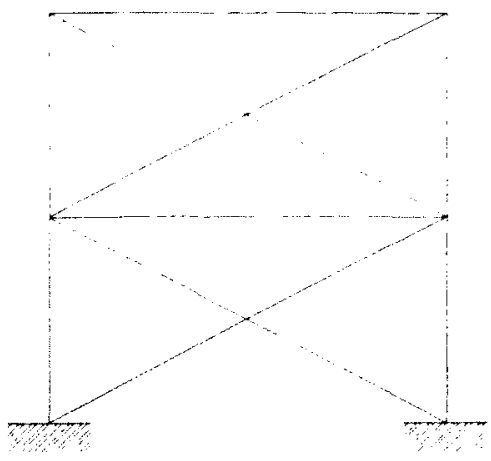

$\mathrm{X}$ bracing

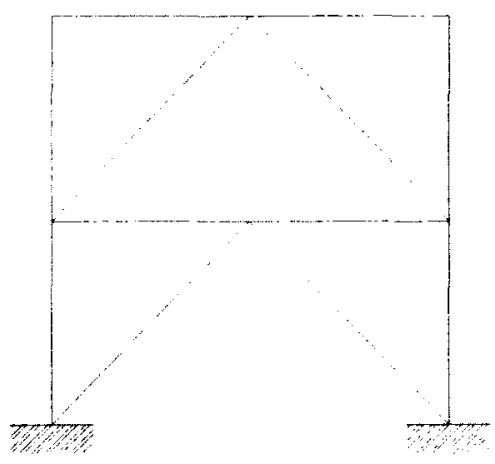

Inverted-V bracing

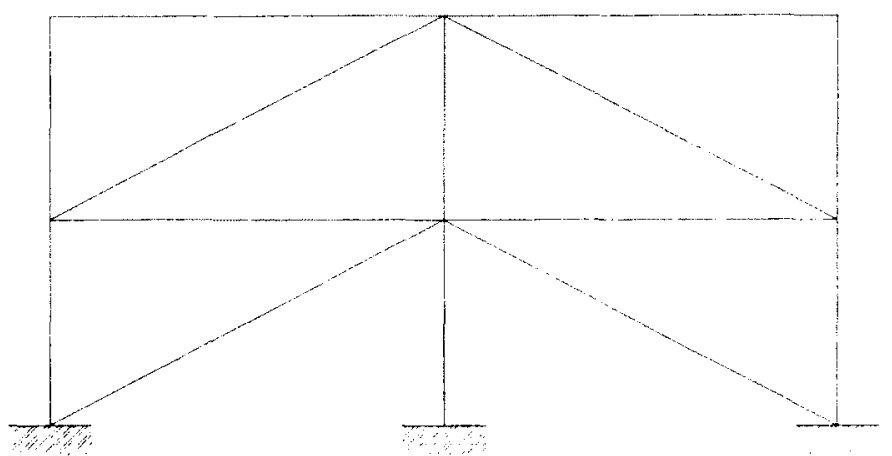

Diagonal bracing

Figure 5.1 Bracing systems (Aghdam 2009) 

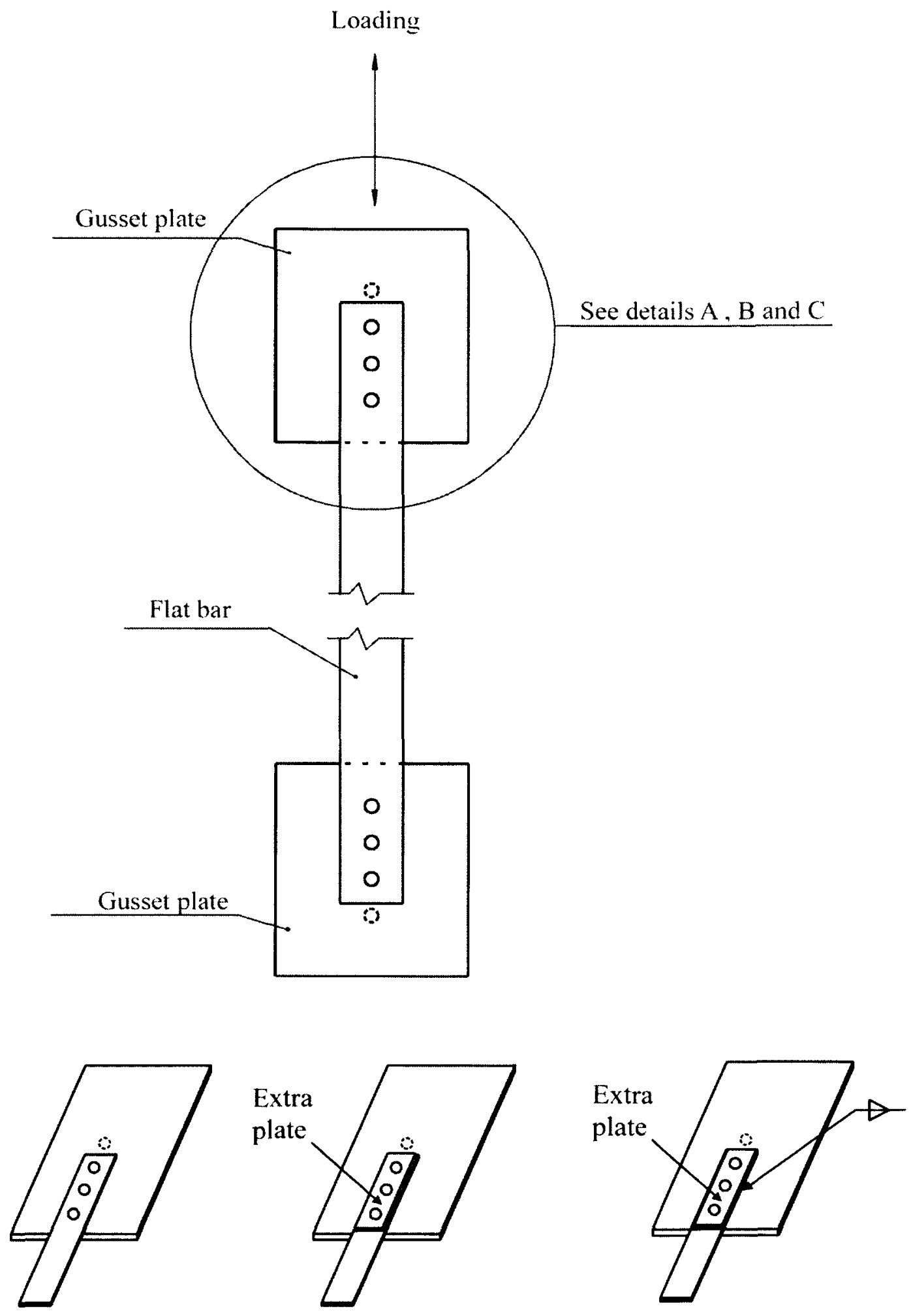

Detail A

Detail B

Detail C

$\overline{\text { No reinforcement }} \overline{\text { Clamped down with extra plate }} \overline{\text { Welded with extra plate }}$

Figure 5.2 Steel strap specimen with various connection details (Aghdam 2009) 


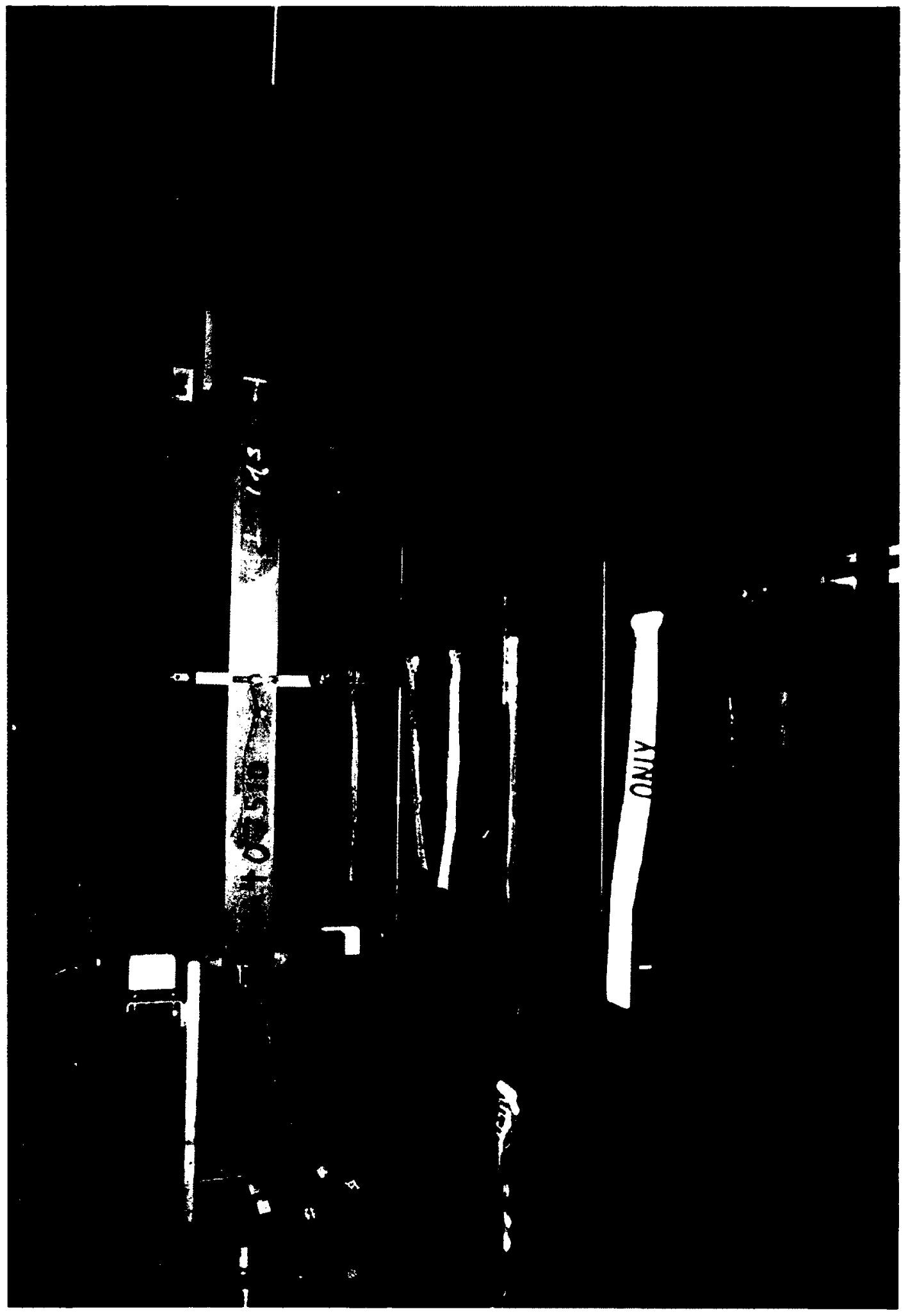

Figure 5.3 Test setup (Aghdam 2009) 


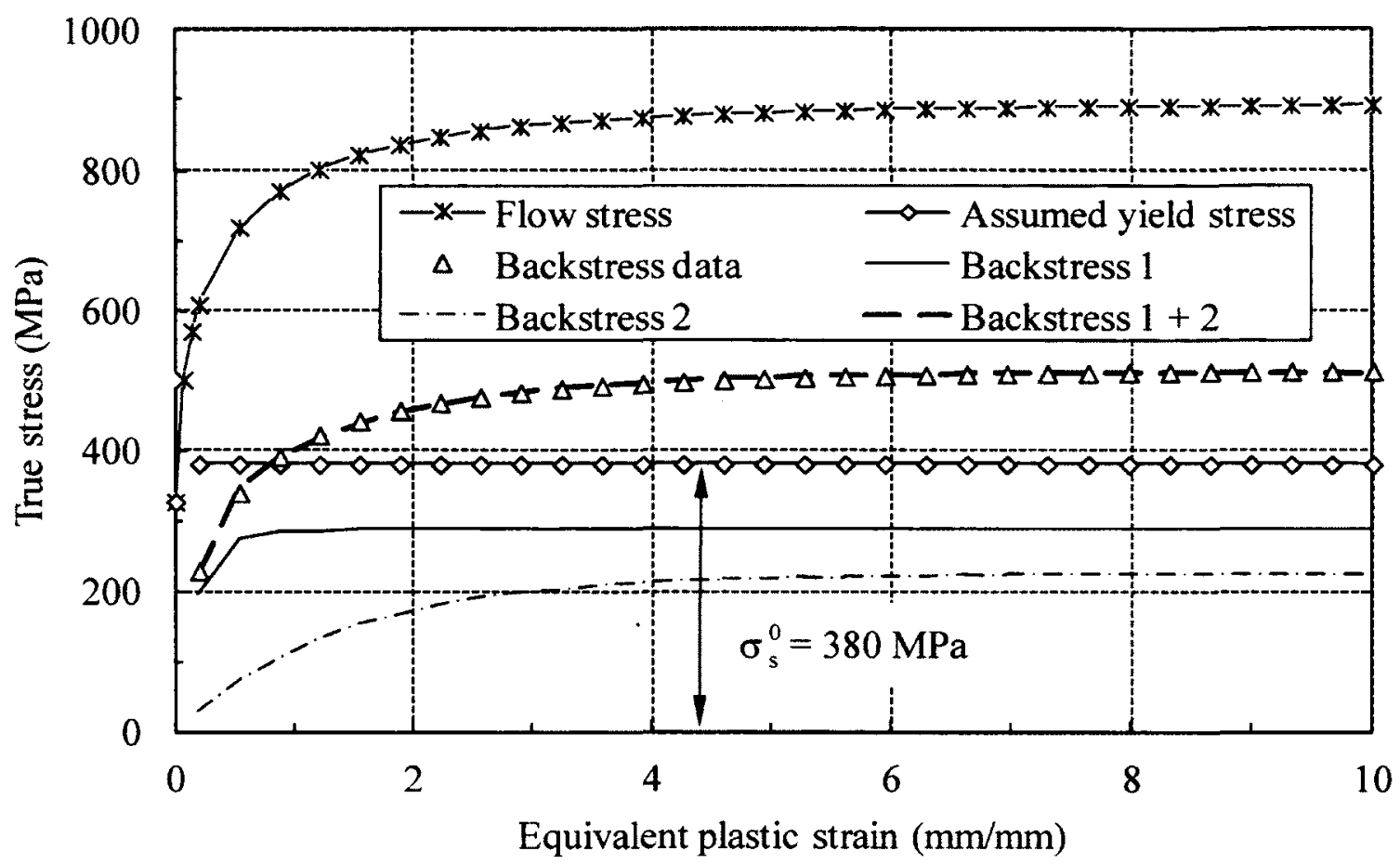

Figure 5.4 Curve fitting of backstress for large strain using assumed saturated yield stress of $380 \mathrm{MPa}$ (two terms)

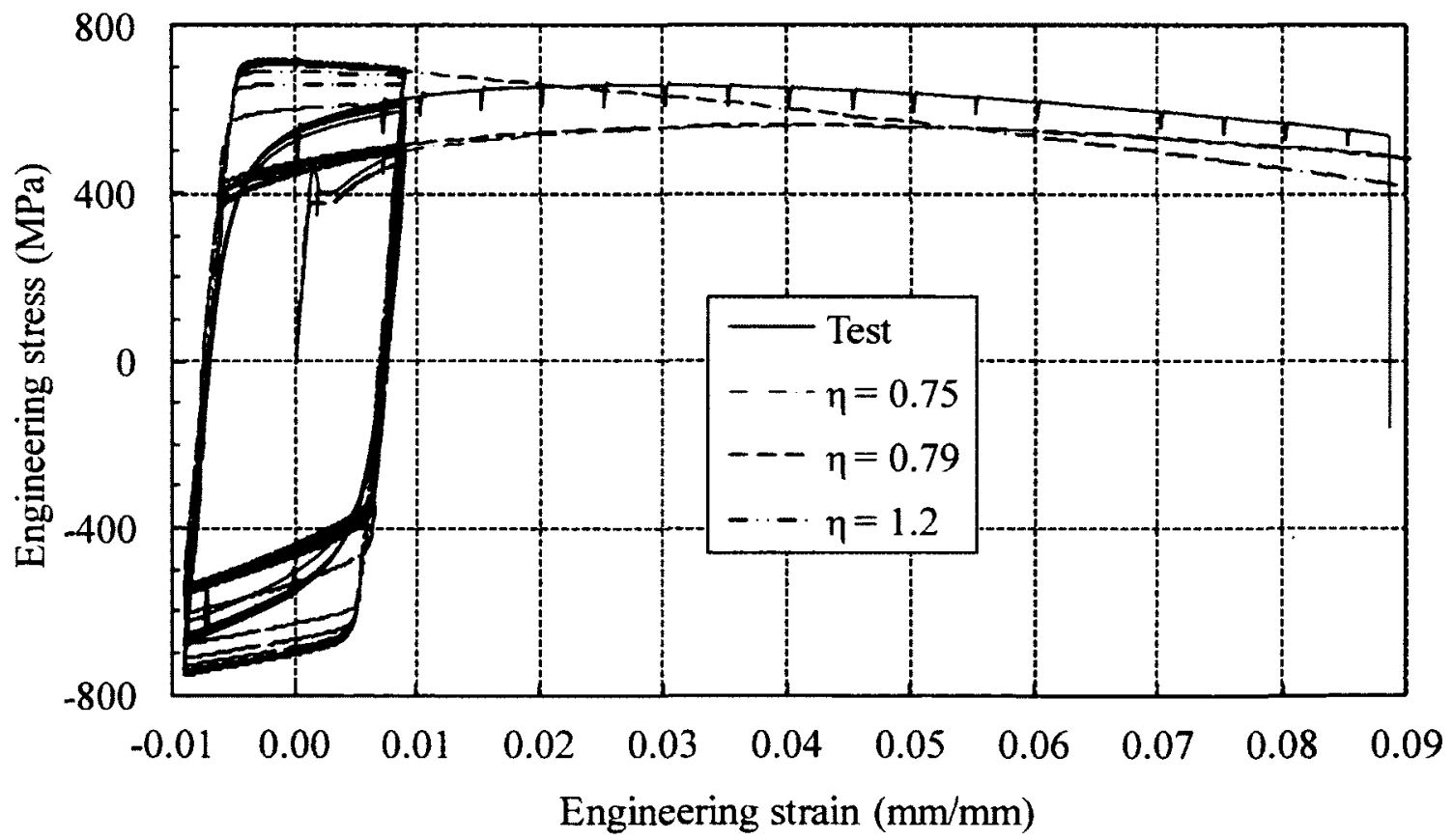

Figure 5.5 Test and predicted engineering stress versus engineering strain for notched specimen BBC20404 with bound $\eta$ of $0.75,0.79$ and 1.2 


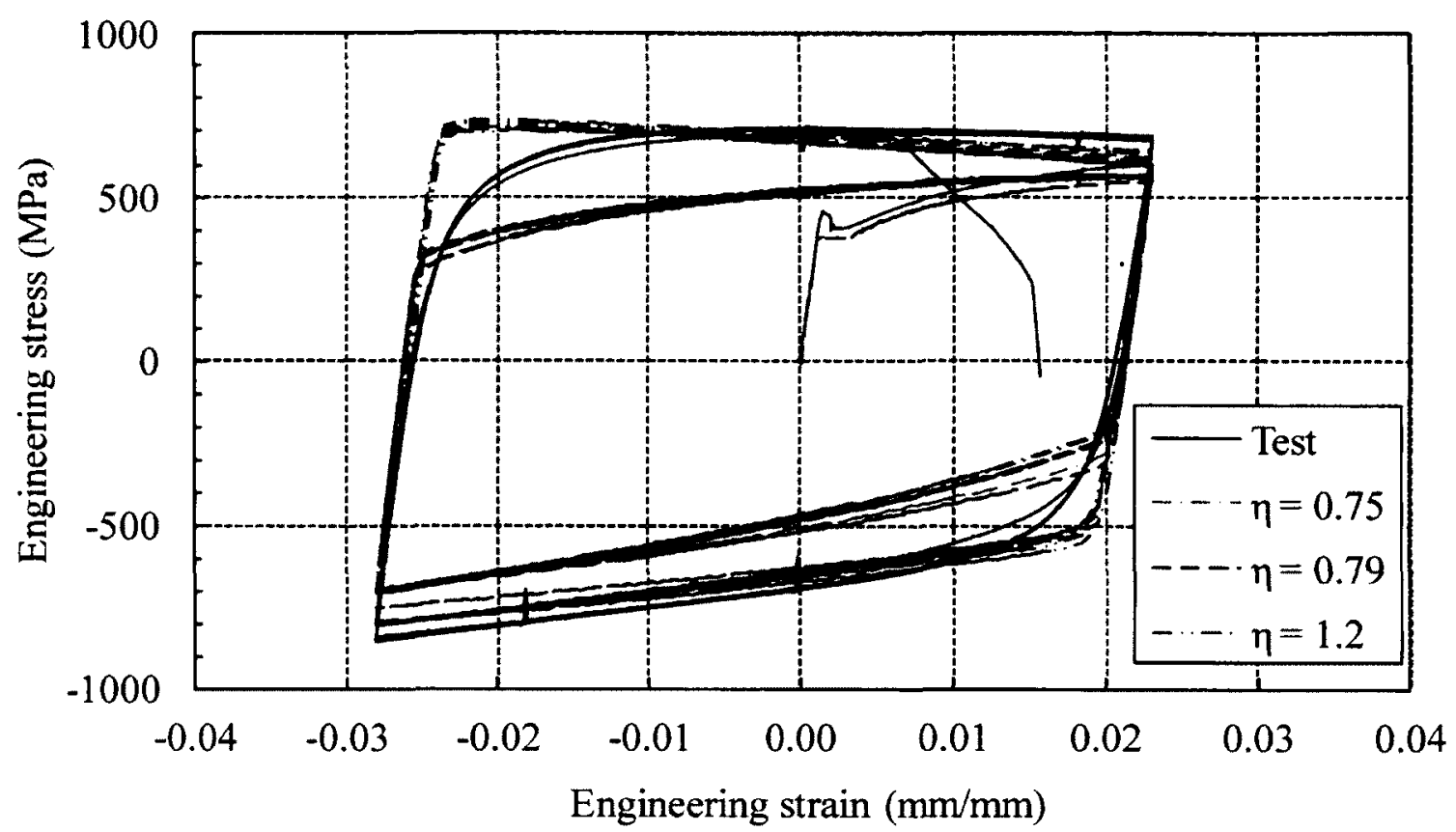

Figure 5.6 Test and predicted engineering stress versus engineering strain for notched specimen BBCF1212 with bound $\eta$ of $0.75,0.79$ and 1.2

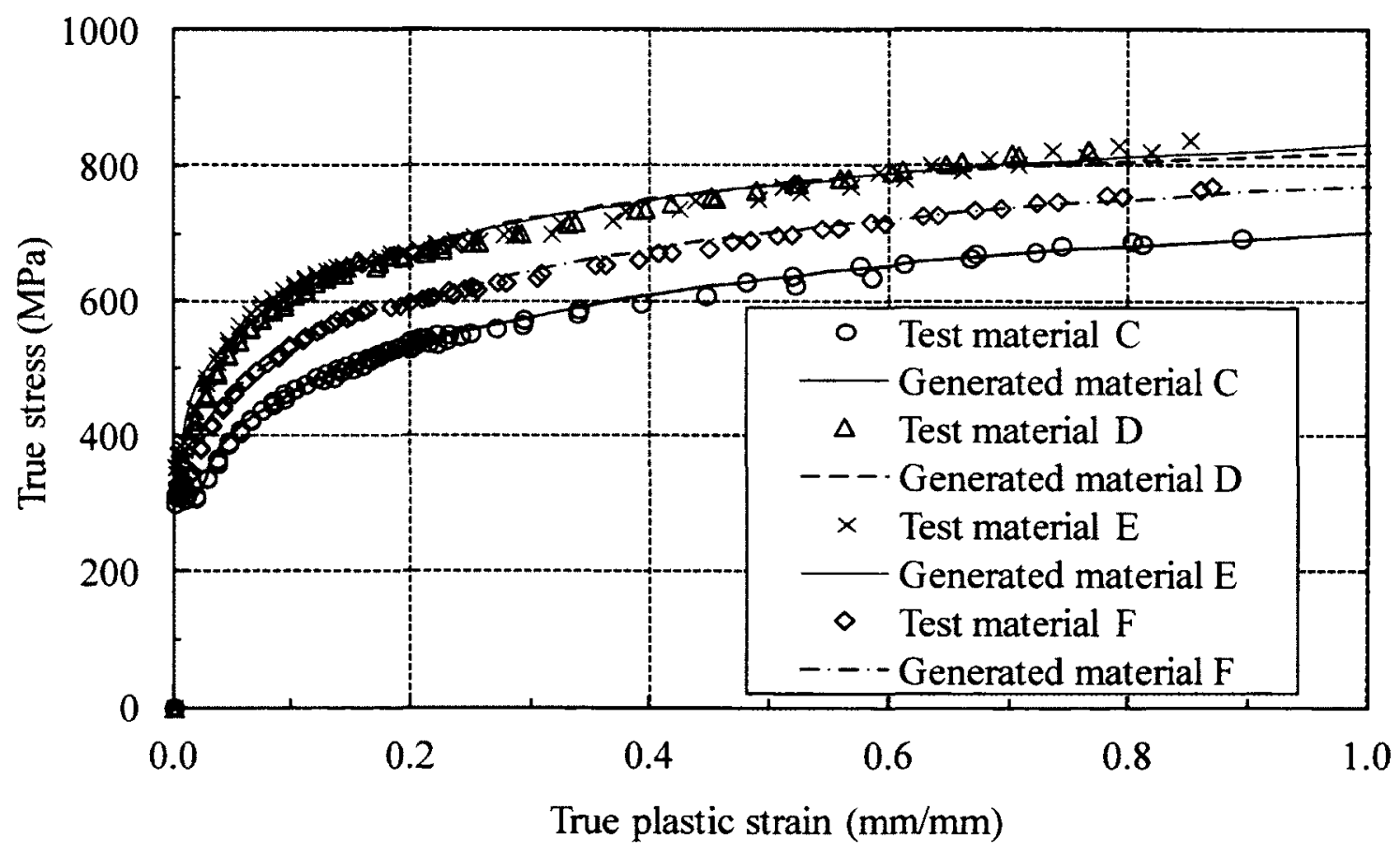

Figure 5.7 Test and generated flow stress versus true plastic strain for materials $\mathrm{C}$ to $\mathrm{F}$ 


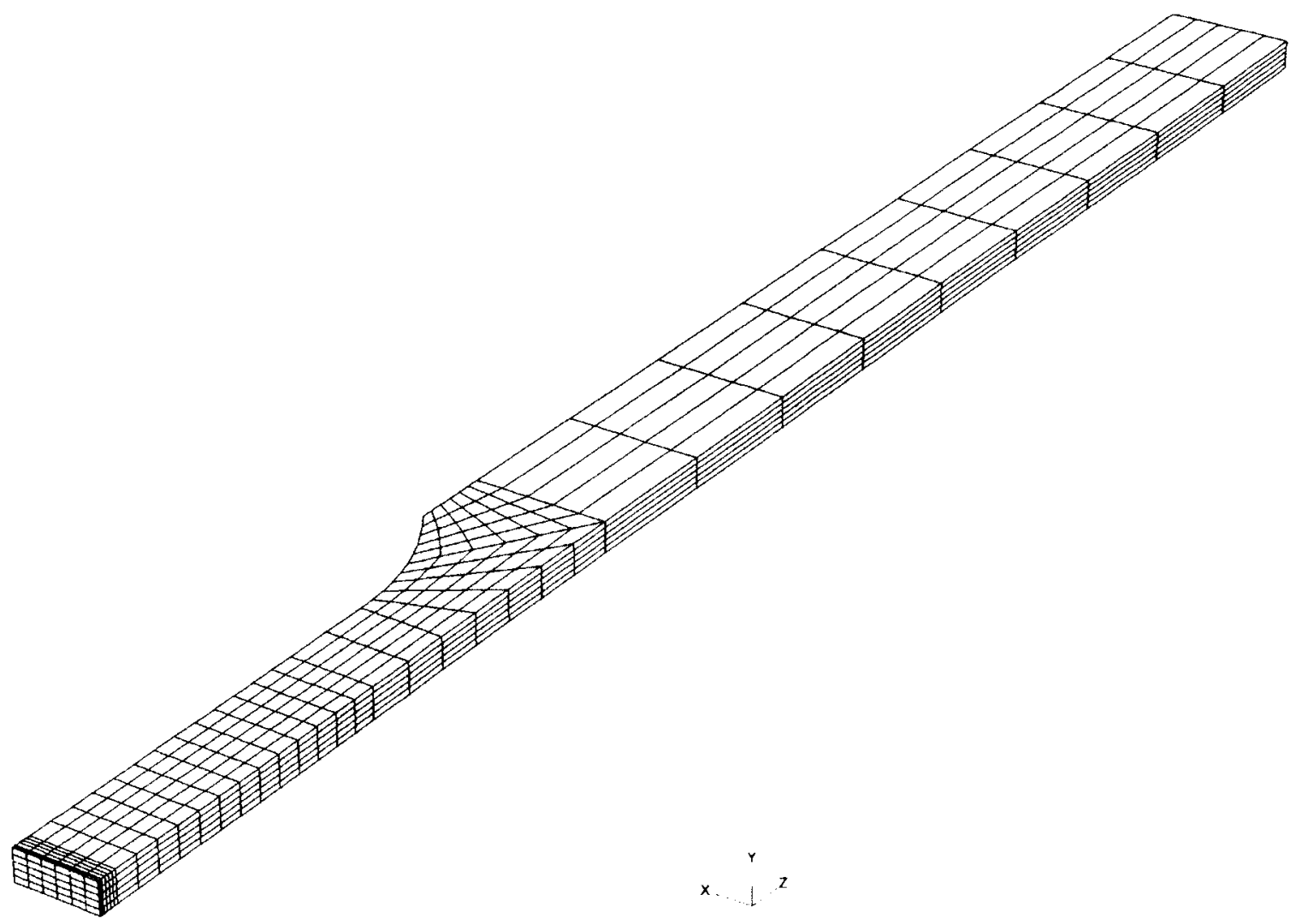

Figure 5.8 Typical mesh for the FEA model of $1 / 8$ rectangular specimen for materials $\mathrm{C}$ to $\mathrm{F}$ (Chen 2010) 


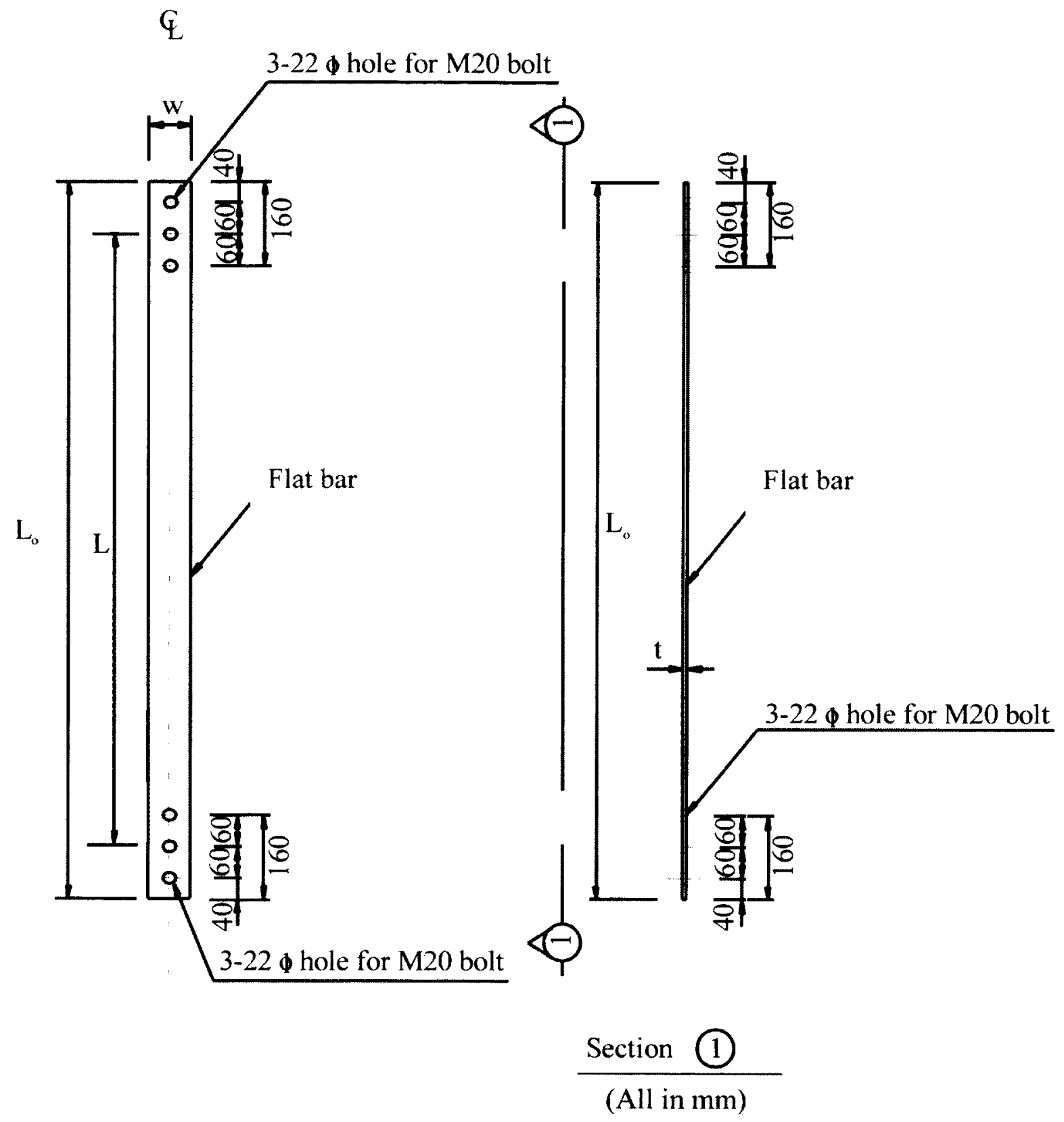

Figure 5.9 Type A strap specimen (Aghdam 2009) 

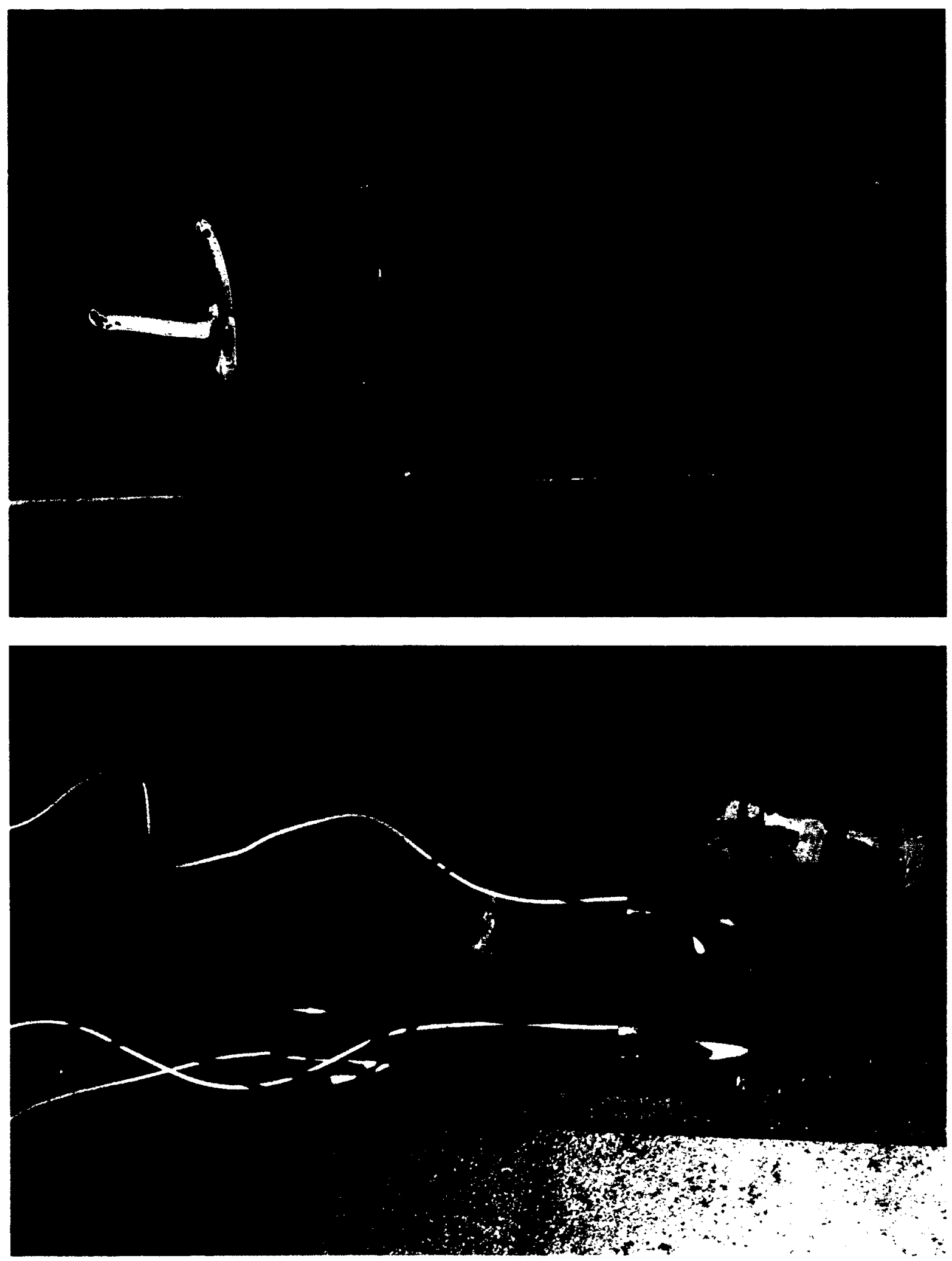

Figure 5.10 Both ends of strap specimen A4078b of material D at the end of the test (Aghdam 2009) 

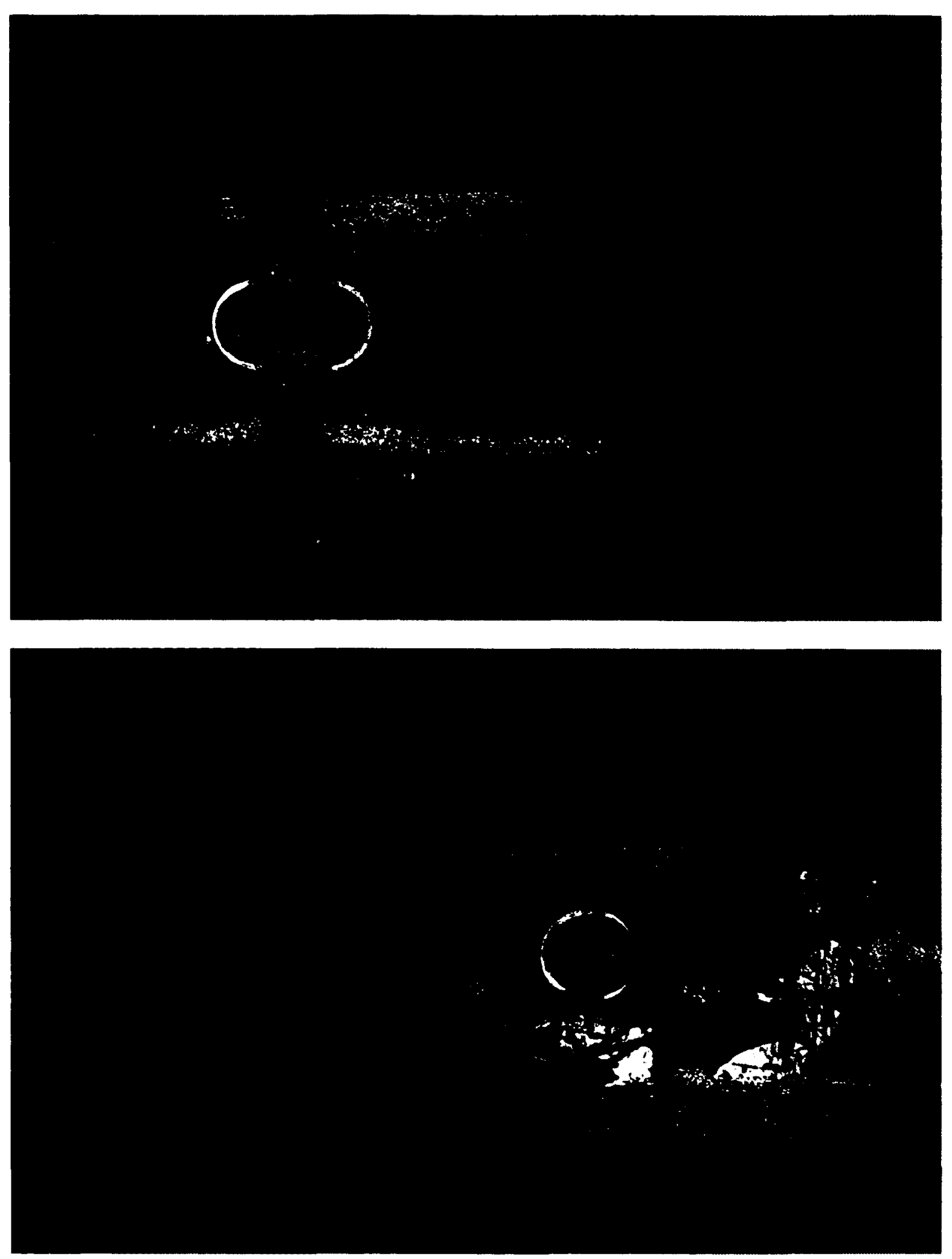

Figure 5.11 Both ends of strap specimen A8075b of material C at the end of the test (Aghdam 2009) 


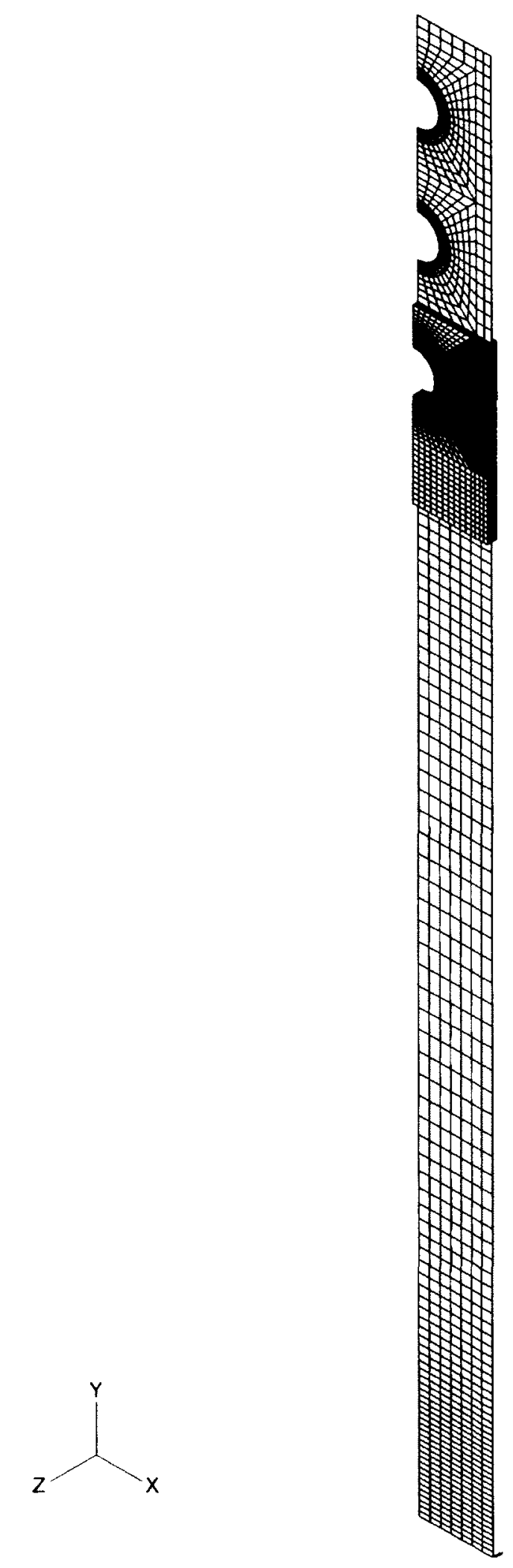

Figure 5.12 FEA mesh of the 1/4 model, shown for strap specimen A4075 of material C 


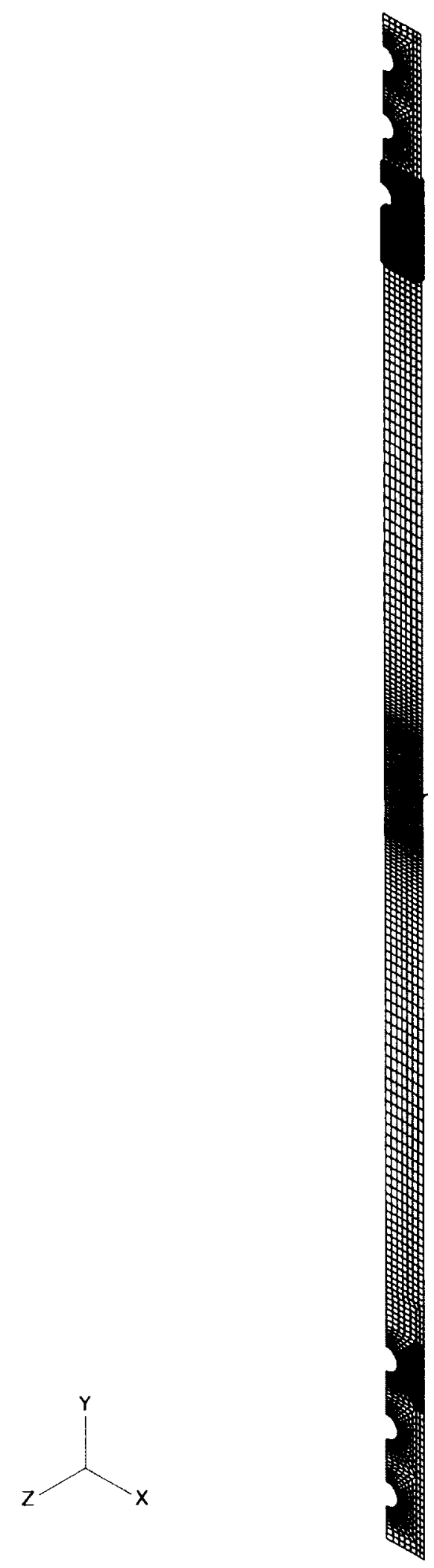

Figure 5.13 FEA mesh of the 1/2 model, shown for strap specimen A4075 of material C 

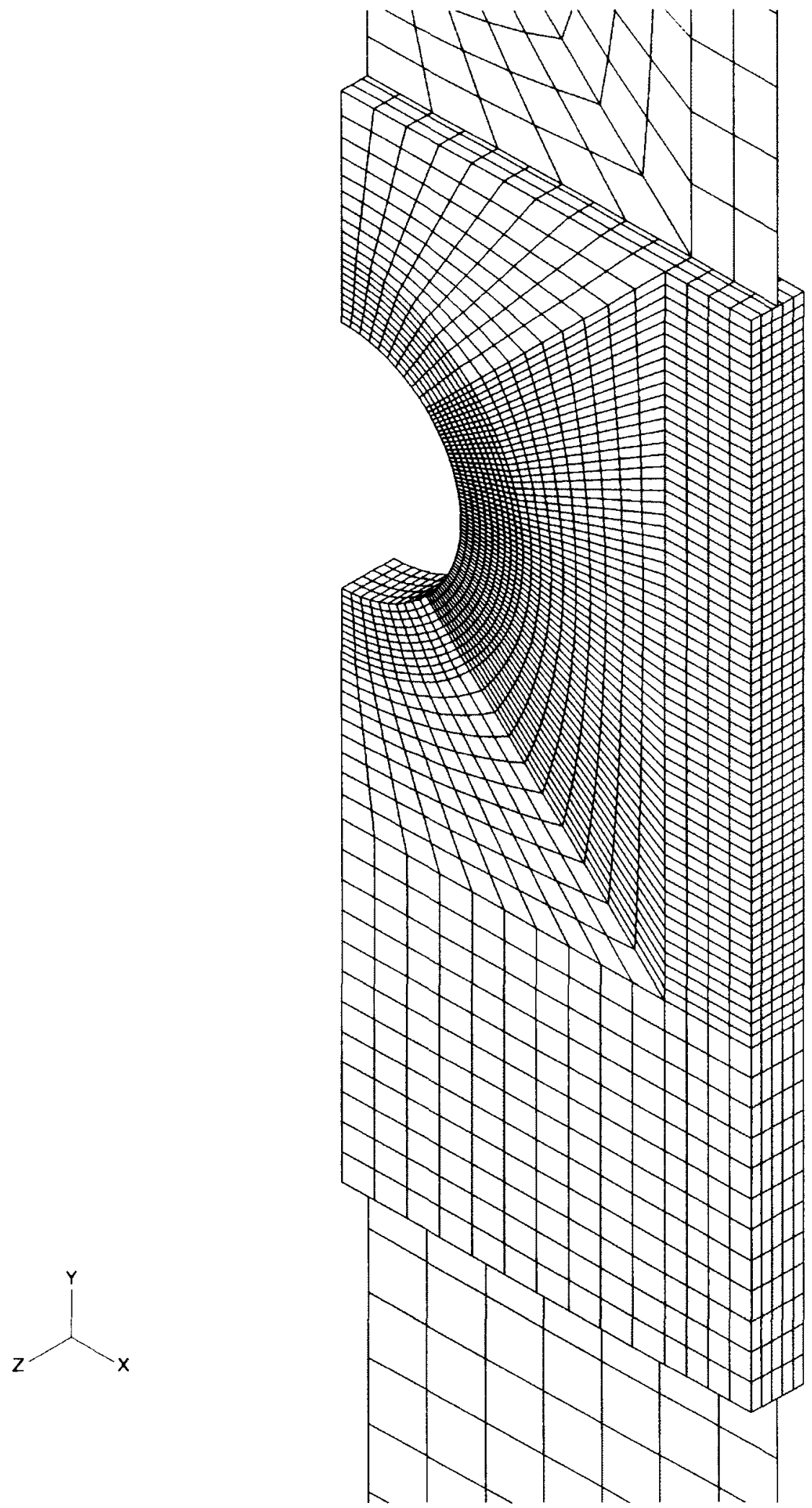

Figure 5.14 The region around the innermost bolt hole modeled with solid element, shown for strap specimen A 4075 


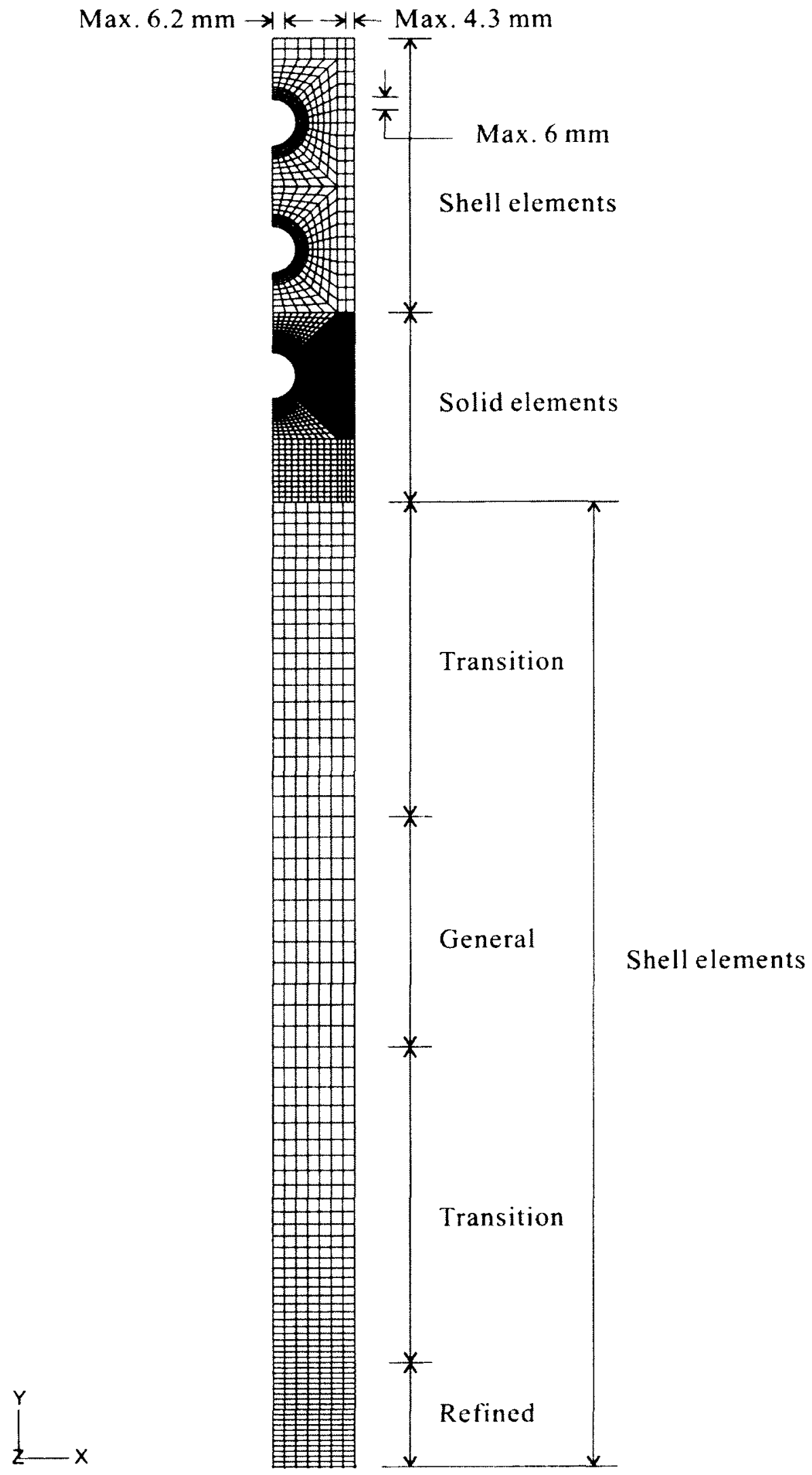

Figure 5.15 Plan view of the 1/4 FEA model, shown for strap specimen A4075 


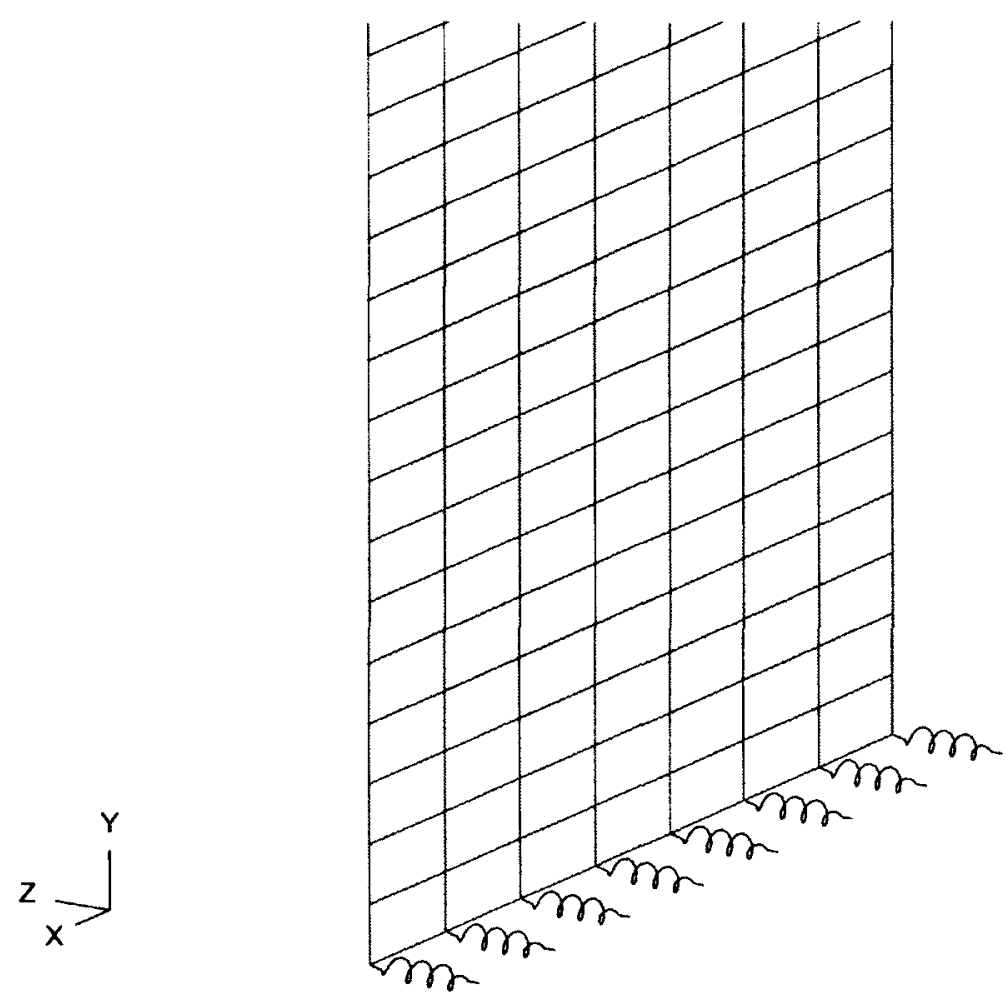

(a) Spring elements of the 1/4 model, shown for strap specimen A4075

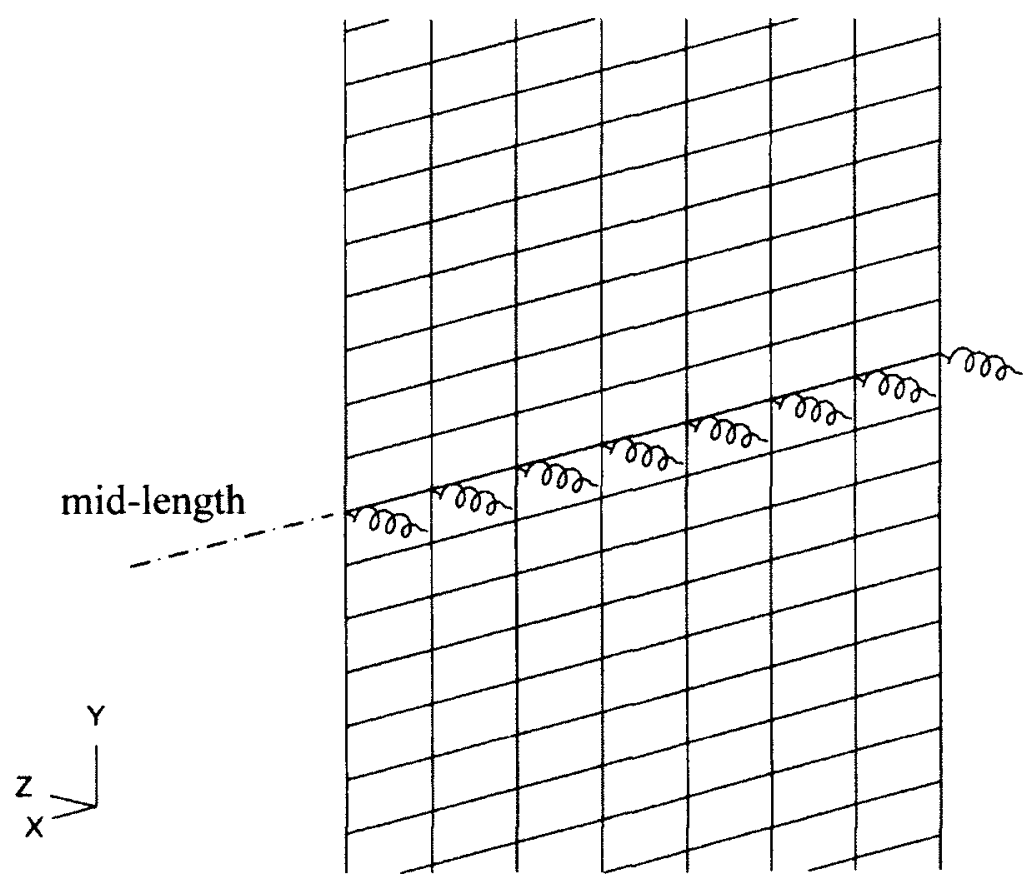

(b) Spring elements of the 1/2 model, shown for strap specimen A4075

Figure 5.16 Spring elements to induce buckling to the appropriate direction 


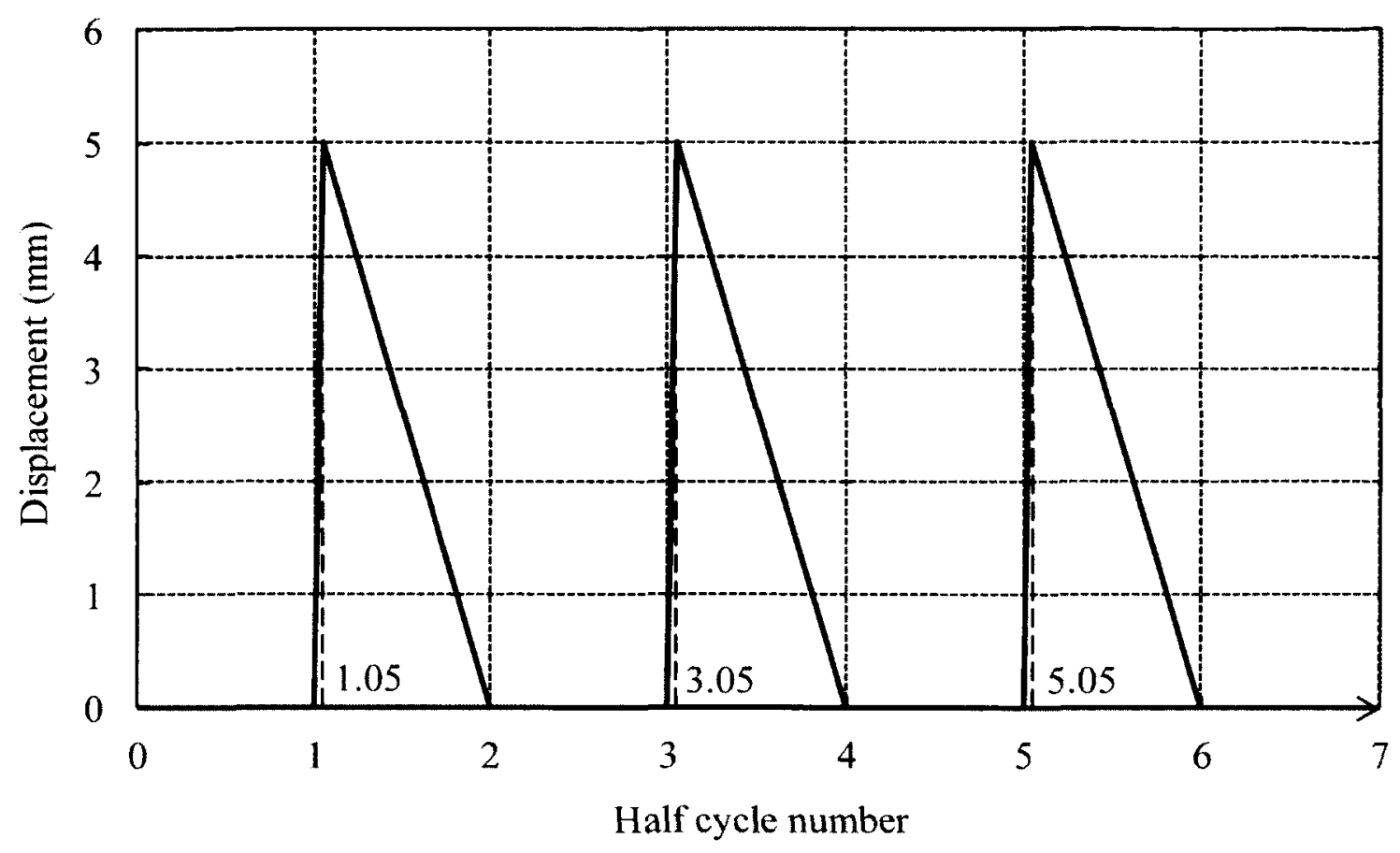

Figure 5.17 Applied transverse displacement ( $Z$ direction) at the spring element support (odd half cycle number corresponds to the start of compression leg) 


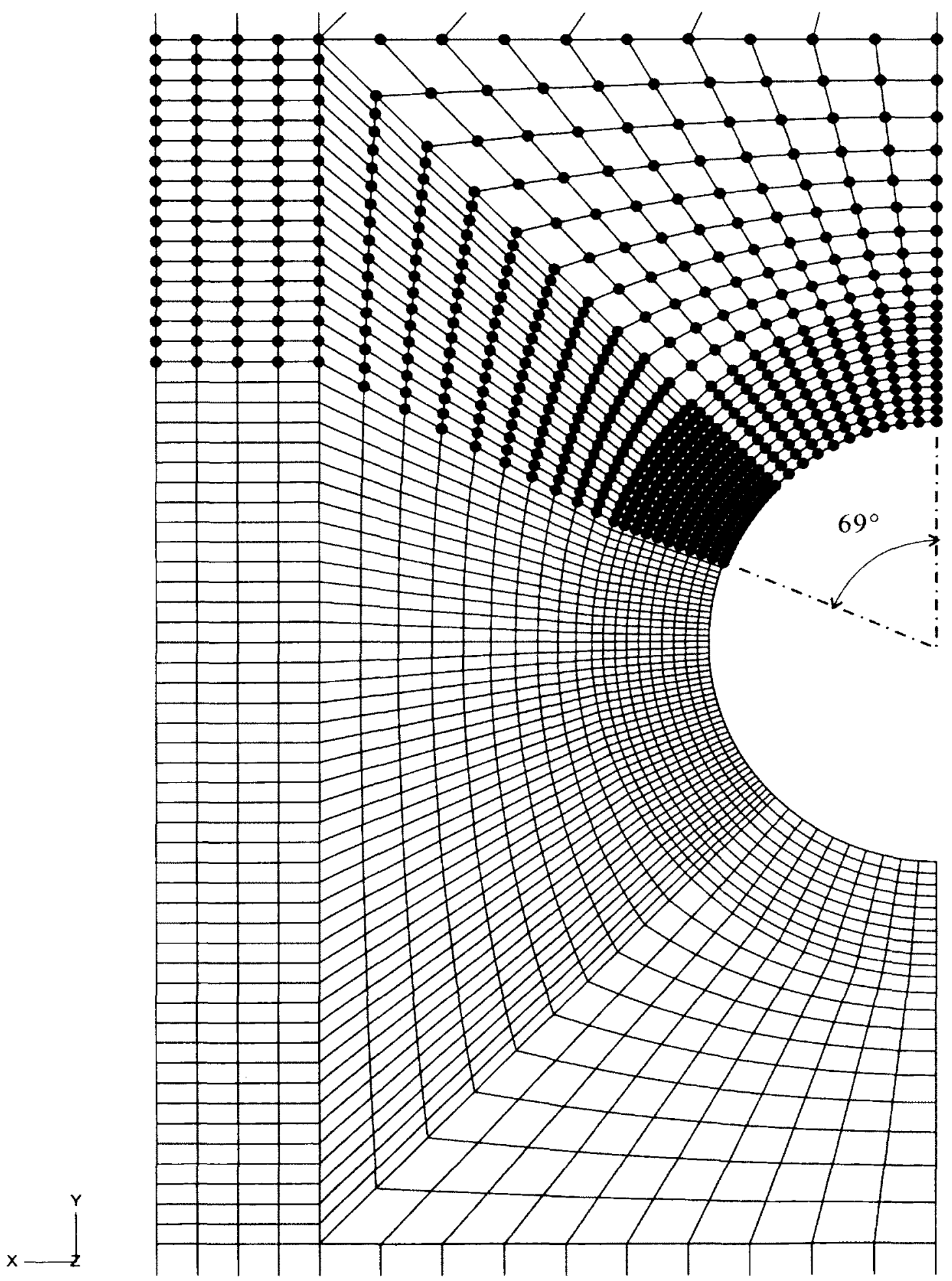

Figure 5.18 Nodes on the bottom face of the solid element around the innermost bolt hole at the upper (loading) end of the specimen constrained in the $\mathrm{Z}$ direction, shown for strap specimen A4075 (same constraint is applied to nodes of the shell element in the similar region at the lower (fixed) end for the $1 / 2$ model) 


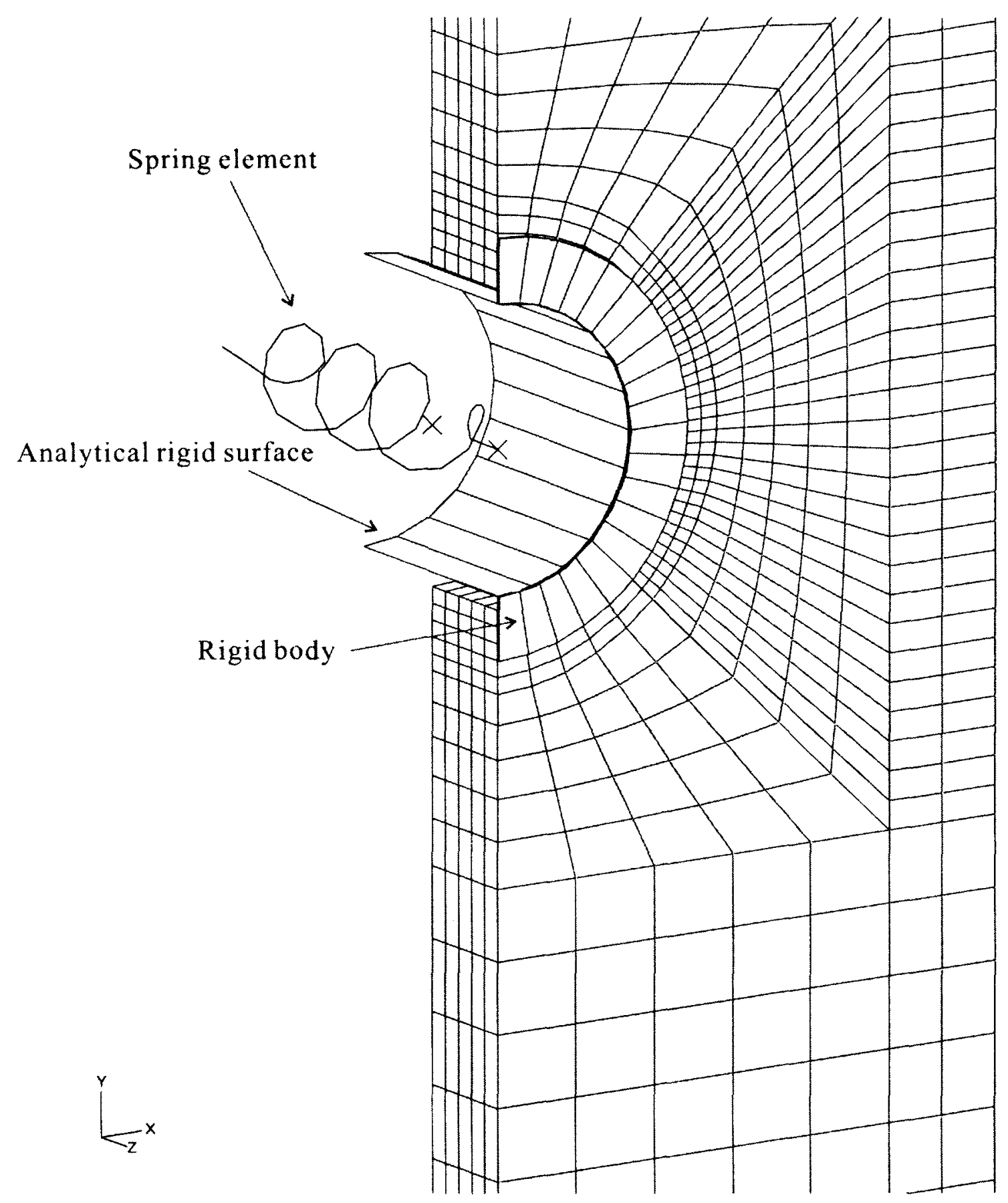

Figure 5.19 Modeling of the bolt and contact surface at the bolt hole 

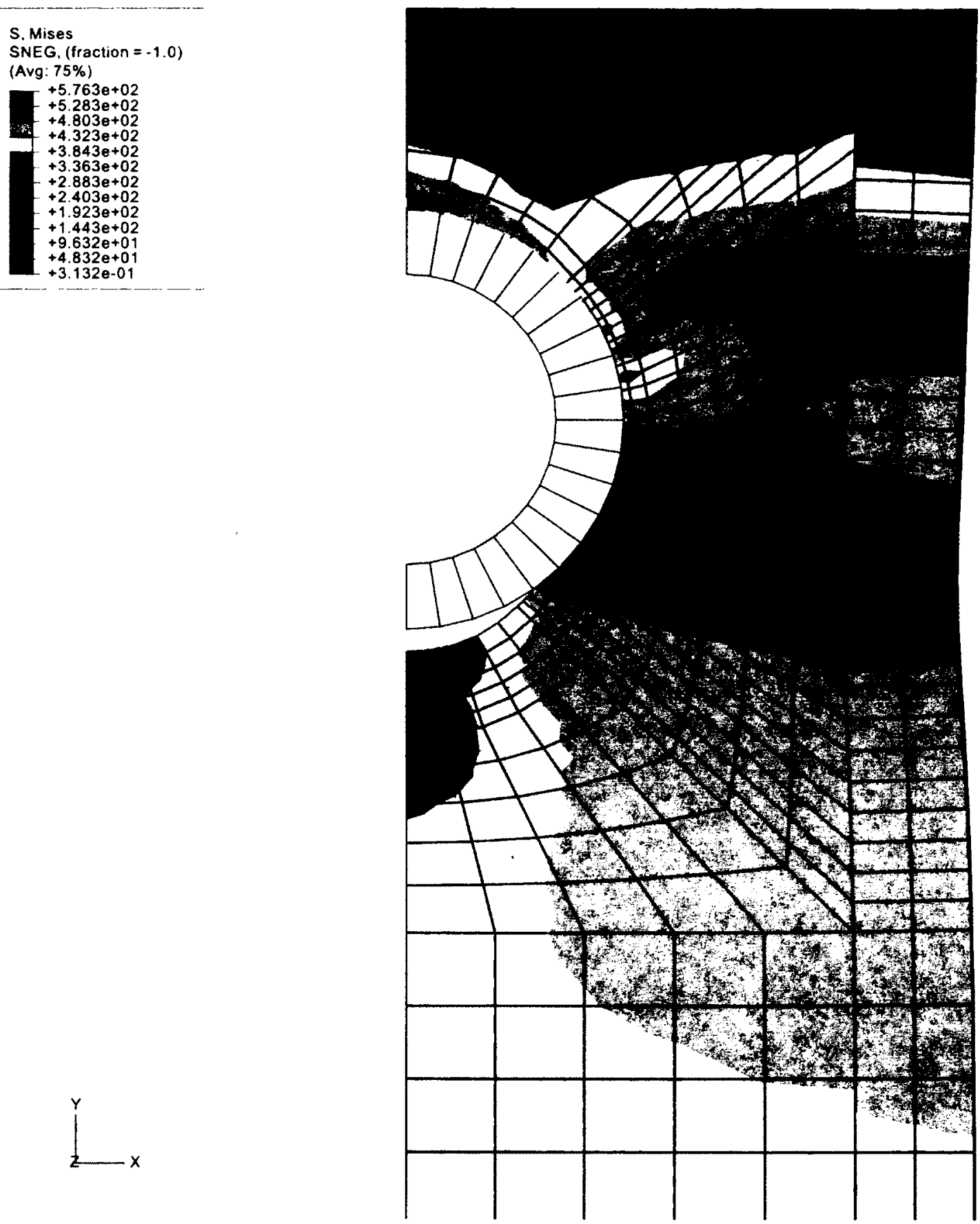

Figure 5.20 Results of FEA simulation with the bolt and contact surfaces at the bolt hole 


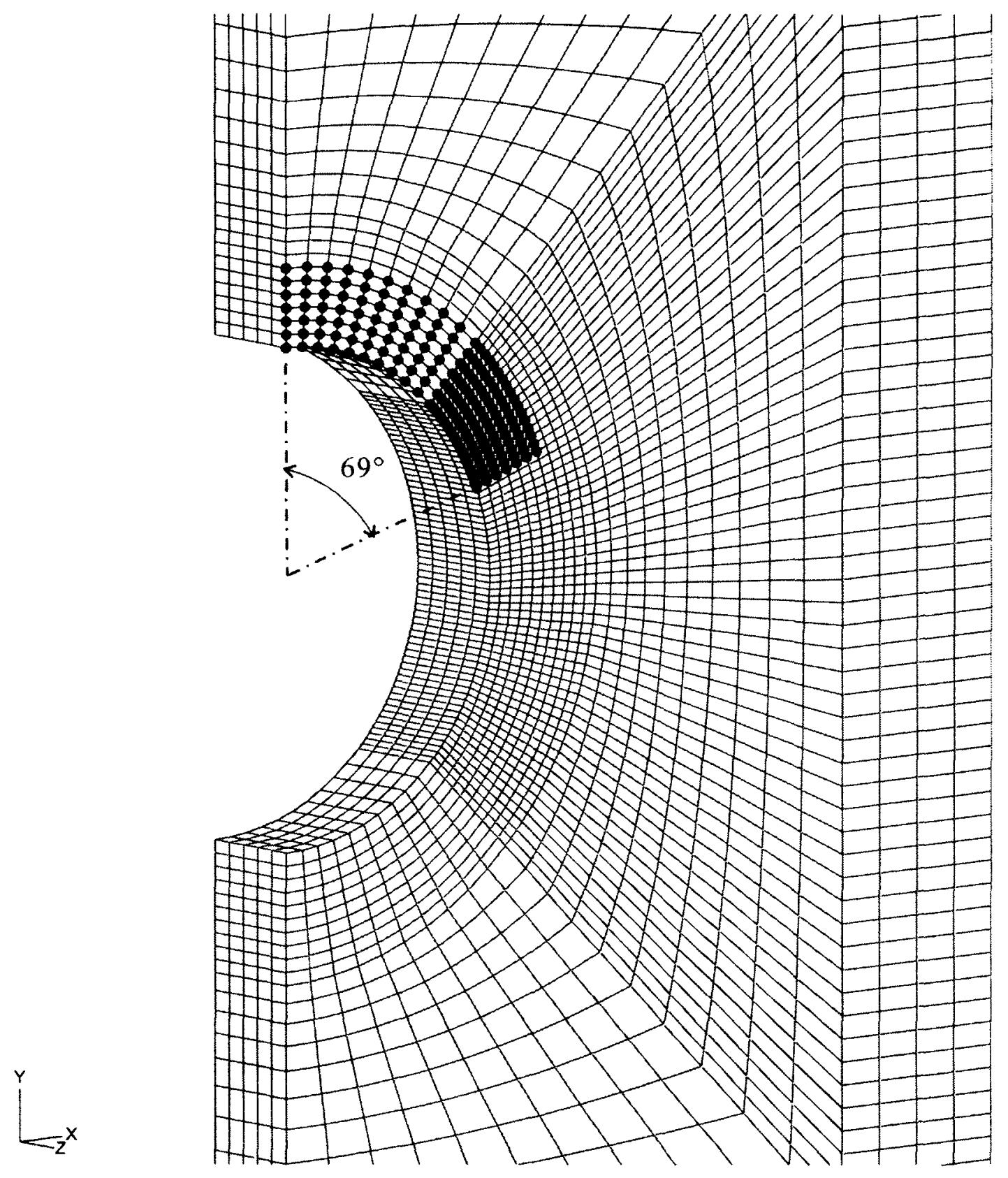

Figure 5.21 Nodes on the surface around the innermost bolt hole constrained in the $\mathrm{Z}$ direction due to clamping of the bolt, shown for strap specimen A4075 

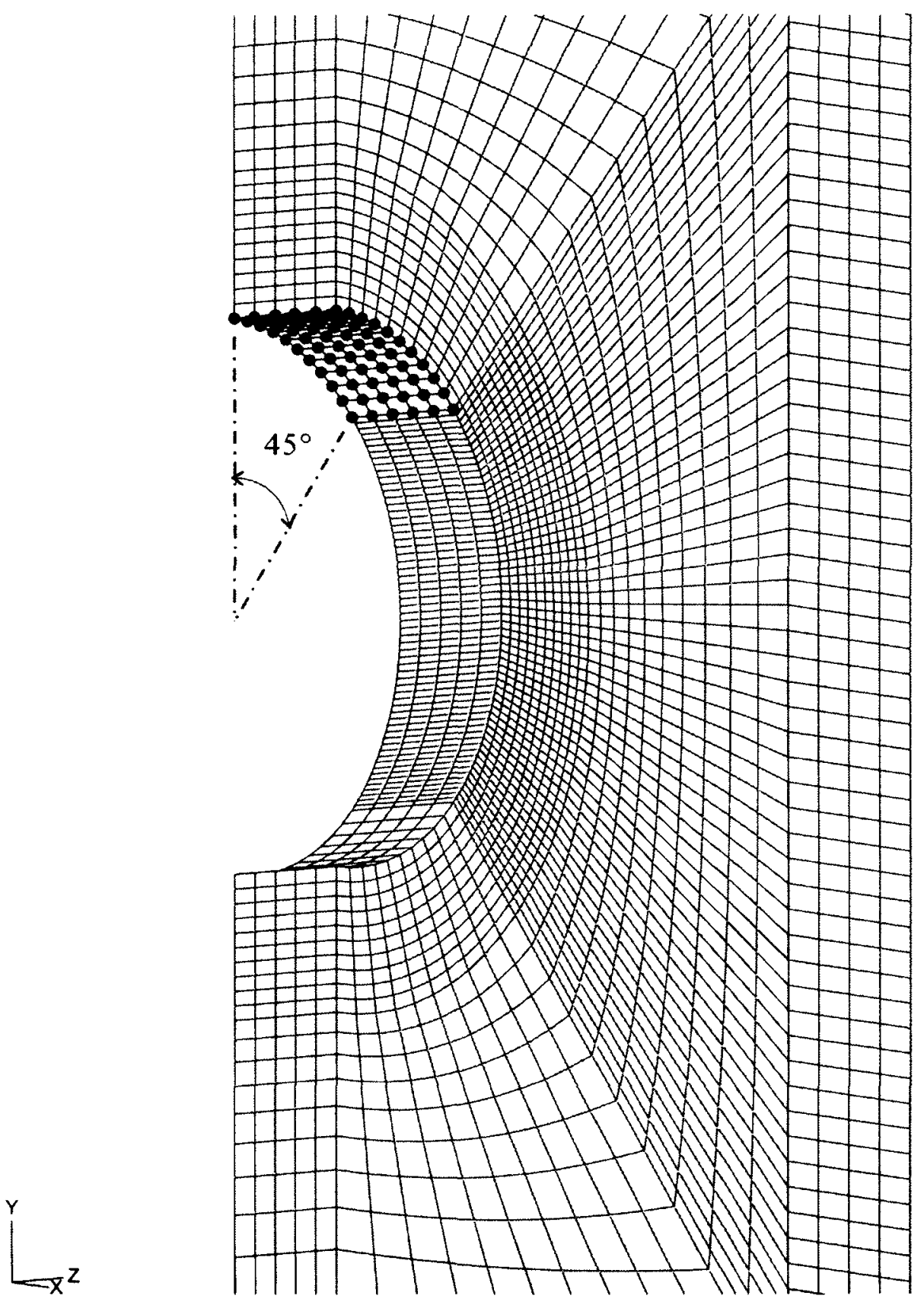

Figure 5.22 Nodes around the innermost bolt hole at the upper end constrained to have the same displacement in the $\mathrm{Y}$ direction, shown for strap specimen A4075 
$\operatorname{Max} .6 .2 \mathrm{~mm} \mathrm{~K} \longrightarrow 1 \quad \longleftrightarrow M a x .4 .3 \mathrm{~mm}$

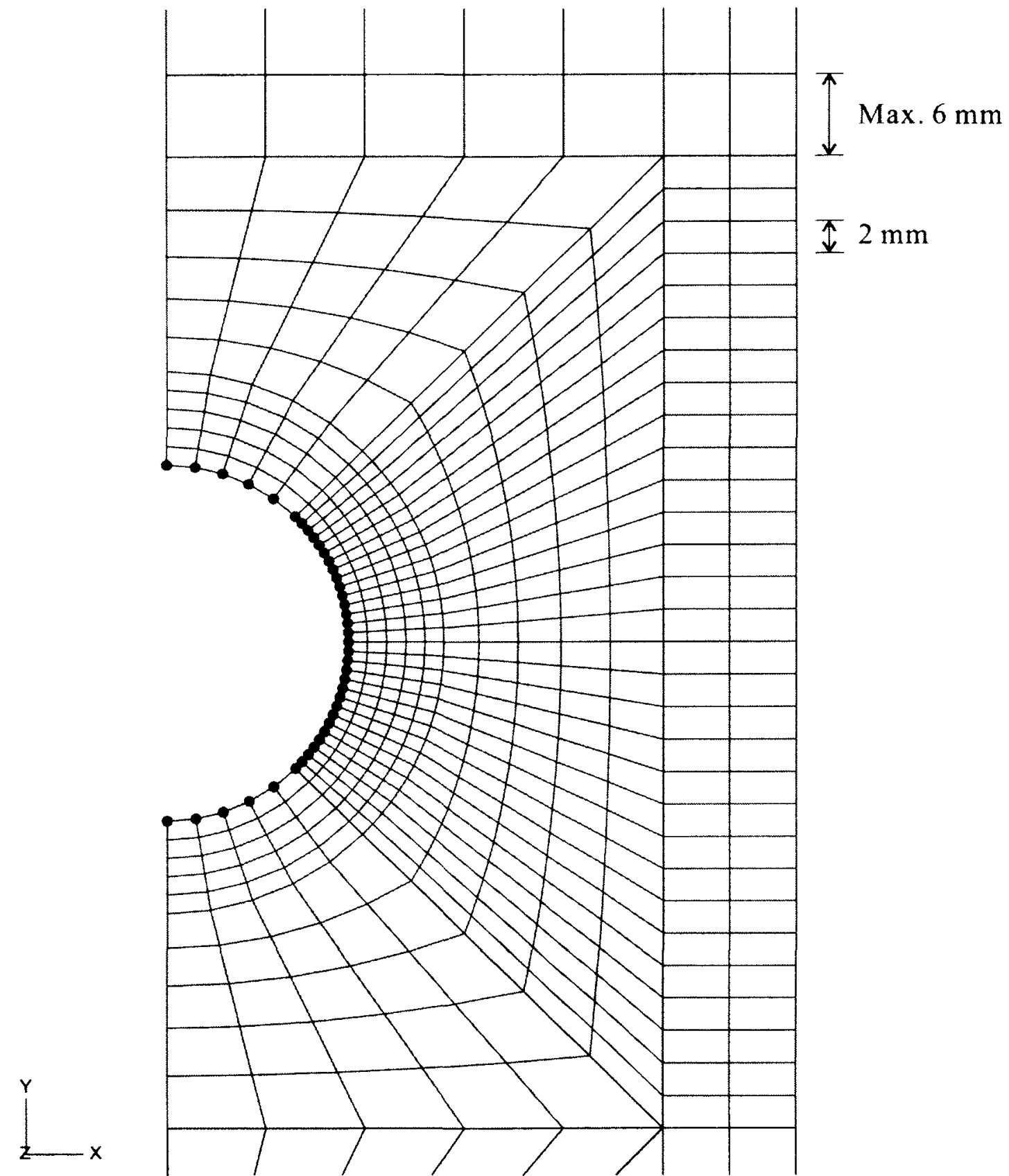

Figure 5.23 Nodes around the innermost bolt hole at the lower (fixed) end constrained against deformation in the $Y$ direction, shown for strap specimen A4075 


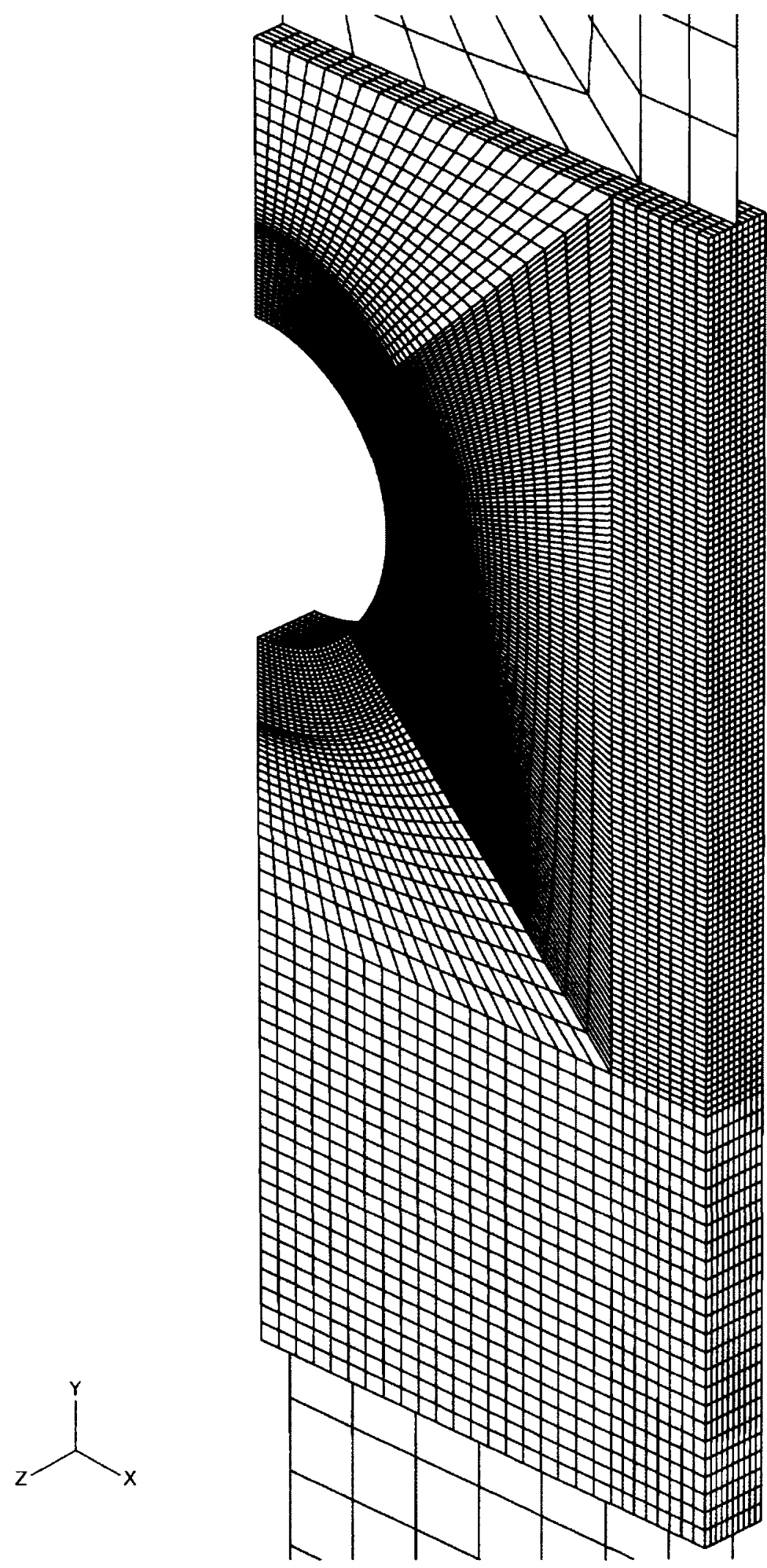

Figure 5.24 Scheme-2 mesh of the critical region of strap specimen A4075 modeled with solid element 


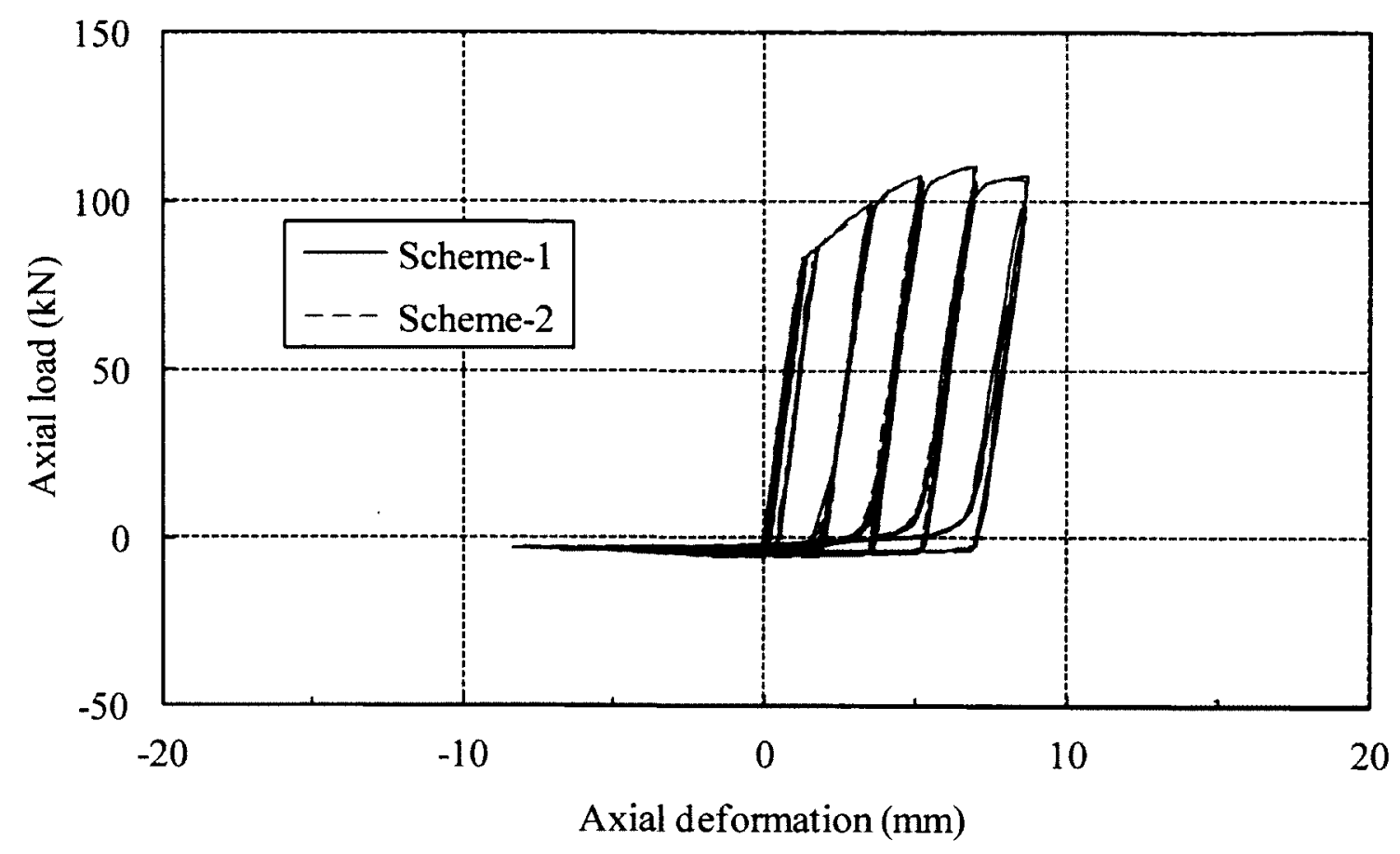

Figure 5.25 FEA simulation of load versus deformation curves for strap specimen A4075 using the $1 / 2$ model with two different mesh schemes

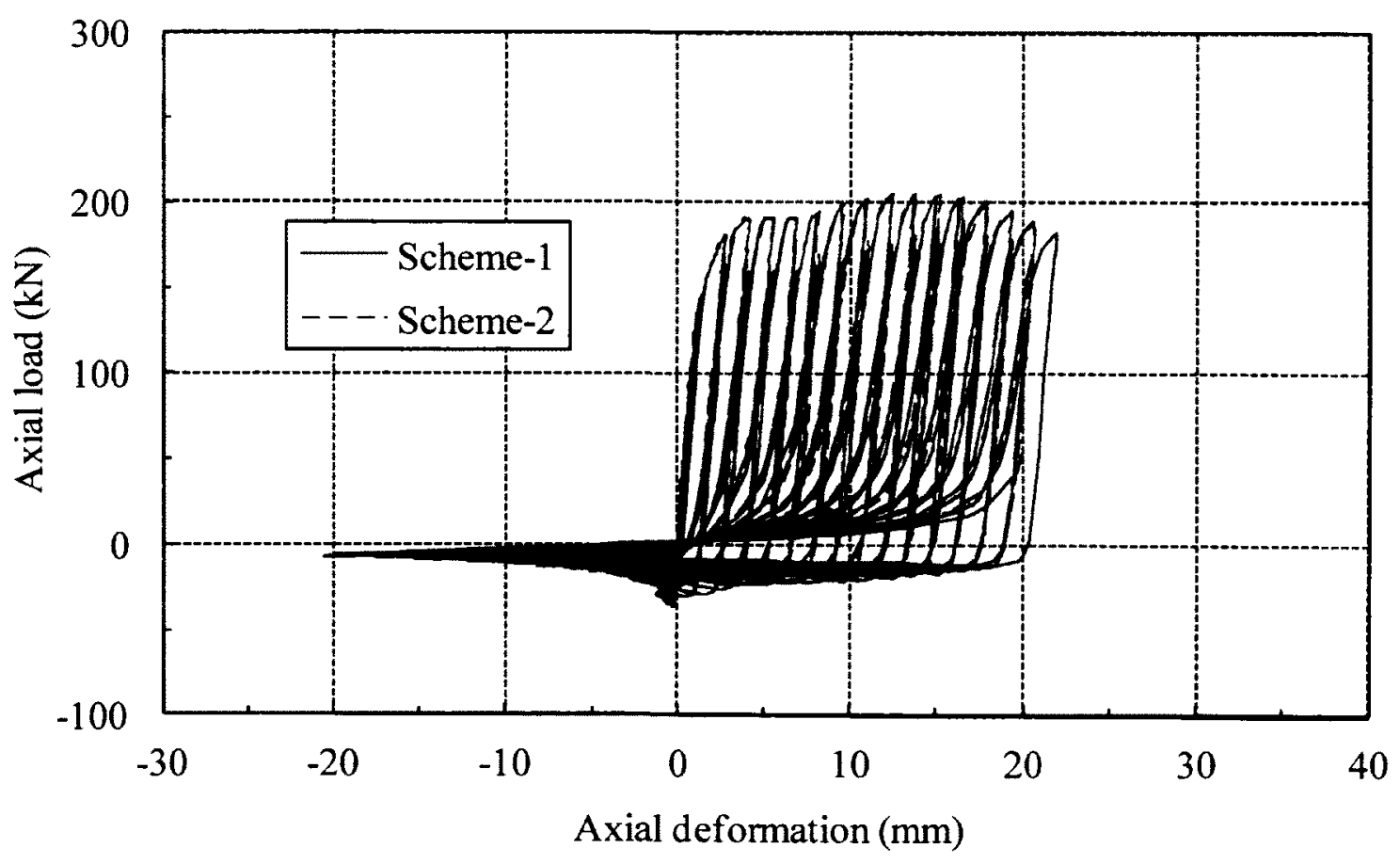

Figure 5.26 FEA simulation of load versus deformation curves for strap specimen A2078 using the 1/4 model with two different mesh schemes 


SDV 10(damage)
SNEG, (fraction $=-1.0)$
(Avg: $75 \%$ )
$+1.134 \mathrm{e}+00$
$+1.039 \mathrm{e}+00$
$+9.450 \mathrm{e}-01$
$+8.505 \mathrm{e}-01$
$+7.560 \mathrm{e}-01$
$+6.615 e-01$
$+5.670 \mathrm{e}-01$
$+4.725 \mathrm{e}-01$
$+3.780 \mathrm{e}-01$
$+2.835 \mathrm{e}-01$
$+1.890 \mathrm{e}-01$
$+9.450 \mathrm{e}-02$
$+0.000 \mathrm{e}+00$

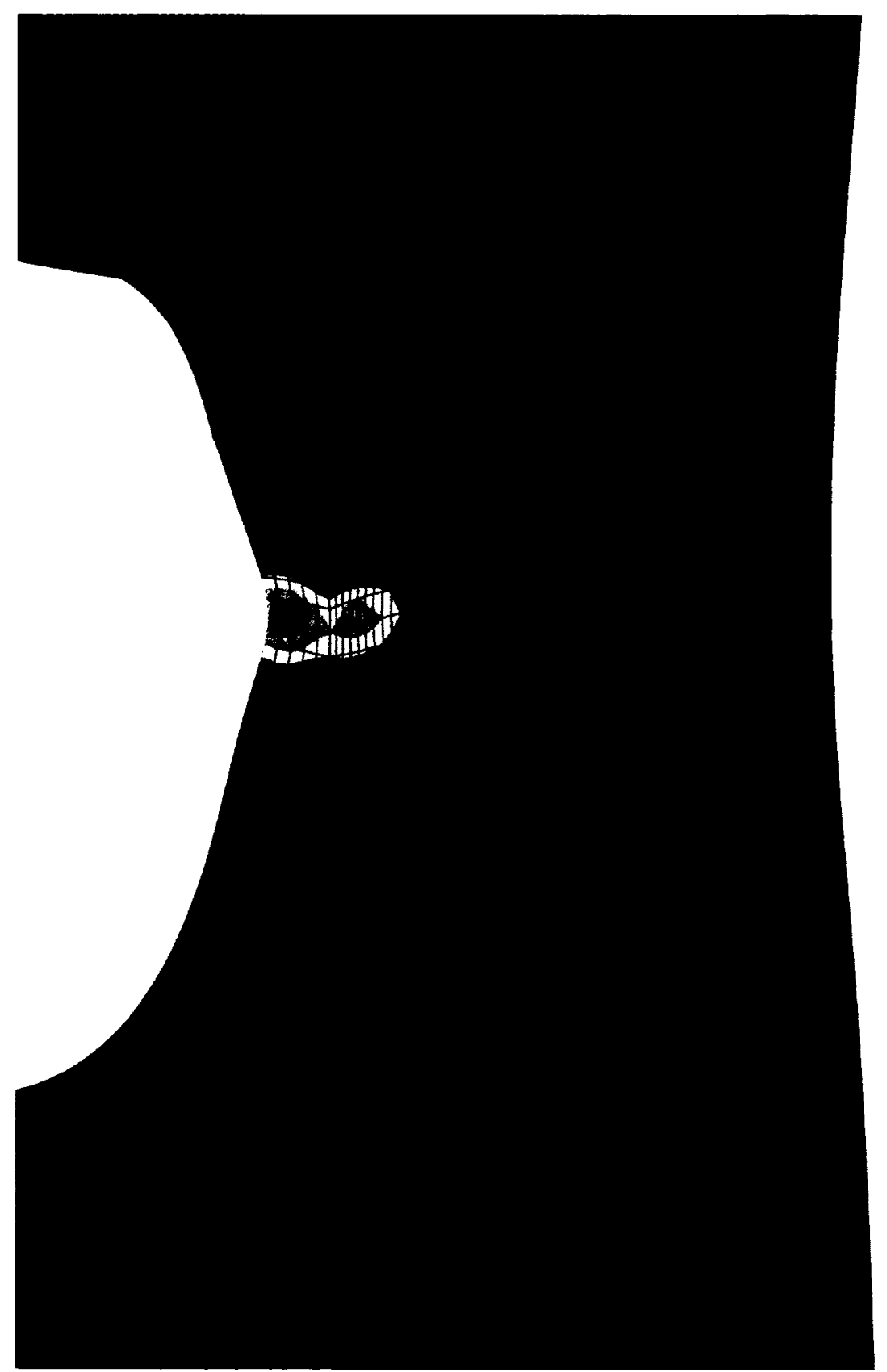

Figure 5.27 Deformation around the region of the innermost bolt hole at close to fracture showing the contour plot of the damage, shown for strap specimen A4075 with $\eta=0.75$ 


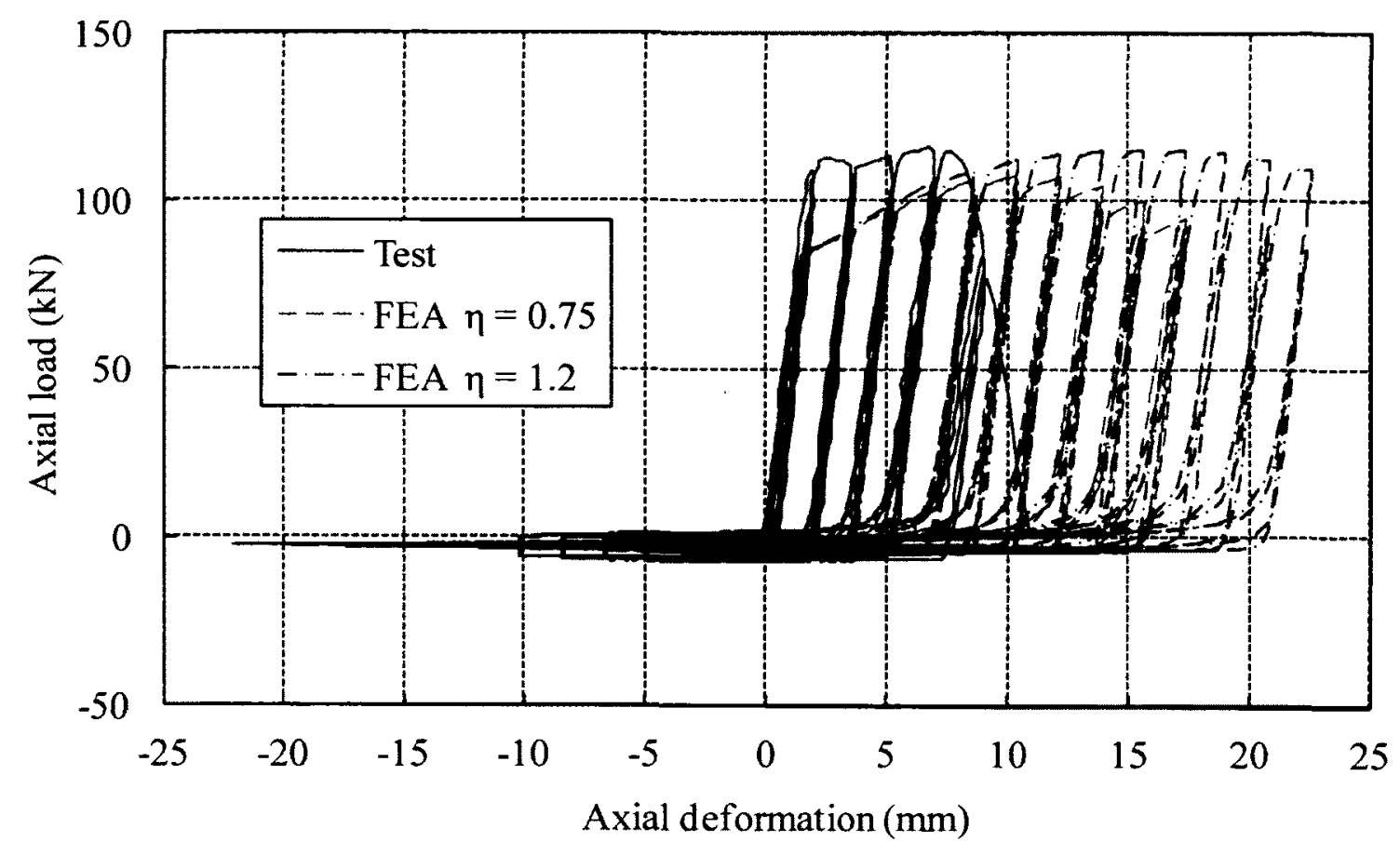

Figure 5.28 Test and predicted load versus deformation curves for strap specimen A4075a using the $1 / 4$ model

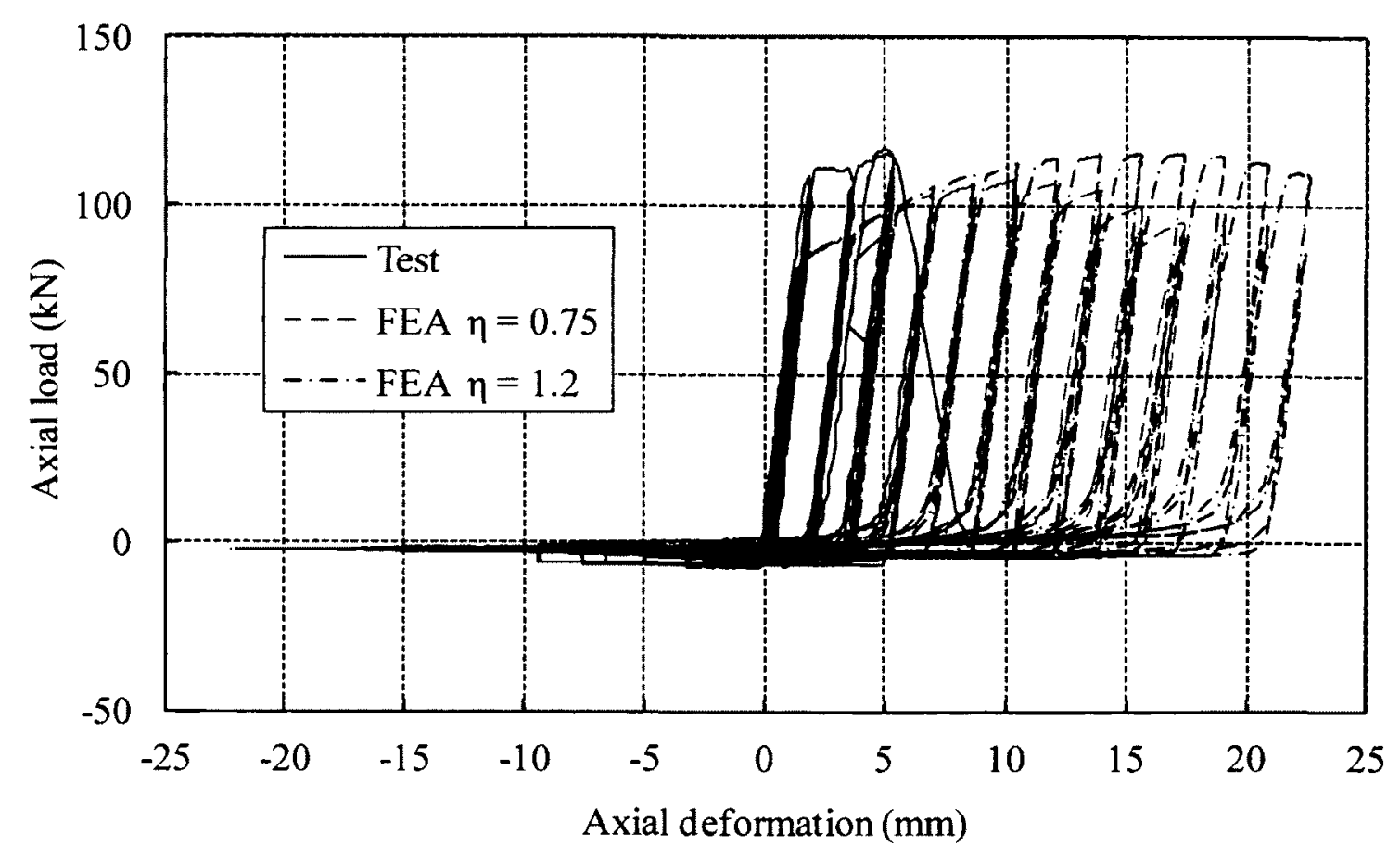

Figure 5.29 Test and predicted load versus deformation curves for strap specimen A $4075 \mathrm{~b}$ using the $1 / 4$ model 


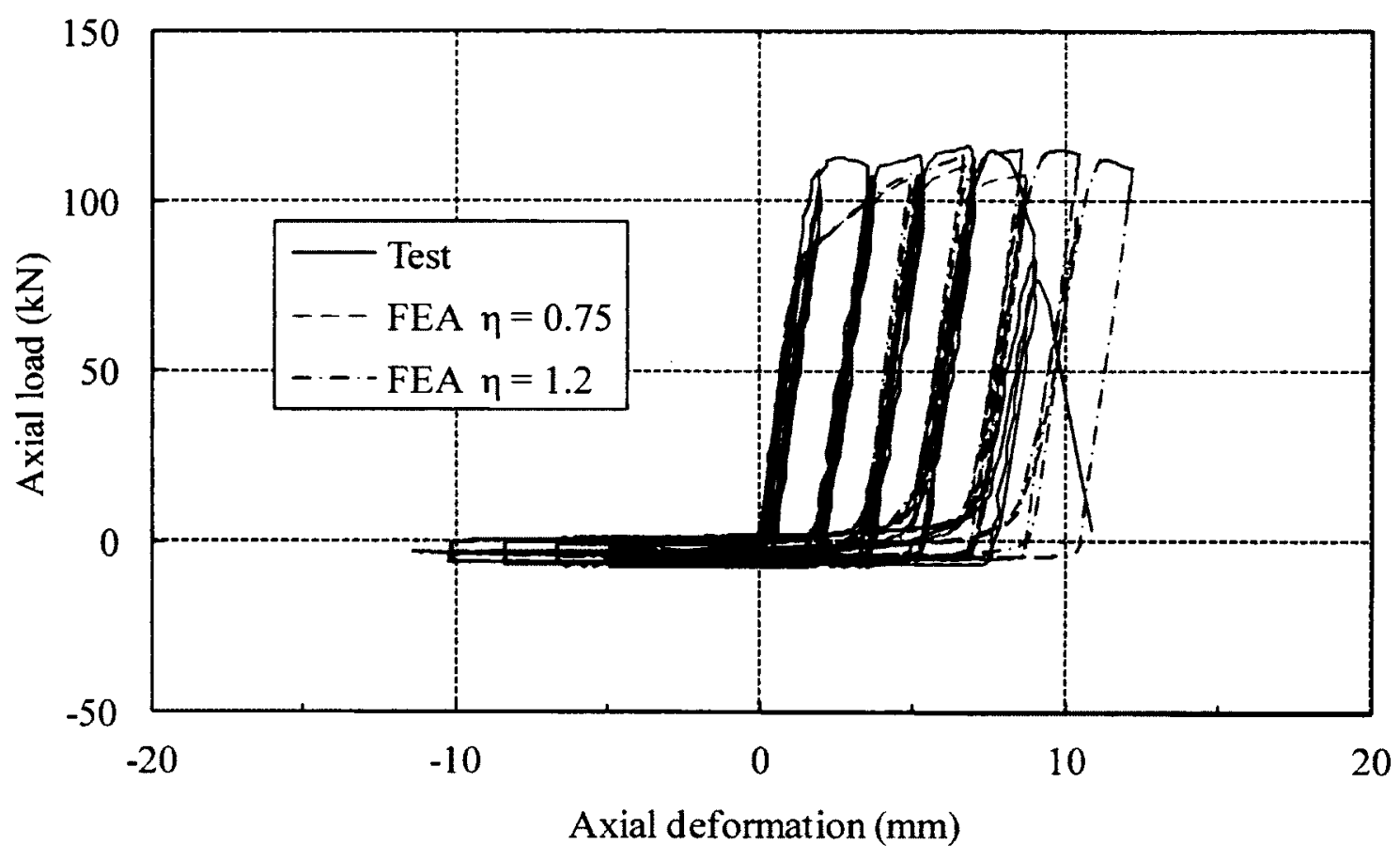

Figure 5.30 Test and predicted load versus deformation curves for strap specimen A $4075 \mathrm{a}$ using the $1 / 2$ model

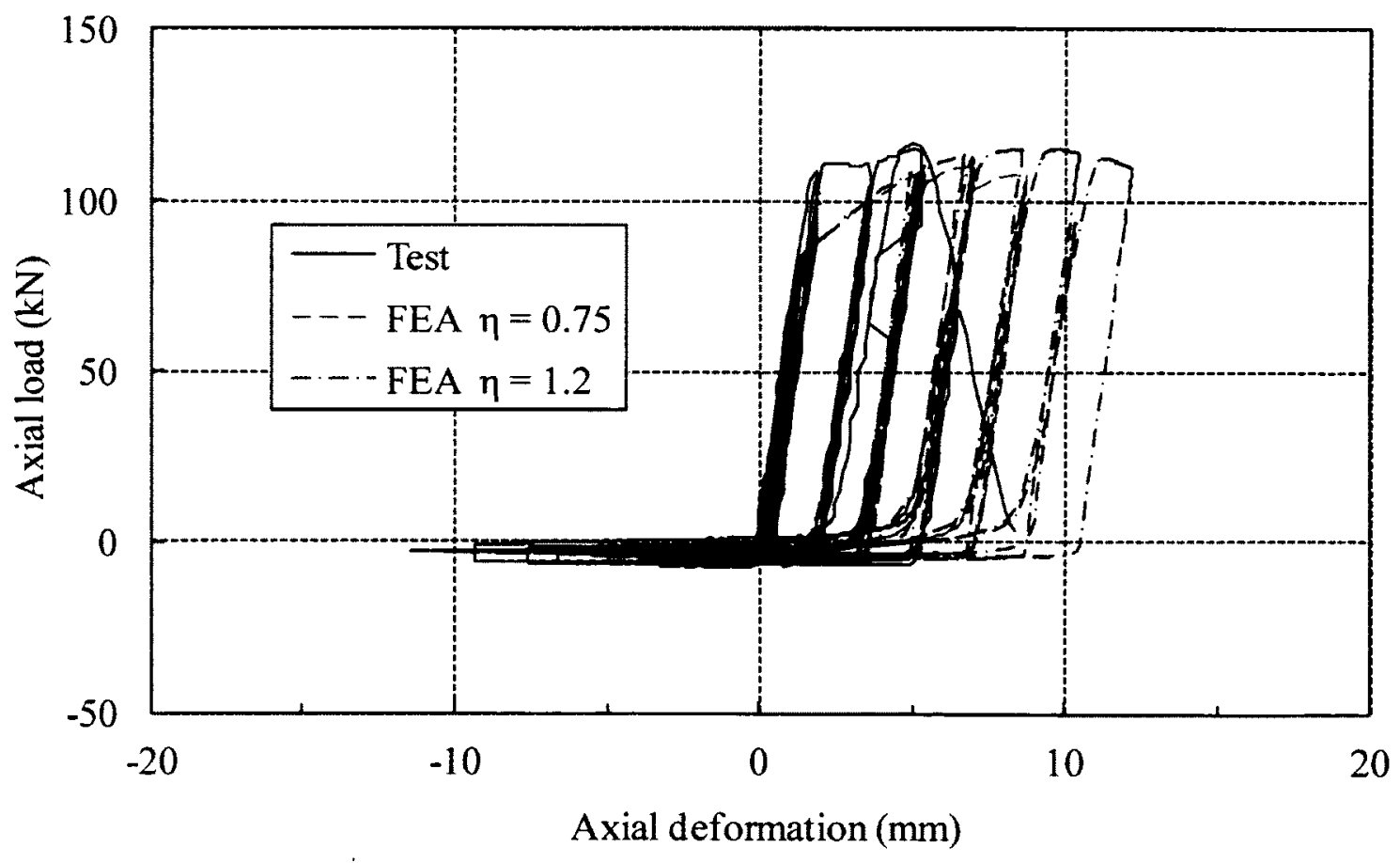

Figure 5.31 Test and predicted load versus deformation curves for strap specimen A4075b using the $1 / 2$ model 


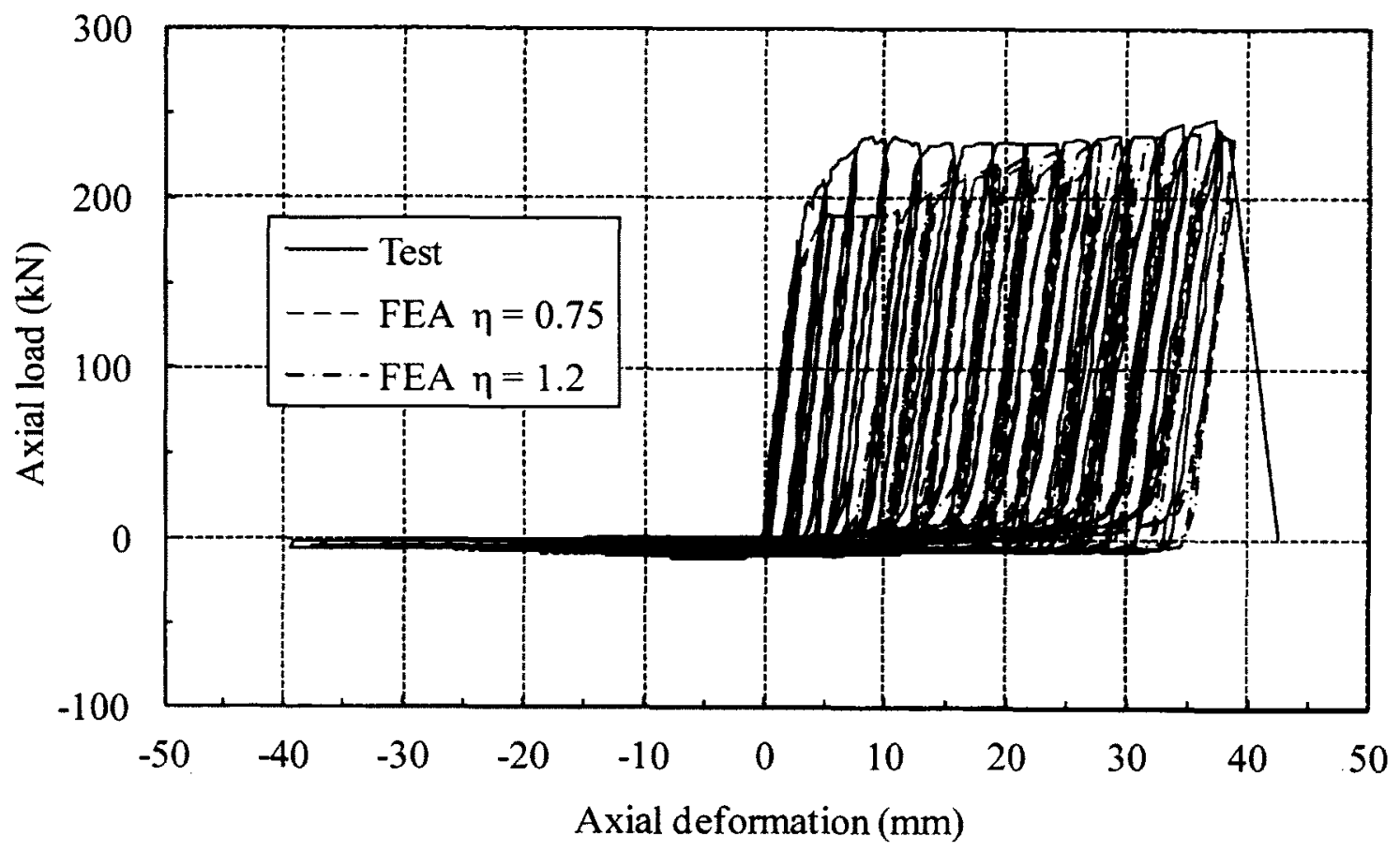

Figure 5.32 Test and predicted load versus deformation curves for strap specimen A4078a using the $1 / 4$ model

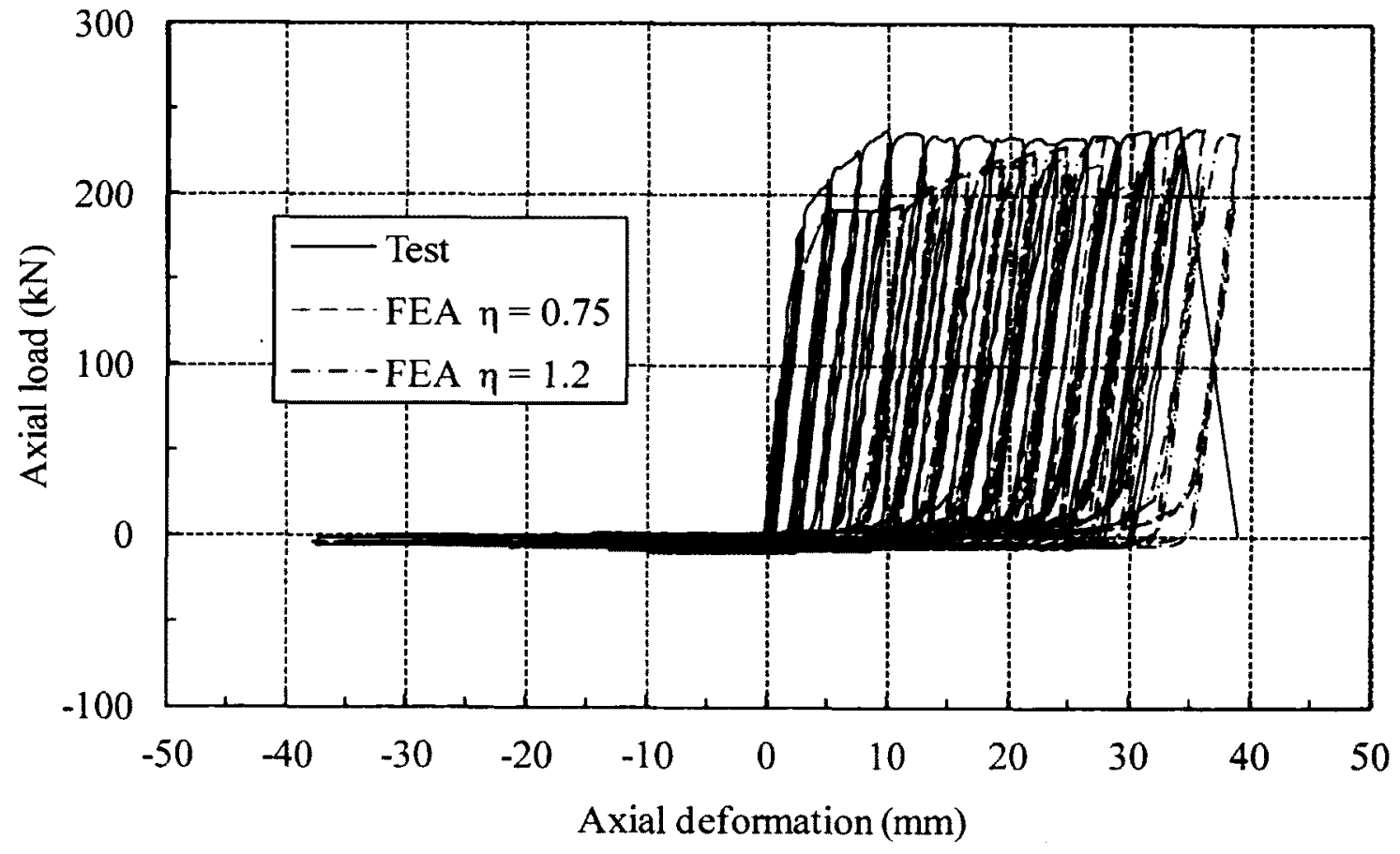

Figure 5.33 Test and predicted load versus deformation curves for strap specimen A $4078 \mathrm{~b}$ using the $1 / 4$ model 


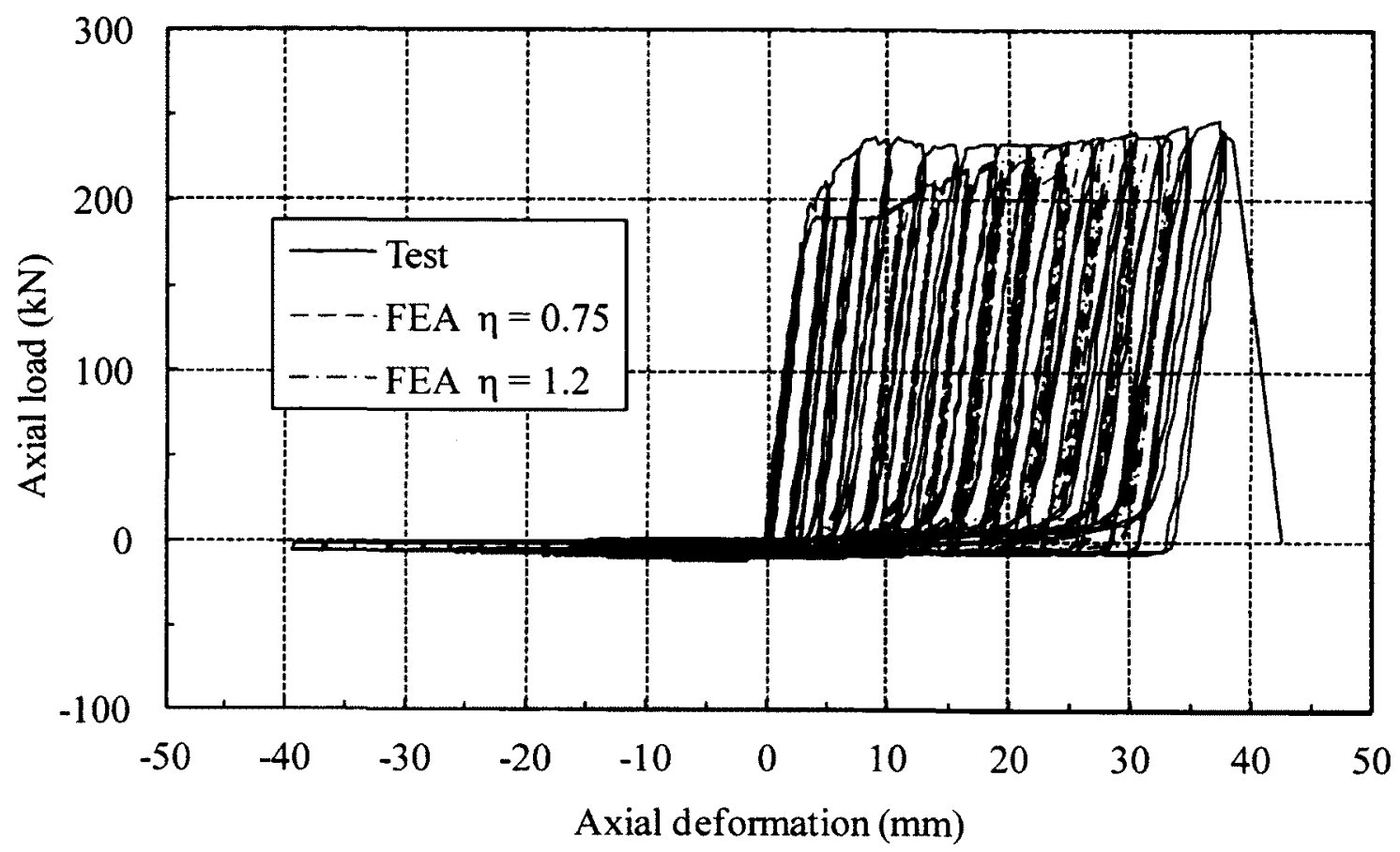

Figure 5.34 Test and predicted load versus deformation curves for strap specimen A4078a using the $1 / 2$ model

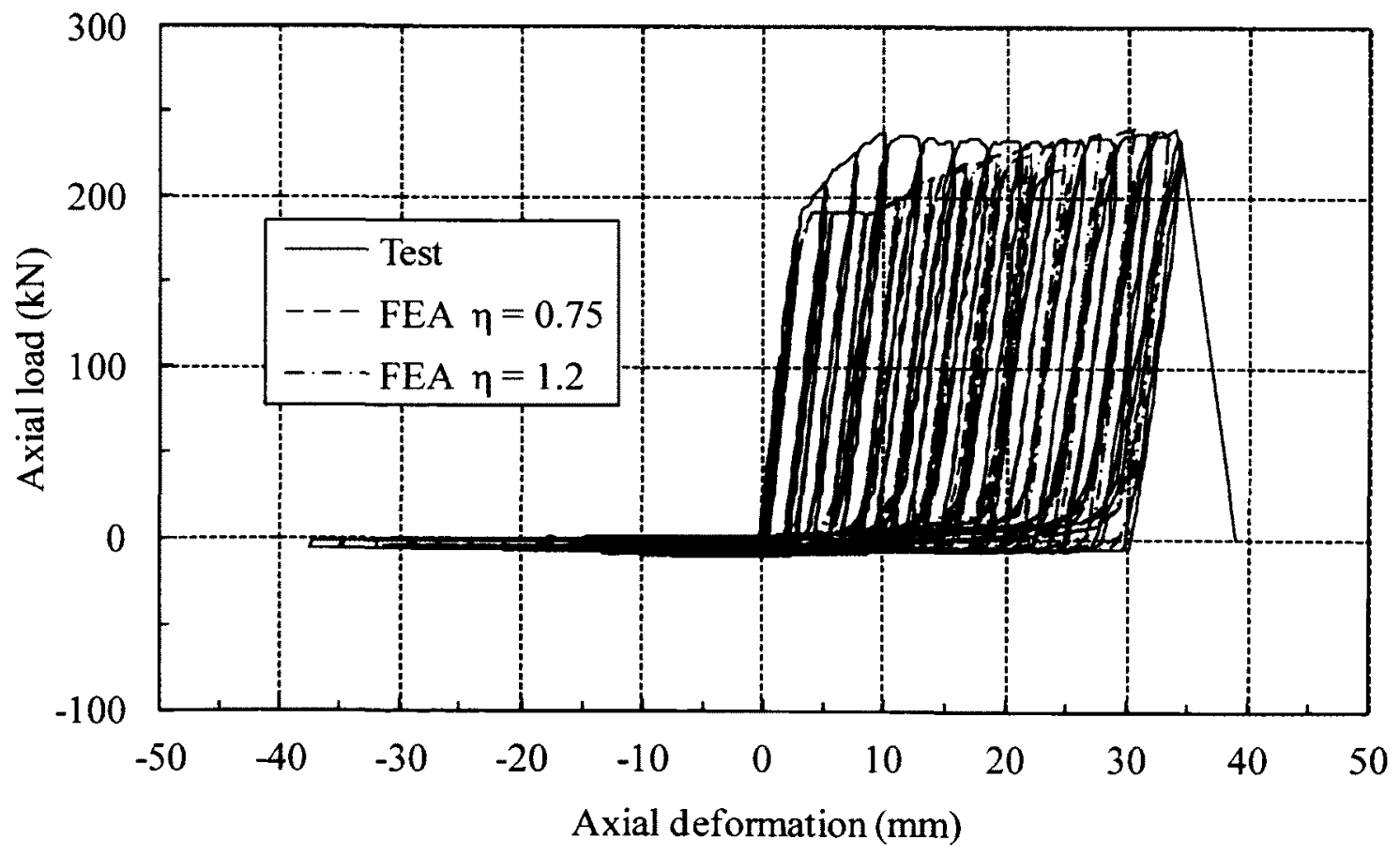

Figure 5.35 Test and predicted load versus deformation curves for strap specimen A $4078 \mathrm{~b}$ using the $1 / 2$ model 


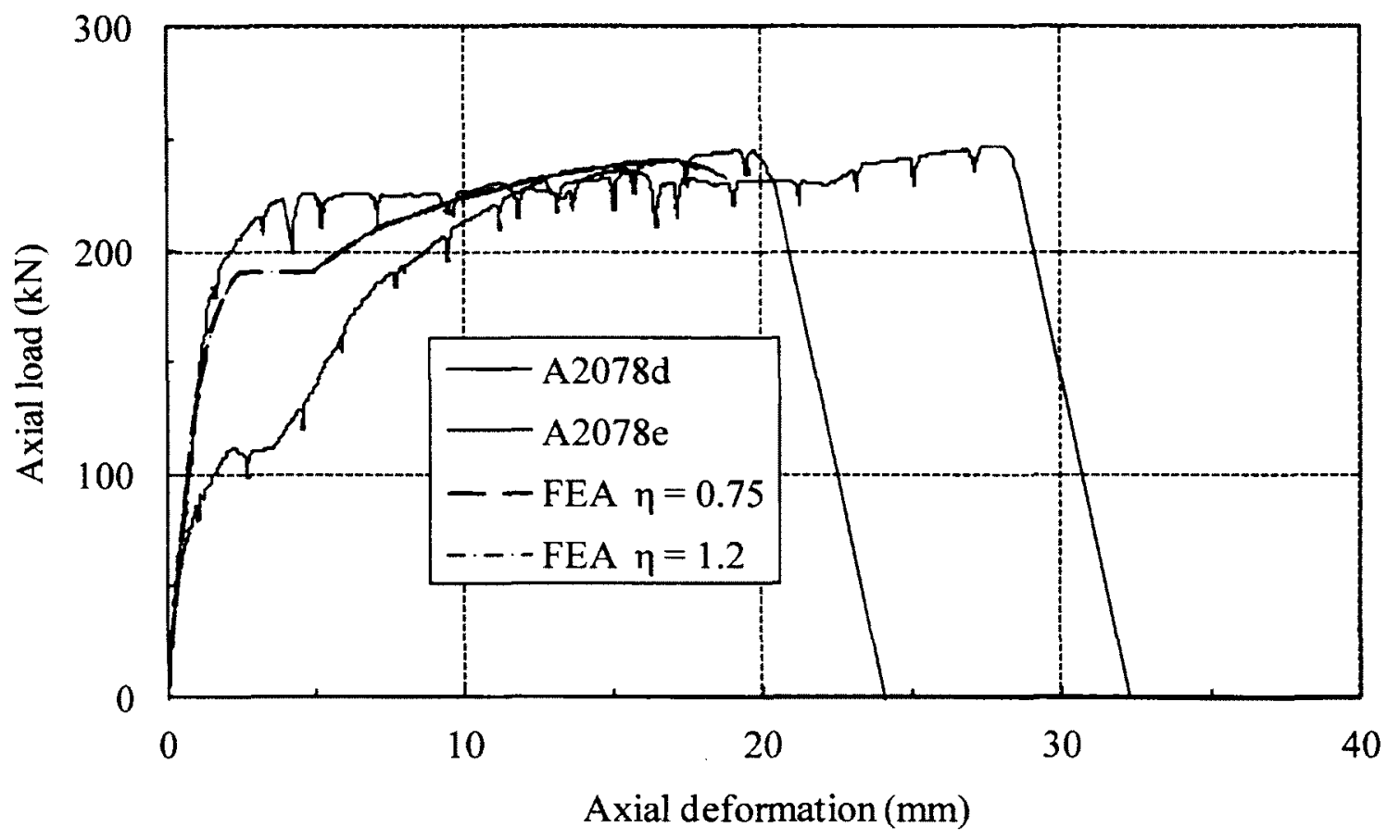

Figure 5.36 Test and predicted load versus deformation curves for strap specimens A2078d and A2078e using the 1/2 model

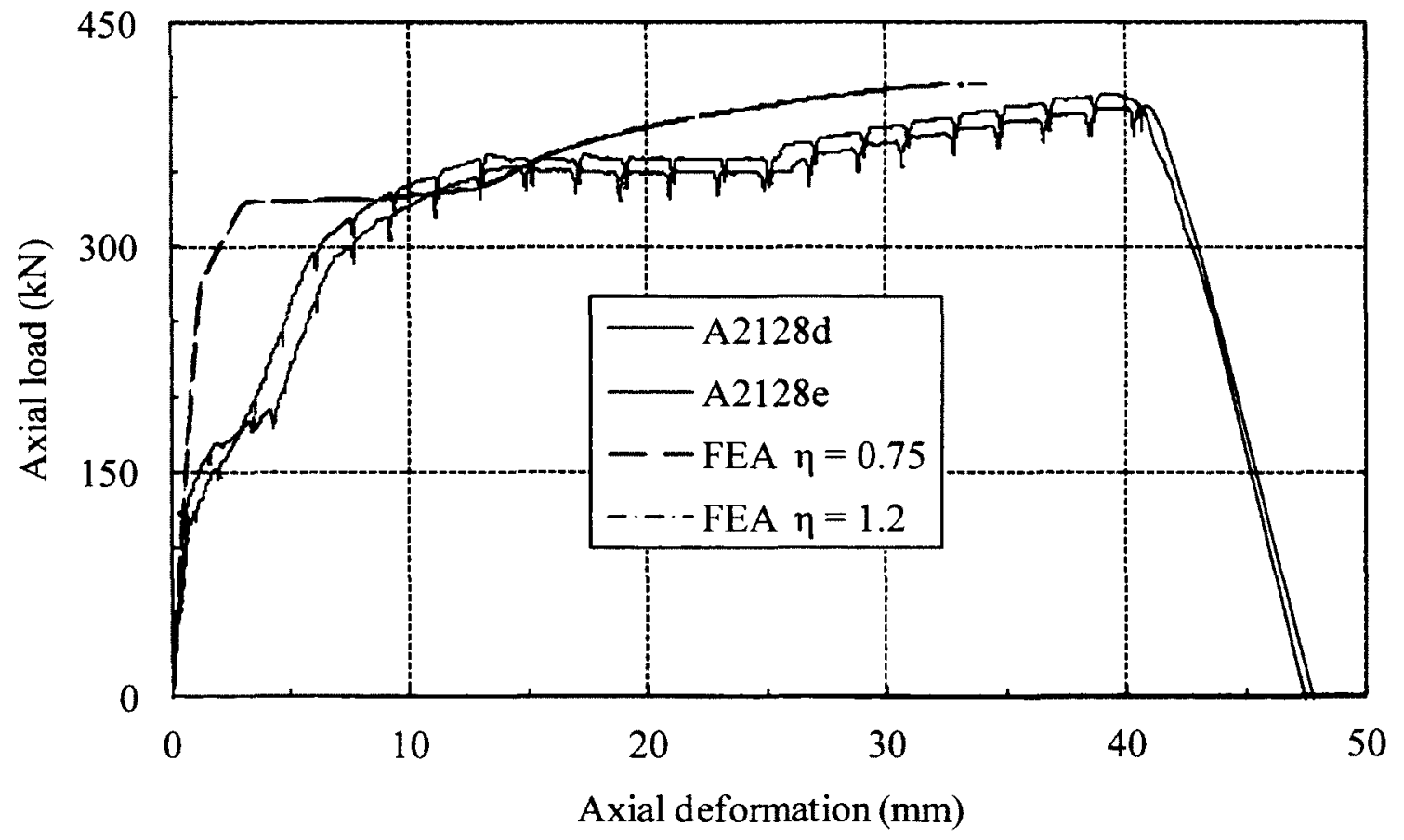

Figure 5.37 Test and predicted load versus deformation curves for strap specimens A2128d and A2128e using the $1 / 2$ model 


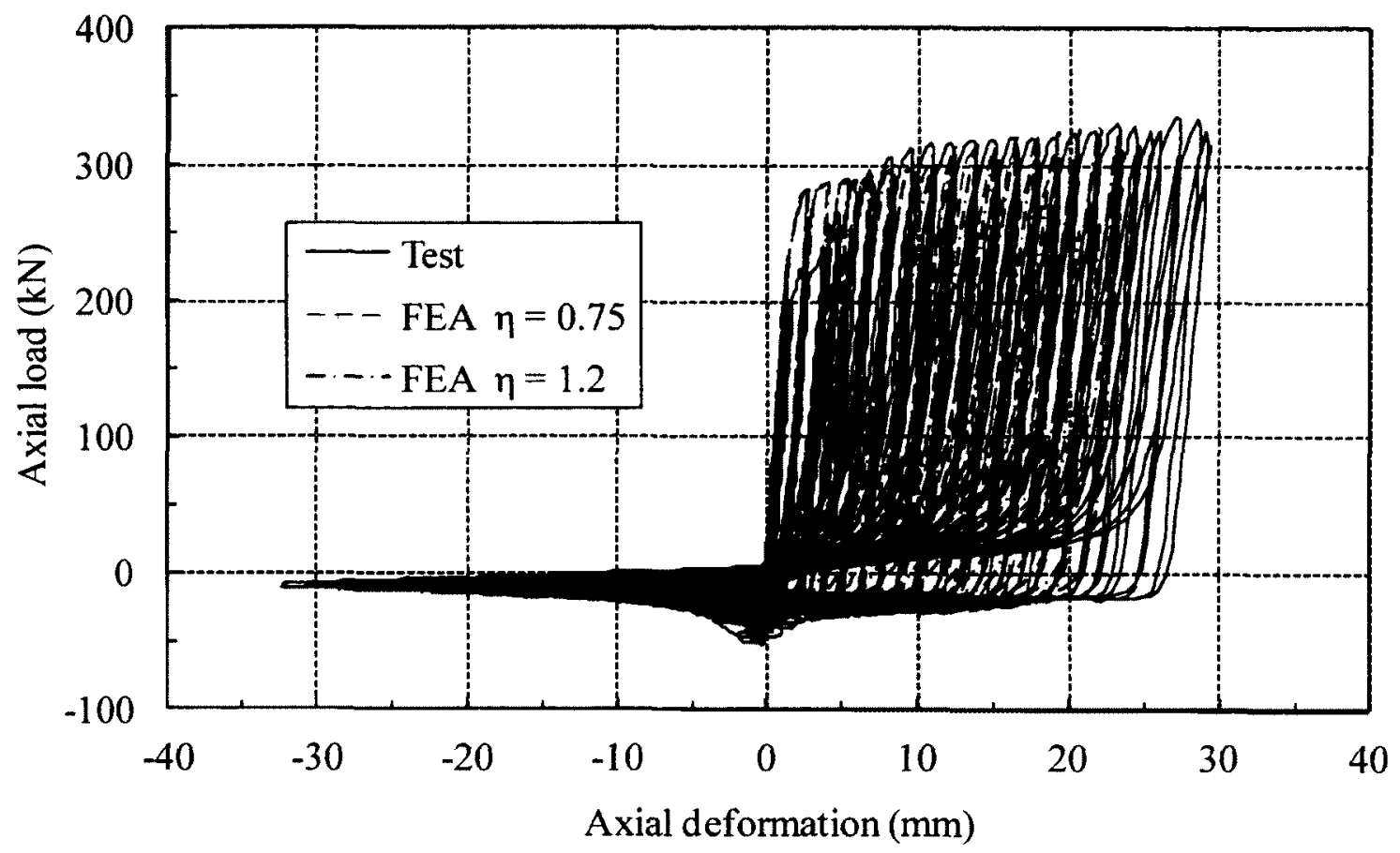

Figure 5.38 Test and predicted load versus deformation curves for strap specimen A2108b using the $1 / 2$ model

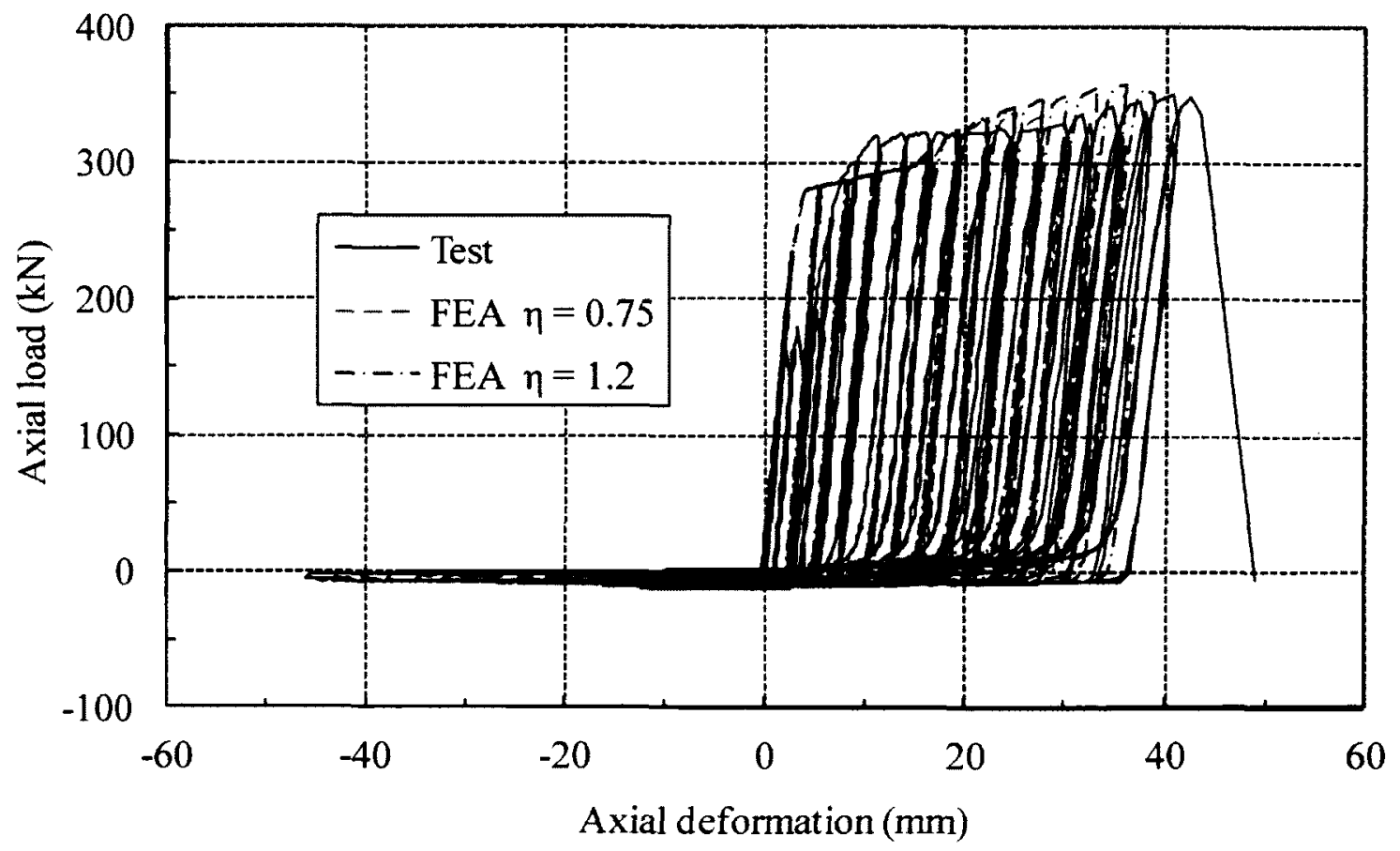

Figure 5.39 Test and predicted load versus deformation curves for strap specimen A4108a using the $1 / 2$ model 


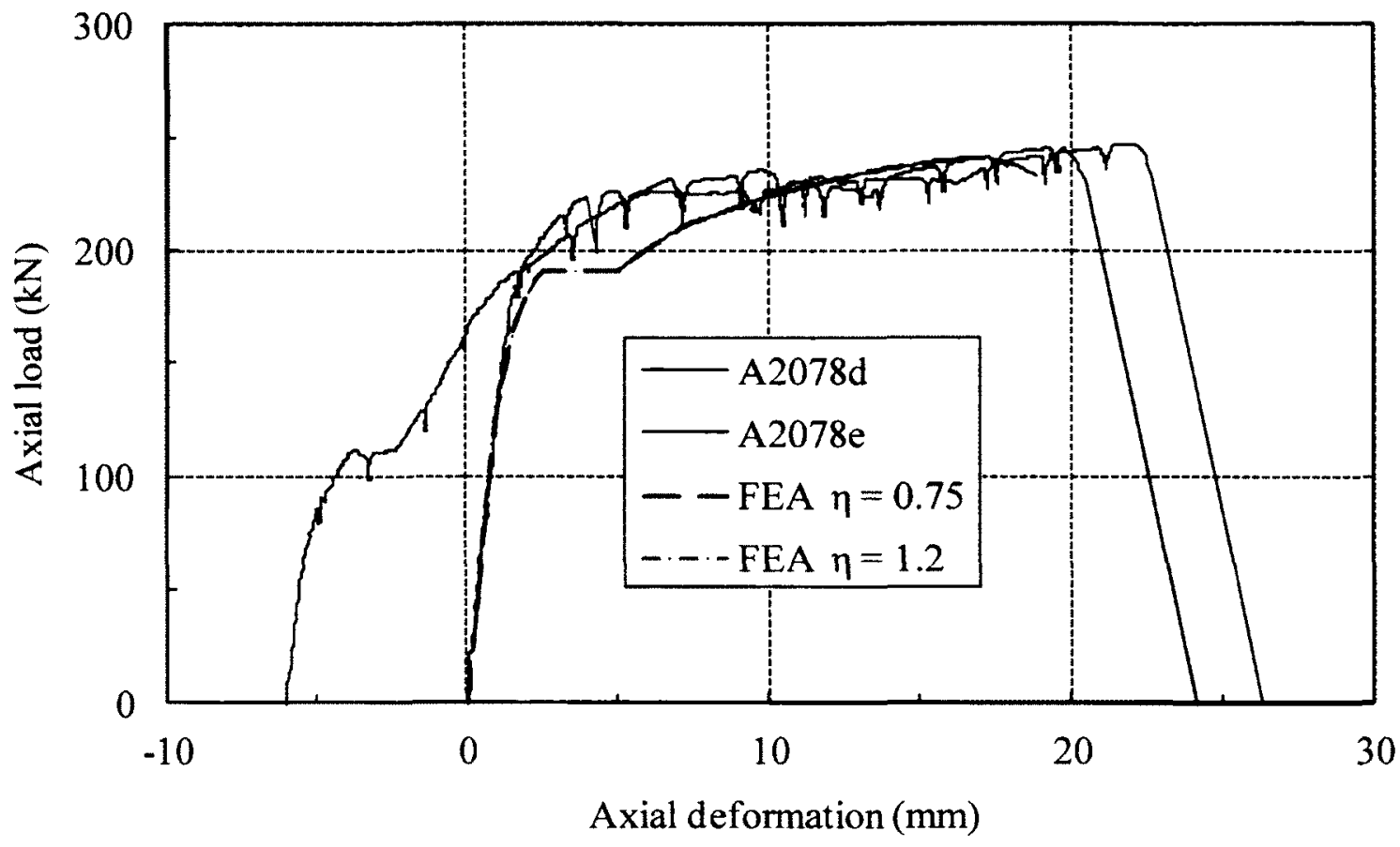

Figure 5.40 Test and predicted load versus deformation curves for strap specimens A2078d and A2078e using the $1 / 2$ model with the test deformation reduced by $6 \mathrm{~mm}$ for $\mathrm{A} 2078 \mathrm{~d}$

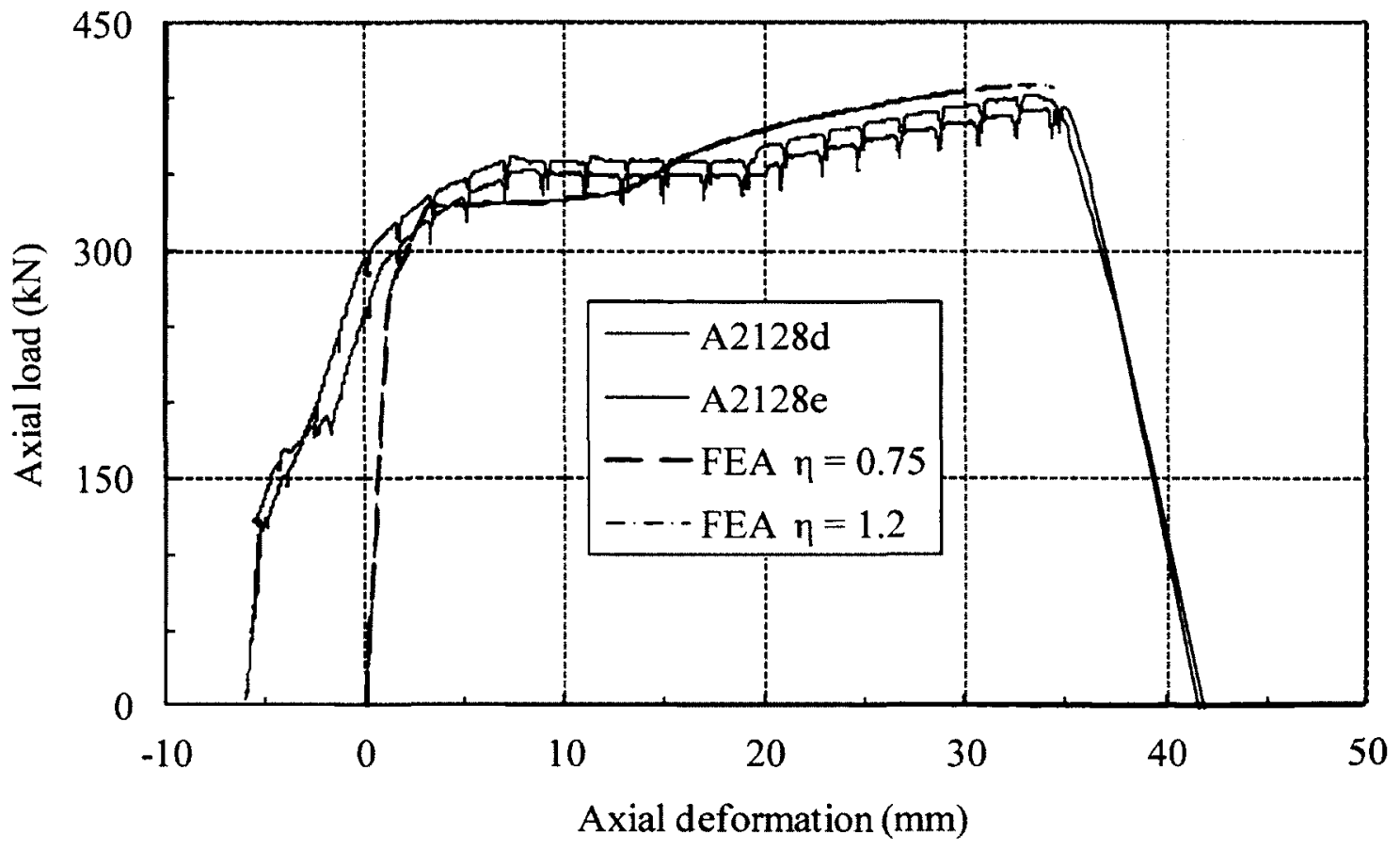

Figure 5.41 Test and predicted load versus deformation curves for strap specimens A2128d and A2128e using the $1 / 2$ model with the test deformation reduced by $6 \mathrm{~mm}$ 


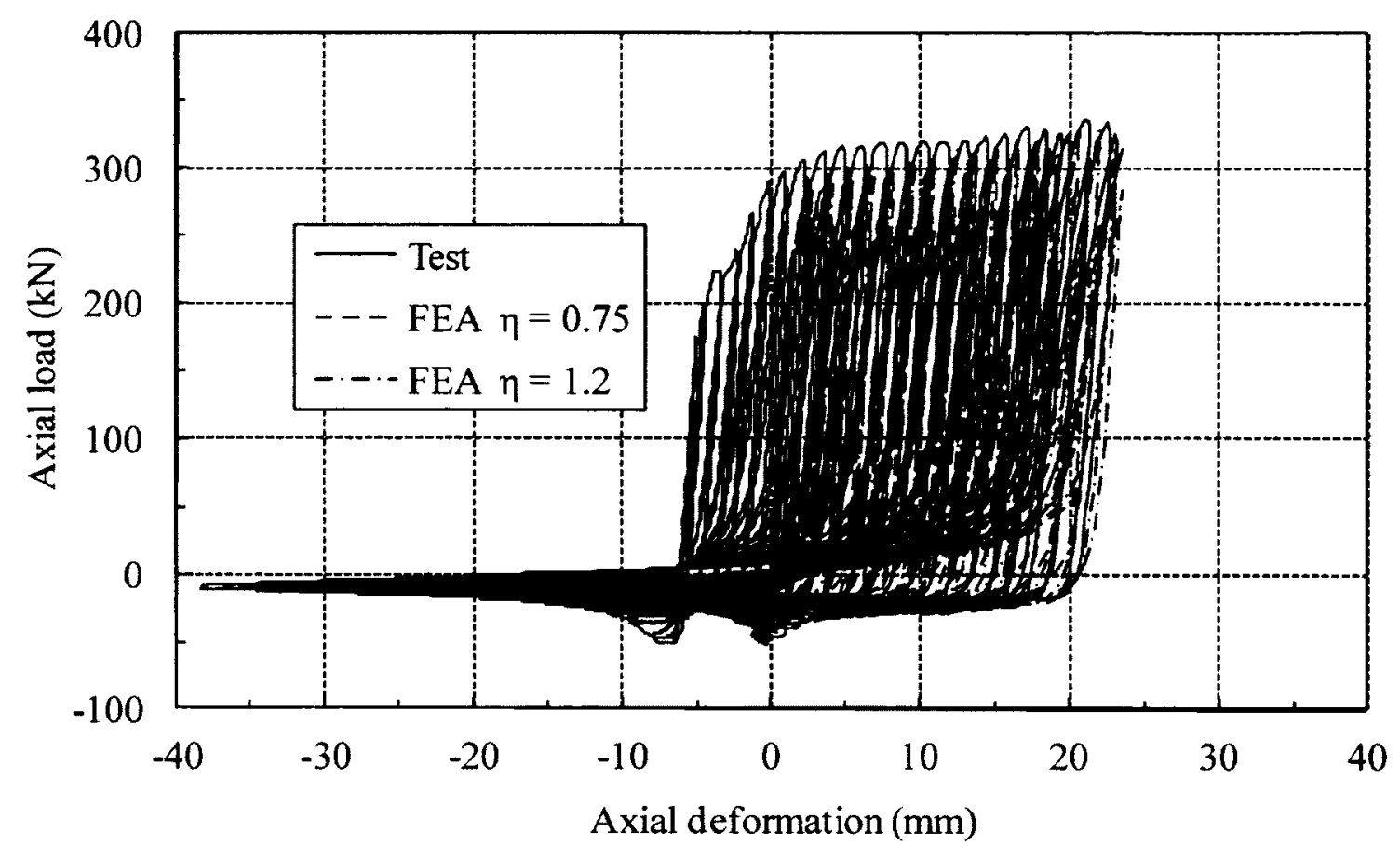

Figure 5.42 Test and predicted load versus deformation curves for strap specimen A2108b using the $1 / 2$ model with the test deformation reduced by $6 \mathrm{~mm}$

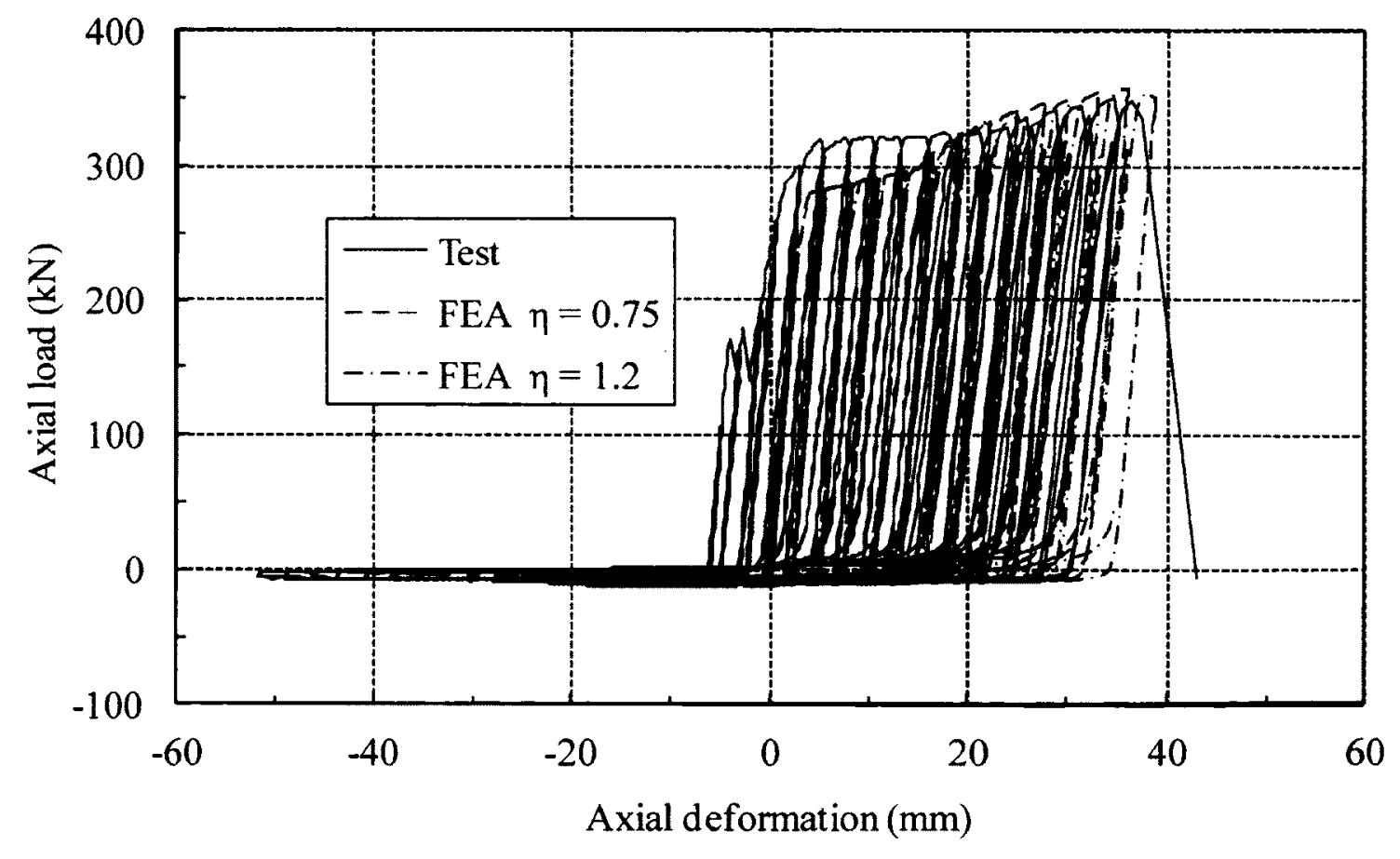

Figure 5.43 Test and predicted load versus deformation curves for strap specimen A4108a using the $1 / 2$ model with the test deformation reduced by $6 \mathrm{~mm}$ 


\section{CHAPTER 6 PARAMETRIC STUDY OF STRAP TENSION-ONLY BRACE}

In Chapter 5, procedures have been established to estimate parameters for the material model and damage evolution using results of a simple material tension test. Numerical simulations of the steel strap using those estimated parameters with the mixedmode hardening model by Chaboche (1986) and the fracture criterion from Eqs. (4.3) and (4.4) have also been validated against results of the tests by Aghdam (2009). Based on these, a parametric finite element analysis study has been carried out to study the performance of steel strap tension-only brace for a range of material behaviour and geometric configurations. Results of the study are used in developing equations to predict the ductility of the steel strap and in helping establish recommendations in designing strap tension-only brace in a Seismic Force Resisting System (SFRS).

\subsection{Generation of Material Properties}

In a standard material test, only the engineering stress-strain data are recorded. However, the flow stress-true plastic strain data together with values of other parameters for the material model are required in a finite element analysis. An engineering stressstrain curve can be characterized by the length of the yield plateau, ultimate to yield strength ratio, and strain at ultimate stress. Thus, these characteristics can be transformed into the equivalent parameters of $\sigma_{\infty}^{y}, b, n, \sigma_{0}^{y}$ and $\varepsilon_{0}^{p}$ for Eq. (3.28) to generate the relevant flow stress-true plastic strain curve.

The shape of an engineering stress-strain curve up to the ultimate (peak) stress can be approximately represented by the engineering stress, and engineering strain at the 
start of strain hardening (proportional limit or end of yield plateau) and at the peak point on the curve. Setting the slope of stress-strain curve to zero yields

$$
\left.\frac{\mathrm{d} \sigma_{\mathrm{e}}}{\mathrm{d} \varepsilon_{\mathrm{e}}}\right|_{\left(\varepsilon_{\mathrm{cut} . \tau_{\mathrm{eu}}}\right)}=0
$$

where $\varepsilon_{\mathrm{e}}$ and $\sigma_{\mathrm{e}}$ are the engineering strain and stress, $\varepsilon_{\mathrm{eu}}$ and $\sigma_{\mathrm{eu}}$ are the corresponding engineering strain and stress at ultimate stress of the stress-strain curve. Up to the ultimate stress, the relationship of the flow stress-true strain curve to the engineering stress-strain curve can be expressed as

$$
\begin{aligned}
& \sigma_{\mathrm{t}}=\sigma_{\mathrm{e}}\left(1+\varepsilon_{\mathrm{e}}\right), \\
& \varepsilon_{\mathrm{t}}=\ln \left(1+\varepsilon_{\mathrm{e}}\right), \\
& \varepsilon_{\mathrm{t}}^{\mathrm{p}}=\varepsilon_{\mathrm{t}}-\frac{\sigma_{\mathrm{t}}}{E},
\end{aligned}
$$

where $\varepsilon_{\mathrm{t}}^{\mathrm{p}}$ is the true plastic strain. Rearranging Eq. (3.28) gives

$$
\varepsilon_{\mathrm{t}}^{\mathrm{p}}=\left[-\frac{1}{\mathrm{~b}} \ln \left|\frac{\sigma_{0}^{y}+\sigma_{\infty}^{y}-\sigma_{\mathrm{t}}}{\sigma_{\infty}^{y}}\right|\right]^{\frac{1}{n}}+\varepsilon_{o}^{\mathrm{p}} .
$$

Substituting Eqs. (6.2), (6.3) and (6.4) into (6.5) gives

$$
\ln \left(1+\varepsilon_{\mathrm{e}}\right)-\frac{\sigma_{\mathrm{c}}\left(1+\varepsilon_{\mathrm{c}}\right)}{\mathrm{E}}=\left[-\frac{1}{\mathrm{~b}} \ln \left|\frac{\sigma_{0}^{\mathrm{y}}+\sigma_{\infty}^{y}-\sigma_{\mathrm{e}}\left(1+\varepsilon_{\mathrm{c}}\right)}{\sigma_{\infty}^{\mathrm{y}}}\right|\right]^{\frac{1}{\mathrm{n}}}+\varepsilon_{\mathrm{o}}^{\mathrm{p}}
$$

Equation (6.6) can be rewritten as

$$
\begin{aligned}
U\left(\varepsilon_{\mathrm{e}}, \sigma_{\mathrm{e}}\right)= & \ln \left(1+\varepsilon_{\mathrm{e}}\right)-\frac{\sigma_{\mathrm{e}}\left(1+\varepsilon_{\mathrm{e}}\right)}{\mathrm{E}}-\left[-\frac{1}{\mathrm{~b}} \ln \left|\frac{\sigma_{0}^{y}+\sigma_{\kappa}^{y}-\sigma_{\mathrm{e}}\left(1+\varepsilon_{\mathrm{e}}\right)}{\sigma_{x}^{y}}\right|\right]^{\frac{1}{n}} \\
& -\varepsilon_{\mathrm{o}}^{\mathrm{p}}=0 .
\end{aligned}
$$

The derivative of Eq. (6.1) can be established from Eq. (6.7) through 


$$
\frac{\mathrm{dU}}{\mathrm{d} \varepsilon_{\mathrm{c}}}=\frac{\partial \mathrm{U}}{\partial \varepsilon_{\mathrm{e}}}+\frac{\partial \mathrm{U}}{\partial \sigma_{\mathrm{e}}} \cdot \frac{\mathrm{d} \sigma_{\mathrm{e}}}{\mathrm{d} \varepsilon_{\mathrm{e}}}=0
$$

Rearranging Eq. (6.8) leads to

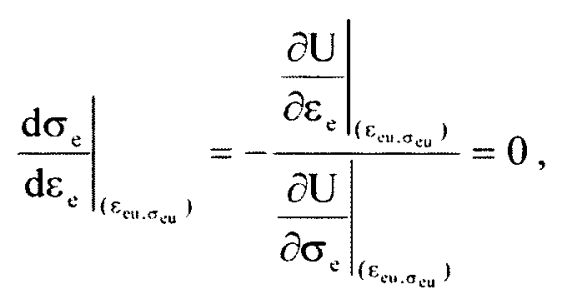

which gives

$$
\left.\frac{\mathrm{d} \sigma_{\mathrm{e}}}{\mathrm{d} \varepsilon_{\mathrm{e}}}\right|_{\left(\varepsilon_{\mathrm{cu}, \sigma_{\mathrm{cu}}}\right)}=\left.\frac{\partial \mathrm{U}}{\partial \varepsilon_{\mathrm{e}}}\right|_{\left(\varepsilon_{\mathrm{ev}, \sigma_{\mathrm{cu}}}\right)}=0 .
$$

From Eq. (6.7),

$$
\frac{\partial U}{\partial \varepsilon_{\mathrm{e}}}=\frac{1}{1+\varepsilon_{\mathrm{e}}}-\frac{\sigma_{\mathrm{e}}}{\mathrm{E}}-\frac{\sigma_{\mathrm{e}}\left[-\frac{1}{\mathrm{~b}} \ln \left|\frac{\sigma_{0}^{y}+\sigma_{s}^{y}-\sigma_{\mathrm{e}}\left(1+\varepsilon_{\mathrm{e}}\right)}{\sigma_{x}^{y}}\right|\right]^{\frac{1}{n}-1}}{\mathrm{bn}\left|\sigma_{0}^{y}+\sigma_{\infty}^{y}-\sigma_{\mathrm{e}}\left(1+\varepsilon_{\mathrm{e}}\right)\right|} .
$$

The corresponding point at ultimate engineering stress in terms of flow stress and true plastic strain is identified as $\sigma_{u}^{y}$ and $\varepsilon_{u}^{p}$. This provides another point $\left(\sigma_{u}^{y}, \varepsilon_{u}^{p}\right)$ on the flow stress-true plastic strain curve to solve $\sigma_{\infty}^{y}, \mathrm{~b}$ and $\mathrm{n}$ for Eq. (3.28). As a result, the parameter $\sigma_{x}^{y}$ can be expressed according to Eq. (3.30) as

$$
\sigma_{x}^{y}=\frac{\sigma_{u}^{y}-\sigma_{0}^{y}}{1-\mathrm{e}^{-\mathrm{b}\left(\varepsilon_{u}^{p}-\varepsilon_{u}^{p}\right)^{n}}},
$$

where $\sigma_{0}^{y}$ and $\varepsilon_{0}^{p}$ can be considered as material constants. Combining Eqs. (6.10) to (6.12) gives 


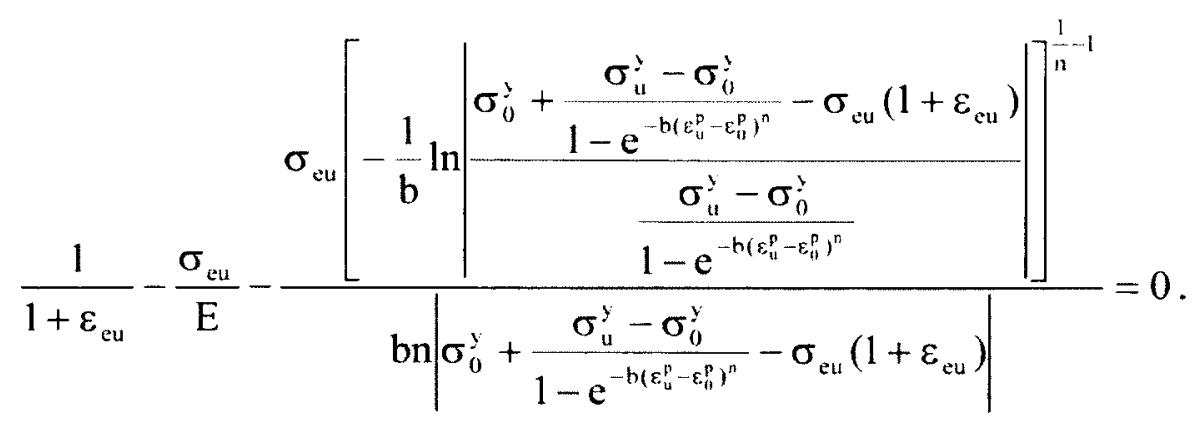

In the situation when either the value of parameter $\mathrm{b}$ or $\mathrm{n}$ is known or given, the value of the unknown either $\mathrm{b}$ or $\mathrm{n}$ can then be determined by solving Eq. (6.13) for the given stress and strain at the starting point of strain hardening (proportional limit or end of the yield plateau) and at the ultimate stress.

\subsection{Parameters Used in the Parametric Study}

Based on findings in Chapter 5, all the finite element analyses in the parametric study are carried out using the $1 / 2$ model of the strap specimen with $\eta=0.75$ for the saturated yield stress. Since the ductility of an actual strap varies, it has to be defined in order to carry out the parametric study. The defined ductility is translated to the corresponding damage limit of a strap brace that can be used in the simulation. In order to reduce the number of material behaviour types that have to be considered in the parametric study, a preliminary study has been carried out to investigate the effect of varying the flow stress parameter $n$, yield plateau, and engineering strain at ultimate stress on the fracture prediction of the strap brace.

\subsubsection{Rectangular Specimen for Calculating Damage Limit}

While there are many aspects of the material behaviour that can be considered, the ductility of the material, which translates to the damage limit, is one aspect of the 
material behaviour that certainly has a significant effect on the predicted ductility of a strap brace. The ductility of the material can be partly characterized by $\ln \left(A_{i} / A_{f}\right)$ measured from a tension material test. The process and determination of the damage limit used in the parametric study is discussed below.

In Chapter 5, the measured cross-sectional area change at fracture, $\ln \left(A_{0} / A_{f}\right)$, in a tension material test (straight specimen) of the strap has been used to calculate the damage limit used in the simulations. Thus, the measured $\ln \left(A_{0} / A_{f}\right)$ for materials $A$ to $F$ are used to help in defining a conservative $\ln \left(A_{0} / A_{f}\right)$ for the material used in the parametric study. However, the cross-sectional profiles used in the material tests for materials $\mathrm{A}$ to $\mathrm{F}$ were not the same. In order to use these measurements to define the value of $\ln \left(A_{0} / A_{f}\right)$ for the material used in the parametric study, the measured material $\ln \left(A_{0} / A_{f}\right)$ has to be translated to a value for the cross-sectional profile that is common to all materials. To this end, the cross-sectional dimension of a $7.9 \mathrm{~mm}$ thick rectangular specimen has been selected as the reference cross-sectional profile in the calculation. The translation of $\ln \left(A_{0} / A_{f}\right)$ across different cross-sectional profiles has been carried out on the basis that the damage limit does not change with the profile. In Chapters 4 and 5 , parameters (c, $\mathrm{m}$ and $\mathrm{k}$ ) for the damage evolution Eqs. (4.3) and (4.4) have been determined for materials A to F. Instead of using the optimum damage limit established in Chapters 4 and 5 , the calculated damage with the previously determined damage evolution parameters in Tables $4.3,4.5$ and 5.8 corresponding to the point in the numerical simulation where $\ln \left(\mathrm{A}_{0} / \mathrm{A}_{\mathrm{f}}\right)$ matches that of the actual tension material test is taken as a new damage limit. This damage limit is then applied to the numerical simulation of a tension material test for a different cross-sectional profile (rectangular 
specimen of a plate with $7.9 \mathrm{~mm}$ thickness) to predict the point of fracture and the corresponding translated $\ln \left(\mathrm{A}_{0} / \mathrm{A}_{1}\right)$ for that cross-sectional profile. Table 6.1 shows the measured and translated $\ln \left(A_{0} / A_{f}\right)$ for materials $A$ to $F$. Material $G$ in Table 6.1 is from the material test by Chen (2010) for CSA G40.20/21 (CSA 2004) Grade 300W FB $64 x$ $7.9 \mathrm{~mm}$ flat bar. It is added to the list of materials to increase the pool of data. For materials $A$ to $C, \ln \left(A_{0} / A_{1}\right)$ has been translated to a value for a plate with $7.9 \mathrm{~mm}$ thickness. Based on the limited data in Table 6.1 , a $99 \%$ probability of exceedance for $\ln \left(A_{0} / A_{f}\right)$ of 0.710 has been selected to define the damage limit for the materials used in the parametric study.

\subsubsection{Flow Stress Parameter n}

Flow stress parameter $\mathrm{n}$ varies for different materials. As seen in Table 6.2, parameter $\mathrm{n}$ varies from 0.48 to 0.64 for materials $\mathrm{A}$ to $\mathrm{F}$. To study the effect of $\mathrm{n}$ on the predicted maximum deformation, comparisons have been made between results of FEA simulations for strap specimens A2128 and A4128 of material F using a n value of 0.75 and its optimum value of 0.57 . The difference of $n$ is 0.18 and the material model parameters for material $F$ with $n=0.75$ are $\left(\sigma_{0}^{y}=333 \mathrm{MPa}, \varepsilon_{0}^{\mathrm{p}}=0.0133, \sigma_{\infty}^{y}=367 \mathrm{MPa}\right.$, $\mathrm{b}=4.55, \mathrm{C}_{1}=1868 \mathrm{MPa}, \gamma_{1}=8.68, \mathrm{C}_{2}=420 \mathrm{MPa}, \gamma_{2}=3.39, \mathrm{c}=1, \mathrm{~m}=2.13, \mathrm{k}=1.50$ and $D_{c}=2.39$ ). As can be seen in Table 6.3, the predicted maximum deformations with a larger flow stress parameter $\mathrm{n}$ are larger than those using a lower value. However, the difference between both predictions is not very large, which is less than $12 \%$ for the two cases considered. The test and predicted engineering stress versus cross-sectional area change curves for monotonic tension tests of material $F$ are shown in Fig. 6.1. There is 
only a small difference between the predicted curves with $\mathbf{n}$ of 0.57 and 0.75 . The mean $\mathrm{n}$ for materials $\mathrm{A}$ to $\mathrm{F}$ is 0.55 , and the lowest $\mathrm{n}$ is only 0.07 from the mean. Considering that the difference in the predicted maximum deformation is less than $12 \%$ for a difference in $\mathrm{n}$ of 0.18 , the mean $\mathrm{n}$ of 0.55 has been adopted to generate flow stress versus true plastic strain data in the parametric study.

\subsubsection{Yield Plateau and Engineering Strain at Ultimate Stress}

Four materials are considered to study the effect of the yield plateau length and the engineering strain at ultimate stress defined in generating the material stress-strain relationship according to the procedure outlined in Section 6.1 for the flow stress Eq. (3.28) with $n=0.55$ on the predicted maximum deformation of the brace. Listed in Table 6.4 , these four materials have the same yield and ultimate strengths, but have a different combination of the yield plateau length and the engineering strain at ultimate stress. The materials with the engineering strain at ultimate stress of 0.15 are designated as MA for no yield plateau and MB for a yield plateau length of 0.02 . Similarly, for the materials with the engineering strain at ultimate stress of 0.22 , they are designated as NA (no yield plateau) and NB (a yield plateau length of 0.02). These values have been adopted based on materials $A$ to $F$ in Table 5.2 where the engineering strain at ultimate stress for materials $A$ to $F$ ranges from 0.151 to 0.216 and the longest yield plateau is 0.0236 . Material model parameters calculated for these materials are shown in Table 6.5. The damage limit has been calculated based on $\ln \left(\mathrm{A}_{0} / \mathrm{A}_{\mathrm{f}}\right)$ of 0.710 . Eight strap specimens with two net section fracture to gross section yielding strength ratios $\left(A_{n} F_{u} / A_{g} F_{y}\right)$ have been simulated with ABAQUS (Simulia 2009) using the 1/2 model and parameters listed 
in Table 6.5. The input initial yield stress in the simulations of strap specimens with the $\mathrm{A}_{n} \mathrm{~F}_{\mathrm{u}} / \mathrm{A}_{\mathrm{g}} \mathrm{F}_{\mathrm{y}}$ ratio of 1.0 has been increased by $5 \%$ in order to delay the yielding of the gross section before the net section fractures as discussed in Chapter 5. The FEA predictions including the ductility measure $\delta_{\mathrm{L}} / \delta_{\mathrm{y}}$ are listed in Table 6.6 where $\delta_{\mathrm{L}}$ is the predicted maximum deformation and $\delta_{y}$ is defined by Eq. (5.1). $F_{y}$ in Eq. (5.1) is taken as $300 \mathrm{MPa}$ to calculate $\delta_{y}$ in Table 6.6 in order to facilitate the discussions of the effect of yield strength on the predicted specimen ductility in Section 6.4.4.

It can be seen from the simulation results that the yield plateau has a significant effect on the fracture prediction when the $A_{n} F_{u} / A_{g} F_{y}$ ratio is greater than 1.0. On the other hand, yield plateau has almost no effect on the predicted ductility when the $\mathrm{A}_{\mathrm{n}} \mathrm{F}_{\mathrm{u}} / \mathrm{A}_{\mathrm{g}} \mathrm{F}_{\mathrm{y}}$ ratio is 1.0 because the initial yield stress has been increased by $5 \%$. Although the engineering strain at ultimate stress has an effect on the predicted maximum deformation, its effect is small. The predicted ductility is lower with a smaller strain at ultimate stress. Therefore, for the parametric finite element analysis study, only one engineering strain at ultimate stress is used while two yield plateau lengths are considered.

\subsection{FEA Simulations and Results Analyses}

Ninety six tension-only braces with various geometric configurations combined with four different materials have been simulated with ABAQUS (Simulia 2009). Results of the simulations are discussed and analyzed. Based on these results, equations have been developed to predict the ductility of the strap tension-only brace. 


\subsubsection{Materials Used in the Parametric Study}

Four materials listed in Table 6.7 are used in the final parametric study. Based on the material properties listed in Table 5.2, two ultimate to yield strength $\left(F_{u} / F_{3}\right)$ ratios of 1.4 and 1.75 have been considered. All four materials have the same yield strength of $300 \mathrm{MPa}$ and engineering strain at ultimate stress of 0.15 using the lower bound for materials $A$ to $F$ in Table 5.2. Materials designated as $K A$ and $K B$ have a higher $F_{u} / F_{y}$ ratio than that of materials JA and JB. As discussed in Section 6.2.3, materials with two yield plateau lengths, one without a yield plateau and the other with a yield plateau length of 0.02 are considered in the parametric study. Materials JB and KB have a yield plateau length of 0.02 , while there is no yield plateau for materials JA and KA.

Based on these material properties together with the flow stress parameter $n$ of 0.55 , the flow stress versus true plastic strain data can be generated using the method proposed in Section 6.1. The saturated yield stress bound parameter $\eta=0.75$ is used to calculate the saturated yield stress in the determination of the parameters $\left(C_{1}, \gamma_{1}, C_{2}, \gamma_{2}\right)$ for the two kinematic hardening terms. Applying the procedure in Section 5.2.3, the parameters for the damage evolution and damage limit for each material are calculated using the $1 / 8$ model of rectangular specimen with $7.9 \mathrm{~mm}$ thickness and $\ln \left(\mathrm{A}_{v} / \mathrm{A}_{\mathrm{f}}\right)$ of 0.710. Material model parameters of each material for the flow stress, kinematic hardening, damage evolution with $\mathrm{c}$ taken as 1 , and damage limit are listed in Table 6.8.

\subsubsection{List of Braces}

Ninety six strap tension-only braces listed in Tables 6.9 and 6.10 have been simulated in the parametric study. Since steel straps used in the structures as braces are 
usually long, most of the strap braces in the parametric study have a length of $4 \mathrm{~m}$ or $6 \mathrm{~m}$, which are much longer than those tested by Aghdam (2009). The slenderness ratio of the specimen is given by $\mathrm{KL} / \mathrm{r}$ where $\mathrm{K}$ is taken as 0.5 for fixed end and $\mathrm{r}$ is the radius of gyration. The following designation is adopted to describe the brace in the parametric study:

$\alpha \beta \eta \xi \chi \omega \rho$

(1) $\alpha \beta$

The first two letters represent the material. The first letter represents materials with the same $F_{u} / F_{y}$ ratio. The second letter of $A$ denotes no yield plateau and $B$ with a yield plateau.

(2) $\eta \xi$

The third and fourth digits denote the slenderness ratio from 400 to 1600 with 04 , $06,08,16$ representing $400,600,800$ and 1600 respectively.

(3) $\chi \omega$

The fifth and sixth digits identify the net section fracture to the gross section yielding strength ratio $\left(\mathrm{A}_{\mathrm{n}} \mathrm{F}_{\mathrm{u}} / \mathrm{A}_{\mathrm{g}} \mathrm{F}_{\mathrm{y}}\right)$ from 0.90 to 1.20 , with $09,10,11,12$ representing the ratio of $0.90,1.00,1.10$ and 1.20 respectively.

(4) $\rho$

This final digit indicates the brace length with 2 for $2 \mathrm{~m}, 3$ for $2.51 \mathrm{~m}, 4$ for $4 \mathrm{~m}$ and 6 for $6 \mathrm{~m}$.

Dimensions of the braces are not restricted by the minimum or maximum edge distance requirement according to CSA S16-09 (CSA 2009) in order to enable a more thorough parametric study to be conducted. 


\subsubsection{FEA Simulations and Discussions}

As discussed in Section 5.3.2, all the numerical analyses for the strap braces have been carried out with ABAQUS (Simulia 2009) through a user-defined material subroutine with two kinematic hardening terms that incorporated the modified Huang and Mahin fracture criterion based on Eqs. (4.3) and (4.4). For the strap brace of material JB or $\mathrm{KB}$ that has a yield plateau, and when the net section fracture strength is equal to the gross section yielding strength $\left(\mathrm{A}_{n} \mathrm{~F}_{\mathrm{u}} / \mathrm{A}_{\mathrm{g}} \mathrm{F}_{\mathrm{y}}=1.0\right)$, the initial yielding stress has been increased by $5 \%$ in the input data for the numerical simulation to account for the possible reduction in the $A_{n} F_{u} / A_{g} F_{y}$ ratio with the loading rate, which may induce fracture at the net section before yielding of the gross section, as in specimens of material $\mathrm{C}$ by Aghdam (2009). The $5 \%$ reduction is adopted based on the reduction of close to $5 \%$ in the $\mathrm{A}_{\mathrm{n}} \mathrm{F}_{\mathrm{u}} / \mathrm{A}_{\mathrm{g}} \mathrm{F}_{\mathrm{y}}$ ratio for materials $\mathrm{C}$ to $\mathrm{F}$ going from a strain rate of $0.0001 \mathrm{~s}^{-1}$ to $0.01 \mathrm{~s}^{-1}$.

Since some strap braces in the parametric study are thicker than those tested by Aghdam (2009), an additional mesh study has been carried out to investigate the influence of mesh density of the solid element in the thickness direction of the strap on the predicted ductility. The number of layer of solid element for scheme-1 shown in Fig. 5.21 is double of that for scheme-3. Numerical simulations are carried out for braces JA04124 and JB04124 for this study. The FEA predictions using two mesh schemes are listed in Table 6.11. As can be seen in the comparisons, there is only a small difference in the predicted ductility using the two mesh schemes for the close to the thickest brace in the parametric study. For this reason, the same number of 5 layers of solid element has been used in the numerical simulations of all the strap braces. All the FEA predictions 
are listed in Tables 6.12 to 6.15 . Analyses of the results are discussed in the following sections.

\subsubsection{Effect of Yield Plateau}

Comparing results in Table 6.12 with 6.13 and Table 6.14 with 6.15 , the ductility of brace can be seen to increase significantly for materials with a yield plateau when the net section fracture strength is greater than the gross section yielding strength $\left(A_{n} F_{u} / A_{u} F_{y}\right.$ $>1.0$ ). This big difference is due to the contribution from the plastic deformation attributed to the yield plateau as the fracture at the net section is preceded by the yielding of the gross section. This difference depends greatly on the length of the yield plateau. While in the case of $A_{n} F_{u} / A_{\mathrm{g}} F_{y}$ ratio no greater than 1.0 , the braces of both materials with and without a yield plateau are predicted to have about the same ductility.

\subsubsection{Effect of $A_{n} F_{u} / A_{g} F_{y}$}

The predicted ductility $\left(\delta_{\mathrm{L}} / \delta_{\mathrm{y}}\right)$ versus the net section fracture to gross section yielding strength ratio $\left(\mathrm{A}_{\mathrm{n}} \mathrm{F}_{\mathrm{u}} / \mathrm{A}_{\mathrm{g}} \mathrm{F}_{\mathrm{y}}\right)$ for $4 \mathrm{~m}$ and $6 \mathrm{~m}$ braces are shown in Figs. 6.3 to 6.10 . A higher $A_{n} F_{u} / A_{y} F_{y}$ ratio allows for a greater overall yielding of the brace. It can be seen that the ductility increases with the $A_{n} F_{u} / A_{g} F_{y}$ ratio for all four materials with and without a yield plateau. For materials JA and KA without a yield plateau, the rate of increase increases with the $A_{n} F_{u} / A_{g} F_{y}$ ratio. However, for materials $J B$ and $K B$ with a yield plateau, there is a big increase in the ductility from the $A_{n 1} F_{u} / A_{g} F_{y}$ ratio of 1.0 to 1.1 due to the contribution of the yield plateau. With the $A_{n} F_{u} / A_{g} F_{y}$ ratio greater than 1.1 , the rate of increase appears to decrease with the $A_{n} F_{u} / A_{g} F_{y}$ ratio. Additional results at an $A_{n} F_{u} / A_{y} F_{y}$ 
ratio greater than 1.2 are needed to properly determine the trend of the rate of increase in the ductility beyond that point for materials JB and KB.

\subsubsection{Effect of Slenderness Ratio}

The slenderness ratio $\mathrm{KL} / \mathrm{r}$ can be varied by either changing the length or the thickness of the brace. Effects on the predicted ductility by changing the slenderness ratio through both approaches are discussed below. Increasing the slenderness ratio reduces the bending stress (strain) induced during buckling in compression. This increases the ductility of the brace. However, other factors may counter the beneficial effect of increasing the slenderness ratio depending on how the slenderness ratio is changed.

Braces of $16.5 \mathrm{~mm}$ thick and $4 \mathrm{~m}$ long $(\mathrm{xx} 04 \times \mathrm{x} 4)$ and $16.7 \mathrm{~mm}$ thick and $6 \mathrm{~m}$ long $(x \times 06 \times x 6)$ have almost the same thickness but a slenderness ratio of 400 and 600 respectively. Comparing results of the simulations for these braces in Tables 6.12 to 6.15 , there does not appear to be any appreciable difference in the predicted ductility by increasing the slenderness ratio from 400 to 600 through changing the length, except for braces of materials with a yield plateau at the $A_{n} F_{u} / A_{g} F_{y}$ ratio of 1.1 (JB04114, JB06116, $\mathrm{KB} 04114$ and $\mathrm{KB} 06116)$. In general, there is a slight drop in the ductility by increasing the length from $4 \mathrm{~m}$ to $6 \mathrm{~m}$ when the $A_{n} F_{u} / A_{g} F_{y}$ ratio is not greater than 1.0. The beneficial effect of having a higher slenderness ratio with a longer length cannot compensate for the reduction in the average deformation because the localized deformation at the bolt hole has to be averaged over a longer length. However, when the $\mathrm{A}_{\mathrm{n}} \mathrm{F}_{\mathrm{u}} / \mathrm{A}_{\mathrm{g}} \mathrm{F}_{\mathrm{y}}$ ratio is greater than 1.0 , the proportion of the deformation at the bolt hole over 
the total deformation is smaller because there is more deformation contribution from the region outside the bolt hole as gross section yielding has occurred before the net section fractures. The increase in slenderness ratio allows for a larger deformation in the gross section of the brace that has the $A_{n} F_{u} / A_{g} F_{y}$ ratio of 1.1 and larger. However, the deformation in the gross section is small when the net section fractures before yielding of the gross section. For this reason, the predicted ductility shown in Tables 6.12 to 6.15 increases slightly when the length increases from $4 \mathrm{~m}$ to $6 \mathrm{~m}$ for the braces with the $A_{n} F_{u} / A_{y} F_{y}$ ratio greater than 1.0 .

The relationship between the predicted ductility and slenderness ratio of $4 \mathrm{~m}$ and $6 \mathrm{~m}$ long braces by changing the thickness are shown in Figs. 6.11 to 6.18 . It can be seen that the ductility increases with the slenderness ratio when the $A_{n 1} F_{u} / A_{g} F_{y}$ ratio is greater than 1.0 for braces of materials with a yield plateau (JB and $\mathrm{KB}$ ), but the trend is not clear for braces of materials without a yield plateau (JA and KA). However, when the $A_{n} F_{u^{\prime}} A_{g} F_{y}$ ratio is not greater than 1.0 , the predicted ductility appears to decrease slightly with a larger slenderness ratio. The effect is similar for $4 \mathrm{~m}$ and $6 \mathrm{~m}$ braces. It appears that increasing the slenderness ratio by reducing the thickness may not always improve the ductility. Increasing the thickness of a brace provides a better bridging to transfer the force across the bolt hole when necking starts. This increases the deformation at fracture compared to a thinner brace under monotonic tension. However, increasing the thickness also increases the range of bending strain due to buckling under cyclic loading and thus reduces the fatigue life of a brace. Depending on the relative impact of these two opposing effects for different configurations and materials, increasing the slenderness ratio by reducing the thickness may or may not improve the ductility. When the 
$A_{n} F_{u} / A_{g} F_{3}$ ratio is not greater than 1.0 , there is a slight reduction in the ductility if the slenderness ratio is increased by reducing the thickness. At the $A_{n} F_{u} / A_{g} F_{y}$ ratio of 1.1 and to a lesser extent at 1.2 , braces of materials with a yield plateau shows a significant improvement in the ductility as the slenderness ratio increases. When the overall deformation is high, the deformation is spread more evenly over the full brace. This may help reduce the localization of the bending at the bolt hole, and thus yields the sharp ductility improvement with the slenderness ratio at the $A_{n} F_{u} / A_{g} F_{y}$ ratio of 1.1 and a slenderness ratio of less than 800, as shown in Figs. 6.13, 6.14, 6.17 and 6.18. Overall, there is only a small improvement in the ductility once the slenderness ratio is larger than 800.

Figures 6.19 to 6.20 show the effect on the ductility by changing the length and thickness while maintaining the same slenderness ratio. Again, due to the various opposing effects of increasing both the length and thickness, there is a slight increase in the ductility with length when the $A_{n} F_{u} / A_{g} F_{y}$ ratio is at least 1.1 , but there is a slight decrease when the $A_{n} F_{u} / A_{g} F_{y}$ ratio is at 1.0.

\subsubsection{Effect of $F_{u} / F_{y}$}

The ultimate to yield strength ratio $\left(F_{w} / F_{y}\right)$ affects the brace in terms of the net to gross section area ratio $\left(A_{n} / A_{g}\right)$ required to achieve the same $A_{n} F_{u} / A_{g} F_{y}$ ratio. As the $A_{n} F_{u} / A_{g} F_{y}$ ratio increases, the $A_{n} / A_{g}$ ratio increases with the reduction in $F_{u} / F_{y}$ ratio. Table 6.16 and Fig. 6.21 show comparisons of the $A_{n} / A_{g}$ ratio at different $A_{n} F_{u} / A_{g} F_{y}$ ratios for various $F_{u} / F_{y}$. In Fig. 6.21, the lines appear to be close to parallel. Therefore, the relative difference of $A_{n} / A_{g}$ with $F_{u} / F_{y}$ does not appear to vary too much at different 
$A_{n} F_{u} / A_{g} F_{z}$ ratios. However, $A_{n} / A_{g}$ gets closer to 1.0 for a smaller $F_{u} / F_{y}$ as $A_{n} F_{u} / A_{g} F_{y}$ increases. As $A_{n} / A_{\underline{g}}$ approaches 1.0 , the strain of the gross section of the brace is closer to the ultimate stress and the flatter region of the engineering stress-strain curve under monotonic tension. The same change in $A_{n} / A_{g}$ (average gross section stress/net section stress) will result in a larger change in the strain of the gross section as $A_{n} / A_{y}$ gets closer to 1.0 . Thus, this yields the difference in the predicted ductility between materials $\mathrm{J}$ and $\mathrm{K}$ as $\mathrm{A}_{\mathrm{n}} \mathrm{F}_{\mathrm{u}} / \mathrm{A}_{\mathrm{g}} \mathrm{F}_{\mathrm{y}}$ increases.

The typical predicted ductility $\delta_{\mathrm{u}} / \delta_{\mathrm{y}}$ versus $\mathrm{F}_{\mathrm{u}} / \mathrm{F}_{\mathrm{y}}$ are shown for some of the braces in Figs. 6.22 to 6.27 . It can be seen that the difference in the predicted ductility between materials $J$ and $K$ increases as $A_{n} F_{u} / A_{g} F_{y}$ increases. All materials of $J$ and $K$ are assumed to have the same engineering strain at ultimate stress. As a result, the material without a yield plateau has a flatter engineering stress versus engineering strain curve (shallower slope) than the one with a yield plateau, as can be seen in Fig. 6.2. Thus, the difference in the predicted ductility for different $F_{u} / F_{y}$ as $A_{n} F_{u} / A_{g} F_{y}$ increases is larger for materials with a yield plateau.

\subsection{Generation of Ductility Prediction Equations}

Using results of the parametric study, the equations for predicting the ductility limit have been generated through two multi-variable nonlinear data regressions. Braces at the $A_{n} F_{u} / A_{g} F_{y}$ ratio of 0.9 for material $J B$ are not included in the regression calculation since the ductility of these braces are very close to those at the $A_{n} F_{u} / A_{g} F_{y}$ ratio of 1.0 due to the $5 \%$ increase in the yield strength used in the simulations for materials with a yield plateau. The first regression $(\mathrm{RI})$ has been conducted for each of the four materials with 
respect to the slenderness ratio $(\mathrm{KL} / \mathrm{r})$, specimen length, net section fracture to gross section yielding strength ratio $\left(A_{n} F_{u} / A_{g} F_{y}\right)$,

$$
\left(\frac{\delta_{\mathrm{L}}}{\delta_{y}}\right)=\mathrm{f}\left(\frac{\mathrm{KL}}{\mathrm{r}}, \mathrm{L}, \frac{\mathrm{A}_{\mathrm{n}} \mathrm{F}_{\mathrm{u}}}{\mathrm{A}_{\mathrm{g}} \mathrm{F}_{\mathrm{y}}}\right)
$$

The second regression (RII) has also been conducted to include the effect of material properties by combining results of materials without a yield plateau (JA and KA) or with a yield plateau (JB and $\mathrm{KB})$,

$$
\left(\frac{\delta_{L}}{\delta_{y}}\right)=f\left(\frac{K L}{r}, L, \frac{A_{n} F_{u}}{A_{g} F_{y}}, \frac{F_{u}}{F_{y}}\right) .
$$

\subsubsection{First Regression (RI) for Ductility Prediction Equations}

Using the trend of the ductility versus various parameters observed in the figures presented in the previous sections, the following equation has been proposed for predicting the ductility of the brace for materials without a yield plateau, JA and KA, as

$$
\frac{\delta_{L}}{\delta_{y}}=P_{1}\left(10+P_{2} \frac{K L}{r}\right)\left(1+P_{3} L\right)\left(\frac{A_{n} F_{u}}{A_{g} F_{y}}\right)^{P_{1}},
$$

where $P_{1}$ to $P_{4}$ are constants to be determined. For materials with a yield plateau, JB and $\mathrm{KB}$, the proposed equation is slightly more complicated in order to consider the effect of the slenderness ratio on the shape of the ductility versus $A_{n} F_{u} / A_{g} F_{y}$ curve as observed in Figs. 6.5, 6.6, 6.9 and 6.10. Thus for materials $\mathrm{JB}$ and $\mathrm{KB}$, the equation is taken as,

$$
\frac{\delta_{L}}{\delta_{y}}=P_{1}\left(\frac{K L}{r}\right)^{P_{2}}\left(1+P_{3} L\right)\left[P_{4}+\left(\frac{K L}{r}\right)^{P_{5}} \ln \left(P_{6}+\frac{A_{n} F_{u}}{A_{g} F_{y}}\right)\right] \text {, }
$$

where $P_{1}$ to $P_{6}$ are constants to be determined. For each of the equations, two sets of constants corresponding to two materials have been determined through multi-variable 
regression and are listed in Tables 6.17 and 6.18. Comparisons between the predicted ductility from FEA simulations, and Eqs. (6.16) and (6.17) are shown in Figs. 6.28 to 6.31. Statistics of the comparisons of the predicted ductility from FEA simulations, and Eqs. (6.16) and (6.17) in terms of the ratio and difference are listed in Tables 6.19 and 6.20. The standard deviation on the ratio is within 0.2 , and less than $0.4 \delta_{\mathrm{l}} / \delta_{\mathrm{y}}$ on the difference for materials $\mathrm{JA}$ and $\mathrm{KA}$, and around $1.0 \delta_{\mathrm{L}} / \delta_{\mathrm{y}}$ for materials JB and KB. There is a lager standard deviation on the difference in the predicted ductility for materials JB and $\mathrm{KB}$ as the predicted ductility for braces of materials JB and $\mathrm{KB}$ is about double that for materials JA and $K A$ when $A_{n} F_{w} / A_{g} F_{y} \geq 1.1$.

The comparisons show that there is a good agreement between the ductility from FEA simulations, and Eqs. (6.16) and (6.17). For materials without a yield plateau, JA and KA, the maximum difference of the predicted ductility $\delta_{1} / \delta_{y}$ between FEA simulations and the equations is within $1.0 \delta_{y}$ (Fig. 6.30), while for materials with a yield plateau, JB and KB, the difference is within $1.5 \delta_{y}$ (Fig. 6.31) except for two data points at $\delta_{\mathrm{L}} / \delta_{\mathrm{y}}$ around 9 . These two points correspond to the predicted ductility for the braces at $\mathrm{KL} / \mathrm{r}=400$, which is most likely less than the slenderness ratio of a strap tension-only brace used in a structure. The ratio of the ductility from FEA simulations to that by the equations is within $12 \%$ except when the $\delta_{\mathrm{L}} / \delta_{\mathrm{y}}$ is less than 4.0 for materials JA and KA (Fig. 6.28), and less than 10.0 for materials JB and KB (Fig. 6.29). Nevertheless, the ratio is generally within $35 \%$ for materials $\mathrm{JA}$ and $\mathrm{KA}$ when $\delta_{\mathrm{L}} / \delta_{\mathrm{y}}$ is less than 4.0 , and within $30 \%$ for materials $\mathrm{JB}$ and $\mathrm{KB}$ when $\delta_{\mathrm{L}} / \delta_{y}$ is less than 10.0 . However, the difference in the predicted ductility between FEA simulations and the equations is small. 


\subsubsection{Second Regression (RII) for Ductility Prediction Equations}

The second regression (RII) has also been conducted for materials with and without a yield plateau to include the effect of ultimate to yield strength ratio $\left(F_{u} / F_{y}\right)$ on the ductility. For materials without a yield plateau, combining JA and KA, Eq. (6.16) is modified to

$$
\frac{\delta_{L}}{\delta_{y}}=P_{1}\left(10+P_{2} \frac{K L}{r}\right)\left(1+P_{3} L\right)\left(\frac{A_{n} F_{u}}{A_{g} F_{y}}\right)^{P_{+}}\left(1-P_{5} \frac{F_{u}}{F_{y}}\right)
$$

where $P_{1}$ to $P_{5}$ are constants to be determined. For materials with a yield plateau, combining JB and $\mathrm{KB}$, Eq. (6.17) is modified to

$$
\frac{\delta_{\mathrm{L}}}{\delta_{y}}=\mathrm{P}_{1}\left(\frac{\mathrm{KL}}{\mathrm{r}}\right)^{\mathrm{P}_{2}}\left(1+\mathrm{P}_{3} \mathrm{~L}\right)\left[\mathrm{P}_{4}+\left(\frac{\mathrm{KL}}{\mathrm{r}}\right)^{\mathrm{P}_{5}} \ln \left(\mathrm{P}_{6}+\frac{\mathrm{A}_{\mathrm{n}} \mathrm{F}_{\mathrm{u}}}{\mathrm{A}_{\mathrm{g}} \mathrm{F}_{\mathrm{y}}}\right)\right]\left(1-\mathrm{P}_{7} \frac{\mathrm{F}_{\mathrm{u}}}{\mathrm{F}_{\mathrm{y}}}\right),
$$

where $P_{1}$ to $P_{7}$ are constants to be determined. The constants determined through data regressions for each of the equations are shown in Tables 6.21 and 6.22. Comparisons between predicted ductility from FEA simulations, and Eqs. (6.19) and (6.20) are shown in Figs. 6.30 and 6.31. Statistics of the comparisons of the predicted ductility from FEA simulations, and Eqs. (6.18) and (6.19) in terms of the ratio and difference are listed in Tables 6.23 and 6.24 .

Since the second regression is essentially a linear interpolation of $F_{u} / F_{y}$, comparisons of the predicted ductility from the FEA simulations and the second regression equations are very similar to that for the first regression equations. Conclusions drawn on the comparisons with the first regression equations are equally applicable here. 


\subsubsection{Comparisons of Test and Predicted Ductility}

Comparisons of test and predicted ductility are carried out with the second regression equations. Results for a different yield plateau length are either interpolated or extrapolated from these equations. Due to the slippage observed in the test, the maximum measured deformation is subtracted by $6 \mathrm{~mm}$ for specimens of materials $\mathrm{D}$ to $\mathrm{F}$ in the comparisons. In numerical simulations of braces for materials with a yield plateau, the initial yield strength has been increased by $5 \%$ when $A_{n} F_{u} / A_{g} F_{y}=1.0$. To account for the possible strain rate effect on the $A_{n} F_{u} / A_{g} F_{y}$ ratio, it is proposed that when the static $\mathrm{A}_{\mathrm{n}} \mathrm{F}_{\mathrm{u}} / \mathrm{A}_{\mathrm{g}} \mathrm{F}_{\mathrm{y}}$ ratio is greater than 0.9 but less than 1.05 , the predicted ductility is interpolated or extrapolated with $A_{n} F_{w} / A_{g} F_{y}=1.0$. Alternatively, the ductility is predicted using Eq. (6.18) for materials without a yield plateau. This applies to specimens of material C. In the comparisons, $\delta_{\mathrm{y}}$ is calculated with $\mathrm{F}_{\mathrm{y}}=300 \mathrm{MPa}$.

Comparisons of test and predicted ductility are listed in Tables 6.25 and 6.26 where the exact $\mathrm{KL} / \mathrm{r}$ value is used instead of the nominal value given by Aghdam (2009) in Table 5.9. Shown in Table 6.25, the predicted ductility through interpolating and extrapolating from Eqs. (6.18) and (6.19) is less than that from the test. The predicted to test ductility ratio varies from 0.30 to 0.79 . For specimens of materials $D$ to $F$, the ratio varies from 0.39 to 0.60 at $\mathrm{KL} / \mathrm{r}=202$ and 0.60 to 0.79 at $\mathrm{KL} / \mathrm{r}=406$. As expected, the prediction is better at $\mathrm{KL} / \mathrm{r}=406$ since no data at $\mathrm{KL} / \mathrm{r}$ less than 400 has been used in the generation of the equations. Although conservative, the predicted maximum deformation (ductility) is not overly conservative for $\mathrm{KL} / \mathrm{r}$ at 400 compared to 200. As shown in Table 6.26, the predicted ductility for specimens of material $\mathrm{C}$ through interpolating and extrapolating from Eqs. (6.18) and (6.19) is more conservative compared to that from Eq. 
(6.18) directly. The prediction is still conservative when using Eq. (6.18) directly to estimate the ductility for specimens of material $C$, although the predicted to test ductility ratio is close to 1.0. A better prediction is achieved for higher $\mathrm{KL} / \mathrm{r}$ ratios of 624 and 834 than 419 .

\subsubsection{Effect of Yield Strength}

The parametric study has been carried out for a yield strength of $300 \mathrm{MPa}$. In order to investigate the applicability of the established ductility prediction equation for other yield strengths, comparisons are made between results of FEA simulations for materials $\mathrm{J}(\mathrm{A} / \mathrm{B})$ and $\mathrm{M}(\mathrm{A} / \mathrm{B})$. Material properties of $\mathrm{MA}$ and $\mathrm{MB}$ are listed in Table 6.4. The only difference between materials $\mathrm{J}(\mathrm{A} / \mathrm{B})$ and $\mathrm{M}(\mathrm{A} / \mathrm{B})$ is the former two have a yield strength of $300 \mathrm{MPa}$ and $350 \mathrm{MPa}$ for the latter two. Comparisons of the predicted ductility listed in Table 6.27 for the simulations using the same loading protocol $\left(\delta_{y}\right.$ for the loading calculated with $F_{y}=300 \mathrm{MPa}$ ) show that the predicted ductility (maximum deformation) is not significantly affected by the yield strength difference of $50 \mathrm{MPa}$. The maximum difference is less than the larger of $4 \%$ of the predicted $\delta_{1} / \delta_{y}$ or 0.3 in the predicted $\delta_{\mathrm{l}} / \delta_{y}$, with the predicted ductility being lower at the yield strength of $300 \mathrm{MPa}$. Based on the conservative prediction of the ductility (maximum deformation) in Tables 6.25 and 6.26 , and the small difference in the predicted ductility at the yield strength of $300 \mathrm{MPa}$ and $350 \mathrm{MPa}$, Eqs. (6.16) to (6.19) can be extended to predict the ductility of the strap tension-only brace of a material with the yield strength higher than $350 \mathrm{MPa}$ and down to $250 \mathrm{MPa}$. 


\subsubsection{Limitations of Ductility Prediction Equations}

The parametric study has been carried out for a certain set of conditions. Therefore, there are some limitations to the applicability of the equations developed for predicting the ductility.

Equations (6.16) to (6.19) should only be used to predict the ductility for braces with the $\mathrm{KL} / \mathrm{r}$ no less than 400 since no data points of $\mathrm{KL} / \mathrm{r}$ lower than 400 have been used in developing the equations. The prediction for $\mathrm{KL} / \mathrm{r}$ lower than 400 may be overly conservative based on the comparisons to the tests by Aghdam (2009). The numerical simulations have been carried out for M20 bolts. Although the effect of the bolt size (hole) on the ductility has not been quantified, it is not expected to be significant if a slightly larger M20 bolt is used. However, caution should be exercised when using Eqs. (6.16) to (6.19) with bolts that are significantly larger than M20. These equations also may not (not likely) be applicable when there is more than one bolt per row. The ductility for the configuration with two or more bolts per row should be investigated to verify the applicability of Eqs. (6.16) to (6.19). In developing the equations, the length of the brace is taken as the distance between the middle bolt hole of a single line three-bolt connection similar to that tested by Aghdam (2009). When the connection has more than three rows of bolts, the length of the brace may be taken as the distance between the bolt next to the innermost bolt hole to determine the slenderness ratio and the maximum deformation based on the predicted $\delta_{\mathrm{L}} / \delta_{y}$ ratio. For the connection with two rows of bolts, the length may be taken as the distance between the mid-points of the bolts. 


\subsection{Recommendations for Designing Strap Tension-Only Brace}

A member in the SFRS designed to dissipate energy is required to have the ductility to accommodate the deformation without losing significant strength. In the tests by Aghdam (2009), there is no reduction in the maximum tension strength of the specimen under an increasing deformation range cyclic loading until fracture occurred. Thus, the ductility not the strength degradation becomes an important consideration for the strap tension-only brace used. In NBCC 2010 (NRCC 2010), the maximum storey drift limit is $2.5 \%$ for a normal building. At the same storey drift, the maximum ductility demand occurs for a brace at $45^{\circ}$. This translates to a ductility of $8.3 \delta_{\mathrm{L}} / \delta_{\mathrm{y}}$ for a storey drift limit of $2.5 \%$ with $\delta_{y}$ calculated based on a yield strength of $300 \mathrm{MPa}$ and the elastic modulus of $200000 \mathrm{MPa}$. At a different angle, the maximum ductility demand for the brace is less than $8.3 \delta_{\mathrm{L}} / \delta_{\mathrm{y}}$.

The parametric study on the ductility of a single diagonal strap tension-only brace has considered influences of the yield plateau and ultimate to yield strength ratio $\left(F_{u} / F_{y}\right)$ of the material, net section fracture to gross section yielding strength ratio $\left(A_{n} F_{u} / A_{g} F_{y}\right)$, slenderness ratio and brace length. Equations have been developed to help predict the ductility of the brace for different material properties and geometric configurations. These equations can be used to help design the brace for the actual ductility requirement. The $A_{n} F_{v} / A_{g} F_{y}$ ratio and the yield plateau length have been found to be the important factors that affect the ductility of the brace since the slenderness ratio is expected to be very high. Based on the results of the parametric study, the following recommendations have been put forward to assist in designing the strap tension-only brace. 
(1) It is useful to know the material properties of the steel strap such as the ultimate and yield strengths, or better to have the engineering stress versus engineering strain curve of the material and the yield plateau length. The information will be helpful in providing a better prediction of the ductility of the brace.

(2) The brace has very little ductility when the net section fracture to gross section yielding strength ratio $\left(A_{n} F_{u} / A_{g} F_{y}\right)$ is less than 1.0. However, the static $A_{n} F_{u} / A_{g} F_{y}$ ratio of the connection should be at least 1.1 in order to partially account for the possible reduction in the effective $A_{n} F_{u} / A_{g} F_{y}$ ratio due to the effect of loading rate on the dynamic $F_{u} / F_{y}$ ratio in order to ensure that gross section yielding precedes fracture of the net section. For the strap specimens tested by Aghdam (2009), the $A_{n} F_{u} / A_{g} F_{y}$ ratio based on the dynamic $\mathrm{F}_{\mathrm{u}} / \mathrm{F}_{\mathrm{y}}$ at strain rates of $0.01 \mathrm{~s}^{-1}$ and $0.5 \mathrm{~s}^{-1}$ are about $5 \%$ and $10 \%$ lower than the ratio based on the static $F_{u} / F_{y}$.

(3) Using a material with a yield plateau improves the ductility. A significant improvement in the ductility of the brace can only be achieved when facture across the net section is preceded by gross section yielding for a material with a significant yield plateau length or conservatively using static $\mathrm{A}_{n} \mathrm{~F}_{\mathrm{u}} / \mathrm{A}_{\mathrm{g}} \mathrm{F}_{\mathrm{y}}$ no less than 1.2 in order to have at least close to half the ductility demand for a $2.5 \%$ storey drift limit even if there is no yield plateau.

(4) Generally, there is little improvement in the ductility beyond the slenderness ratio of 800 . However, the slenderness ratio should be kept greater than 400 
in order to benefit from the increase in ductility with the slenderness ratio for the material with a yield plateau.

(5) If the yield plateau length of the material is not known, the ductility should be conservatively predicted using Eqs. (6.16) and (6.18) for materials without a yield plateau.

(6) Using a material with a lower $F_{u} / F_{y}$ improves the brace ductility. However, this may not be an economical option since a lower $F_{u} / F_{y}$ will require a larger $A_{n} / A_{y}$ ratio or cross-sectional area for the same strap thickness in order to maintain the same $A_{n} F_{u} / A_{g} F_{y}$ ratio.

\subsection{Summary}

A procedure to generate the material input data for the specific material properties characterized by yield plateau length, ultimate to yield strength ratio, and strain at ultimate stress has been developed in this chapter. Four materials were considered in the parametric finite element analysis study, which consist of the combination of the ultimate to yield strength ratio of 1.4 and 1.75 , and a yield plateau length of 0.02 and no yield plateau. A total of ninety six strap tension-only braces similar to the Type A strap specimen tested by Aghdam (2009) with the combination of these four materials and various lengths, slenderness ratios, $A_{n} F_{u} / A_{y} F_{y}$ ratios have been designed and simulated.

Numerical simulations have been carried out with ABAQUS (Simulia 2009) through a user-defined material subroutine with two kinematic hardening terms that incorporated the modified Huang and Mahin fracture criterion based on Eqs. (4.3) and 
(4.4). Effects of yield plateau, $A_{n} F_{u} / A_{g} F_{z}$ ratio, slenderness ratio as well as $F_{u} / F_{z}$ ratio on the ductility of brace have been analyzed based on the simulation results.

Two levels of multi-variable nonlinear data regressions have been conducted using results of the parametric study to predict the ductility of the brace with a slenderness ratio no less than 400 . The first regression (RI) developed the ductility prediction equation for each of the four materials with respect to the slenderness ratio, specimen length, and $A_{n} F_{u} / A_{9} F_{y}$ ratio. The second regression (RII) combined the effect of $F_{u} / F_{y}$ ratio into the ductility prediction equation for each of the materials with and without a yield plateau. Comparisons of the results have shown that there is a good agreement between the ductility from FEA simulations and ductility prediction equations. These equations were further applied to predict the ductility of Type A strap specimens by Aghdam (2009). The prediction was found to be conservative and provide a better prediction at a slenderness ratio no less than 400. A few recommendations for designing steel strap tension-only braces in a Seismic Force Resisting System have also been provided. 
Table 6.1 The cross-sectional area change $\ln \left(\mathrm{A}_{0} / \mathrm{A}\right)$ at fracture for different materials

\begin{tabular}{|c|c|c|c|}
\hline Material & Thickness & Test $\ln \left(\mathrm{A}_{0} / \mathrm{A}_{\mathrm{f}}\right)$ & Translated $\ln \left(\mathrm{A}_{0} / \mathrm{A}_{\mathrm{f}}\right)$ for $\mathrm{t}=7.9 \mathrm{~mm}$ \\
\hline $\mathrm{A}$ & Round $\phi 25.4 \mathrm{~mm}$ & 0.986 & 0.909 \\
\hline $\mathrm{B}$ & Round $\phi 25.4 \mathrm{~mm}$ & 0.857 & 0.808 \\
\hline $\mathrm{C}$ & $4.8 \mathrm{~mm}$ & 0.867 & 0.957 \\
\hline $\mathrm{D}$ & $7.9 \mathrm{~mm}$ & 0.791 & 0.791 \\
\hline $\mathrm{E}$ & $7.9 \mathrm{~mm}$ & 0.870 & 0.870 \\
\hline $\mathrm{F}$ & $7.9 \mathrm{~mm}$ & 0.896 & 0.896 \\
\hline $\mathrm{G}$ & $7.9 \mathrm{~mm}$ & 0.792 & 0.792 \\
\hline \multirow{4}{*}{-} & \multicolumn{2}{|c|}{ Minimum } & 0.791 \\
\cline { 2 - 4 } & \multicolumn{2}{|c|}{ Mean } & 0.860 \\
\cline { 2 - 4 } & \multicolumn{2}{|c|}{ Standard deviation } & 0.065 \\
\cline { 2 - 4 } & \multicolumn{2}{|c|}{ Coefficient of variation } & $7.5 \%$ \\
\cline { 2 - 4 } & At 90\% probability of exceedance & 0.777 \\
\cline { 2 - 4 } & At 99\% probability of exceedance & 0.710 \\
\hline
\end{tabular}

Table 6.2 Flow stress parameter $\mathrm{n}$ for materials $\mathrm{A}$ to $\mathrm{F}$

\begin{tabular}{|c|c|}
\hline Material & Flow stress parameter $\mathrm{n}$ \\
\hline $\mathrm{A}$ & 0.48 \\
\hline $\mathrm{B}$ & 0.52 \\
\hline $\mathrm{C}$ & 0.64 \\
\hline $\mathrm{D}$ & 0.57 \\
\hline $\mathrm{E}$ & 0.52 \\
\hline $\mathrm{F}$ & 0.57 \\
\hline Mean & 0.55 \\
\hline
\end{tabular}

Table 6.3 FEA predictions for strap specimens A2128 and A4128 with different values of flow stress parameter $n(\eta=0.75)$

\begin{tabular}{|c|c|c|c|c|c|c|}
\hline \multirow{2}{*}{ Specimen } & \multicolumn{2}{|c|}{$\mathrm{n}=0.57$} & \multicolumn{2}{c|}{$\mathrm{n}=0.75$} & \multicolumn{2}{c|}{ Comparison } \\
\cline { 2 - 7 } & $\begin{array}{c}\text { Half cycle } \\
\text { no. at } \\
\text { fracture (A) }\end{array}$ & $\begin{array}{c}\text { Max. } \\
\text { deformation } \\
(\mathrm{mm})(\mathrm{B})\end{array}$ & $\begin{array}{c}\text { Half cycle } \\
\text { no. at } \\
\text { fracture (C) }\end{array}$ & $\begin{array}{c}\text { Max. } \\
\text { deformation } \\
(\mathrm{mm})(\mathrm{D})\end{array}$ & $(\mathrm{A}) /(\mathrm{C})$ & $(\mathrm{B}) /(\mathrm{D})$ \\
\hline $\mathrm{A} 2128$ & 103 & 24.81 & 105 & 26.12 & 0.98 & 0.95 \\
\hline $\mathrm{A} 4128$ & 91 & 41.60 & 97 & 47.17 & 0.94 & 0.88 \\
\hline
\end{tabular}


Table 6.4 Material properties to study effect of yield plateau length and engineering strain at ultimate stress

\begin{tabular}{|c|c|c|c|c|c|c|}
\hline Material & $\begin{array}{c}\text { Elastic modulus } \\
(\mathrm{MPa})\end{array}$ & $\begin{array}{c}\text { Yield plateau } \\
\varepsilon_{\mathrm{ep}}\end{array}$ & $\begin{array}{c}\text { Yield strength } \\
\mathrm{F}_{\mathrm{y}}(\mathrm{MPa})\end{array}$ & $\begin{array}{c}\text { Engineering strain at } \\
\text { ultimate stress, } \varepsilon_{\mathrm{eu}}\end{array}$ & $\begin{array}{c}\text { Ultimate strength } \\
\mathrm{F}_{\mathrm{u}}(\mathrm{MPa})\end{array}$ & $\mathrm{F}_{\mathrm{u}} / \mathrm{F}_{\mathrm{y}}$ \\
\hline $\mathrm{MA}$ & 200000 & 0.00 & 350 & 0.15 & 490 & 1.40 \\
\hline $\mathrm{MB}$ & 200000 & 0.02 & 350 & 0.15 & 490 & 1.40 \\
\hline $\mathrm{NA}$ & 200000 & 0.00 & 350 & 0.22 & 490 & 1.40 \\
\hline $\mathrm{NB}$ & 200000 & 0.02 & 350 & 0.22 & 490 & 1.40 \\
\hline
\end{tabular}

Table 6.5 Material model parameters for materials MA, MB, NA and NB

\begin{tabular}{|c|c|c|c|c|c|c|c|c|c|c|c|c|}
\hline \multirow{2}{*}{ Material } & \multicolumn{4}{|c|}{ Flow stress parameters } & \multicolumn{3}{c|}{ Kinematic hardening parameters } & \multicolumn{2}{c}{$\begin{array}{c}\text { Damage evolution } \\
\text { parameters }\end{array}$} & $\begin{array}{c}\text { Damage } \\
\text { limit }\end{array}$ \\
\cline { 2 - 15 } & $\sigma_{0}^{\mathrm{y}}(\mathrm{MPa})$ & $\varepsilon_{0}^{\mathrm{p}}$ & $\sigma_{x}^{y}(\mathrm{MPa})$ & $\mathrm{b}$ & $\mathrm{n}$ & $\mathrm{C}_{1}(\mathrm{MPa})$ & $\gamma_{1}$ & $\mathrm{C}_{2}(\mathrm{MPa})$ & $\gamma_{2}$ & $\mathrm{~m}$ & $\mathrm{k}$ & $\mathrm{D}_{\mathrm{c}}$ \\
\hline $\mathrm{MA}$ & 350.6 & 0.00 & 393.7 & 2.32 & 0.55 & 2022 & 8.41 & 165 & 1.22 & 2.36 & 1.68 & 1.44 \\
\hline $\mathrm{MB}$ & 357.6 & 0.02 & 327.4 & 3.22 & 0.55 & 2247 & 9.82 & 162 & 1.83 & 2.40 & 1.59 & 1.46 \\
\hline $\mathrm{NA}$ & 350.6 & 0.00 & 993.8 & 0.70 & 0.55 & 1323 & 4.00 & 194 & 0.34 & 2.15 & 1.90 & 1.27 \\
\hline $\mathrm{NB}$ & 357.6 & 0.02 & 684.5 & 1.12 & 0.55 & 1492 & 4.80 & 181 & 0.52 & 2.14 & 1.86 & 1.25 \\
\hline
\end{tabular}


Table 6.6 Effects of yield plateau length and engineering strain at ultimate stress on the FEA prediction

\begin{tabular}{|c|c|c|c|c|c|c|c|c|c|c|c|}
\hline \multirow[b]{2}{*}{ Specimen } & \multirow[b]{2}{*}{$\begin{array}{c}\text { Dimension }(\mathrm{mm}) \\
\text { Lo } \times \mathrm{w} \times \mathrm{t}\end{array}$} & \multirow[b]{2}{*}{$\mathrm{KL} / \mathrm{r}$} & \multirow[b]{2}{*}{$A_{n} F_{u} / A_{g} F_{y}$} & \multicolumn{3}{|c|}{$\varepsilon_{\mathrm{eu}}=0.15$} & \multicolumn{3}{|c|}{$\varepsilon_{\mathrm{eu}}=0.22$} & \multicolumn{2}{|c|}{ Comparison } \\
\hline & & & & $\begin{array}{c}\text { Half cycle } \\
\text { no. at } \\
\text { fracture (A) }\end{array}$ & $\begin{array}{c}\text { Max. } \\
\text { deformation } \\
(\mathrm{mm})(\mathrm{B})\end{array}$ & $\delta_{\mathrm{L}} / \delta_{\mathrm{y}}$ & $\begin{array}{c}\text { Half cycle } \\
\text { no. at } \\
\text { fracture (C) }\end{array}$ & $\begin{array}{c}\text { Max. } \\
\text { deformation } \\
(\mathrm{mm})(\mathrm{D})\end{array}$ & $\delta_{\mathrm{L}} / \delta_{\mathrm{y}}$ & $(\mathrm{A}) /(\mathrm{C})$ & $(\mathrm{B}) /(\mathrm{D})$ \\
\hline$(\mathrm{M} / \mathrm{N}) \mathrm{A} 08103$ & $2510 \times 77 \times 4.8$ & 800 & 1.00 & 37 & 8.55 & 2.47 & 43 & 10.39 & 3.00 & 0.86 & 0.82 \\
\hline$(\mathrm{M} / \mathrm{N}) \mathrm{A} 08123$ & $2510 \times 154 \times 4.8$ & 800 & 1.20 & 63 & 28.58 & 8.25 & 67 & 31.15 & 8.99 & 0.94 & 0.92 \\
\hline$(\mathrm{M} / \mathrm{N}) \mathrm{B} 08103$ & $2510 \times 77 \times 4.8$ & 800 & 1.00 & 37 & 9.30 & 2.68 & 39 & 10.39 & 3.00 & 0.95 & 0.89 \\
\hline$(\mathrm{M} / \mathrm{N}) \mathrm{B} 08123$ & $2510 \times 154 \times 4.8$ & 800 & 1.20 & 99 & 61.59 & 17.77 & 103 & 62.37 & 18.00 & 0.96 & 0.99 \\
\hline
\end{tabular}


Table 6.7 Mechanical properties of materials for the parametric study

\begin{tabular}{|c|c|c|c|c|c|c|}
\hline Material & $\begin{array}{c}\text { Elastic modulus } \\
(\mathrm{MPa})\end{array}$ & $\begin{array}{c}\text { Yield plateau } \\
\varepsilon_{\mathrm{ep}}\end{array}$ & $\begin{array}{c}\text { Yield strength } \\
\mathrm{F}_{\mathrm{y}}(\mathrm{MPa})\end{array}$ & $\begin{array}{c}\text { Engineering strain at } \\
\text { ultimate stress, } \varepsilon_{\mathrm{eu}}\end{array}$ & $\begin{array}{c}\text { Ultimate strength } \\
\mathrm{F}_{\mathrm{u}}(\mathrm{MPa})\end{array}$ & $\mathrm{F}_{\mathrm{u}} / \mathrm{F}_{\mathrm{y}}$ \\
\hline $\mathrm{JA}$ & 200000 & 0.00 & 300 & 0.15 & 420 & 1.40 \\
\hline JB & 200000 & 0.02 & 300 & 0.15 & 420 & 1.40 \\
\hline KA & 200000 & 0.00 & 300 & 0.15 & 525 & 1.75 \\
\hline KB & 200000 & 0.02 & 300 & 0.15 & 525 & 1.75 \\
\hline
\end{tabular}

Table 6.8 Material model parameters for materials JA, JB, KA and KB

\begin{tabular}{|c|c|c|c|c|c|c|c|c|c|c|c|c|}
\hline \multirow{2}{*}{ Material } & \multicolumn{4}{|c|}{ Flow stress parameters } & \multicolumn{3}{c|}{ Kinematic hardening parameters } & \multicolumn{2}{c|}{$\begin{array}{c}\text { Damage evolution } \\
\text { parameters }\end{array}$} & $\begin{array}{c}\text { Damage } \\
\text { limit }\end{array}$ \\
\cline { 2 - 15 } & $\sigma_{0}^{y}(\mathrm{MPa})$ & $\varepsilon_{0}^{\mathrm{p}}$ & $\sigma_{x}^{y}(\mathrm{MPa})$ & $\mathrm{b}$ & $\mathrm{n}$ & $\mathrm{C}_{1}(\mathrm{MPa})$ & $\gamma_{1}$ & $\mathrm{C}_{2}(\mathrm{MPa})$ & $\gamma_{2}$ & $\mathrm{~m}$ & $\mathrm{k}$ & $\mathrm{D}_{\mathrm{c}}$ \\
\hline JA & 300.5 & 0.00 & 338.7 & 2.31 & 0.55 & 1734 & 8.39 & 142 & 1.21 & 2.42 & 1.62 & 0.71 \\
\hline JB & 306.5 & 0.02 & 281.4 & 3.20 & 0.55 & 1923 & 9.77 & 138 & 1.82 & 2.48 & 1.49 & 0.73 \\
\hline KA & 300.5 & 0.00 & 422.0 & 3.79 & 0.55 & 2441 & 10.15 & 192 & 2.19 & 2.41 & 1.46 & 2.17 \\
\hline KB & 306.5 & 0.02 & 383.7 & 4.85 & 0.55 & 2680 & 11.42 & 182 & 2.96 & 2.47 & 1.41 & 2.21 \\
\hline
\end{tabular}


Table 6.9 List of braces for materials JA and JB

\begin{tabular}{|c|c|c|c|c|c|}
\hline Specimen & $\begin{array}{c}\text { Length } \mathrm{L}_{v} \\
(\mathrm{~mm})\end{array}$ & $\begin{array}{c}\text { Width } \\
(\mathrm{mm})\end{array}$ & $\begin{array}{c}\text { Thickness } \\
(\mathrm{mm})\end{array}$ & $\mathrm{KL} / \mathrm{r}$ & $\mathrm{A}_{\mathrm{n}} \mathrm{F}_{\mathrm{u}} / \mathrm{A}_{\mathrm{g}} \mathrm{F}_{\mathbf{~}}$ \\
\hline $\mathrm{J}(\mathrm{A} / \mathrm{B}) 04094$ & 4000 & 61.6 & 16.5 & 400 & 0.90 \\
\hline $\mathrm{J}(\mathrm{A} / \mathrm{B}) 08094$ & 4000 & 61.6 & 8.2 & 800 & 0.90 \\
\hline $\mathrm{J}(\mathrm{A} / \mathrm{B}) 16094$ & 4000 & 61.6 & 4.1 & 1600 & 0.90 \\
\hline $\mathrm{J}(\mathrm{A} / \mathrm{B}) 06096$ & 6000 & 61.6 & 16.7 & 600 & 0.90 \\
\hline $\mathrm{J}(\mathrm{A} / \mathrm{B}) 08096$ & 6000 & 61.6 & 12.6 & 800 & 0.90 \\
\hline $\mathrm{J}(\mathrm{A} / \mathrm{B}) 16096$ & 6000 & 61.6 & 6.3 & 1600 & 0.90 \\
\hline $\mathrm{J}(\mathrm{A} / \mathrm{B}) 08103$ & 2510 & 77.0 & 4.8 & 800 & 1.00 \\
\hline $\mathrm{J}(\mathrm{A} / \mathrm{B}) 04104$ & 4000 & 77.0 & 16.5 & 400 & 1.00 \\
\hline $\mathrm{J}(\mathrm{A} / \mathrm{B}) 08104$ & 4000 & 77.0 & 8.2 & 800 & 1.00 \\
\hline $\mathrm{J}(\mathrm{A} / \mathrm{B}) 16104$ & 4000 & 77.0 & 4.1 & 1600 & 1.00 \\
\hline $\mathrm{J}(\mathrm{A} / \mathrm{B}) 06106$ & 6000 & 77.0 & 16.7 & 600 & 1.00 \\
\hline $\mathrm{J}(\mathrm{A} / \mathrm{B}) 08106$ & 6000 & 77.0 & 12.6 & 800 & 1.00 \\
\hline $\mathrm{J}(\mathrm{A} / \mathrm{B}) 16106$ & 6000 & 77.0 & 6.3 & 1600 & 1.00 \\
\hline $\mathrm{J}(\mathrm{A} / \mathrm{B}) 06112$ & 2000 & 102.7 & 5.2 & 600 & 1.10 \\
\hline $\mathrm{J}(\mathrm{A} / \mathrm{B}) 08112$ & 2000 & 102.7 & 3.9 & 800 & 1.10 \\
\hline $\mathrm{J}(\mathrm{A} / \mathrm{B}) 04114$ & 4000 & 102.7 & 16.5 & 400 & 1.10 \\
\hline $\mathrm{J}(\mathrm{A} / \mathrm{B}) 06114$ & 4000 & 102.7 & 11.0 & 600 & 1.10 \\
\hline $\mathrm{J}(\mathrm{A} / \mathrm{B}) 08114$ & 4000 & 102.7 & 8.2 & 800 & 1.10 \\
\hline $\mathrm{J}(\mathrm{A} / \mathrm{B}) 16114$ & 4000 & 102.7 & 4.1 & 1600 & 1.10 \\
\hline $\mathrm{J}(\mathrm{A} / \mathrm{B}) 06116$ & 6000 & 102.7 & 16.7 & 600 & 1.10 \\
\hline $\mathrm{J}(\mathrm{A} / \mathrm{B}) 08116$ & 6000 & 102.7 & 12.6 & 800 & 1.10 \\
\hline $\mathrm{J}(\mathrm{A} / \mathrm{B}) 16116$ & 6000 & 102.7 & 6.3 & 1600 & 1.10 \\
\hline $\mathrm{J}(\mathrm{A} / \mathrm{B}) 08123$ & 2510 & 154.0 & 4.8 & 800 & 1.20 \\
\hline $\mathrm{J}(\mathrm{A} / \mathrm{B}) 04124$ & 4000 & 154.0 & 16.5 & 400 & 1.20 \\
\hline $\mathrm{J}(\mathrm{A} / \mathrm{B}) 08124$ & 4000 & 154.0 & 8.2 & 800 & 1.20 \\
\hline $\mathrm{J}(\mathrm{A} / \mathrm{B}) 16124$ & 4000 & 154.0 & 4.1 & 1600 & 1.20 \\
\hline $\mathrm{J}(\mathrm{A} / \mathrm{B}) 06126$ & 6000 & 154.0 & 16.7 & 600 & 1.20 \\
\hline $\mathrm{J}(\mathrm{A} / \mathrm{B}) 08126$ & 6000 & 154.0 & 12.6 & 800 & 1.20 \\
\hline $\mathrm{J}(\mathrm{A} / \mathrm{B}) 16126$ & 6000 & 154.0 & 6.3 & 1600 & 1.20 \\
\hline
\end{tabular}


Table 6.10 List of braces for materials KA and KB

\begin{tabular}{|c|c|c|c|c|c|}
\hline Specimen & $\begin{array}{c}\text { Length } \mathrm{L}_{0} \\
(\mathrm{~mm})\end{array}$ & $\begin{array}{c}\text { Width } \\
(\mathrm{mm})\end{array}$ & $\begin{array}{c}\text { Thickness } \\
(\mathrm{mm})\end{array}$ & $\mathrm{KL} / \mathrm{r}$ & $\mathrm{A}_{n} \mathrm{~F}_{\mathrm{u}} / \mathrm{A}_{\mathrm{g}} \mathrm{F}_{\mathrm{g}}$ \\
\hline $\mathrm{K}(\mathrm{A} / \mathrm{B}) 04104$ & 4000 & 51.3 & 16.5 & 400 & 1.00 \\
\hline $\mathrm{K}(\mathrm{A} / \mathrm{B}) 08104$ & 4000 & 51.3 & 8.2 & 800 & 1.00 \\
\hline $\mathrm{K}(\mathrm{A} / \mathrm{B}) 16104$ & 4000 & 51.3 & 4.1 & 1600 & 1.00 \\
\hline $\mathrm{K}(\mathrm{A} / \mathrm{B}) 06106$ & 6000 & 51.3 & 16.7 & 600 & 1.00 \\
\hline $\mathrm{K}(\mathrm{A} / \mathrm{B}) 08106$ & 6000 & 51.3 & 12.6 & 800 & 1.00 \\
\hline $\mathrm{K}(\mathrm{A} / \mathrm{B}) 16106$ & 6000 & 51.3 & 6.3 & 1600 & 1.00 \\
\hline $\mathrm{K}(\mathrm{A} / \mathrm{B}) 04114$ & 4000 & 59.2 & 16.5 & 400 & 1.10 \\
\hline $\mathrm{K}(\mathrm{A} / \mathrm{B}) 06114$ & 4000 & 59.2 & 11.0 & 600 & 1.10 \\
\hline $\mathrm{K}(\mathrm{A} / \mathrm{B}) 08114$ & 4000 & 59.2 & 8.2 & 800 & 1.10 \\
\hline $\mathrm{K}(\mathrm{A} / \mathrm{B}) 16114$ & 4000 & 59.2 & 4.1 & 1600 & 1.10 \\
\hline $\mathrm{K}(\mathrm{A} / \mathrm{B}) 06116$ & 6000 & 59.2 & 16.7 & 600 & 1.10 \\
\hline $\mathrm{K}(\mathrm{A} / \mathrm{B}) 08116$ & 6000 & 59.2 & 12.6 & 800 & 1.10 \\
\hline $\mathrm{K}(\mathrm{A} / \mathrm{B}) 16116$ & 6000 & 59.2 & 6.3 & 1600 & 1.10 \\
\hline $\mathrm{K}(\mathrm{A} / \mathrm{B}) 04124$ & 4000 & 70.0 & 16.5 & 400 & 1.20 \\
\hline $\mathrm{K}(\mathrm{A} / \mathrm{B}) 08124$ & 4000 & 70.0 & 8.2 & 800 & 1.20 \\
\hline $\mathrm{K}(\mathrm{A} / \mathrm{B}) 16124$ & 4000 & 70.0 & 4.1 & 1600 & 1.20 \\
\hline $\mathrm{K}(\mathrm{A} / \mathrm{B}) 06126$ & 6000 & 70.0 & 16.7 & 600 & 1.20 \\
\hline $\mathrm{K}(\mathrm{A} / \mathrm{B}) 08126$ & 6000 & 70.0 & 12.6 & 800 & 1.20 \\
\hline $\mathrm{K}(\mathrm{A} / \mathrm{B}) 16126$ & 6000 & 70.0 & 6.3 & 1600 & 1.20 \\
\hline
\end{tabular}

Table 6.11 FEA predictions for braces JA04124 and JB04124 using two different mesh schemes

\begin{tabular}{|c|c|c|c|c|c|}
\hline \multirow{2}{*}{ Specimen } & Mesh & $\begin{array}{c}\text { Solid element } \\
\text { layer }\end{array}$ & $\begin{array}{c}\text { Half cycle } \\
\text { no. at fracture }\end{array}$ & $\begin{array}{c}\text { Max. deformation } \\
(\mathrm{mm})\end{array}$ & $\delta_{\mathrm{L}} / \delta_{\mathrm{y}}$ \\
\hline \multirow{3}{*}{ JA04124 } & Scheme-1 (A) & 5 & 63 & 47.04 & 8.25 \\
\cline { 2 - 6 } & Scheme-3(B) & 10 & 63 & 45.56 & 7.99 \\
\cline { 2 - 6 } & $(\mathrm{A}) /(\mathrm{B})$ & - & 1.00 & 1.03 & 1.03 \\
\hline \multirow{3}{*}{ JB04124 } & Scheme-1 (C) & 5 & 95 & 94.58 & 16.59 \\
\cline { 2 - 7 } & Scheme-3(D) & 10 & 95 & 94.69 & 16.61 \\
\cline { 2 - 7 } & $(\mathrm{C}) /(\mathrm{D})$ & - & 1.00 & 1.00 & 1.00 \\
\hline
\end{tabular}


Table 6.12 FEA predictions for braces of material JA that has no yield plateau

\begin{tabular}{|c|c|c|c|}
\hline \multirow{2}{*}{ Specimen } & \multicolumn{3}{|c|}{$1 / 2$ model $(\eta=0.75)$} \\
\cline { 2 - 4 } & $\begin{array}{c}\text { Predicted half } \\
\text { cycle no. }\end{array}$ & $\begin{array}{c}\text { Predicted max. } \\
\text { deformation }(\mathrm{mm})\end{array}$ & $\delta_{\mathrm{I}} / \delta$ \\
\hline JA04094 & 35 & 11.40 & 2.00 \\
\hline JA08094 & 31 & 8.27 & 1.45 \\
\hline JA16094 & 31 & 8.04 & 1.41 \\
\hline JA06096 & 31 & 13.27 & 1.53 \\
\hline JA08096 & 31 & 10.77 & 1.24 \\
\hline JA16096 & 31 & 12.42 & 1.43 \\
\hline JA08103 & 37 & 7.50 & 2.17 \\
\hline JA04104 & 37 & 14.47 & 2.54 \\
\hline JA08104 & 37 & 12.68 & 2.23 \\
\hline JA16104 & 37 & 11.40 & 2.00 \\
\hline JA06106 & 37 & 22.32 & 2.57 \\
\hline JA08106 & 37 & 18.57 & 2.13 \\
\hline JA16106 & 33 & 17.37 & 2.00 \\
\hline JA06112 & 47 & 13.07 & 4.84 \\
\hline JA08112 & 47 & 12.62 & 4.67 \\
\hline JA04114 & 47 & 26.22 & 4.60 \\
\hline JA06114 & 47 & 27.41 & 4.81 \\
\hline JA08114 & 47 & 25.84 & 4.53 \\
\hline JA16114 & 47 & 22.80 & 4.00 \\
\hline JA06116 & 51 & 43.48 & 5.00 \\
\hline JA08116 & 51 & 45.30 & 5.21 \\
\hline JA16116 & 47 & 34.76 & 4.00 \\
\hline JA08123 & 63 & 27.72 & 8.00 \\
\hline JA04124 & 63 & 47.04 & 8.25 \\
\hline JA08124 & 67 & 51.28 & 9.00 \\
\hline JA16124 & 61 & 45.38 & 7.96 \\
\hline JA06126 & 67 & 78.29 & 9.00 \\
\hline JA08126 & 71 & 86.96 & 10.00 \\
\hline JA16126 & 65 & 78.21 & 8.99 \\
\hline
\end{tabular}


Table 6.13 FEA predictions for braces of material JB that has a yield plateau

\begin{tabular}{|c|c|c|c|}
\hline \multirow{2}{*}{ Specimen } & \multicolumn{3}{|c|}{$1 / 2$ model $(\eta=0.75)$} \\
\cline { 2 - 4 } & $\begin{array}{c}\text { Predicted half } \\
\text { cycle no. }\end{array}$ & $\begin{array}{c}\text { Predicted max. } \\
\text { deformation }(\mathrm{mm})\end{array}$ & $\delta_{\mathrm{I}} / \delta_{\mathrm{y}}$ \\
\hline JB04094 & 37 & 11.39 & 2.00 \\
\hline JB08094 & 31 & 10.03 & 1.76 \\
\hline JB16094 & 31 & 8.69 & 1.52 \\
\hline JB06096 & 31 & 13.00 & 1.49 \\
\hline JB08096 & 31 & 13.18 & 1.51 \\
\hline JB16096 & 31 & 11.94 & 1.37 \\
\hline JB08103 & 37 & 8.56 & 2.47 \\
\hline JB04104 & 37 & 11.40 & 2.00 \\
\hline JB08104 & 37 & 11.38 & 2.00 \\
\hline JB16104 & 31 & 10.61 & 1.86 \\
\hline JB06106 & 31 & 12.51 & 1.44 \\
\hline JB08106 & 31 & 15.06 & 1.73 \\
\hline JB16106 & 31 & 12.75 & 1.47 \\
\hline JB06112 & 75 & 29.69 & 11.00 \\
\hline JB08112 & 83 & 35.10 & 13.00 \\
\hline JB04114 & 67 & 51.25 & 8.99 \\
\hline JB06114 & 79 & 68.34 & 11.99 \\
\hline JB08114 & 87 & 83.39 & 14.63 \\
\hline JB16114 & 91 & 88.78 & 15.57 \\
\hline JB06116 & 83 & 113.95 & 13.10 \\
\hline JB08116 & 87 & 129.94 & 14.94 \\
\hline JB16116 & 91 & 136.39 & 15.68 \\
\hline JB08123 & 99 & 58.89 & 17.00 \\
\hline JB04124 & 95 & 94.58 & 16.59 \\
\hline JB08124 & 101 & 102.59 & 18.00 \\
\hline JB16124 & 103 & 102.59 & 18.00 \\
\hline JB06126 & 99 & 148.62 & 17.08 \\
\hline JB08126 & 103 & 157.80 & 18.14 \\
\hline JB16126 & 107 & 165.06 & 18.97 \\
\hline
\end{tabular}


Table 6.14 FEA predictions for braces of material KA that has no yield plateau

\begin{tabular}{|c|c|c|c|}
\hline \multirow{2}{*}{ Specimen } & \multicolumn{3}{|c|}{$1 / 2$ model $(\eta=0.75)$} \\
\cline { 2 - 4 } & $\begin{array}{c}\text { Predicted half } \\
\text { cycle no. }\end{array}$ & $\begin{array}{c}\text { Predicted max. } \\
\text { deformation }(\mathrm{mm})\end{array}$ & $\delta_{\mathrm{I}} / \delta_{\mathrm{y}}$ \\
\hline KA04104 & 37 & 12.00 & 2.10 \\
\hline KA08104 & 37 & 11.40 & 2.00 \\
\hline KA16104 & 31 & 9.04 & 1.59 \\
\hline KA06106 & 37 & 17.40 & 2.00 \\
\hline KA08106 & 37 & 17.39 & 2.00 \\
\hline KA16106 & 31 & 14.08 & 1.62 \\
\hline KA04114 & 43 & 17.37 & 3.05 \\
\hline KA06114 & 43 & 17.10 & 3.00 \\
\hline KA08114 & 43 & 17.55 & 3.08 \\
\hline KA16114 & 37 & 15.13 & 2.65 \\
\hline KA06116 & 43 & 27.50 & 3.16 \\
\hline KA08116 & 43 & 26.74 & 3.07 \\
\hline KA16116 & 37 & 23.10 & 2.66 \\
\hline KA04124 & 47 & 22.75 & 3.99 \\
\hline KA08124 & 47 & 24.74 & 4.34 \\
\hline KA16124 & 47 & 22.79 & 4.00 \\
\hline KA06126 & 51 & 43.49 & 5.00 \\
\hline KA08126 & 47 & 40.59 & 4.67 \\
\hline KA16126 & 47 & 34.79 & 4.00 \\
\hline
\end{tabular}


Table 6.15 FEA predictions for braces of material KB that has a yield plateau

\begin{tabular}{|c|c|c|c|}
\hline \multirow{2}{*}{ Specimen } & \multicolumn{3}{|c|}{$1 / 2$ model $(\eta=0.75)$} \\
\cline { 2 - 4 } & $\begin{array}{c}\text { Predicted half } \\
\text { cycle no. }\end{array}$ & $\begin{array}{c}\text { Predicted max. } \\
\text { deformation }(\mathrm{mm})\end{array}$ & $\delta_{1} / \delta_{y}$ \\
\hline KB04104 & 37 & 13.61 & 2.39 \\
\hline KB08104 & 31 & 10.90 & 1.91 \\
\hline KB16104 & 31 & 9.62 & 1.69 \\
\hline KB06106 & 37 & 18.81 & 2.16 \\
\hline KB08106 & 31 & 14.64 & 1.68 \\
\hline KB16106 & 31 & 12.51 & 1.44 \\
\hline KB04114 & 63 & 46.05 & 8.08 \\
\hline KB06114 & 71 & 57.52 & 10.09 \\
\hline KB08114 & 83 & 75.58 & 13.26 \\
\hline KB16114 & 91 & 85.50 & 15.00 \\
\hline KB06116 & 75 & 95.70 & 11.00 \\
\hline KB08116 & 87 & 121.80 & 14.00 \\
\hline KB16116 & 91 & 130.47 & 15.00 \\
\hline KB04124 & 87 & 79.79 & 14.00 \\
\hline KB08124 & 93 & 91.19 & 16.00 \\
\hline KB16124 & 95 & 91.92 & 16.13 \\
\hline KB06126 & 91 & 132.03 & 15.18 \\
\hline KB08126 & 91 & 136.77 & 15.72 \\
\hline KB16126 & 95 & 142.42 & 16.37 \\
\hline
\end{tabular}

Table 6.16 $\quad A_{n} / A_{\mathrm{g}}$ ratio at the same thickness but different $F_{\mathrm{u}} / \mathrm{F}_{\mathrm{y}}$ with drilled hole for M20 bolt

\begin{tabular}{|c|c|c|c|}
\hline \multirow{2}{*}{$\mathrm{A}_{\mathrm{n}} \mathrm{F}_{\mathrm{u}} / \mathrm{A}_{\mathrm{g}} \mathrm{F}_{\mathrm{y}}$} & \multicolumn{3}{|c|}{$\mathrm{A}_{\mathrm{n}} / \mathrm{A}_{\mathrm{g}}$} \\
\cline { 2 - 4 } & $\mathrm{F}_{\mathrm{u}} / \mathrm{F}_{\mathrm{y}}=1.4$ & $\mathrm{~F}_{\mathrm{u}} / \mathrm{F}_{\mathrm{y}}=1.575$ & $\mathrm{~F}_{\mathrm{u}} / \mathrm{F}_{\mathrm{y}}=1.75$ \\
\hline 1.0 & 0.71 & 0.63 & 0.57 \\
\hline 1.1 & 0.79 & 0.70 & 0.63 \\
\hline 1.2 & 0.86 & 0.76 & 0.69 \\
\hline
\end{tabular}


Table 6.17 Constants for the ductility prediction equations of materials without a yield plateau, JA and KA

\begin{tabular}{|c|c|c|c|c|}
\hline \multirow{2}{*}{ Material } & \multicolumn{4}{|c|}{ Constants } \\
\cline { 2 - 5 } & $\mathrm{P}_{1}$ & $\mathrm{P}_{2}$ & $\mathrm{P}_{3}$ & $\mathrm{P}_{4}$ \\
\hline $\mathrm{JA}$ & 0.219 & $-7.70 \mathrm{E}-04$ & 0.042 & 7.049 \\
\hline $\mathrm{KA}$ & 0.176 & $-1.17 \mathrm{E}-03$ & 0.046 & 4.521 \\
\hline
\end{tabular}

Table 6.18 Constants for the ductility prediction equations of materials with a yield plateau, JB and $\mathrm{KB}$

\begin{tabular}{|c|c|c|c|c|c|c|}
\hline \multirow{2}{*}{ Material } & \multicolumn{6}{|c|}{ Constants } \\
\cline { 2 - 7 } & $\mathrm{P}_{1}$ & $\mathrm{P}_{2}$ & $\mathrm{P}_{3}$ & $\mathrm{P}_{4}$ & $\mathrm{P}_{5}$ & $\mathrm{P}_{6}$ \\
\hline $\mathrm{JB}$ & 2.201 & 0.153 & 0.025 & 4.050 & -0.002 & -0.979 \\
\hline $\mathrm{KB}$ & 0.780 & 0.213 & 0.022 & 6.085 & 0.025 & -0.991 \\
\hline
\end{tabular}

Table 6.19 Statistics of the ratio of the predicted ductility from FEA simulation to that by the first regression equation for all four materials

\begin{tabular}{|c|c|c|c|}
\hline \multirow{2}{*}{ Material } & \multicolumn{3}{|c|}{ Ratio of FEA to equation prediction $\left(\delta_{\mathrm{l}} / \delta_{\mathrm{y}}\right)$} \\
\cline { 2 - 4 } & Mean & Standard deviation & Coefficient of variation \\
\hline JA & 1.04 & 0.18 & $17.1 \%$ \\
\hline KA & 1.00 & 0.05 & $5.4 \%$ \\
\hline JB & 1.01 & 0.16 & $15.5 \%$ \\
\hline KB & 1.00 & 0.12 & $12.4 \%$ \\
\hline
\end{tabular}

Table 6.20 Statistics of the difference of the predicted ductility between FEA simulation and that by the first regression equation for all four materials

\begin{tabular}{|c|c|c|}
\hline \multirow{2}{*}{ Material } & \multicolumn{2}{|c|}{ Difference of FEA to equation prediction $\left(\delta_{\mathrm{L}} / \delta_{\mathrm{y}}\right)$} \\
\cline { 2 - 3 } & Mean & Standard deviation \\
\hline JA & 0.02 & 0.38 \\
\hline KA & 0.00 & 0.17 \\
\hline JB & 0.00 & 0.95 \\
\hline KB & 0.00 & 1.06 \\
\hline
\end{tabular}


Table 6.21 Constants for the ductility prediction equation of materials without a yield plateau, combining JA and KA

\begin{tabular}{|c|c|c|c|c|c|}
\hline \multirow{2}{*}{ Material } & \multicolumn{5}{|c|}{ Constants } \\
\cline { 2 - 6 } & $\mathbf{P}_{1}$ & $\mathbf{P}_{2}$ & $\mathbf{P}_{3}$ & $\mathbf{P}_{4}$ & $\mathbf{P}_{5}$ \\
\hline $\mathrm{JA}, \mathrm{KA}$ & 0.666 & $-8.70 \mathrm{E}-04$ & 0.043 & 6.585 & 0.462 \\
\hline
\end{tabular}

Table 6.22 Constants for the ductility prediction equation of materials with a yield plateau, combining JB and $\mathrm{KB}$

\begin{tabular}{|c|c|c|c|c|c|c|c|}
\hline \multirow{2}{*}{ Material } & \multicolumn{7}{|c|}{ Constants } \\
\cline { 2 - 8 } & $\mathrm{P}_{1}$ & $\mathrm{P}_{2}$ & $\mathrm{P}_{3}$ & $\mathrm{P}_{4}$ & $\mathrm{P}_{5}$ & $\mathrm{P}_{6}$ & $\mathrm{P}_{7}$ \\
\hline $\mathrm{JB}, \mathrm{KB}$ & 2.159 & 0.179 & 0.024 & 4.721 & 0.008 & -0.984 & 0.215 \\
\hline
\end{tabular}

Table 6.23 Statistics of the ratio of the predicted ductility from FEA simulation to that by the second regression equation for the combined materials

\begin{tabular}{|c|c|c|c|}
\hline \multirow{2}{*}{ Material } & \multicolumn{3}{|c|}{ Ratio of FEA to equation prediction $\left(\delta_{\mathrm{L}} / \delta_{\mathrm{y}}\right)$} \\
\cline { 2 - 4 } & Mean & Standard deviation & Coefficient of variation \\
\hline JA, KA & 1.05 & 0.17 & $16.3 \%$ \\
\hline JB, KB & 1.01 & 0.15 & $15.1 \%$ \\
\hline
\end{tabular}

Table 6.24 Statistics of the difference of the predicted ductility between FEA simulation to that by the second regression equation for the combined materials

\begin{tabular}{|c|c|c|}
\hline \multirow{2}{*}{ Material } & \multicolumn{2}{|c|}{ Difference of FEA to equation prediction $\left(\delta_{1} / \delta_{y}\right)$} \\
\cline { 2 - 3 } & Mean & Standard deviation \\
\hline JA, KA & 0.04 & 0.41 \\
\hline JB, KB & 0.00 & 1.01 \\
\hline
\end{tabular}


Table 6.25 Comparisons of test and predicted ductility through interpolating and extrapolating from Eqs. (6.18) and (6.19) with the measured maximum deformation reduced by $6 \mathrm{~mm}$ (except specimens of material $\mathrm{C}$ )

\begin{tabular}{|c|c|c|c|c|c|c|c|c|c|c|c|c|c|c|}
\hline \multirow[b]{2}{*}{ Material } & \multirow[b]{2}{*}{ Specimen } & \multirow[b]{2}{*}{$\mathrm{KL} / \mathrm{r}$} & \multirow[b]{2}{*}{$\mathrm{L}(\mathrm{m})$} & \multirow[b]{2}{*}{$A_{n} F_{u} / A_{g} F_{y}$} & \multirow[b]{2}{*}{$\mathrm{F}_{\mathrm{u}} / \mathrm{F}_{\mathrm{y}}$} & \multirow[b]{2}{*}{$\begin{array}{c}\text { Yield } \\
\text { plateau } \\
\varepsilon_{\mathrm{cp}}\end{array}$} & \multicolumn{4}{|c|}{ Test } & \multicolumn{3}{|c|}{ Regression (RII) } & \multirow[b]{2}{*}{ RII/Test } \\
\hline & & & & & & & $\begin{array}{c}\text { Max. } \\
\text { deformation } \\
(\mathrm{mm})\end{array}$ & $\delta_{\mathrm{L}} / \delta_{\mathrm{y}}$ & $\begin{array}{c}\text { Adjusted } \\
\text { max. } \\
\text { deformation } \\
(\mathrm{mm})\end{array}$ & $\begin{array}{c}\text { Adjusted } \\
\delta_{\mathrm{L}} / \delta_{\mathrm{y}}\end{array}$ & $\begin{array}{c}\delta_{\mathrm{L}} / \delta_{\mathrm{y}} \\
\text { by Eq. } \\
(6.18)\end{array}$ & $\begin{array}{c}\delta_{1} / \delta_{y} \\
\text { by Eq. } \\
(6.19)\end{array}$ & $\delta_{L} / \delta_{y}$ & \\
\hline \multirow{6}{*}{$\mathrm{C}$} & A4075a & \multirow{2}{*}{419} & \multirow{2}{*}{1.16} & \multirow{6}{*}{1.00} & \multirow{6}{*}{1.45} & \multirow{6}{*}{0.0236} & 9.14 & 5.25 & - & - & \multirow{2}{*}{2.24} & \multirow{2}{*}{1.66} & \multirow{2}{*}{1.55} & 0.30 \\
\hline & $\mathrm{A} 4075 \mathrm{~b}$ & & & & & & 6.74 & 3.87 & - & - & & & & 0.40 \\
\hline & $\mathrm{A} 6075 \mathrm{a}$ & \multirow{2}{*}{624} & \multirow{2}{*}{1.73} & & & & 7.52 & 2.90 & - & - & \multirow{2}{*}{2.25} & \multirow{2}{*}{1.74} & \multirow{2}{*}{1.64} & 0.57 \\
\hline & $\mathrm{A} 6075 \mathrm{~b}$ & & & & & & 8.53 & 3.29 & - & - & & & & 0.50 \\
\hline & $\mathrm{A} 8075 \mathrm{a}$ & \multirow{2}{*}{834} & \multirow{2}{*}{2.31} & & & & 11.75 & 3.39 & - & - & \multirow{2}{*}{2.26} & \multirow{2}{*}{1.80} & \multirow{2}{*}{1.72} & 0.51 \\
\hline & $\mathrm{A} 8075 \mathrm{~b}$ & & & & & & 9.92 & 2.86 & - & - & & & & 0.60 \\
\hline \multirow{5}{*}{$\mathrm{D}$} & $\mathrm{A} 2078 \mathrm{a}$ & \multirow{3}{*}{202} & & & & & 23.95 & 17.36 & 17.95 & 13.01 & & & & 0.50 \\
\hline & $\mathrm{A} 2078 \mathrm{~b}$ & & 0.92 & & & & 26.48 & 19.19 & 20.48 & 14.84 & 5.35 & 11.71 & 6.47 & 0.44 \\
\hline & $\mathrm{A} 2078 \mathrm{c}$ & & & 1.23 & 1.73 & 0.0035 & 28.96 & 20.99 & 22.96 & 16.64 & & & & 0.39 \\
\hline & A4078a & 406 & 185 & & & & 37.83 & 13.63 & 31.83 & 11.47 & 5.46 & 1353 & 688 & 0.60 \\
\hline & $\mathrm{A} 4078 \mathrm{~b}$ & 400 & 1.00 & & & & 34.36 & 12.38 & 28.36 & 10.22 & 3.40 & S.J.5 & 0.00 & 0.67 \\
\hline & $\mathrm{A} 2108 \mathrm{a}$ & 202 & 0 ? & & & & 28.32 & 20.52 & 22.32 & 16.17 & & & & 0.60 \\
\hline $\mathrm{E}$ & $A 2108 b$ & 202 & 0.92 & 127 & 161 & & 29.45 & 21.34 & 23.45 & 16.99 & 8.18 & 12.09 & 9.05 & 0.57 \\
\hline L & A4108a & 406 & 5 & 1.21 & 1.01 & 0.0005 & 42.48 & 15.31 & 36.48 & 13.15 & 35 & 66 & 1041 & 0.79 \\
\hline & $\mathrm{A} 4108 \mathrm{~b}$ & 400 & 1.05 & & & & 47.35 & 17.06 & 41.35 & 14.90 & 0.55 & 14.00 & 10.41 & 0.70 \\
\hline & $\mathrm{A} 2128 \mathrm{a}$ & & & & & & 32.82 & 23.78 & 26.82 & 19.43 & & & & 0.59 \\
\hline & $\mathrm{A} 2128 \mathrm{~b}$ & 202 & 0.92 & & & & 35.11 & 25.44 & 29.11 & 21.09 & 8.73 & 12.81 & 11.47 & 0.54 \\
\hline $\mathrm{F}$ & $\mathrm{A} 2128 \mathrm{c}$ & & & 1.24 & 1.50 & 0.0134 & 32.17 & 23.31 & 26.17 & 18.96 & & & & 0.60 \\
\hline & $\mathrm{A} 4128 \mathrm{a}$ & 106 & 185 & & & & 55.61 & 20.04 & 49.61 & 17.88 & 80 & 1481 & 1786 & 0.72 \\
\hline & $\mathrm{A} 4128 \mathrm{~b}$ & 400 & 1.05 & & & & 54.23 & 19.54 & 48.23 & 17.38 & 8.90 & 14.81 & 12.80 & 0.74 \\
\hline
\end{tabular}


Table 6.26 Comparisons of test and predicted ductility using Eq. (6.18) for specimens of material C

\begin{tabular}{|c|c|c|c|c|c|c|c|c|c|c|c|c|}
\hline \multirow[b]{2}{*}{ Material } & \multirow[b]{2}{*}{ Specimen } & \multirow[b]{2}{*}{$\mathrm{KL} / \mathrm{r}$} & \multirow[b]{2}{*}{$\mathrm{L}(\mathrm{m})$} & \multirow[b]{2}{*}{$\mathrm{A}_{n} \mathrm{~F}_{\mathrm{u}} / \mathrm{A}_{\mathrm{g}} \mathrm{F}_{\mathrm{y}}$} & \multirow[b]{2}{*}{$\mathrm{F}_{\mathrm{u}} / \mathrm{F}_{\mathrm{y}}$} & \multirow[b]{2}{*}{$\begin{array}{c}\text { Yield } \\
\text { plateau } \\
\varepsilon_{\text {epp }}\end{array}$} & \multicolumn{4}{|c|}{ Test } & \multirow{2}{*}{\begin{tabular}{|c|} 
Regression (RII) \\
$\delta_{\mathrm{L}} / \delta_{\mathrm{y}}$ by Eq. \\
$(6.18)$
\end{tabular}} & \multirow[b]{2}{*}{ RIL/Test } \\
\hline & & & & & & & $\begin{array}{c}\text { Max. } \\
\text { deformation } \\
\text { (mm) }\end{array}$ & $\delta_{\mathrm{L}} / \delta_{\mathrm{y}}$ & \begin{tabular}{|c|} 
Adjusted \\
max. \\
deformation \\
$(\mathrm{mm})$
\end{tabular} & $\begin{array}{c}\text { Adjusted } \\
\delta_{1} / \delta_{y}\end{array}$ & & \\
\hline \multirow{6}{*}{ C } & $\mathrm{A} 4075 \mathrm{a}$ & \multirow{2}{*}{419} & \multirow{2}{*}{1.16} & \multirow{6}{*}{1.03} & \multirow{6}{*}{1.45} & \multirow{6}{*}{0.0236} & 9.14 & 5.25 & - & - & \multirow{2}{*}{2.67} & 0.51 \\
\hline & $\mathrm{A} 4075 \mathrm{~b}$ & & & & & & 6.74 & 3.87 & - & $\overline{-}$ & & 0.69 \\
\hline & \begin{tabular}{|l|} 
A6075a \\
\end{tabular} & \multirow{2}{*}{624} & \multirow{2}{*}{1.73} & & & & 7.52 & 2.90 & - & - & \multirow{2}{*}{2.68} & 0.93 \\
\hline & \begin{tabular}{|l|}
$\mathrm{A} 6075 \mathrm{~b}$ \\
\end{tabular} & & & & & & 8.53 & 3.29 & - & - & & 0.82 \\
\hline & A8075a & \multirow{2}{*}{34} & \multirow{2}{*}{2.31} & & & & 11.75 & 3.39 & - & - & \multirow{2}{*}{2.69} & 0.79 \\
\hline & A8075b & & & & & & 9.92 & 2.86 & - & - & & 0.94 \\
\hline
\end{tabular}

Table 6.27 Comparisons of the predicted ductility for materials with different yield strengths

\begin{tabular}{|c|c|c|c|}
\hline \multirow{2}{*}{ Specimen } & $\mathrm{J}(\mathrm{A} / \mathrm{B}), \mathrm{F}_{\mathrm{y}}=300 \mathrm{MPa}$ & $\mathrm{M}(\mathrm{A} / \mathrm{B}), \mathrm{F}_{\mathrm{y}}=350 \mathrm{MPa}$ & \multirow{2}{*}{$(\mathrm{D}) /(\mathrm{E})$} \\
\cline { 2 - 3 } & $\delta_{\mathrm{L}} / \delta_{\mathrm{y}}(\mathrm{D})$ & $\delta_{\mathrm{L}} / \delta_{\mathrm{y}}(\mathrm{E})$ & \\
\hline$(\mathrm{J} / \mathrm{M}) \mathrm{A} 08103$ & 2.17 & 2.47 & 0.88 \\
\hline$(\mathrm{J} / \mathrm{M}) \mathrm{A} 08123$ & 8.00 & 8.25 & 0.97 \\
\hline$(\mathrm{J} / \mathrm{M}) \mathrm{B} 08103$ & 2.47 & 2.68 & 0.92 \\
\hline (J/M)B08123 & 17.00 & 17.77 & 0.96 \\
\hline
\end{tabular}




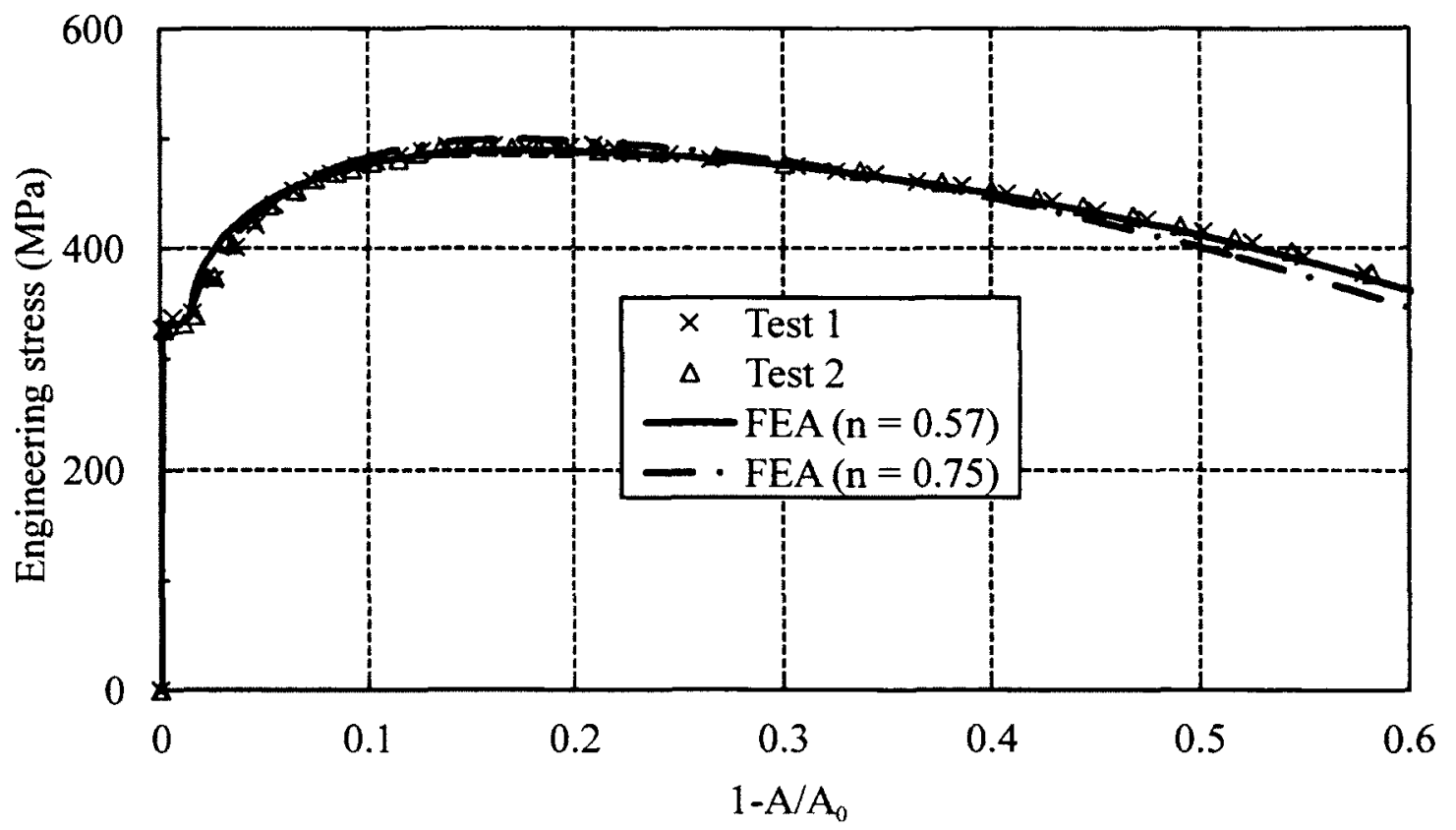

Figure 6.1 Test and predicted engineering stress versus cross-sectional area change curves for monotonic tension tests of material $\mathrm{F}$ with different values of flow stress parameter $n(\eta=0.75)$

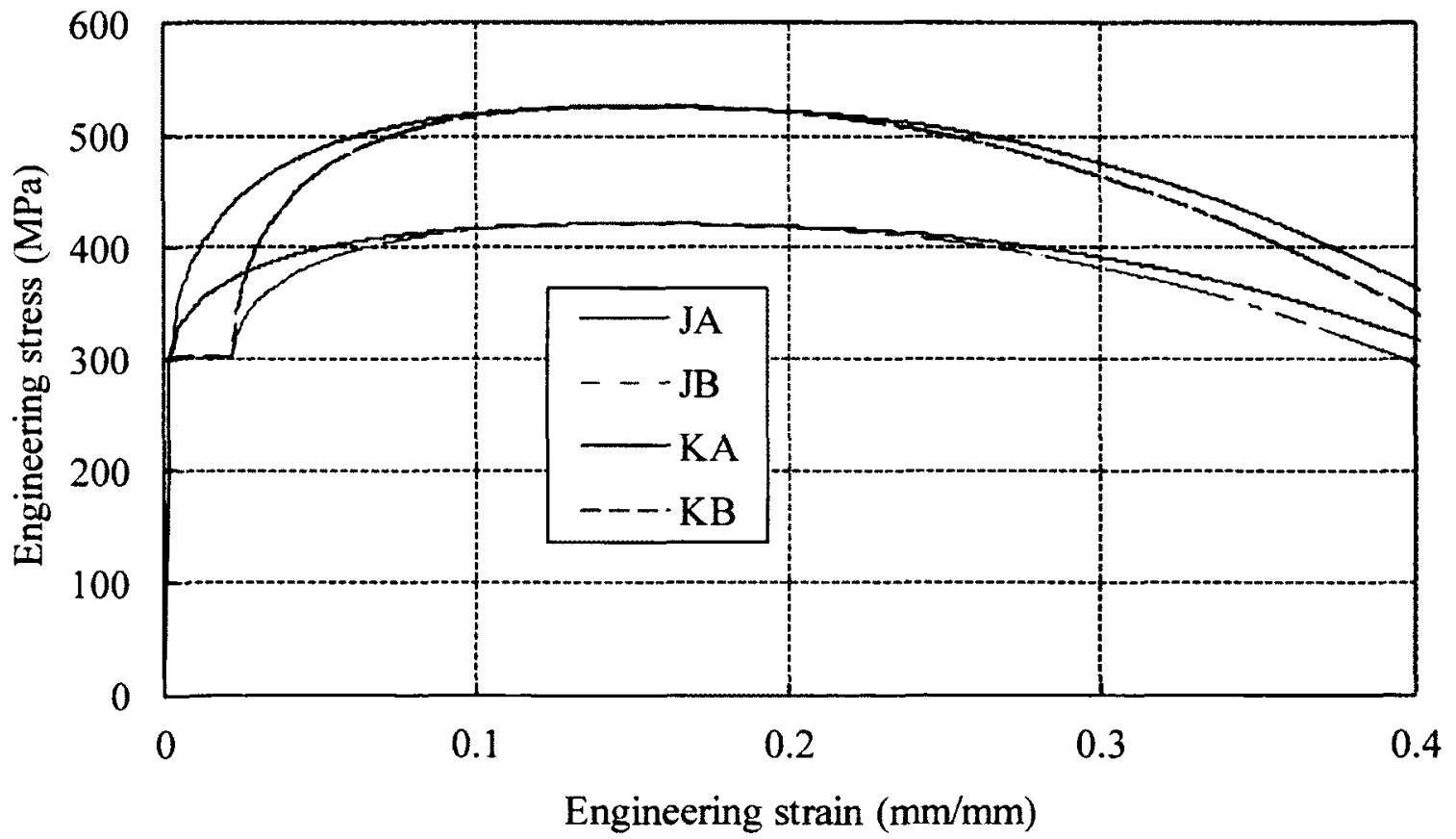

Figure 6.2 FEA simulations of engineering stress versus engineering strain curves for monotonic tension tests of four materials considered in the parametric study, JA, JB, KA and KB 


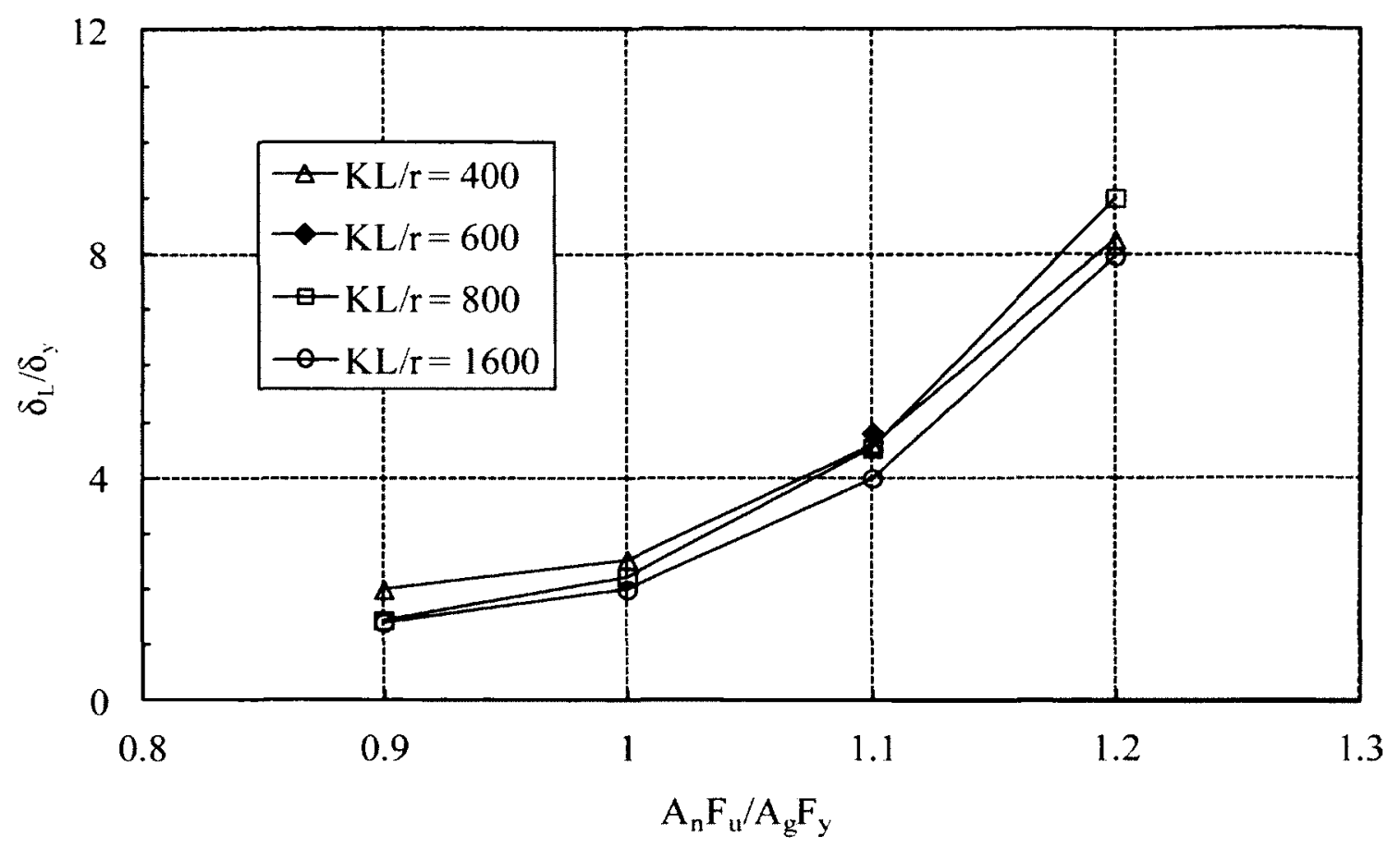

Figure 6.3 Predicted ductility versus $\mathrm{A}_{\mathrm{n}} \mathrm{F}_{\mathrm{u}} / \mathrm{A}_{\mathrm{g}} \mathrm{F}_{\mathrm{y}}$ for $4 \mathrm{~m}$ braces of material JA

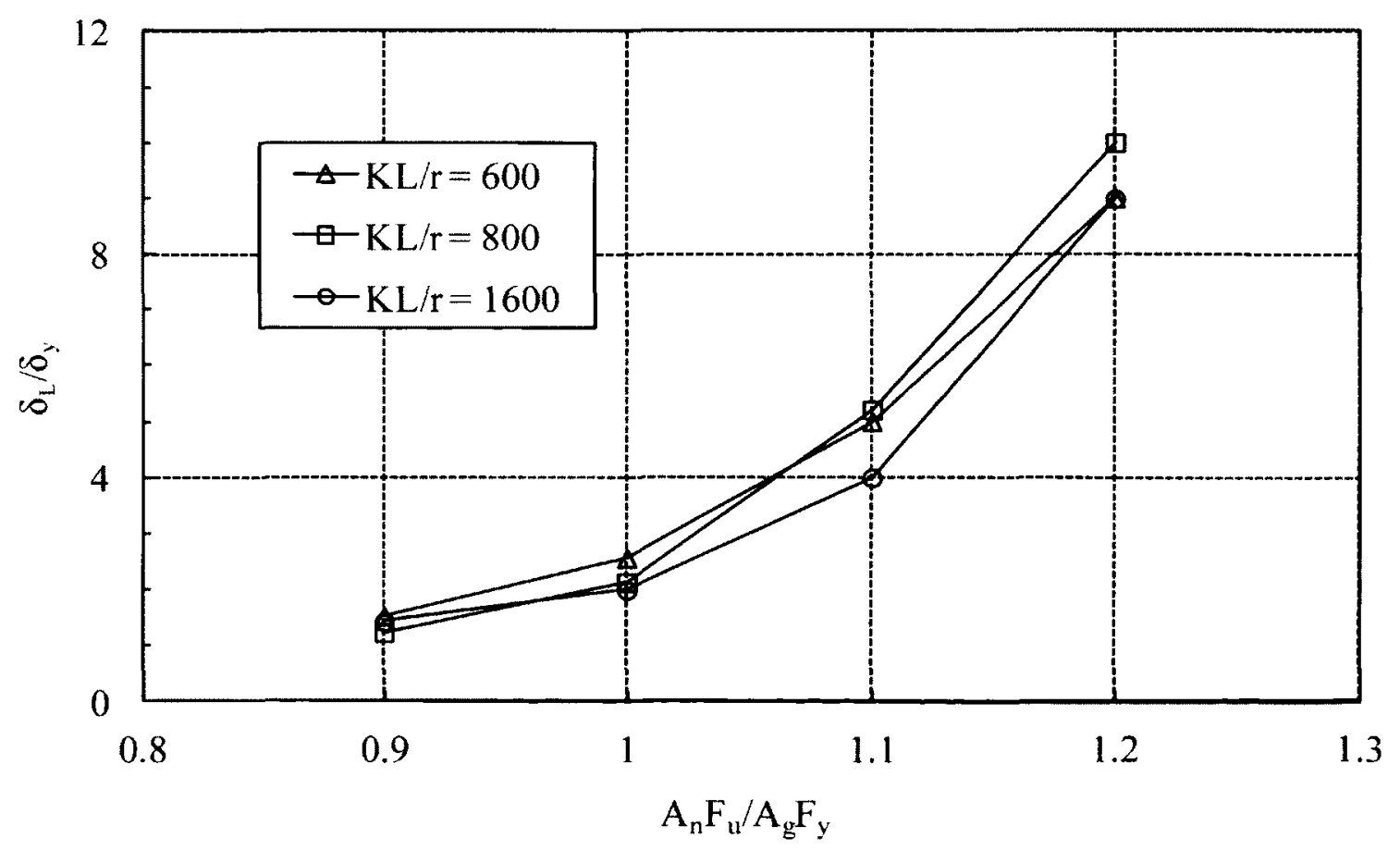

Figure 6.4 Predicted ductility versus $\mathrm{A}_{\mathrm{n}} \mathrm{F}_{\mathrm{u}} / \mathrm{A}_{\mathrm{g}} \mathrm{F}_{\mathrm{y}}$ for $6 \mathrm{~m}$ braces of material JA 


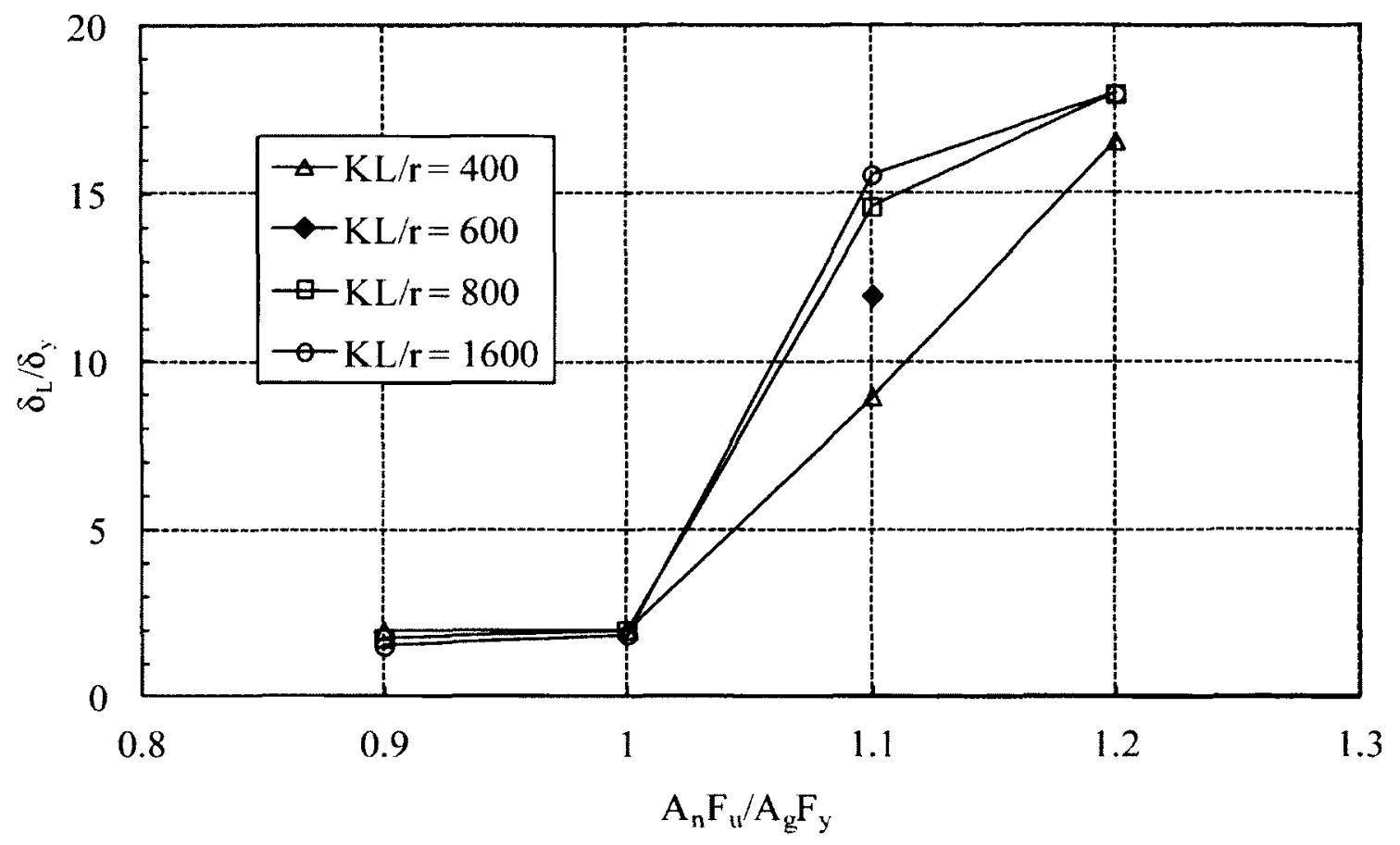

Figure 6.5 Predicted ductility versus $\mathrm{A}_{\mathrm{n}} \mathrm{F}_{\mathrm{u}} / \mathrm{A}_{\mathrm{g}} \mathrm{F}_{\mathrm{y}}$ for $4 \mathrm{~m}$ braces of material JB

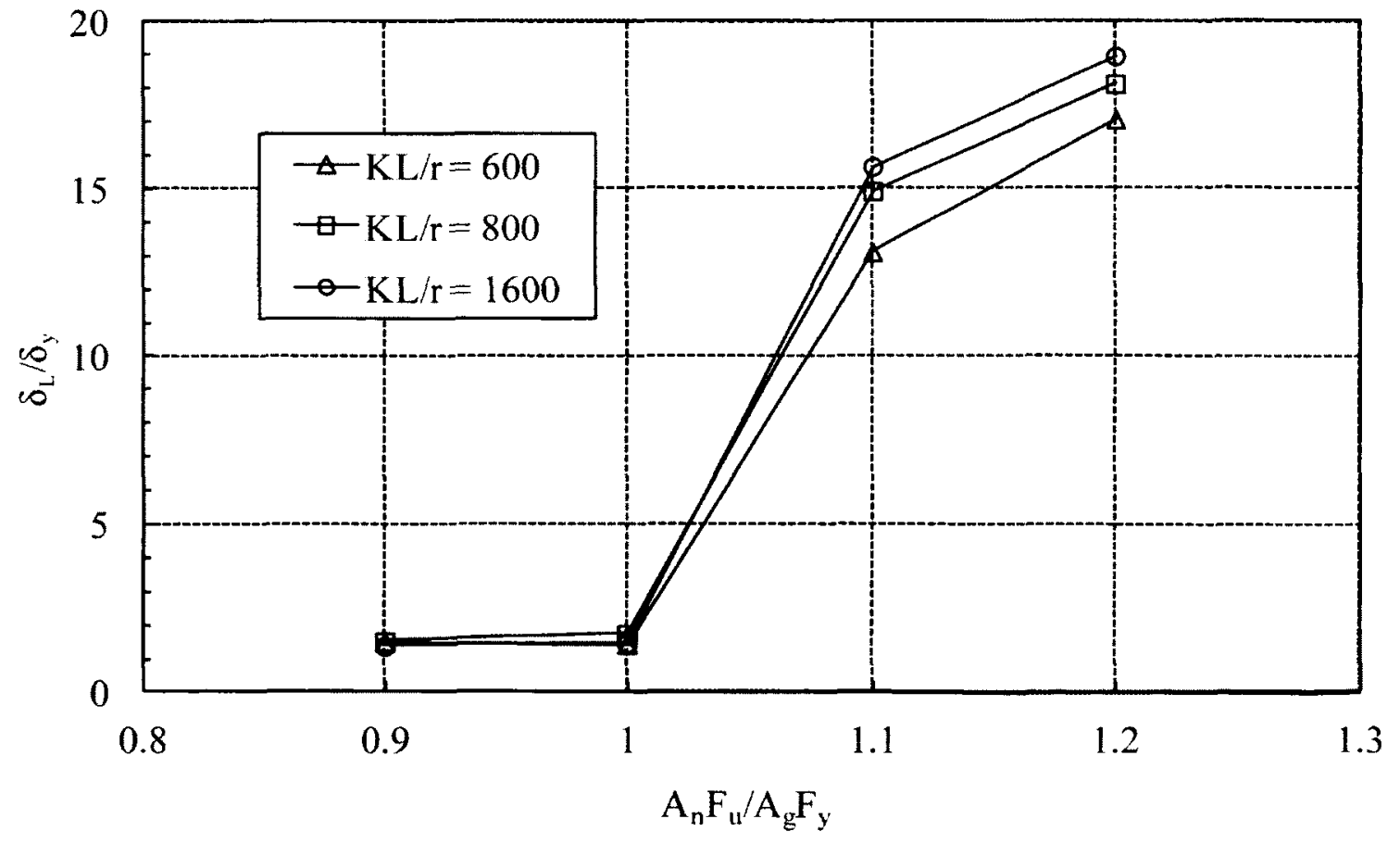

Figure 6.6 Predicted ductility versus $\mathrm{A}_{\mathrm{n}} \mathrm{F}_{\mathrm{u}} / \mathrm{A}_{\mathrm{g}} \mathrm{F}_{\mathrm{y}}$ for $6 \mathrm{~m}$ braces of material JB 


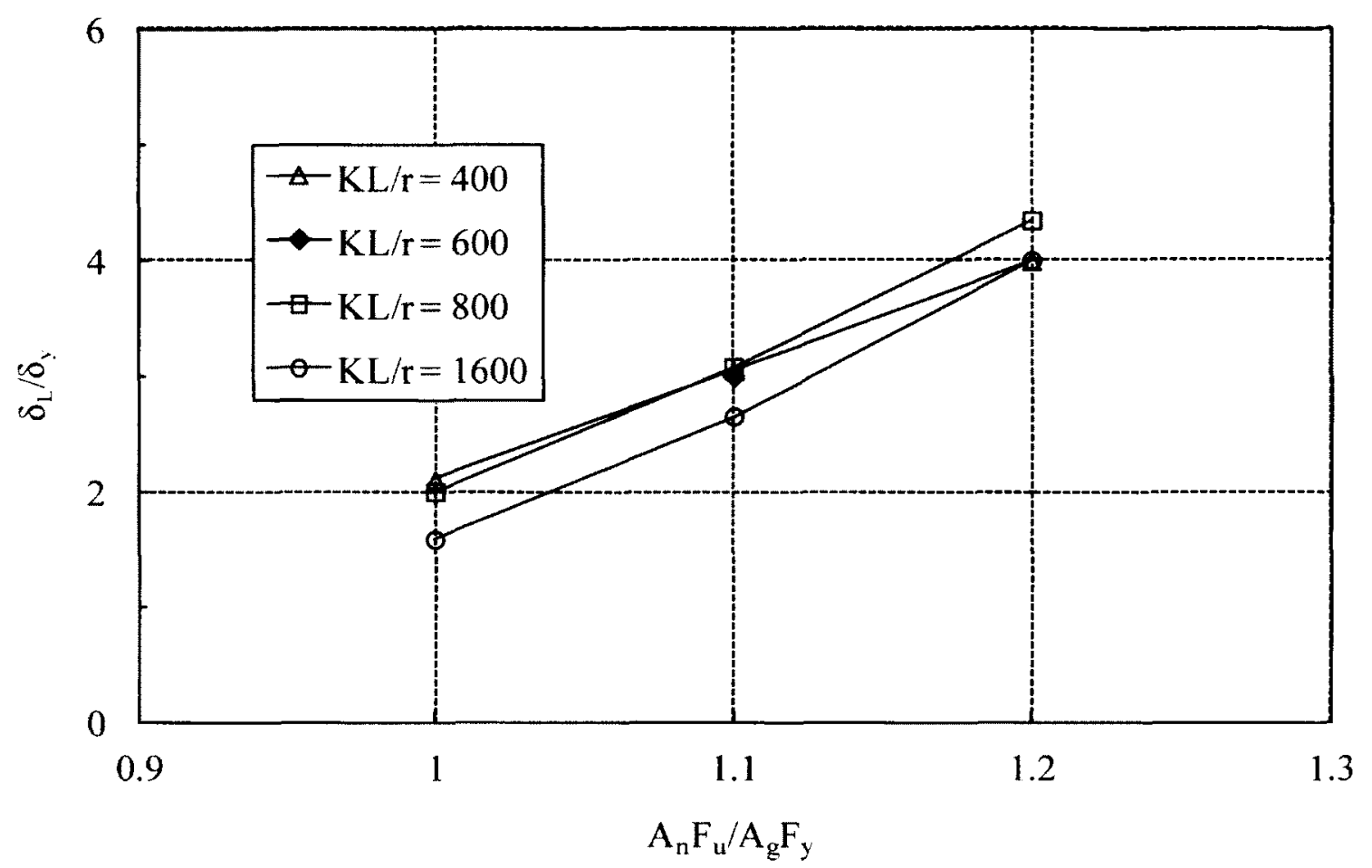

Figure 6.7 Predicted ductility versus $A_{n} F_{u} / A_{g} F_{y}$ for $4 \mathrm{~m}$ braces of material KA

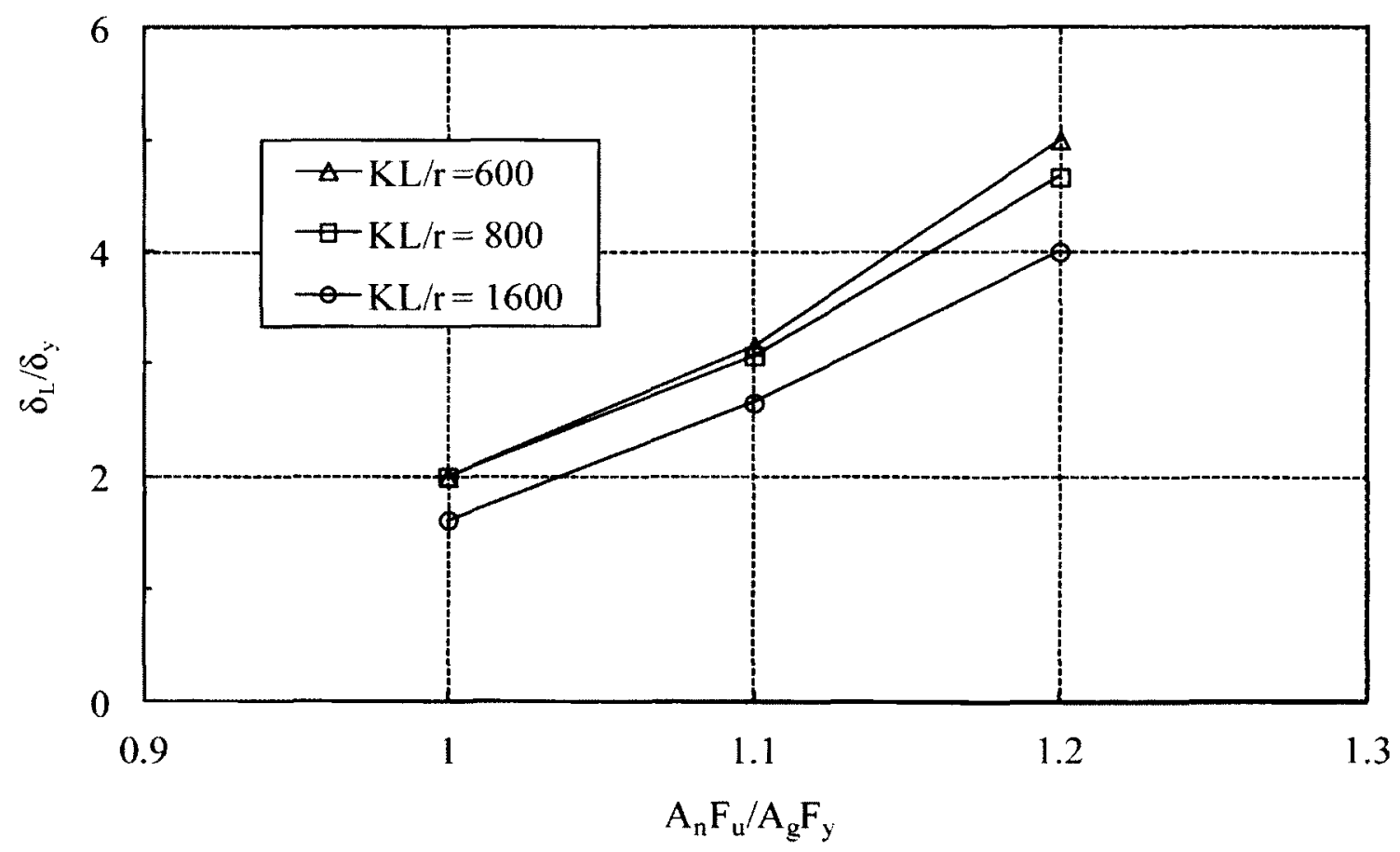

Figure 6.8 Predicted ductility versus $\mathrm{A}_{\mathrm{n}} \mathrm{F}_{\mathrm{u}} / \mathrm{A}_{\mathrm{g}} \mathrm{F}_{\mathrm{y}}$ for $6 \mathrm{~m}$ braces of material KA 


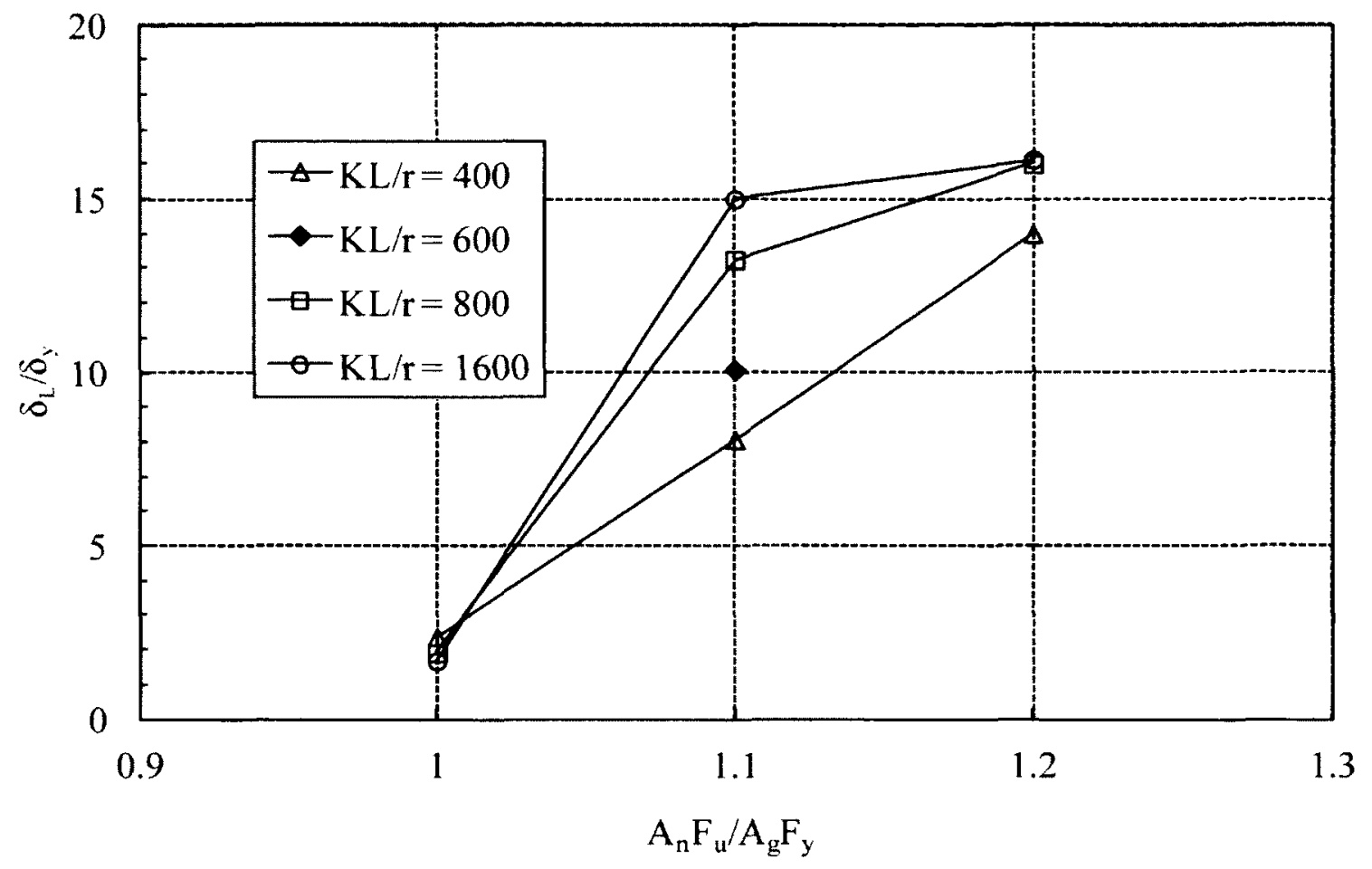

Figure 6.9 Predicted ductility versus $\mathrm{A}_{\mathrm{n}} \mathrm{F}_{\mathrm{u}} / \mathrm{A}_{\mathrm{g}} \mathrm{F}_{\mathrm{y}}$ for $4 \mathrm{~m}$ braces of material $\mathrm{KB}$

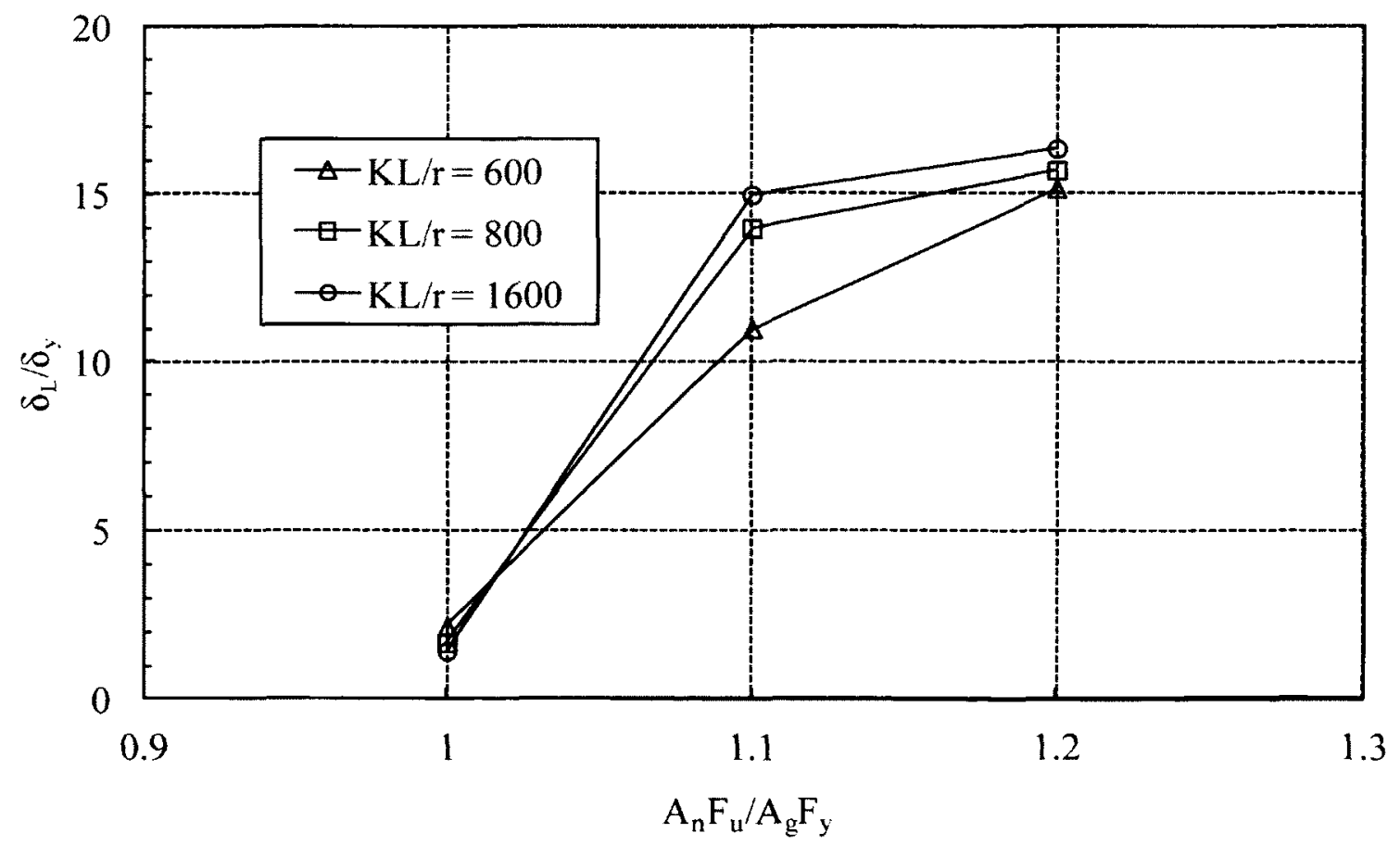

Figure 6.10 Predicted ductility versus $\mathrm{A}_{\mathrm{n}} \mathrm{F}_{\mathrm{u}} / \mathrm{A}_{\mathrm{g}} \mathrm{F}_{\mathrm{y}}$ for $6 \mathrm{~m}$ braces of material KB 


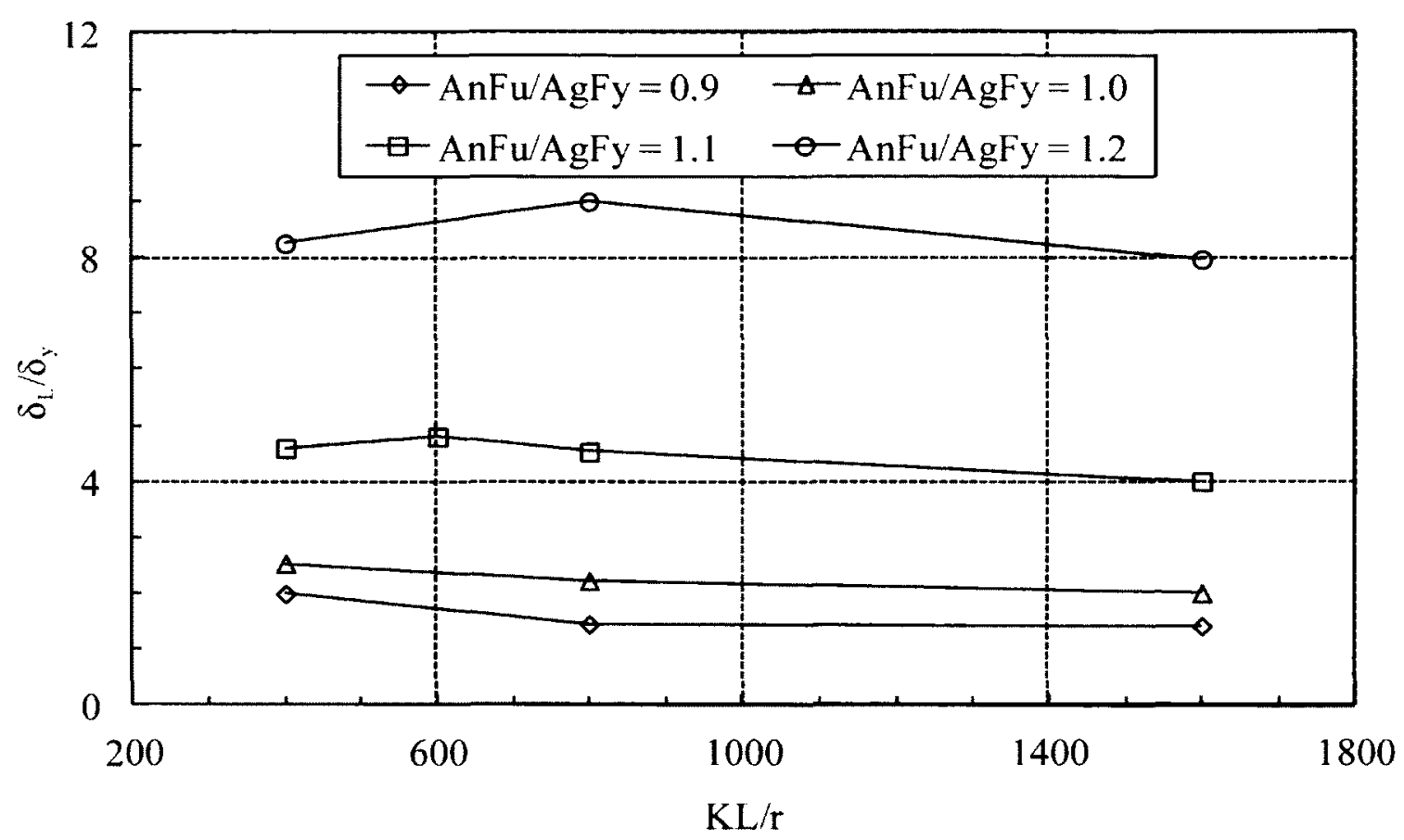

Figure 6.11 Predicted ductility versus slenderness ratio for $4 \mathrm{~m}$ braces of material JA

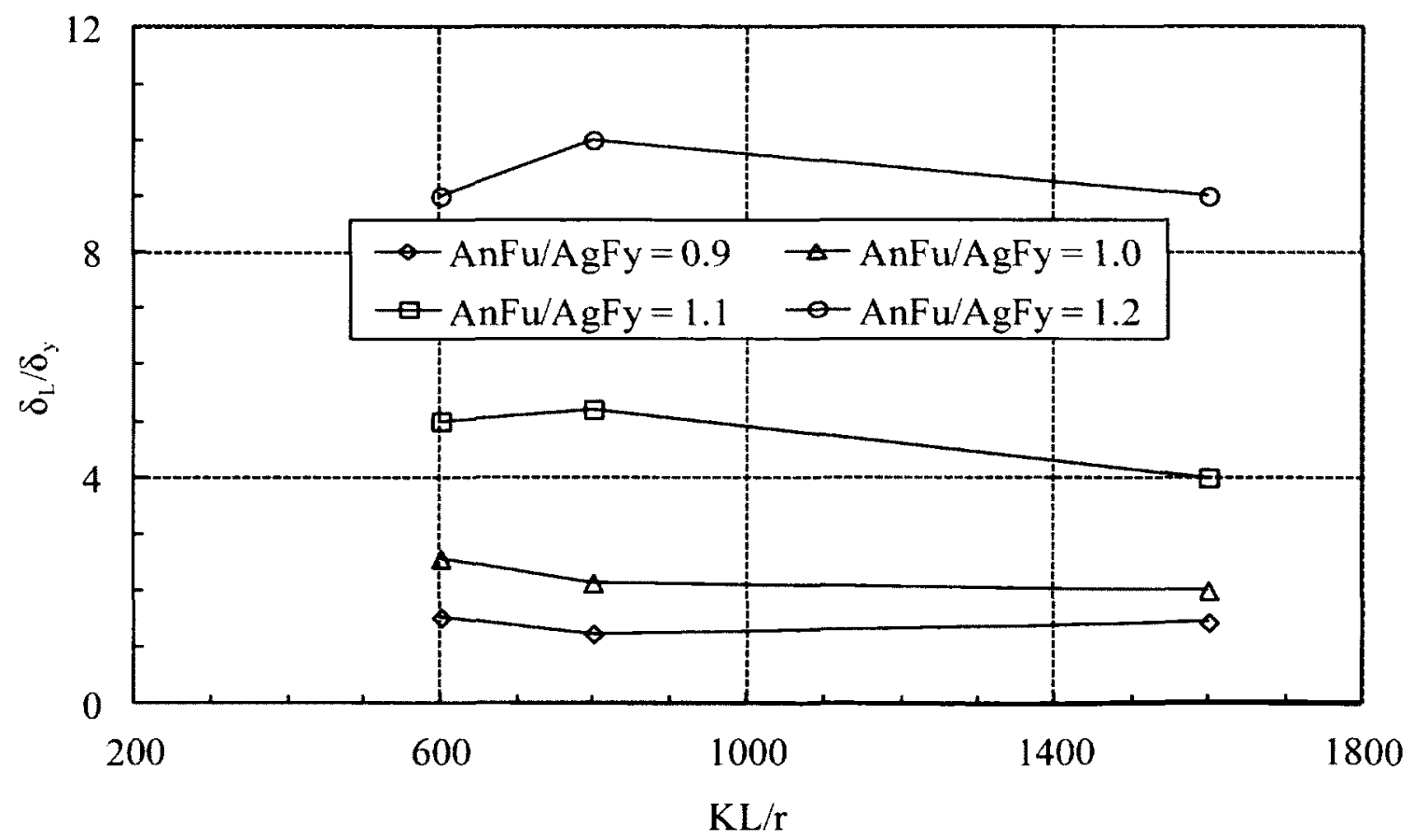

Figure 6.12 Predicted ductility versus slenderness ratio for $6 \mathrm{~m}$ braces of material JA 


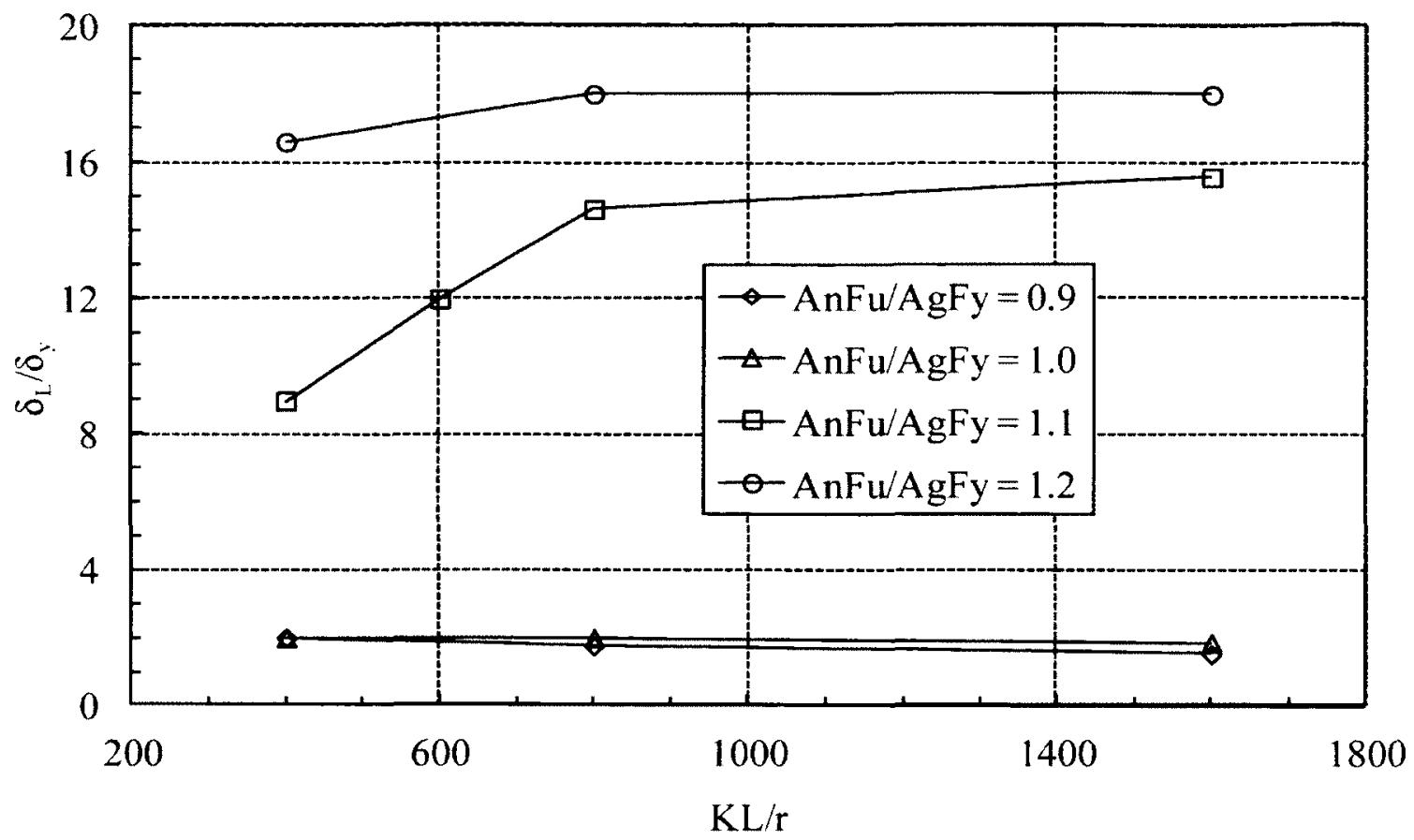

Figure 6.13 Predicted ductility versus slenderness ratio for $4 \mathrm{~m}$ braces of material JB

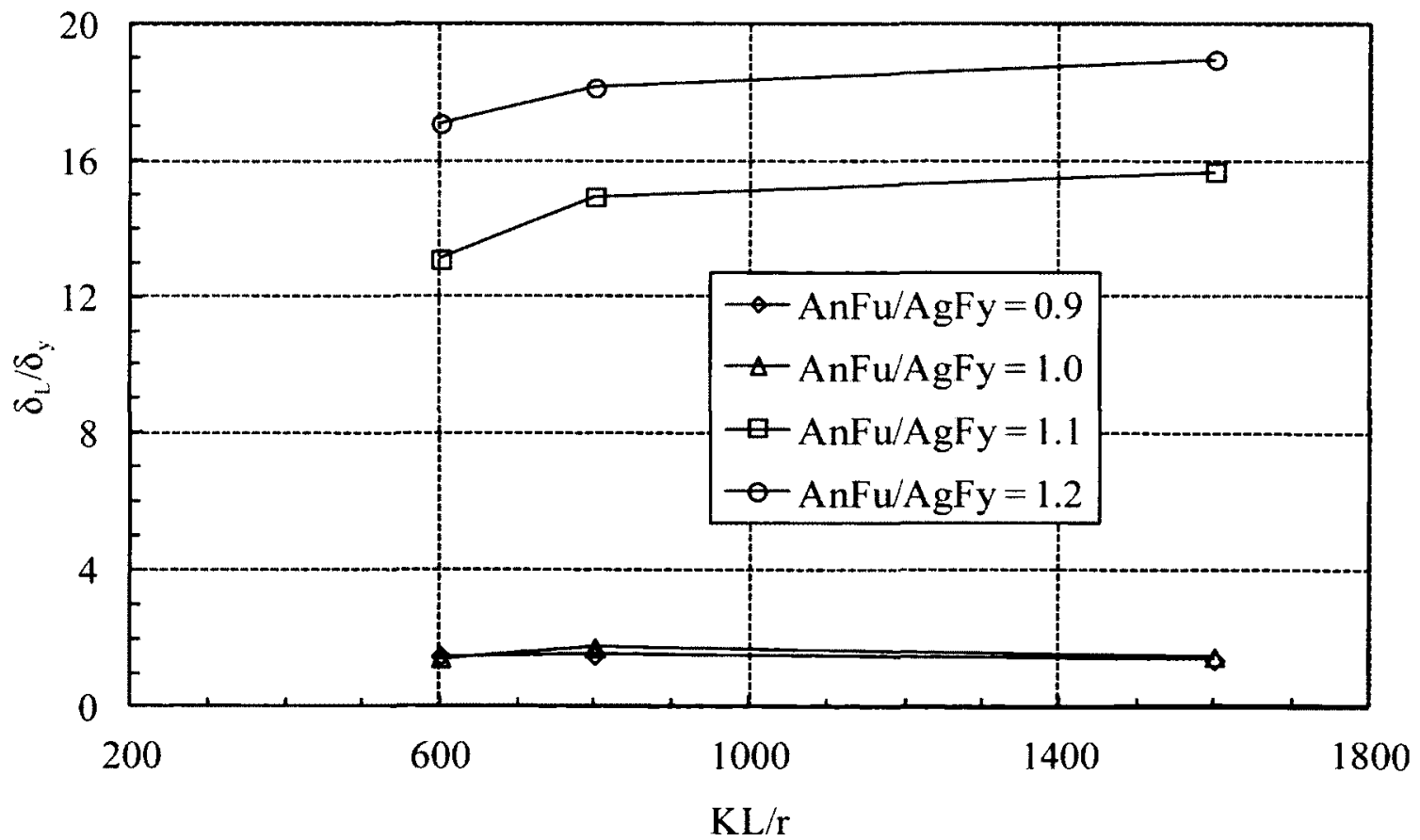

Figure 6.14 Predicted ductility versus slenderness ratio for $6 \mathrm{~m}$ braces of material JB 


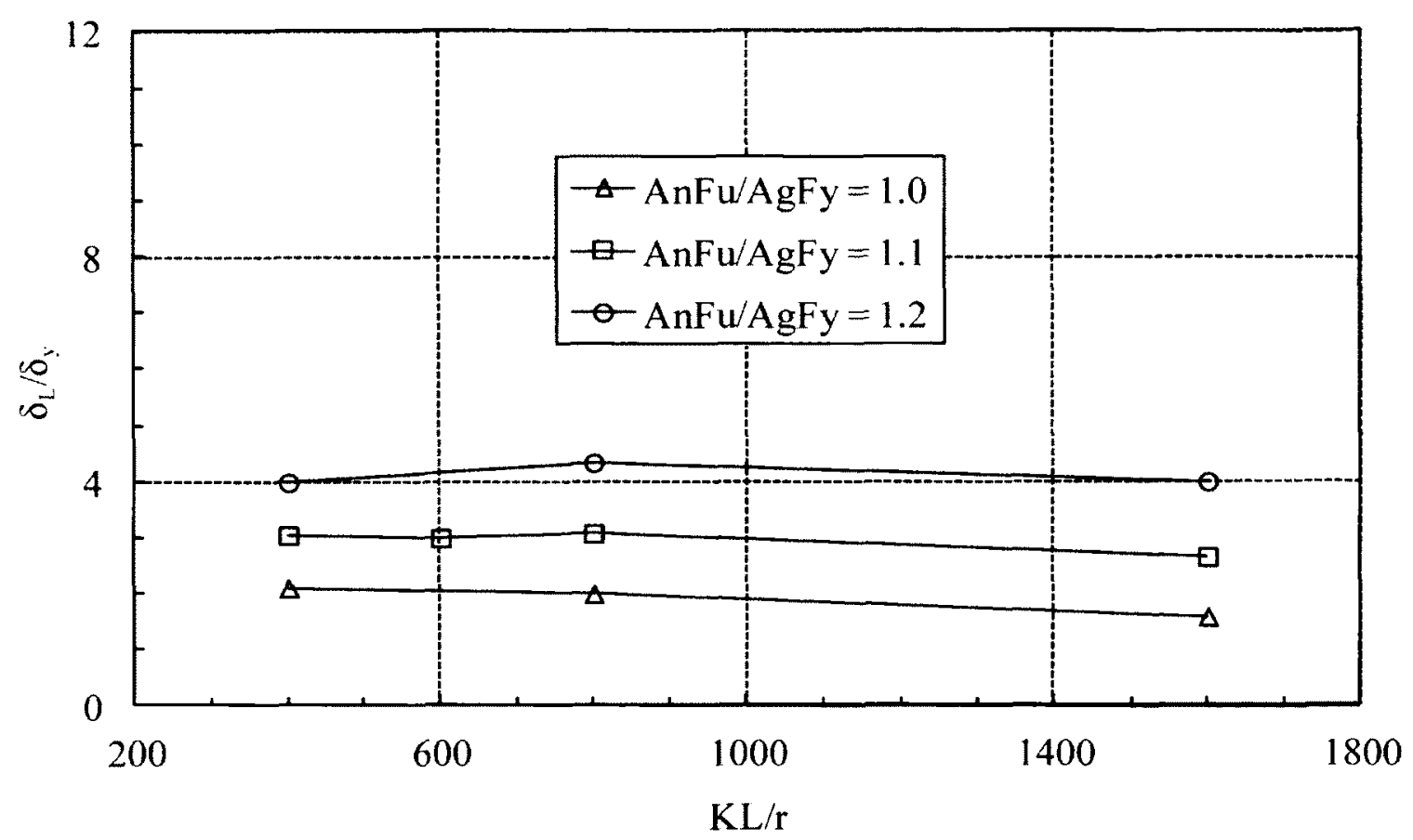

Figure 6.15 Predicted ductility versus slenderness ratio for $4 \mathrm{~m}$ braces of material KA

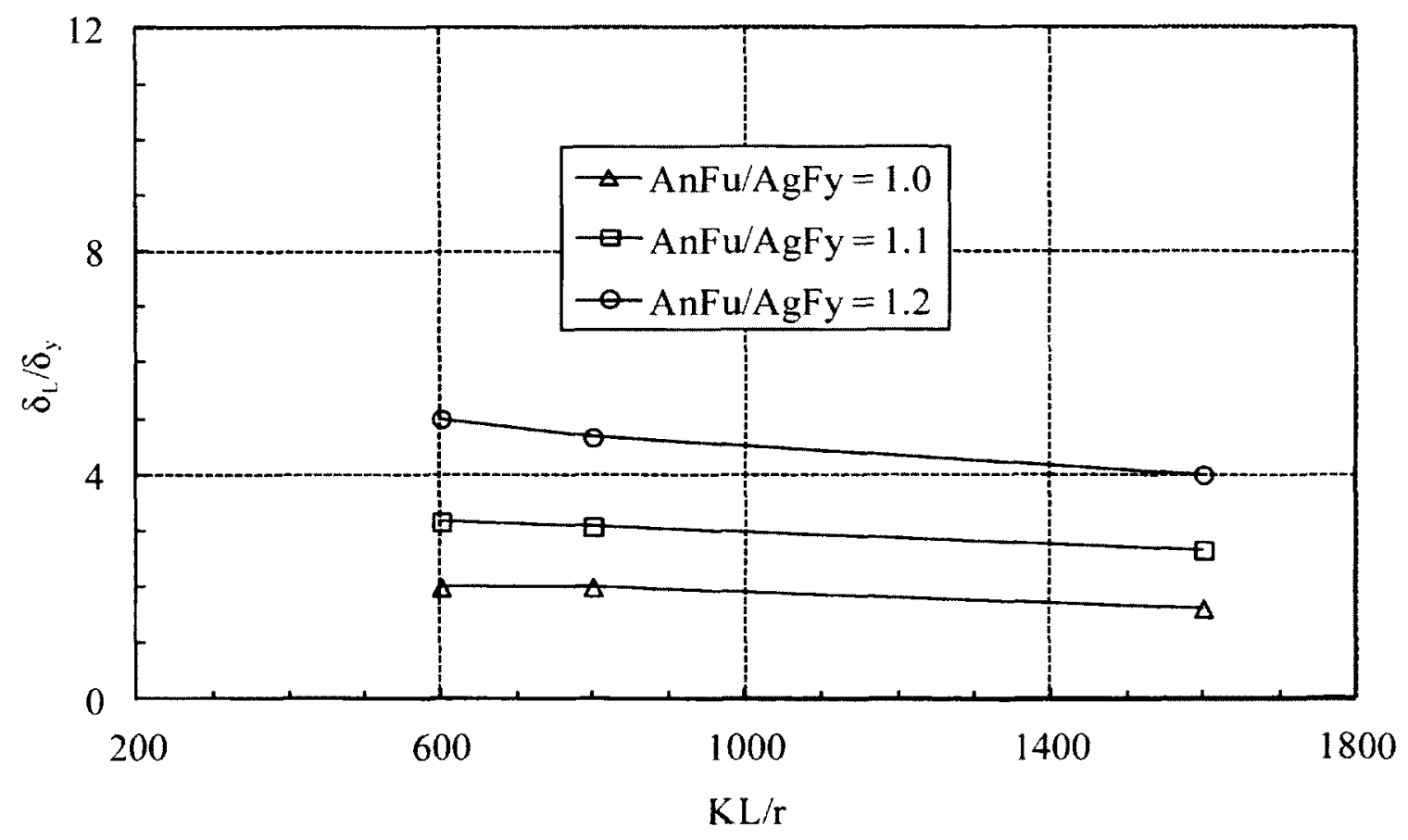

Figure 6.16 Predicted ductility versus slenderness ratio for $6 \mathrm{~m}$ braces of material KA 


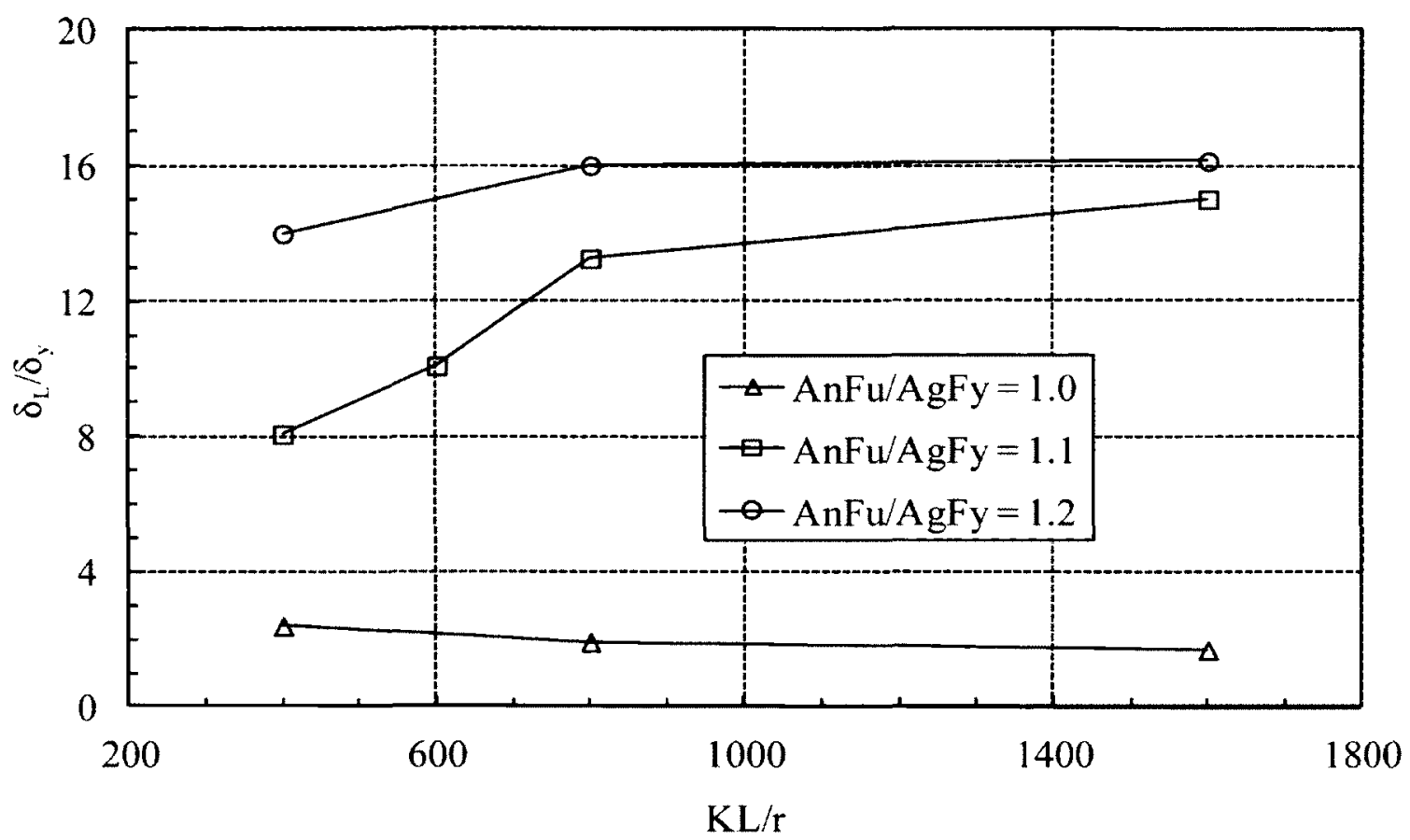

Figure 6.17 Predicted ductility versus slenderness ratio for $4 \mathrm{~m}$ braces of material KB

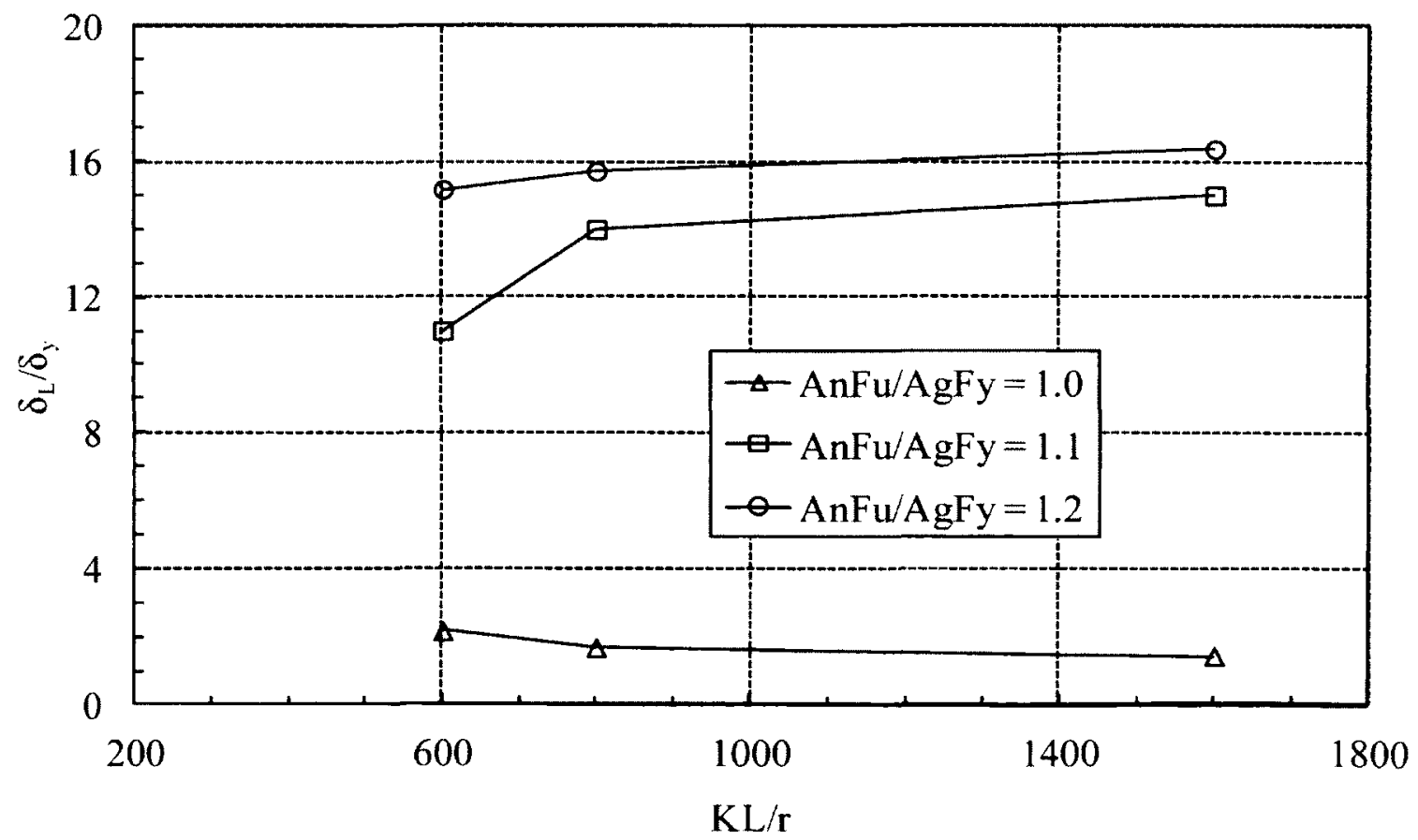

Figure 6.18 Predicted ductility versus slenderness ratio for $6 \mathrm{~m}$ braces of material KB 


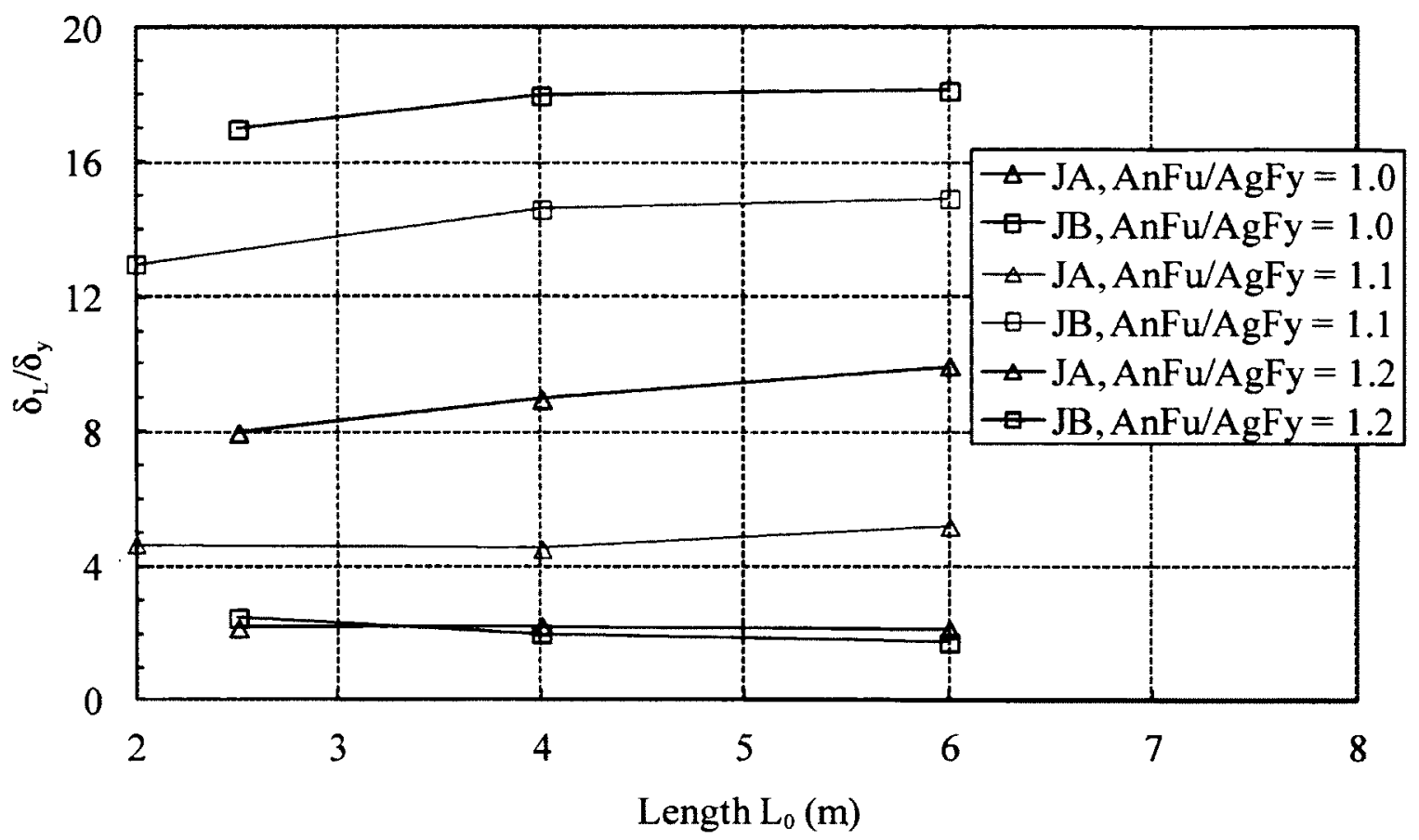

Figure 6.19 Predicted ductility versus brace length for $\mathrm{KL} / \mathrm{r}=800$

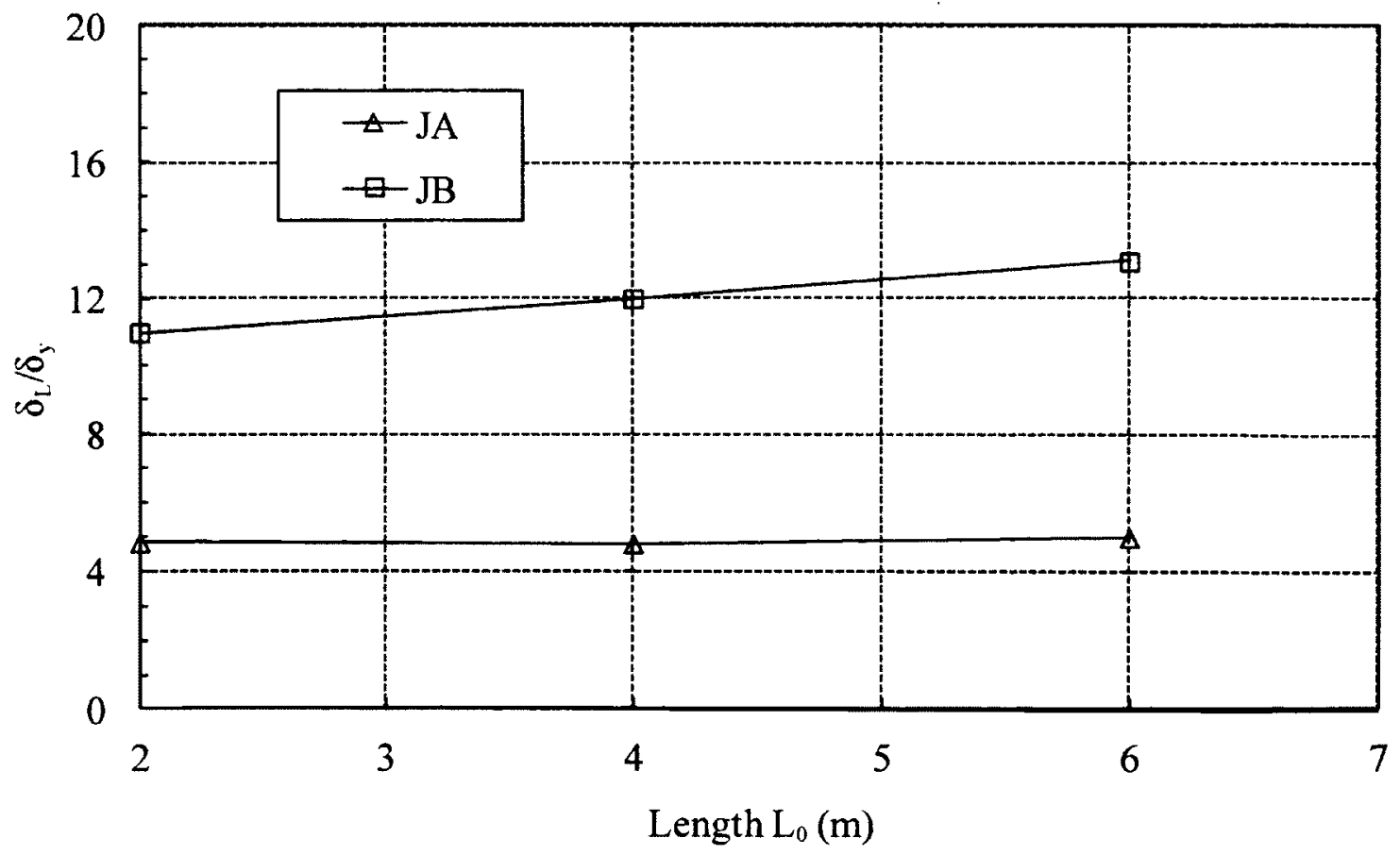

Figure 6.20 Predicted ductility versus brace length for $\mathrm{KL} / \mathrm{r}=600$ and $\mathrm{A}_{\mathrm{n}} \mathrm{F}_{\mathrm{u}} / \mathrm{A}_{\mathrm{g}} \mathrm{F}_{\mathrm{y}}=1.1$ 


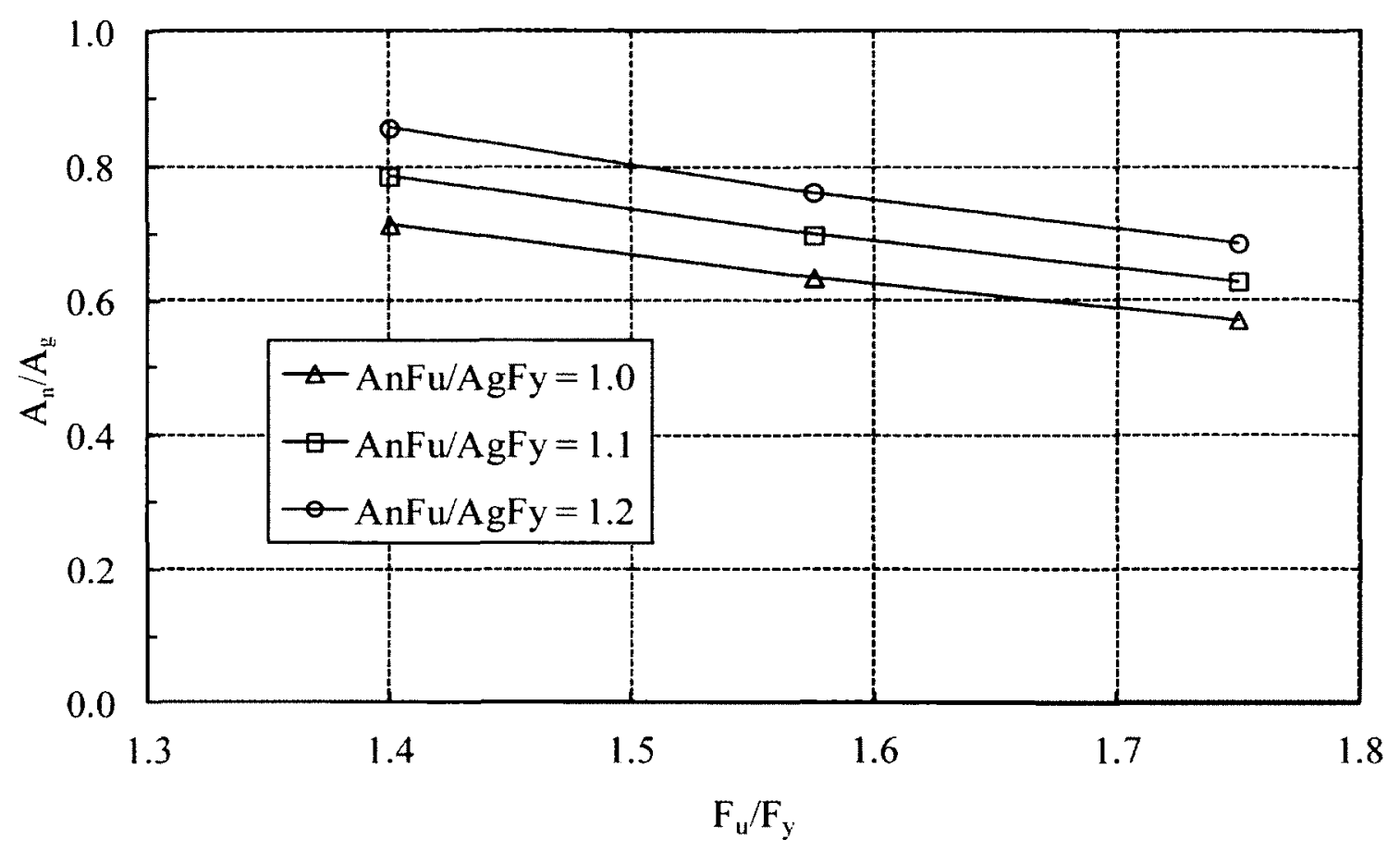

Figure 6.21 $\quad \mathrm{A}_{\mathrm{n}} / \mathrm{A}_{\mathrm{g}}$ versus $\mathrm{F}_{\mathrm{u}} / \mathrm{F}_{\mathrm{y}}$ at the same thickness for different $\mathrm{A}_{n} \mathrm{~F}_{\mathrm{u}} / \mathrm{A}_{\mathrm{g}} \mathrm{F}_{\mathrm{y}}$

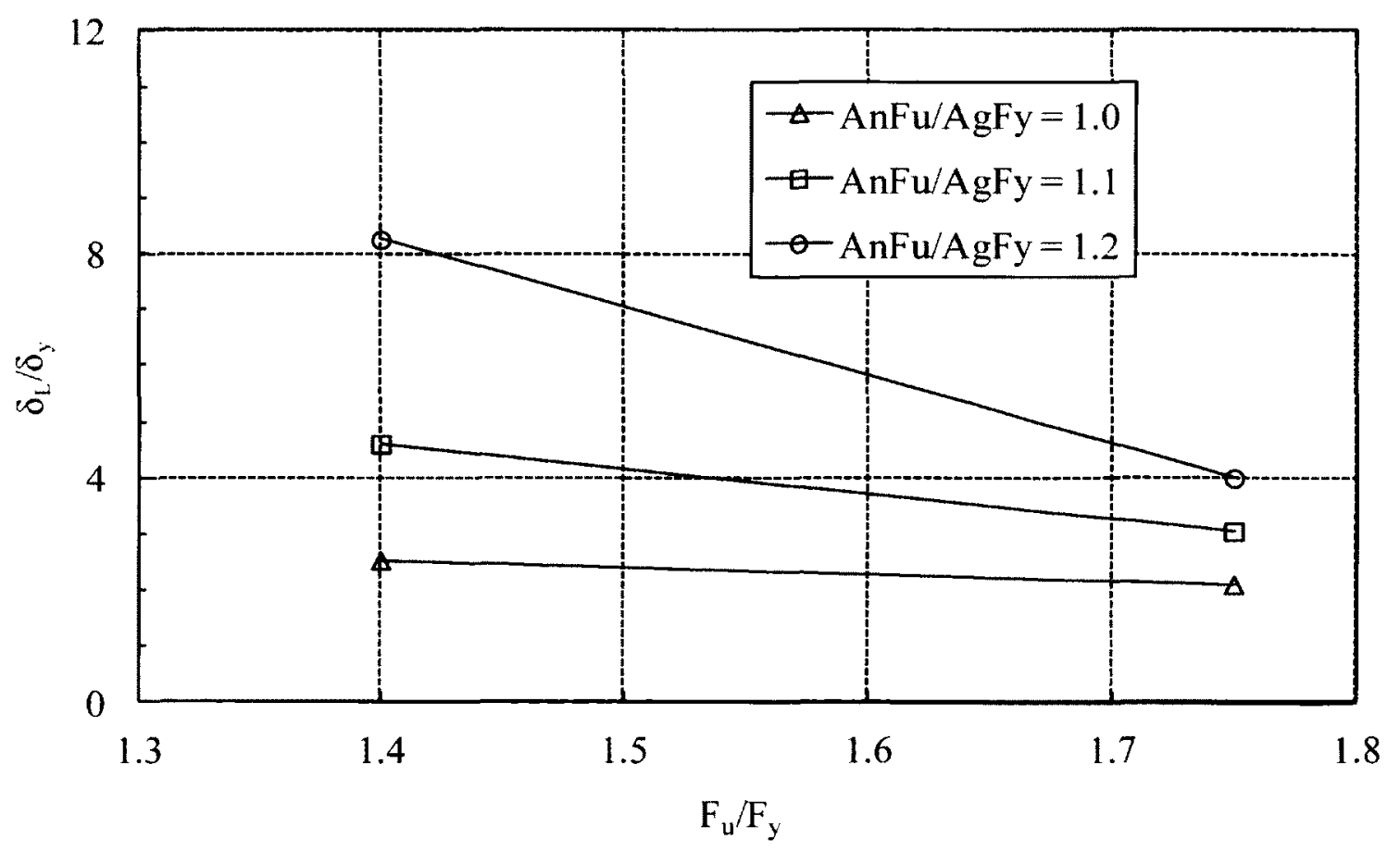

Figure 6.22 Predicted ductility versus $F_{d} / F_{y}$ for braces with $4 \mathrm{~m}$ length and $\mathrm{KL} / \mathrm{r}=400$ of materials without a yield plateau, JA and KA 


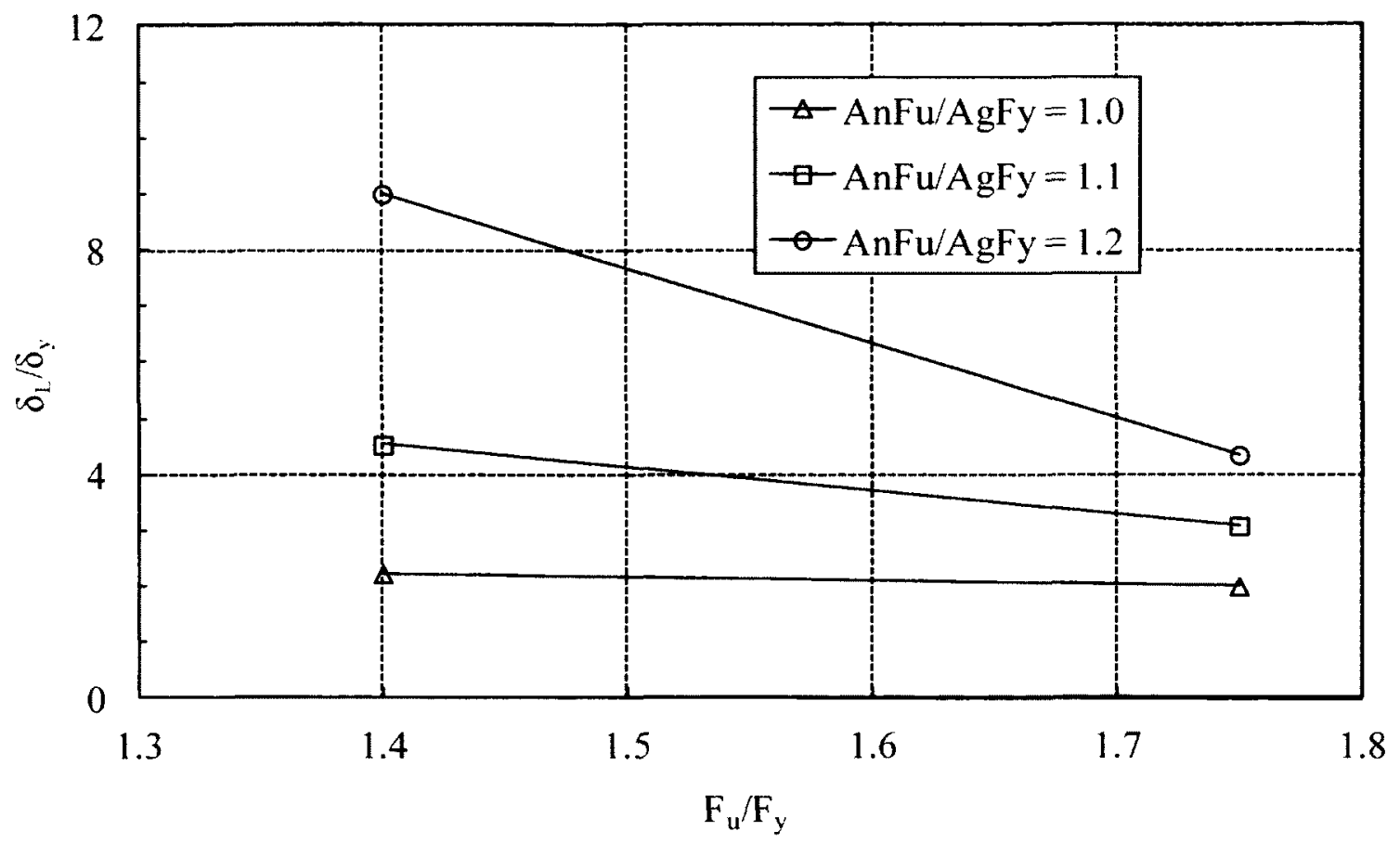

Figure 6.23 Predicted ductility versus $F_{u} / F_{y}$ for braces with $4 \mathrm{~m}$ length and $\mathrm{KL} / \mathrm{r}=800$ of materials without a yield plateau, JA and KA

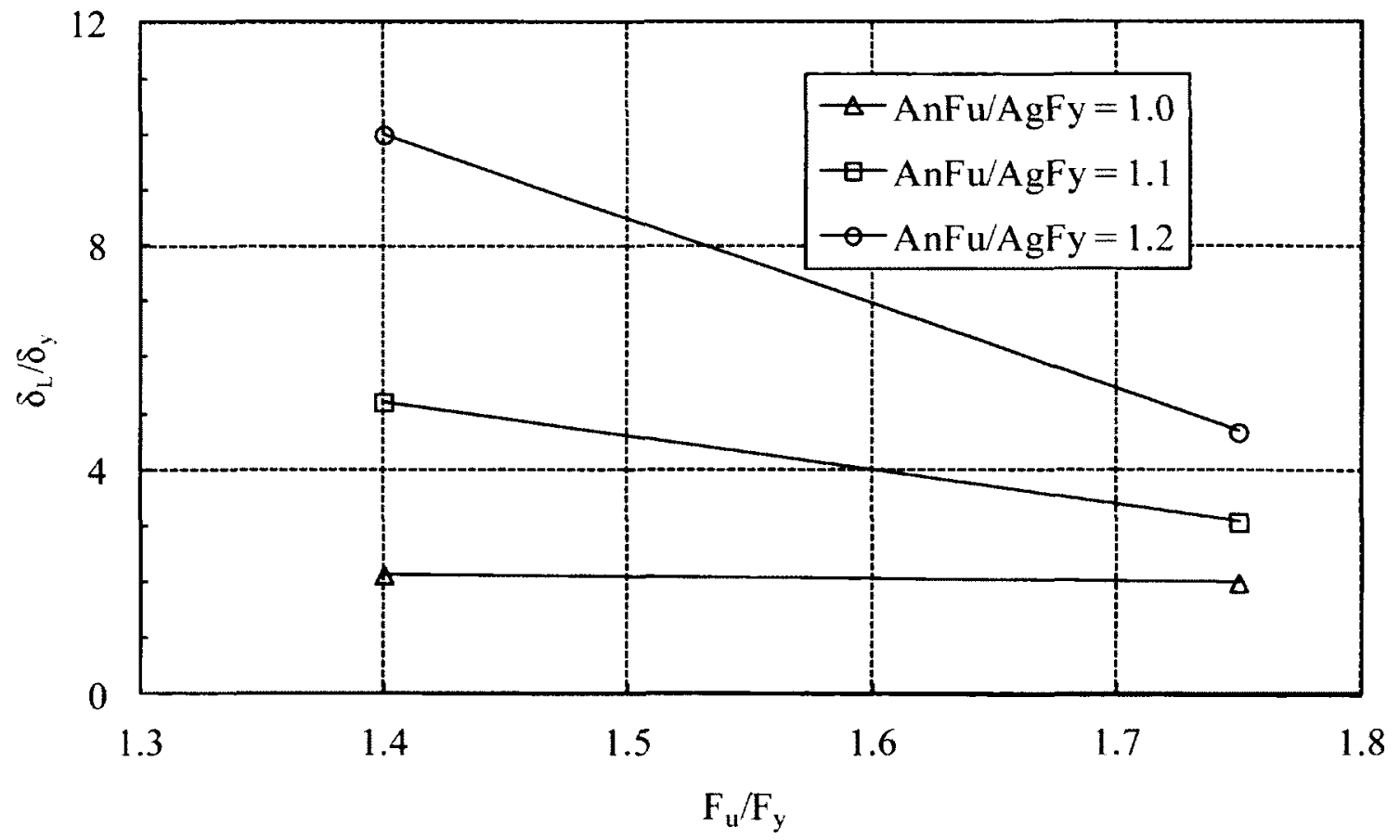

Figure 6.24 Predicted ductility versus $F_{u} / F_{y}$ for braces with $6 \mathrm{~m}$ length and $\mathrm{KL} / \mathrm{r}=800$ of materials without a yield plateau, JA and $\mathrm{KA}$ 


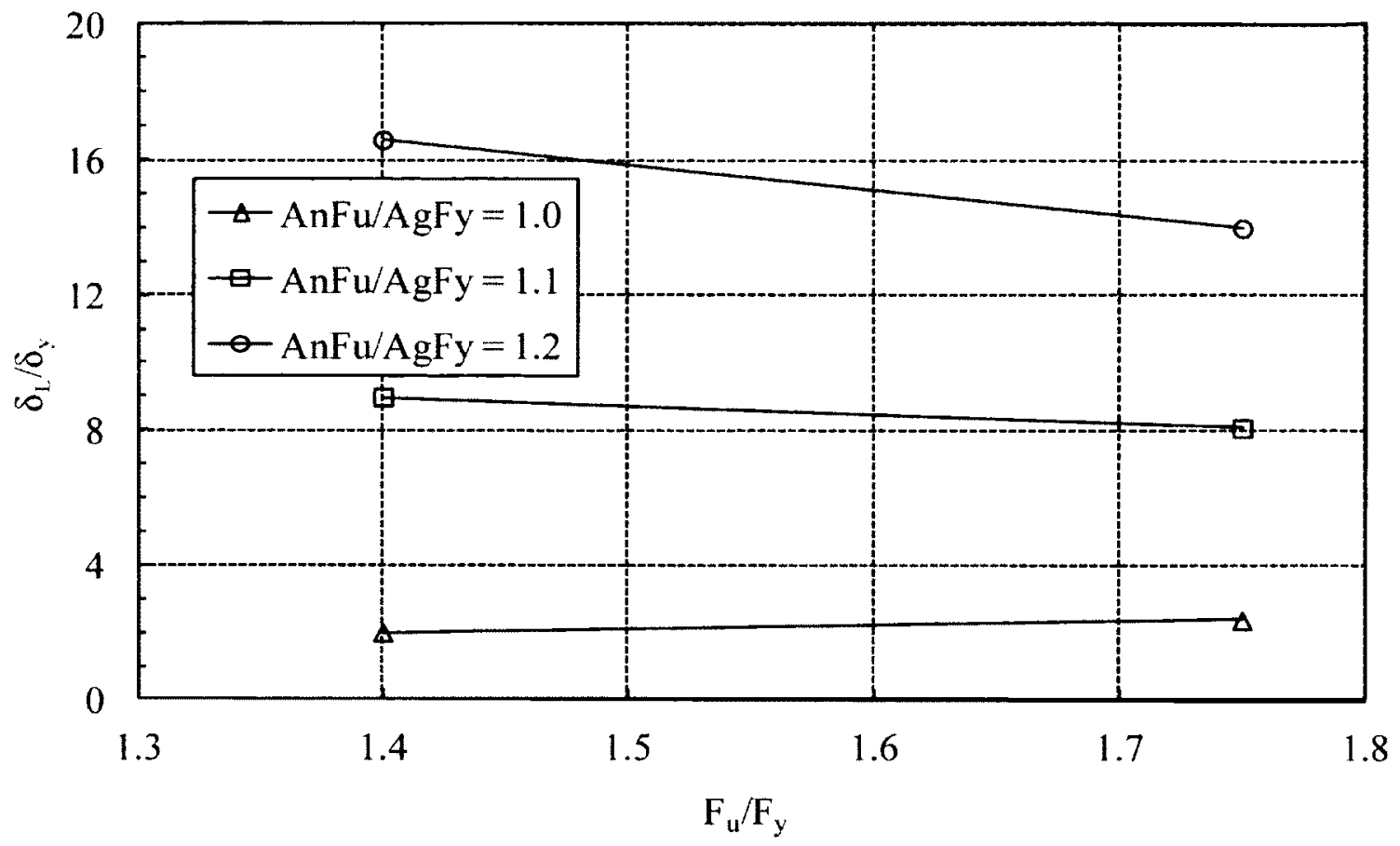

Figure 6.25 Predicted ductility versus $F_{u} / F_{y}$ for braces with $4 \mathrm{~m}$ length and $\mathrm{KL} / \mathrm{r}=400$ of materials with a yield plateau, JB and KB

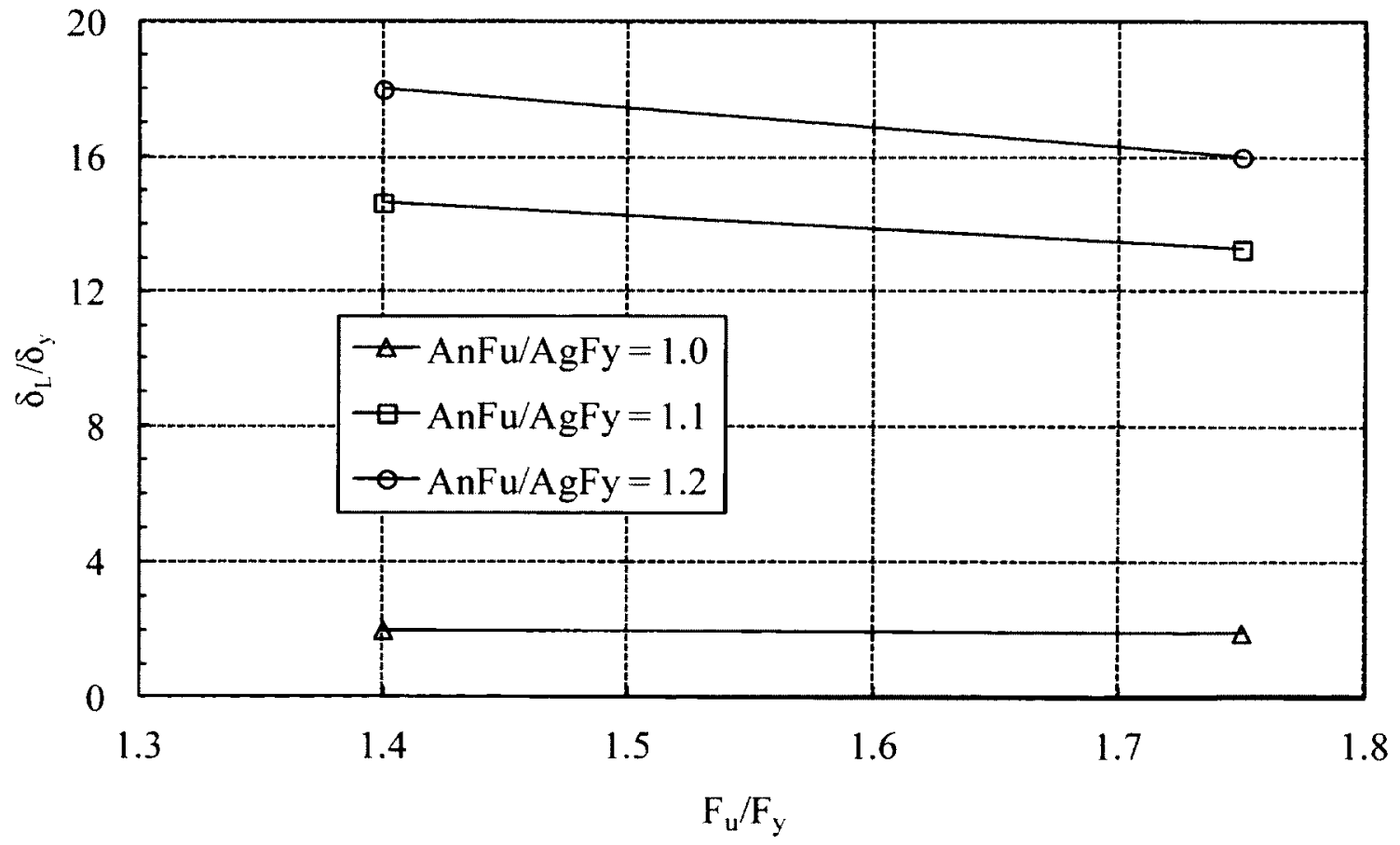

Figure 6.26 Predicted ductility versus $F_{u} / F_{y}$ for braces with $4 \mathrm{~m}$ length and $\mathrm{KL} / \mathrm{r}=800$ of materials with a yield plateau, JB and KB 


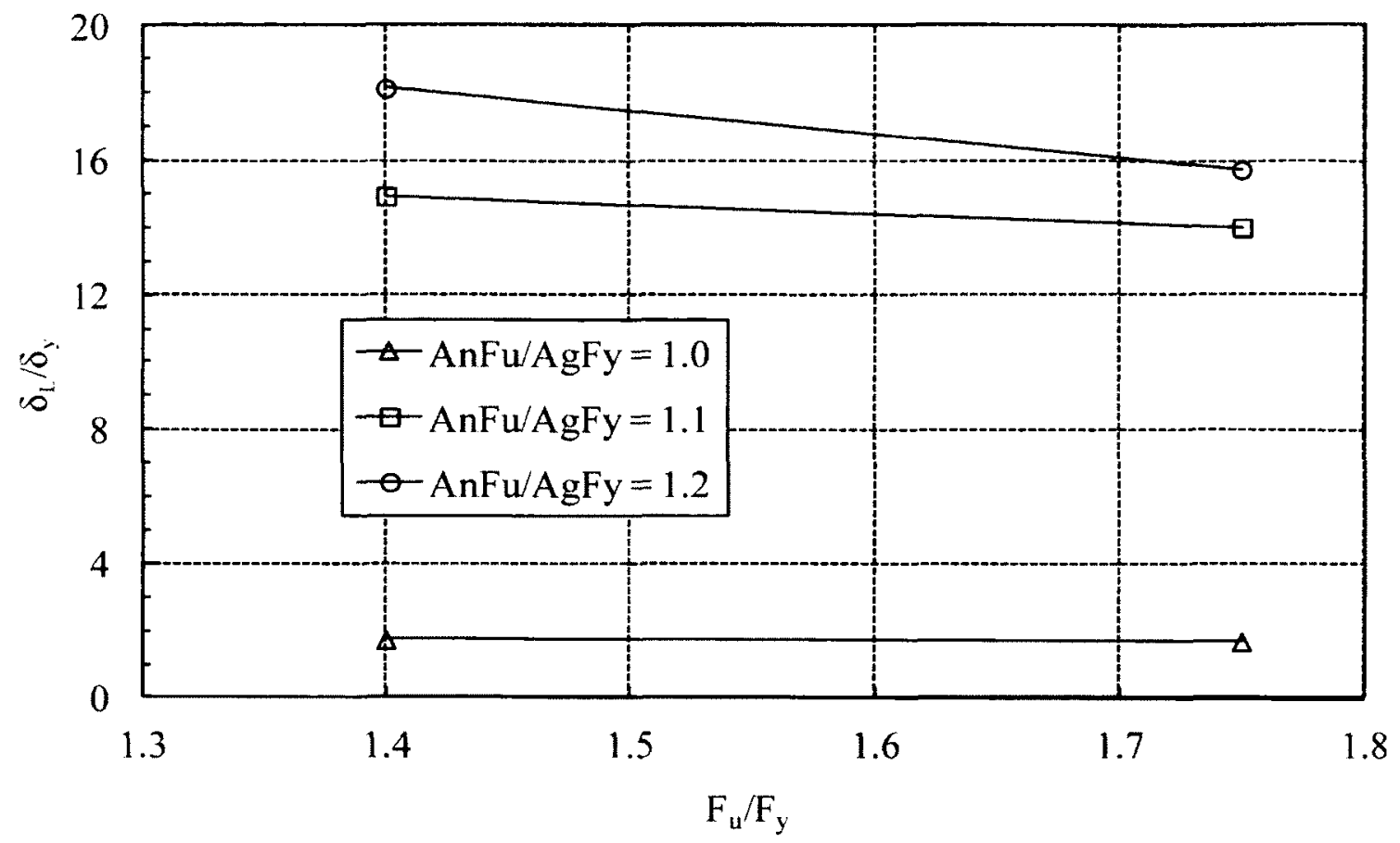

Figure 6.27 Predicted ductility versus $F_{u} / F_{y}$ for braces with $6 \mathrm{~m}$ length and $\mathrm{KL} / \mathrm{r}=800$ of materials with a yield plateau, JB and $\mathrm{KB}$

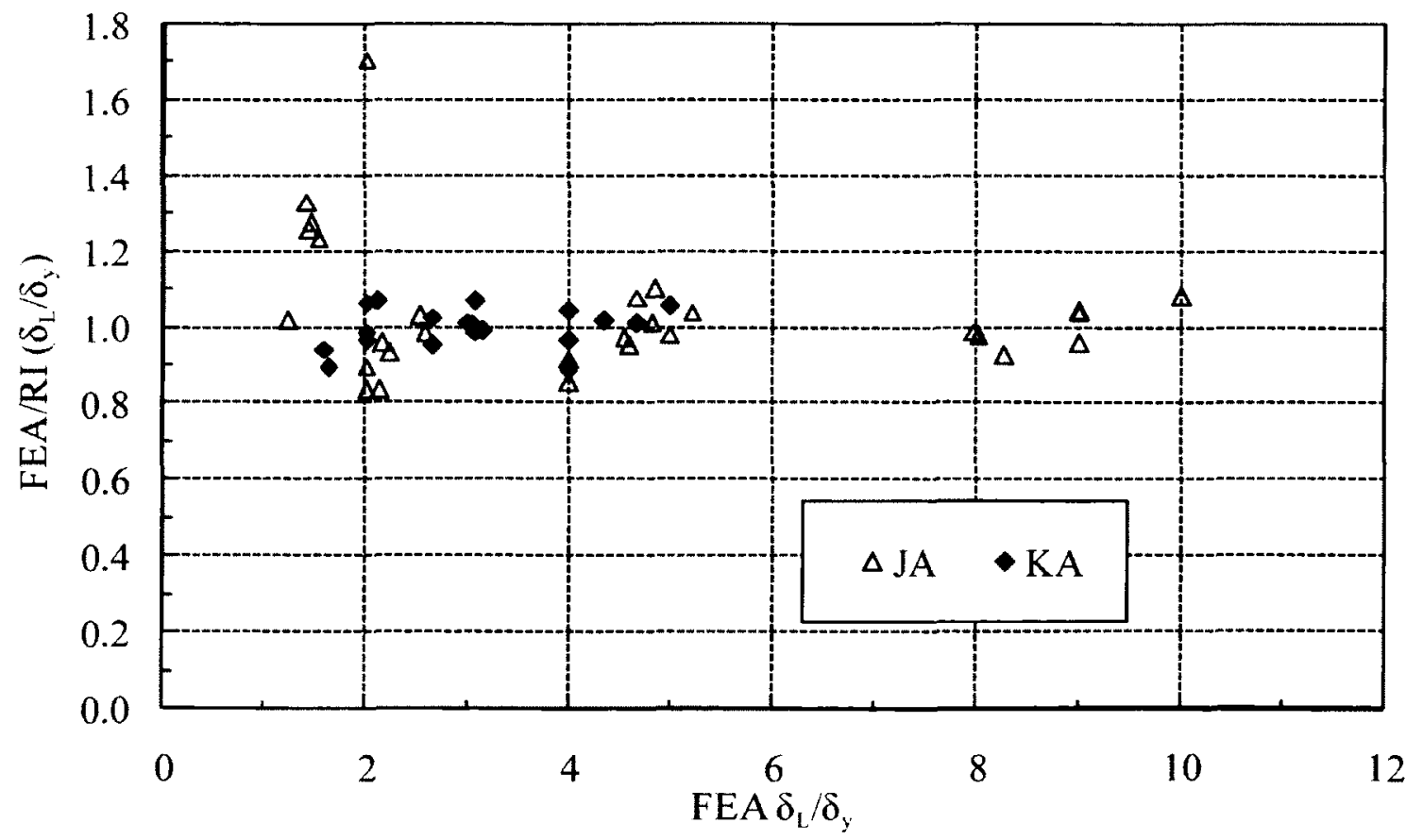

Figure 6.28 FEA/RI of ductility for materials without a yield plateau, JA and KA 


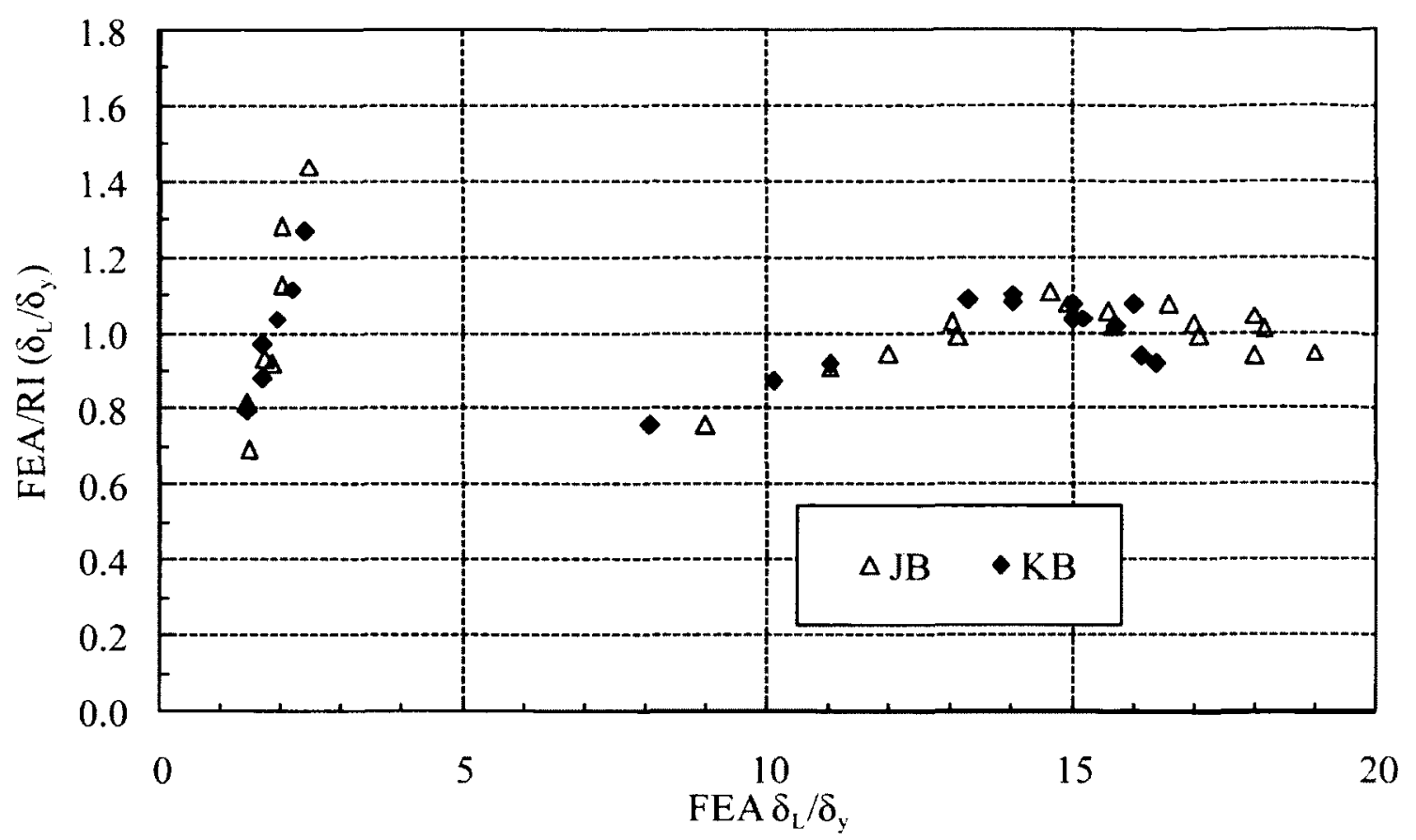

Figure 6.29 FEA/RI of ductility for materials with a yield plateau, JB and KB

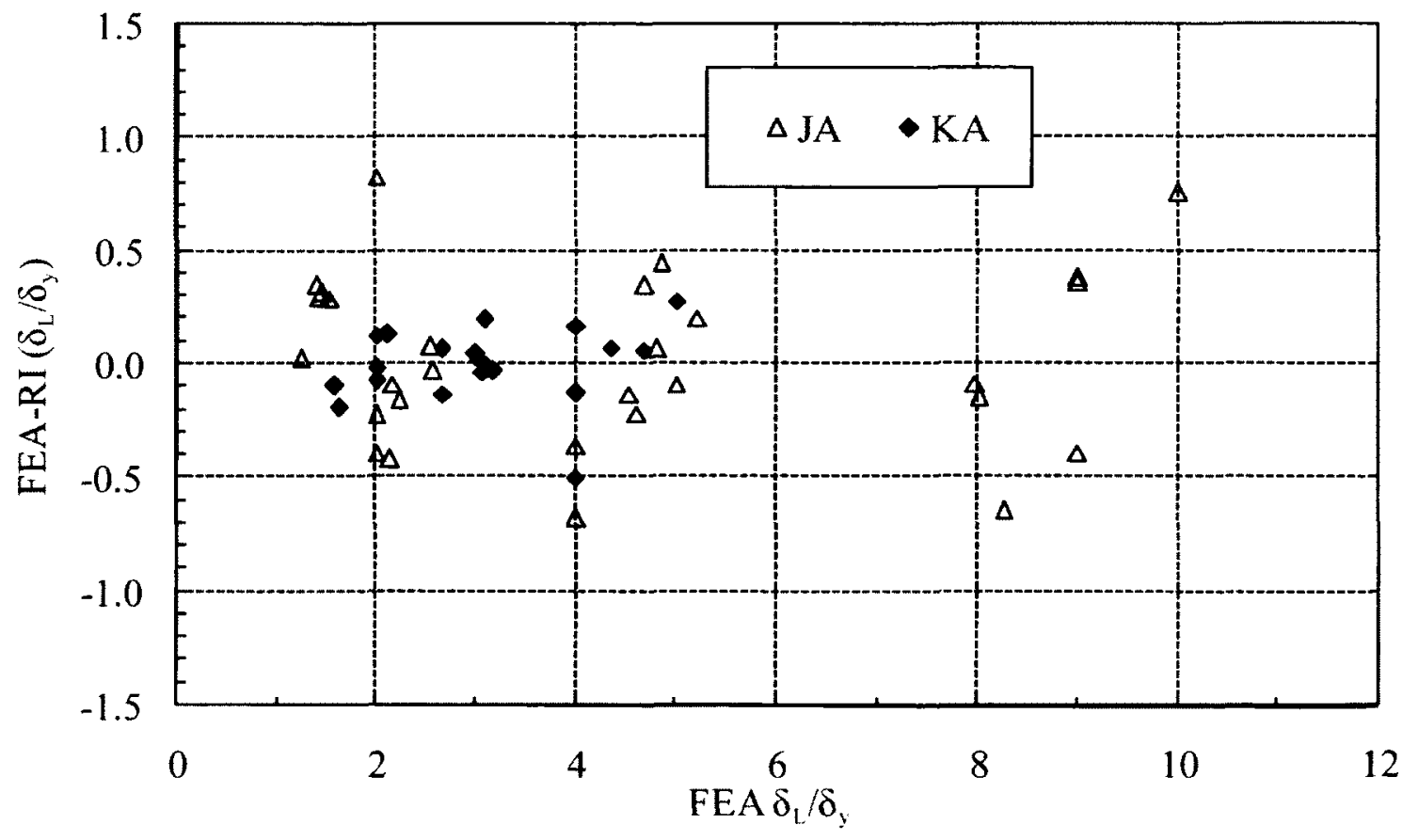

Figure 6.30 FEA-RI of ductility for materials without a yield plateau, JA and KA 


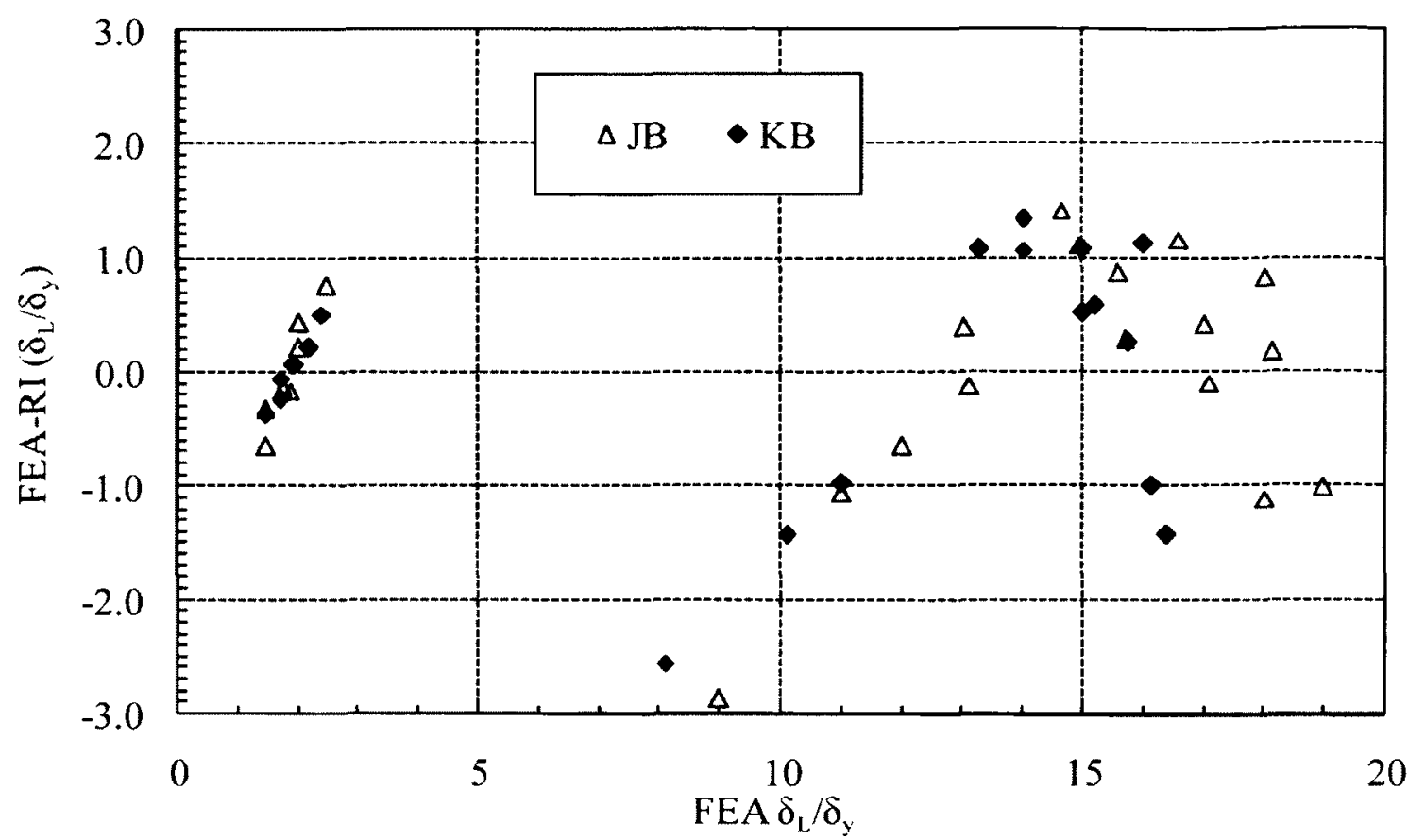

Figure 6.31 FEA-RI of ductility for materials with a yield plateau, JB and KB

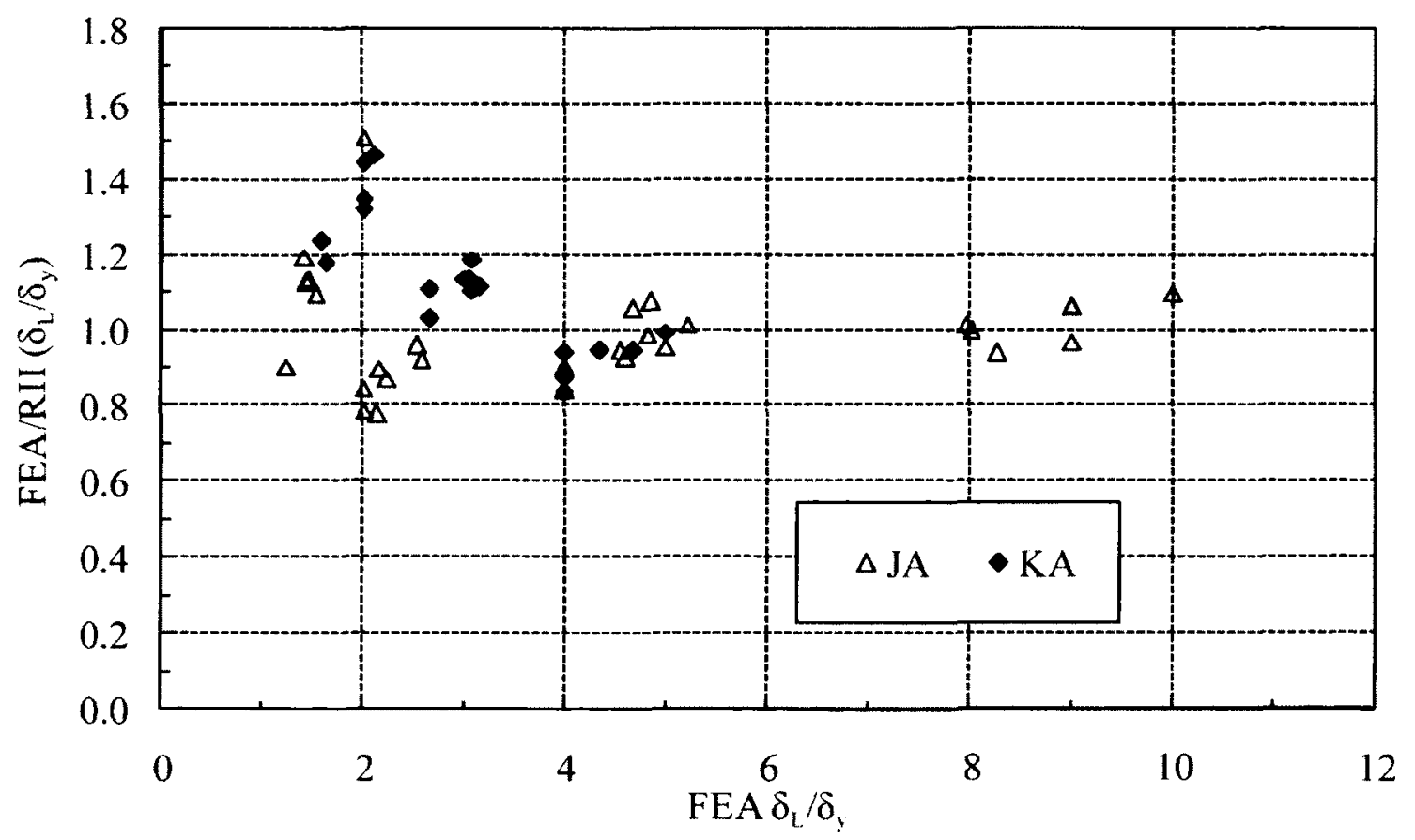

Figure 6.32 FEA/RII of ductility for materials without a yield plateau, JA and KA 


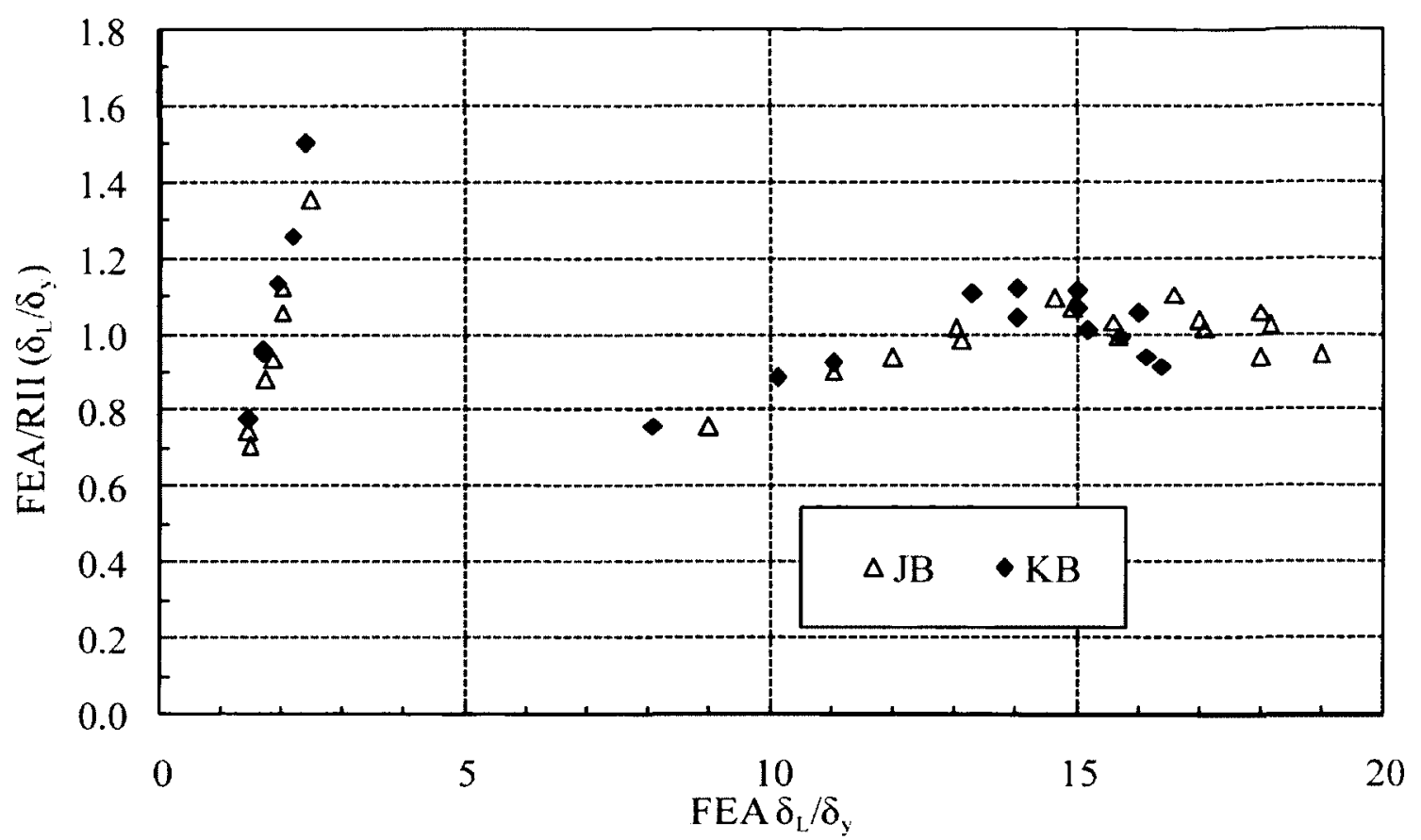

Figure 6.33 FEA/RII of ductility for materials with a yield plateau, JB and KB

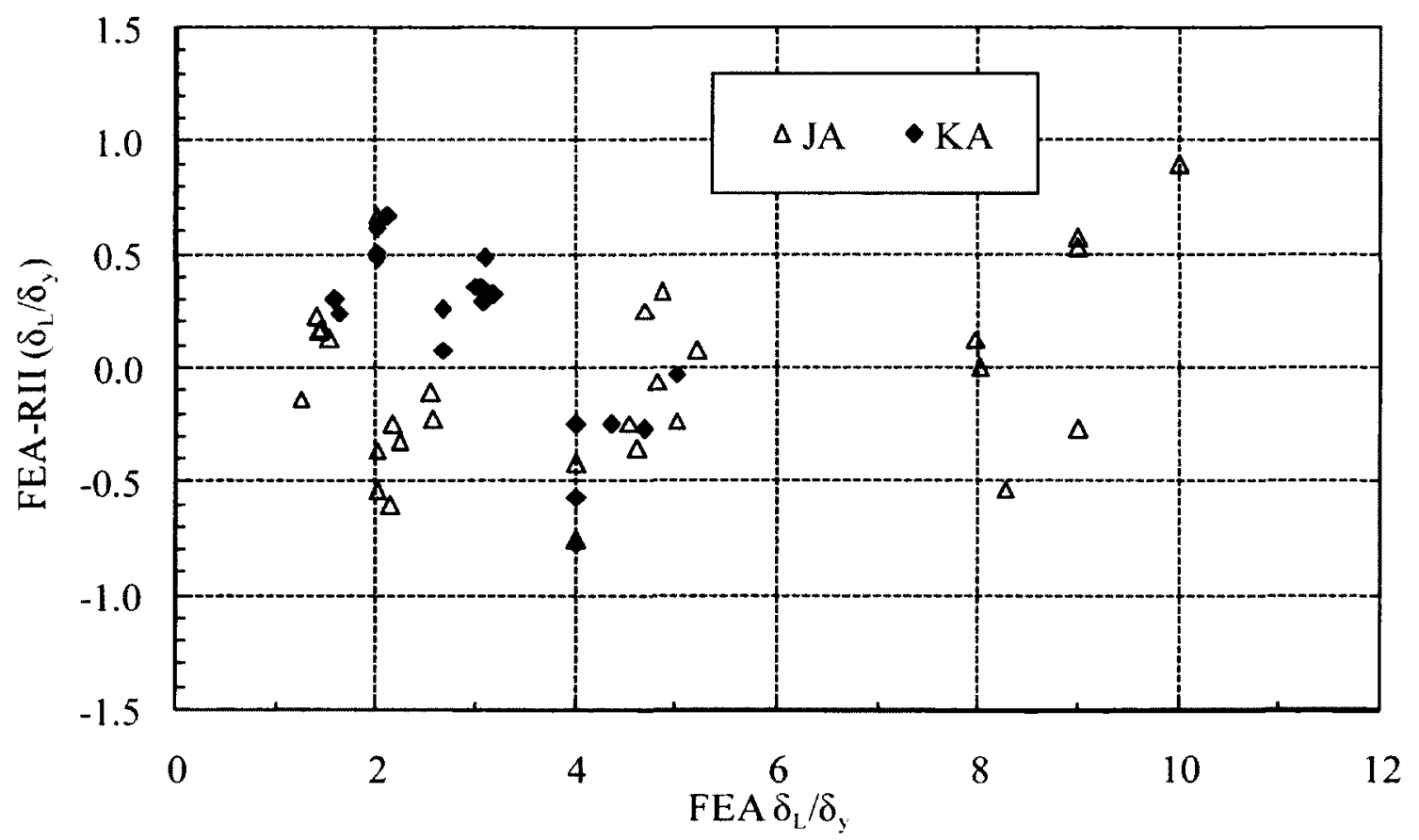

Figure 6.34 FEA-RII of ductility for materials without a yield plateau, JA and KA 


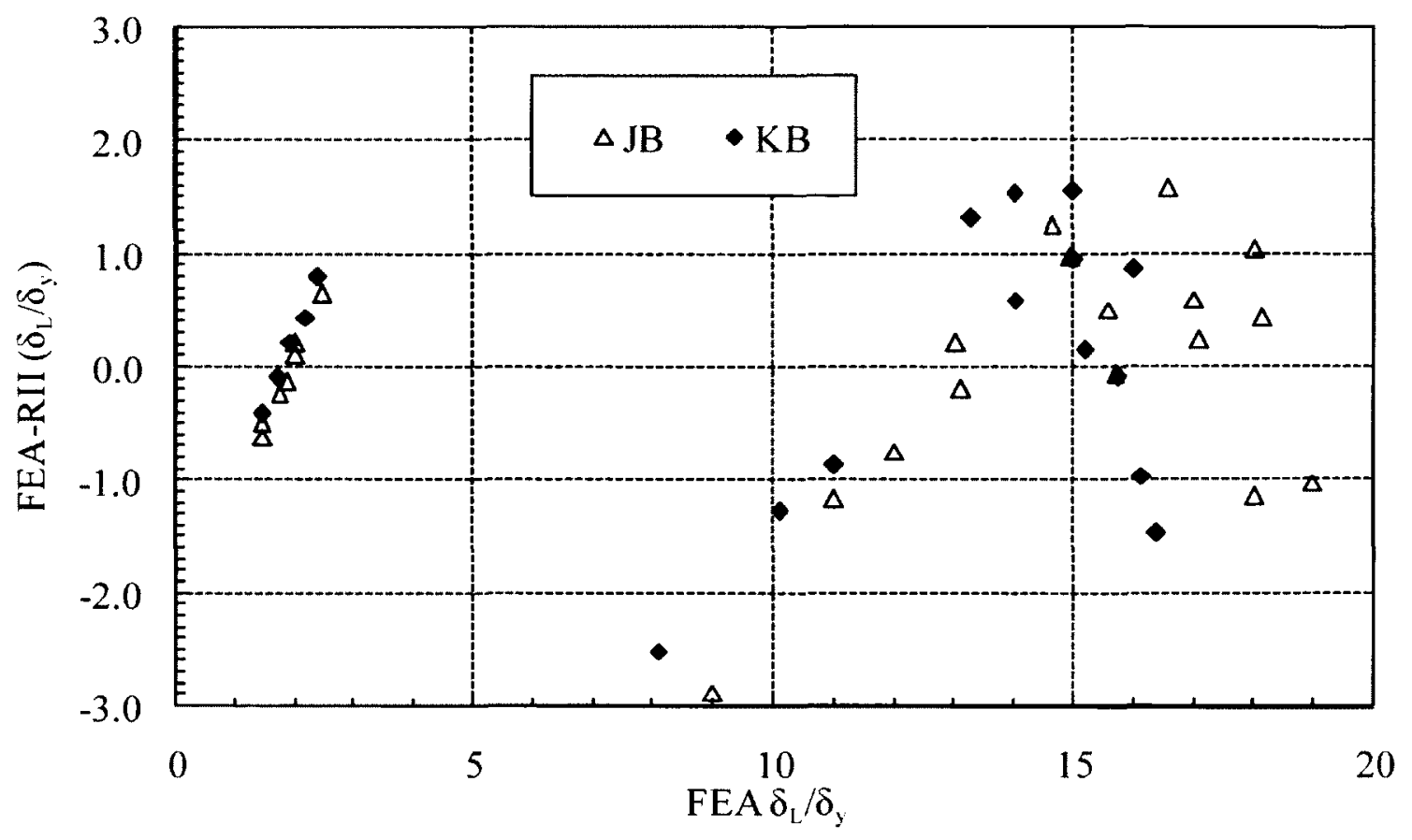

Figure 6.35 FEA-RII of ductility for materials with a yield plateau, JB and KB 


\section{CHAPTER 7 SUMMARY, CONCLUSIONS AND RECOMMENDATIONS}

\subsection{Summary and Conclusions}

A brief literature review of plasticity models and fracture criteria for steel has been carried out. In the literature review, a few of the cyclic hardening models and ductile fracture criteria were discussed. It was found that the combined isotropic and nonlinear kinematic hardening model proposed by Chaboche (1986) appeared to be a reasonable model to be used in the numerical simulations of steel structures. Following the literature review, four key tasks have been completed for the thesis, which include (1) the development of a procedure to calibrate and determine the parameters for the plasticity model by Chaboche (1986), (2) the evaluation of a number of fracture criteria and the adoption of a modified Huang and Mahin criterion for predicting fracture in steel, (3) the validation of the FEA model, the adapted procedures to calibrate and generate material model parameters and the fracture prediction using the modified fracture criterion for strap tension-only brace, and (4) a parametric finite element analysis study on strap tension-only brace.

\subsubsection{Calibration and Determination of Plasticity Model Parameters}

A total of thirty nine round cross-sectional shape specimens from two steel grades with mainly three profiles and various loading sequences have been designed and tested to furnish test data for the evaluation of the plasticity model and fracture criteria. Using results of round specimen tests, a procedure has been developed to calibrate and determine the parameters for the plasticity model by Chaboche (1986). This procedure 
mainly uses the flow stress versus true plastic strain relationship and an assumed saturated yield stress to determine parameters $\mathrm{C}$ and $\gamma$ for kinematic hardening. The true stress versus true plastic strain data used are generated using the exponential law flow stress equation by Chinh et al. (2004) with parameters of the equation calibrated against the load versus cross-sectional area change data of a monotonic tension material test of a straight specimen. It is found that the predicted load versus cross-sectional deformation of a round specimen under monotonic tension by the finite element analysis can be considered to depend only on the flow stress versus true plastic strain relationship and be independent of the kinematic hardening parameters of the plasticity model. Using the calibrated material model parameters, FEA simulations have been carried out for all the round specimens. There is a good agreement between test and predicted load versus cross-sectional deformation of straight specimens and load versus axial deformation of notched specimens. However, there are some differences between test and predicted load versus cross-sectional deformation of notched specimens. The following conclusions can be drawn from the comparisons of test and predicted results.

(1) It is feasible to use the combined isotropic and nonlinear kinematic hardening model by Chaboche (1986) with multiple nonlinear kinematic hardening terms to simulate both monotonic and cyclic tests using one set of input data for the material.

(2) The developed procedure is an effective way of finding a single set of the material model parameters that are applicable to both monotonic and cyclic loading. A minimum of two kinematic hardening terms are required to simulate the tests with reasonable accuracy. 


\subsubsection{Evaluation of Fracture Criteria}

A number of fracture criteria have been evaluated in the preliminary study. The preliminary study showed that the fracture criterion based on void growth with void closing in compression by Kanvinde and Deierlein $(2004,2007)$ and the Huang and Mahin (2008) criterion with gross strain energy density can provide a reasonable good fracture prediction for the limited number of tests. These two criteria have further been evaluated using results of thirty nine round cross-sectional shape specimens tested. The Huang and Mahin (2008) criterion with gross strain energy density assumes that damage is small before fracture occurs, and thus enables damage to uncouple from the constitutive model. It has been found that there is no significant drop in the elastic stiffness of the specimen until fracture occurs. An additional modification has also been made to the Huang and Mahin (2008) criterion to include the effect of $\sigma_{\mathrm{n}} / \sigma_{\mathrm{eq}}$ more generally in Eq. (4.4). The evaluation of the fracture criteria shows that the modified Huang and Mahin criterion based on Eqs. (4.3) and (4.4) is able to reasonably predict the point of fracture for all the tests, whereas the cumulative strain at fracture predicted by the Kanvinde and Deierlein $(2004,2007)$ criterion is off by more than $100 \%$ for some cyclic tests.

\subsubsection{Validation of Strap Tension-Only Brace Modeling}

The experimental study on steel strap specimens by Aghdam (2009) has been briefly discussed. Since only monotonic tension material tests have been carried out for the steel straps, the material model parameters have been determined using the assumed saturated yield stress based on the bound established with the calibrated material model 
parameters from the current and other studies on various grades of steel. A procedure has also been developed to establish the damage limit and the damage evaluation parameters for the modified Huang and Mahin fracture criterion using results of monotonic tension material tests of steel straps and current round specimen tests. FEA models of the steel strap specimen have been constructed using both solid and shell elements. The model considers there is no slippage at the bolted connection. Numerical simulations of steel strap specimens have been carried out using the established material model parameters with the modified Huang and Mahin fracture criterion and the mixed-mode plasticity model by Chaboche (1986) with two kinematic hardening terms. The following conclusions can be drawn from the comparisons of test and predicted results.

(1) A full-length FEA model is required to simulate the behaviour of the brace for all geometric configurations. The FEA simulation with the full-length model on average underestimates the maximum deformation of the brace by $6 \mathrm{~mm}$ if slippage occurs at the bolted connection.

(2) The test results in general are bounded by the FEA simulations of the saturated yield stress bound parameter $\eta$ of 0.75 and 1.2 with the maximum deformation of the test closer to the predicted value with $\eta=0.75$. The simulation with $\eta=0.75$ predicts a lower maximum deformation compared to $\eta=1.2$.

(3) The proposed procedure using the limits of the saturated yield stress bound parameter $\eta$ is an effective way to establish the material model parameters to be used in the numerical simulation where there are only data from the monotonic tension material test. 


\subsubsection{Parametric Study on Strap Tension-Only Brace}

A procedure to generate the material input data for the specific material property characteristics has been developed. A total of ninety six braces similar to the Type A strap specimen tested by Aghdam (2009) have been simulated for four materials and various geometric configurations. The four materials consist of the combination of the ultimate to yield strength ratio of 1.4 and 1.75 , and a yield plateau length of 0.02 and no yield plateau, and are generated with $\eta=0.75$. Results from the FEA simulations have been analyzed and equations to predict the ductility of the brace have been proposed for a slenderness ratio no less than 400. Recommendations for designing steel strap tensiononly braces in a Seismic Force Resisting System have also been provided. A few conclusions can be drawn from results of the simulations.

(1) The yield plateau length has a great effect on the ductility when the net section fracture strength is greater than the gross section yielding strength, with the ductility increasing with the yield plateau length.

(2) The ductility increases with the net section fracture to gross section yielding strength ratio $\left(A_{n} F_{u} / A_{g} F_{y}\right)$.

(3) The slenderness ratio has little effect on the ductility when its value is greater than 800 . Increasing the slenderness ratio from 400 to 600 by increasing the length or reducing the thickness of specimen increases the ductility when the $A_{n} F_{u} / A_{k} F_{y}$ ratio is greater than 1 for the material with a yield plateau length of 0.02 . The difference in the ductility is not appreciable when there is no yield plateau. 
(4) The ductility reduces as the ultimate to yield strength ratio $\left(F_{u} / F_{y}\right)$ increases when the $A_{n} F_{u} / A_{g} F_{y}$ ratio is greater than 1.0.

(5) The proposed ductility equations predict a conservative maximum deformation compared to the test, with the prediction improved for the slenderness ratio no less than 400 .

(6) The ductility improves with the higher yield strength. Thus, the proposed equations are applicable to a yield strength greater than $300 \mathrm{MPa}$.

\subsection{Recommendations for Future Studies}

(1) Current parametric study has only considered two yield plateau lengths and ultimate to yield strength $\left(\mathrm{F}_{\mathrm{u}} / \mathrm{F}_{\mathrm{y}}\right)$ ratios. Numerical simulations with materials at additional yield plateau lengths and $\mathrm{F}_{\mathrm{u}} / \mathrm{F}_{\mathrm{y}}$ ratios can better quantify the effect of these two factors on the ductility of a steel strap tension-only brace.

(2) The parametric study has only considered an isolated diagonal brace with a single longitudinal line of bolts. Additional experimental and numerical studies can be carried out to consider $\mathrm{X}$ bracing and connections with more than a single longitudinal line of bolts.

(3) Numerical simulations of the strap specimens have been carried out for an assumed range of material behaviour under cyclic loading. Since no cyclic material test has been conducted for the strap and these may have indirectly validated the plasticity model and the damage criterion, a more direct 
validation with the material model parameters determined from the known cyclic material properties is desirable.

(4) Current approach of generating material input data for specific material property characteristics can be applied to study the response of other structural components for a range of material behaviour.

(5) The structural response to seismic loading is affected by the dynamic behaviour of the material. A study can be conducted to explore how to generate the material input data for the strain rate sensitive plasticity model to account for the strain rate effect in the numerical simulations.

(6) In the current study, there is a difference between test and predicted crosssectional area change of the round material test specimens. Additional studies may be conducted to explore if any improvement can be made to the predicted load versus cross-sectional area change if other material models are considered.

(7) In the evaluation of the fracture criteria (calibration of fracture limit), only results of mainly three specimen profiles have been considered. Results of more specimens with different notched profiles should be used to better study the fracture criterion, and calibrate the fracture limit and damage evolution parameters for the effect of mean stress or $\sigma_{m} / \sigma_{\text {eq. }}$. A variation of Eq. (4.4) can also be considered for the damage evolution with the term in the brackets taken as $\left[1+\mathrm{b}\left(\sigma_{\mathrm{m}} / \sigma_{\mathrm{eq}}\right)^{\mathrm{k}}\right]$, with $\mathrm{b}$ and $\mathrm{k}$ being material constants.

(8) In Chapter 5, procedures have been developed to generate material model parameters only using data of the standard tension material test of a straight 
specimen with $\eta=0.75$ and 1.2 . A study may be conducted to explore if a single set of material model parameters that is representative of the material can be obtained by adjusting $\eta$ until the simulations agree with the cyclic tests of the structural component (member) without having to conduct any cyclic material test. 
APPENDIX A COMPARISONS OF TESTS AND FEA SIMULTIONS FOR MATERIALS A AND B (TWO TERMS)

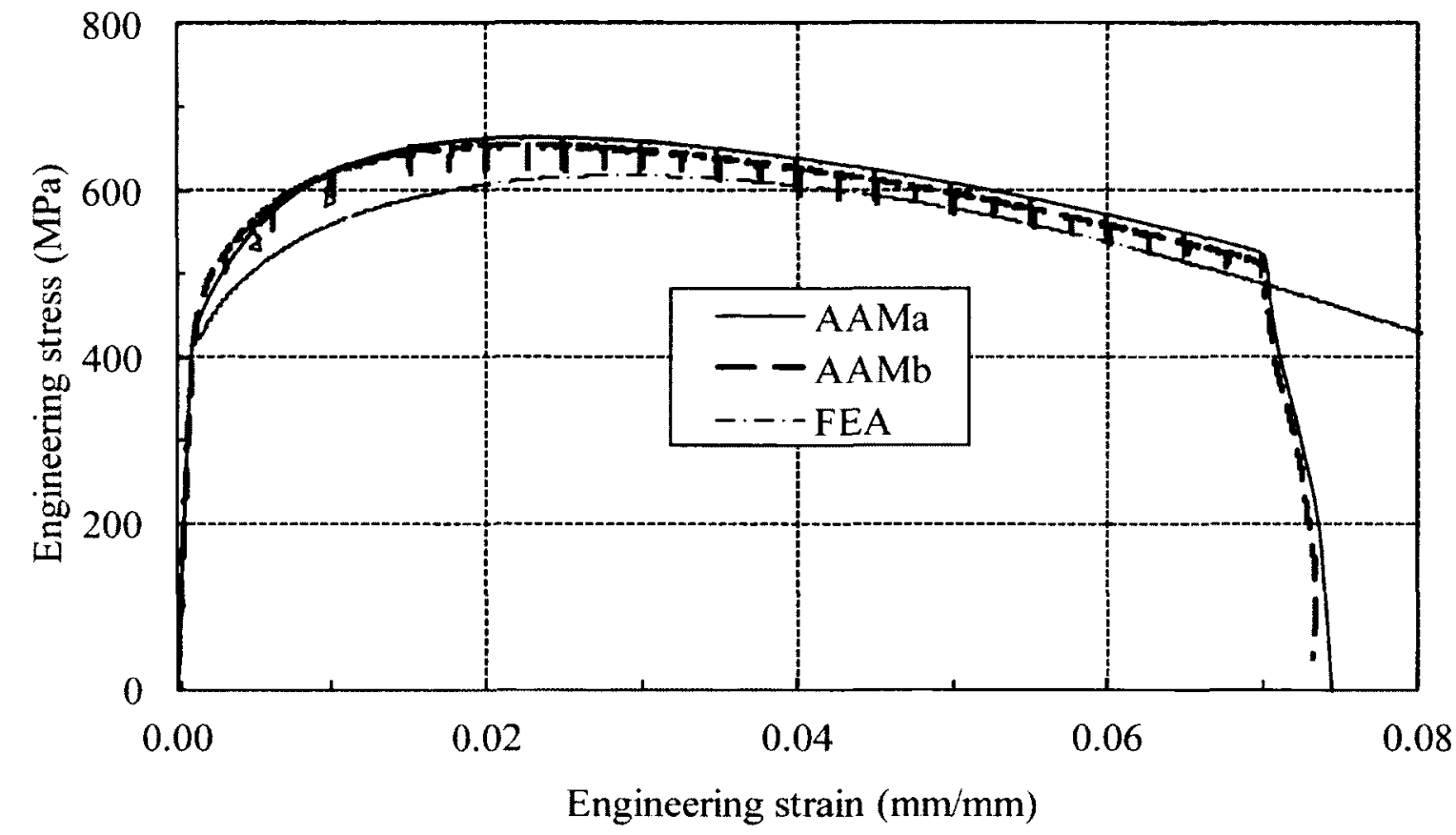

Figure A.1 Test and predicted engineering stress versus engineering strain curves for notched specimens AAMa and AAMb (two terms)

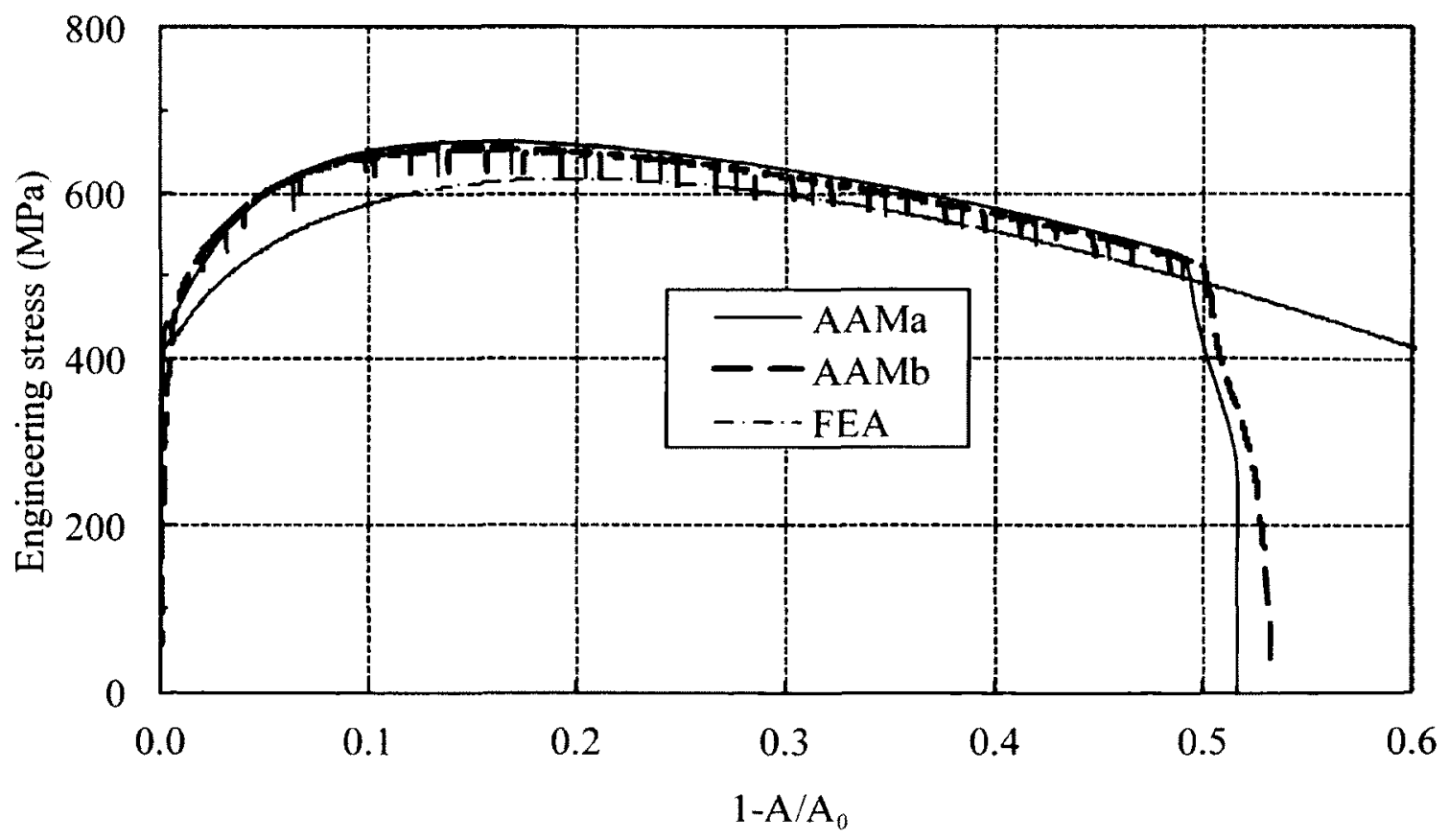

Figure A.2 Test and predicted engineering stress versus cross-sectional area change curves for notched specimens AAMa and AAMb (two terms) 


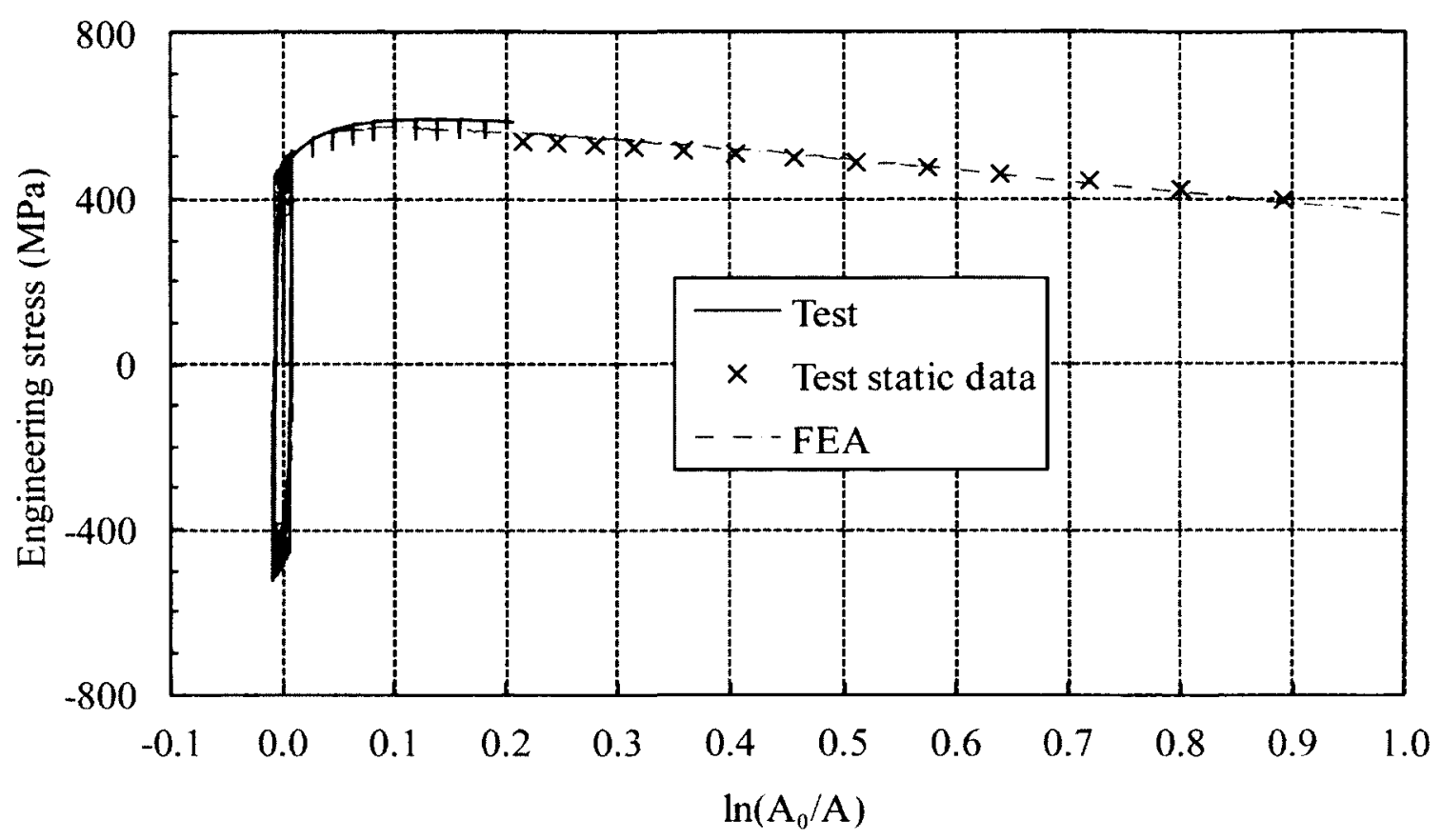

Figure A.3 Test and predicted engineering stress versus cross-sectional area change curves for straight specimen ADC40101 (two terms)

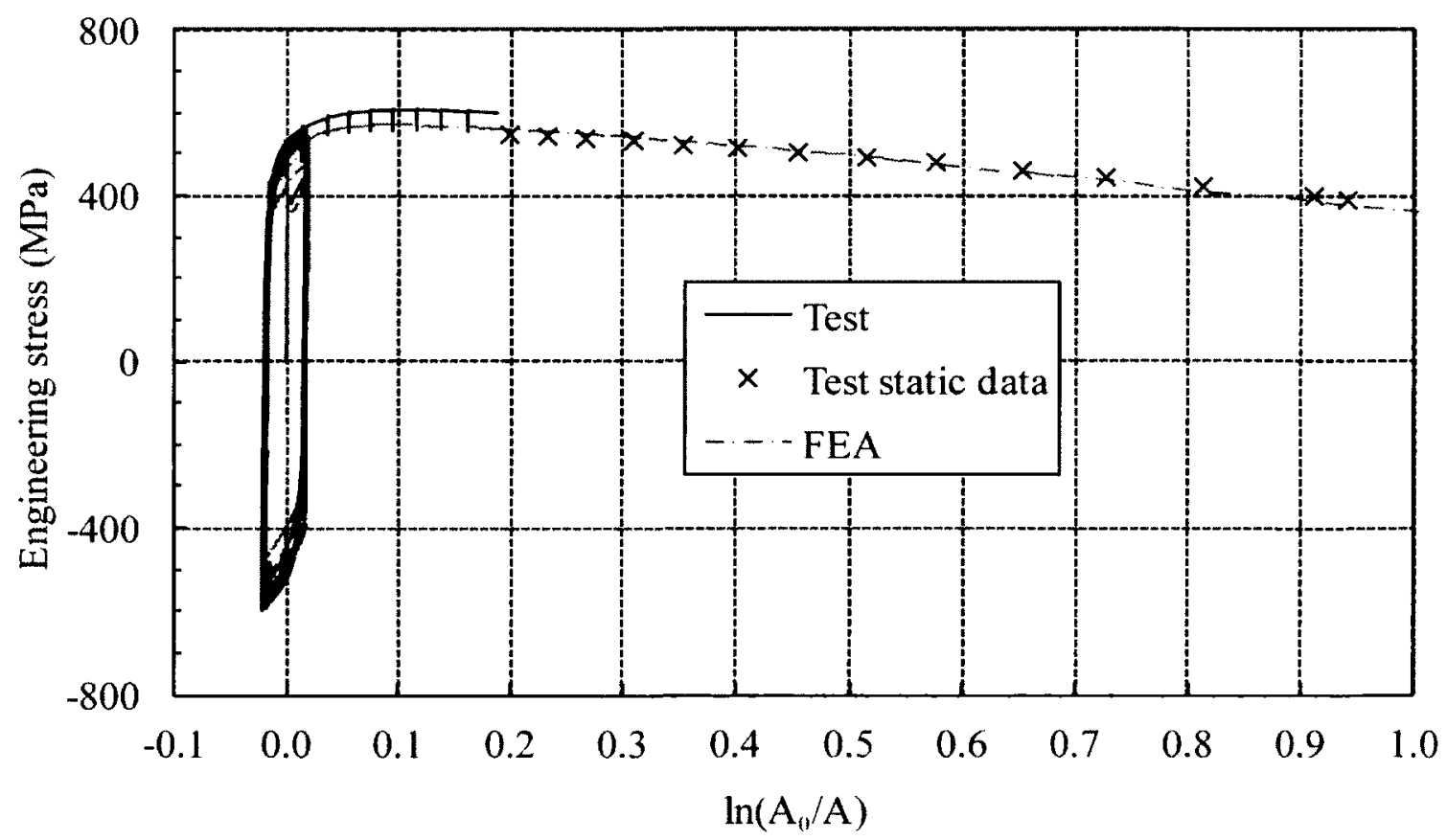

Figure A.4 Test and predicted engineering stress versus cross-sectional area change curves for straight specimen ADC40202 (two terms) 


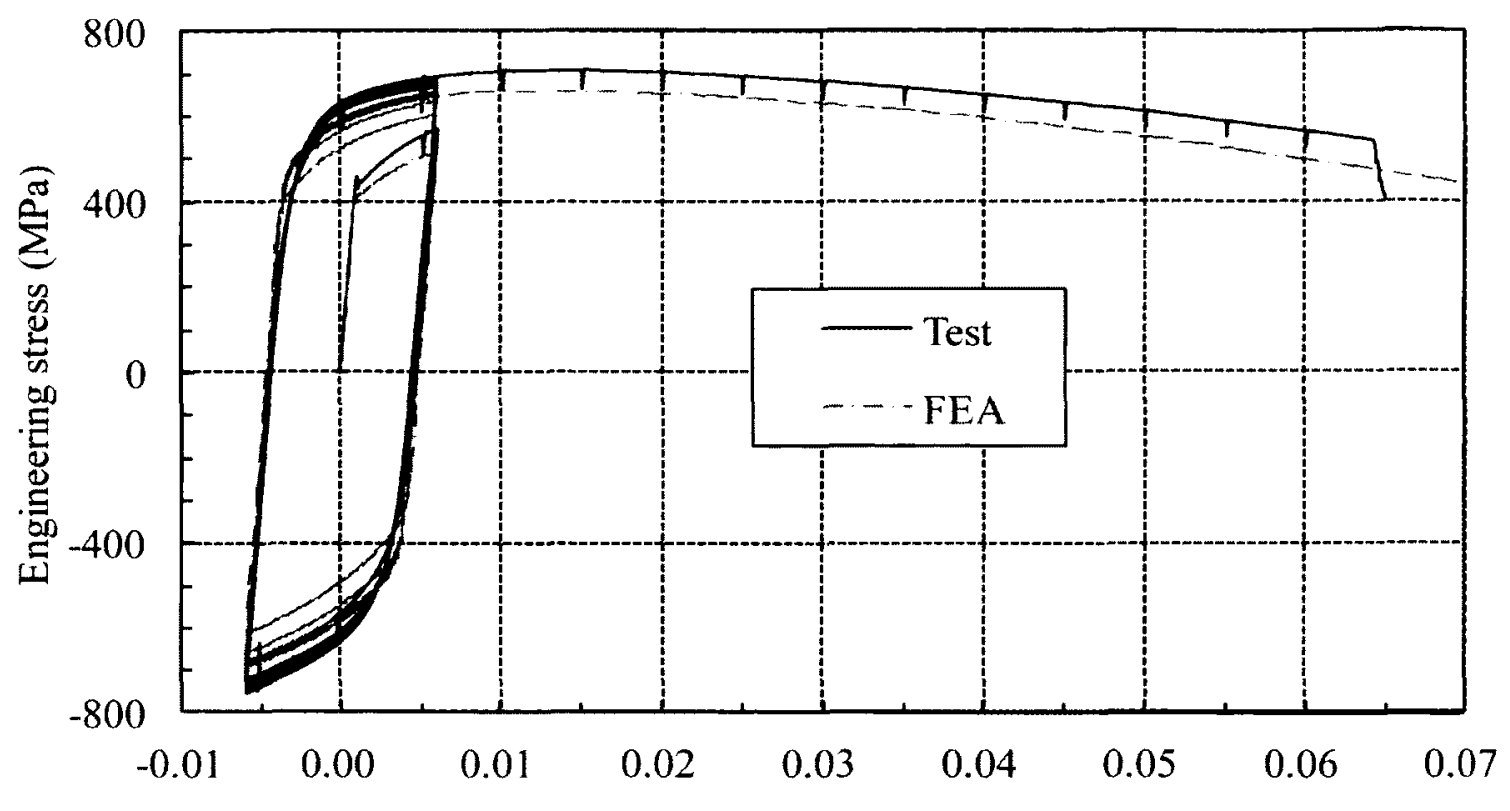

Engineering strain $(\mathrm{mm} / \mathrm{mm})$

Figure A.5 Test and predicted engineering stress versus engineering strain curves for notched specimen AAC20404 (two terms)

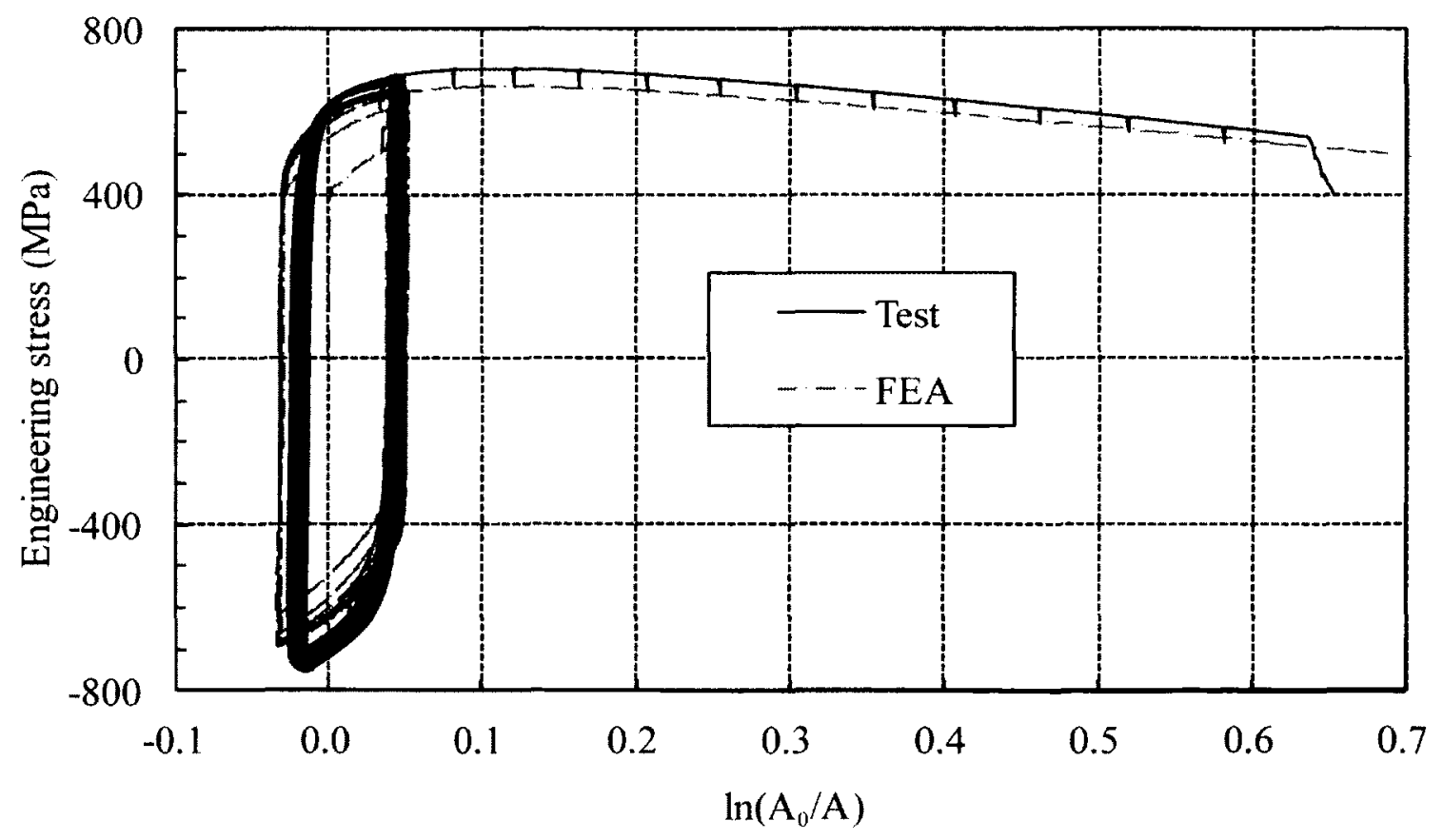

Figure A.6 Test and predicted engineering stress versus cross-sectional area change curves for notched specimen AAC20404 (two terms) 


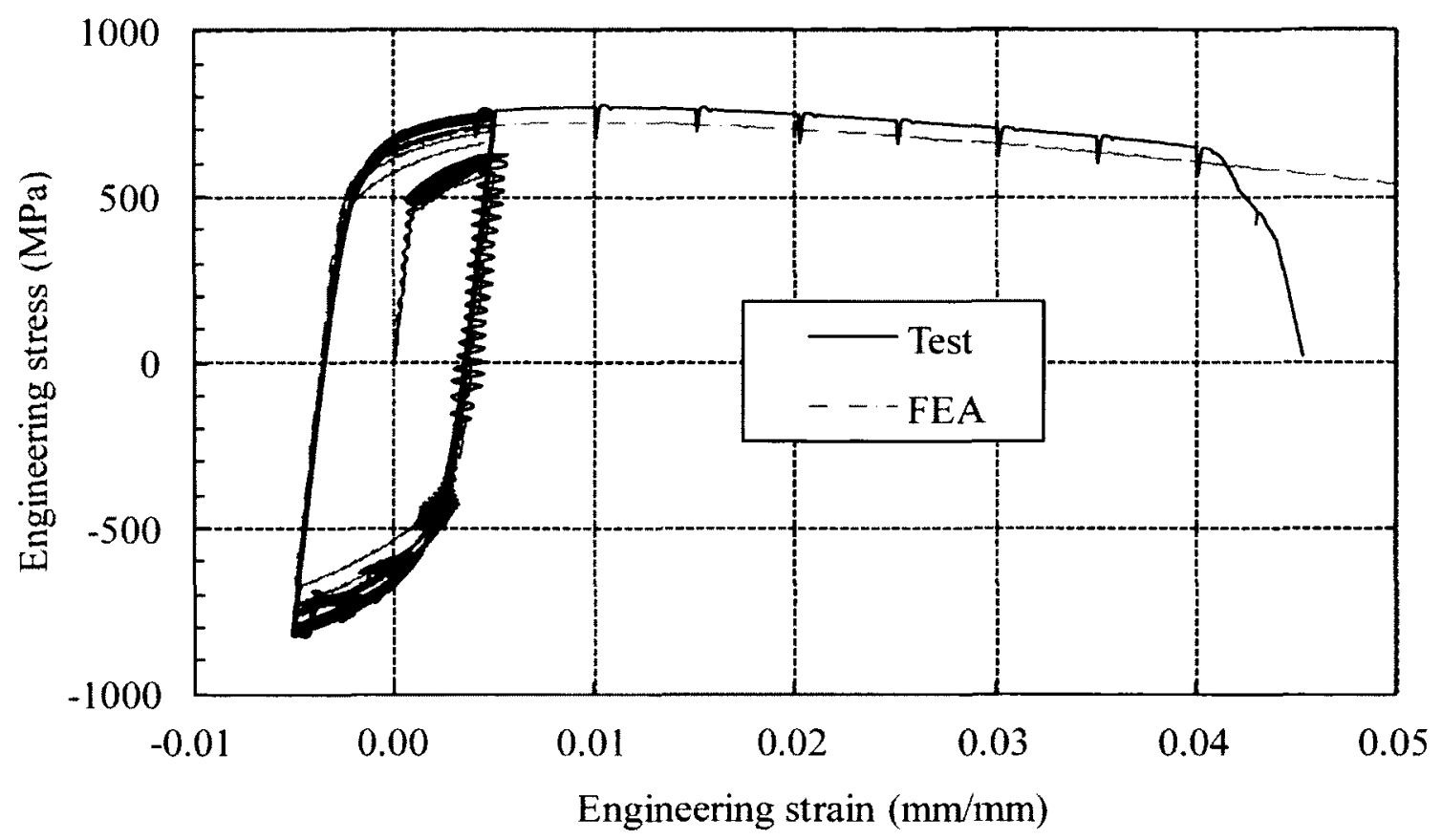

Figure A.7 Test and predicted engineering stress versus engineering strain curves for notched specimen ACC20404 (two terms)

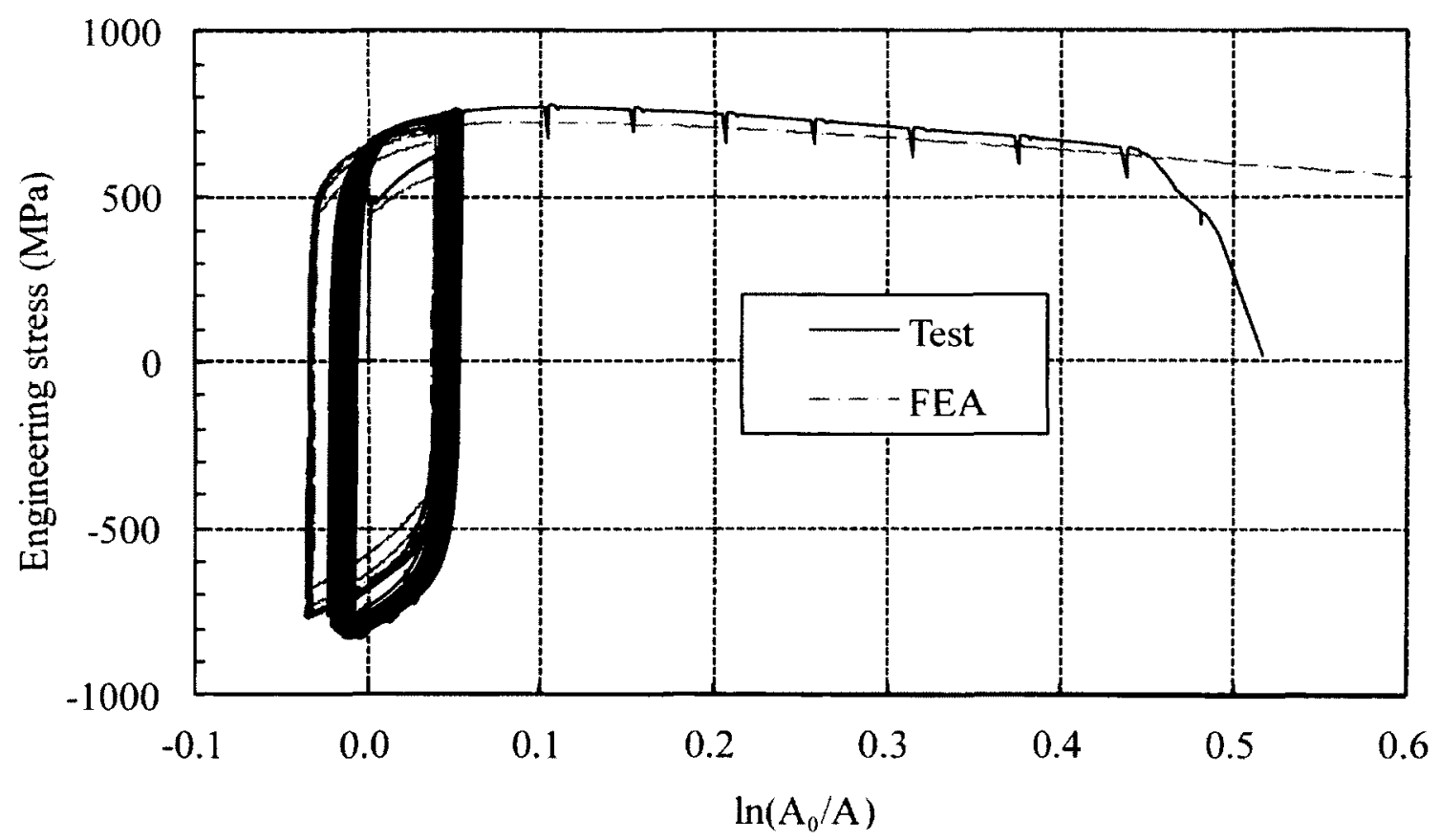

Figure A.8 Test and predicted engineering stress versus cross-sectional area change curves for notched specimen ACC20404 (two terms) 


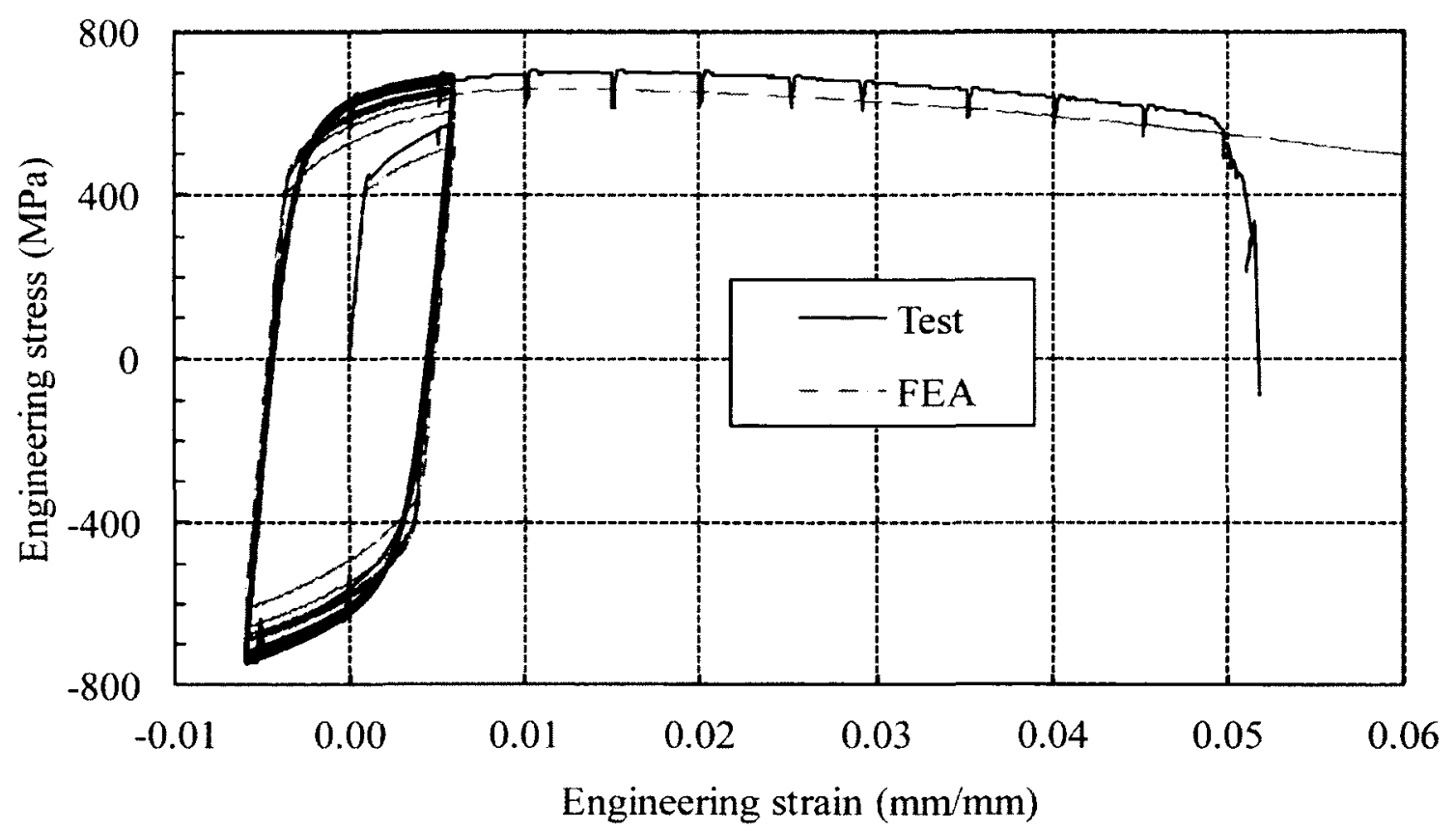

Figure A.9 Test and predicted engineering stress versus engineering strain curves for notched specimen AAC70404 (two terms)

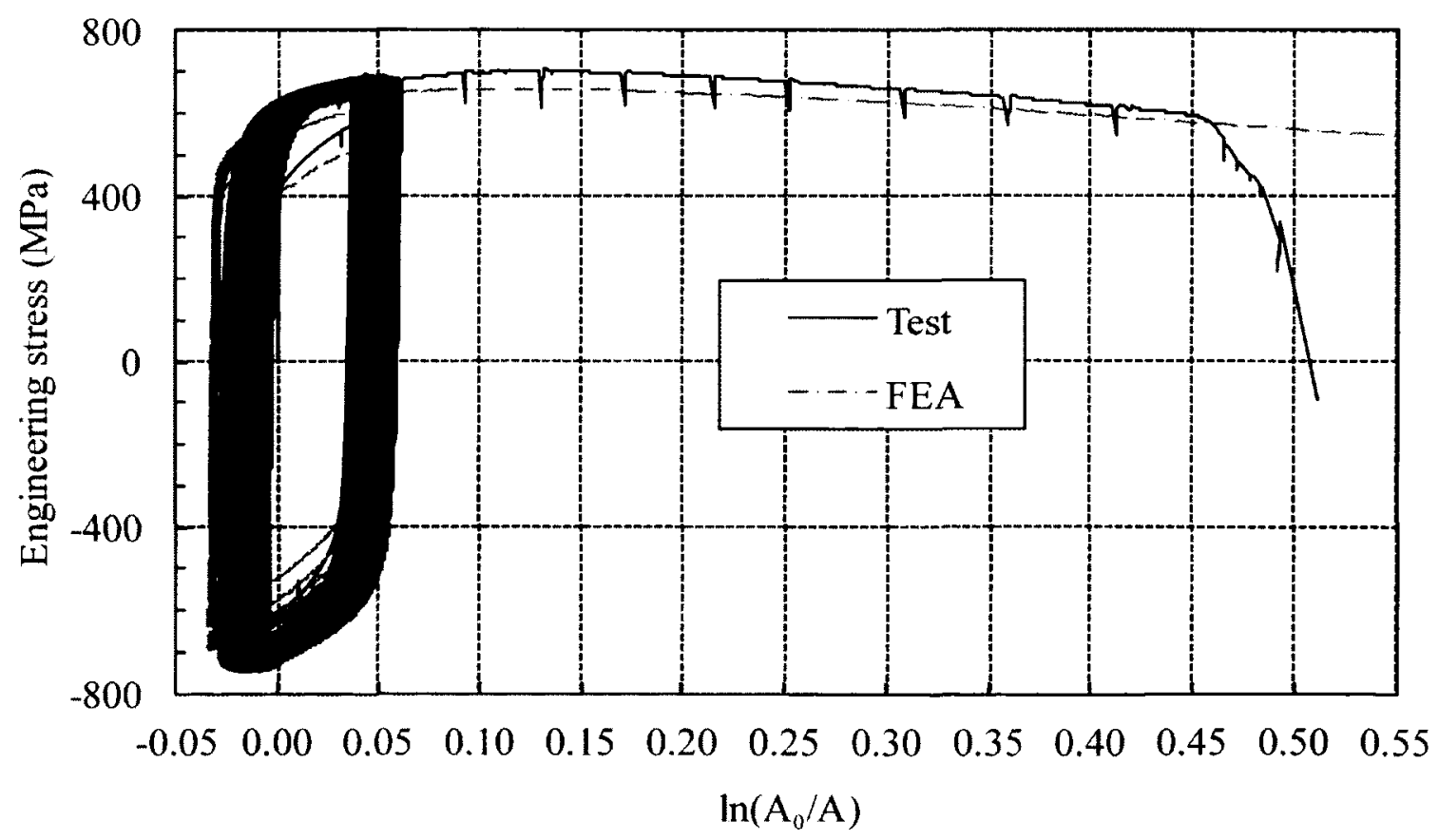

Figure A.10 Test and predicted engineering stress versus cross-sectional area change curves for notched specimen AAC70404 (two terms) 


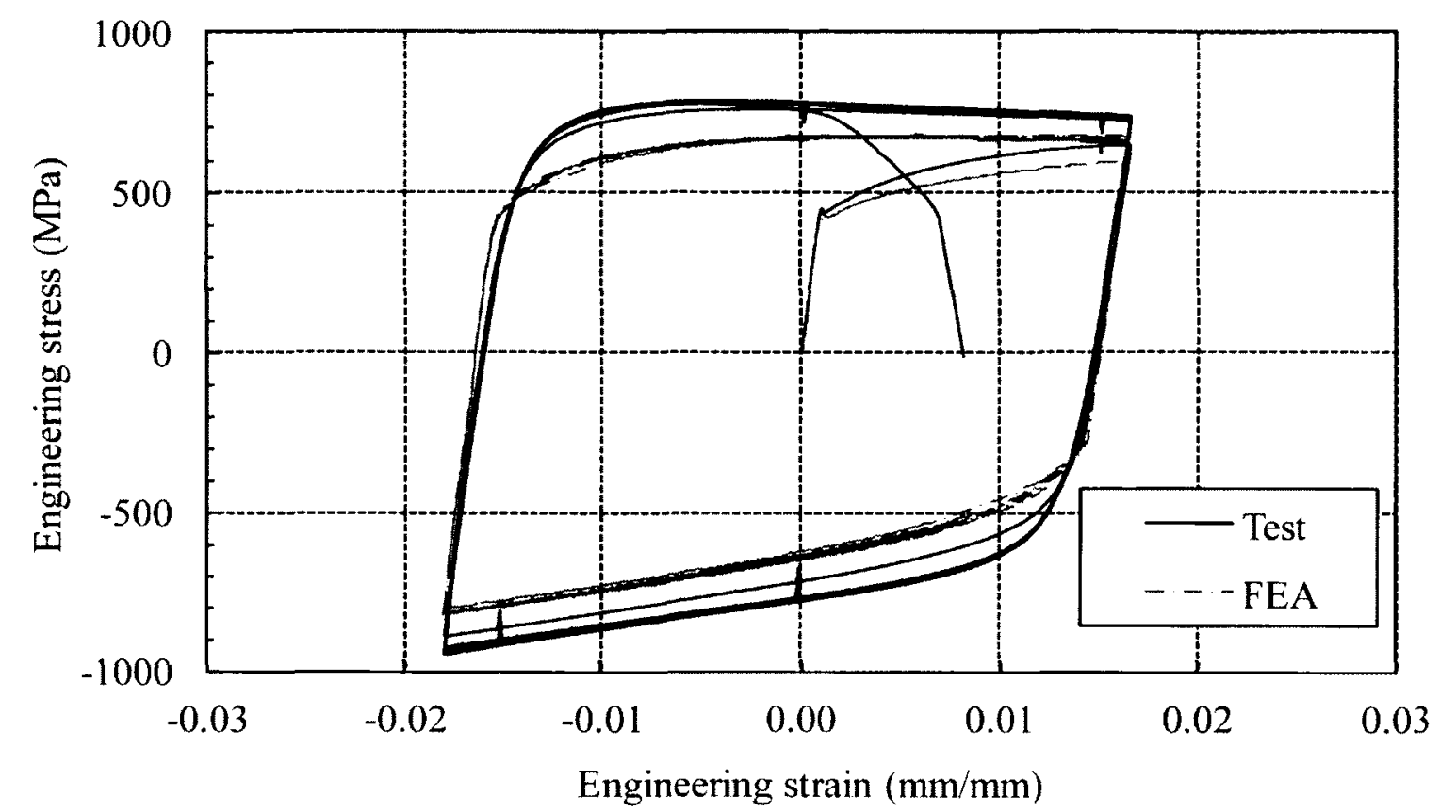

Figure A.11 Test and predicted engineering stress versus engineering strain curves for notched specimen AACF 1212 (two terms)

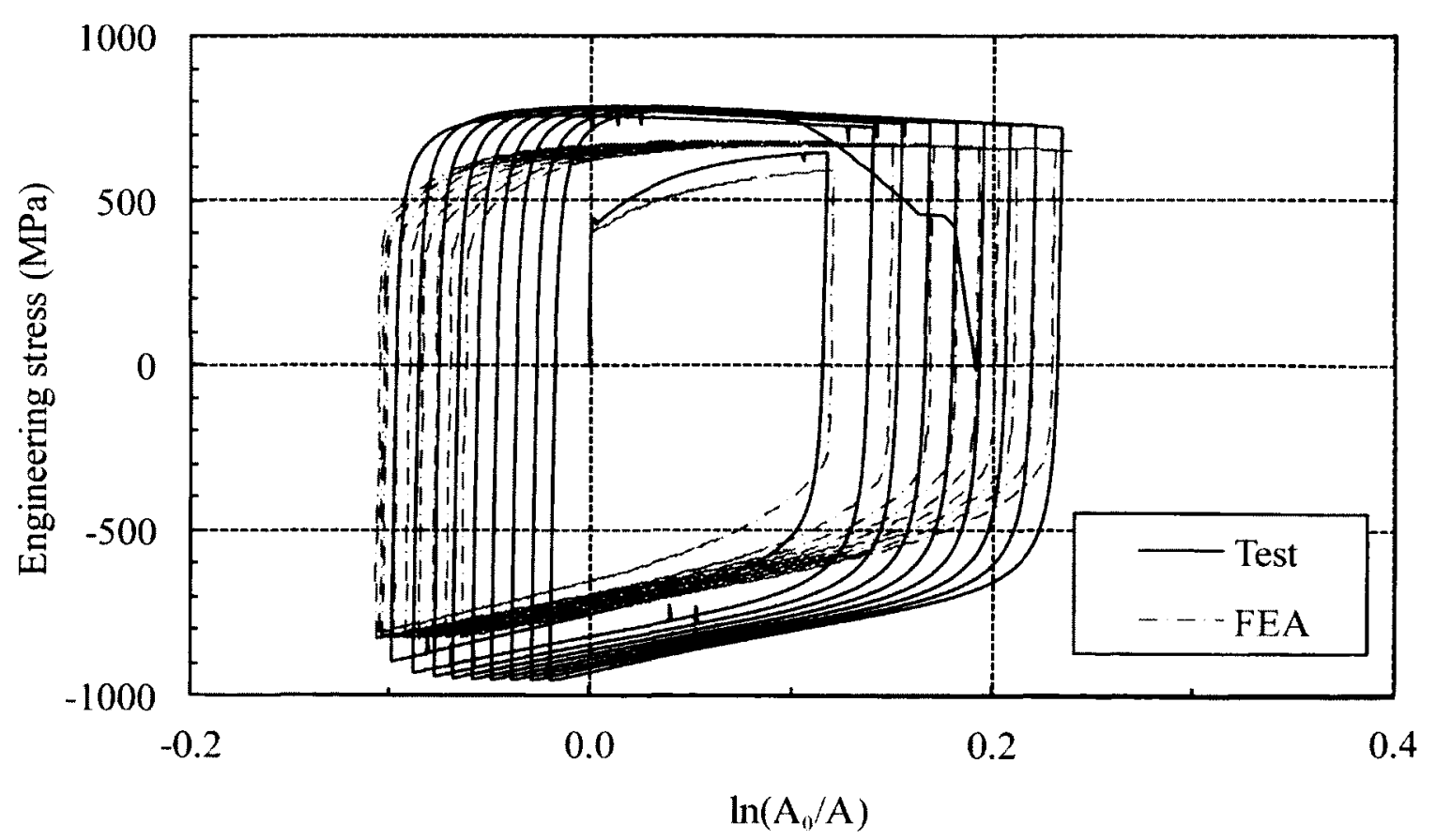

Figure A.12 Test and predicted engineering stress versus cross-sectional area change curves for notched specimen AACF 1212 (two terms) 


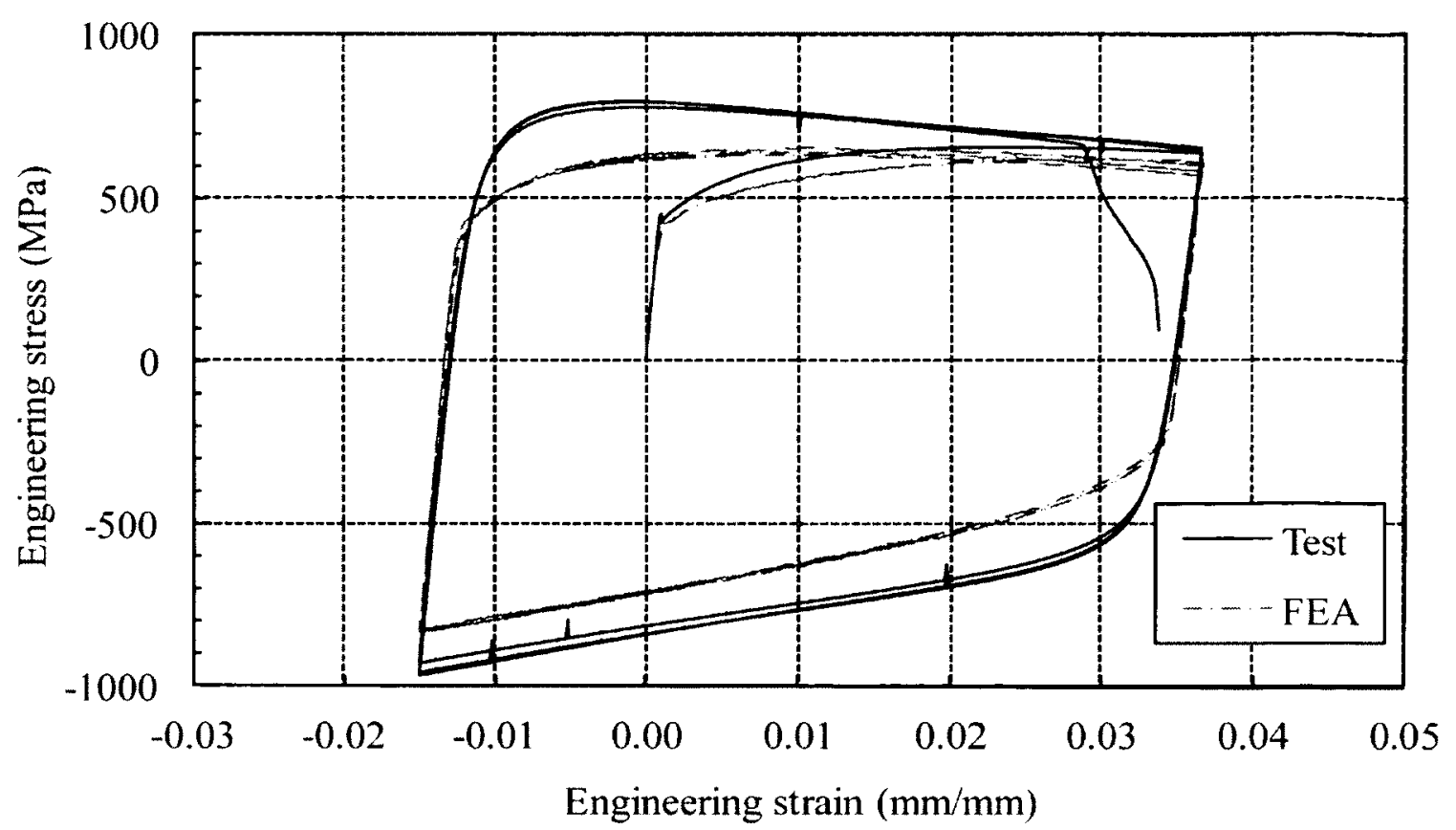

Figure A.13 Test and predicted engineering stress versus engineering strain curves for notched specimen AACF3010 (two terms)

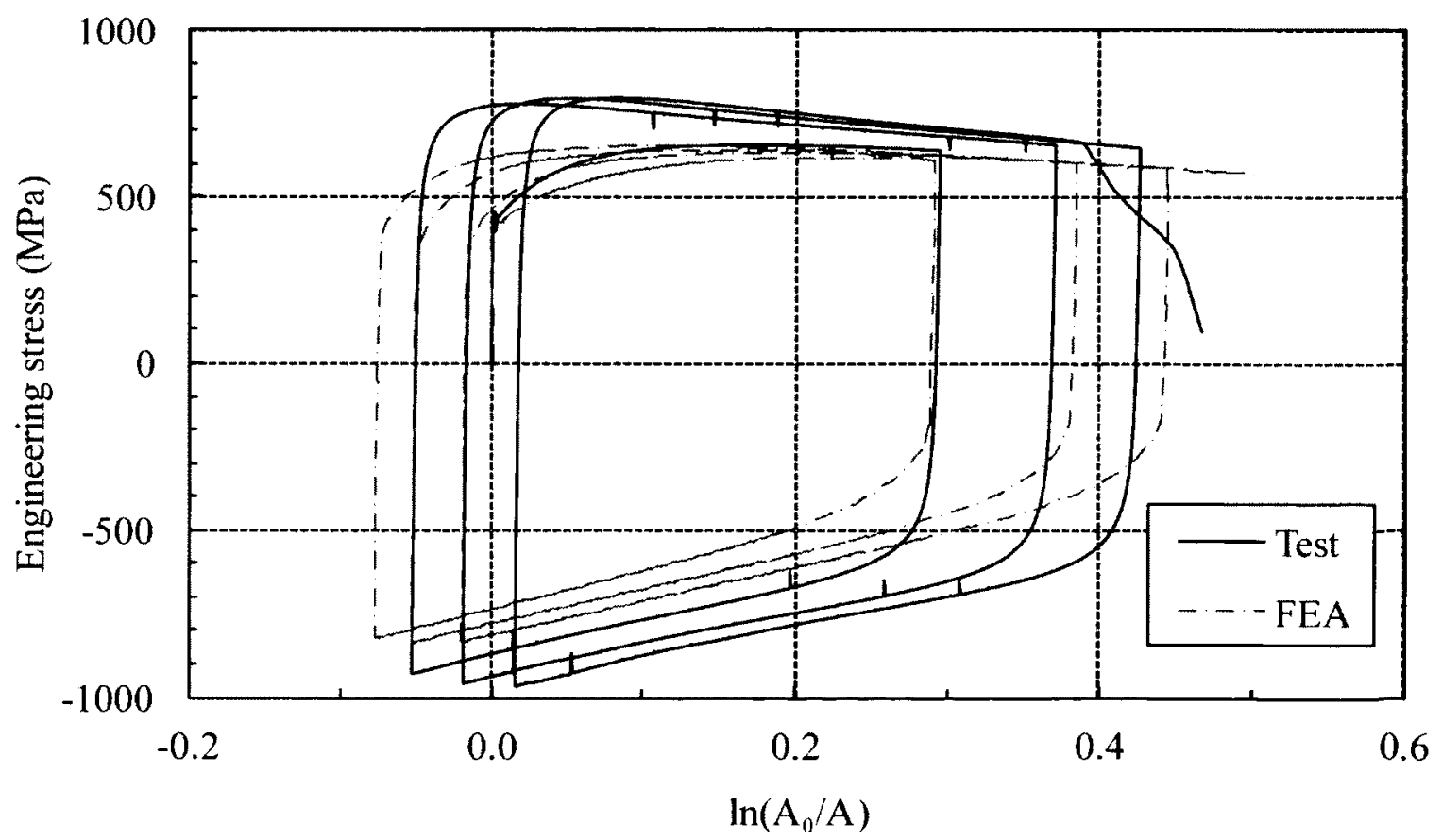

Figure A.14 Test and predicted engineering stress versus cross-sectional area change curves for notched specimen AACF3010 (two terms) 


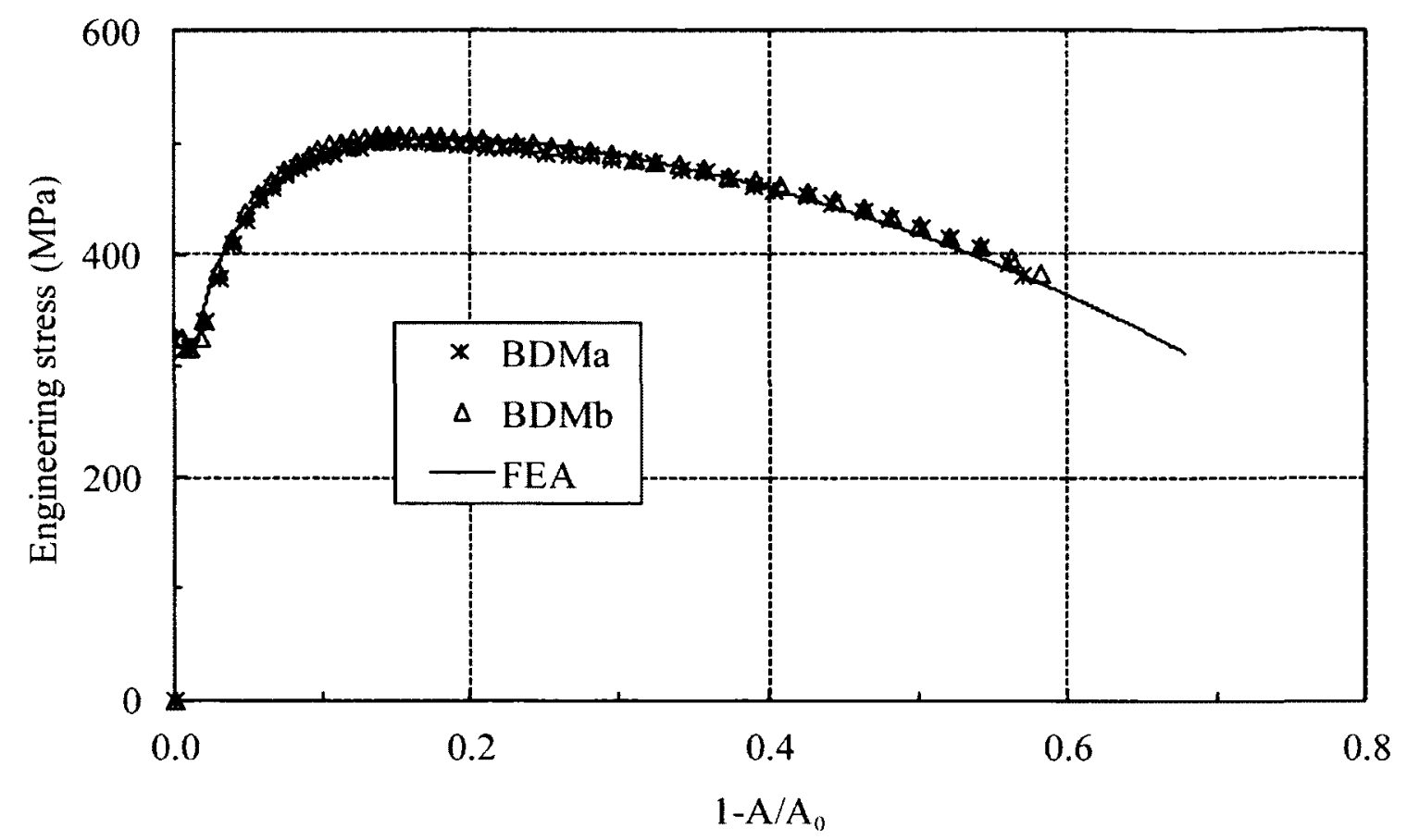

Figure A.15 Test and predicted engineering stress versus cross-sectional area change curves for straight specimens BDMa and BDMb (two terms)

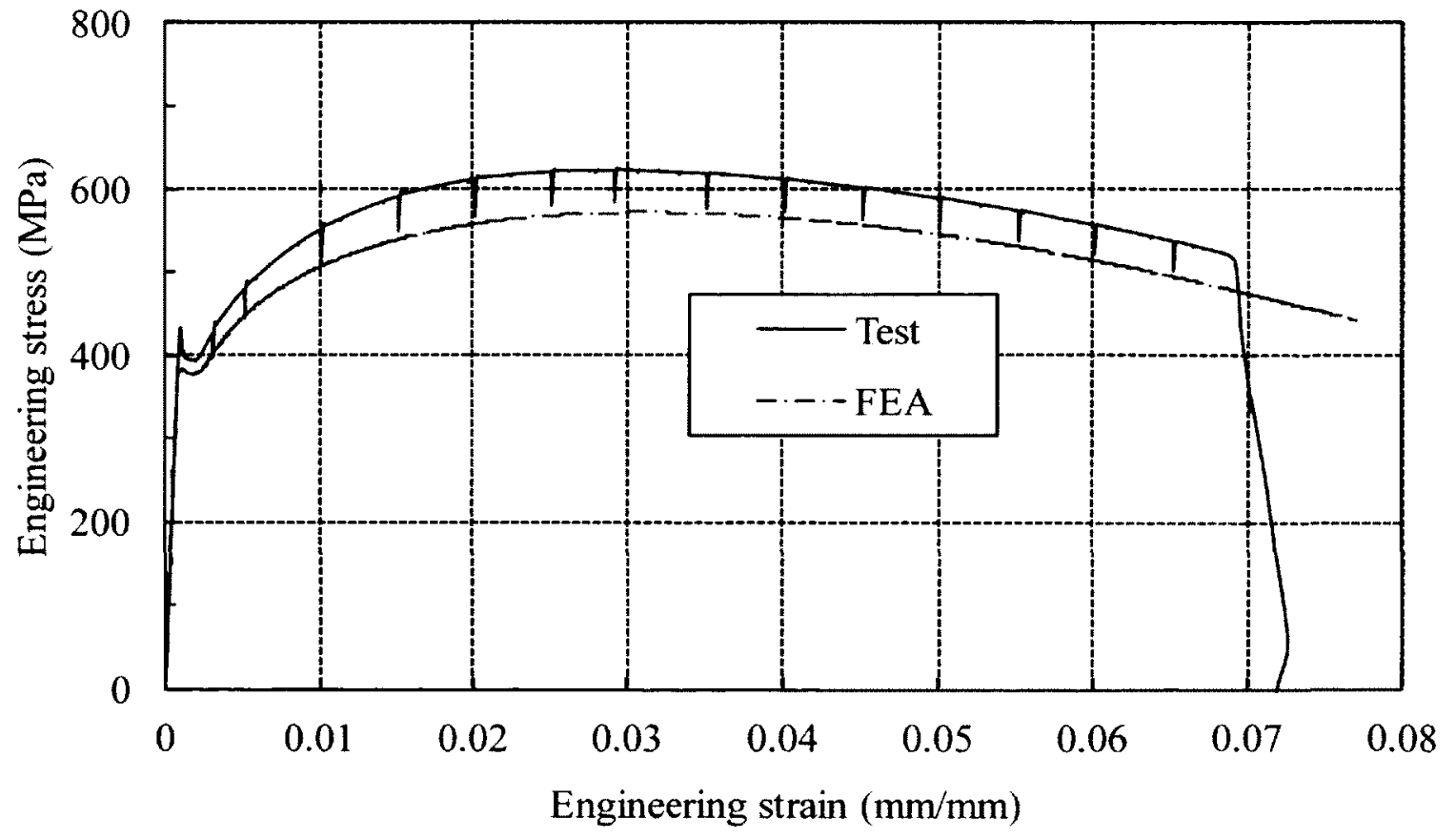

Figure A.16 Test and predicted engineering stress versus engineering strain curves for notched specimen BAM (two terms) 


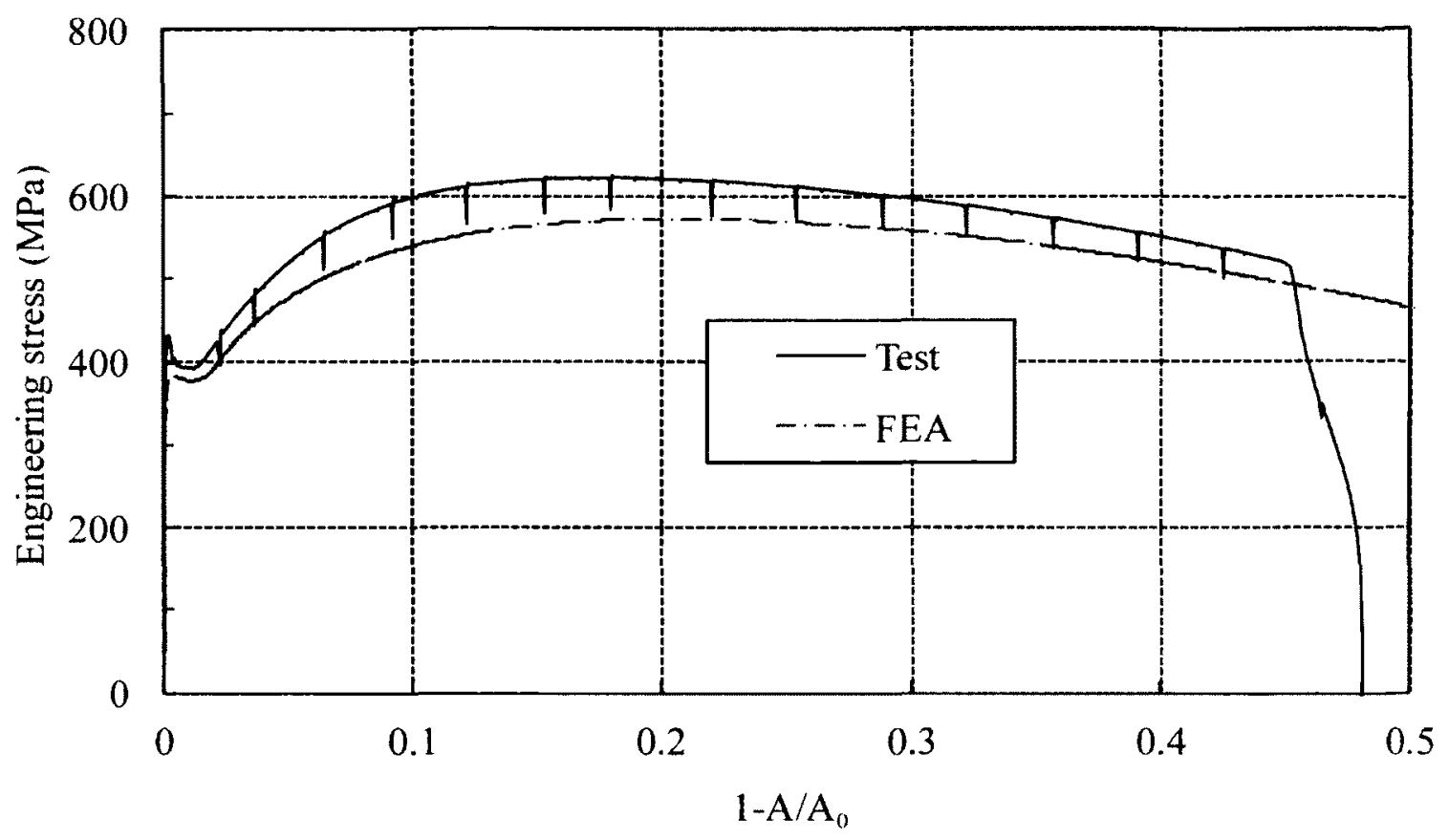

Figure A.17 Test and predicted engineering stress versus cross-sectional area change curves for notched specimen BAM (two terms)

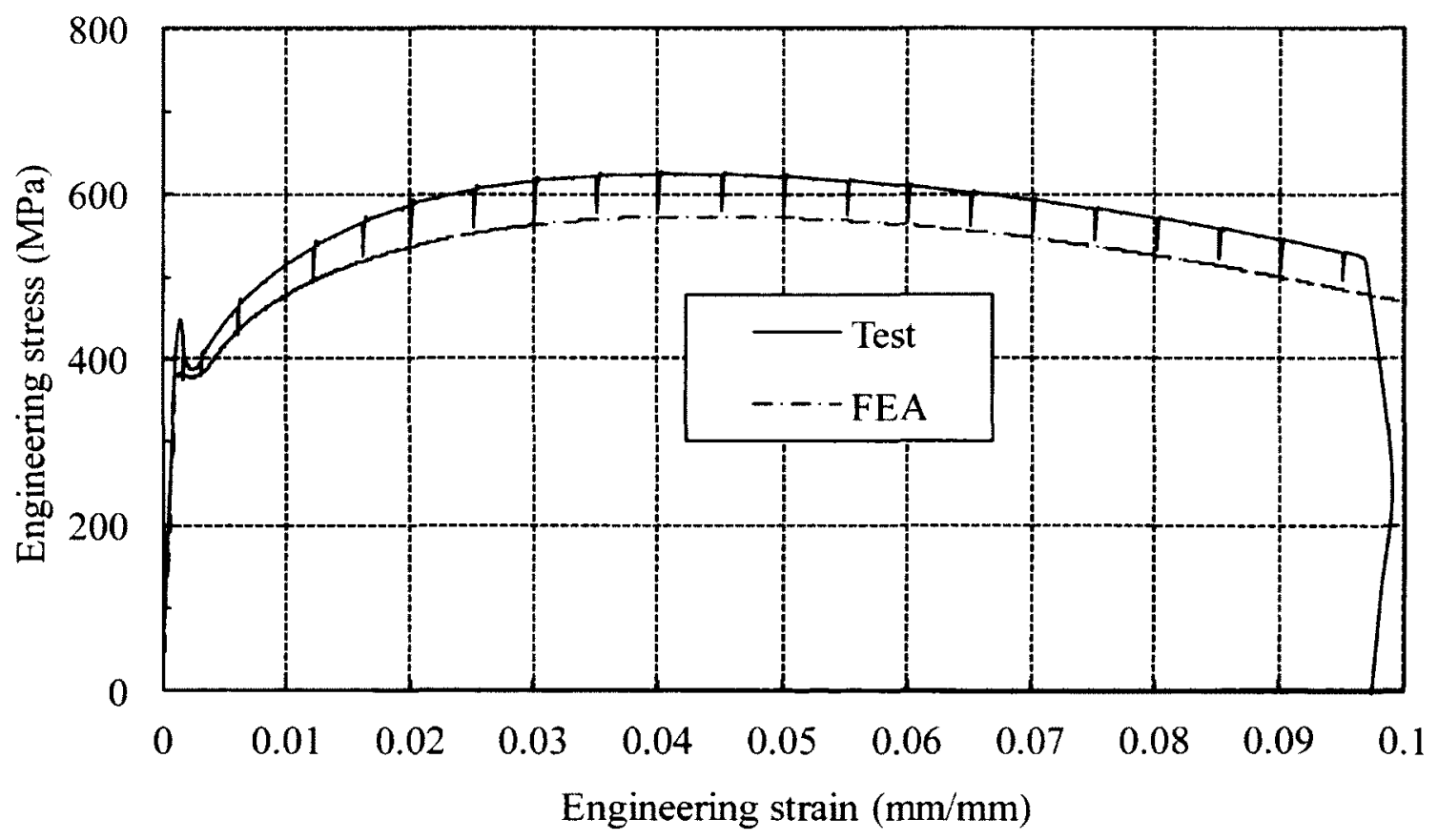

Figure A.18 Test and predicted engineering stress versus engineering strain curves for notched specimen BBM (two terms) 


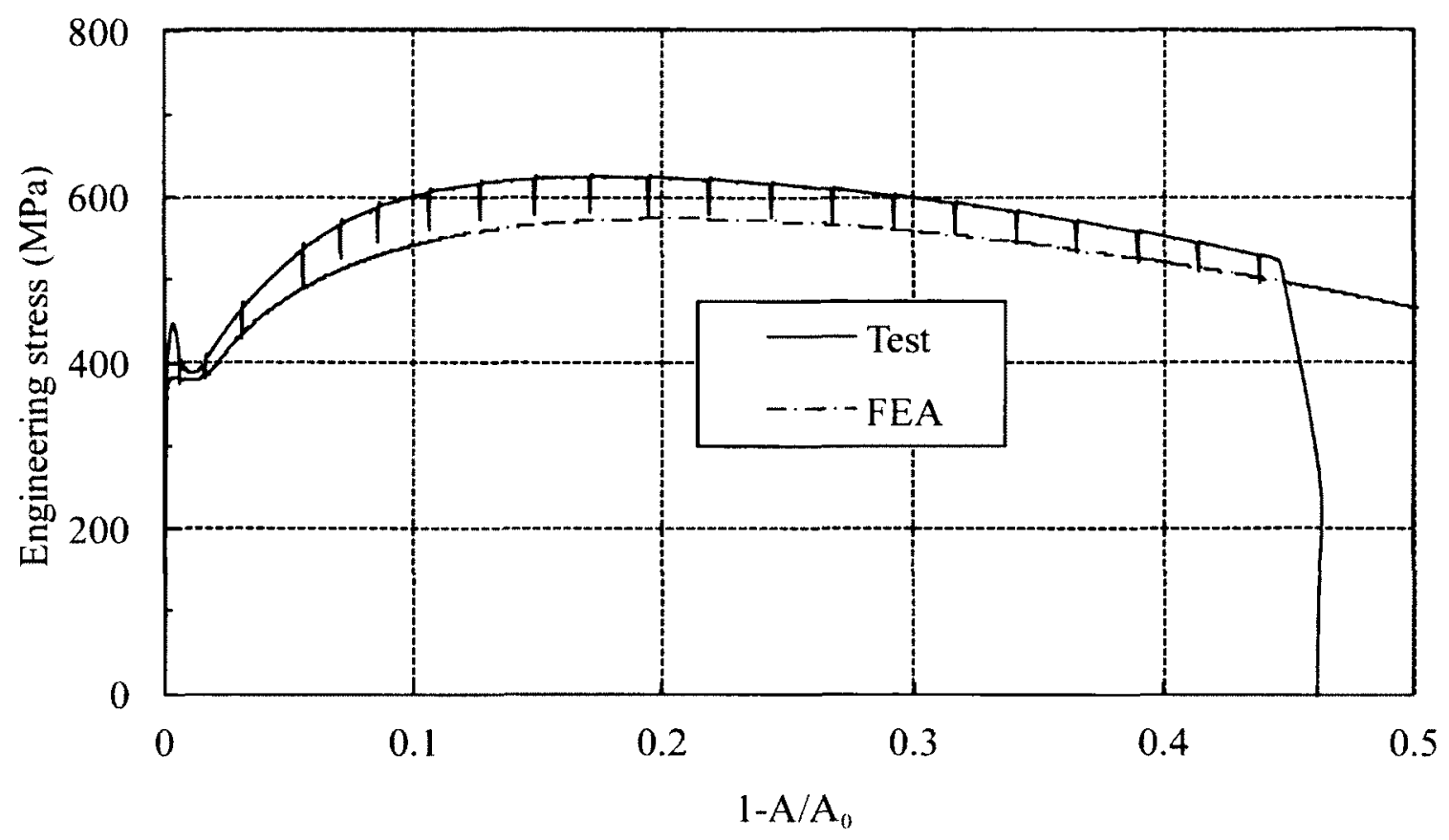

Figure A.19 Test and predicted engineering stress versus cross-sectional area change curves for notched specimen BBM (two terms)

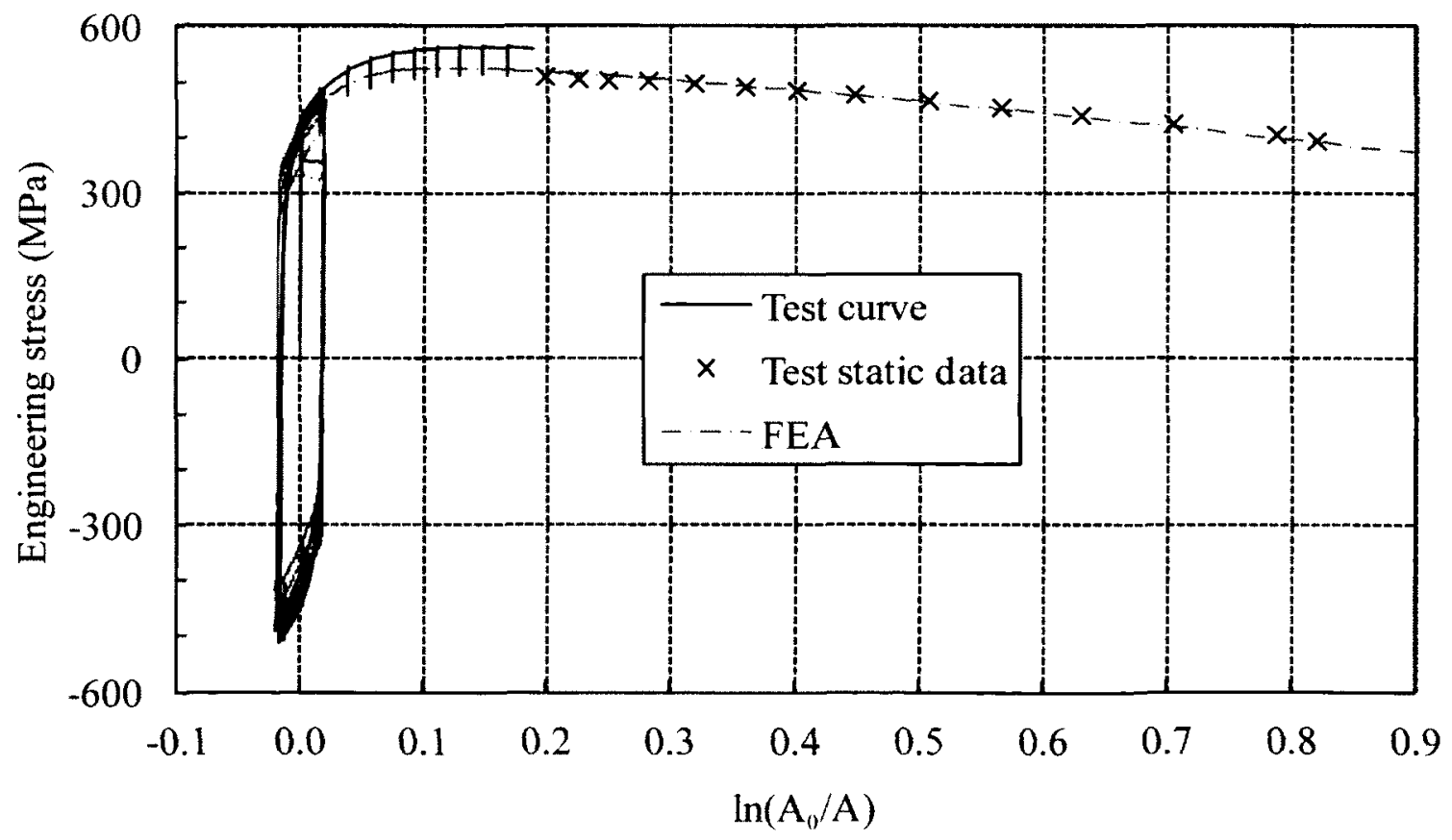

Figure A.20 Test and predicted engineering stress versus cross-sectional area change curves for straight specimen BDC20202 (two terms) 


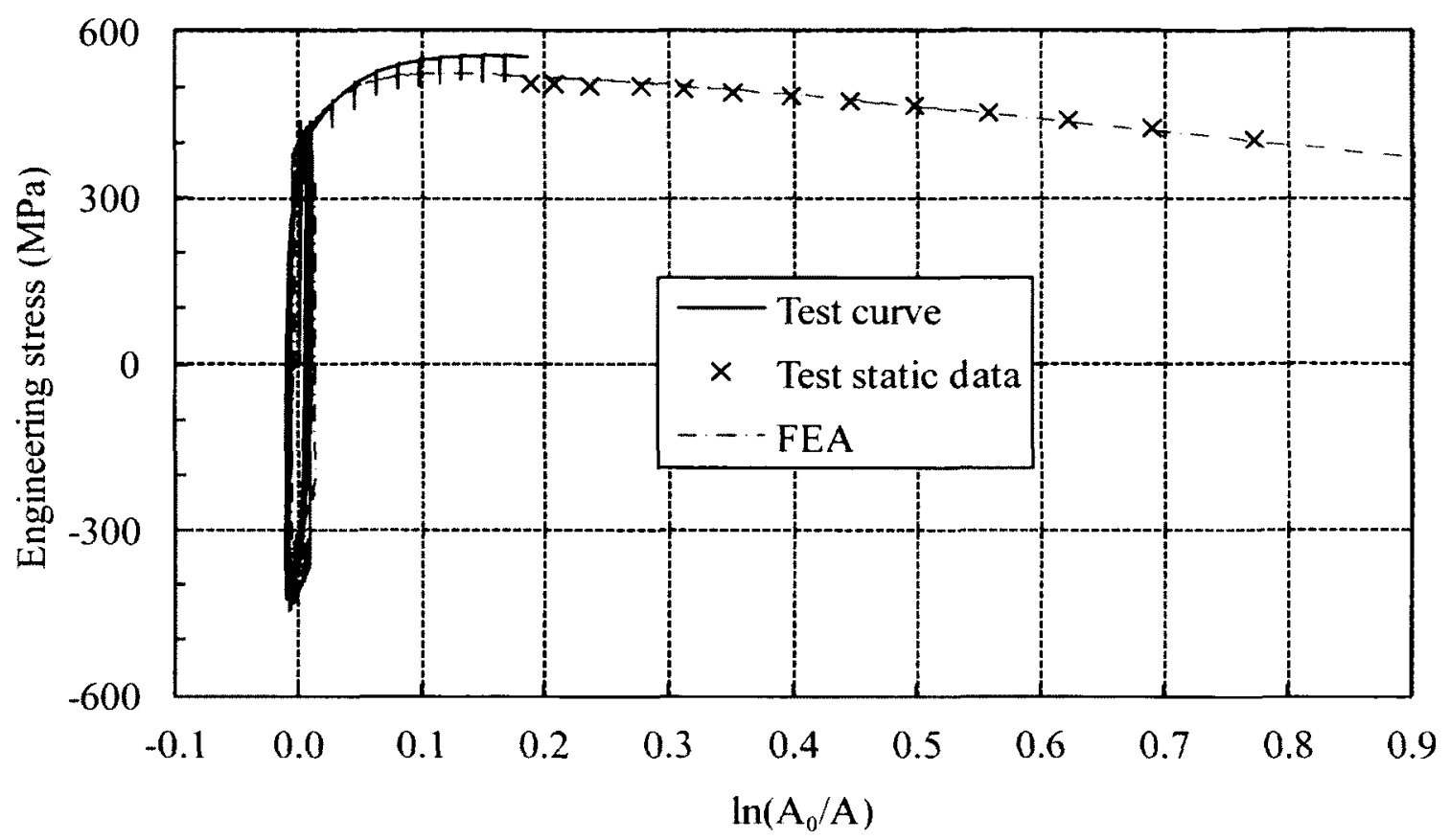

Figure A.21 Test and predicted engineering stress versus cross-sectional area change curves for straight specimen BDC40101 (two terms)

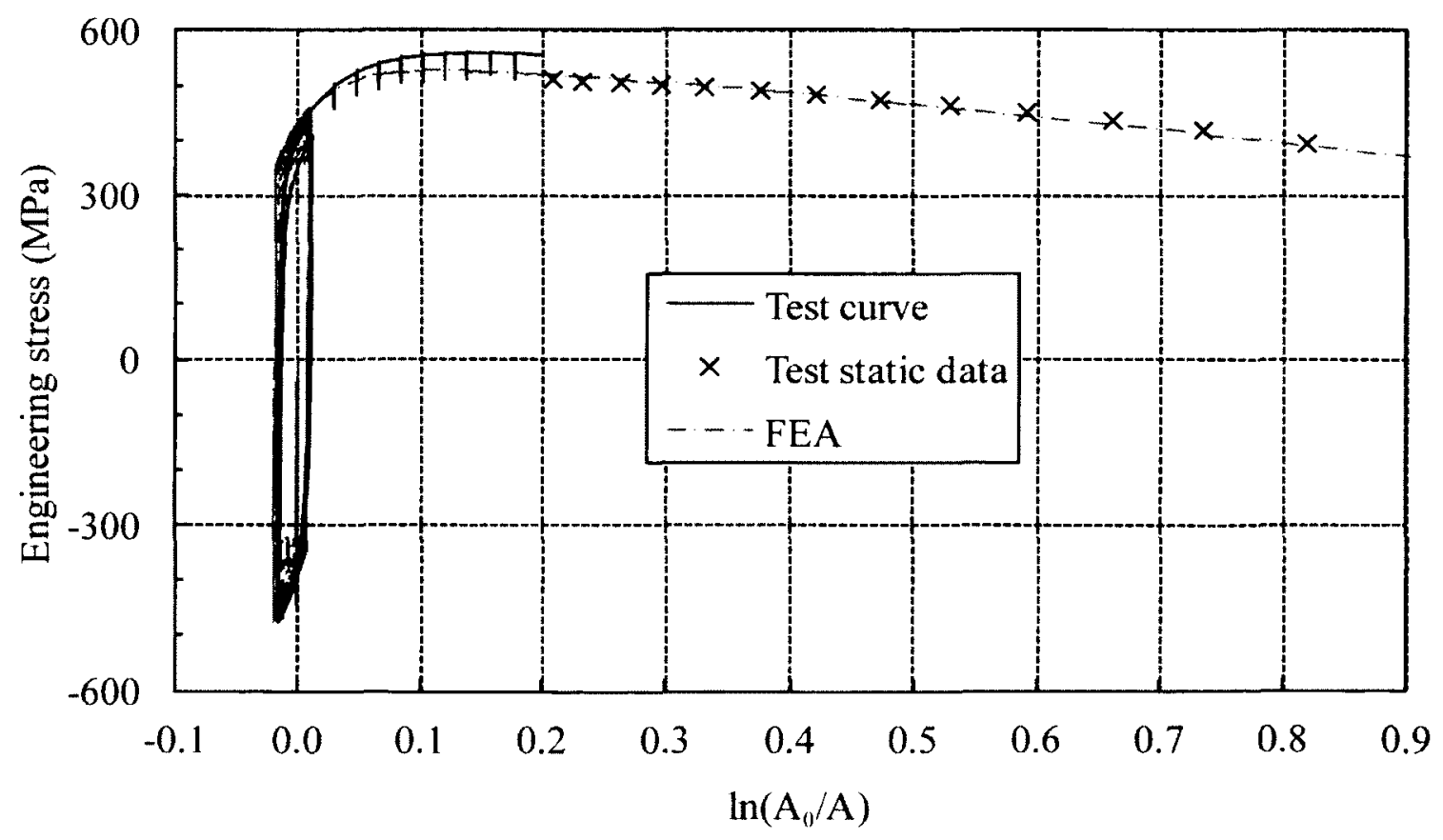

Figure A.22 Test and predicted engineering stress versus cross-sectional area change curves for straight specimen BDC40102 (two terms) 


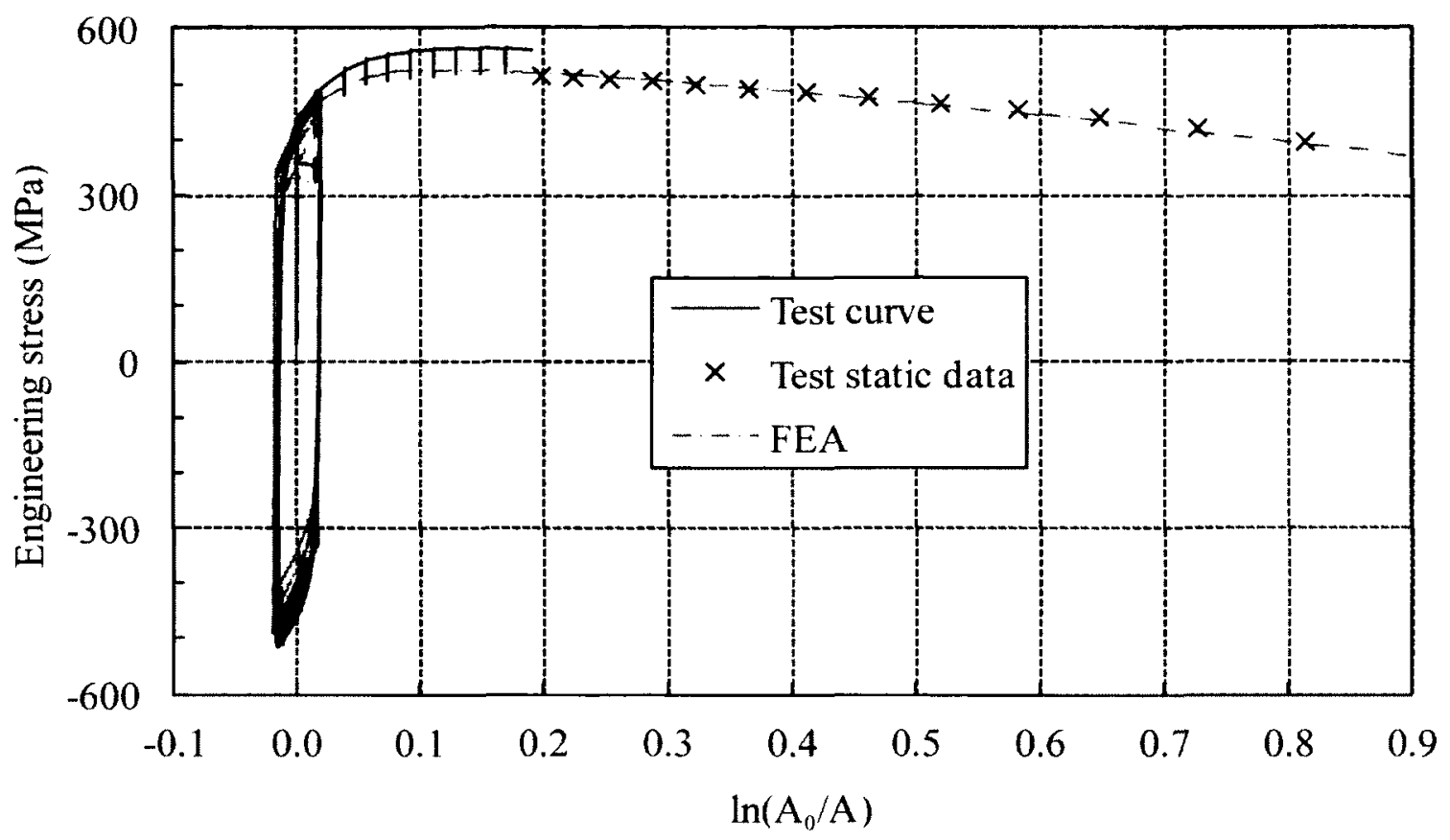

Figure A.23 Test and predicted engineering stress versus cross-sectional area change curves for straight specimen BDC40202 (two terms)

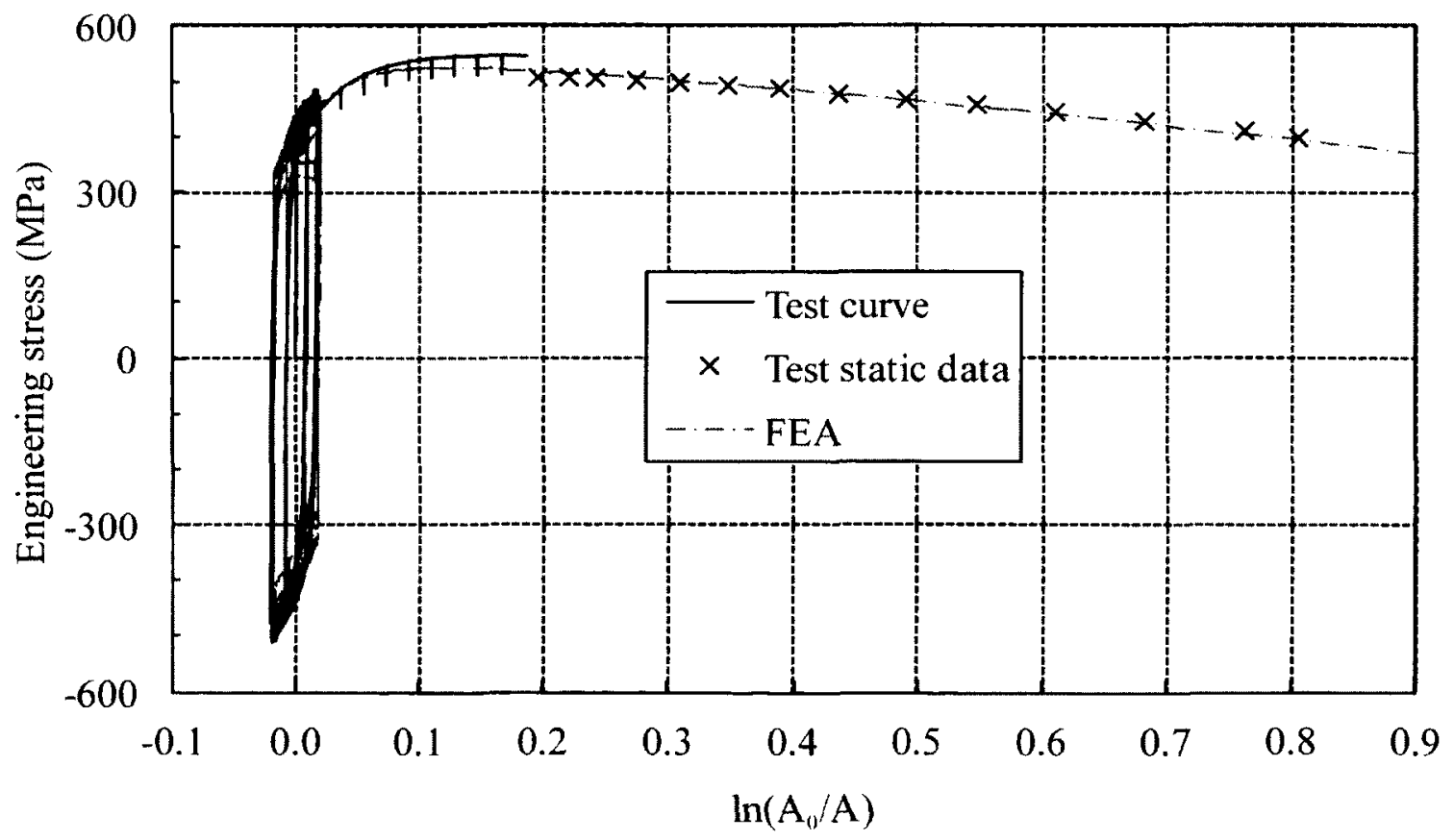

Figure A.24 Test and predicted engineering stress versus cross-sectional area change curves for straight specimen BDCR0201 (two terms) 


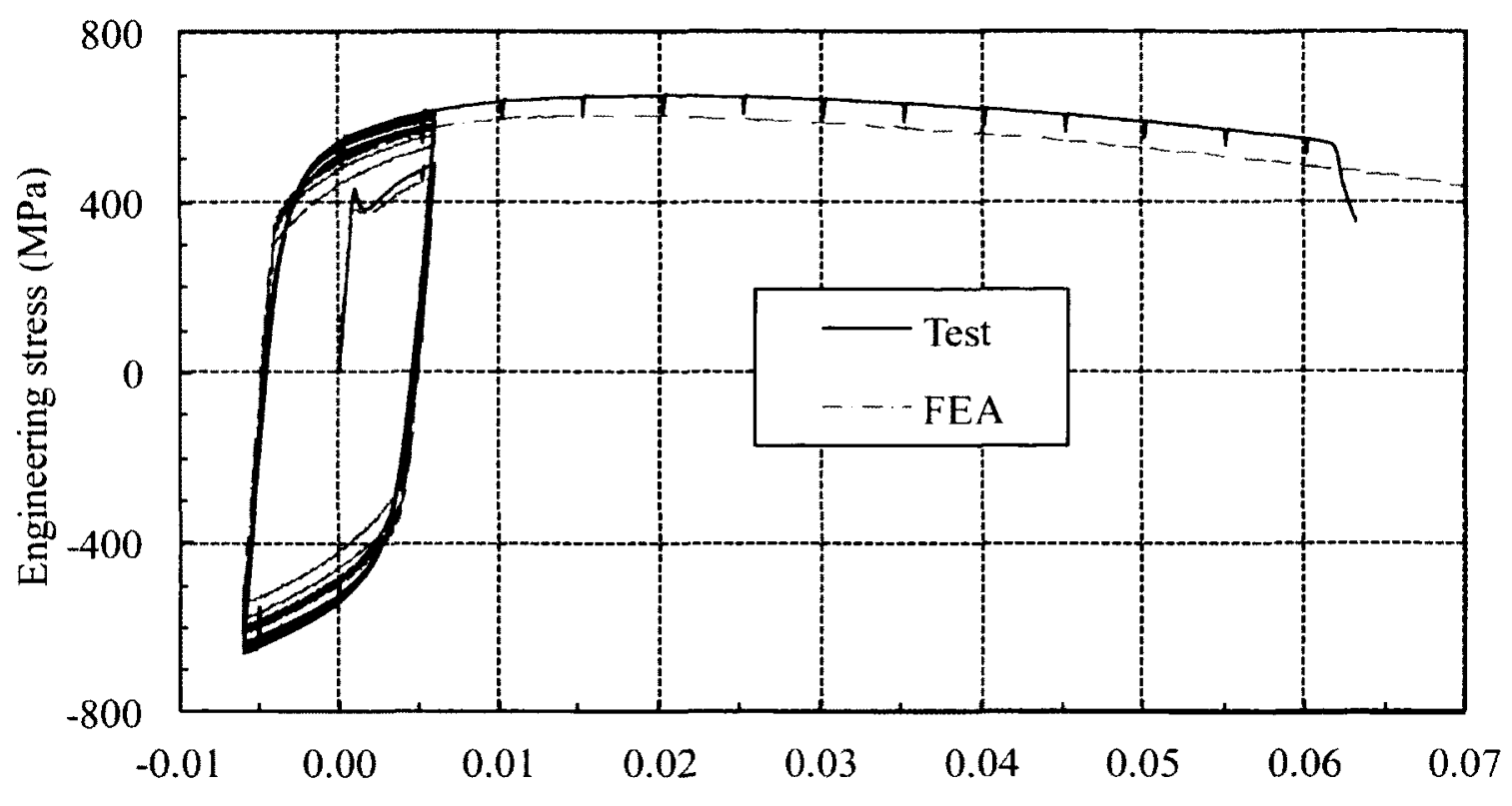

Engineering strain $(\mathrm{mm} / \mathrm{mm})$

Figure A.25 Test and predicted engineering stress versus engineering strain curves for notched specimen BAC20404 (two terms)

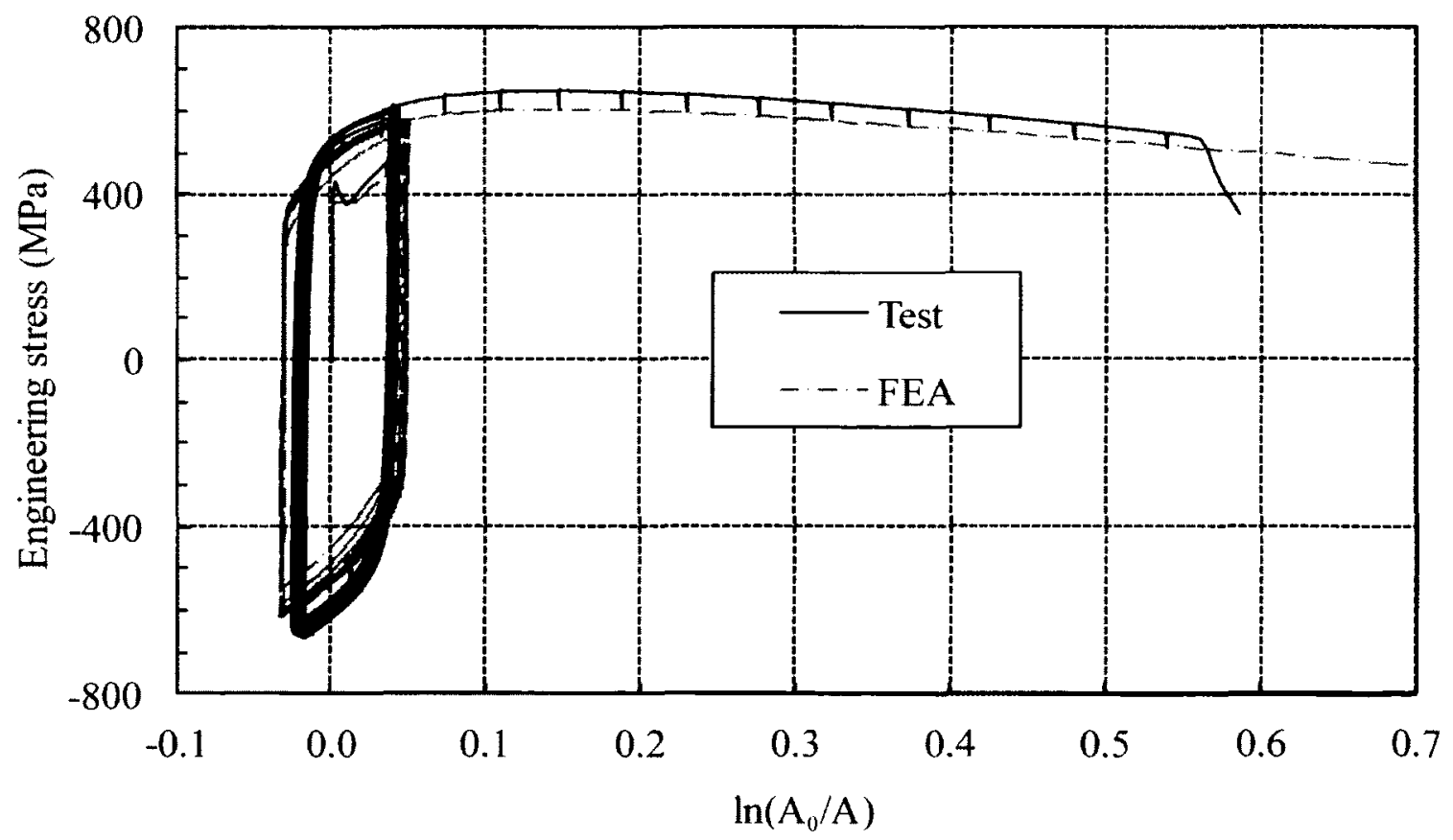

Figure A.26 Test and predicted engineering stress versus cross-sectional area change curves for notched specimen BAC20404 (two terms) 


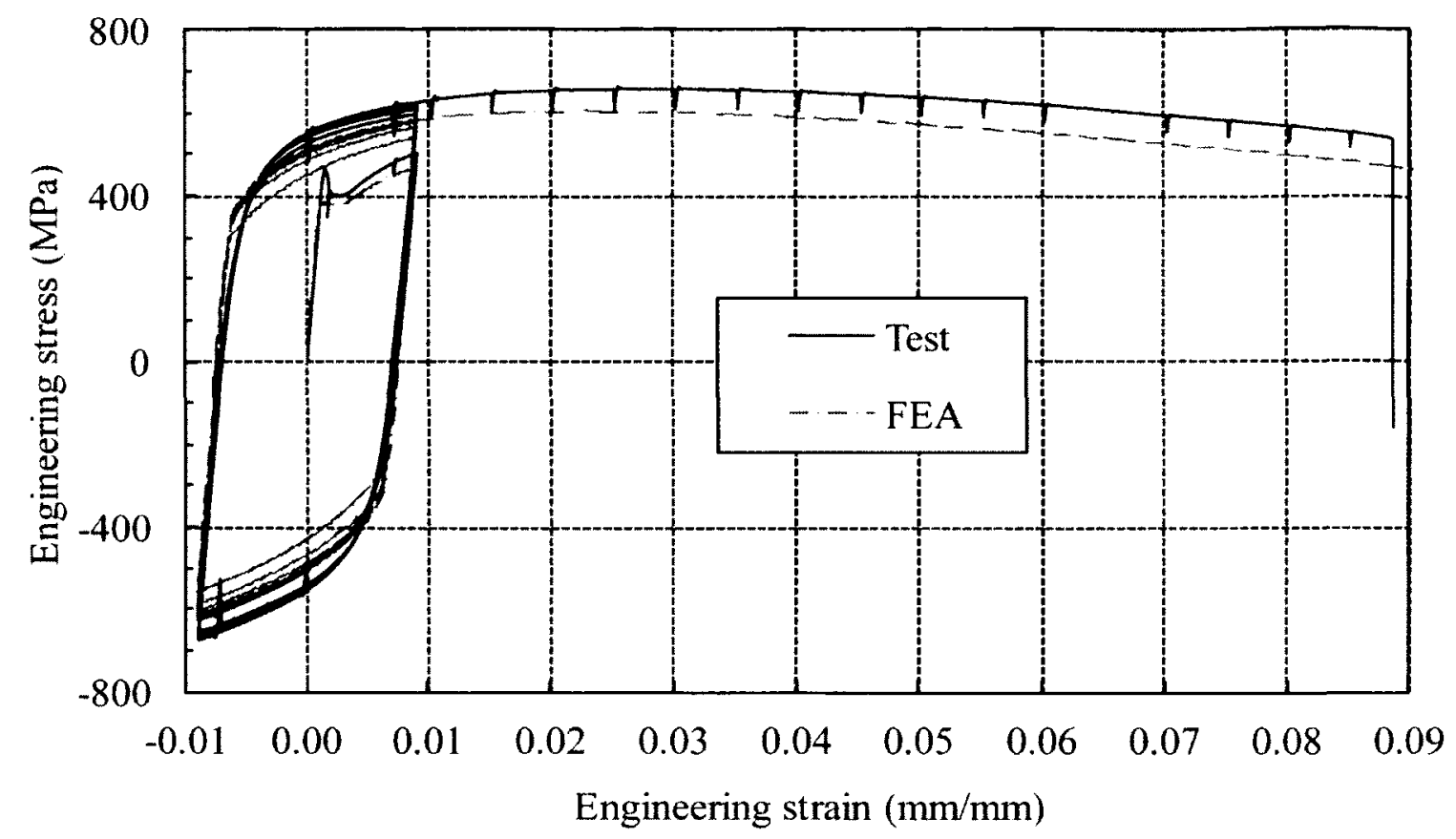

Figure A.27 Test and predicted engineering stress versus engineering strain curves for notched specimen BBC20404 (two terms)

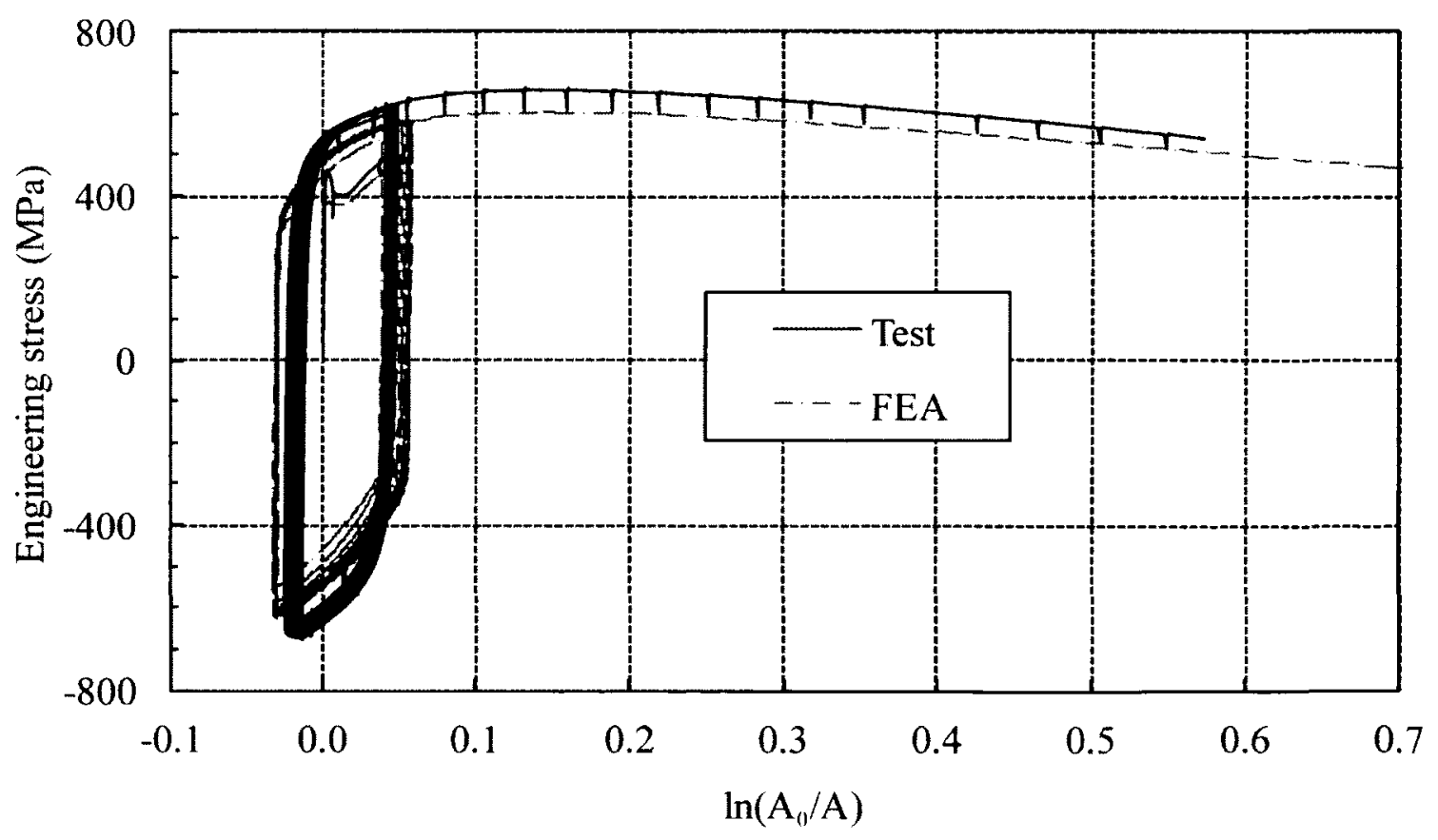

Figure A.28 Test and predicted engineering stress versus cross-sectional area change curves for notched specimen BBC20404 (two terms) 


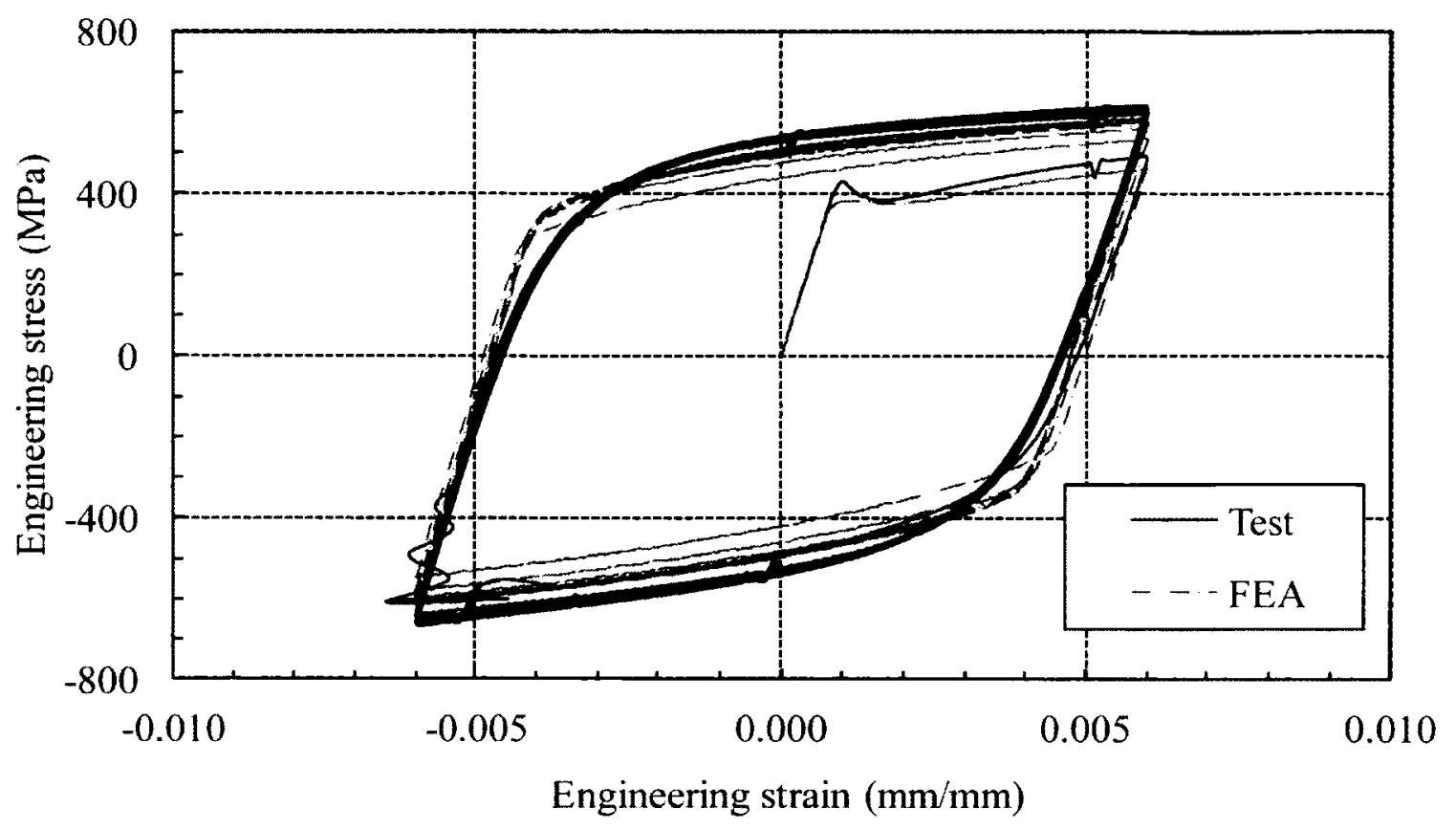

Figure A.29 Test and predicted engineering stress versus engineering strain curves for notched specimen BAC70404 (two terms)

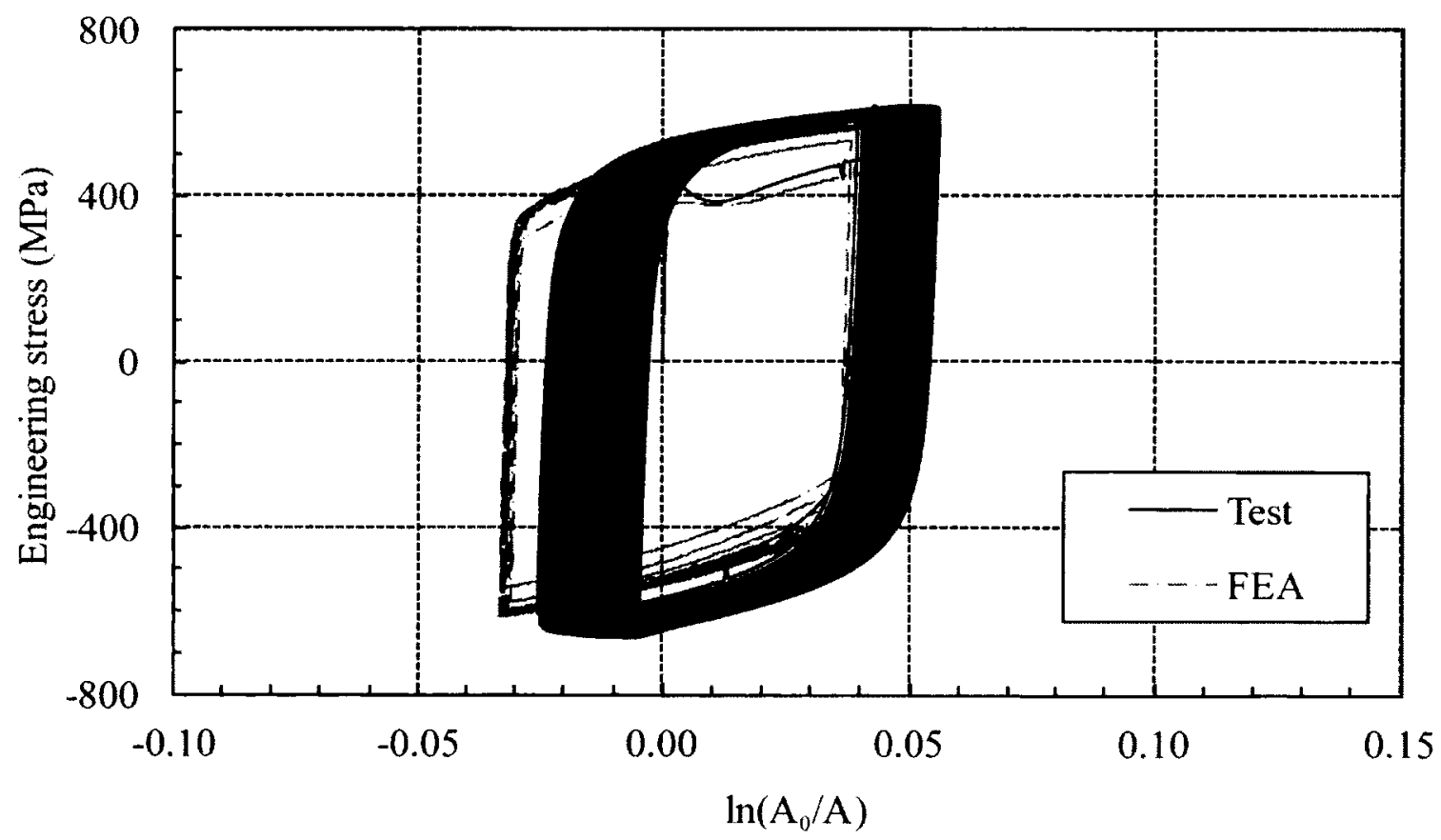

Figure A.30 Test and predicted engineering stress versus cross-sectional area change curves for notched specimen BAC70404 (two terms) 


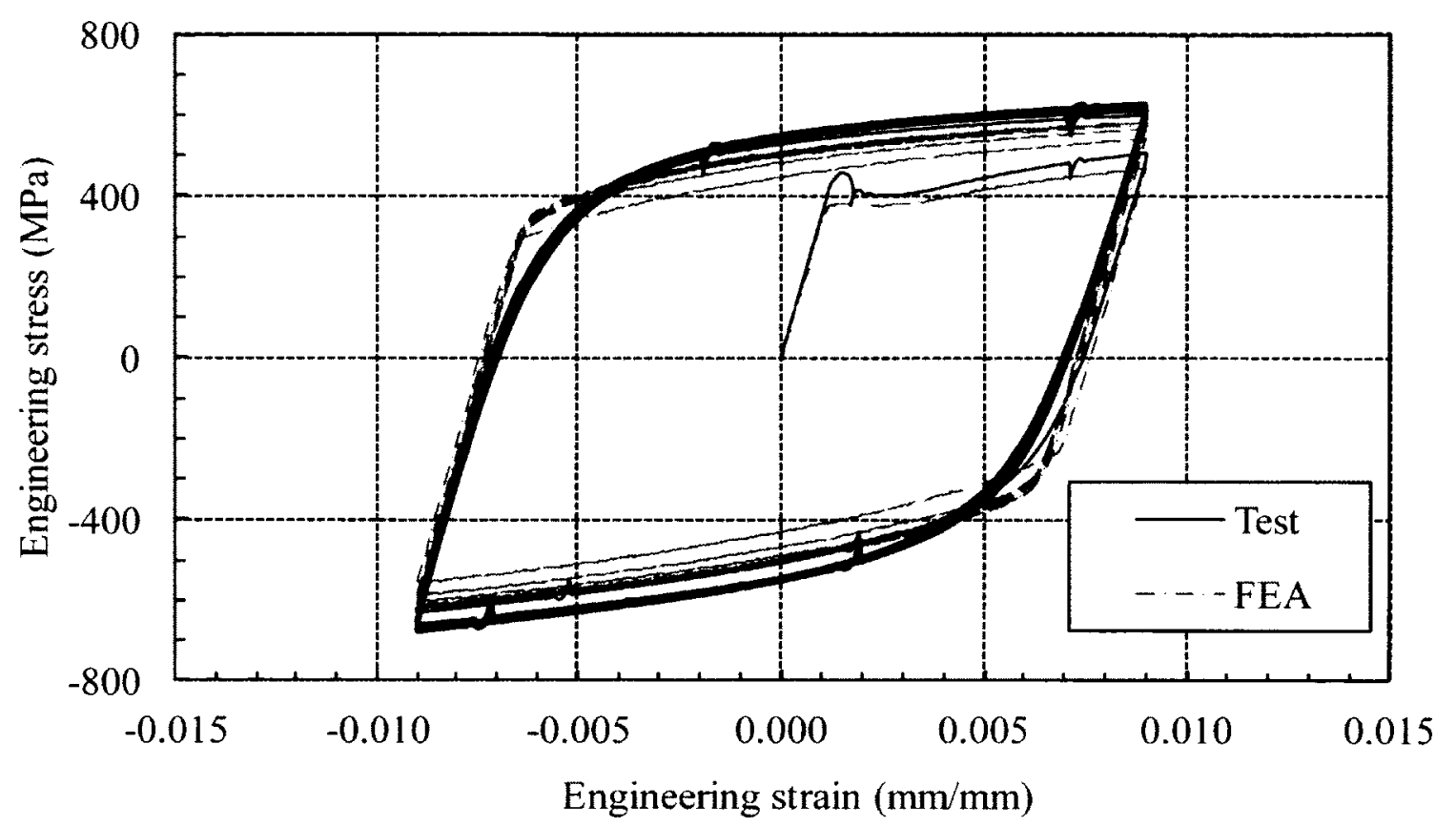

Figure A.31 Test and predicted engineering stress versus engineering strain curves for notched specimen BBC70404 (two terms)

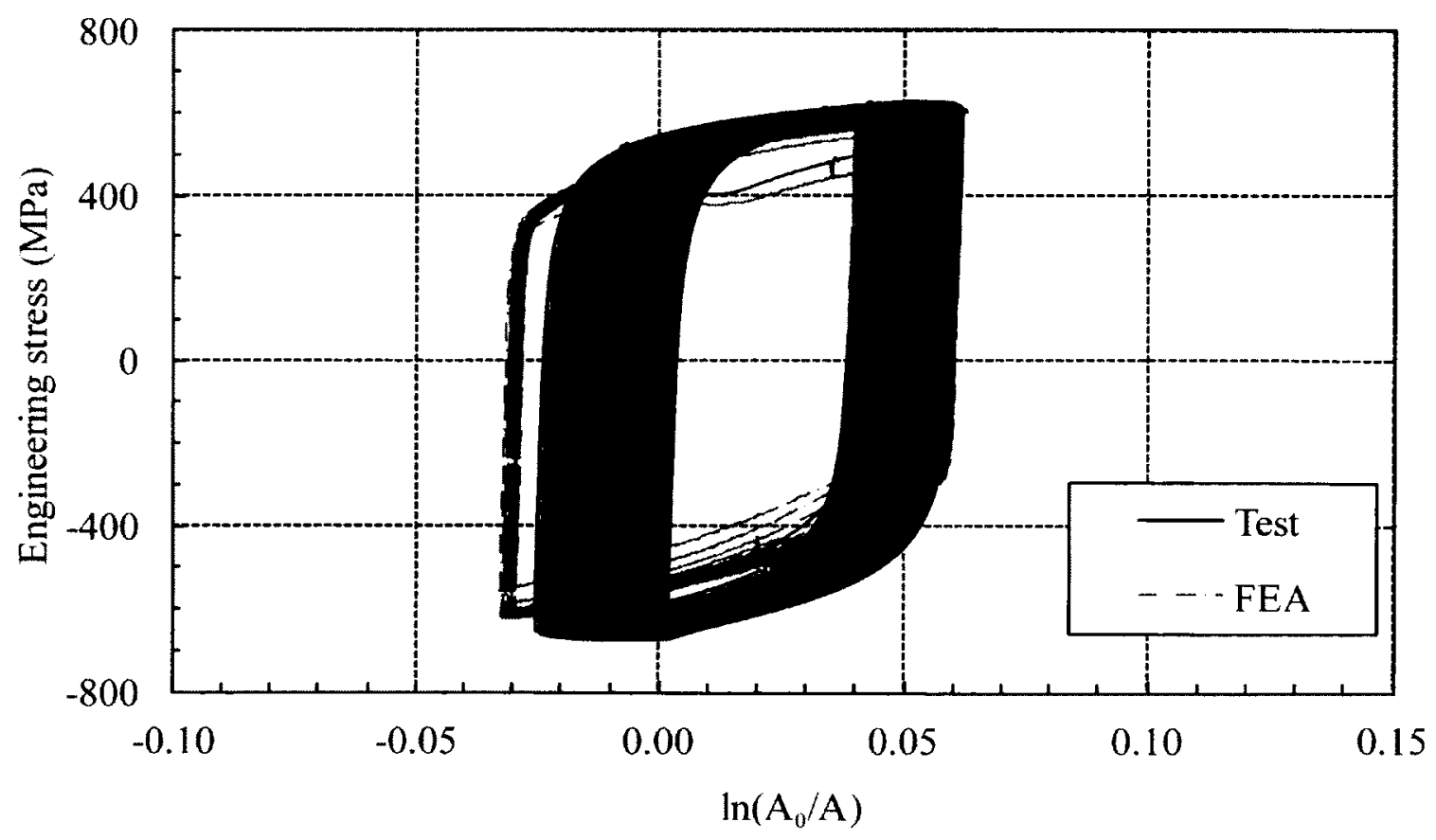

Figure A.32 Test and predicted engineering stress versus cross-sectional area change curves for notched specimen BBC70404 (two terms) 


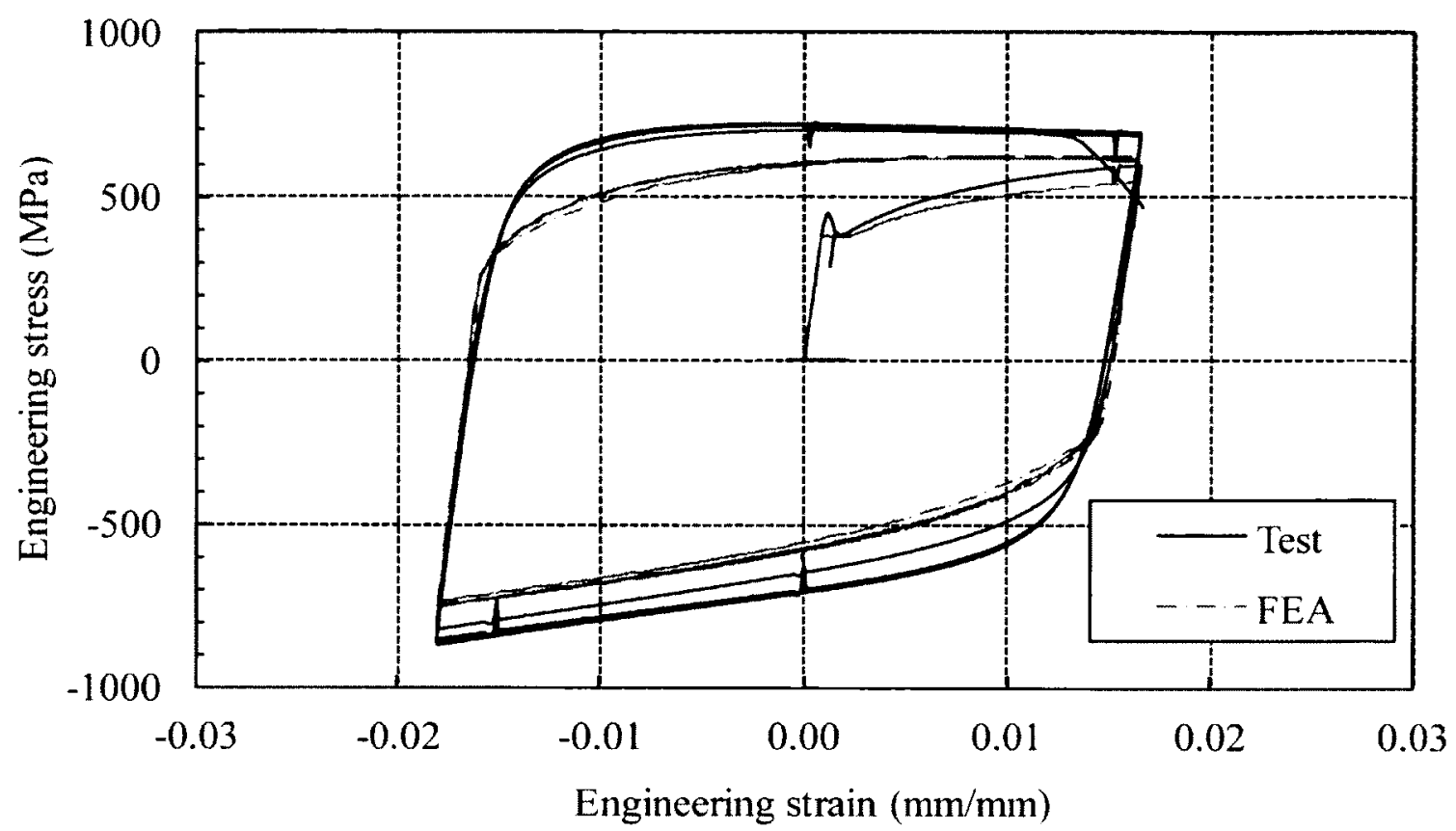

Figure A.33 Test and predicted engineering stress versus engineering strain curves for notched specimen BACF 1212 (two terms)

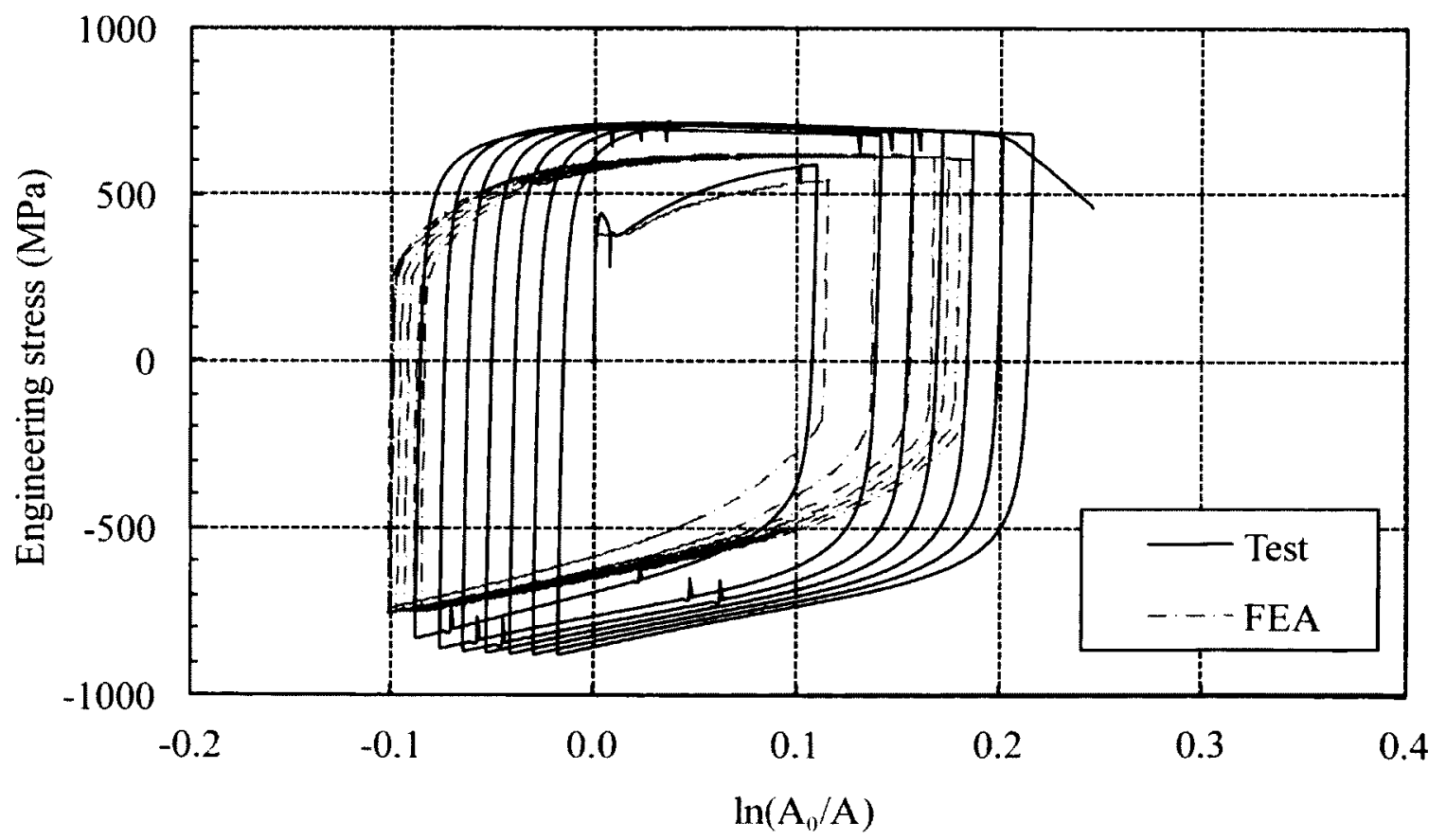

Figure A.34 Test and predicted engineering stress versus cross-sectional area change curves for notched specimen BACF1212 (two terms) 


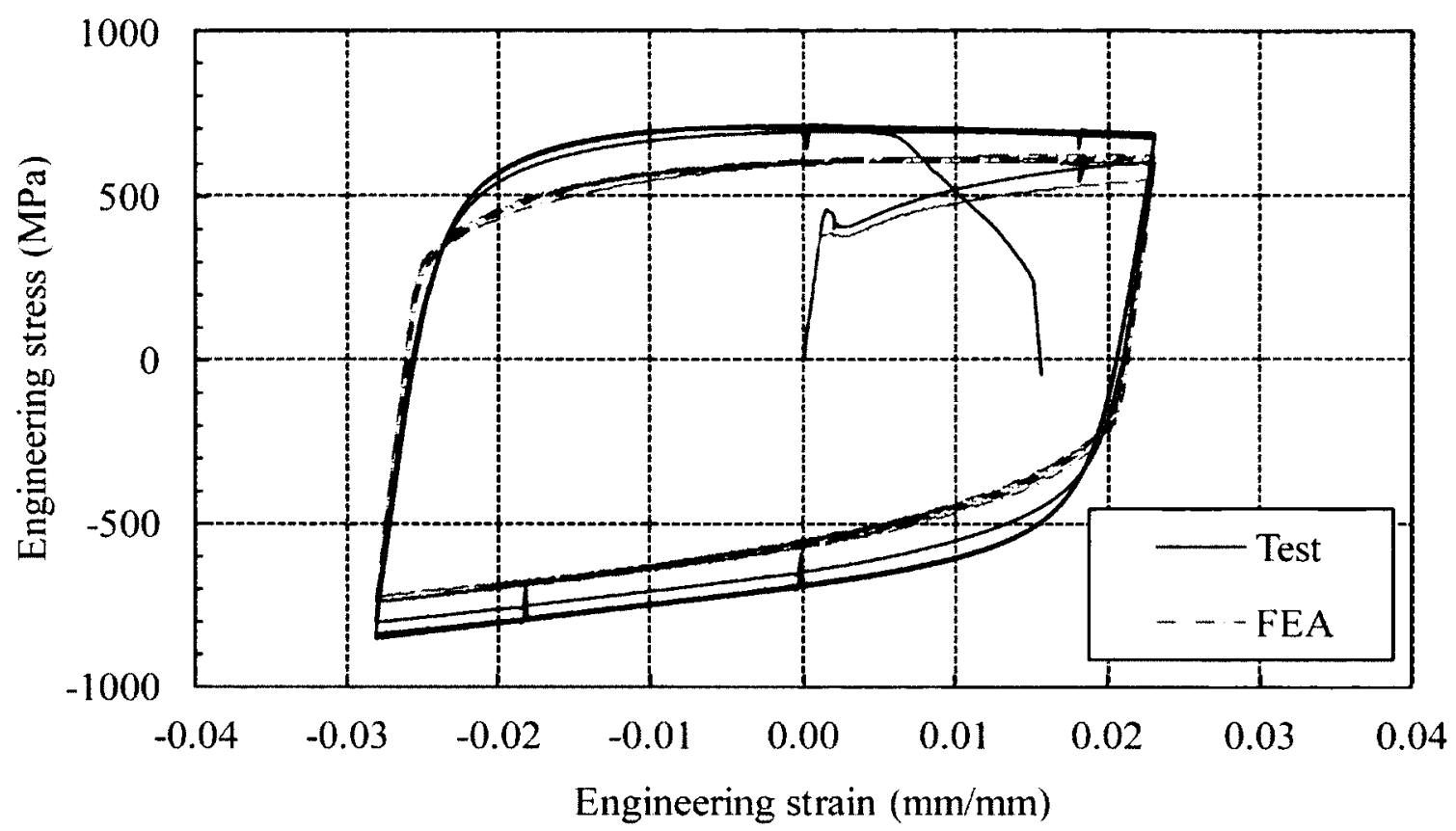

Figure A.35 Test and predicted engineering stress versus engineering strain curves for notched specimen BBCF1212 (two terms)

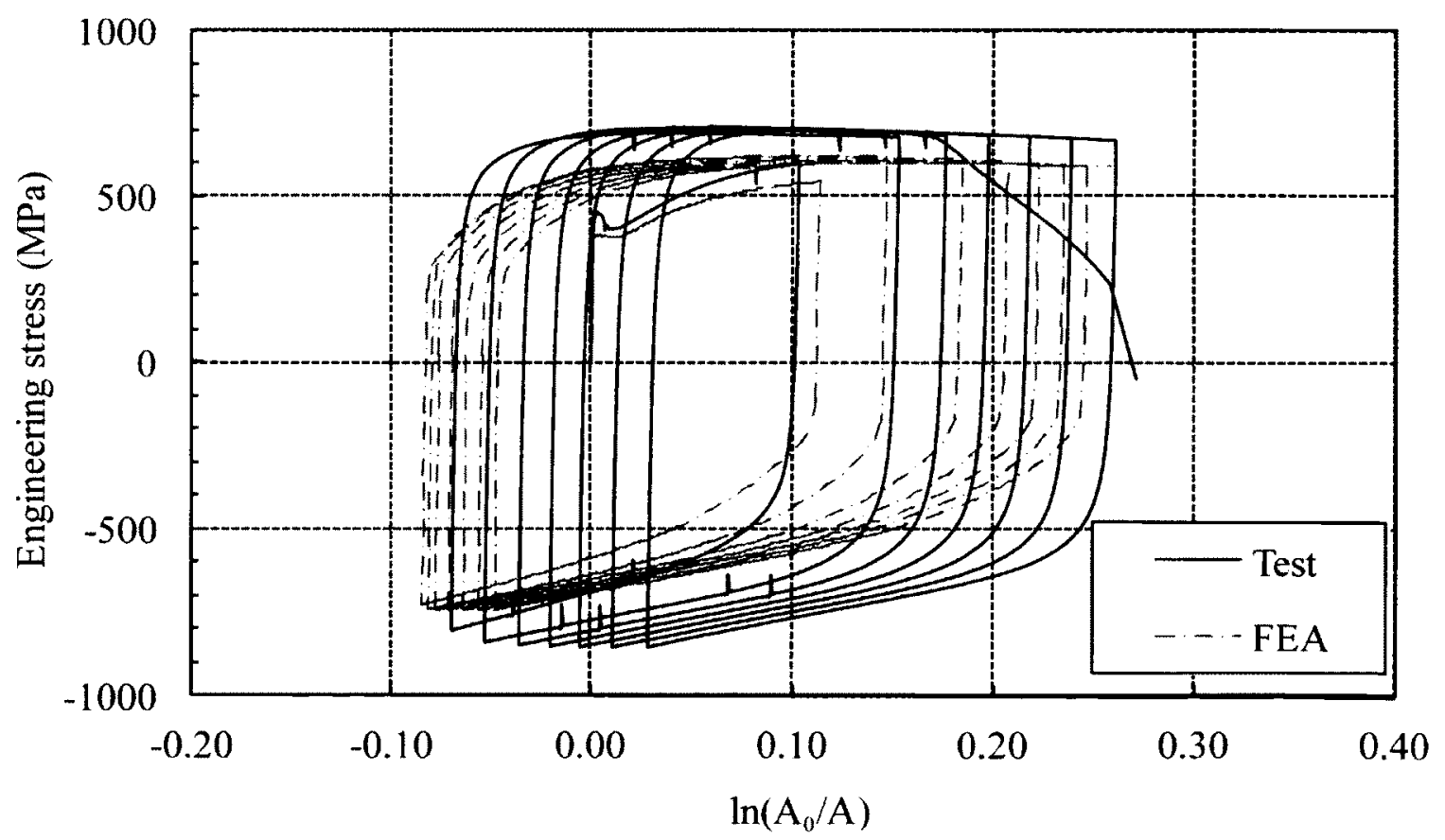

Figure A.36 Test and predicted engineering stress versus cross-sectional area change curves for notched specimen BBCF 1212 (two terms) 


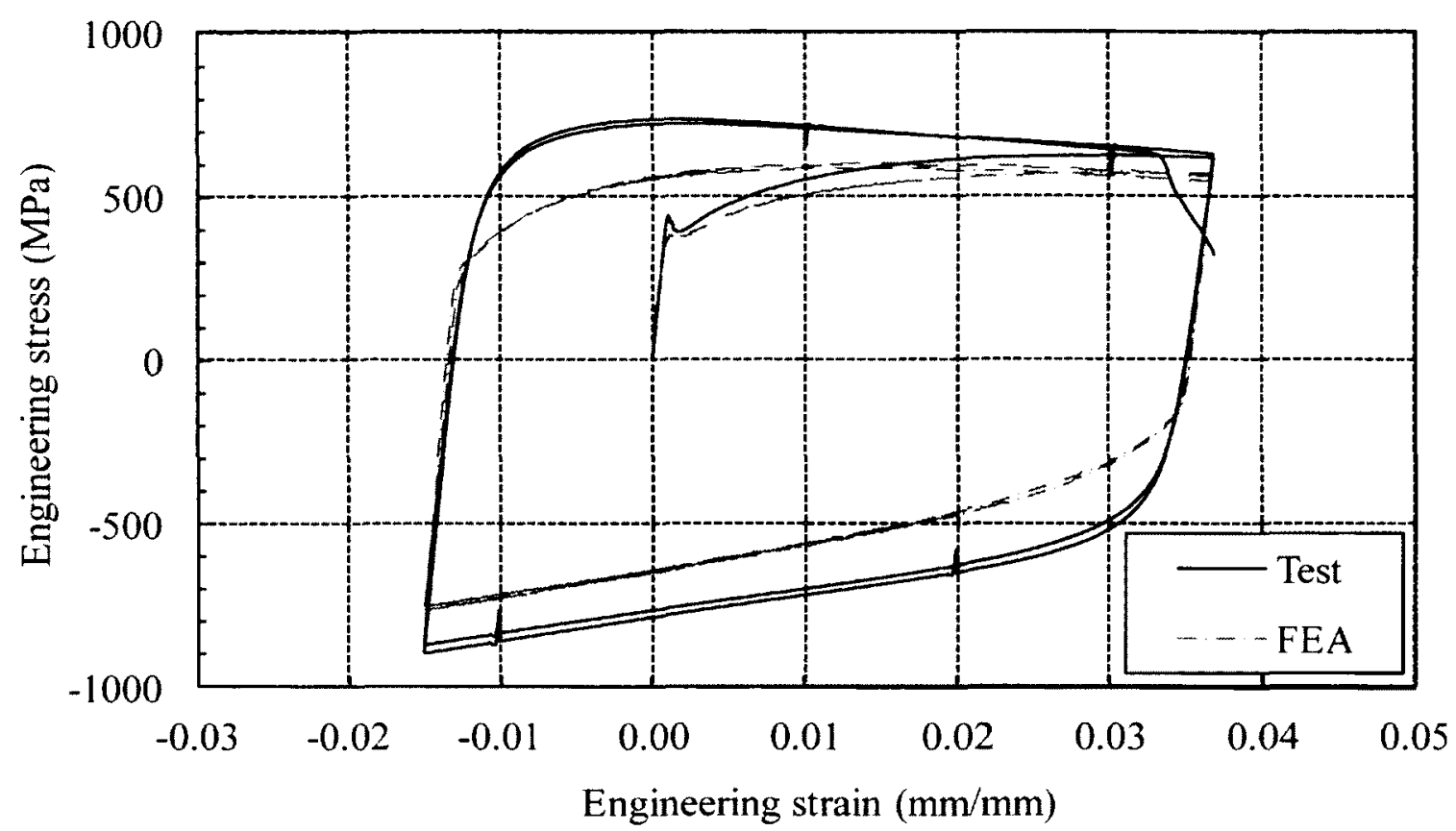

Figure A.37 Test and predicted engineering stress versus engineering strain curves for notched specimen BACF3010 (two terms)

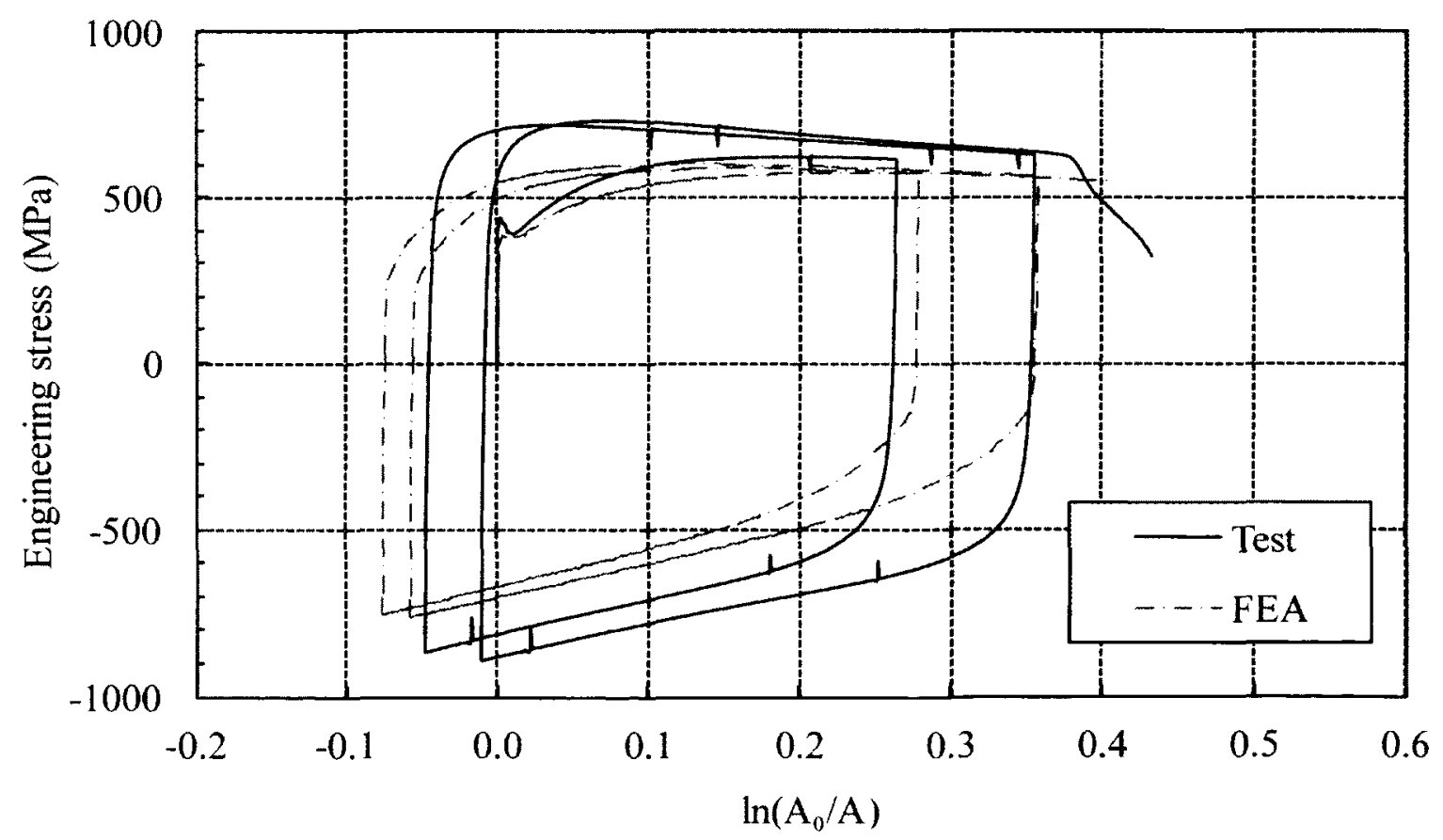

Figure A.38 Test and predicted engineering stress versus cross-sectional area change curves for notched specimen BACF3010 (two terms) 


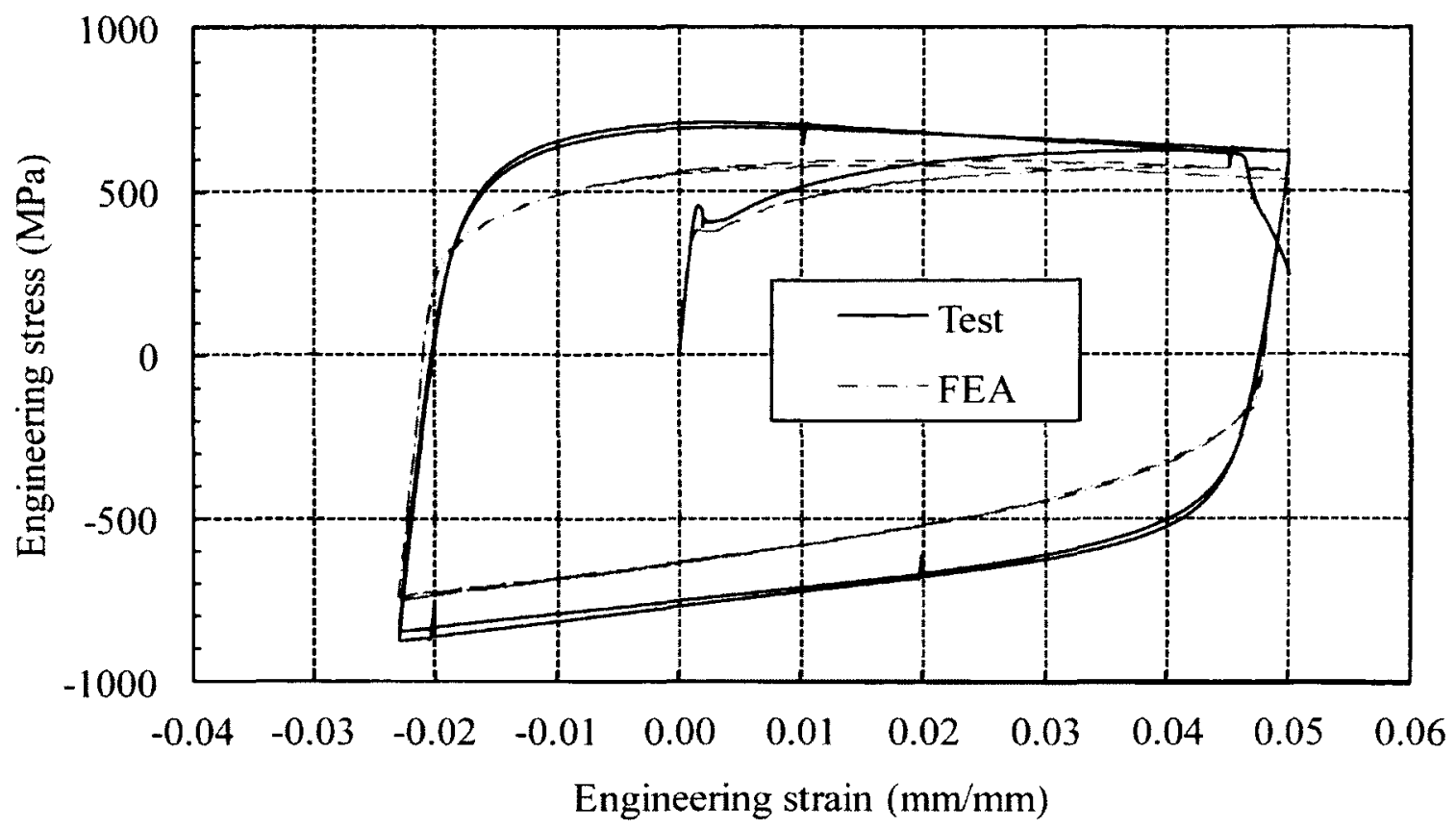

Figure A.39 Test and predicted engineering stress versus engineering strain curves for notched specimen BBCF3010 (two terms)

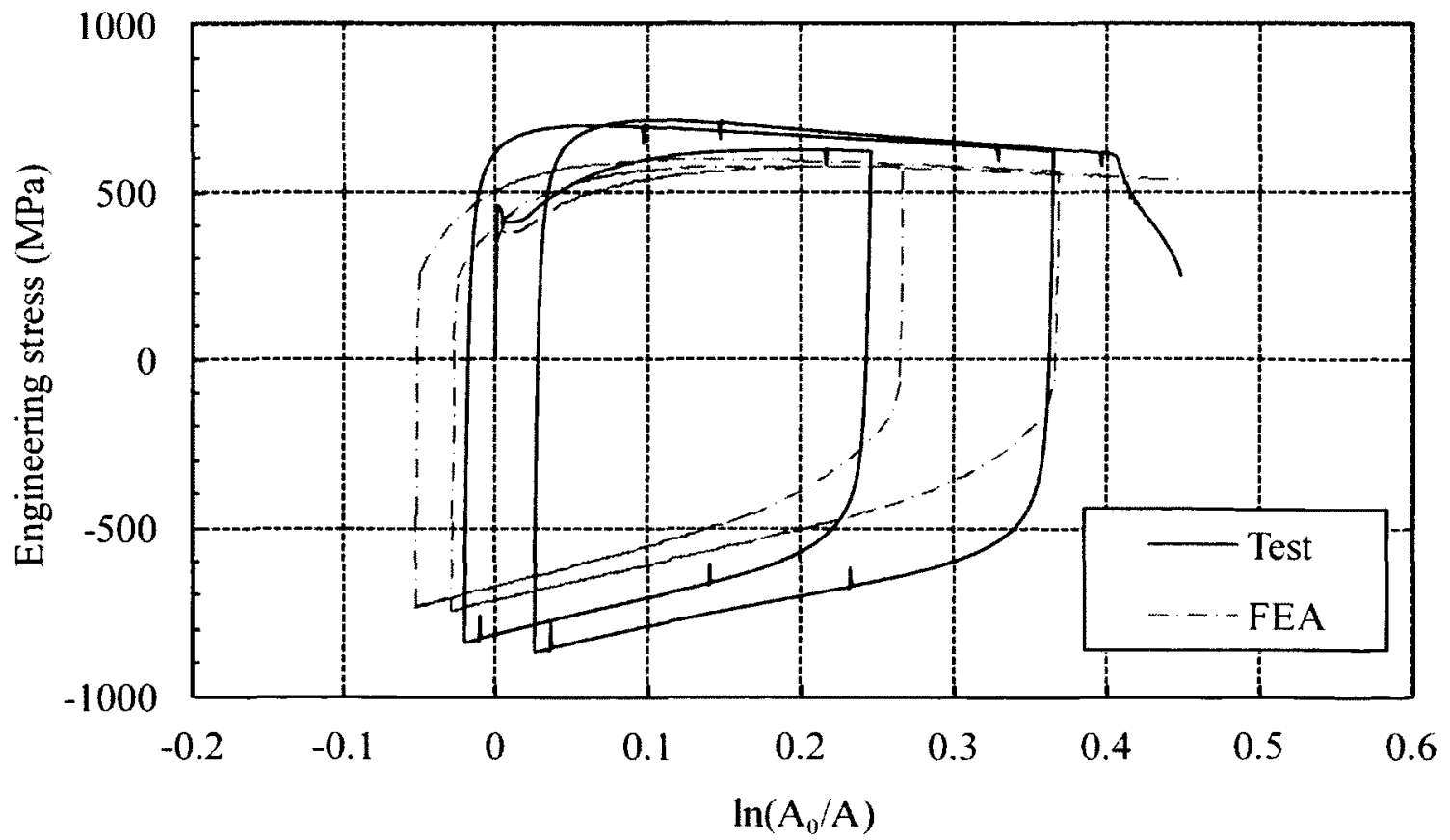

Figure A.40 Test and predicted engineering stress versus cross-sectional area change curves for notched specimen BBCF3010 (two terms) 


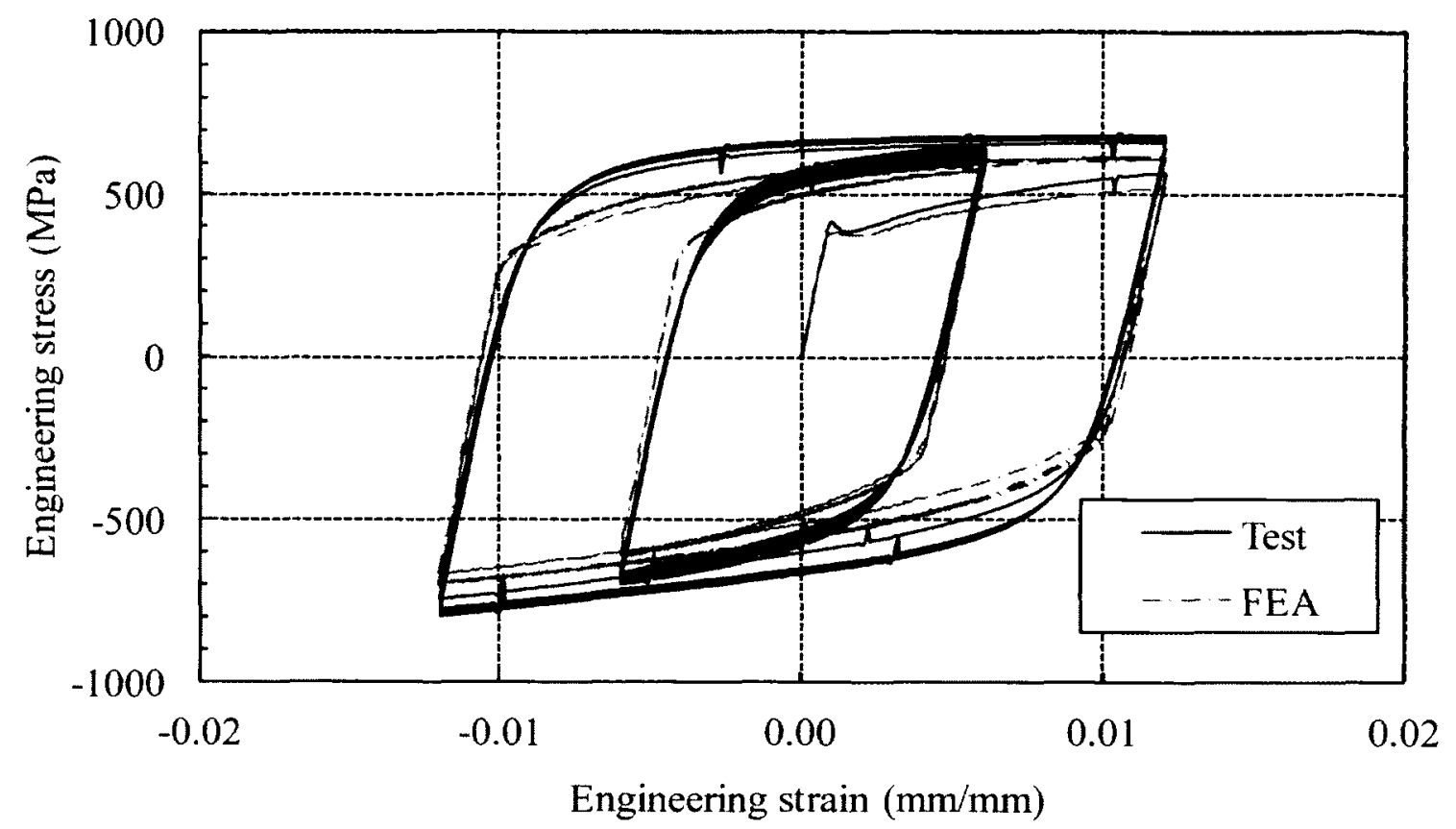

Figure A.41 Test and predicted engineering stress versus engineering strain curves for notched specimen BACR0804 (two terms)

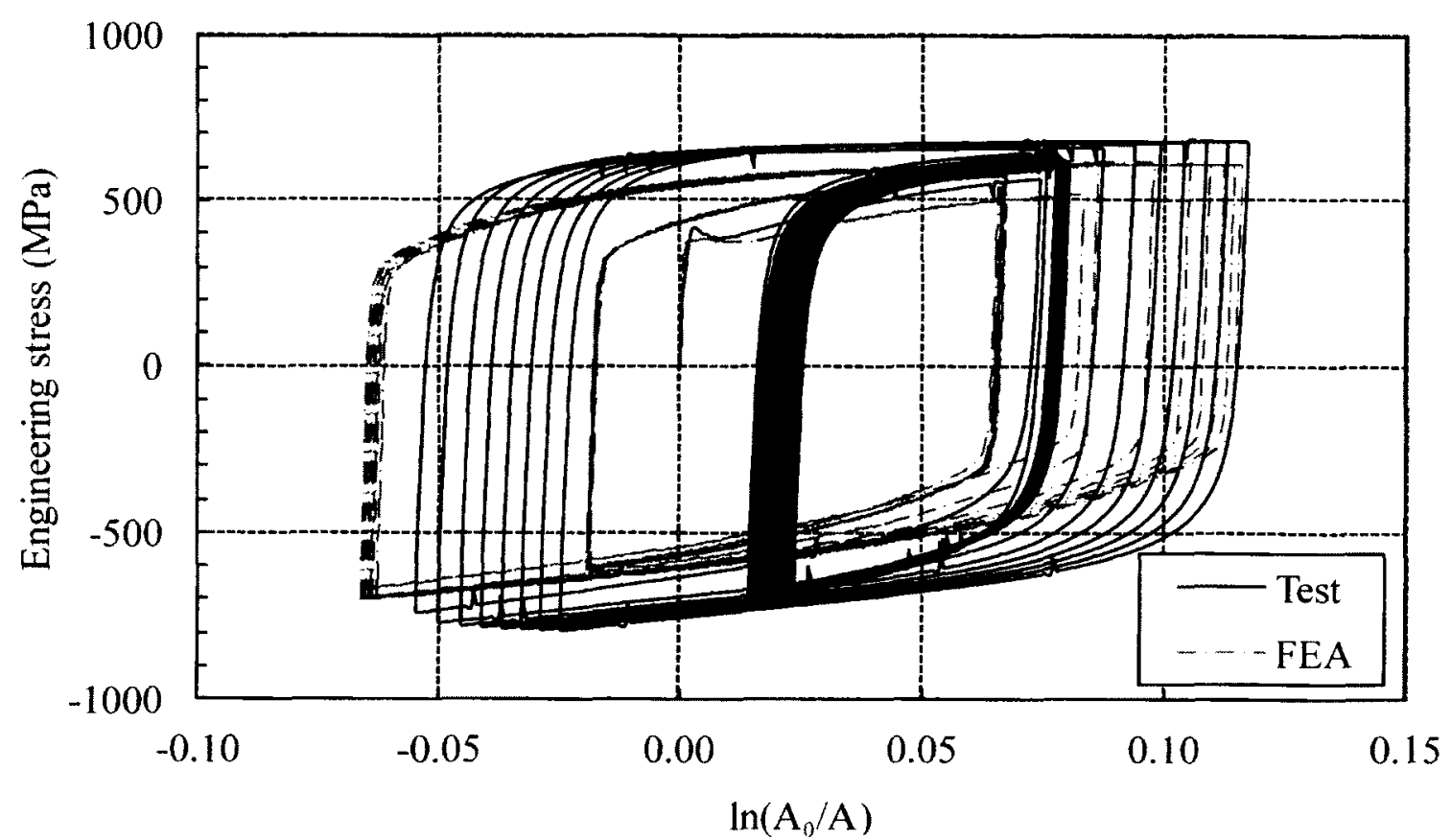

Figure A.42 Test and predicted engineering stress versus cross-sectional area change curves for notched specimen BACR0804 (two terms) 


\section{APPENDIX B COMPARISONS OF TESTS AND FEA SIMULTIONS FOR MATERIAL A (ONE TERM)}

For material $\mathrm{A}$, the optimum hardening parameters can be determined with $\sigma_{\mathrm{s}}^{0}=$ $489 \mathrm{MPa}, \Delta \sigma_{\mathrm{s}}^{0}=24 \mathrm{MPa}$ and $\mathrm{b}=5.43$. With an assumed Poisson's ratio $v=0.3$, the measured elastic modulus $E=205600 \mathrm{MPa}, \sigma_{0}^{y}=362 \mathrm{MPa}$ and $\varepsilon_{0}^{\mathrm{p}}=0.0053$, the rest of the parameters for the hardening model have been determined to be $\sigma_{\sigma}^{y}=407 \mathrm{MPa}, \mathrm{n}=$ $0.83, \mathrm{C}_{1}=1489 \mathrm{MPa}$ and $\gamma_{1}=5.83$. The yield stress versus equivalent plastic strain data are calculated by subtracting the backstress from the flow stress generated with Eq. (3.28). Various components of stress for material A are shown in Fig. B.1 and the yield stress versus equivalent plastic strain data are listed in Table B.1.

Using the above parameters, numerical simulations have been carried out for some test specimens of material A. Comparisons of the test results with numerical simulations for these specimens are shown in Figs. B.2 to B.11. 
Table B.1 Yield stress versus equivalent plastic strain data for material A (one term)

\begin{tabular}{|c|c|}
\hline Equivalent plastic strain $(\mathrm{mm} / \mathrm{mm})$ & Yield stress (MPa) \\
\hline 0.000 & 360.3 \\
\hline 0.005 & 354.4 \\
\hline 0.010 & 370.9 \\
\hline 0.019 & 391.8 \\
\hline 0.028 & 407.6 \\
\hline 0.038 & 420.1 \\
\hline 0.047 & 430.6 \\
\hline 0.056 & 439.1 \\
\hline 0.066 & 446.5 \\
\hline 0.075 & 452.6 \\
\hline 0.084 & 458.0 \\
\hline 0.093 & 462.5 \\
\hline 0.106 & 468.2 \\
\hline 0.115 & 471.7 \\
\hline 0.124 & 474.9 \\
\hline 0.135 & 478.1 \\
\hline 0.145 & 481.0 \\
\hline 0.156 & 483.6 \\
\hline 0.168 & 486.2 \\
\hline 0.176 & 487.6 \\
\hline 0.189 & 489.7 \\
\hline 0.203 & 491.8 \\
\hline 0.244 & 496.5 \\
\hline 0.295 & 500.3 \\
\hline 0.313 & 501.3 \\
\hline 0.354 & 503.2 \\
\hline 0.413 & 505.3 \\
\hline 0.501 & 507.5 \\
\hline 0.597 & 509.1 \\
\hline 0.701 & 510.4 \\
\hline 0.818 & 511.4 \\
\hline 0.915 & 512.0 \\
\hline 0.991 & 512.4 \\
\hline 1.200 & 512.9 \\
\hline 2.000 & 513.4 \\
\hline 5.000 & 513.5 \\
\hline 10.000 & 513.5 \\
\hline 20.000 & 513.5 \\
\hline
\end{tabular}




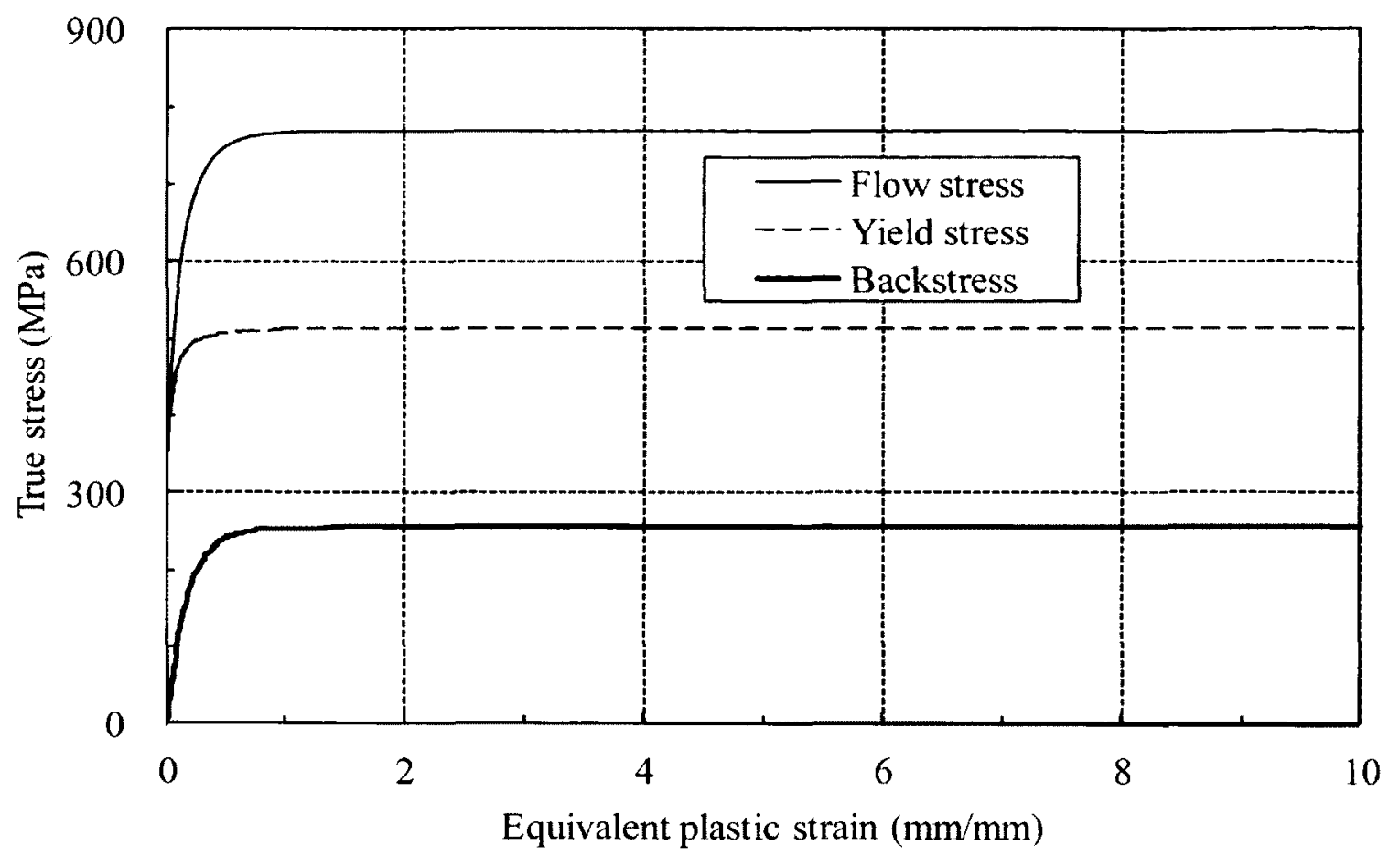

Figure B.1 Various components of stresses for material A (one terms)

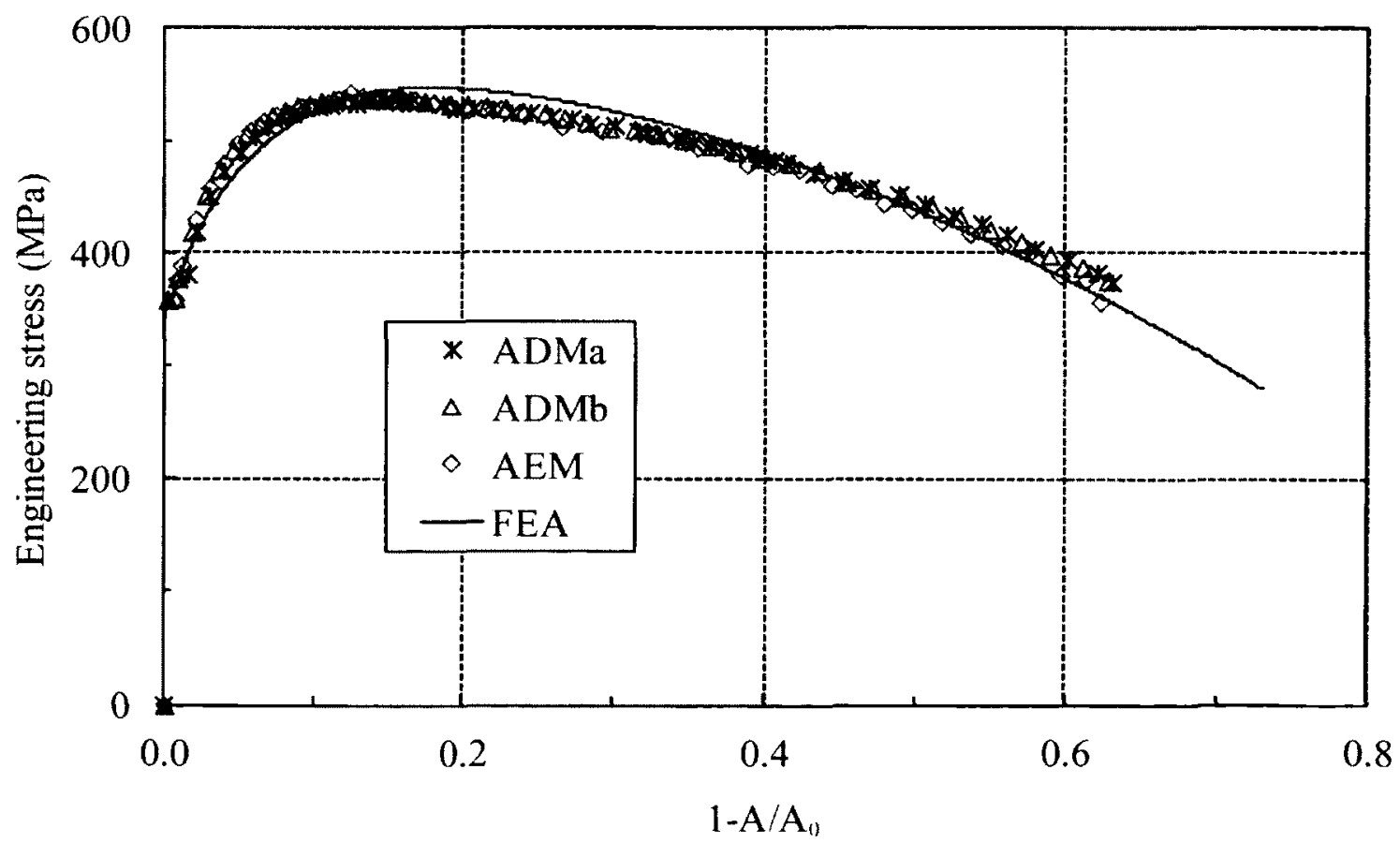

Figure B.2 Test and predicted engineering stress versus cross-sectional area change curves for straight specimens ADMa, ADMb and AEM (one term) 


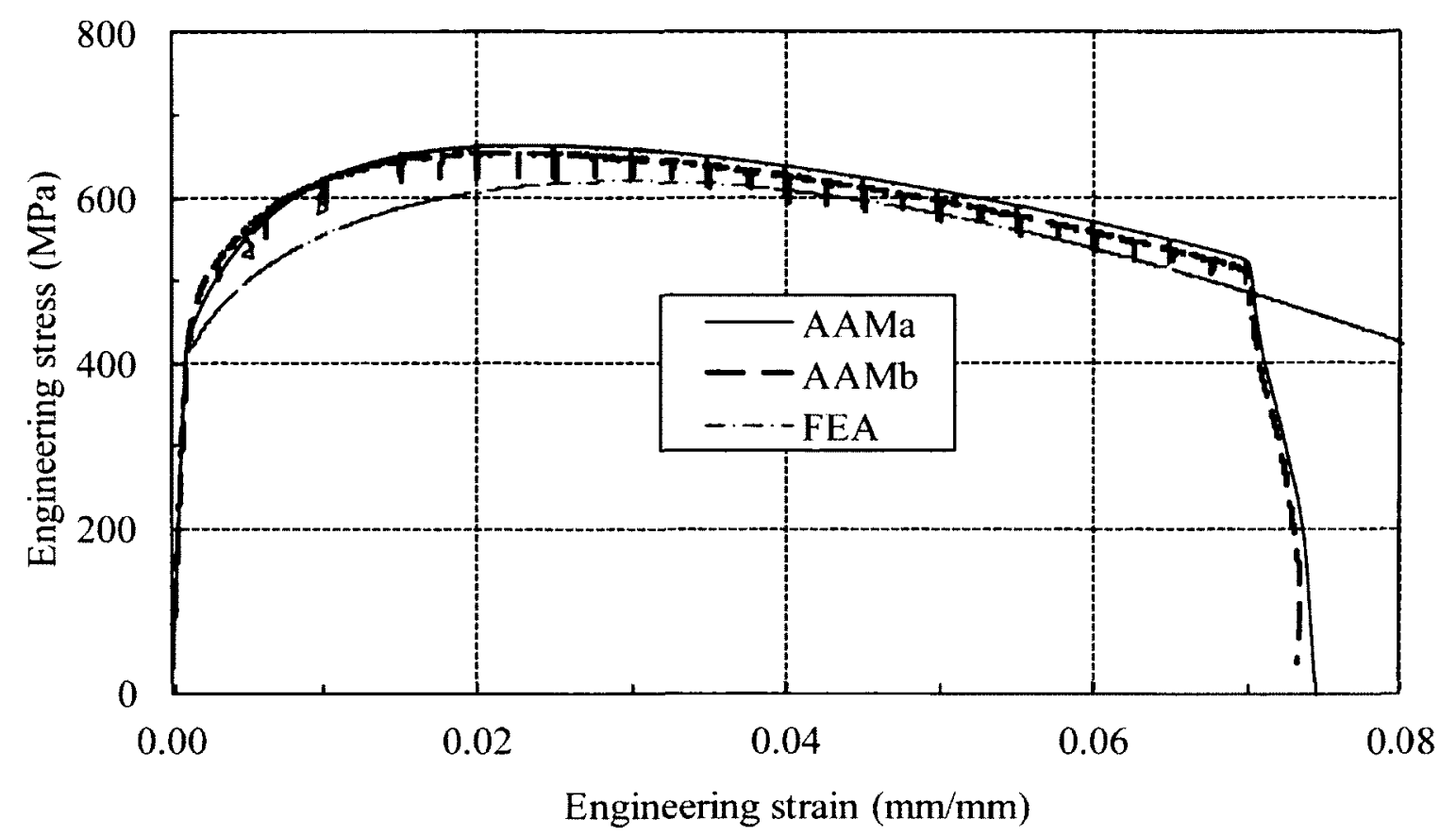

Figure B.3 Test and predicted engineering stress versus engineering strain curves for notched specimens AAMa and AAMb (one term)

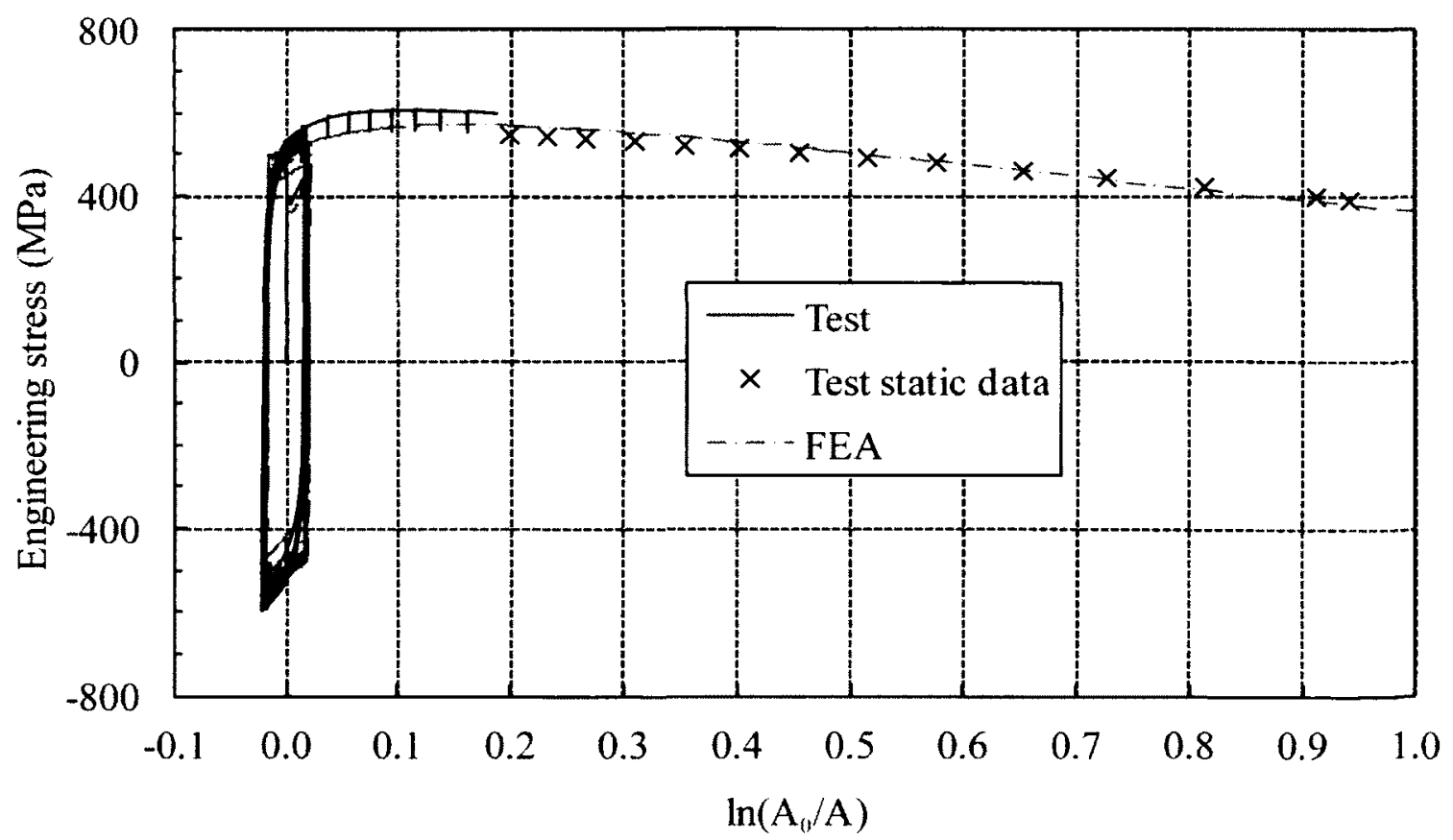

Figure B.4 Test and predicted engineering stress versus cross-sectional area change curves for straight specimen ADC40202 (one term) 


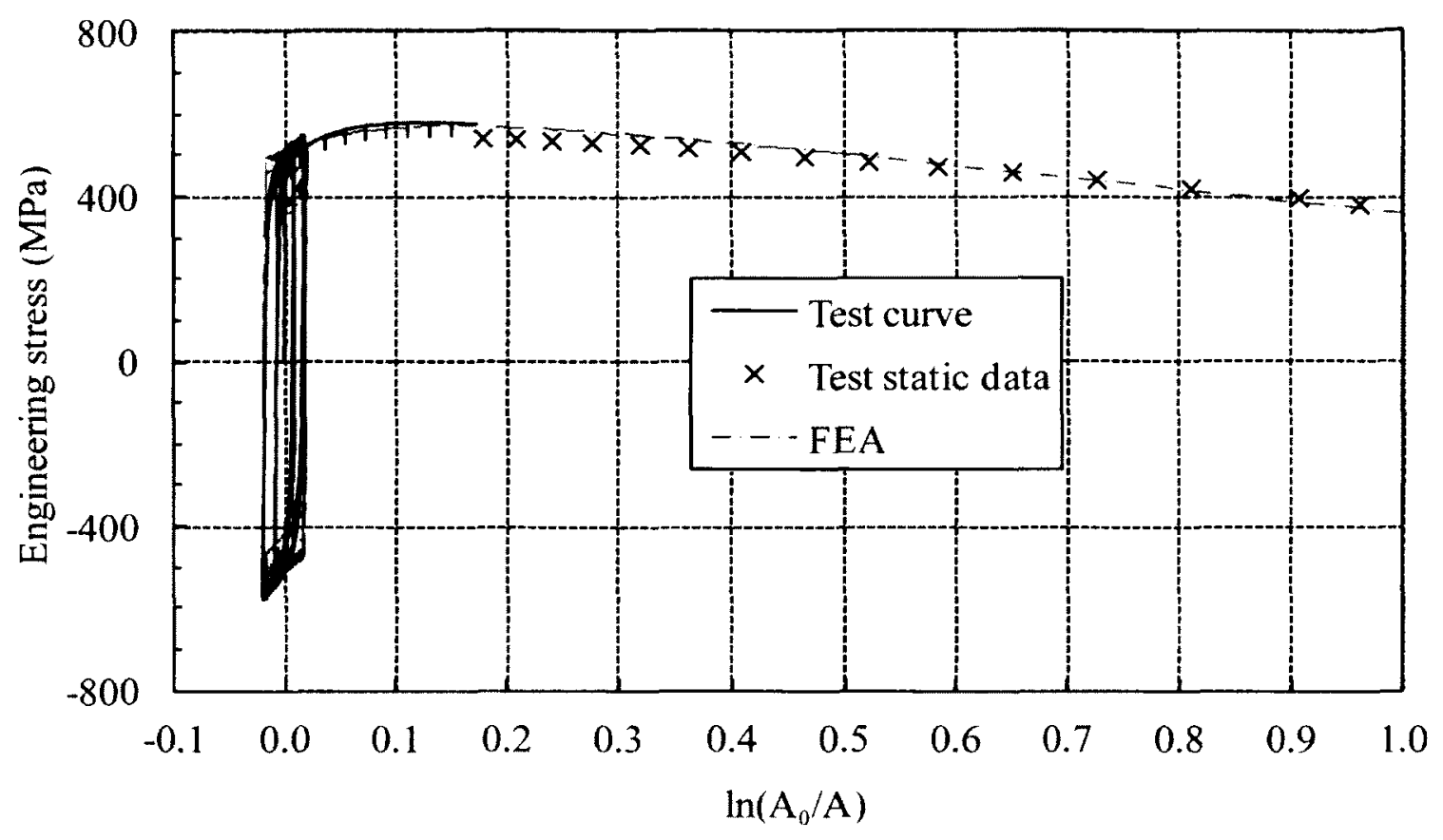

Figure B.5 Test and predicted engineering stress versus cross-sectional area change curves for straight specimen ADCR0201 (one term)

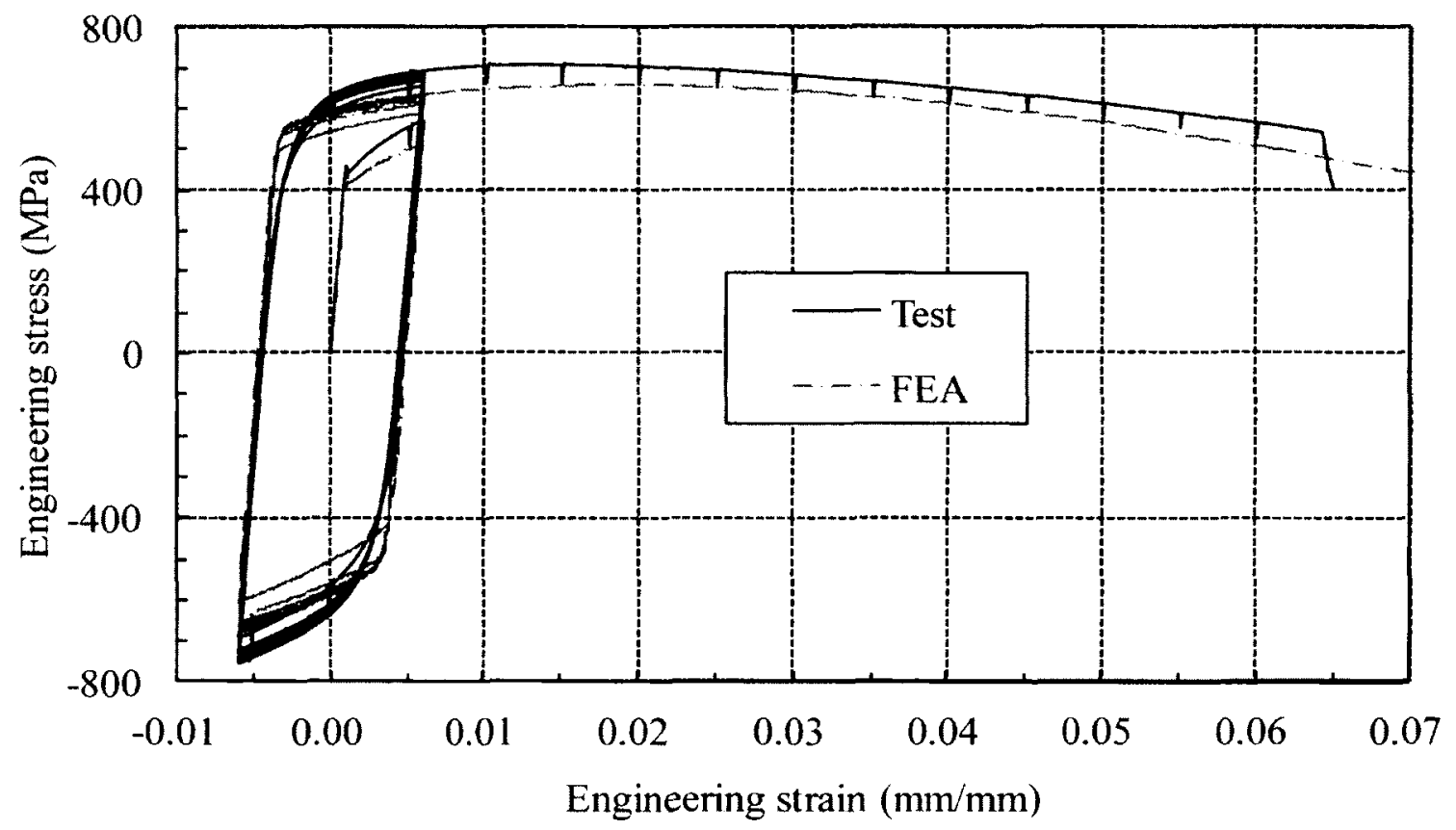

Figure B.6 Test and predicted engineering stress versus engineering strain curves for notched specimen AAC20404 (one term) 


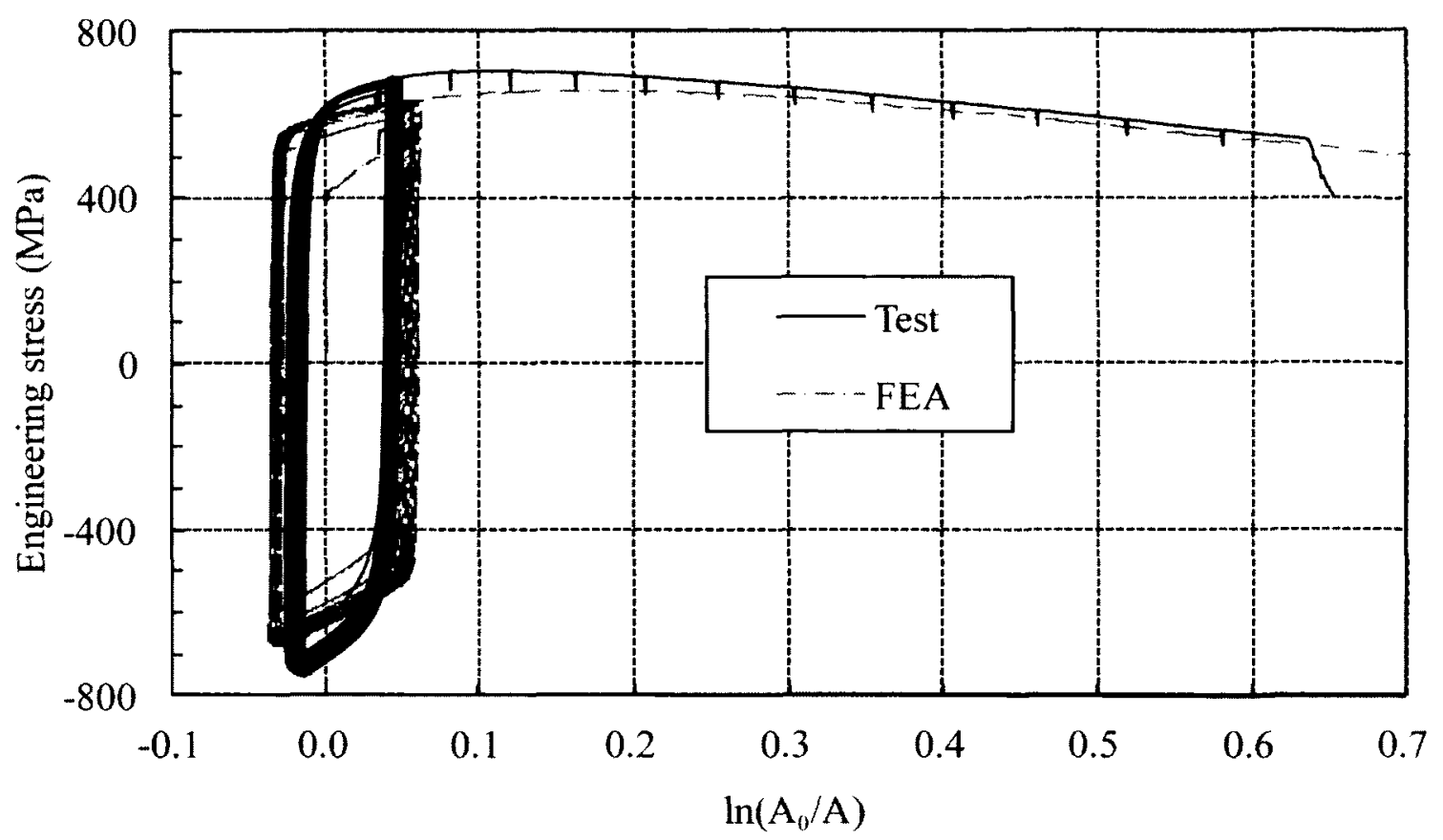

Figure B.7 Test and predicted engineering stress versus cross-sectional area change curves for notched specimen AAC20404 (one term)

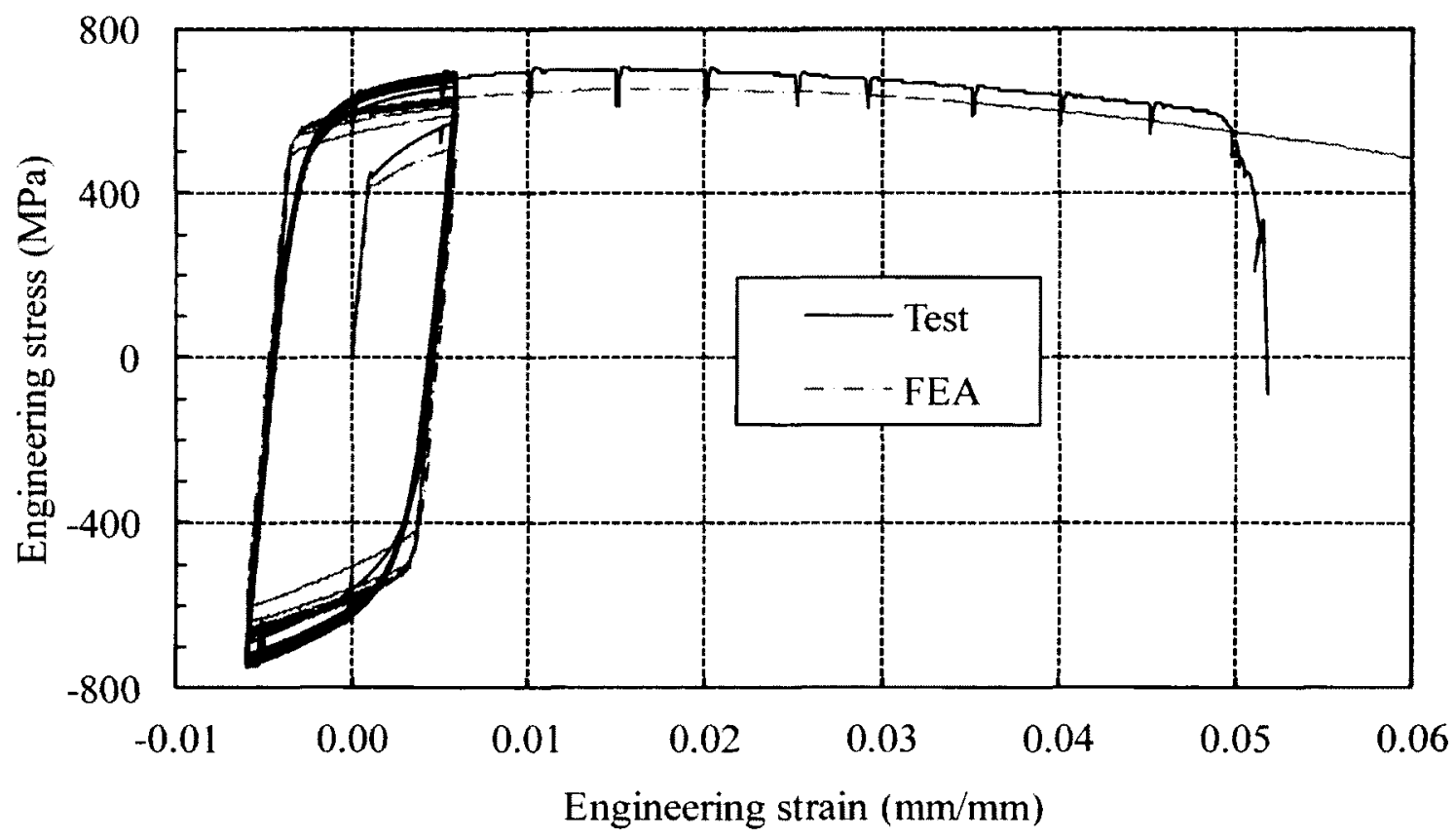

Figure B.8 Test and predicted engineering stress versus engineering strain curves for notched specimen AAC70404 (one term) 


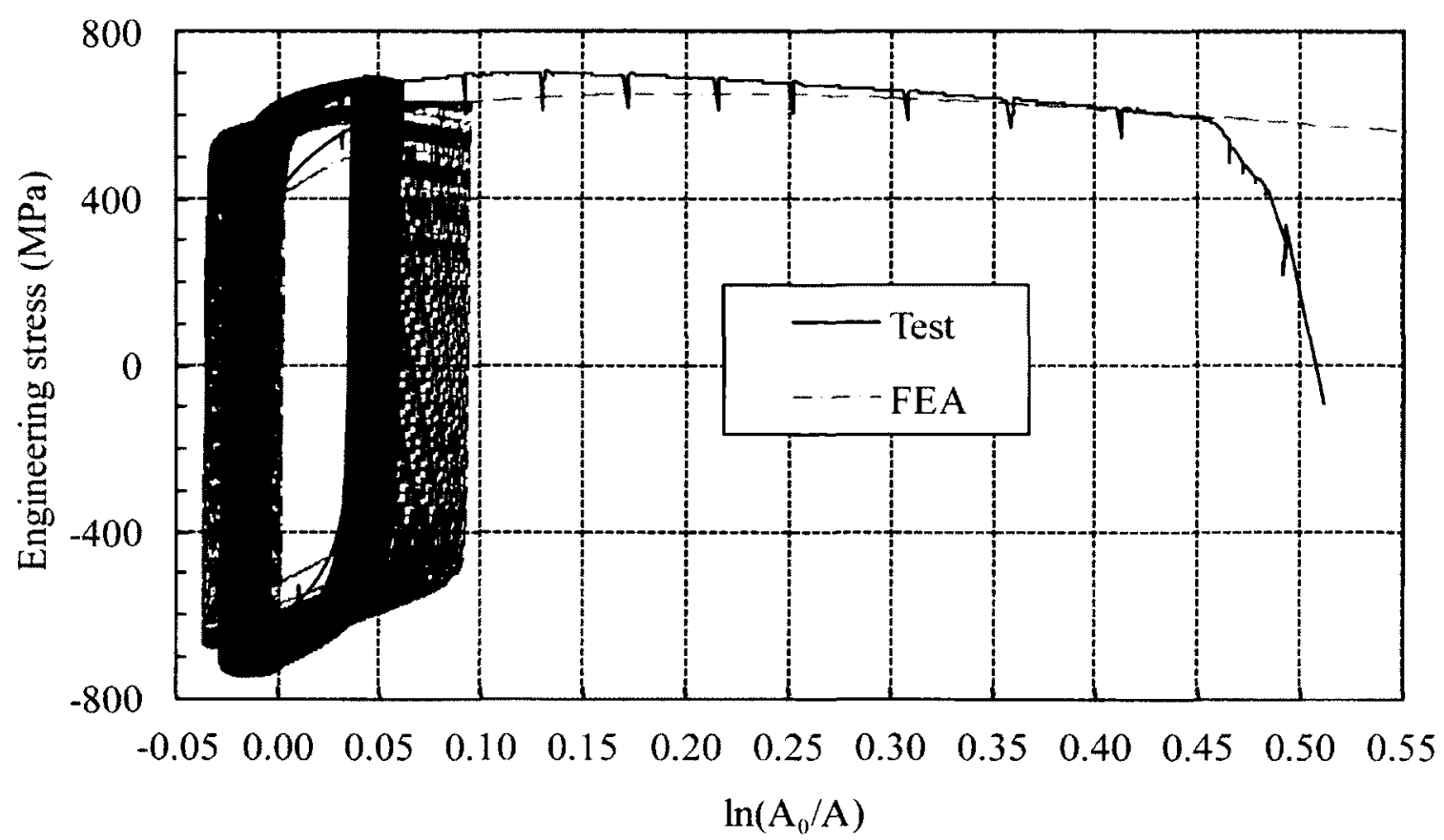

Figure B.9 Test and predicted engineering stress versus cross-sectional area change curves for notched specimen AAC70404 (one term)

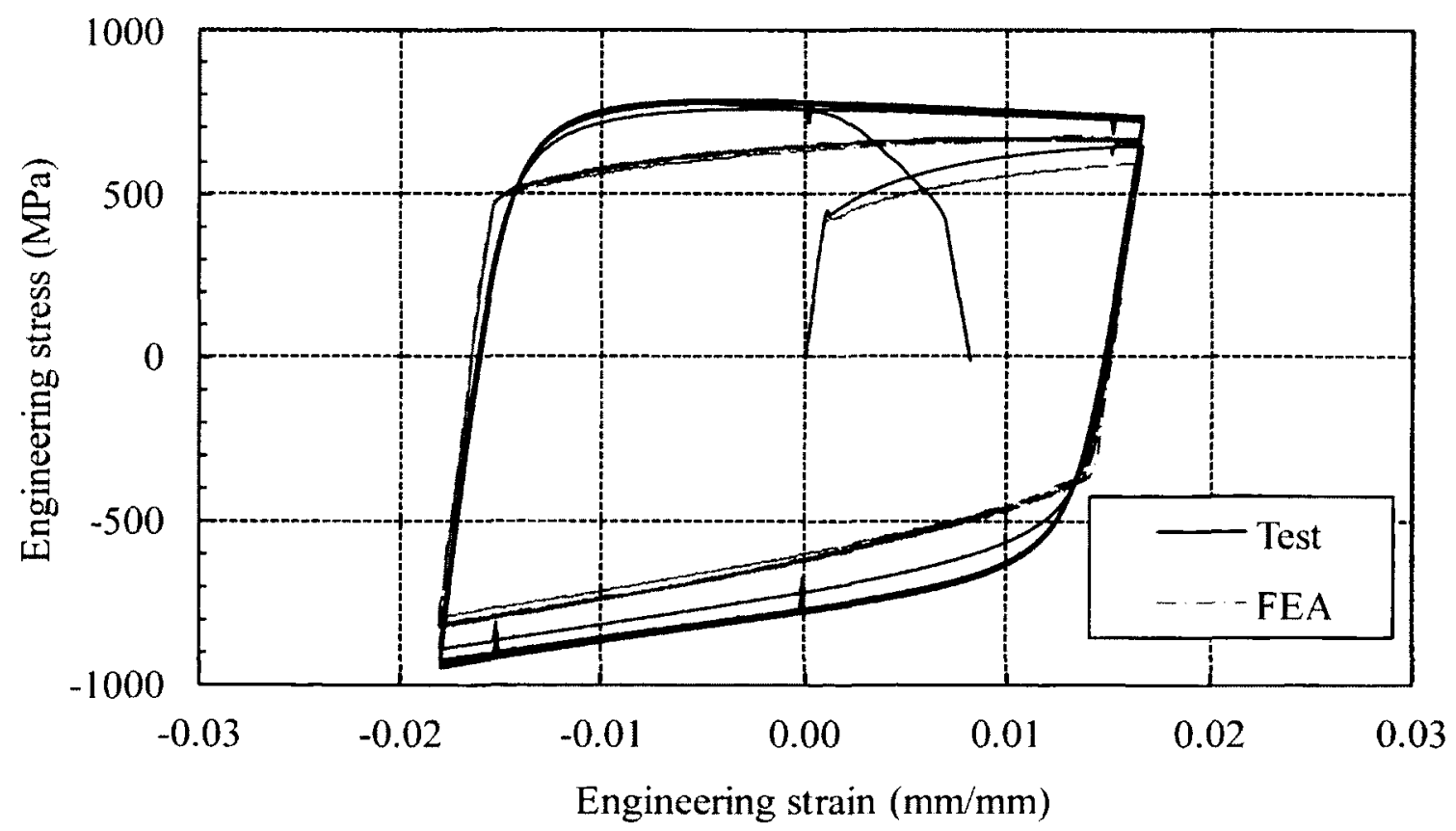

Figure B.10 Test and predicted engineering stress versus engineering strain curves for notched specimen AACF1212 (one term) 


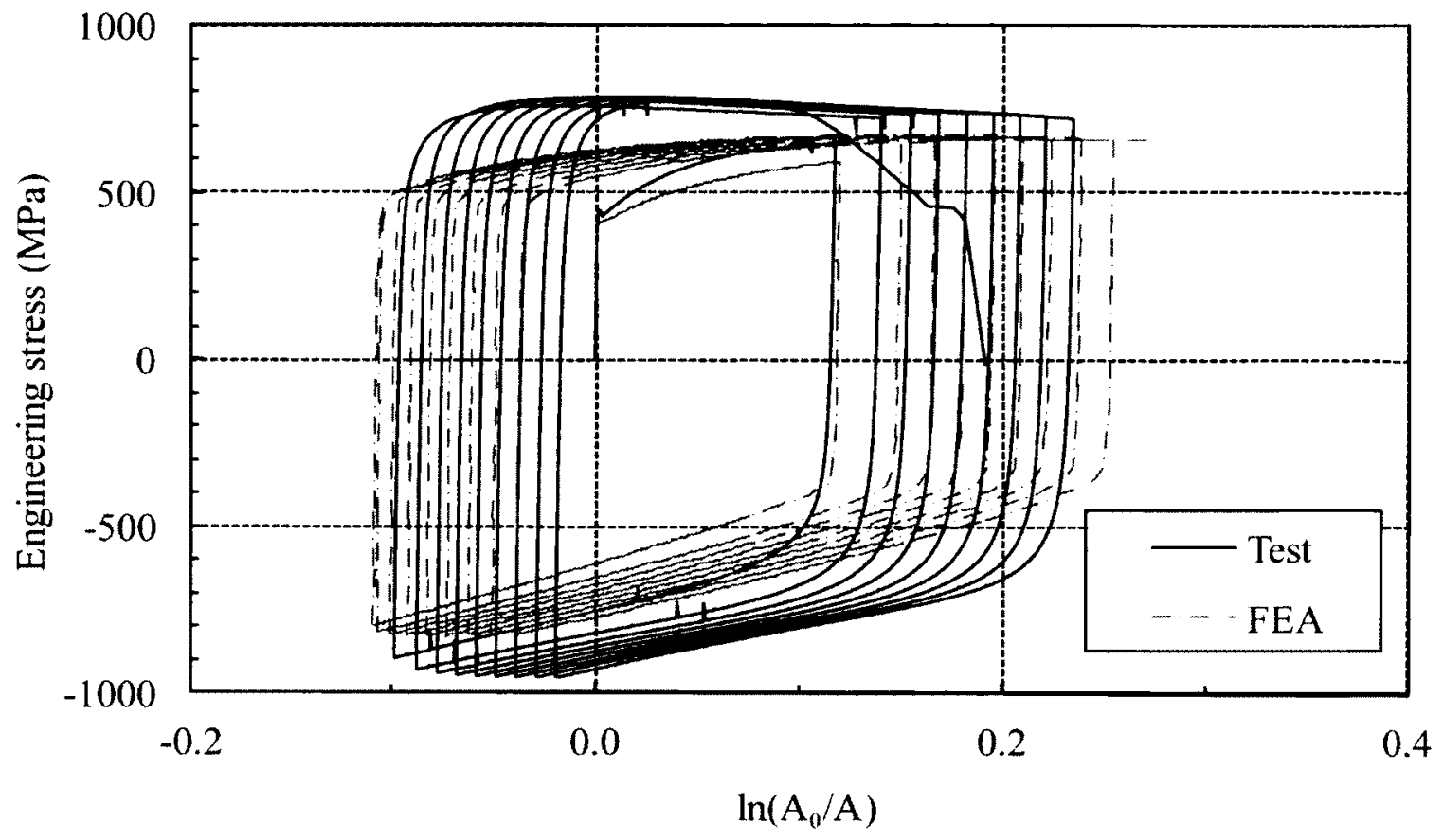

Figure B.11 Test and predicted engineering stress versus cross-sectional area change curves for notched specimen AACF1212 (one term) 


\section{APPENDIX C PRELIMINARY STUDY ON FRACTURE CRITERIA}

In the preliminary study, nine fracture criteria were assessed in the numerical studies. These criteria are summarized in Table C.1 including those by Rice and Tracey (1969), Kanvinde and Deierlein (2004, 2007), Huang and Mahin (2008), Huang and Mahin (2008) with gross strain energy density, Freudenthal (1950), Cockcroft and Latham (1968), Brozzo et al. (1972), Norris et al. (1978) and Oyane et al. (1980). Numerical simulations of the specimens were carried out with ABAQUS (Simulia 2009) using material model parameters with two kinematic hardening terms listed in Section 3.3.2.1.3. In the following tables, the listed specimen $\mathrm{AEM}^{*}$ is a duplicate specimen of AEM and has only been included in the preliminary study. There were some differences in the measurement procedure used in this initial trial test compared to other specimens. Therefore, this specimen has not been included in the final study.

Numerical predictions using these criteria are listed in Tables C.2 to C.10. For monotonic tests, the fracture criteria are evaluated based on the test and predicted diameter at fracture, $d_{p} / d_{f}$ and $\left(d_{0}-d_{p}\right) /\left(d_{0}-d_{f}\right)$ where $d_{0}$ is the initial diameter of the specimen, $d_{f}$ and $d_{p}$ are the measured and predicted diameters at fracture. However for cyclic tests, the cycle number at fracture may be a more important indicator than the diameter at fracture when the specimen failed under cyclic loading since the change in the diameter is small within the loading cycle. The methodology used in calculating the damage limit is described in Section 4.2.2. Except for the criterion by Kanvinde and Deierlein $(2004,2007)$, the damage measure for other criteria only increases when the damage evolution function $f(\sigma)$ in Eq. (4.6) is larger than zero. 
It can be seen in Tables C.2, C.6 to C.8, and C.10 that the criteria by Rice and Tracey (1969), Freudenthal (1950), Cockcroft and Latham (1968), Brozzo et al. (1972), and Oyane et al. (1980) cannot predict the point of fracture for the specimens of both monotonic and cyclic tests simultaneously. All these criteria significantly overestimate the diameter reduction at fracture for monotonic tests, but underestimate the ductility of cyclic tests, especially the number of loading cycle that can be applied to specimen ABC 70404 before fracture occurs. There is a significant difference in the calculated damage at fracture between monotonic and cyclic tests. The prediction in Table C.9 for the criterion by Norris et al. (1978) shows a better agreement with both monotonic and cyclic tests when considering only the diameter reduction at fracture. However, specimen $\mathrm{ABC} 70404$ is predicted to fracture at half the number of the cycle that was applied in the test. Thus, it does not appear that the damage limit for all the above criteria can be calibrated to be applicable to both monotonic and cyclic tests.

Comparisons of the test and predicted diameter and loading cycle number at fracture based on criteria by Kanvinde and Deierlein $(2004,2007)$ with void closing in compression, Huang and Mahin (2008), and Huang and Mahin (2008) with gross strain energy density are shown in Tables C.3 to C.5. These three criteria appear to be able to provide a reasonable prediction of the point of fracture for both monotonic and cyclic tests. The two fracture criteria that are based on the criterion by Huang and Mahin (2008) have almost the same fracture prediction. Nevertheless, the damage limit for the criterion using the gross strain energy density is much easier to calibrate since the damage evolution function is independent of the state of damage. Kanvinde and Deierlein (2004, 2007) also proposed an additional modification to the damage limit with Eq. (2.28) in 
terms of the equivalent plastic strain accumulated in each tension cycle leg. This modification has not been explored in the preliminary study. 
Table C.1 Fracture criteria for the preliminary study

\begin{tabular}{|c|c|}
\hline Criterion & Equation \\
\hline $\begin{array}{l}\text { Rice and Tracey } \\
\text { (1969) }\end{array}$ & $\ln \left(\frac{\mathrm{R}_{\mathrm{c}}}{\mathrm{R}_{0}}\right)=\int_{0}^{\varepsilon_{\mathrm{c}}^{\mathrm{p}}} \operatorname{cexp}(1.5 \mathrm{~T}) \mathrm{d} \varepsilon_{\mathrm{eq}}^{\mathrm{p}}$ \\
\hline $\begin{array}{c}\text { Kanvinde and } \\
\text { Deierlein (2004, } \\
\text { 2007) }\end{array}$ & 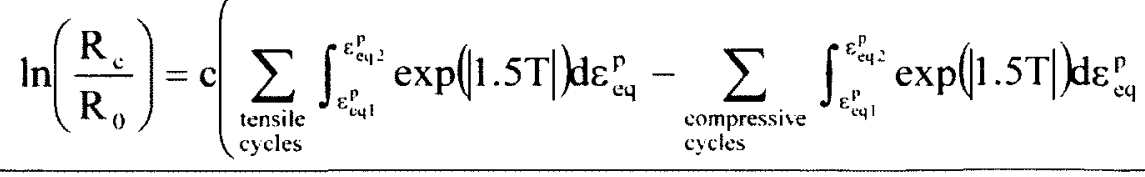 \\
\hline $\begin{array}{l}\text { Huang and Mahin } \\
\qquad(2008)\end{array}$ & $\begin{array}{c}\mathrm{dD}=\left\{\begin{array}{cc}(\mathrm{cy})^{\mathrm{m}} \mathrm{d} \varepsilon_{\mathrm{eq}}^{\mathrm{p}} & \text { for } \frac{\sigma_{\mathrm{m}}}{\sigma_{\mathrm{eq}}}>-\frac{1}{3}, \text { and } \\
0 & \text { otherwise }\end{array}\right. \\
\mathrm{y}=\frac{\sigma_{\mathrm{eq}}{ }^{2}}{2 \mathrm{E}(1-\mathrm{D})^{2}}\left[\frac{2}{3}(1+v)+3(1-2 v)\left(\frac{\sigma_{\mathrm{m}}}{\sigma_{\mathrm{eq}}}\right)^{2}\right] \\
\mathrm{D}_{\mathrm{c}}=\int_{0}^{\mathrm{e}_{\mathrm{eq}}^{\mathrm{rf}}} \mathrm{dD}\end{array}$ \\
\hline $\begin{array}{l}\text { Huang and Mahin } \\
\text { (2008) with gross } \\
\text { strain energy } \\
\text { density }\end{array}$ & $\begin{array}{c}\mathrm{dD}=\left\{\begin{array}{cc}(\mathrm{cy})^{\mathrm{m}} \mathrm{d} \varepsilon_{\mathrm{eq}}^{\mathrm{p}} & \text { for } \frac{\sigma_{\mathrm{m}}}{\sigma_{\mathrm{eq}}}>-\frac{1}{3}, \text { and } \\
0 & \text { otherwise }\end{array}\right. \\
\mathrm{y}=\frac{\sigma_{\mathrm{eq}}{ }^{2}}{2 \mathrm{E}}\left[\frac{2}{3}(1+v)+3(1-2 v)\left(\frac{\sigma_{\mathrm{m}}}{\sigma_{\mathrm{eq}}}\right)^{2}\right] \\
\mathrm{D}_{\mathrm{c}}=\int_{0}^{\varepsilon_{\mathrm{eq}}^{\mathrm{p}}} \mathrm{dD}\end{array}$ \\
\hline $\begin{array}{l}\text { Freudenthal } \\
(1950)\end{array}$ & $\mathrm{D}_{\mathrm{c}}=\int_{0}^{\varepsilon_{\mathrm{eq}}^{\mathrm{p}}} \sigma_{\mathrm{eq}} \mathrm{d} \varepsilon_{\mathrm{eq}}^{\mathrm{p}}$ \\
\hline $\begin{array}{l}\text { Cockcroft and } \\
\text { Latham (1968) }\end{array}$ & $D_{c}=\int_{0}^{e_{e 4}^{p g}} \sigma_{1} d \varepsilon_{e q}^{p}$ \\
\hline $\begin{array}{c}\text { Brozzo et al. } \\
(1972)\end{array}$ & $\mathrm{D}_{\mathrm{c}}=\int_{0}^{\varepsilon_{\mathrm{eq}}^{\mathrm{pr}}} \frac{2 \sigma_{1}}{3\left(\sigma_{1}-\sigma_{\mathrm{m}}\right)} \mathrm{d} \varepsilon_{\mathrm{eq}}^{\mathrm{p}}$ \\
\hline $\begin{array}{l}\text { Norris et al. } \\
\quad(1978)\end{array}$ & $\mathrm{D}_{\mathrm{c}}=\int_{0}^{\mathrm{v}_{\mathrm{eq}}^{\mathrm{p}}}\left(\frac{1}{1-\mathrm{co} \sigma_{\mathrm{m}}}\right) \mathrm{d} \varepsilon_{\mathrm{eq}}^{\mathrm{p}}$ \\
\hline $\begin{array}{l}\text { Oyane et al. } \\
\quad(1980)\end{array}$ & $D_{c}=\int_{0}^{g_{c 4}^{r e}}\left(1+\frac{\sigma_{m}}{c \sigma_{e q}}\right) d \varepsilon_{c q}^{p}$ \\
\hline
\end{tabular}


Table C.2 Test and predicted fracture using criterion by Rice and Tracey (1969) with $c=1$

\begin{tabular}{|c|c|c|c|c|c|c|c|}
\hline Specimen & $\begin{array}{c}\text { Damage } \\
\text { at fracture }\end{array}$ & $\begin{array}{c}\mathrm{d}_{\mathrm{o}} \\
(\mathrm{mm})\end{array}$ & $\begin{array}{c}\mathrm{d}_{\mathrm{f}} \\
(\mathrm{mm})\end{array}$ & $\begin{array}{c}\mathrm{d}_{\mathrm{p}} \\
(\mathrm{mm})\end{array}$ & $\mathrm{d}_{\mathrm{p}} / \mathrm{d}_{\mathrm{f}}$ & $\left(\mathrm{d}_{0}-\mathrm{d}_{\mathrm{p}}\right) /\left(\mathrm{d}_{0}-\mathrm{d}_{\mathrm{f}}\right)$ & $\begin{array}{c}\text { Predicted } \\
\text { fracture } \\
\text { cycle }\end{array}$ \\
\hline AEM & 3.04 & 13.94 & 8.53 & 5.41 & 0.63 & 1.58 & - \\
\hline AEM & 3.00 & 13.96 & 8.57 & 5.42 & 0.63 & 1.59 & - \\
\hline ABM & 2.23 & 14.00 & 10.18 & 6.28 & 0.62 & 2.02 & - \\
\hline ABC20404 & 11.11 & 14.00 & 10.04 & 11.08 & 1.10 & 0.74 & $20+$ \\
\hline ACC20404 & 12.48 & 10.00 & 7.92 & 9.80 & 1.24 & 0.09 & 20 \\
\hline ABC70404 & 30.50 & 14.00 & 12.57 & 13.87 & 1.10 & 0.09 & 24 \\
\hline Mean & 10.39 & \multicolumn{7}{|c|}{-} \\
\hline
\end{tabular}

Table C.3 Test and predicted fracture using criterion by Kanvinde and Deierlein $(2004,2007)$ with void closing in compression and $\mathrm{c}=1$

\begin{tabular}{|c|c|c|c|c|c|c|c|}
\hline Specimen & $\begin{array}{c}\text { Damage } \\
\text { at fracture }\end{array}$ & $\begin{array}{c}\mathrm{d}_{0} \\
(\mathrm{~mm})\end{array}$ & $\begin{array}{c}\mathrm{d}_{\mathrm{f}} \\
(\mathrm{mm})\end{array}$ & $\begin{array}{c}\mathrm{d}_{\mathrm{p}} \\
(\mathrm{mm})\end{array}$ & $\mathrm{d}_{\mathrm{p}} / \mathrm{d}_{\mathrm{f}}$ & $\left(\mathrm{d}_{0}-\mathrm{d}_{\mathrm{p}}\right) /\left(\mathrm{d}_{0}-\mathrm{d}_{\mathrm{f}}\right)$ & $\begin{array}{c}\text { Predicted } \\
\text { fracture } \\
\text { cycle }\end{array}$ \\
\hline AEM & 3.04 & 13.94 & 8.53 & 9.20 & 1.08 & 0.88 & - \\
\hline AEM & 3.00 & 13.96 & 8.57 & 9.21 & 1.07 & 0.88 & - \\
\hline ABM & 2.23 & 14.00 & 10.18 & 10.07 & 0.99 & 1.03 & - \\
\hline ABC20404 & 2.53 & 14.00 & 10.04 & 10.45 & 1.04 & 0.90 & $20+$ \\
\hline ACC20404 & 2.31 & 10.00 & 7.92 & 7.92 & 1.00 & 1.00 & $20+$ \\
\hline ABC70404 & 0.82 & 14.00 & 12.57 & 10.37 & 0.82 & 2.55 & $70+$ \\
\hline Mean & 2.32 & \multicolumn{7}{|c|}{-} \\
\hline
\end{tabular}

Table C.4 Test and predicted fracture using criterion by Huang and Mahin (2008) with $\mathrm{c}=0.15$ and $\mathrm{m}=4$

\begin{tabular}{|c|c|c|c|c|c|c|c|}
\hline Specimen & $\begin{array}{c}\text { Damage } \\
\text { at fracture }\end{array}$ & $\begin{array}{c}\mathrm{d}_{0} \\
(\mathrm{~mm})\end{array}$ & $\begin{array}{c}\mathrm{d}_{\mathrm{f}} \\
(\mathrm{mm})\end{array}$ & $\begin{array}{c}\mathrm{d}_{\mathrm{p}} \\
(\mathrm{mm})\end{array}$ & $\mathrm{d}_{\mathrm{p}} / \mathrm{d}_{\mathrm{f}}$ & $\left(\mathrm{d}_{\mathrm{r}}-\mathrm{d}_{\mathrm{p}}\right) /\left(\mathrm{d}_{0}-\mathrm{d}_{\mathrm{f}}\right)$ & $\begin{array}{c}\text { Predicted } \\
\text { fracture } \\
\text { cycle }\end{array}$ \\
\hline AEM & 0.0086 & 13.94 & 8.53 & 8.43 & 0.99 & 1.02 & - \\
\hline AEM & 0.0083 & 13.96 & 8.57 & 8.45 & 0.99 & 1.02 & - \\
\hline ABM & 0.0065 & 14.00 & 10.18 & 9.80 & 0.96 & 1.10 & - \\
\hline ABC20404 & 0.0090 & 14.00 & 10.04 & 10.16 & 1.01 & 0.97 & $20+$ \\
\hline ACC20404 & 0.0137 & 10.00 & 7.92 & 8.75 & 1.11 & 0.60 & $20+$ \\
\hline ABC70404 & 0.0099 & 14.00 & 12.57 & 13.55 & 1.08 & 0.32 & 68 \\
\hline Mean & 0.0094 & \multicolumn{7}{|c|}{-} \\
\hline
\end{tabular}


Table C.5 Test and predicted fracture using criterion by Huang and Mahin (2008) with gross strain energy density, and $\mathrm{c}=0.44$ and $\mathrm{m}=4$

\begin{tabular}{|c|c|c|c|c|c|c|c|}
\hline Specimen & $\begin{array}{c}\text { Damage } \\
\text { at fracture }\end{array}$ & $\begin{array}{c}\mathrm{d}_{\mathrm{o}} \\
(\mathrm{mm})\end{array}$ & $\begin{array}{c}\mathrm{d}_{\mathrm{f}} \\
(\mathrm{mm})\end{array}$ & $\begin{array}{c}\mathrm{d}_{\mathrm{p}} \\
(\mathrm{mm})\end{array}$ & $\mathrm{d}_{\mathrm{p}} / \mathrm{d}_{\mathrm{f}}$ & $\left(\mathrm{d}_{0}-\mathrm{d}_{\mathrm{p}}\right) /\left(\mathrm{d}_{0}-\mathrm{d}_{\mathrm{f}}\right)$ & $\begin{array}{c}\text { Predicted } \\
\text { fracture } \\
\text { cycle }\end{array}$ \\
\hline AEM & 0.617 & 13.94 & 8.53 & 8.44 & 0.99 & 1.02 & - \\
\hline AEM & 0.598 & 13.96 & 8.57 & 8.45 & 0.99 & 1.02 & - \\
\hline ABM & 0.472 & 14.00 & 10.18 & 9.80 & 0.96 & 1.10 & - \\
\hline ABC20404 & 0.644 & 14.00 & 10.04 & 10.17 & 1.01 & 0.97 & $20+$ \\
\hline ACC20404 & 0.962 & 10.00 & 7.92 & 8.76 & 1.11 & 0.60 & $20+$ \\
\hline ABC70404 & 0.705 & 14.00 & 12.57 & 13.65 & 1.09 & 0.25 & 68 \\
\hline Mean & 0.666 & \multicolumn{7}{|c|}{-} \\
\hline
\end{tabular}

Table C.6 Test and predicted fracture using criterion by Freudenthal (1950)

\begin{tabular}{|c|c|c|c|c|c|c|c|}
\hline Specimen & $\begin{array}{c}\text { Damage } \\
\text { at fracture }\end{array}$ & $\begin{array}{c}\mathrm{d}_{0} \\
(\mathrm{~mm})\end{array}$ & $\begin{array}{c}\mathrm{d}_{\mathrm{f}} \\
(\mathrm{mm})\end{array}$ & $\begin{array}{c}\mathrm{d}_{\mathrm{p}} \\
(\mathrm{mm})\end{array}$ & $\mathrm{d}_{\mathrm{p}} / \mathrm{d}_{\mathrm{f}}$ & $\left(\mathrm{d}_{0}-\mathrm{d}_{\mathrm{p}}\right) /\left(\mathrm{d}_{0}-\mathrm{d}_{\mathrm{f}}\right)$ & $\begin{array}{c}\text { Predicted } \\
\text { fracture } \\
\text { cycle }\end{array}$ \\
\hline AEM* & 794 & 13.94 & 8.53 & 5.45 & 0.64 & 1.57 & - \\
\hline AEM & 786 & 13.96 & 8.57 & 5.46 & 0.64 & 1.58 & - \\
\hline ABM & 472 & 14.00 & 10.18 & 5.56 & 0.55 & 2.21 & - \\
\hline ABC20404 & 1766 & 14.00 & 10.04 & 11.09 & 1.10 & 0.74 & $20+$ \\
\hline ACC20404 & 1404 & 10.00 & 7.92 & 6.87 & 0.87 & 1.51 & $20+$ \\
\hline ABC70404 & 4604 & 14.00 & 12.57 & 13.79 & 1.10 & 0.15 & 25 \\
\hline Mean & 1638 & \multicolumn{7}{|c|}{-} \\
\hline
\end{tabular}

Table C.7 Test and predicted fracture using criterion by Cockcroft and Latham (1968)

\begin{tabular}{|c|c|c|c|c|c|c|c|}
\hline Specimen & $\begin{array}{c}\text { Damage } \\
\text { at fracture }\end{array}$ & $\begin{array}{c}\mathrm{d}_{\mathrm{o}} \\
(\mathrm{mm})\end{array}$ & $\begin{array}{c}\mathrm{d}_{\mathrm{f}} \\
(\mathrm{mm})\end{array}$ & $\begin{array}{c}\mathrm{d}_{\mathrm{p}} \\
(\mathrm{mm})\end{array}$ & $\mathrm{d}_{\mathrm{p}} / \mathrm{d}_{\mathrm{f}}$ & $\left(\mathrm{d}_{\mathrm{p}}-\mathrm{d}_{\mathrm{p}}\right) /\left(\mathrm{d}_{\mathrm{d}_{1}}-\mathrm{d}_{\mathrm{f}}\right)$ & $\begin{array}{c}\text { Predicted } \\
\text { fracture } \\
\text { cycle }\end{array}$ \\
\hline AEM $*$ & 1040 & 13.94 & 8.53 & 5.68 & 0.67 & 1.53 & - \\
\hline AEM & 1027 & 13.96 & 8.57 & 5.69 & 0.66 & 1.54 & - \\
\hline ABM & 680 & 14.00 & 10.18 & 6.23 & 0.61 & 2.03 & - \\
\hline ABC20404 & 2657 & 14.00 & 10.04 & 11.07 & 1.10 & 0.74 & $20+$ \\
\hline ACC20404 & 2415 & 10.00 & 7.92 & 7.81 & 0.99 & 1.05 & $20+$ \\
\hline ABC70404 & 6915 & 14.00 & 12.57 & 13.83 & 1.10 & 0.12 & 25 \\
\hline Mean & 2456 & \multicolumn{7}{|c|}{-} \\
\hline
\end{tabular}


Table C.8 Test and predicted fracture using criterion by Brozzo et al. (1972)

\begin{tabular}{|c|c|c|c|c|c|c|c|}
\hline Specimen & $\begin{array}{c}\text { Damage } \\
\text { at fracture }\end{array}$ & $\begin{array}{c}\mathrm{d}_{0} \\
(\mathrm{~mm})\end{array}$ & $\begin{array}{c}\mathrm{d}_{\mathrm{f}} \\
(\mathrm{mm})\end{array}$ & $\begin{array}{c}\mathrm{d}_{\mathrm{p}} \\
(\mathrm{mm})\end{array}$ & $\mathrm{d}_{\mathrm{p}} / \mathrm{d}_{\mathrm{f}}$ & $\left(\mathrm{d}_{1}-\mathrm{d}_{\mathrm{p}}\right) /\left(\mathrm{d}_{1}-\mathrm{d}_{\mathrm{f}}\right)$ & $\begin{array}{c}\text { Predicted } \\
\text { fracture } \\
\text { cycle }\end{array}$ \\
\hline AEM $^{*}$ & 1.47 & 13.94 & 8.53 & 4.83 & 0.57 & 1.68 & - \\
\hline AEM & 1.45 & 13.96 & 8.57 & 4.84 & 0.56 & 1.69 & - \\
\hline ABM & 0.99 & 14.00 & 10.18 & 5.35 & 0.53 & 2.26 & - \\
\hline ABC20404 & 4.73 & 14.00 & 10.04 & 11.46 & 1.14 & 0.64 & $20+$ \\
\hline ACC20404 & 4.36 & 10.00 & 7.92 & 7.99 & 1.01 & 0.97 & $20+$ \\
\hline ABC70404 & 13.00 & 14.00 & 12.57 & 13.52 & 1.08 & 0.34 & 23 \\
\hline Mean & 4.33 & \multicolumn{7}{|c|}{-} \\
\hline
\end{tabular}

Table C.9 Test and predicted fracture using criterion by Norris et al. (1978) with $\mathbf{c}=$ $1.37 \mathrm{GPa}^{-1}$

\begin{tabular}{|c|c|c|c|c|c|c|c|}
\hline Specimen & $\begin{array}{c}\text { Damage } \\
\text { at fracture }\end{array}$ & $\begin{array}{c}\mathrm{d}_{0} \\
(\mathrm{~mm})\end{array}$ & $\begin{array}{c}\mathrm{d}_{\mathrm{f}} \\
(\mathrm{mm})\end{array}$ & $\begin{array}{c}\mathrm{d}_{\mathrm{p}} \\
(\mathrm{mm})\end{array}$ & $\mathrm{d}_{\mathrm{p}} / \mathrm{d}_{\mathrm{f}}$ & $\left(\mathrm{d}_{0}-\mathrm{d}_{\mathrm{p}}\right) /\left(\mathrm{d}_{\mathrm{p}}-\mathrm{d}_{\mathrm{f}}\right)$ & $\begin{array}{c}\text { Predicted } \\
\text { fracture } \\
\text { cycle }\end{array}$ \\
\hline AEM & 6.14 & 13.94 & 8.53 & 8.40 & 0.98 & 1.02 & - \\
\hline AEM & 5.67 & 13.96 & 8.57 & 8.42 & 0.98 & 1.03 & - \\
\hline ABM & 11.91 & 14.00 & 10.18 & 10.17 & 1.00 & 1.00 & - \\
\hline ABC20404 & 12.56 & 14.00 & 10.04 & 10.26 & 1.02 & 0.95 & $20+$ \\
\hline ACC20404 & 12.64 & 10.00 & 7.92 & 8.98 & 1.13 & 0.49 & $20+$ \\
\hline ABC70404 & 22.66 & 14.00 & 12.57 & 13.84 & 1.10 & 0.11 & 37 \\
\hline Mean & 11.93 & \multicolumn{7}{|c|}{-} \\
\hline
\end{tabular}

Table C.10 Test and predicted fracture using criterion by Oyane et al. (1980) with $\mathrm{c}=$ 0.74

\begin{tabular}{|c|c|c|c|c|c|c|c|}
\hline Specimen & $\begin{array}{c}\text { Damage } \\
\text { at fracture }\end{array}$ & $\begin{array}{c}\mathrm{d}_{0} \\
(\mathrm{~mm})\end{array}$ & $\begin{array}{c}\mathrm{d}_{\mathrm{f}} \\
(\mathrm{mm})\end{array}$ & $\begin{array}{c}\mathrm{d}_{\mathrm{p}} \\
(\mathrm{mm})\end{array}$ & $\mathrm{d}_{\mathrm{p}} / \mathrm{d}_{\mathrm{f}}$ & $\left(\mathrm{d}_{\mathrm{o}}-\mathrm{d}_{\mathrm{p}}\right) /\left(\mathrm{d}_{0}-\mathrm{d}_{\mathrm{f}}\right)$ & $\begin{array}{c}\text { Predicted } \\
\text { fracture } \\
\text { cycle }\end{array}$ \\
\hline AEM* & 2.09 & 13.94 & 8.53 & 4.81 & 0.56 & 1.69 & - \\
\hline AEM & 2.07 & 13.96 & 8.57 & 4.82 & 0.56 & 1.70 & - \\
\hline ABM & 1.41 & 14.00 & 10.18 & 5.32 & 0.52 & 2.27 & - \\
\hline ABC20404 & 6.70 & 14.00 & 10.04 & 11.46 & 1.14 & 0.64 & $20+$ \\
\hline ACC20404 & 6.14 & 10.00 & 7.92 & 7.93 & 1.00 & 1.00 & $20+$ \\
\hline ABC70404 & 18.43 & 14.00 & 12.57 & 13.52 & 1.08 & 0.34 & 23 \\
\hline Mean & 6.14 & \multicolumn{7}{|c|}{-} \\
\hline
\end{tabular}




\section{APPENDIX D MESH STUDY OF FEA MODEL FOR PROFILES D10R14N AND D14R19N}

A mesh study has also been carried out to ensure that the calculated damage is not significantly affected by a further mesh refinement or increased mesh density.

Figures D.1 and D.2 show the scheme-2 meshes for D10R14N and D14R19N notched specimens. Although not shown, their boundary conditions are similar to scheme- 1 meshes in Figs. 3.7 and 3.8 respectively. The element size of scheme-2 is half that of scheme-1. Figures D. 3 to D.22 are results of FEA simulations on the engineering stress versus engineering strain, engineering stress versus cross-sectional area change and the calculated damage state $\mathrm{D}$ based on Eqs. (4.3) and (4.4) with $\mathrm{c}=1, \mathrm{~m}=3.07$ and $\mathrm{k}=$ 1.44 using both mesh schemes for various loading conditions. The comparisons show that the simulation results using scheme- 1 and scheme- 2 meshes are almost identical. Thus, the mesh for scheme- 1 has been considered to be sufficiently fine that no further refinement is required. 


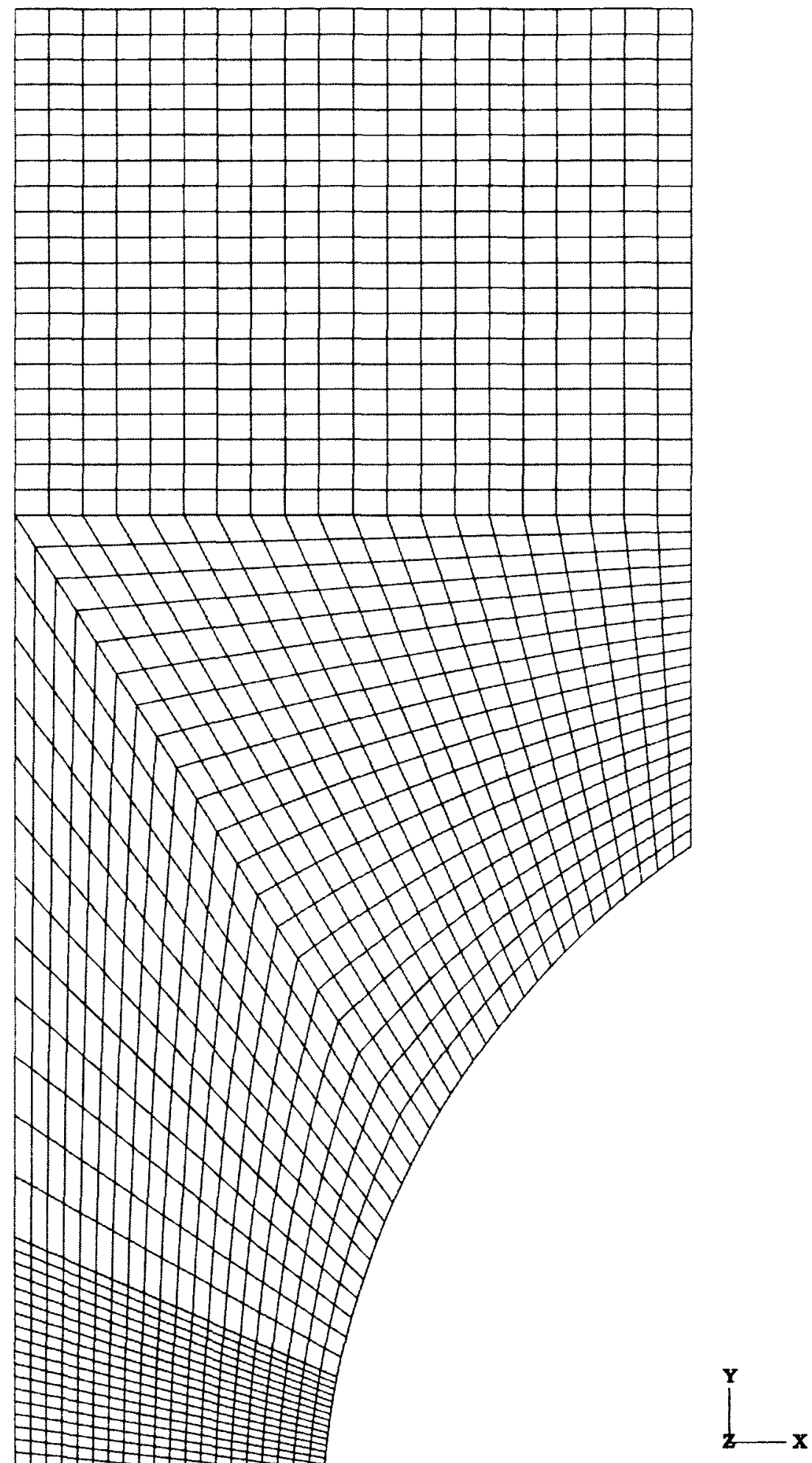

Figure D.1 Scheme-2 mesh of $25.4 \mathrm{~mm}$ (one-inch) FEA model for D10R14N notched specimen (boundary condition not shown) 

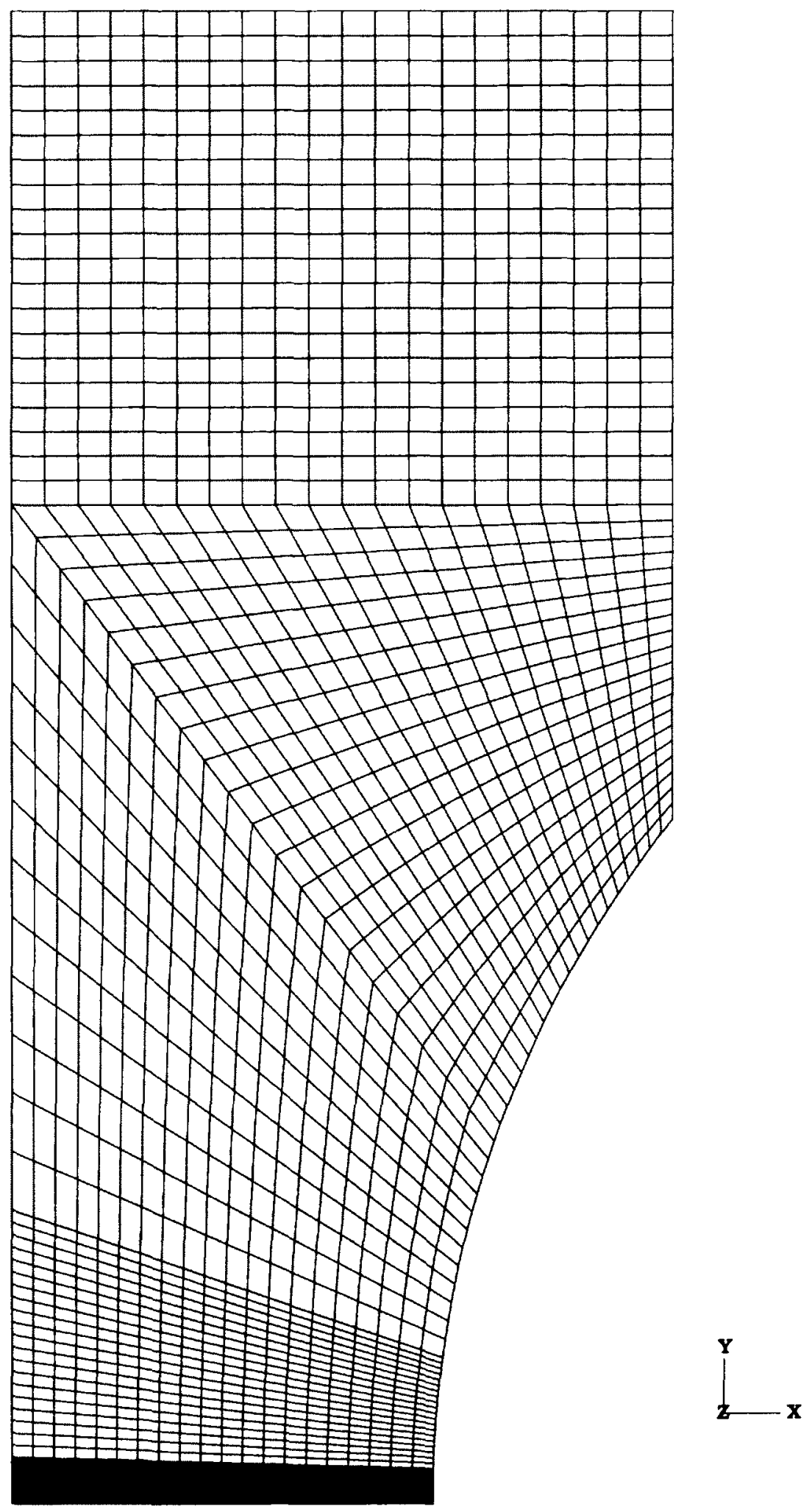

Figure D.2 Scheme-2 mesh of $25.4 \mathrm{~mm}$ (one-inch) FEA model for D14R19N notched specimen (boundary condition not shown) 


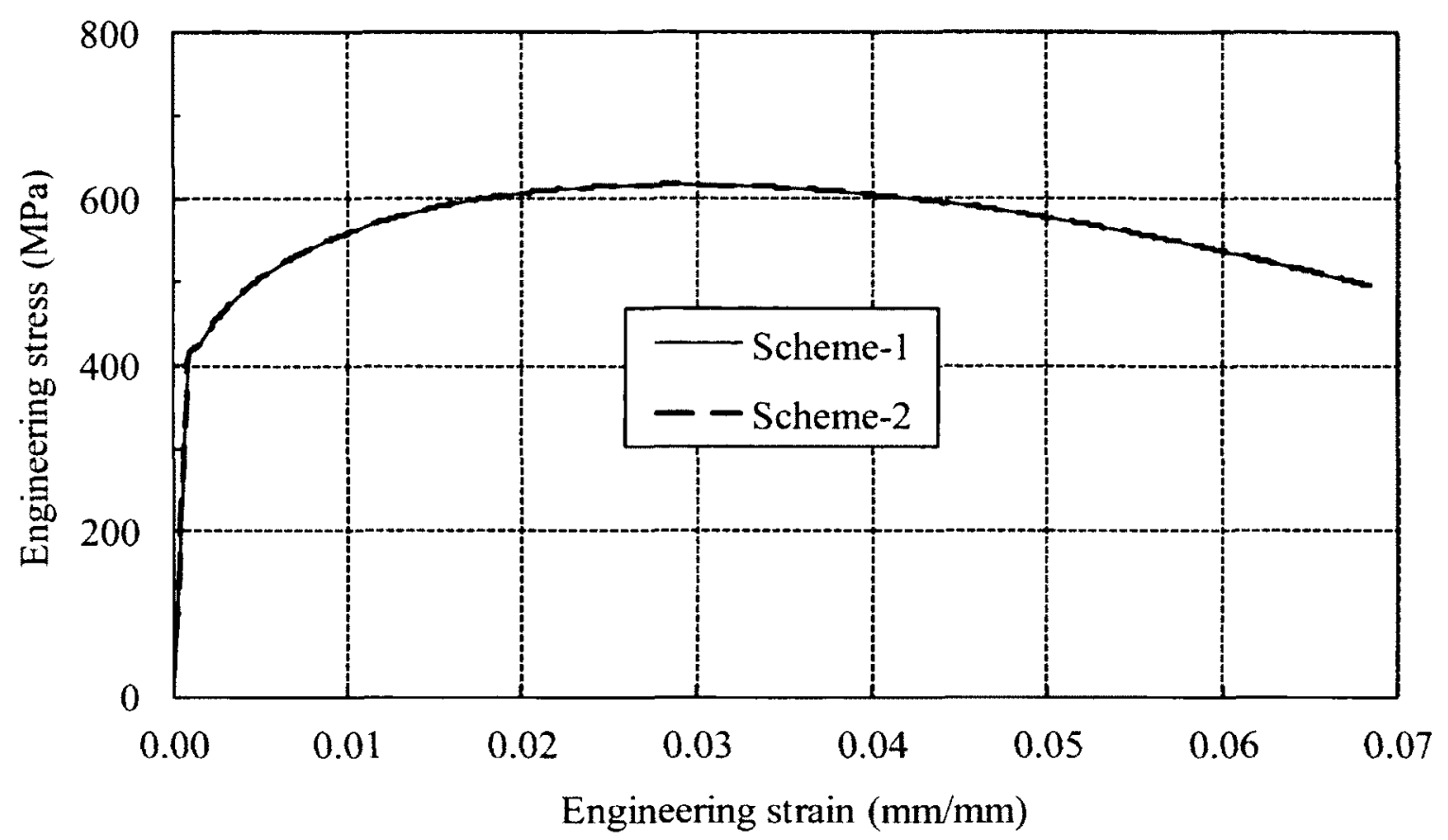

Figure D.3 Engineering stress versus engineering strain curve from FEA simulations for notched specimens AAMa and AAMb with two mesh schemes

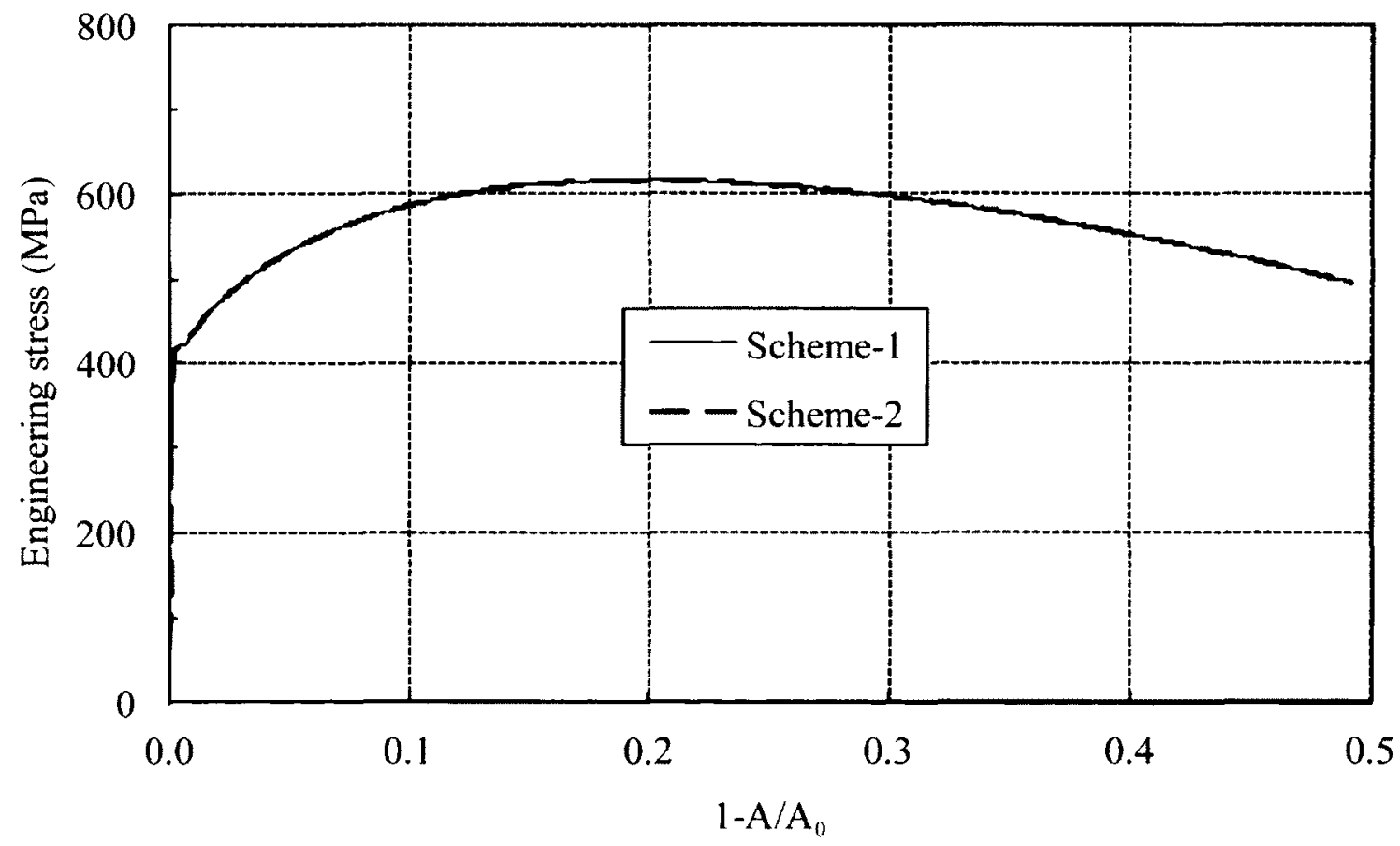

Figure D.4 Engineering stress versus cross-sectional area change curves from FEA simulations for notched specimens AAMa and AAMb with two mesh schemes 


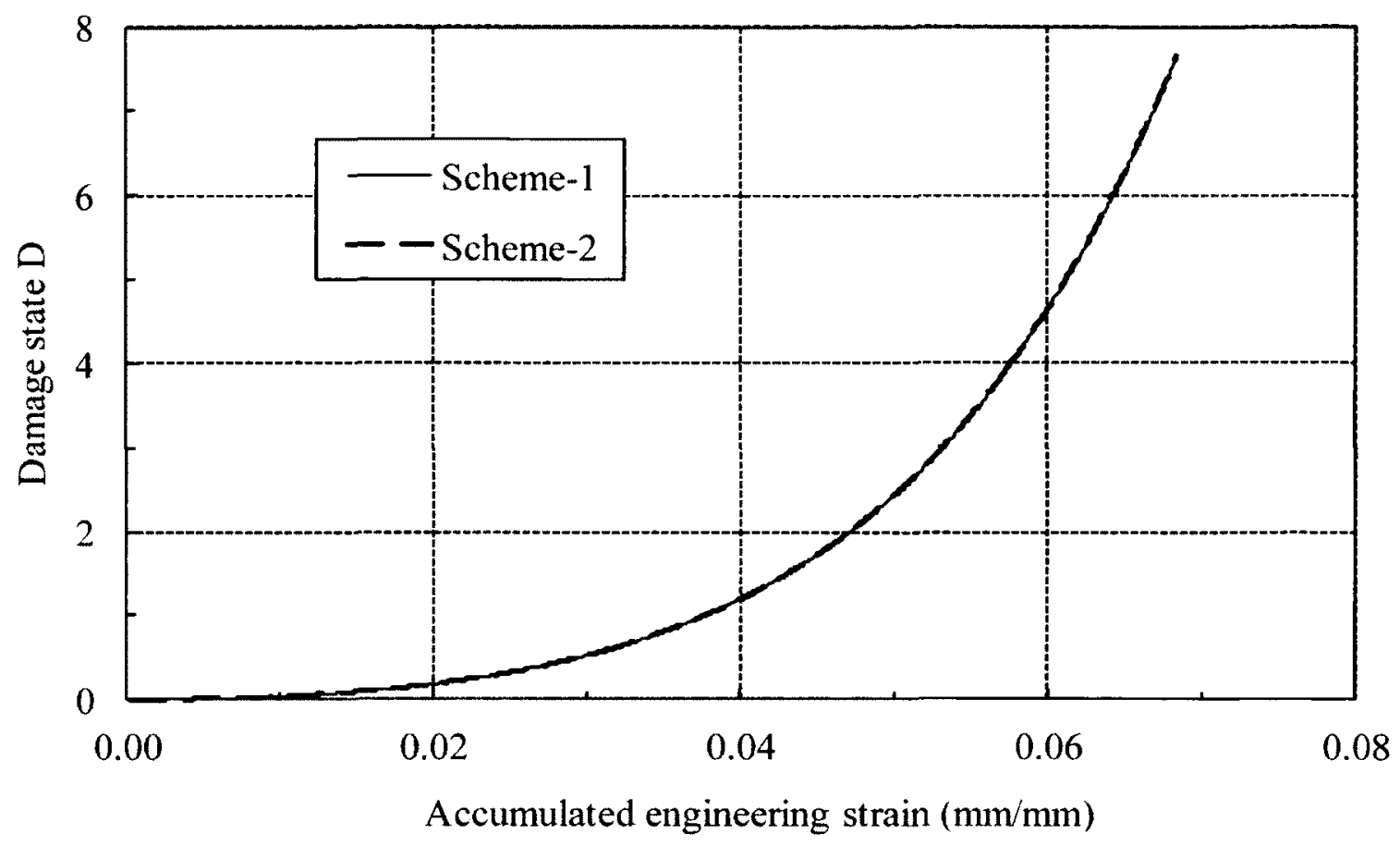

Figure D.5 Calculated damage state D from FEA simulations for notched specimens $\mathrm{AAMa}$ and $\mathrm{AAMb}$ with two mesh schemes

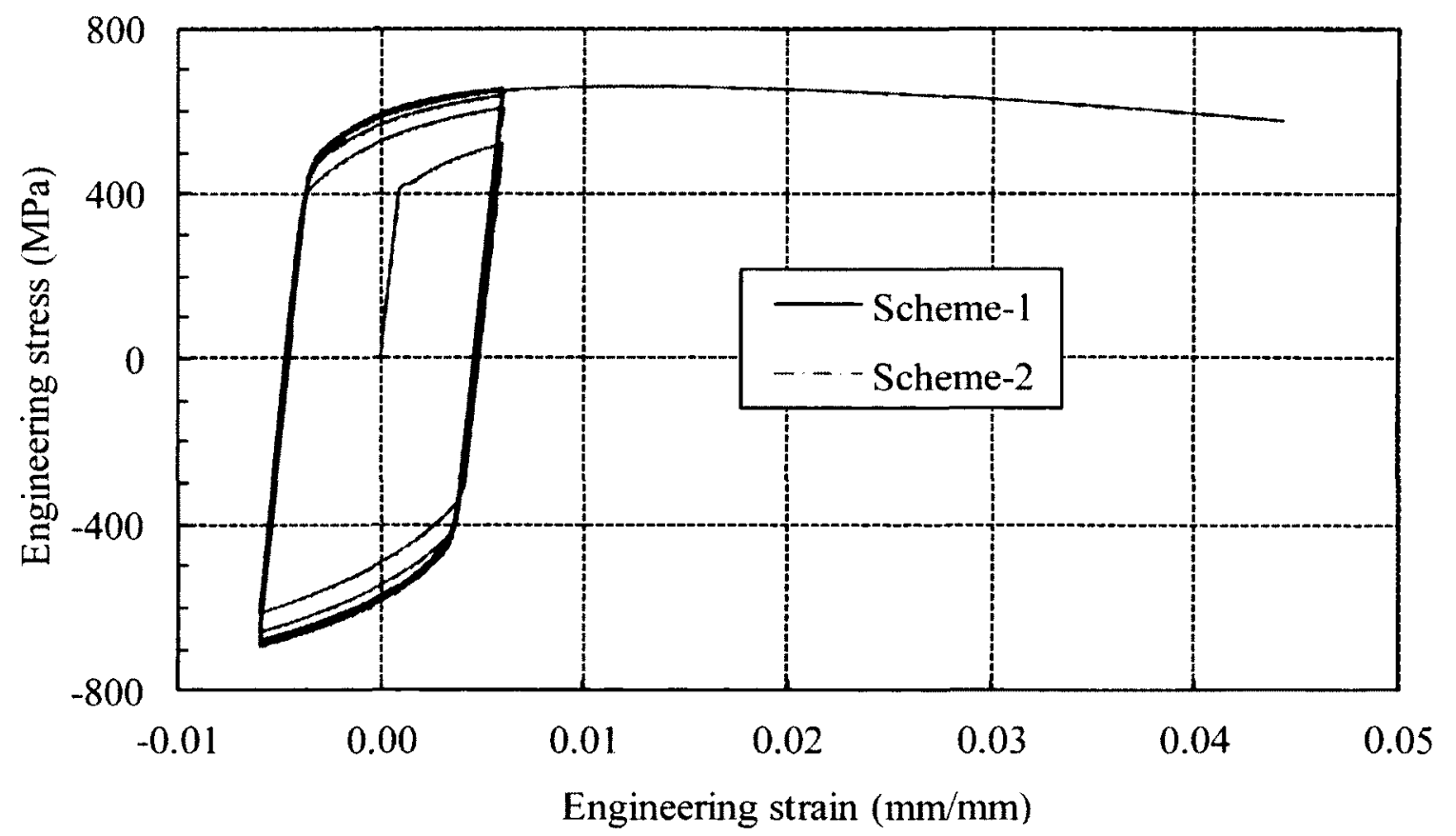

Figure D.6 Engineering stress versus engineering strain curve from FEA simulations for notched specimen AAC70404 with two mesh schemes 


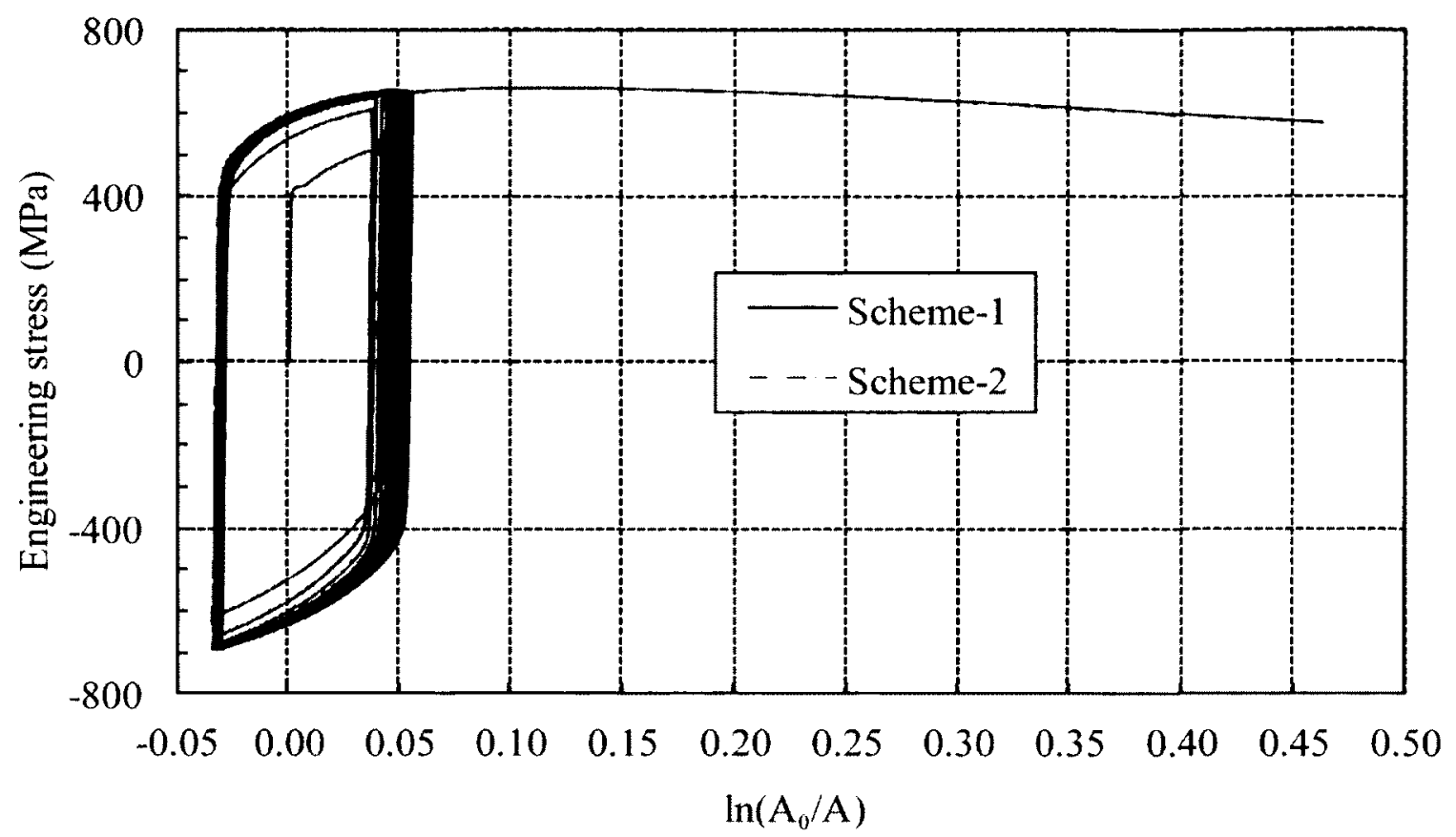

Figure D.7 Engineering stress versus cross-sectional area change curves from FEA simulations for notched specimen AAC70404 with two mesh schemes

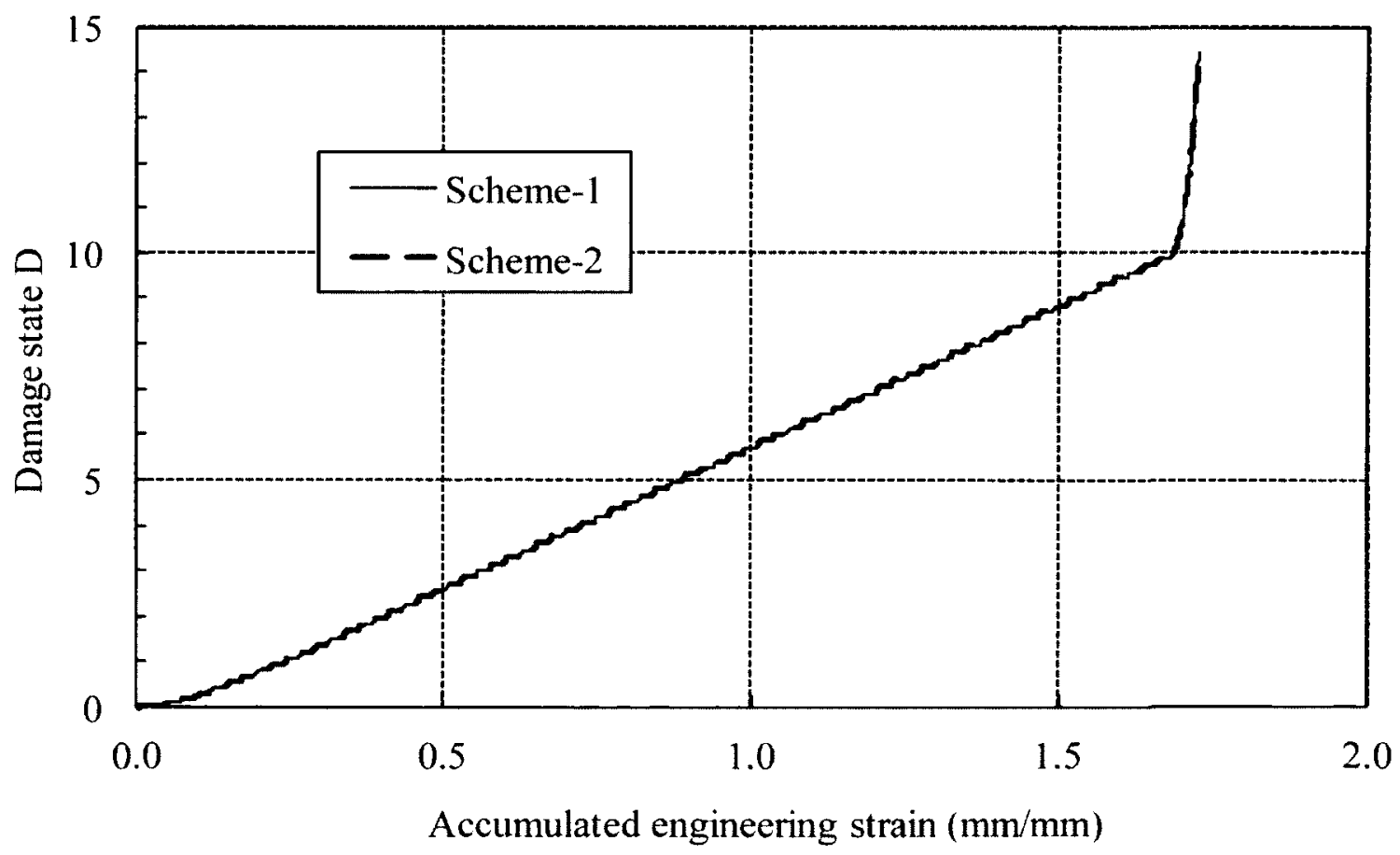

Figure D.8 Calculated damage state D from FEA simulations for notched specimen AAC70404 with two mesh schemes 


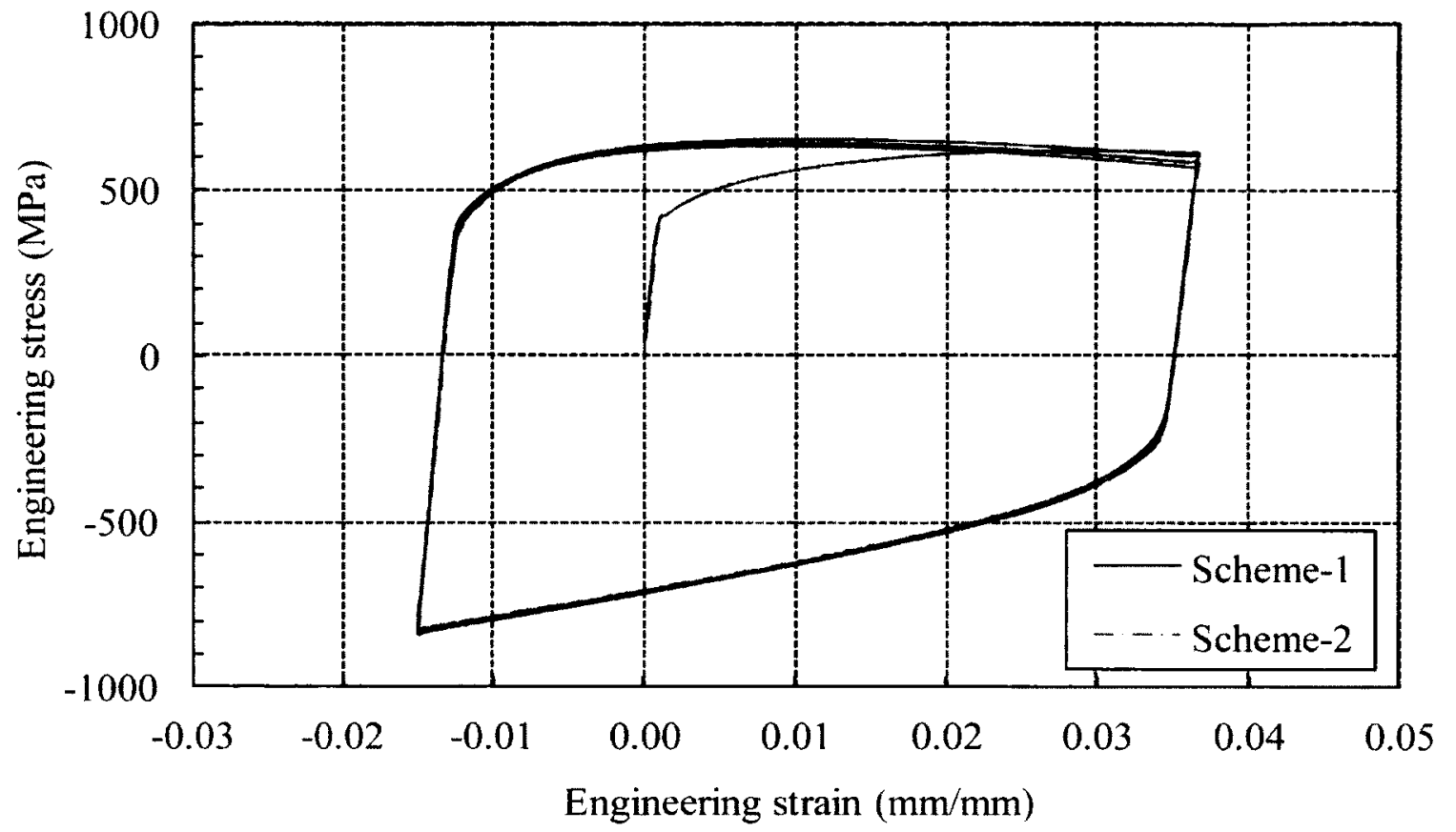

Figure D.9 Engineering stress versus engineering strain curve from FEA simulations for notched specimen AACF3010 with two mesh schemes

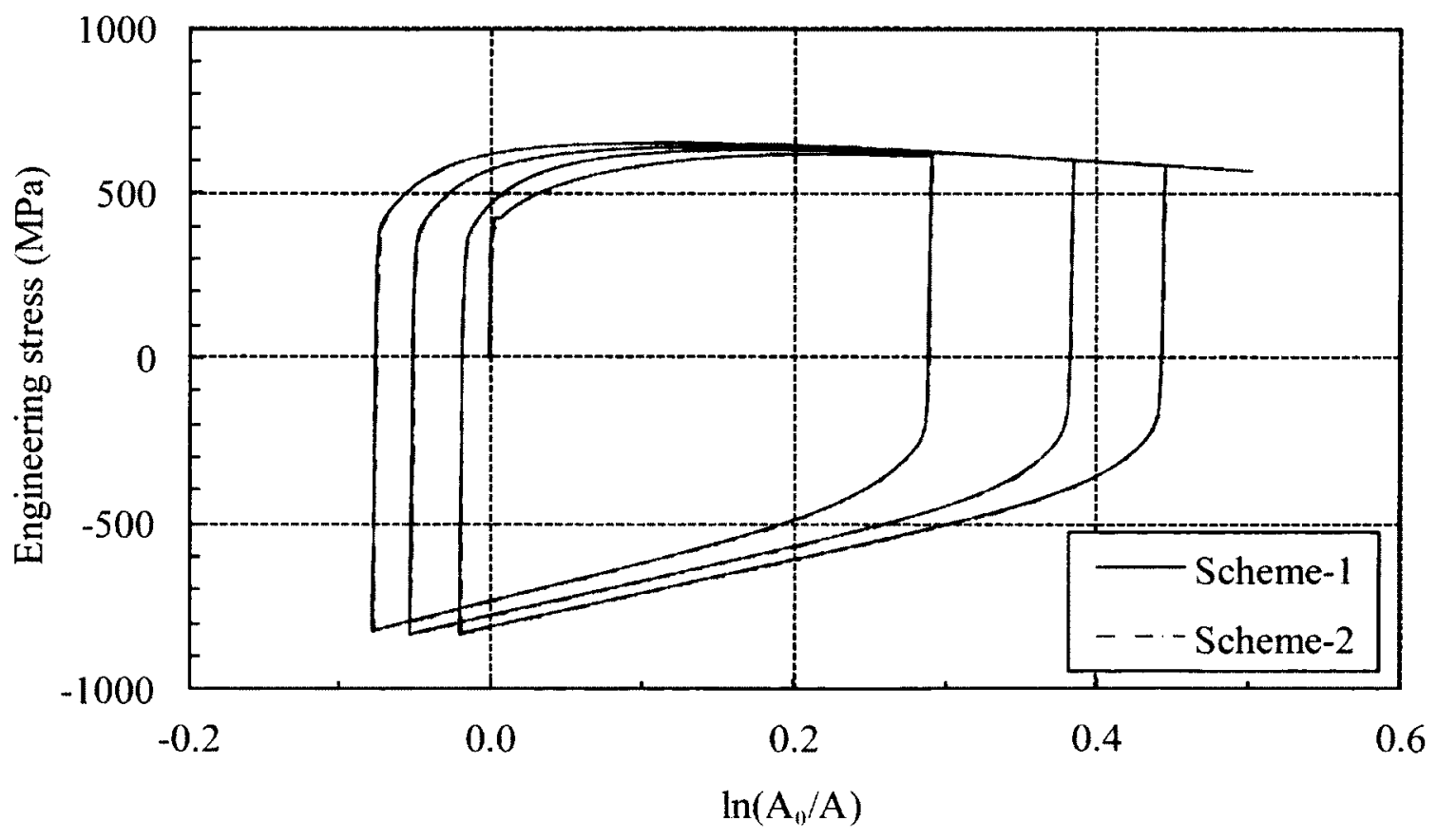

Figure D.10 Engineering stress versus cross-sectional area change curves from FEA simulations for notched specimen AACF3010 with two mesh schemes 


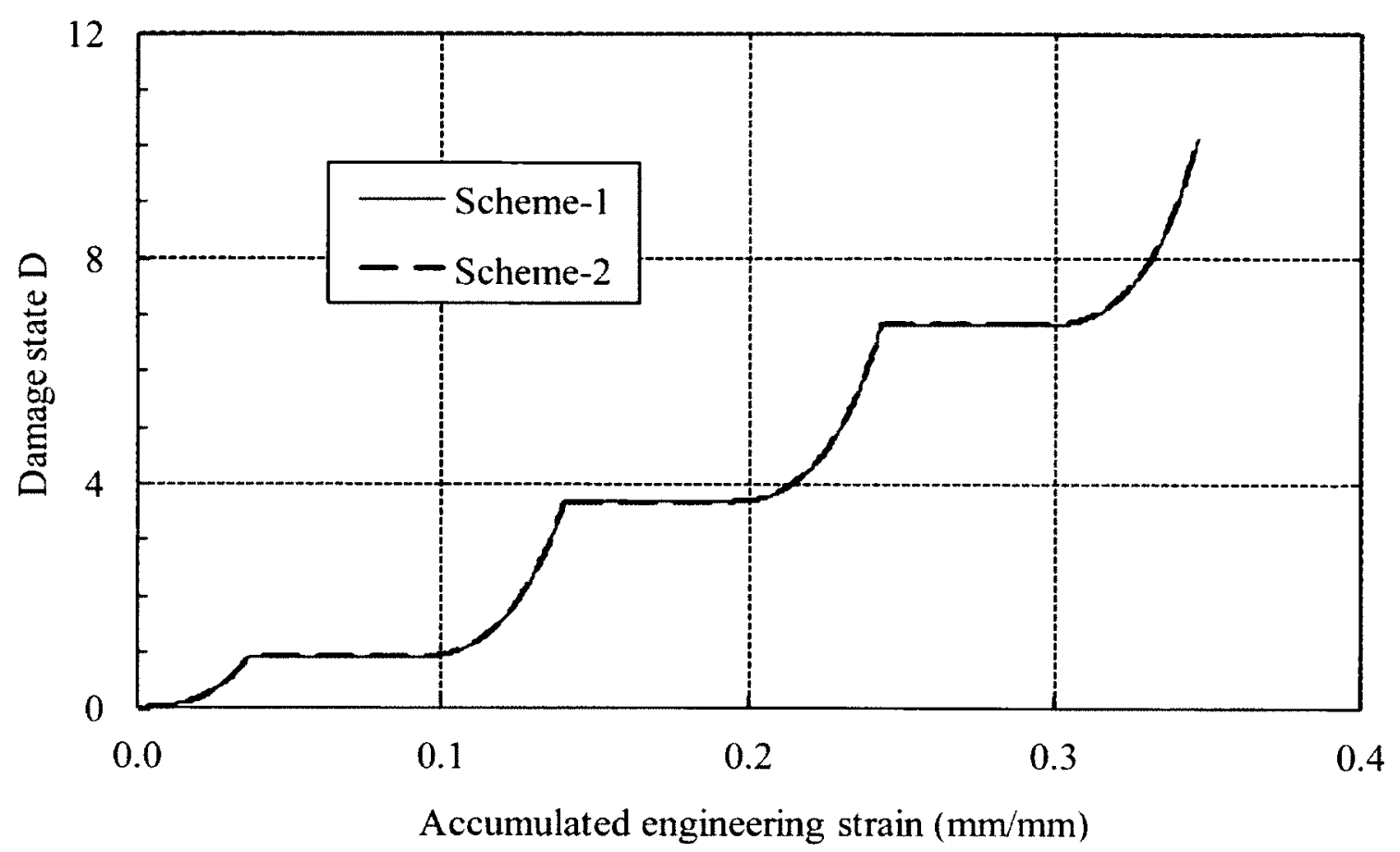

Figure D.11 Calculated damage state D from FEA simulations for notched specimen AACF3010 with two mesh schemes

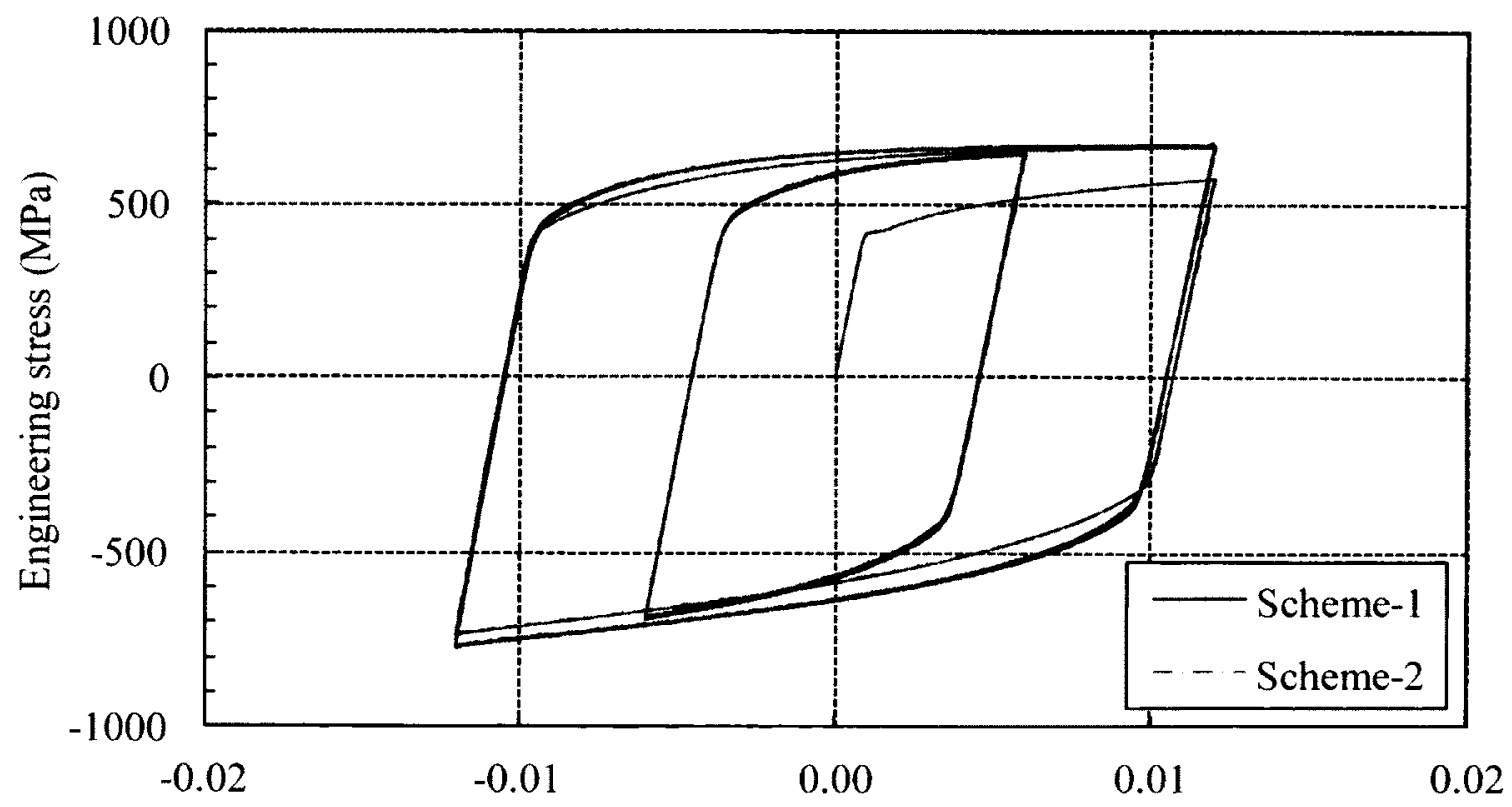

Engineering strain $(\mathrm{mm} / \mathrm{mm})$

Figure D.12 Engineering stress versus engineering strain curve from FEA simulations for notched specimen AACR0804 with two mesh schemes 


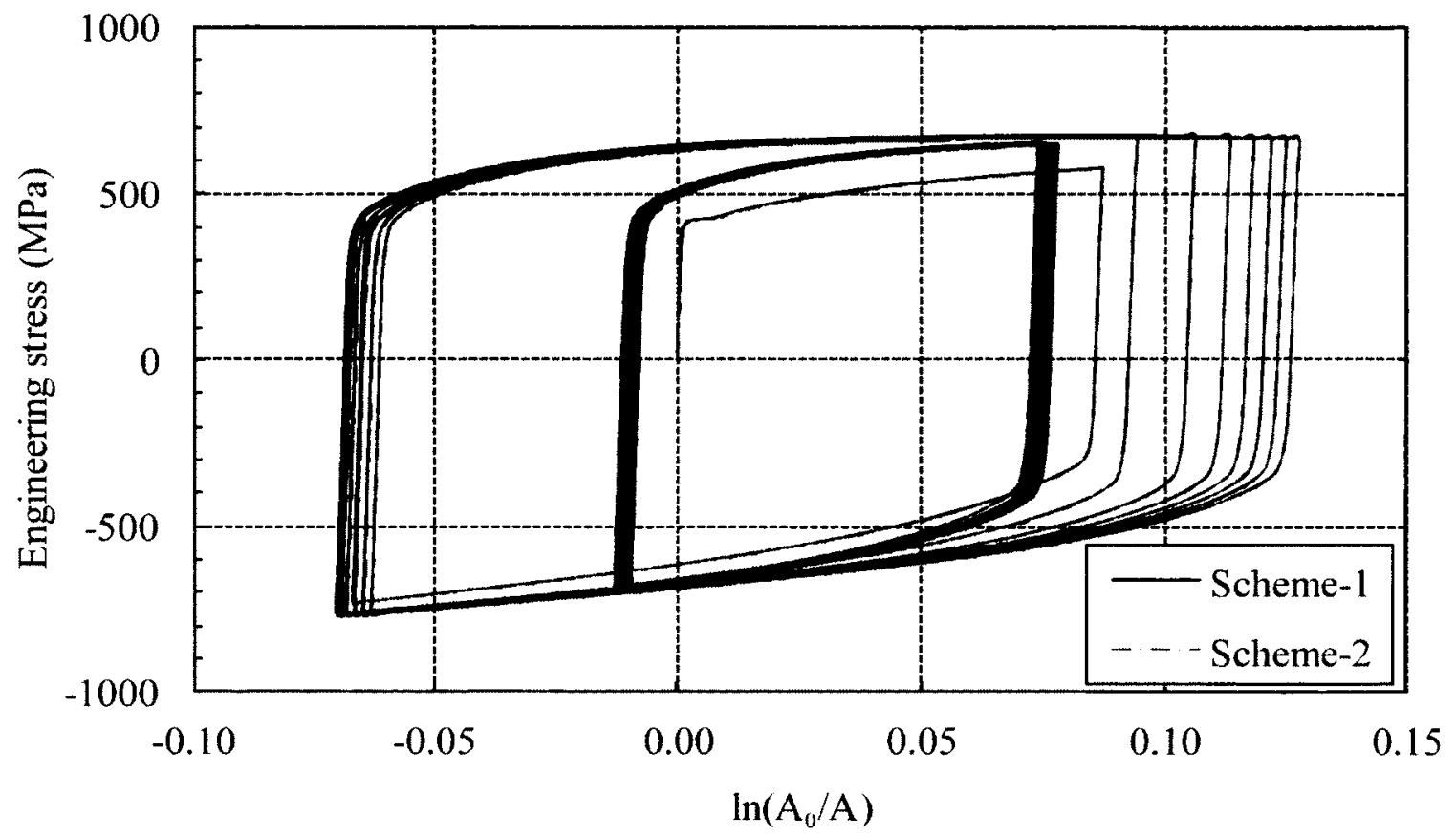

Figure D.13 Engineering stress versus cross-sectional area change curves from FEA simulations for notched specimen AACR0804 with two mesh schemes

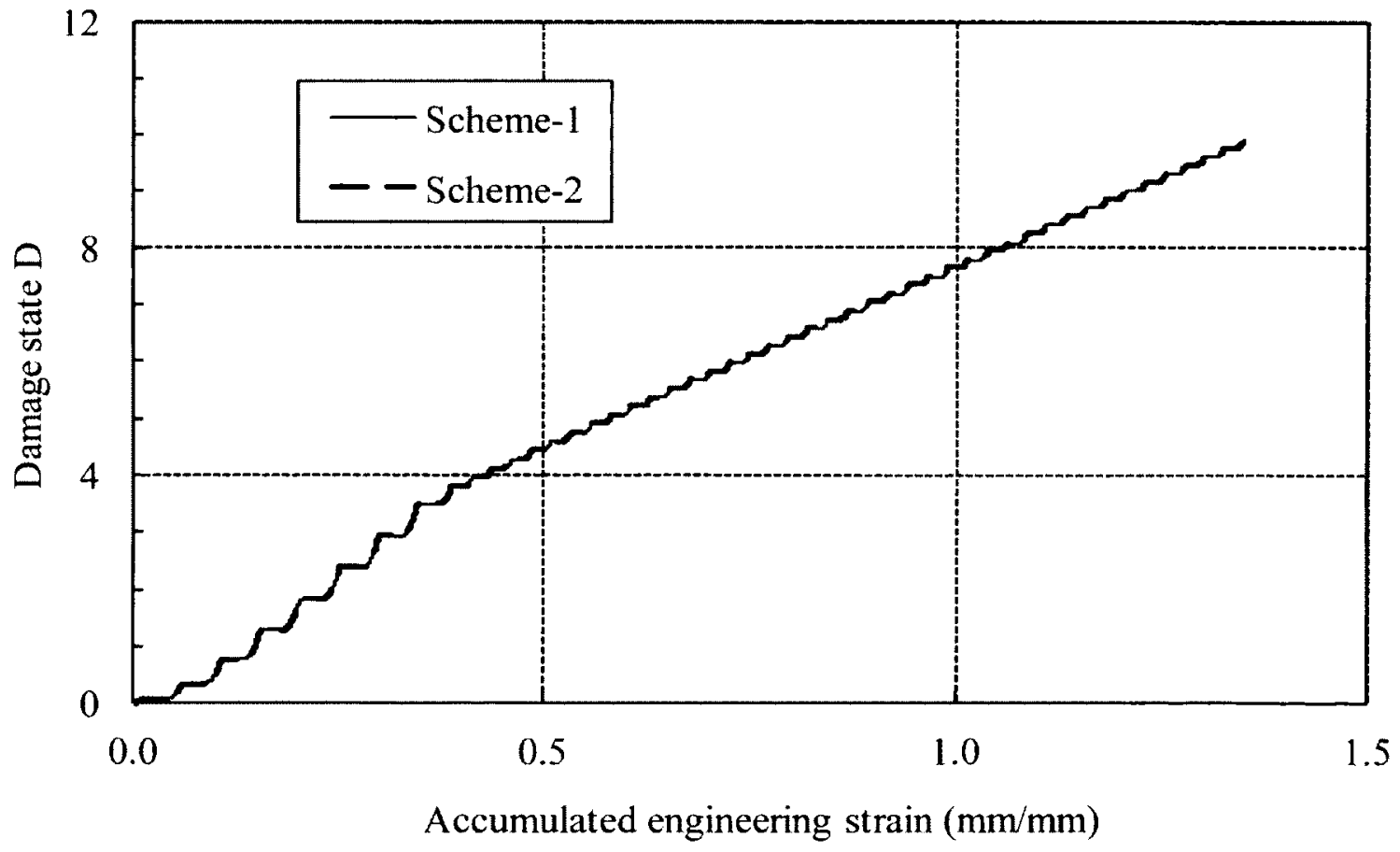

Figure D.14 Calculated damage state D from FEA simulations for notched specimen AACR0804 with two mesh schemes 


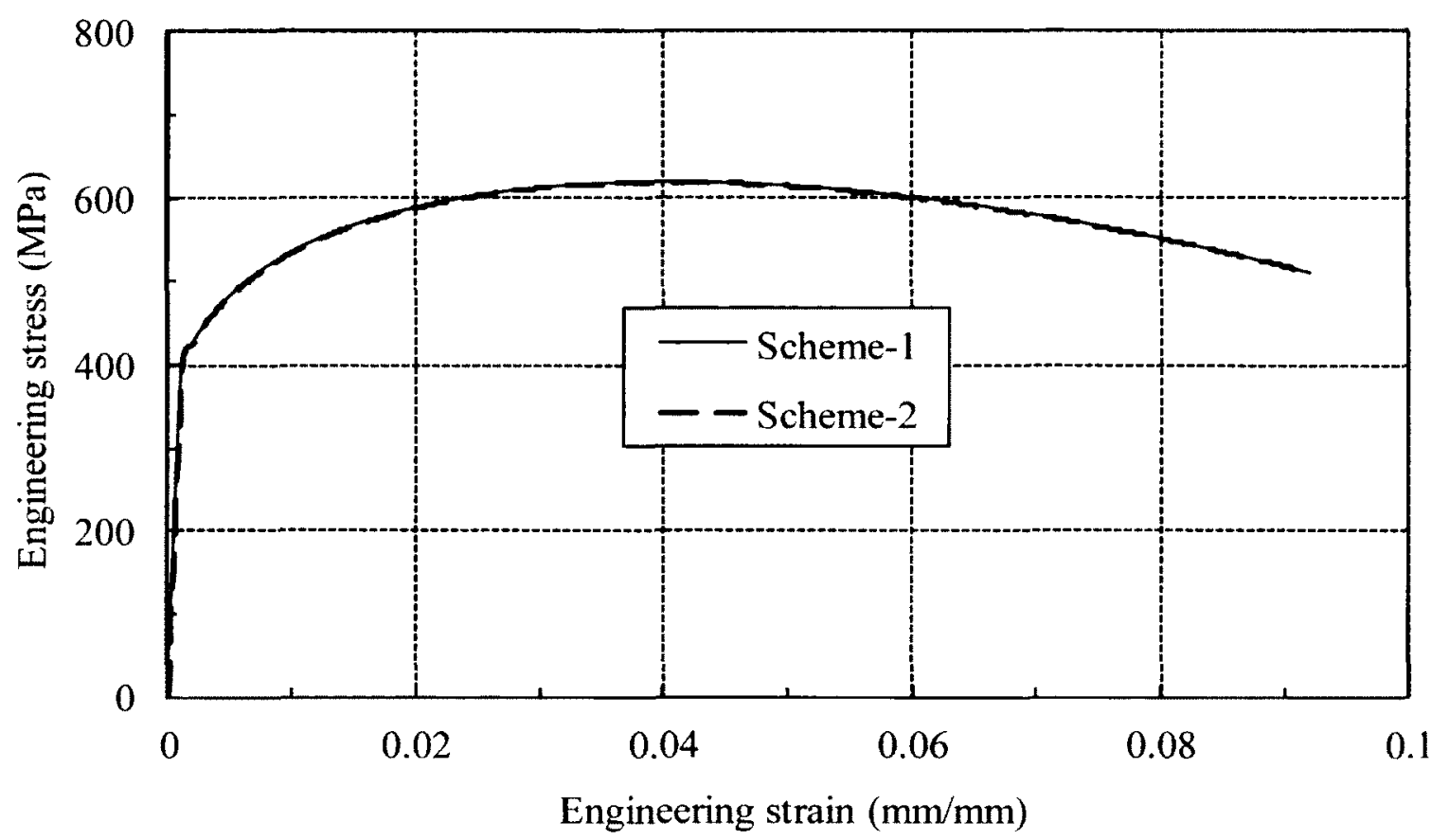

Figure D.15 Engineering stress versus engineering strain curve from FEA simulations for notched specimen ABM with two mesh schemes

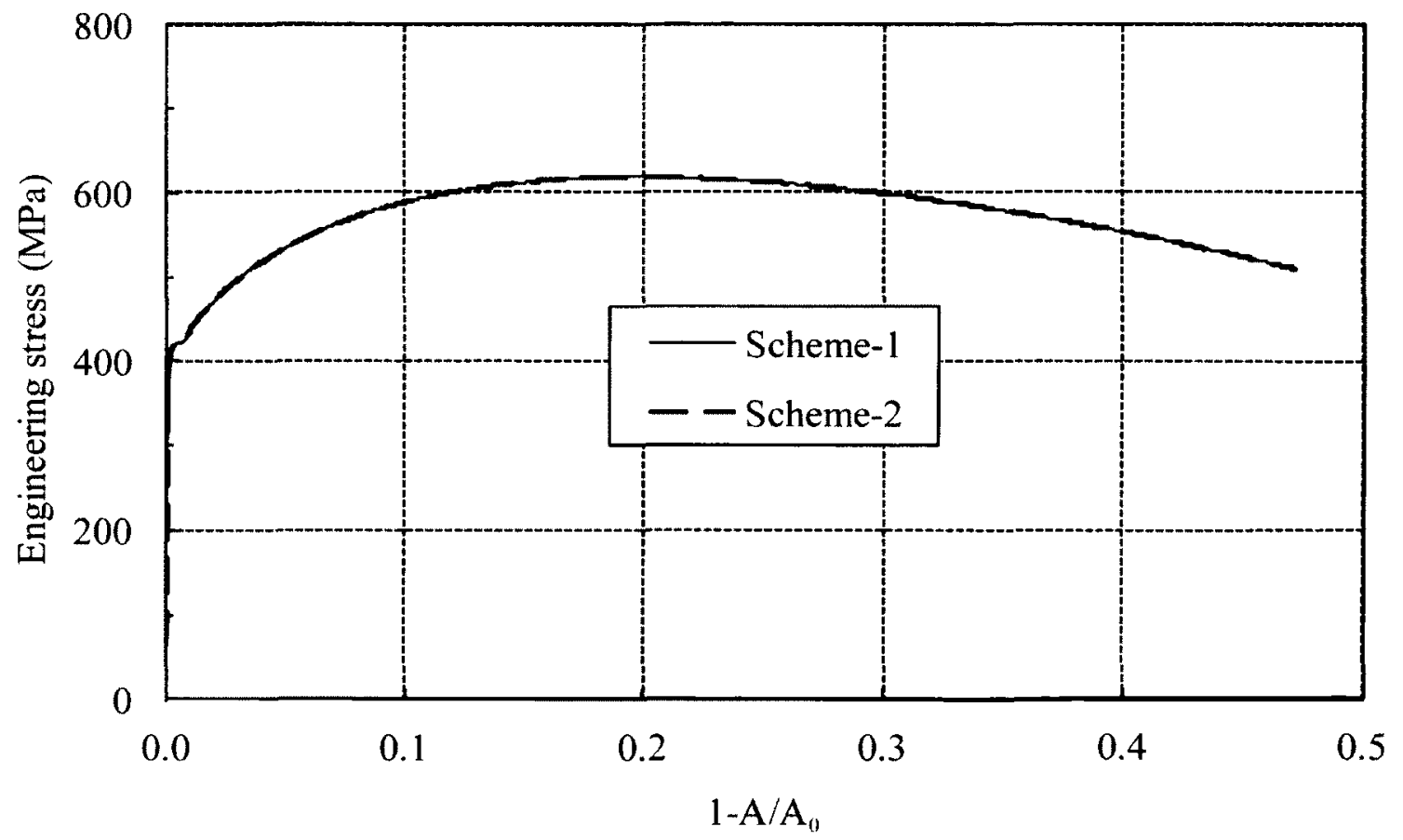

Figure D.16 Engineering stress versus cross-sectional area change curves from FEA simulations for notched specimen ABM with two mesh schemes 


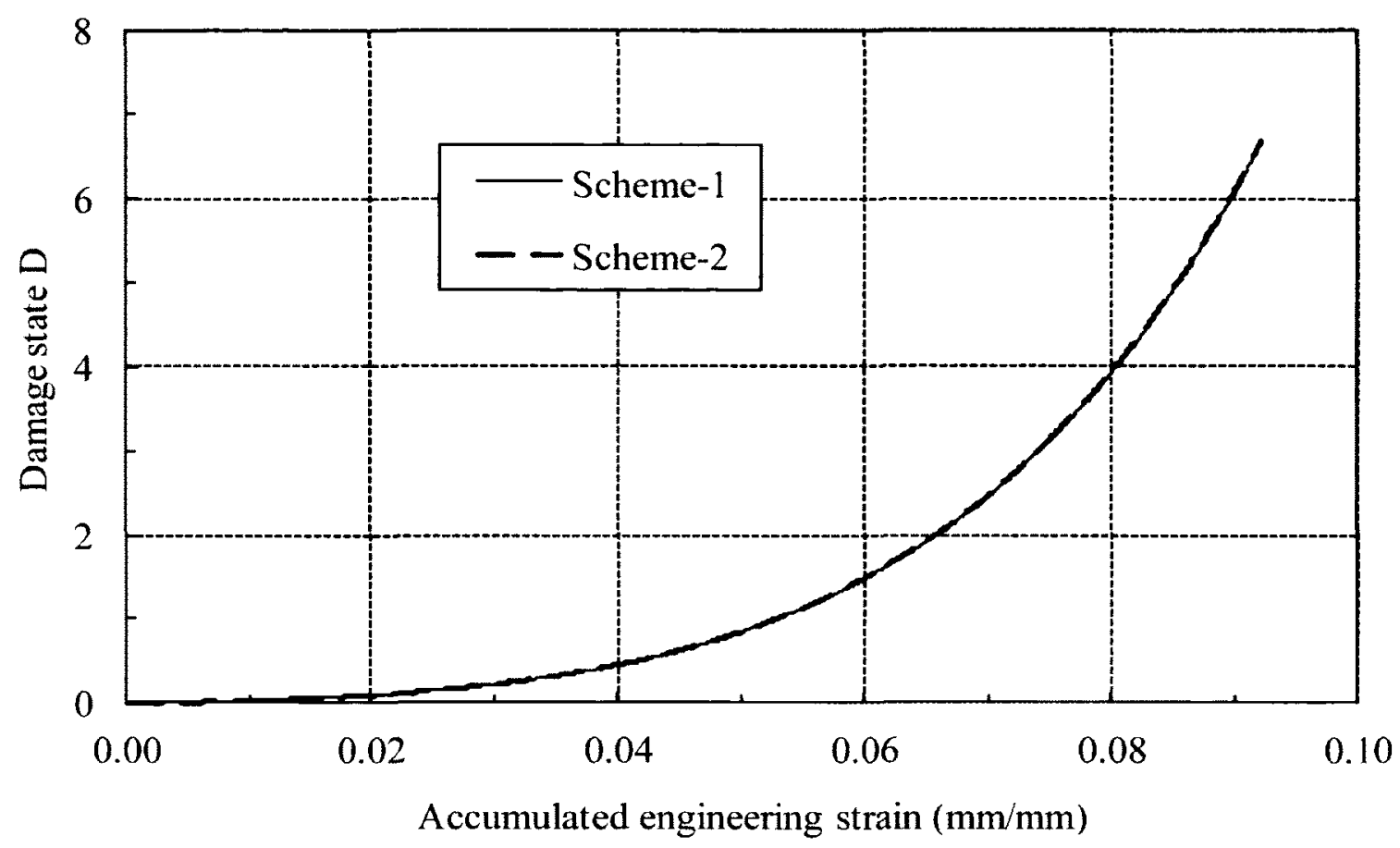

Figure D.17 Calculated damage state D from FEA simulations for notched specimen ABM with two mesh schemes

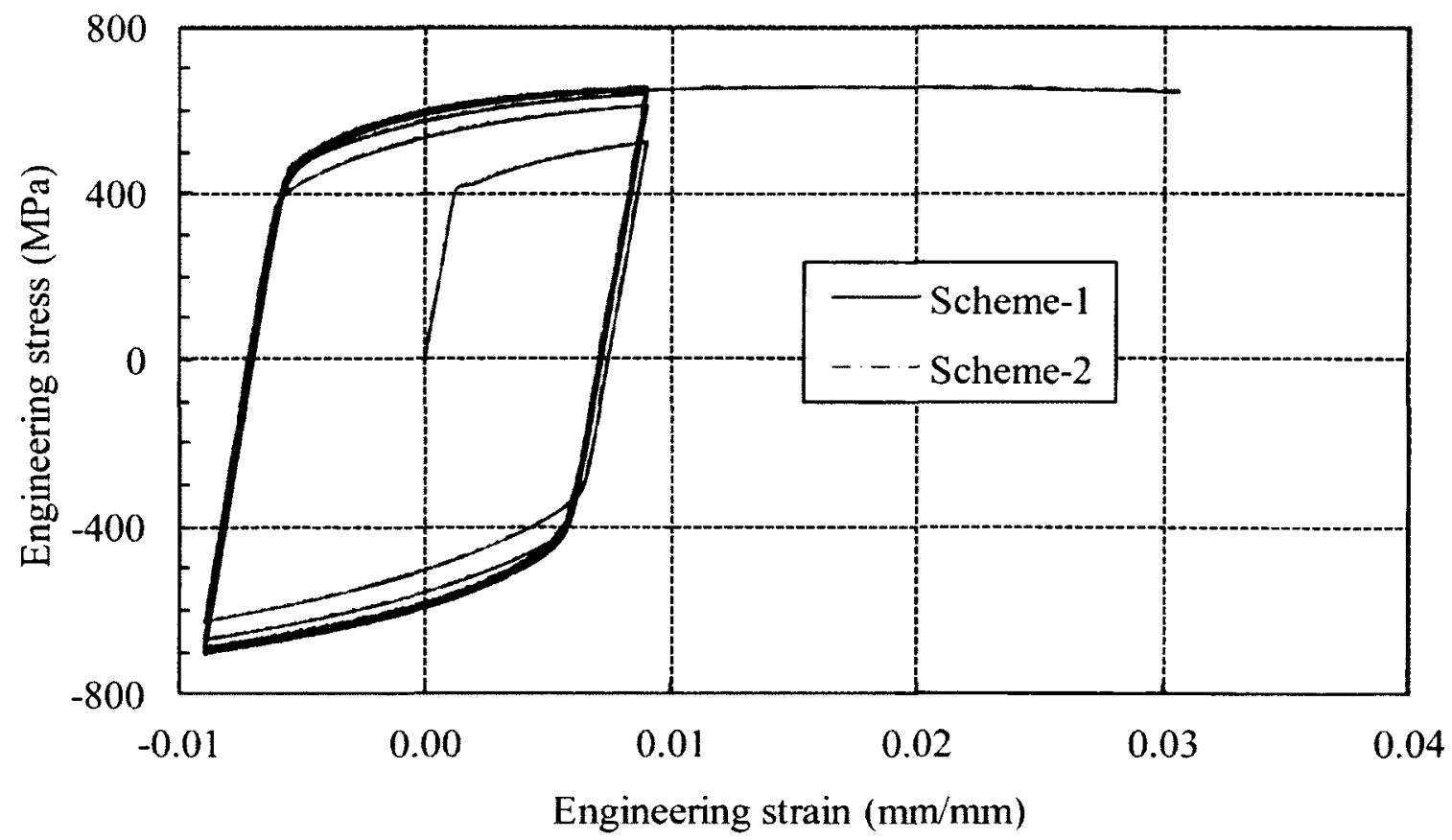

Figure D.18 Engineering stress versus engineering strain curve from FEA simulations for notched specimen $\mathrm{ABC} 70404$ with two mesh schemes 


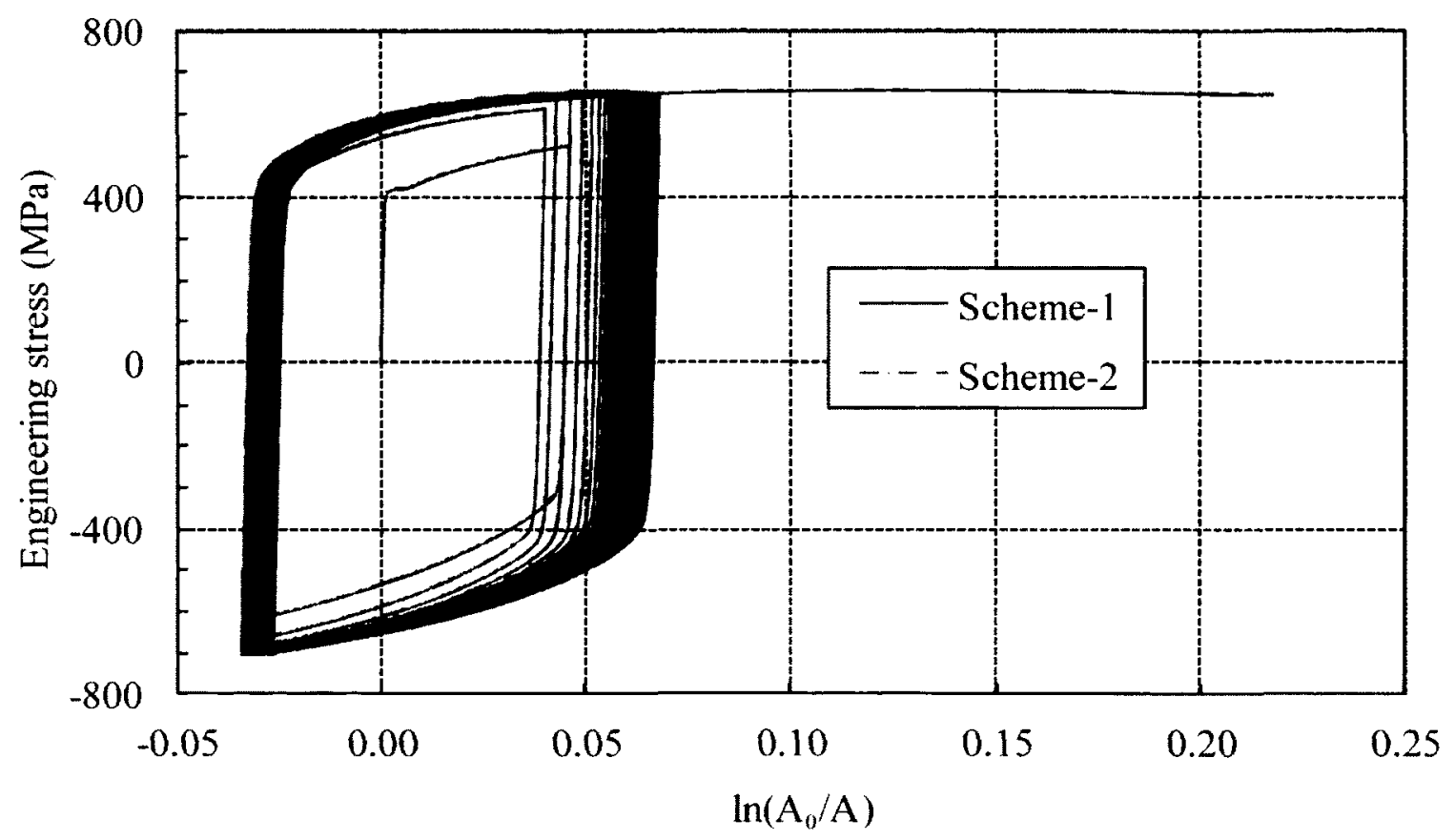

Figure D.19 Engineering stress versus cross-sectional area change curves from FEA simulations for notched specimen $\mathrm{ABC} 70404$ with two mesh schemes

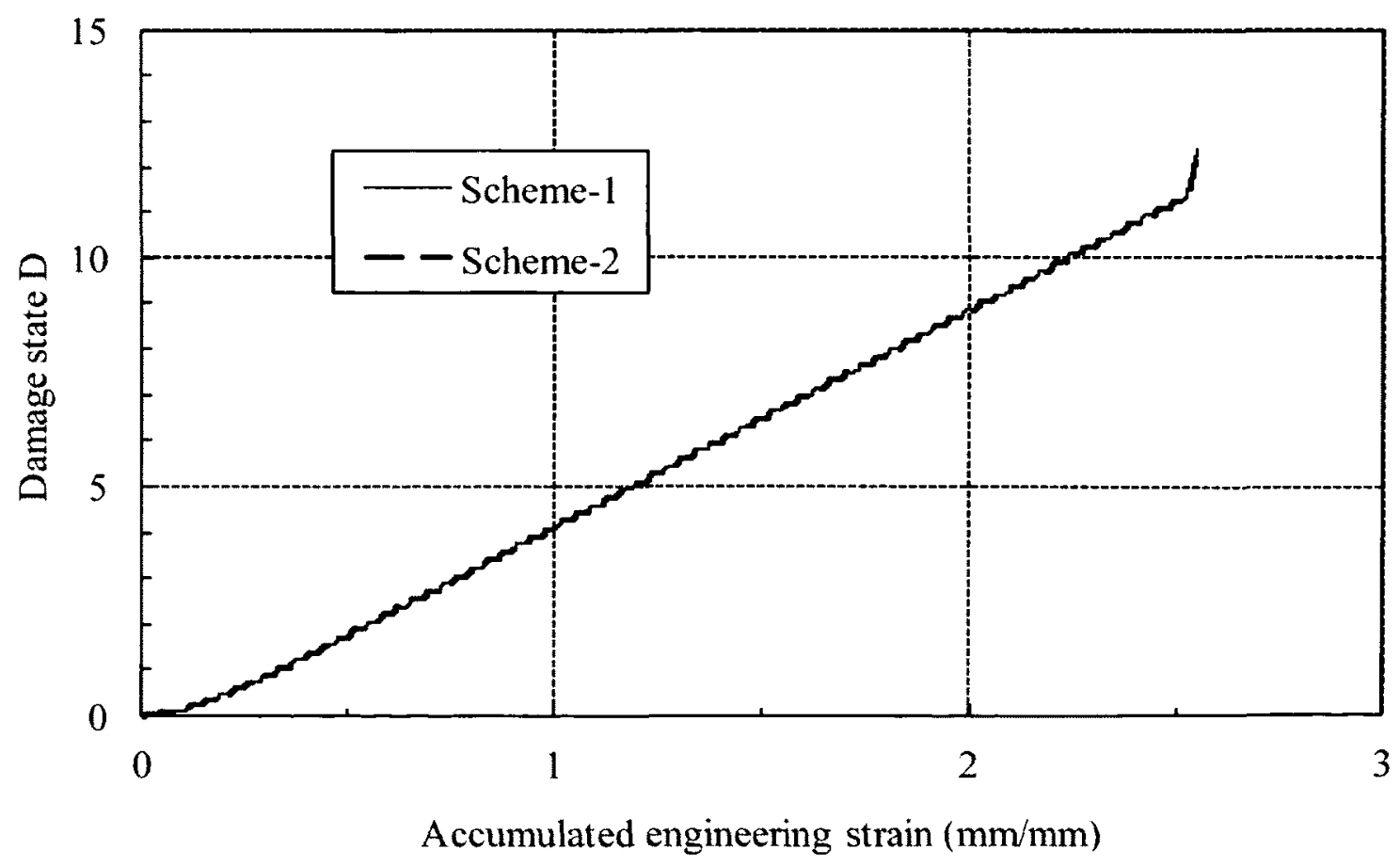

Figure D.20 Calculated damage state D from FEA simulations for notched specimen $\mathrm{ABC} 70404$ with two mesh schemes 


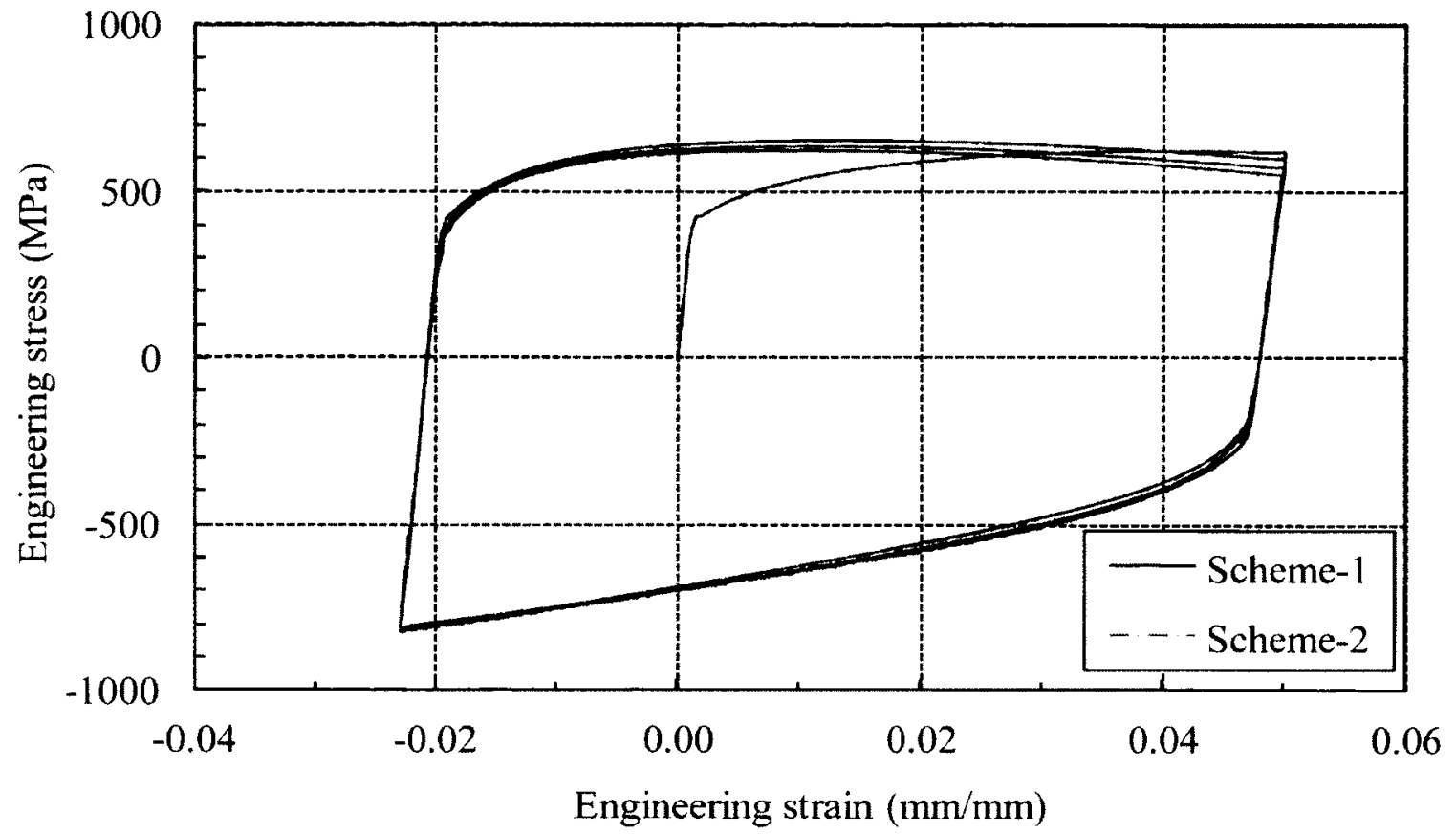

Figure D.21 Engineering stress versus engineering strain curve from FEA simulations for notched specimen $\mathrm{ABCF} 3010$ with two mesh schemes

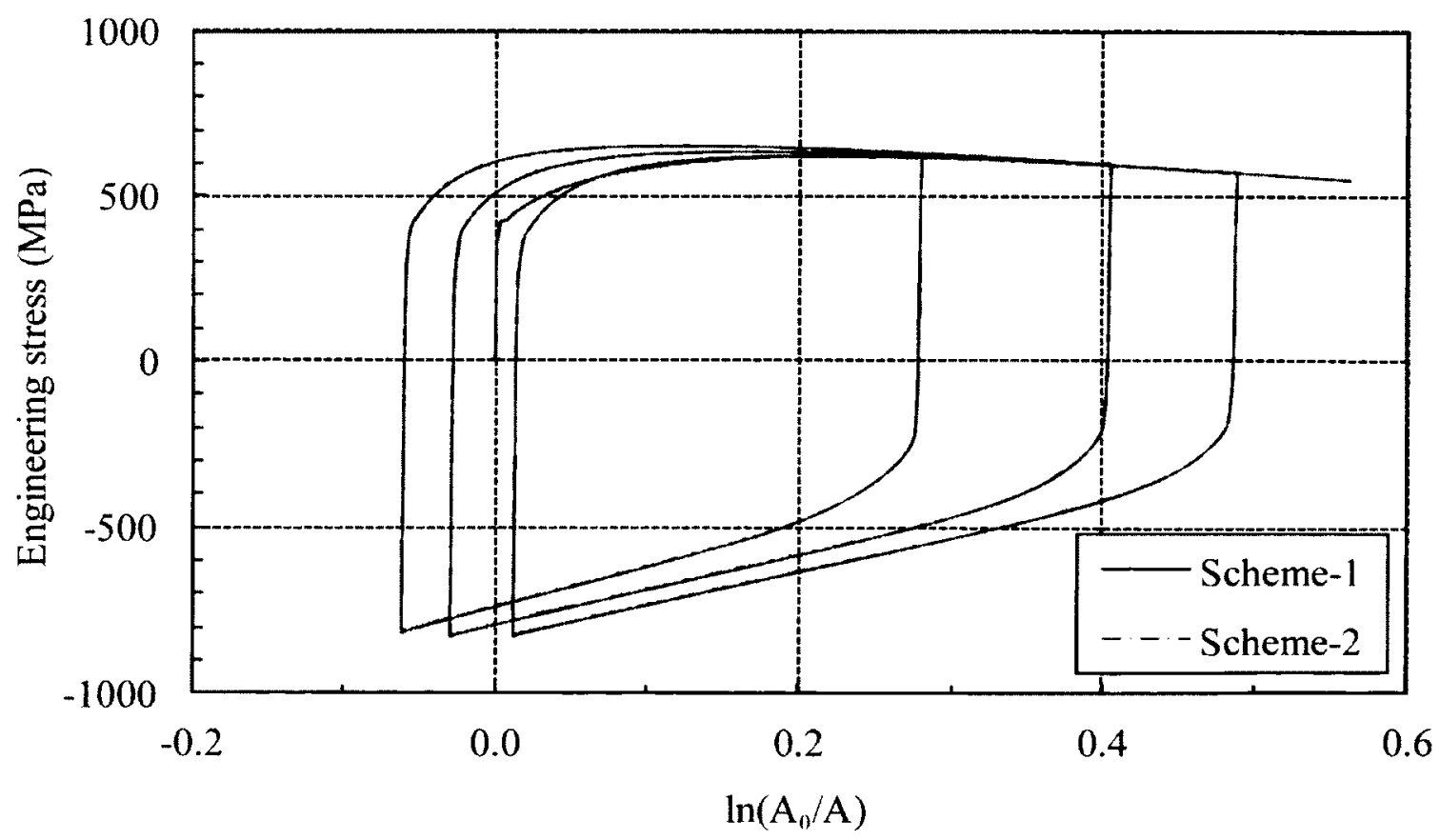

Figure D.22 Engineering stress versus cross-sectional area change curves from FEA simulations for notched specimen ABCF 3010 with two mesh schemes 


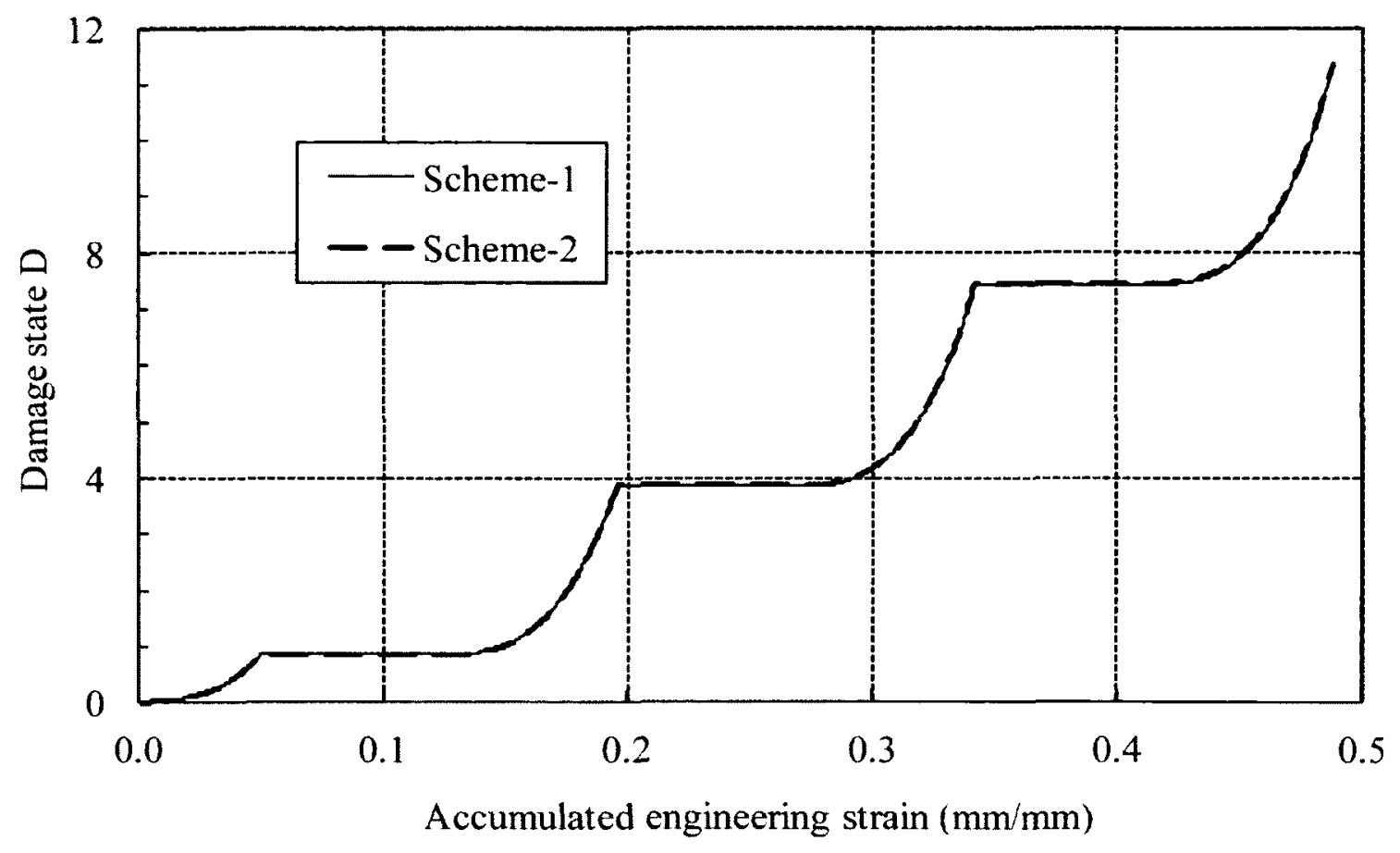

Figure D.23 Calculated damage state D from FEA simulations for notched specimen ABCF3010 with two mesh schemes 


\section{APPENDIX E FEA SIMULATIONS FOR MONOTONIC TENSION AND CYCLIC TESTS OF STRAP SPECIMENS}

The test and predicted load versus deformation curves of the specimens using the 1/2 model are shown in the following Figs. E.1 to E.14. The slippage due to the clearance at the bolt hole has caused some specimens appear to be stiffer in the simulation compared to the test. Since numerical simulations have been based on material model parameters for the static behaviour and after taking into consideration of the possible slippage at the bolt hole, there is a general good agreement between the test and predicted load versus deformation curves. 


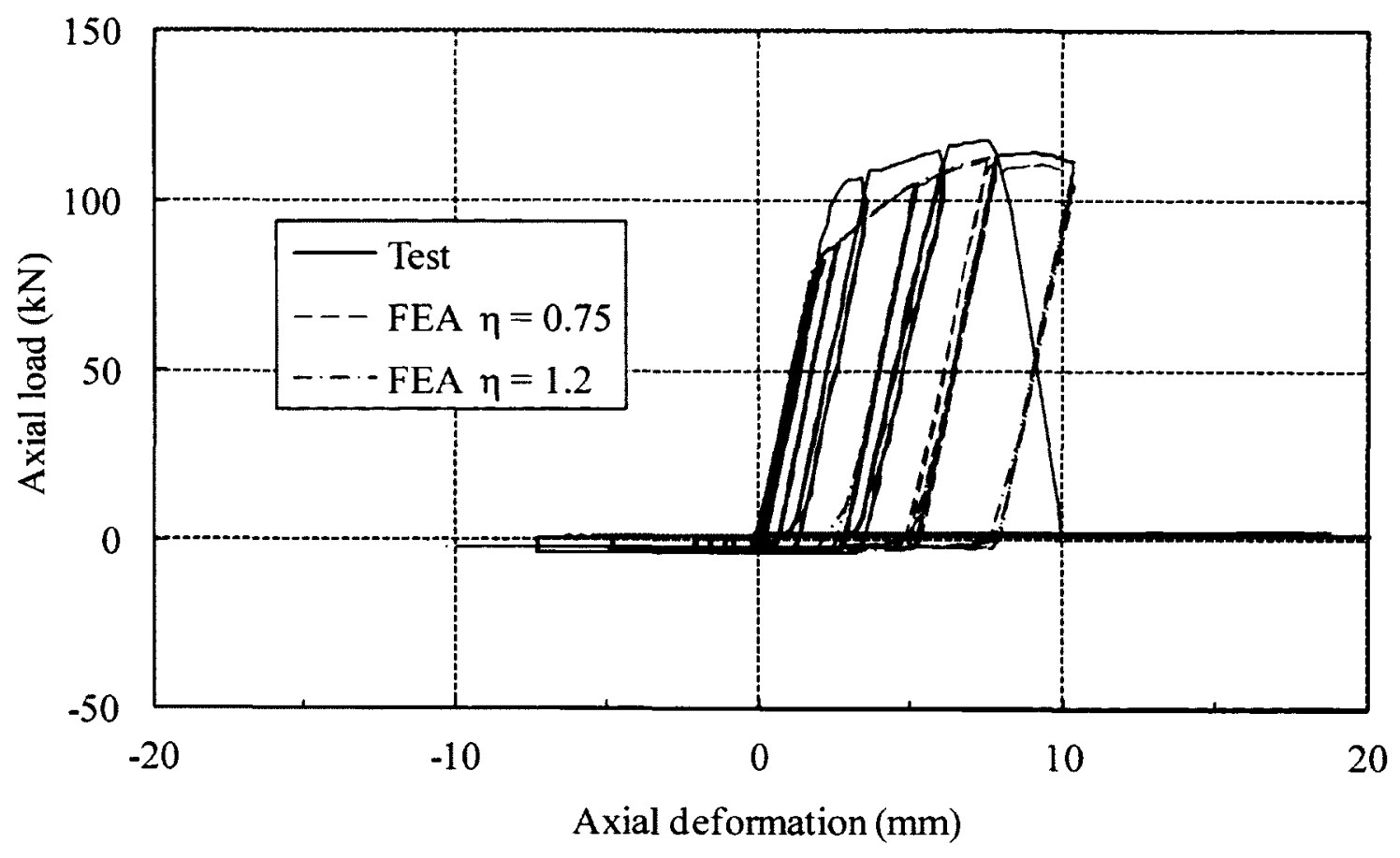

Figure E.1 Test and predicted load versus deformation curves for strap specimen A6075a using the $1 / 2$ model

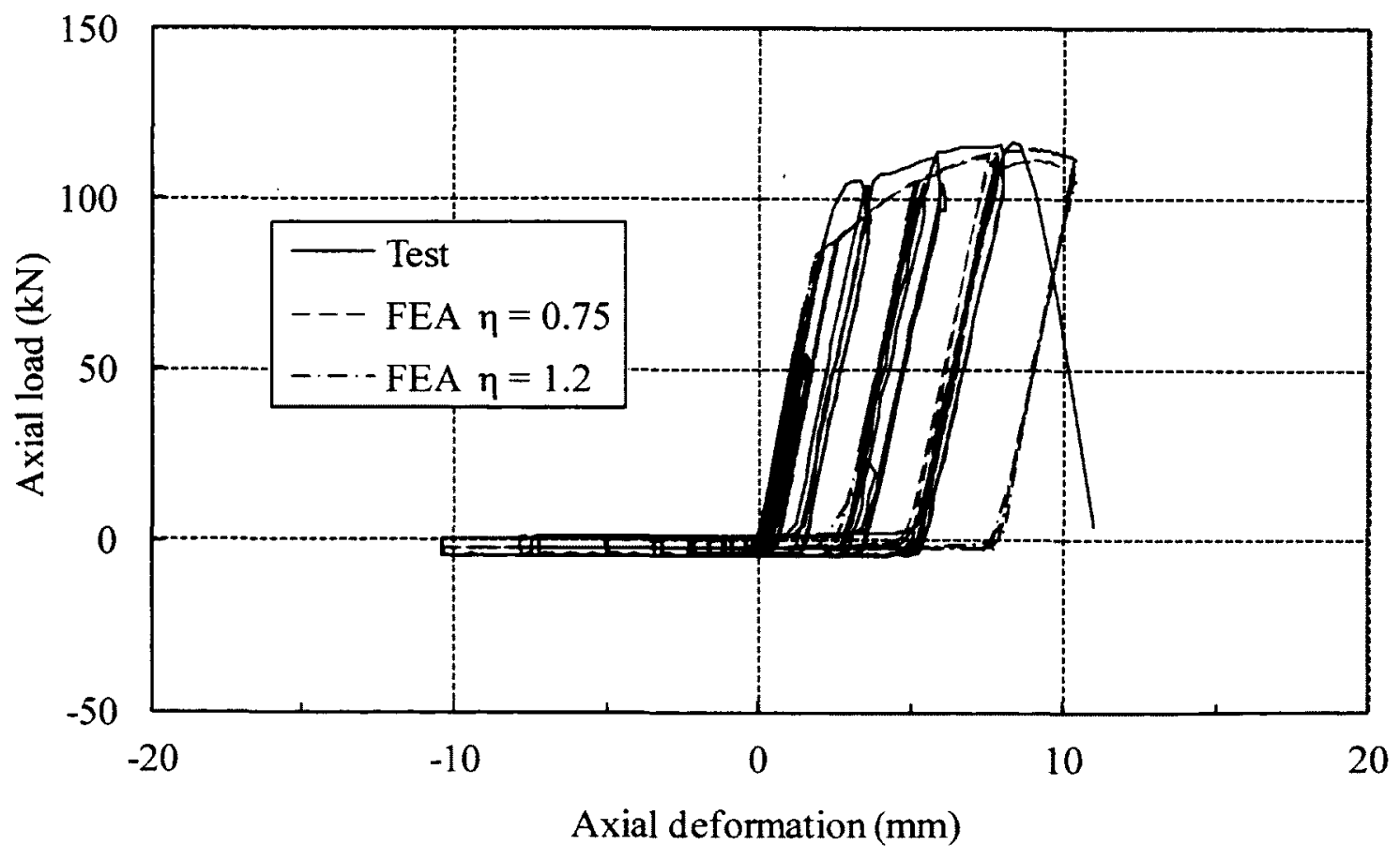

Figure E.2 Test and predicted load versus deformation curves for strap specimen A6075b using the $1 / 2$ model 


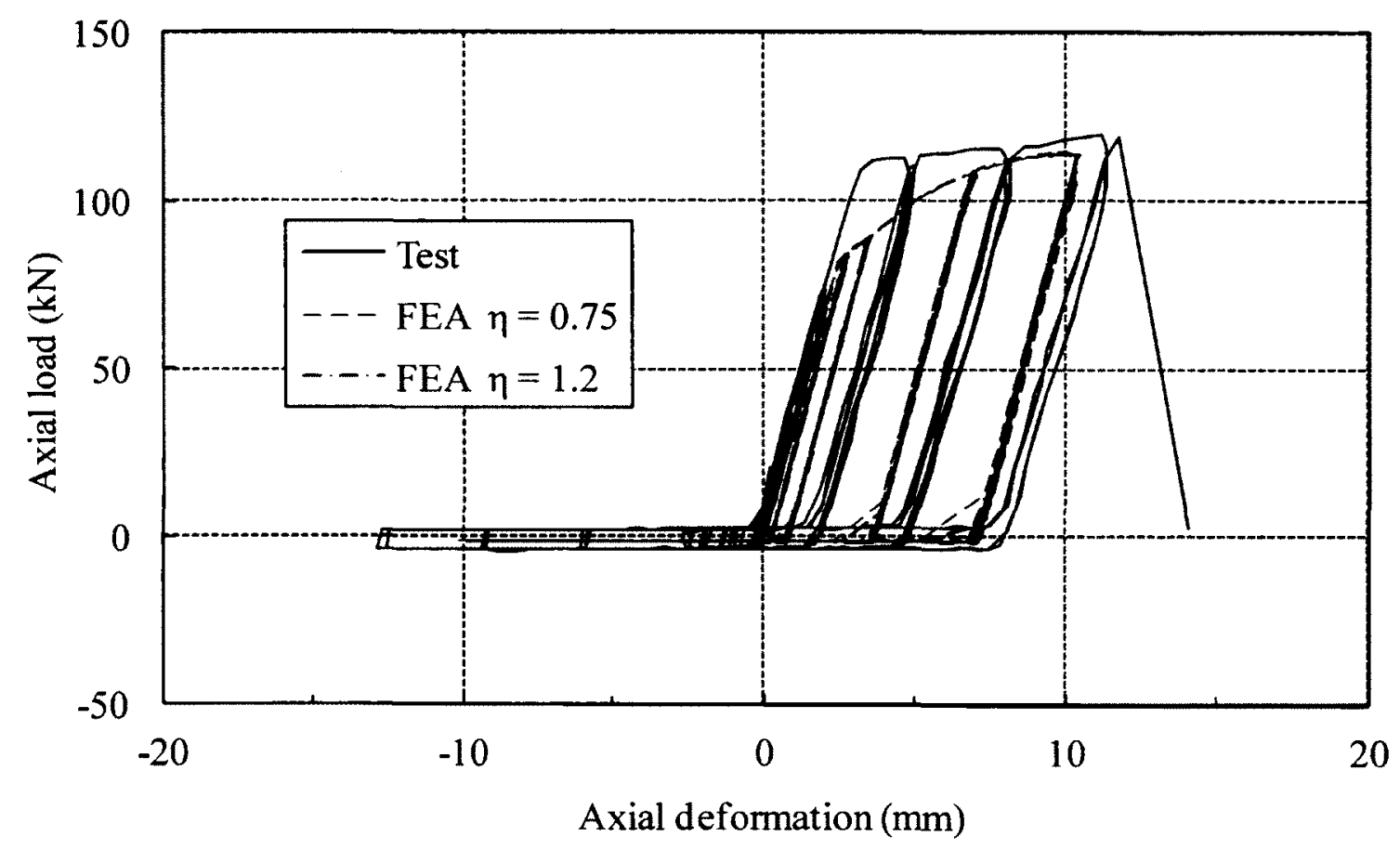

Figure E.3 Test and predicted load versus deformation curves for strap specimen A8075a using the $1 / 2$ model

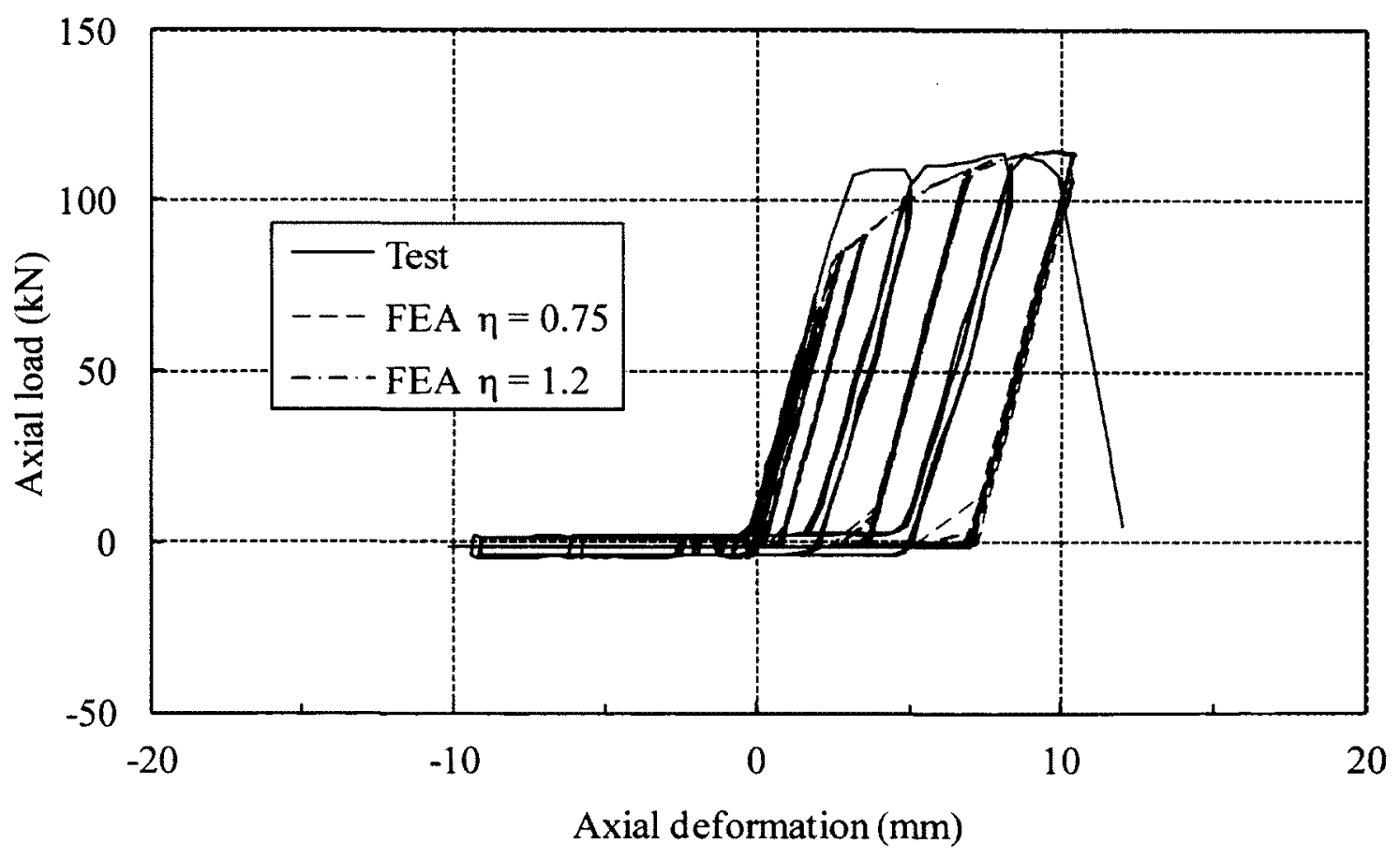

Figure E.4 Test and predicted load versus deformation curves for strap specimen A8075b using the $1 / 2$ model 


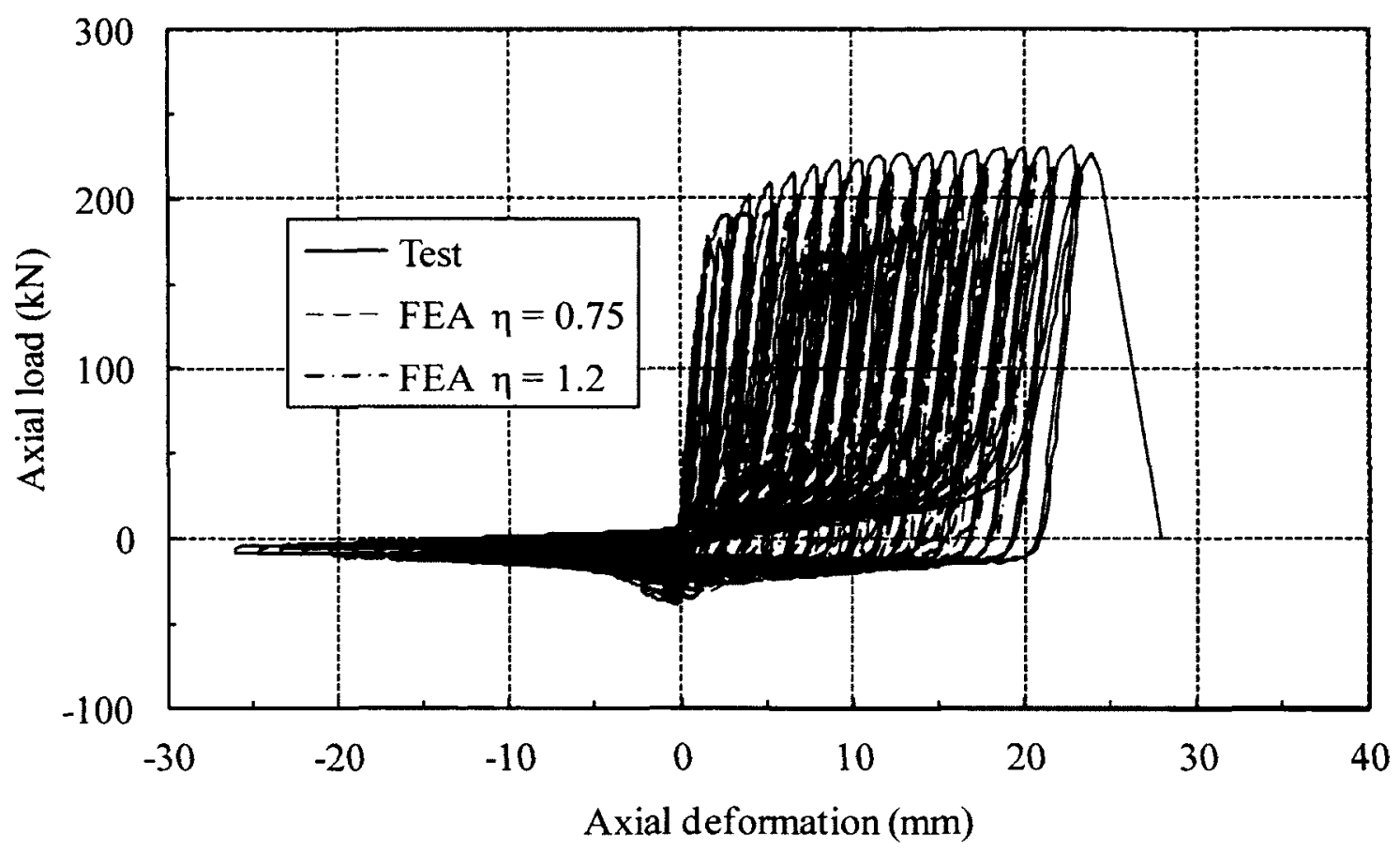

Figure E.5 Test and predicted load versus deformation curves for strap specimen A2078a using the $1 / 2$ model

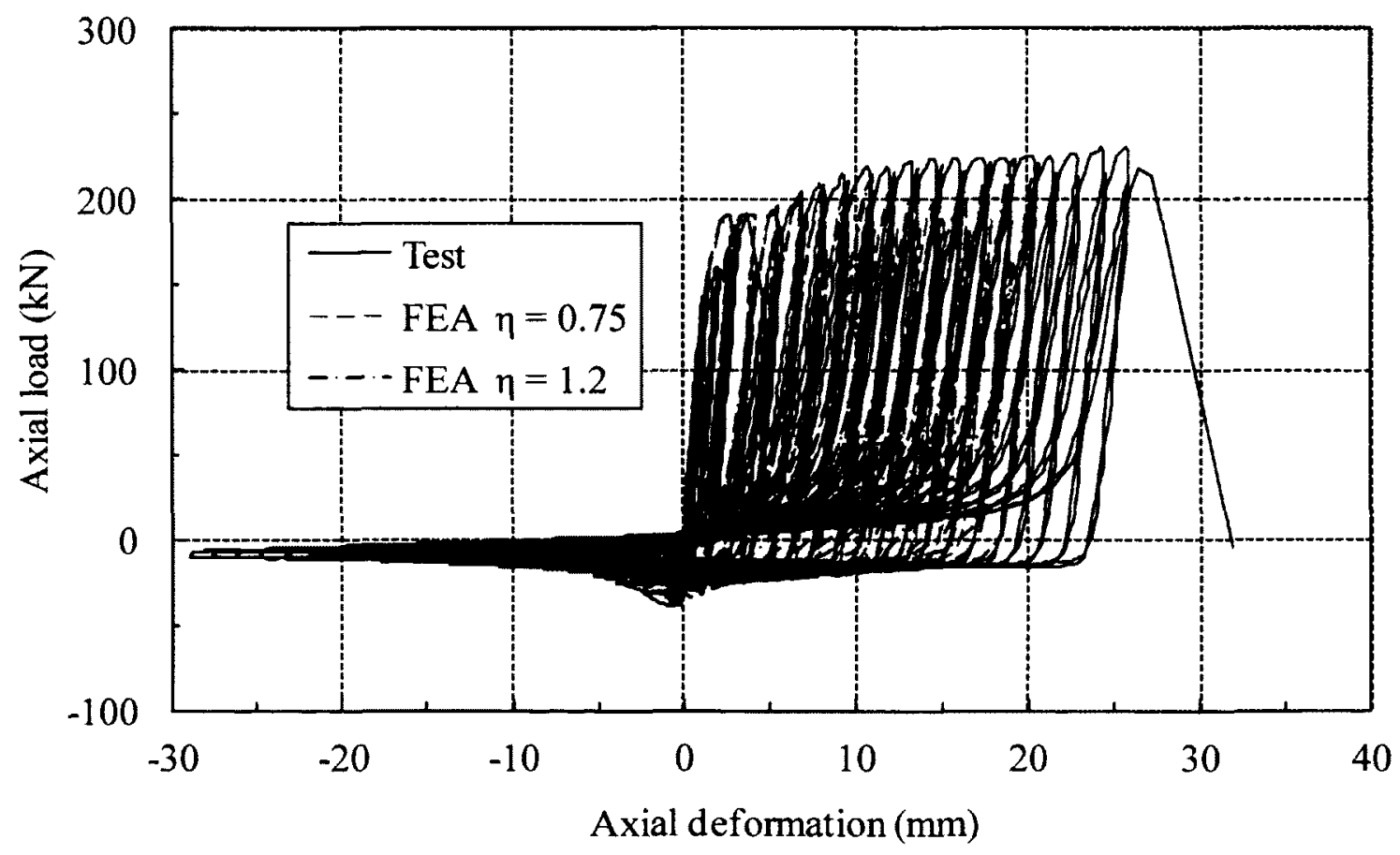

Figure E.6 Test and predicted load versus deformation curves for strap specimen A2078b using the $1 / 2$ model 


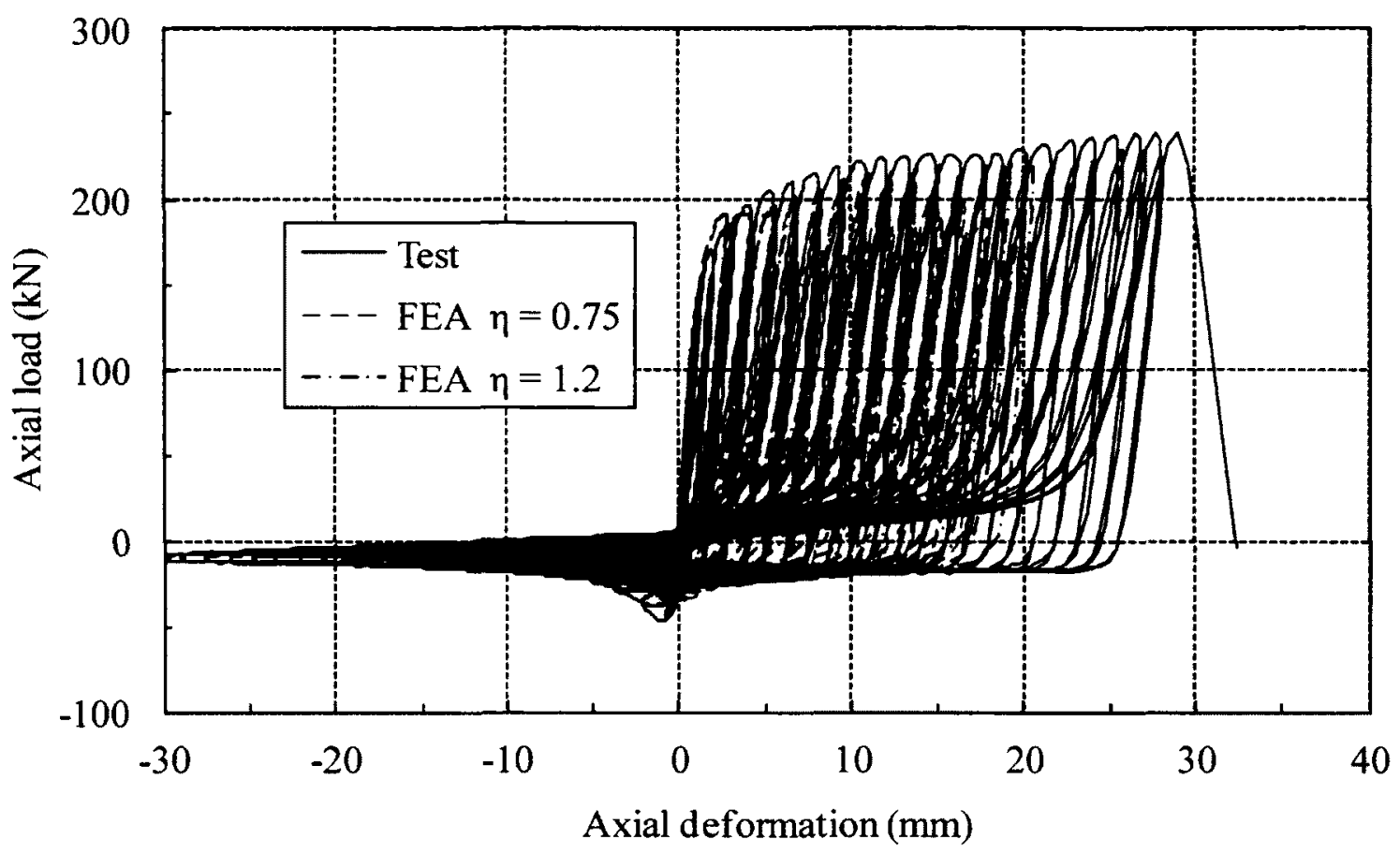

Figure E.7 Test and predicted load versus deformation curves for strap specimen A2078c using the $1 / 2$ model

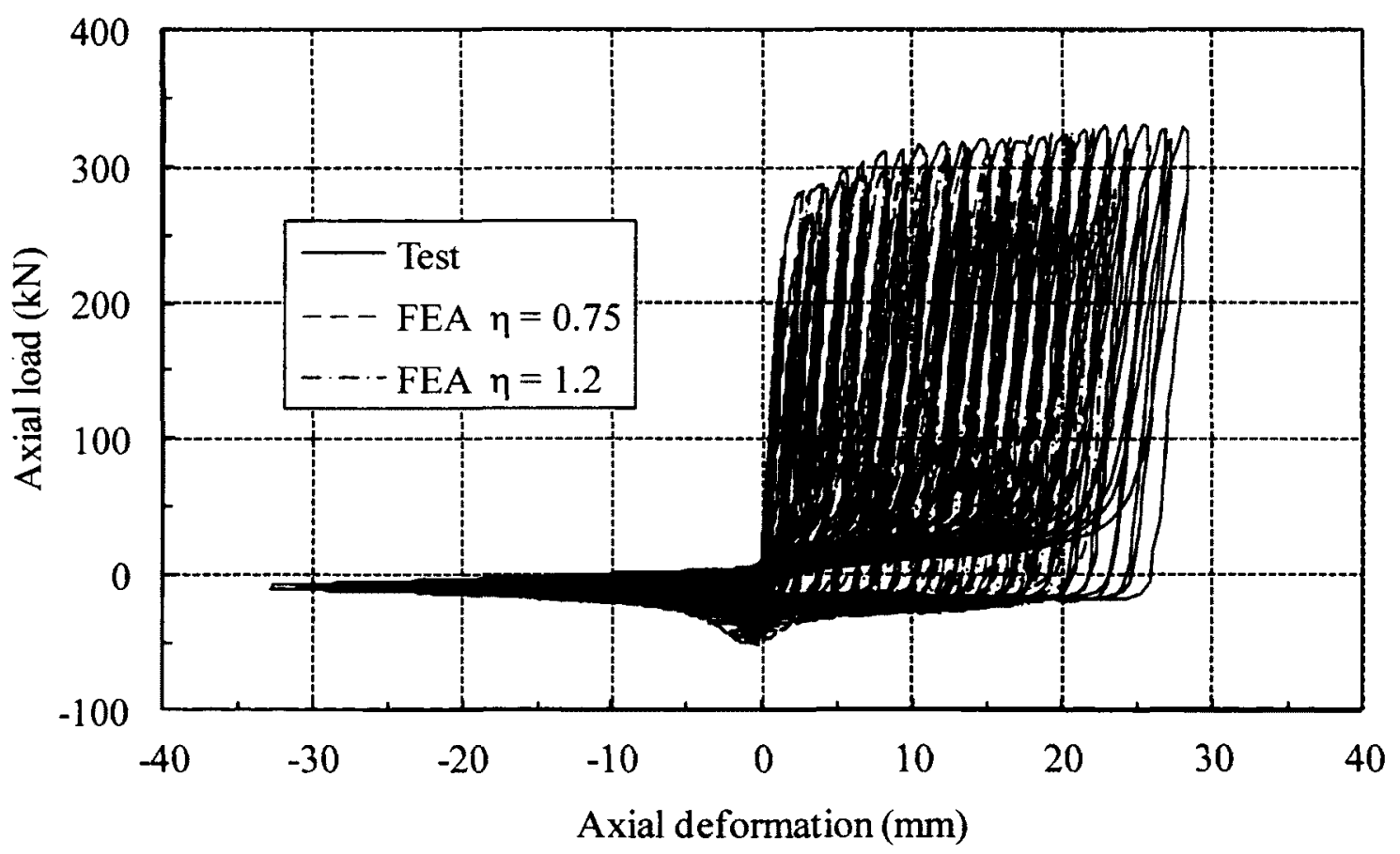

Figure E.8 Test and predicted load versus deformation curves for strap specimen A2108a using the $1 / 2$ model 


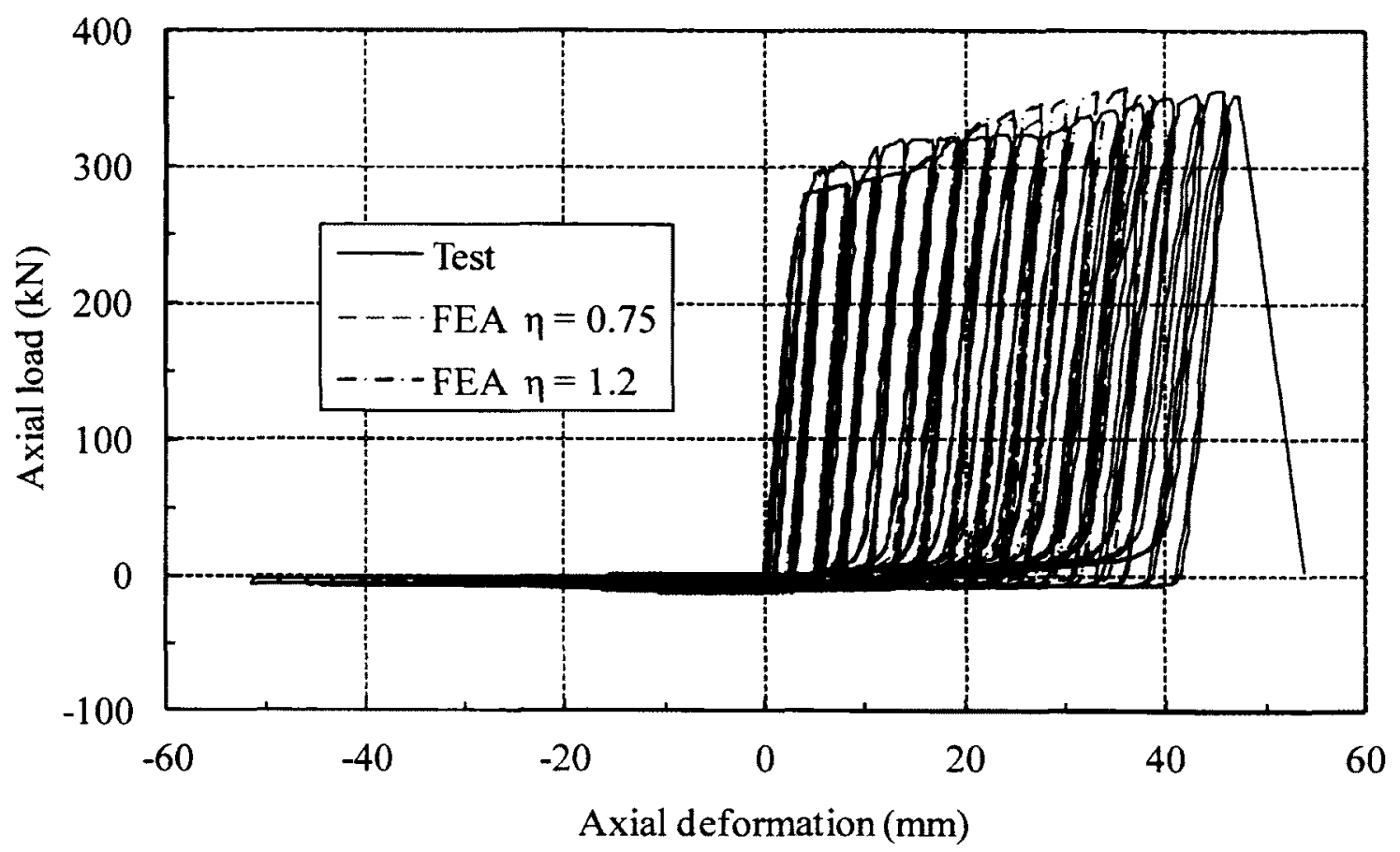

Figure E.9 Test and predicted load versus deformation curves for strap specimen A $4108 \mathrm{~b}$ using the $1 / 2$ model

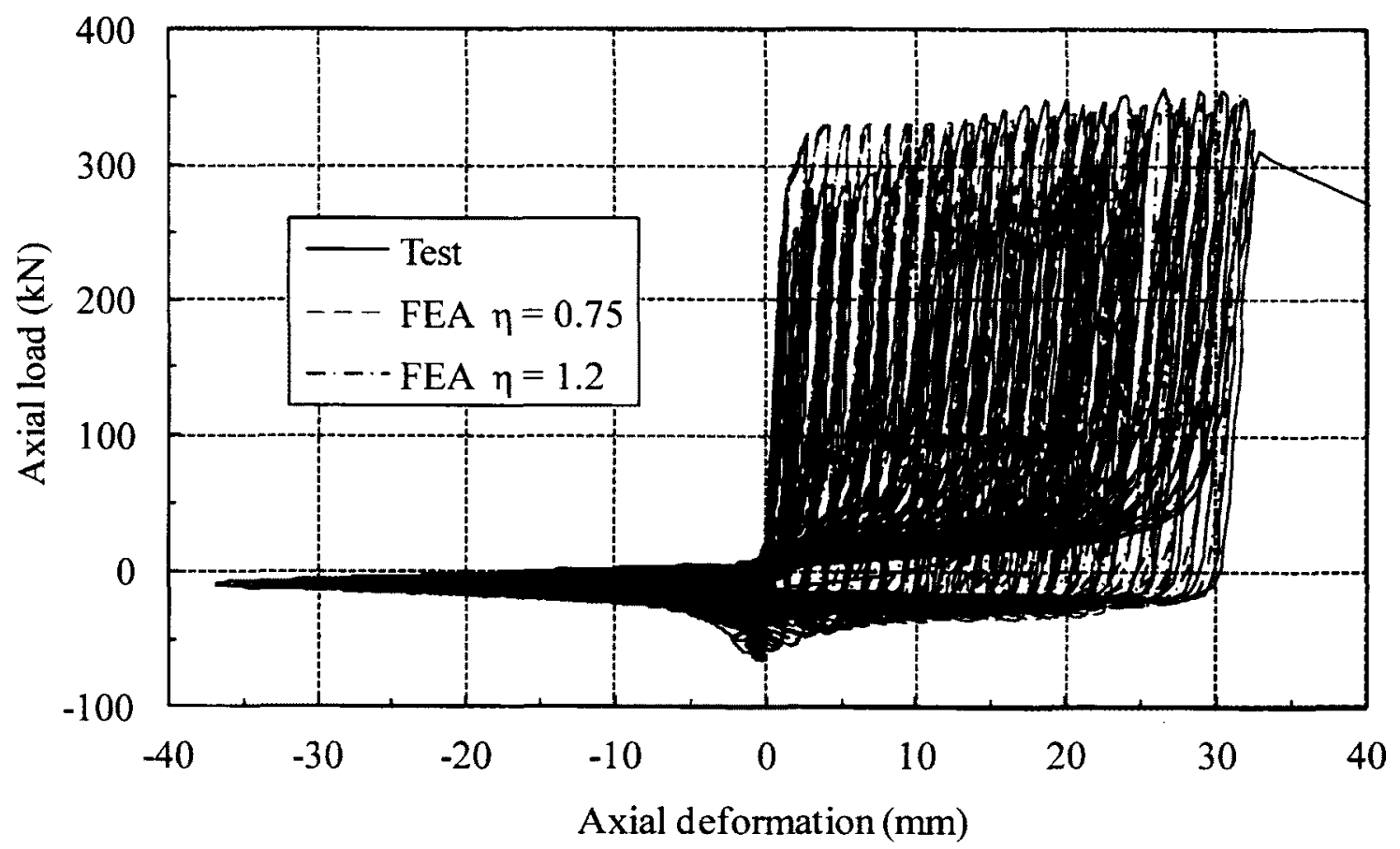

Figure E.10 Test and predicted load versus deformation curves for strap specimen A2128a using the $1 / 2$ model 


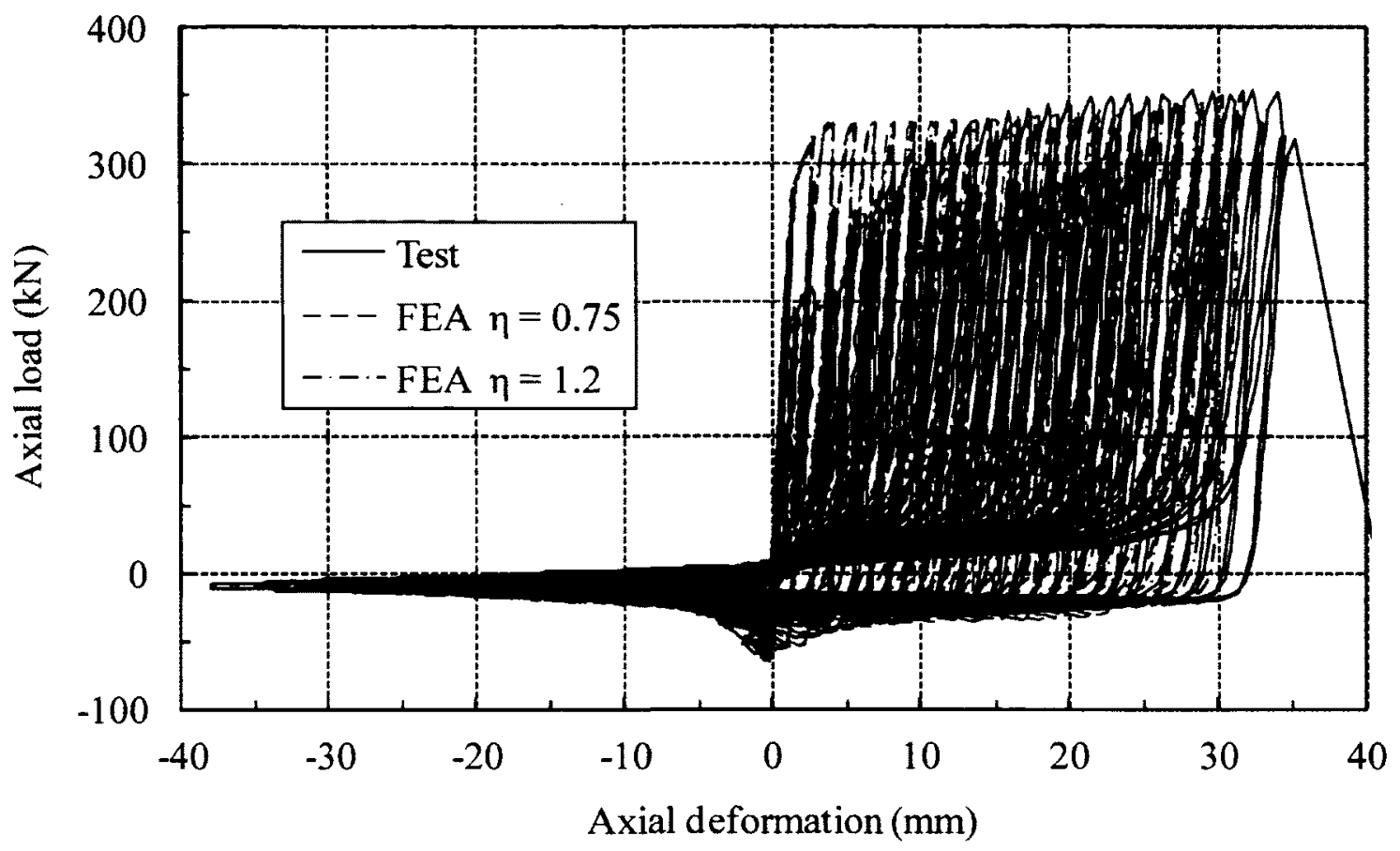

Figure E.11 Test and predicted load versus deformation curves for strap specimen A2128b using the $1 / 2$ model

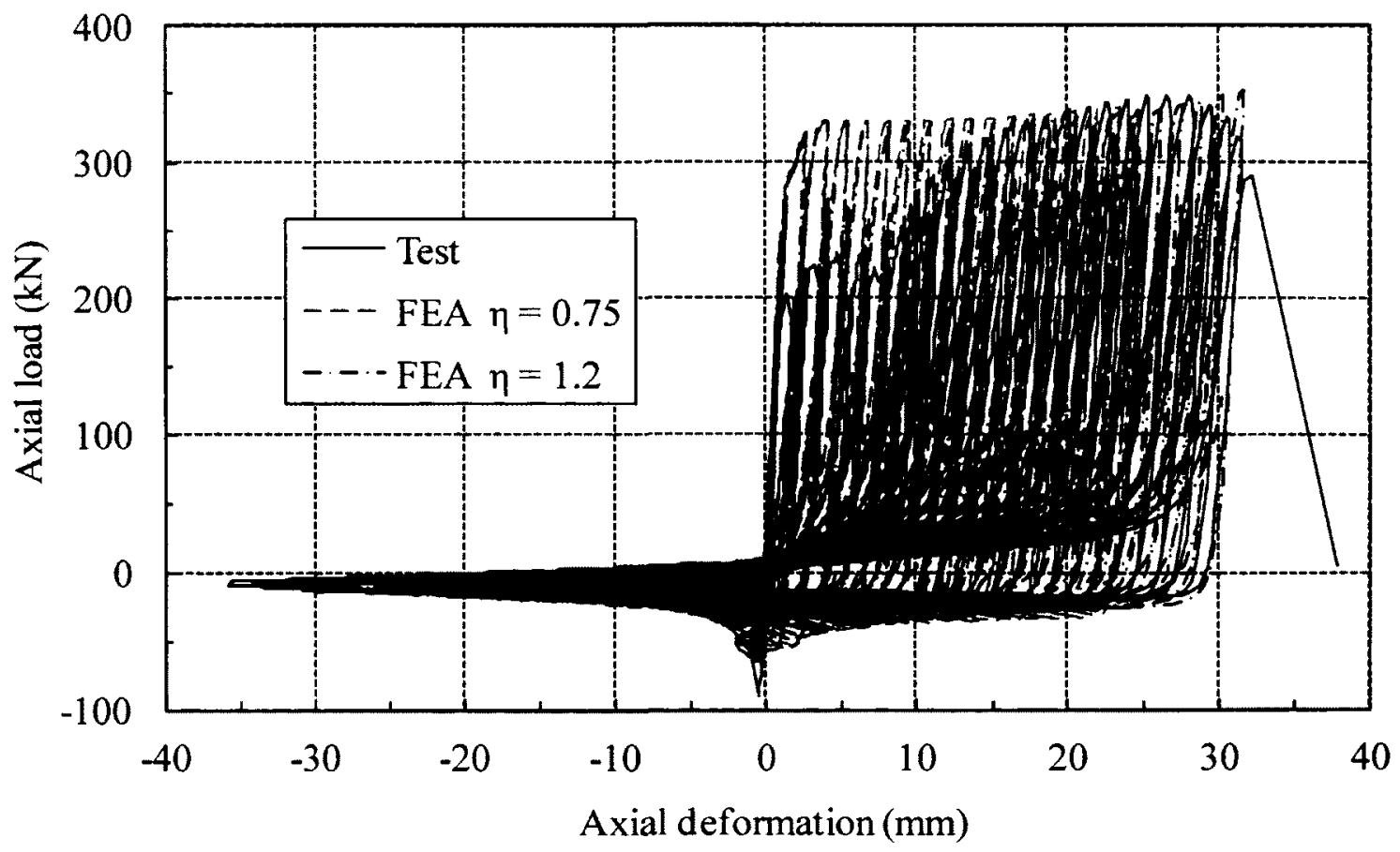

Figure E.12 Test and predicted load versus deformation curves for strap specimen A2128c using the $1 / 2$ model 


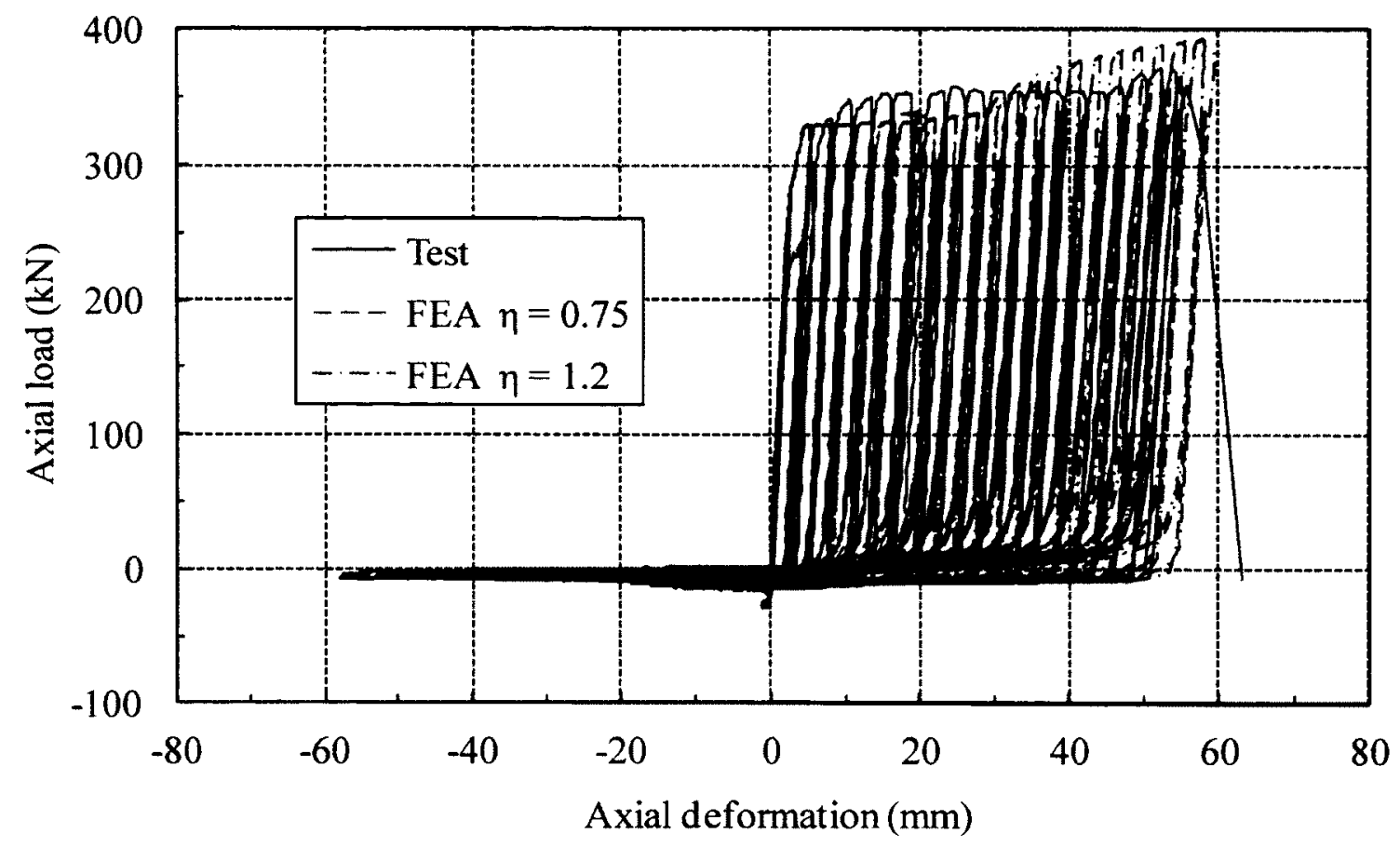

Figure E.13 Test and predicted load versus deformation curves for strap specimen A4128a using the $1 / 2$ model

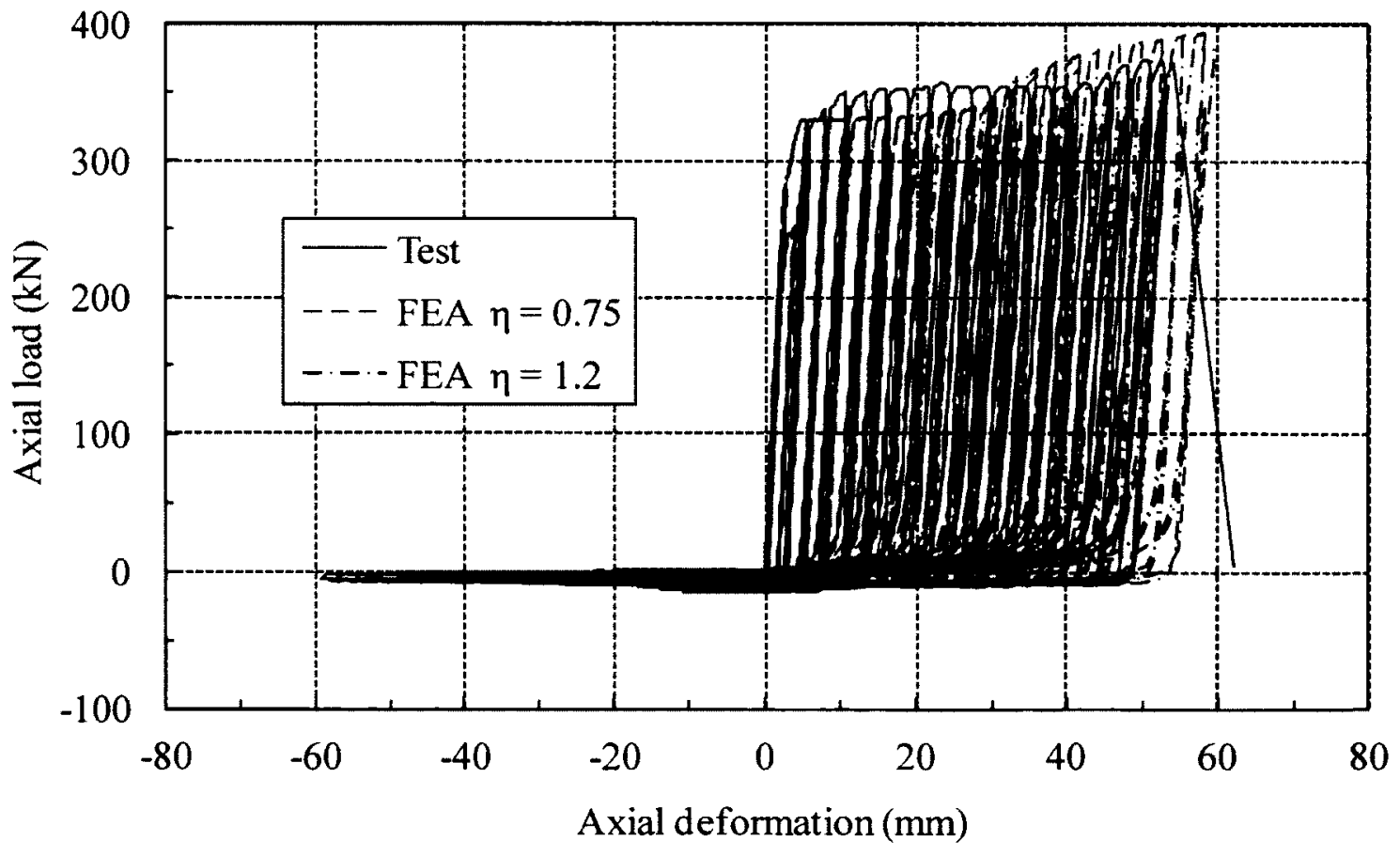

Figure E.14 Test and predicted load versus deformation curves for strap specimen A $4128 \mathrm{~b}$ using the $1 / 2$ model 


\section{REFERENCES}

1. ABAQUS (Simulia 2009). "ABAQUS Version 6.9 Standard User's Manual." Dassault Systèmes Simulia Corporation, Providence, Rhode Island, USA.

2. Aghdam, M. R. (2009). "An Experimental Study on Strap Tension-Only Brace." Master Thesis, Carleton University, Ottawa, Ontario, Canada.

3. Al-Kharat, M. and Rogers (2007). "Inelastic Performance of Cold-Formed Steel Strap Braced Walls." Journal of Construction Steel Research, Vol. 63, No. 4, pp. $460-474$.

4. Armstrong, P. J. and Frederick, C. O. (1966). "A Mathematical Representation of the Multiaxial Bauschinger Effect." CEGB Report, RD/B/N/731, Berkeley Nuclear Laboratories, R\&D Department, California, USA.

5. ASTM (2007). "ASTM A572/A572M, Standard Specification for High-Strength Low-Alloy Columbium-Vanadium Structural Steel." American Society for Testing and Materials, Pennsylvania, USA.

6. ATC (1992). "ATC-24, Guidelines for Cyclic Seismic Testing of Components of Steel Structures." Applied Technology Council, California, USA.

7. Atkins, A. G. (1981). "Possible Explanation for Unexpected Departures in Hydrostatic Tension-Fracture Strain Relations.” Metal Science, Vol. 15, No. 2 , pp. 81-83.

8. Banks-Sills and Budiansky (1982). "On Void Collapse in Viscous Solids." Mechanics of Materials, Vol. 1, No. 3, pp. 209-218. 
9. Barbier,G., Desmorat, R., Sermage, J.-P., Du Tertre, A., Courtin, S., Dehouve, J. and Tchou-Kien, D. (2008). "Mean Stress Effect by Incremental Two Scale Damage Model." The 6th International Conference on Low Cycle Fatigue, Berlin, Germany, pp. 539-544.

10. Basuroychowdhury, I. N. and Voyiadjis, G. Z. (1998). "A Multiaxial Cyclic Plasticity Model for Non-Proportional Loading Cases." International Journal of Plasticity, Vol. 14, No. 9, pp. 855-870.

11. Benzerga, A. A., Besson, J. and Pineau, A. (2004). "Anisotropic Ductile Fracture Part II: Theory." Acta Materialia, Vol. 52, No. 15, pp. 4639-4650.

12. Berman, J. W. and Bruneau, M. (2006). "Proof-of-Concept Testing and Finite Element Modelling of Self-Stabilizing Hybrid Rectangular Links For Eccentrically Braced Frames." Proceedings of the 8th U.S. National Conference on Earthquake Engineering, San Francisco, California, USA.

13. Besson, J. and Guillemer-Neel, C. (2003). "An Extension of the Green and Gurson Models to Kinematic Hardening." Mechanics of Materials, Vol. 35, No. 1-2, pp. 1-18.

14. Bonora, N. (1997). "A Nonlinear CDM Model for Ductile Failure." Engineering Fracture Mechanics, Vol. 58, No. 1/2, pp. 11-28.

15. Bower, A. F. (1989). "Cyclic Hardening Properties of Hard-Drawn Copper and Rail Steel." Journal of the Mechanics and Physics of Solids, Vol. 37, No. 4, pp. $455-470$. 
16. Bridgman, P. W. (1947). "Fracture and Hydrostatic Pressure." Fracturing of Metals, The Twenty-Ninth National Metal Congress and Exposition, American Society for Metals, Chicago, October 18 to 24, pp. 246-261.

17. Brozzo, P., Deluka, B. and Rendina, R. (1972). "A New Method for the Prediction of Formability in Metal Sheets." Proceedings of the 7th Biennial Conference on Sheet metal Forming and Formability, Vol. 169, pp. 41-57.

18. Cedergren, J., Melin, S. and Lidström, P. (2004). "Numerical Modelling of P/M Steel Bars Subjected to Fatigue Loading Using an Extended Gurson Model." European Journal of Mechanics, A/Solids, Vol. 23, No. 6, pp. 899-908.

19. Chaboche, J. L. (1986). "Time-Independent Constitutive Theories for Cyclic Plasticity." International Journal of Plasticity, Vol. 2, No. 2, pp. 149-188.

20. Chen, J. W. (2010). "An Experimental Study of Strain Rate Effects on Mild Steel." Master Thesis, Carleton University, Ottawa, Ontario, Canada.

21. Chinh, N. Q., Horvath, G., Horita, Z. and Langdon, T. G. (2004). "A New Constitutive Relationship for the Homogeneous Deformation of Metals over a Wide Range of Strain.” Acta Materialia, Vol. 52, No. 12, pp. 3555-3563.

22. Chiou, Y. J. and Hsiao, P. A. (2005). "Large Displacement Analysis of Cyclically Loaded Inelastic Structures." Journal of Structural Engineering, Vol. 131, No. 12, pp. $1803-1810$.

23. Chou, C. C. and Wu, C. C. (2006). "Cyclic Performance of Reduced Flange Plate Moment Connections." Proceedings of the 8th U.S. National Conference on Earthquake Engineering, San Francisco, California, USA. 
24. Chun, B. K., Jinn, J. T. and Lee, J. K. (2002). "Modeling the Bauschinger Effect for Sheet Metals, Part I: Theory." International Journal of Plasticity, Vol. 18, No. $5-6$, pp. $571-595$.

25. Chung, K., Lee, M.-G., Kim, D., Kim, C., Wenner, M. L. and Barlat, F. (2005). "Spring-back Evaluation of Automotive Sheets Based on Isotropic-Kinematic Hardening Laws and Non-Quadratic Anisotropic Yield Functions Part I: Theory and Formulation." International Journal of Plasticity, Vol. 21, No. 5, pp. 861-882.

26. CISC (2010). "Handbook of Steel Construction, Tenth Edition." Canadian Institute of Steel Construction, Markham, Ontario, Canada.

27. Cockcroft, M. G., Latham, D. J. (1968). "Ductility and the Workability of Metals." Journal of the Institute of Metals, Vol. 96, No. 2, pp. 33-39.

28. Coffin, L. F. (1954). "A Study of the Effects of Cyclic Thermal Stresses on a Ductile Metal." Transations, ASME, Vol. 76, pp. 931-950.

29. CSA (2004). "CSA G40.20/21, General Requirements for Rolled or Welded Structural Quality Steel." Canadian Standards Association, Mississauga, Ontario, Canada.

30. Dafalias, Y. F. and Popov, E. P. (1975). “A Model of Nonlinearly Hardening Materials for Complex Loading." Acta Mechanica, Vol. 21, pp. 173-192.

31. Dafalias, Y. F. and Popov, E. P. (1976). "Plastic Internal Variables Formalism of Cyclic Plasticity." Journal of Applied Mechanics, Vol. 43, pp. 645-651.

32. Dufailly, J. and Lemaitre, J. (1995). "Modeling Very Low Cycle Fatigue." International Journal of Damage Mechanics, Vol. 4, No. 2, pp. 153-170. 
33. Feigenbaum, H. P. and Dafalias, Y. F. (2007). "Directional Distortional Hardening in Metal Plasticity within Thermodynamics." International Journal of Solids and Structures, Vol. 44, No. 22-23, pp. 7526-7542.

34. Feigenbaum, H. P. and Dafalias, Y. F. (2008). "Simple Model for Directional Distortional Hardening in Metal Plasticity within Thermodynamics." Journal of Engineering Mechanics, Vol. 134, No. 9, pp. 730-738.

35. Francois, M. (2001). "A Plasticity Model with Yield Surface Distortion for Non Proportional Loading." International Journal of Plasticity, Vol. 17, No. 5, pp. 703-717.

36. Freudenthal, A. M. (1950). "The Inelastic Behavior of Engineering Materials and Structures." John Wiley \& Sons, New York.

37. Geng, L. M. and Wagoner, R. H. (2002). "Role of Plastic Anisotropic and Its Evolution on Springback.” International Journal of Mechanical Sciences, Vol. 44, No. 1, pp. 123-148.

38. Gillemot, L. F. (1976). "Criterion of Crack Initiation and Spreading." Engineering Fracture Mechanics, Vol. 8, No. 1, pp. 239-253.

39. Goijaerts, A. M., Govaert, L. E. and Baaijens, F. P. T. (2000). "Prediction of Ductile Fracture in Metal Blanking." Journal of Manufacturing Science and Engineering, Vol. 122, No. 3, pp. 476-483.

40. Goto, Y., Wang, Q. Y. and Obata, M. (1998). "FEM Analysis for Hysteretic Behavior of Thin-Walled Columns." Journal of Structural Engineering, Vol. 124, No. 11, pp. 1290-1301. 
41. Gurson, A. L. (1977a). "Continuum Theory of Ductile Rupture by Void Nucleation and Growth: Part I - Yield Criteria and Flow Rules for Porous Ductile Media." Journal of Engineering Materials and Technology, Vol. 99, pp. 2-15.

42. Gurson, A. L. (1977b). "Porous Rigid-Plastic Materials Containing Rigid Inclusions - Yield Function, Plastic Potential, and Void Nucleation." Proceeding of the 4th International Conference on Fracture, ICF4, International Congress on Fracture, Waterloo, Canada, Vol. 2A, pp. 357-364.

43. Han, H. N. and Kim, K.-H. (2003). "A Ductile Fracture Criterion in Sheet Metal Forming Process." Journal of Materials Processing Technology, Vol. 142, No. 1, pp. 231-238.

44. Hancock, J. W. and Brown, D. K. (1983). "On the Role of Strain and Stress State in Ductile Failure." Journal of the Mechanics and Physics of Solids, Vol. 31, No. 1, pp. $1-24$

45. Hollomon, J. H. (1945). "Tensile Deformation." American Institute of Mining and Metallurgical Engineers - Transactions, Vol. 162, pp. 268-289.

46. Hori, M. and Nemat-Nasser, S. (1988). "Mechanics of Void Growth and Void Collapse in Crystals." Mechanics of Materials, Vol. 7, No. 1, pp. 1-13.

47. Huang, Y. L. and Mahin, S. A. (2008). "Evaluation of Steel Structure Deterioration with Cyclic Damaged Plasticity." The 14th World Conference on Earthquake Engineering, Beijing, China.

48. Itoh, T. and Ozaki, T. (2008). "Multiaxial Low Cycle Fatigue Life of Notch Specimens for SUS316 Stainless and SGV410 Steels under Non-Proportional 
Loading." The 6th International Conference on Low Cycle Fatigue, Berlin, Germany, pp. 59-64.

49. Jiang, L. Z., Goto, Y. and Obata, M. (2002). "Hysteretic Modeling of ThinWalled Circular Steel Columns under Biaxial Bending." Journal of Structural Engineering, Vol. 128, No. 3, pp. 319-327.

50. Johnson, G. R. and Cook, W. H. (1985). "Fracture Characteristics of Three Metals Subjected to Various Strains, Strain Rates, Temperatures and Pressures." Engineering Fracture Mechanics, Vol. 21, No. 1, pp. 31-48.

51. Kanvinde, A. M. and Deierlein, G. G. (2004). "Micromechanical Simulation of Earthquake-Induced Fracture in Steel Structures." Technical Report 145, John A. Blume Earthquake Engineering Center, Stanford University, California, USA.

52. Kanvinde, A. M. and Deierlein, G. G. (2007). "Cyclic Void Growth Model to Assess Ductile Fracture Initiation in Structural Steels due to Ultra Low Cycle Fatigue." Journal of Engineering Mechanics, Vol. 133, No. 6, pp. 701-712.

53. Khoo, H. (2010). Personal Correspondence, Carleton University, Ottawa, Ontario, Canada.

54. Krieg, R. D. (1975). "A Practical Two Surface Plasticity Theory." Journal of Applied Mechanics, Vol. 42, No. 3, pp. 641-646.

55. Lee, M.-G., Kim, D., Kim, C., Wenner, M. L., Wagoner, R. H. and Chung, K. (2007). “A Practical Two-Surface Plasticity Model and Its Application to SpringBack Prediction.” International Journal of Plasticity, Vol. 23, No. 7, pp. 11891212. 
for Ductile materials." NRL Memorandum Report No. 5936, Naval Research Laboratory, Washington, D. C., USA.

65. Mear, M. E. and Hutchinson J. W. (1985). "Influence of Yield Surface Curvature on Flow Localization in Dilatant Plasticity." Mechanics of Material, Vol. 4, No. 3-4, pp. 395-407.

66. McClintock, F. A., Argon, A. S. (1966). Mechanical Behavior of Materials, Addison-Wesley Publishing Company.

67. McClintock, F. A., Kaplan, S. M. and Berg, C. A. (1966). "Ductile Fracture by Hole Growth in Shear Bands." International Journal of Fracture, Vol. 2, No. 4, pp. 614-627.

68. McClintock, F. A. (1968). "A Criterion for Ductile Fracture by the Growth of Holes." Journal of Applied Mechanics, Vol. 35, No. 2, pp. 363-371.

69. Minagawa, M., Nishiwaki, T. and Masuda, N. (1987). "Modelling Cyclic Plasticity of Structural Steels." Japan Society of Civil Engineers, Structural Engineering / Earthquake Engineering, Vol. 4, No. 2, pp. 361-370.

70. Mizuno, E., Shen, C., Tanaka, Y. and Usami, T. (1992). "A Uniaxial StressStrain Model for Structural Steels under Cyclic Loading." Stability and Ductility of Steel Structures under Cyclic Loading, CRC Press, Boca Raton, Florida, USA.

71. Montáns, F.-J. (2000). "Bounding Surface Plasticity Model with Extended Masing Behavior." Computer Methods in Applied Mechanics and Engineering, Vol. 182, No. 1-2, pp. 135-162.

72. Mroz, Z. (1967). "On the Description of Anisotropic Workhardening." Journal of the Mechanics and Physics of Solids, Vol. 15, pp. 163-175. 
73. Naqvi, Z. and Khoo, H. (2004). "Constitutive Properties of Weld Metal and HeatAffected Zone at A Butt Weld." Proceedings of the 5th Structural Specialty Conference of the Canadian Society for Civil Engineering, Saskatoon, Saskatchewan, Canada, ST-150.

74. Naqvi, Z. and Khoo, H. (2007). "Modelling of Weld Metal and Heat-Affected Zone." CSCE 2007 Annual General Meeting \& Conference, Yellowknife, Northwest Territories, Canada.

75. Norris, D. M., Reaugh, J. E., Moran, B. and Quinones, D. F. (1978). "A PlasticStrain, Mean-Stress Criterion for Ductile Fracture." Journal of Engineering Materials and Technology, Vol. 100, No. 3, pp. 279-286.

76. NRCC (2010). "National Building Code of Canada 2010." National Research Council of Canada, Ottawa, Ontario, Canada.

77. Ohno, N. and Wang, J.-D. (1993a). "Kinematic Hardening Rules with Critical State of Dynamic Recovery, Part I: Formulation and Basic Features for Ratchetting Behavior.” International Journal of Plasticity, Vol. 9, No. 3, pp. 375 390.

78. Ohno, N. and Wang, J.-D. (1993b). "Kinematic Hardening Rules with Critical State of Dynamic Recovery, Part II: Application to Experiments of Ratchetting Behavior.” International Journal of Plasticity, Vol. 9, No. 3, pp. 391-403.

79. Oyane, M., Sato, T., Okimoto, K. and Shima, S. (1980). "Criteria for Ductile Fracture and Their Applications." Journal of Mechanical Working Technology, Vol. 4, No. 1, pp. 65-81. 
80. Pardoen, T. and Hutchinson, J. W. (2000). "An Extended Model for Void Growth and Coalescence." Journal of Mechanics and Physics of Solids, Vol. 48, No. 12, pp. $2467-2512$.

81. Petersson, H. and Popov, E. P. (1977). "Constitutive Relations for Generalized Loadings." Journal of the Engineering Mechanics Division, Vol. 103, No. 4, pp. $611-627$

82. Pirondi, A. and Bonora, N. (2003). "Modeling Ductile Damage under Fully Reversed Cycling." Computational Materials Science, Vol. 26, pp. 129-141.

83. Prager, W. and Providence, R. 1. (1956). "A New Method of Analyzing Stresses and Strains in Work-Hardening Plastic Solids." ASME Journal of Applied Mechanics, Vol. 23, pp. 493-496.

84. Rice, J. R. and Tracey, D. M. (1969). "On the Ductile Enlargement of Voids in Triaxial Stress Fields." Journal of the Mechanics and Physics of Solids, Vol. 17, No. 3, pp. 201-217.

85. Ristinmaa, M. (1997). "Void Growth in Cyclic Loaded Porous Plastic Solid." Mechanics of Materials, Vol. 26, No. 4, pp. 227-245.

86. Shen, C., Mamaghani, I. H. P., Mizuno, E. and Usami, T. (1995). "Cyclic Behavior of Structural Steels. II: Theory." Journal of Engineering Mechanics, Vol. 121, No. 11, pp. 1165-1172.

87. Shi, Y. W., Barnby, J. T. and Nadkarni, A. S. (1991). "Void Growth at Ductile Crack Initiation of a Structural Steel.” Engineering Fracture Mechanics, Vol. 39, No. 1, pp. 37-44. 
88. Steglich, D., Pirondi, A., Bonora, N. and Brocks, W. (2005). "Micromechanical Modelling of Cyclic Plasticity Incorporating Damage." International Journal of Solids and Structures, Vol. 42, No. 2, pp. 337-351.

89. Sun, D.-Z., Siegele, D., Voss, B. and Schmitt, W. (1989). "Application of Local Damage Models to the Numerical Analysis of Ductile Rupture." Fatigue and Fracture of Engineering Materials and Structures, Vol. 12, No. 3, pp. 201-212.

90. Susmel, L., Meneghetti, G. and Atzori, B. (2008). "On the Use of the Modified Manson-Coffin Curve Method to Estimate Low-Cycle Fatigue Lifetime of Notched Components Subjected to Multiaxial Cyclic Loading." The 6th International Conference on Low Cycle Fatigue, Berlin, Germany, pp. 119-124.

91. Tai, W. H. and Yang, B. X. (1987). "A New Damage Mechanics Criterion for Ductile Fracture." Engineering Fracture Mechanics, Vol. 27, No. 4, pp. 371-378.

92. Tremblay, R., Robert, N. and Filiatrault A. (1997). "Tension-Only Bracing: A Viable Earthquake Resistant System for Low-Rise Steel Buildings?" Proceedings of the 5th International Colloquium on Stability and Ductility of Steel Structures, Nagoya, Japan, Vol. 2, pp. 1163-1170.

93. Tseng, N. T. and Lee, G. C. (1983). "Simple Plasticity Model of Two-Surface Type." Journal of Engineering Mechanics, Vol. 109, No. 3, pp. 795-810.

94. Tvergaard, V. (1982). "Ductile Fracture by Cavity Nucleation between Larger Voids." Journal of the Mechanics and Physics of Solids, Vol. 30, No. 4, pp. 265286.

95. Tvergaard, V. and Needleman, A. (1984). "Analysis of the Cup-Cone Fracture in a Round Tensile Bar." Acta Metallurgica, Vol. 32, No. 1, pp. 157-169. 
96. Voce, E. (1955). “A Practical Strain-Hardening Function.” Metallurgia, Vol. 51, pp. 219-226.

97. Yang, T. and Itoh, T. (2008). "Material Dependence of Multiaxial Low Cycle Fatigue Life under Non-Proportional Loading." The 6th International Conference on Low Cycle Fatigue, Berlin, Germany, pp. 41-46.

98. Ziegler, H. (1959). "A Modification of Prager's Hardening Rule." Quarterly of Applied Mathematics, Vol. 17, pp. 55-65.

99. Zhao, R. G. (2005). "A Study on Slotted Square and Rectangular Hollow Structural Section Connections." Master Thesis, Carleton University, Ottawa, Ontario, Canada. 